A UNITED STATES DEPARTMENT OF COMMERCE PUBLICATION

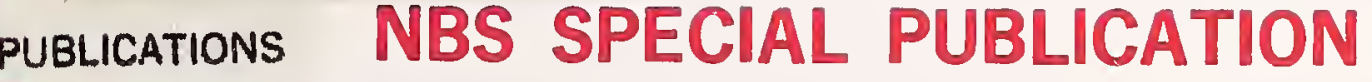

REFERENCE

\title{
Photonuclear Reaction Data, 1973
}

U.S. DEPARTMENT

OF COMMERCE

National

rau

QC of

100 ds

.057

no. 380

1973 
The National Bureau of Standards ${ }^{1}$ was established by an act of Congress March 3 , 1901. The Bureau's overall goal is to strengthen and advance the Nation's science and technology and facilitate their effective application for public benefit. To this end, the Bureau conducts research and provides: (1) a basis for the Nation's physical measurement system, (2) scientific and technological services for industry and government, (3) a technical basis for equity in trade, and (4) technical services to promote public safety. The Bureau consists of the Institute for Basic Standards, the Institute for Materials Research, the Institute for Applied Technology, the Center for Computer Sciences and Technology, and the Office for Information Programs.

THE INSTITUTE FOR BASIC STANDARDS provides the central basis within the United States of a complete and consistent system of physical measurement; coordinates that system with measurement systems of other nations; and furnishes essential services leading to accurate and uniform physical measurements throughout the Nation's scientific community, industry, and commerce. The Institute consists of a Center for Radiation Research, an Office of Measurement Services and the following divisions:

Applied Mathematics - Electricity - Mechanics - Heat - Optical Physics Linac Radiation ${ }^{2}$ - Nuclear Radiation ${ }^{2}$ - Applied Radiation ${ }^{2}$ — Quantum Electronics ${ }^{3}$ - Electromagnetics ${ }^{3}$ - Time and Frequency ${ }^{3}$ — Laboratory Astrophysics : - Cryogenics ${ }^{3}$.

THE INSTITUTE FOR MATERIALS RESEARCH conducts materials research leading to improved methods of measurement, standards, and data on the properties of well-characterized materials needed by industry, commerce, educational institutions, and Government; provides advisory and research services to other Government agencies; and develops, produces, and distributes standard reference materials. The Institute consists of the Office of Standard Reference Materials and the following divisions:

Analytical Chemistry-Polymers-Metallurgy-Inorganic Materials-Reactor Radiation-Physical Chemistry.

THE INSTITUTE FOR APPLIED TECHNOLOGY provides technical services to promote the use of available technology and to facilitate technological innovation in industry and Government; cooperates with public and private organizations leading to the development of technological standards (including mandatory safety standards), codes and methods of test; and provides technical advice and services to Government agencies upon request. The Institute also monitors NBS engineering standards activities and provides liaison between NBS and national and international engineering standards bodies. The Institute consists of a Center for Building Technology and the following divisions and offices:

Engineering and Product Standards-Weights and Measures-Invention and Innovation-Product Evaluation Technology-Electronic Technology-Technical Analysis-Measurement Engineering-Building Standards and Code Services $^{4}$ _- Housing Technology ${ }^{4}$ - Federal Building Technology ${ }^{4}$-Structures, Materials and Life Safety ${ }^{4}$-Building Environment ${ }^{4}$ - Technical Evaluation and Application ${ }^{4}$ - Fire Technology.

THE INSTITUTE FOR COMPUTER SCIENCES AND TECHNOLOGY conducts research and provides technical services designed to aid Government agencies in improving cost effectiveness in the conduct of their programs through the selection, acquisition, and effective utilization of automatic data processing equipment; and serves as the principal focus within the executive branch for the development of Federal standards for automatic data processing equipment, techniques, and computer languages. The Center consists of the following offices and divisions:

Information Processing Standards-Computer Information-Computer Services -Systems Development-Information Processing Technology.

THE OFFICE FOR INFORMATION PROGRAMS promotes optimum dissemination and accessibility of scientific information generated within NBS and other agencies of the Federal Government; promotes the development of the National Standard Reference Data System and a system of information analysis centers dealing with the broader aspects of the National Measurement System; provides appropriate services to ensure that the NBS staff has optimum accessibility to the scientific information of the world, and directs the public information activities of the Bureau. The Office consists of the following organizational units:

Office of Standard Reference Data-Office of Technical Information and Publications-Library-Office of International Relations.

\footnotetext{
1 Headquarters and Laboratories at Gaithersburg, Maryland, unless otherwise noted; mailing address Washington, D.C. 20234.

2 Part of the Center for Radiation Research.

3 Located at Boulder, Colorado 80302.

4 Part of the Center for Building Technology.
} 
E. G. Fuller, H. M. Gerstenberg,

H. Vander Molen, and T. C. Dunn

Center for Radiation Research

Institute for Basic Standards

National Bureau of Standards

Washington, D.C. 20234

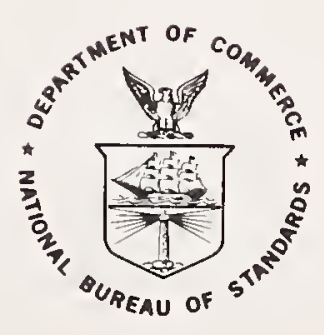

U.S. DEPARTMENT OF COMMERCE, Frederick B. Dent, Secretary NATIONAL BUREAU OF STANDARDS, Richard W. Roberts, Director Issued March 1973 
Library of Congress Catalog Card Number: 72-600057

\section{National Bureau of Standards Special Publication 380}

Nat. Bur. Stand. (U.S.), Spec. Publ. 380,131 pages (Mar. 1973)

CODEN: XNBSAV

(This publication supersedes NBS Miscellaneous Publication 277 and NBS Special Publication 322 which also embraced Supplement 1 to Misc Publ. 277. All information contained in these publications has been combined and merged with new data to form the Photonuclear Data Index and Bibliography contained in this publication.) 


\section{Foreword}

The National Standard Reference Data System was established in 1963 for the purpose of promoting the critical evaluation and dissemination of numerical data of the physical sciences. The program is coordinated by the Office of Standard Reference Data of the National Bureau of Standards but involves the efforts of many groups in universities, government laboratories, and private industry. The primary aim of the program is to provide compilations of critically evaluated physical and chemical property data. These tables are published in the Journal of Physical and Chemical Reference Data, in the NSRDS-NBS series of the National Bureau of Standards, and through other appropriate channels.

The task of critical evaluation is carried out in various data centers, each with a well-defined technical scope. A necessary preliminary step to the critical evaluation process is the retrieval from the world scientific literature of all papers falling within the scope of the center. Each center, therefore, builds up a comprehensive well-indexed bibliographical file which forms the base for the evaluation task. Bibliographies derived from these files are published when they appear to be of value to research workers and others interested in the particular technical area.

Further information on NSRDS and the publications which form the primary output of the program may be obtained by writing to the Office of Standard Reference Data, National Bureau of Standards, Washington, DC 20234.

David R. Lide, Jr., Chief

Office of Standard Reference Data 
1. Introduction - . . . . . . . . . . . . . . . . .

2. Gross Features of the Giant Resonance - - . - . - . - . - -

2.1. General Description - - . - . - . . . - . - .

2.2. Experimental Data - - - - - - - - - - - - - -

2.3. Estimating Yield - - - - - - - - - - - - - -

3. Description of the Photonuclear Data Index and Bibliography - - - - - -

3.1. Introduction - - - - - - - - . - . - . - .

3.2. Scope of the Data Index - - - - - - - - - - - - -

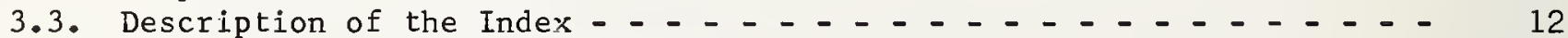

3.4. Description of Column Headings - - - - - - - - - - - - 13

4. Pho ¿onuclear Data Index (1955-1972) _ . . . . . . . . . . . 15

5. Photorluclear Reaction Data Bibliography (1955-1972) - - - - - - - - - 55

6. Definitions of Abbreviations and Symbols _ _ . - . - . - . - - 122

6.1. Symbols Used to Define Incoming Beam and Reaction Products - - - - - 122

6.2. Symbols and Abbreviations - - - - - - . - . - - - - 122

7. References - . . . . . . . . . . . . . . . . . 
E. G. Fuller, H. M. Gerstenberg, H. Vander Molen, ${ }^{*}$ and T. Co Dunn

A brief summary is given of the available data on the gross features of the photonuclear giant resonance. Data are presented in tabular form for all nuclei where measurements have been made. In addition, a comprehensive, annotated data index and bibliography are given which cover experimental data for the field of photonuclear reactions published in scientific and technical journals in the period from 1955 through 1972. Organized by element and isotope, each entry in the index is for a specific reaction reported in a given reference. Information is given on the type of measurement, excitation energies studied, source type and energies, detector type and angular ranges covered for each reaction entry.

Key words: Bibliography; data index; data summary; elements; isotopes; nuclear physics; photonuclear giant resonance; photonuclear reaction.

\section{Introduction}

The field of Photonuclear Reactions is concerned with the interaction of electromagnetic radiation with nuclei. The interactions can take place through the intermediary of either "real" or "virtual" photons. The field was first opened for study forty years ago when Chadwick and Goldhaber $[1]^{1}$ observed protons in an ionization chamber containing deuterium gas that was bombarded with the $2.61 \mathrm{MeV} \gamma$-rays from ${ }^{20} \mathrm{TI}$. This most elementary photonuclear reaction is still the subject of considerable study both experimentally as well as theoretically.

During the first ten years of its history, the study of the field was limited to the use of real photons obtained from radioactive sources or from the $\gamma$-decay of highly excited states formed in nuclear reactions. The range of photon energies at which studies could be made was of necessity very limited. Only after the development of the betatron [2] was it possible to study the photonuclear interaction as a function of photon energy. In the late forties, Baldwin and Klaiber [3] were able to obtain the first evidence for the existence of the photonuclear giant resonance in spite of the fact that the bremsstrahlung beam produced by the betatron was a continuous energy spectrum of photons with only a well-defined upper energy limit. This phenomenon, i.e., the concentration of most of the strength of the photonuclear reaction over a relatively narrow energy band, has been studied extensively over the last 25 years. During the decade of the fifties, improvements in accelerator technology, data analysis techniques, and radiation detectors resulted in a number of significant measurements being made of the cross sections for total photon absorption, quasi-elastic photon scattering, and specific reaction yields. These experiments established the systematics of the photonuclear interaction and showed the dependence of the general features of the giant resonance on nuclear structure, e.g•, the ground-state deformation and the tensor polarizability of the nucleus. Further developments in accelerator and detector technologies in the decade of the sixties provided data that confirmed the general features indicated by the earlier bremsstrahlung data and also permitted measurements to be made in greater detail and with higher precision. To a large extent, the improved data resulted from the development of "quasi-monoenergetic" photon beams using the annihilation-in-flight of positrons generated by electron beams from linear accelerators [4]. Up through the sixties the great majority of data obtained on the photonuclear interaction was "one-dimensional", i.e., a particular reaction was studied as a

\footnotetext{
*A Nuclear Information Research Associate (NIRA) at the National Bureau of Standards.

I Figures in brackets indicate the literature references at the end of this publication.
} 
function of excitation energy only. It had long been realized that studies of inelastic electron scattering could lead to "two-dimensional" data [5]. These experiments could give information not only as a function of excitation energy but also for a fixed excitation energy, as a function of the momentum transferred to the nucleus, $i . e .$, the wavelength of the probe used to study the interaction could be made arbitrarily shorter than that associated with the energy transfer. Only during the last few years have developments in acce1erator facilities, detection systems, and data analysis techniques permitted reliable data of this type to be obtained. Measurements in this area should have a considerable impact on the field of photonuclear reactions during the decade of the seventies.

The objective of this report is twofold. First, to present a brief summary of what is known about the gross features of the giant resonance in such a form that the data may be useful both for individuals interested in the basic interaction as well as those interested in obtaining the yield of a particular reaction under specified conditions; second, to present a cumulative, comprehensive, annotated index and bibliography covering the data on photonuclear reactions that have been published in the world's scientific and technical literature in the period from 1955 through 1972. The report is organized as follows: Section 2 discusses the gross features of the giant resonance and presents data in both graphical as well as tabular form; Section 3 gives a description of the annotated index and bibliography; Section 4 consists of the data index; Section 5, the bibliography; and Section 6 lists the abbreviations used in the various tables and their definitions. the final section, 7 , is a reference list to citations made in the textual parts of this report which are not included in the bibliography given in Section 5.

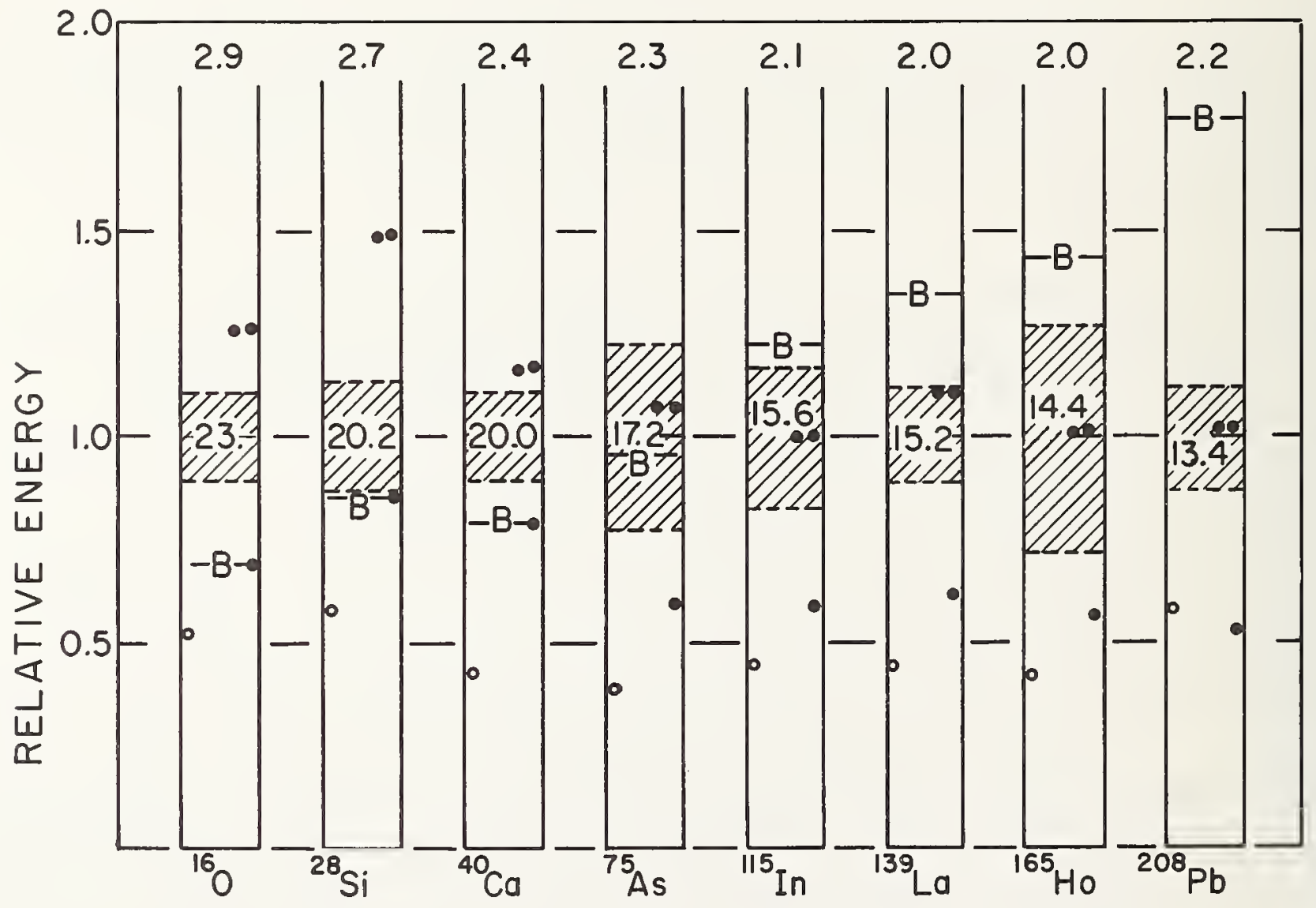

Figure 1. Giant resonance summary. The energy scale for each nucleus has been normalized so that the giant resonance is at one. The width of the giant resonance on this scale is indicated by the shaded regions. Separation energies are indicated by circles, open for protons and closed for neutrons. See text for complete description. 


\section{Gross Features of the Giant Resonance}

\subsection{General Description}

The giant resonance of the photonuclear interaction is an empirical phenomenon exhibited by all nuclei. It is the large peak seen in the plot of the nuclear photon absorption cross section as a function of photon energy. The area under this peak is always found to be approximately that given by the dipole sum-rule result [6]:

$$
\int_{0}^{\infty} \sigma(E) d E=\frac{2 \pi^{2} e^{2} \hbar}{M c} \frac{N Z}{A}=0.06 \mathrm{NZ} / \mathrm{A} \text { (MeV-barns) }
$$

Figure 1 gives a rough "bird's-eye" picture of the giant resonance for nuclei ranging from oxygen to lead. In this figure the energy scale for each nucleus has been normalized so that the mean energy for photon absorption below $30 \mathrm{MeV}$ has been set equal to one. The actual mean energies for the specific nuclei are given in $\mathrm{MeV}$ by the figures in the center of the shaded region indicated for each nucleus. These shaded regions indicate the extent of the giant resonance, i.e., they indicate roughly the region between the half maximum points on the absorption cross section curve. Note that in this figure the overall widths of the giant resonance vary by about a factor of two. The narrowest resonances are for those nuclei with closed shells while the broadest are for those (e.g., $\left.{ }^{165} \mathrm{Ho}\right)$ having large permanent deformations. The overall extent of the giant resonance for the deformed nuclei is determined to a large extent by the splitting of the giant resonance resulting from the deformation. In this respect, the large width indicated for ${ }^{75}$ As is interesting in that it can probably be associated with a dynamic deformation of this nucleus resulting from the zero-point vibrations of the nuclear surface.

The black dots and open circles shown in Figure $l$ represent, respectively, the reutron and proton separation energies. The lines designated by the letter " $B$ " in each diagram indicate the top of the coulomb barrier for protons. In the light nuclei this barrier compensates for the difference in the separation energy for neutrons and protons. The total $(\gamma, p)$ yield might then be expected to equal the total $(\gamma, n)$ yield were it not for the fact that the high neutron separation energy results in there being a paucity of states aval1able for neutron decay. In the heavy nuclei the top of the Couloinb barrier comes well above the giant resonance and the decay is preiominantly by neutron emission. The two black dots indicate the threshold for the $(\gamma, 2 n)$ reaction. In the light nuclei this threshold is well above the giant resonance while in the heavier ones it comes at about its center. For these nuclei the $(\gamma, 2 n)$ reaction can contribute appreciably to the total absorption cross section.

The numbers given across the top of Figure 1 give the energies, in units of the giant resonance energy, at which the ratio of the nuclear radius to the photon wavelength divided by $2 \pi$ is equal to one. This is the energy at which retardation effects might be expected to set in and where the usual multipole expansion of the electromagnetic operator might be expected to lead to difficulties.

Gooi resolution experiments have shown that for the light nuclei, atomic number $A<40$, the giant resonance has considerable fine structure. While some such struciure has been shown to exist for the heaviex nuclei, this is in general not a significant feature of the shape of the absorption curve for nuclei with $A>100$. In any event, all such structure is going to be smoothed over in this discussion. The only structure that will be indicated is that associated with nuclei having permanently deformed nuclear ground states. The giant resonance for these nuclei is very well described by the superposition of two Lorentz shape resonance curves in which the area under the higher energy peak is twice that under the lorver energy peak, i.e.,

$$
\sigma(E)=\frac{\sigma_{a}\left(\Gamma_{a} E\right)}{\left(E^{2}-E_{a}^{2}\right)^{2}+\left(\Gamma_{a} E\right)^{2}}+\frac{\sigma_{b}\left(\Gamma_{b} E\right)}{\left(E^{2}-E_{b}^{2}\right)^{2}+\left(\Gamma_{b} E\right)^{2}}
$$


and $\sigma_{b} \Gamma_{b}=2 \sigma_{a} \Gamma_{a}$. Except for the normalizations used to present the data in some of the figures, little or no attempt will be made to correlate any of the data presented with theory. This aspect of the field is covered in a number of recent reviews [7].

\subsection{Experimental Data}

The gross features of the giant resonance are summarized in Table 1 and Figures 2 and 3. Table 1 presents data for all nuclei for which measurements have been made. No attempt has been made to indicate the fine structure of ten observed in the giant resonance for the lighter nuclei. The data presented for a given nucleus and reaction are the energy at which the giant resonance peaks, $E_{\text {; }}$ the magnitude of the cross section at $\mathrm{E}$, SIG; and the full width of the cross section curve at half maximum, DEL.

Where authors have fit a cross section curve by either one or the superposition of two Lorentz lines, the resulting parameters are listed and a letter $\mathrm{L}$ is entered under REMARKS. Also listed are the integrated cross section up to some upper energy limit $\sigma_{0}\left(E_{m}\right)$, SIG-0, and the bremstrahlung weighted cross section $\sigma_{-1}\left(E_{m}\right)$, SIG-1. These quantities are defined by

$$
\begin{aligned}
& \sigma_{0}\left(E_{m}\right)=\int_{0}^{E_{m}} \sigma(E) d E \\
& \sigma_{-1}\left(E_{m}\right)=\int_{0}^{E_{m}} \frac{\sigma(E)}{E} d E
\end{aligned}
$$

When these integrals have been evaluated from the Lorentz line parameters used to fit the cross section, $\mathrm{F}_{\mathrm{m}}$ is given as $\mathrm{L}$. For the lighter nuclei these quantities are given where possible both for the total cross section as well as for one or more of the partial reaction cross sections which make a major contribution to the total. For the heavier nuclei the data pertain almost exclusively to neutron-producing cross sections. The various reactions listed are indicated by the symbols given in the second column of the table, (INT). These symbols are defined as follows:

$\begin{array}{ll}\text { TOT } & \text { the total nuclear absorption cross section } \\ & \text { for photons } \\ \text { N } & \text { the }(\gamma, \mathrm{n}) \text { cross section } \\ \mathrm{XN} & \text { the neutron yield cross section } \sigma(\mathrm{xn}) \\ & \sigma(\mathrm{xn})=\sigma(\gamma, \mathrm{n})+\sigma(\gamma, \mathrm{np})+2 \sigma(\gamma, 2 \mathrm{n})+\eta \sigma(\gamma, \mathrm{f}) \\ \mathrm{SN} & \text { the sum of the neutron-producing cross sections } \\ & \sigma(\mathrm{SN}) ; \sigma(\mathrm{SN})=\sigma(\gamma, \mathrm{n})+\sigma(\gamma, \mathrm{np})+\sigma(\sigma, 2 \mathrm{n})+\ldots \\ \mathrm{P} & \text { the }(\gamma, \mathrm{p}) \text { cross section } \\ \mathrm{T} & \text { the }(\gamma, \mathrm{t}) \text { cross section }(\mathrm{t}=\text { triton) } \\ \mathrm{D} & \text { the }(\gamma, \mathrm{d}) \text { cross section }(\mathrm{d}=\text { deuteron) } \\ \mathrm{F} & \text { the photofission cross section } \\ \mathrm{N}+\mathrm{P} & \sigma(\gamma, \mathrm{n})+\sigma(\gamma, \mathrm{p}) \\ \mathrm{SNF} & \sigma(\mathrm{SN})+\sigma(\gamma, \mathrm{f})\end{array}$

Where a number of measurements of a particular cross section are available in the literature, the parameters given in Table 1 are taken from a single measurement which has been selected as being the most representative of the data for a particular nucleus. This measurement is identified by the bibliographic reference given for each entry in the table. The notes referred to in the final colum (REMARKS) are listed at the end of the table. These are used to expand on the information given in the table, to indicate the need for 
possible renormalizations of the values quoted, and to give the source of data listed where no bibliographic reference number is available.

The general trends with mass number A of the integrated absorption cross section and the bremsstrahlung weighted cross section are indicated in Figures 2 and 3, respectively. On these figures the data for nuclei having mass numbers less than 70 all come from direct measurements of the total photon absorption cross section. For the lightest nuclei two values are given, one where the upper limit to the integration is $140 \mathrm{MeV}$ (i.e., approximately the meson production threshold) and a second value where the limit is $30 \mathrm{MeV}$ for $\sigma_{0}$ and $35 \mathrm{MeV}$ for $\sigma_{-1}$. The data for the heavier nuclei are all taken from measurements of the neutron-producting cross section, $\sigma(S N)$, that have been fit with Lorentz shape resonance curves. In general, the measurements have extended up to about $25 \mathrm{MeV}$. The values listed all result from carrying out the appropriate analytical integrations $(0<\mathrm{E}<\infty)$ over the resonance curves. None of the data presented in these figures were derived from $\sigma(\mathrm{SN})$ curves that had been obtained by making statistical mosel corrections to measirements of $\sigma(X N)$. The integrated cross section values have been normalized in terms of the value given by the classical dipole sum rule (see Equation (1)). The bremsstrahlung weighted cross section values have been nornalized by dividing by $\mathrm{A}^{4 / 3}$, the mass number dependence given by most elementary models of the nuclear photoeffect [8].

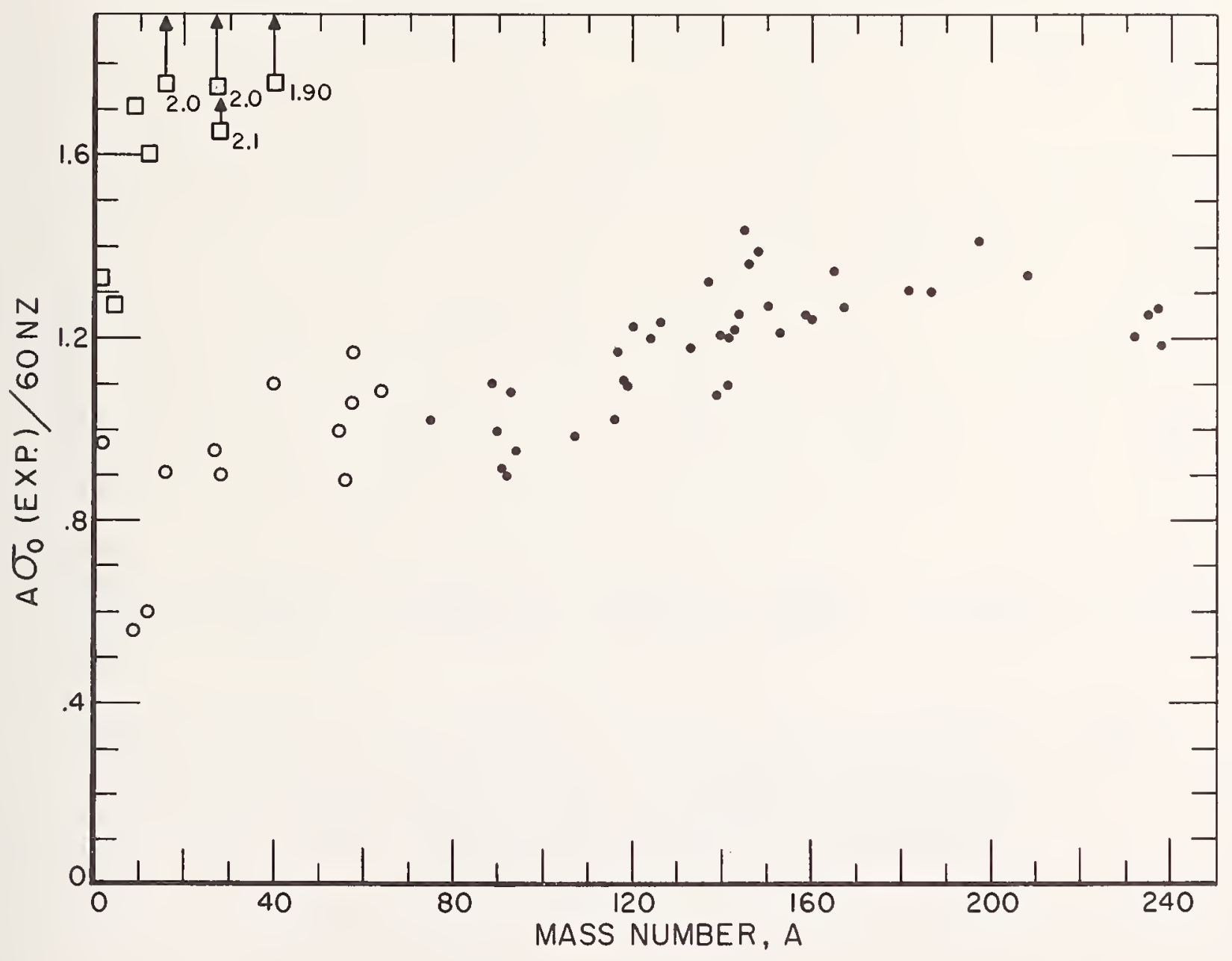

Figure 2. Integrated absorption cross sections normalized to the classical dipole sum rule value. All points are from data selected from those listed in Table 1. Solid dots were obtained from integrating Lorentz line fits to $\sigma(\mathrm{SN})$ data. Open circles and squares are from total absorption cross section measurements. For open circles integration is taken to $30 \mathrm{MeV}$, for the squares it goes to $140 \mathrm{MeV}$. 
Except for some fluctuations associated with the lightest nuclei, the normalized values of both $\sigma_{0}$ and $\sigma_{-1}$ are relatively independent of the mass number. Note that for the light nuclei $(A<40)$, where data are available, that there is a rather large contribution to $\sigma_{0}(140)$ coming from energies above $30 \mathrm{MeV}$. The contribution of the cross section at these ranges to $\sigma_{-1}(140)$, however, is relatively small. The total cross section for these nuclei indicates that $\sigma_{0}(140)$ is about twice the classical sum-rule result. Unfortunately, only very sketchy data are available to give an indication of what this limit might be for the heavier nuclei. The data which do exist are not inconsistent with $\sigma_{0}(140)$ being twice the dipole sum-rule value for these nuclei also (see Equation (1)).

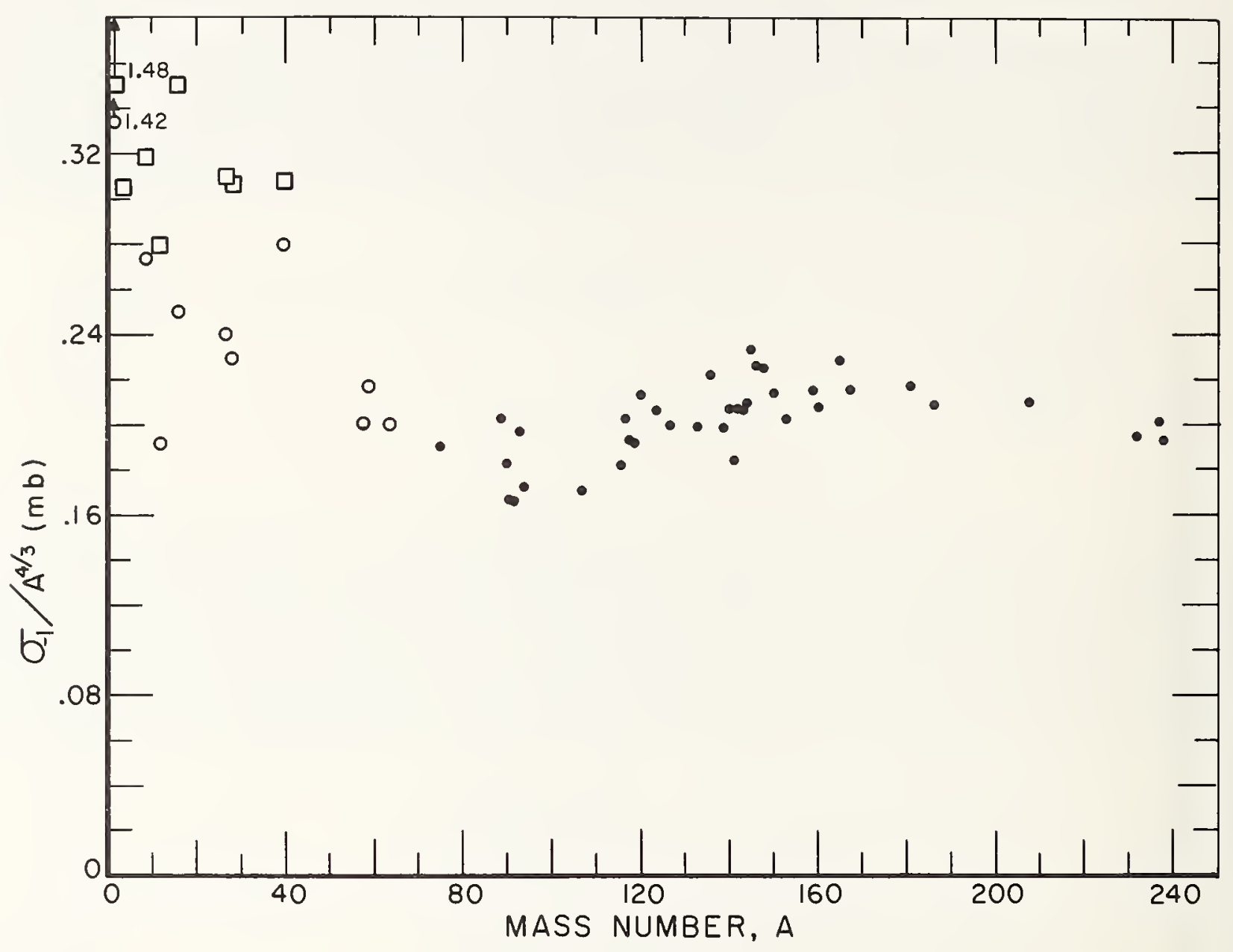

Figure 3. Normalized bremsstrahlung weighted cross sections. Solid dots obtained from integrating Lorentz line fits to $\sigma(\mathrm{SN})$ data. Open circles and squares from total absorption cross section measurements. For open circles integration is to $35 \mathrm{MeV}$, while for squares it goes to $140 \mathrm{MeV}$. 
Table 1. Gross features of the Giant Resonance

\begin{tabular}{|c|c|c|c|c|c|c|c|c|c|}
\hline NUCL & EUS & INT & E & SIG & DEL & Sig-o & $516-1$ & REF $P$ & REMARKS \\
\hline 2 & A & & MEV & ME & MEV & $M E V-M B(M E V)$ & $M E(M E V)$ & & \\
\hline IH & 2 & TOT & 4.48 & 2.5 & 18.1 & $\begin{array}{l}40(140) \\
29(30)\end{array}$ & $\begin{array}{l}3.7(140) \\
3.6(30)\end{array}$ & & $* 1, * 2$ \\
\hline $1 H$ & 3 & D & 12 . & .72 & 12. & $12(33)$ & $.78(33)$ & $68 P F 1$ & \\
\hline $2 \mathrm{HE}$ & & D & $12 \cdot 5$ & .75 & 18. & $16(40)$ & $1.1(40)$ & 65ST? & \\
\hline $2 \mathrm{HE}$ & & $\mathrm{N}$ & 15. & .90 & 13 . & $13(30)$ & $.77(30)$ & $70 B E 6$ & \\
\hline $2 H E$ & & $\mathrm{P}$ & 26.5 & 1.7 & 14 . & $40(170)$ & $1.1(170)$ & 68605 & $* 2$ \\
\hline $2 \mathrm{HE}$ & & $N$ & $26 \cdot 5$ & 1.7 & $14 \cdot$ & $42(170)$ & $1.2(170)$ & 68605 & $\neq 2$ \\
\hline 3\llcorner] & & I & $18 \cdot 5$ & .59 & 11 . & $5.6(35)$ & $.22(35)$ & $71 V E I$ & $685 H 2$ \\
\hline $3 L I$ & & $5 i !$ & 12. & 1.7 & 16. & $27(32)$ & $1.9(32)$ & $658 E 1$ & $* 3$ \\
\hline $3\llcorner 1$ & & $\mathbf{I}$ & 17. & 0.2 & 20 . & $4.9(35)$ & & $670 E_{1}$ & $55 M 11$ \\
\hline $3 L I$ & & $X N$ & $16+$ & 3.0 & $13+$ & $50(29)$ & & $65 \mathrm{HAl}$ & \\
\hline $4 B E$ & & TOT & 24 . & 5.2 & 28 . & $228(140)$ & $7.4(: 40)$ & $71 \mathrm{AH} 1$ & $* 4$ \\
\hline $\begin{array}{l}4 B E \\
4 B E\end{array}$ & & $\times N$ & 28 . & 4.7 & 26. & $\begin{array}{r}75(30) \\
220(70)\end{array}$ & $\begin{array}{l}5.1(35) \\
7.8(70)\end{array}$ & $\begin{array}{l}71 \mathrm{AHI} \\
66 \mathrm{CO}_{4}\end{array}$ & \\
\hline 58 & 10 & $X N$ & 24 . & 6.5 & 10.5 & $67(27)$ & & $65 \mathrm{HAl}$ & \\
\hline $5 B$ & 11 & $X N$ & 26. & 7.5 & 11. & $69(29)$ & & 65HAl & \\
\hline $5 B$ & 11 & $\mathrm{P}$ & 25 . & 15. & 10 . & $98(31)$ & & 69502 & \\
\hline $6 C$ & 12 & TOT & 23. & 20. & 5.2 & $288(140)$ & $\begin{array}{l}7.7(140) \\
5.1(35)\end{array}$ & $\begin{array}{l}71 \mathrm{AHI} \\
71 \mathrm{AHI}\end{array}$ & \\
\hline $6 C$ & 12 & SN & 23. & 7. & $4 \cdot 8$ & $36(30)$ & & 66FU1 & * 5 \\
\hline $7 \mathrm{~N}$ & 14 & TOT & 22.5 & 27. & 7.0 & $195(30)$ & $8.4(30)$ & $698 E 2$ & \\
\hline $7 \mathrm{~N}$ & 14 & SN & $23 \cdot 2$ & 14. & $5 \cdot 1$ & $98(30)$ & & 7OBE 8 & \\
\hline 80 & 16 & TOT & 23.5 & 30. & 5.5 & $280(140)$ & $14(1+0)$ & $71 \mathrm{AHI}$ & \\
\hline 80 & 16 & SN & 33 & 7 & & $216(30)$ & 101351 & $71 \mathrm{AH} 1$ & \\
\hline & & & 20 & . & 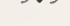 & $46(30)$ & & $648 R 1$ & $* 3$ \\
\hline gF & 19 & TつT & 24. & 18. & $14+$ & $271(30)$ & & $698 E 2$ & \\
\hline
\end{tabular}

IONE

IINA23 TOT 23. 15. 16. 200130) 11(35) 65WY1

IINA23 SN 23. 10. 15+ $119(27)$ TIALI

12 MG TOT 19. 24. 13. 225(30) 12(35) 65WY1

$12 M G 24$ SN $19.5 \quad 7.5 \quad 11 \cdot \quad 50(28) \quad 2.8128) \quad 71 F U 2$

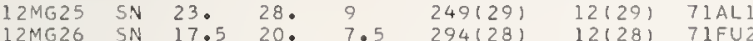

13AL27 TOT 21. 40. 10. 408(140) 26(140) 71AH]

$\begin{array}{llllll}13 A L 27 \text { SN 21. 15. 11. } & 384(30) & 20(35) & 71 \text { AH1 } \\ & 172(37) & & 66 \text { FU1 }\end{array}$

1451 TOT 20.2 52. 5. $882(140) \quad 26(140) 71 \mathrm{AHI}$

$145129 \mathrm{~N}$ 21. 15. 5. $\quad \begin{array}{rrr}378(30) & 20135) \quad 71 \mathrm{AHI}\end{array}$

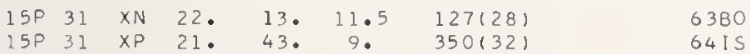

165 TOT 20. 50. 7. 400(30) 22(35) 65WY1

16532 XN 21: 16: 11: $137(32) \quad 22(35)$ TOAN3

18AP.40 TOT 19. 42. 10. 450132) 65EHI

$18 A R 40$ N 19. 37. 5. 200(32) 65EHI

19K $37 \mathrm{~N} \mathrm{20.16.7.} \mathrm{102(30)} 71 \mathrm{WE} 1$ \%6

20CA TOT 20. 92. $4.8 \quad 1140(140) 42(140) 71 \mathrm{AHI}$

2OCA SN 20. 15. $\quad 3.8 \quad 660(30) \quad 38(35) \quad 71 \mathrm{AHI}$

$215 C 45$

NO DATA

$22 T 1 \quad X N \quad 17.0 \quad 607+\quad 67001$

$22 \mathrm{TI}+6 \quad \mathrm{SN} \quad 20.5 \quad 31 \quad 8.5 \quad 269(31) \quad 625 \mathrm{H}$

22T1 $A=47,48,49,50$ NO DATA

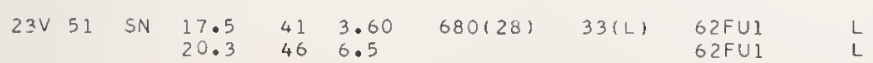

NUCLEUS INT E SIG DEL SIG-O SIG-I REF REMARKS

$24 \mathrm{CR} \quad X N \quad 18.5 \quad 97 \quad 1628(80) \quad 62(801-64 C 02$

$24 C R \quad A=50,52,53,54$

NO DATA

25 MN55 TOT $17.5 \quad 100 \quad 7.5 \quad 816(29) \quad 69001$

26FE TOT $17.5 \quad 90 \quad 9.0 \quad 735(27) \quad 69002$

26FE54 N $19.2 \quad 38 \quad 6.9 \quad 290(31) \quad 57$ CA2

$26 \mathrm{FE} \quad A=56,57,58$

NO UATA

$\begin{array}{lllllll}27 \operatorname{COS9} \text { TOT } & 18.6 & 92 & 9.3 & 1030(35) & 50(35) & 65 \text { WY }\end{array}$

$\begin{array}{lrrrrrrr}28 N I & \text { TOT } & 18.7 & 89 & 7.5 & 920135) & 44(35) & 65 \text { WYI } \\ 28 N I 58 & N+P & 19.5 & 125 & 4.8 & 840132) & & 59 C A 4\end{array}$

$28 N I 60 \quad N+P \quad 19.0 \quad 90 \quad 5.5 \quad 940130) \quad 69 G 0270 I S 4$

$28 \mathrm{NI} \quad A=61,62,64 \quad$ NO DATA

$29 C U$ TOT $17.5 \quad 94 \quad 11.5 \quad 1036(35) \quad 51(35) \quad 65$ WY1

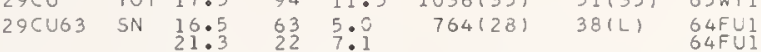

$20 C U 65$ SN $16.8 \quad 88 \quad 5: 0 \quad 766(28) \quad 53(\mathrm{~L}) \quad 64 \mathrm{FUI}$

$64 \mathrm{FUI}$

$\begin{array}{llllllllll}302 N & X N & 16.7 & 91 & 4.6 & 1607180) & 66(80) & 67 \mathrm{CO} & 64 \mathrm{CO}\end{array}$

$302 N 64 \quad N \quad 17.2 \quad 48 \quad 7.0 \quad 330(23) \quad 60 R 04$

NO DATA

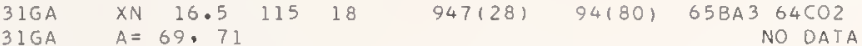

32GE $\quad X N \quad 17.5 \quad 158 \quad 2495(80) \quad 102(80) \quad 64 C 02$

$\begin{array}{lllllll}32 G E 70 & N & 17.5 & 158 & 8.6 & & 6 \text { OFEI } \\ 326 E 76 & N & 18.9 & 243 & 7+ & 1500(21) & 55 B O I\end{array}$

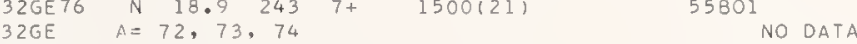

$\begin{array}{llllllll}33 A 575 & 5 N & 15.0 & 39 & 3.63 & 910(30) & 51(30) & 69 B E 1 \\ & 17.5 & 78 & 7.41 & & & 698 E 1 & L\end{array}$

$\begin{array}{llllll}34 S E & X N & 15.5 & 118 & 5.2 & \end{array}$

$345 E \quad A=74,76,77,78,80$

$35 B R$

NO DATA

$36 K R$

NO DATA

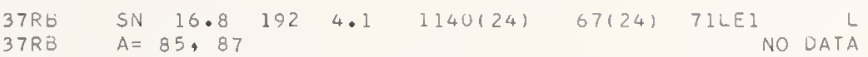

$385 R B 4$

SN $\quad 16.7 \quad 207 \quad 4.2 \quad 1420(27)$

$385286 \quad X N \quad 15.9 \quad 160 \quad 5.0 \quad 920(23)$

38SRE 7 XN $15.8 \quad 146 \quad 5.3 \quad 1000(23)$

$80(27) 71 L E I$ NO DATA

$385 R 88 \times N \quad 16.32014 .01050(231$

$56 Y E 2$

$56 Y E 2$

$56 Y E 2$

39Y $89 \quad$ SN $16.7 \quad 225 \quad 4.1 \quad 1360(27) \quad 77(27) \quad 71 L E I$

\begin{tabular}{llllllll}
\hline $02 R 90$ & SN & 16.7 & 211 & 4.0 & $1260(26)$ & $71(26)$ & $71 L E 1$
\end{tabular}

$40 Z R 91$ SN $16.5 \quad 184 \quad 4.23 \quad 1080(30) \quad 06(30) \quad 670 E 2$ $\begin{array}{llllllll}40 Z R 92 & S N & 16.3 & 165 & 4.73 & 1100(28) & 65(28) & 676 E 2\end{array}$

67822

4IN893 SN $\quad 16.5 \quad 202 \quad 4.70 \quad 1330(24) \quad 79(24) \quad 71 L F ?$

$42 M 092 \times N \quad 16.4 \quad 170 \quad 5.0 \quad 1290(30) \quad 70 I S]$

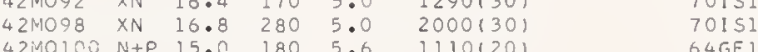

$4290 \quad A=94,95,36,97 \quad$ NO DATA

$44 R U$

NO DATA

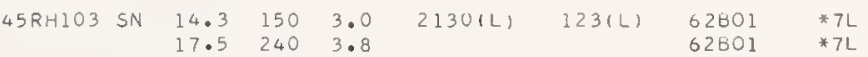

46PO108 5N $15.7 \quad 210 \quad 5.0 \quad 1725(25) \quad 104(25) \quad 690 E 5$ NO DATA PO 102, 104, 105, 106, 110

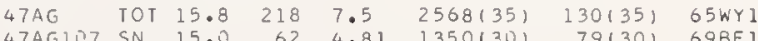
$47 A G 107$ SN $15.0 \quad 62 \quad 4.81 \quad 1350(30) \quad 79(30) 698 E 1$

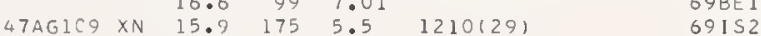

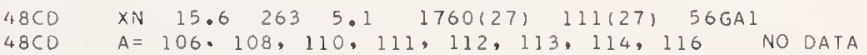
$48 C 0 \quad A=106,108,110,111,112,113,114,116 \quad$ NO DATA $\begin{array}{llllllll}49 I N & 5 N & 15.6 & 266 & 5.24 & 1901(31) & 113(31) & 69 F U 1\end{array}$ $\begin{array}{llllllll}491 N 115 \text { SN } & 14.0 & 116 & 3.0 & 2210(\mathrm{~L}) & 119(\mathrm{~L}) & 62801 & * 7 \mathrm{~L} \\ 16.3 & 240 & 3.8 & & & 62801 & * 7 \mathrm{~L}\end{array}$ 


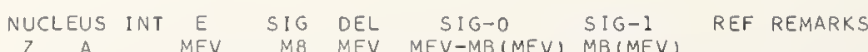

5OSN XN $16.0 \quad 300 \quad 5.0 \quad 1640(40) \quad 134(\mathrm{~L}) \quad 58 \mathrm{FU}]$

50SN112 N $16.0 \quad 340 \quad 5.0 \quad 1820(21) \quad 152(\mathrm{~L})$ 61KUI

5JSN116 SN $15.7 \quad 266 \quad 4.19 \quad 1667(29) \quad 94(29)$ 69FUI

SOSN117 SN $15.7 \quad 254 \quad 5.32 \quad 1939(31) \quad 114(31) \quad 69 F U 1$

50SN118 SN $15.6 \quad 255 \quad 4.76 \quad 1898(30) \quad 110(30)$ 69FU1

50SN119 SN $15.5 \quad 253 \quad 4.812078(31)$ 119(31) $69 F U 1$

$50 S N 124$ SN $15.2 \quad 283 \quad 4.812077(31)$ 1 $23(31)$ (20FU1

50 SN $A=114,115,122$ NO DAIA

5158

NO DATA

$52 T E$

NO DATA

$\begin{array}{llllllllll}53 \text { I } & 127 & \text { SN } & 14.5 & 255 & 3.78 & 2020(25) & 129(25) & 698 E 6 & \text { L }\end{array}$

$54 \times E$

NO DATA

$55 \operatorname{CS133} \mathrm{SN} \quad 15.3 \quad 287 \quad 5.05 \quad 2000(30) \quad 124(30) \quad .698 \mathrm{~B} 1$

$\begin{array}{lllllllll}56 B A & \text { SN } & 15.3 & 356 & 4.70 & 2600(\mathrm{~L}) & 157(\mathrm{~L}) & 718 E 4 & \text { L }\end{array}$ $\begin{array}{lllllllll}568 A 138 & \text { SN } & 15.3 & 327 & 4.61 & 2040(27) & 131(27) & 706 E 8\end{array}$

56BA $\quad A=134,135,136,137 \quad$ NO DATA

57LA139 SN $15.2 \quad 340 \quad 4.45 \quad 2324(28) \quad 146(28) \quad 716 E 468055$ L

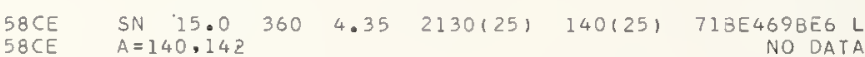

58CE

$\begin{array}{llllllllll}59 P R 141 & 5 N & 15.2 & 320 & 4.49 & 2100(30) & 136(L) & 666 R 1 & \end{array}$

60ND142 SN $14.9 \quad 359 \quad 4.43 \quad 2500(\mathrm{~L}) \quad 153(\mathrm{~L}) \quad 71 \mathrm{CA}$

6ONDI43 SN 15:9 $3594.43-2500(\mathrm{~L}) \quad 153(\mathrm{LI}) \quad 71 \mathrm{CA}$

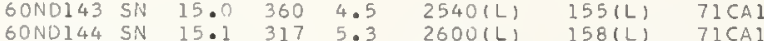

6OND145 SN $15.0 \quad 297 \quad 6.5 \quad 3000(\mathrm{~L}) \quad 178(\mathrm{~L}) \quad 71 \mathrm{CAl}$

6UND146 SN $14.8 \quad 308$ 6. $2900(\mathrm{~L}) \quad 174(\mathrm{~L}) \quad 71 \mathrm{CA}$

60ND148 SN $14.7 \quad 263 \quad 7.2 \quad 3000(\mathrm{~L}) \quad 176(\mathrm{~L}) \quad 71 \mathrm{CA}$

60NO150 SN $12.3 \quad 174 \quad 3.3 \quad 2700(\mathrm{~L}) \quad 171(\mathrm{~L}) \quad 71 \mathrm{CA}$

$\begin{array}{llllllllr}62 S M & \text { SN } & 12.8 & 155 & 4.25 & 2480(25) & 167(25) & 698 E 6 & \mathrm{~L} \\ & & 15.5 & 260 & 4.6 & & & 69 \mathrm{EE} 6 & \mathrm{~L} \\ 62 S M 148 & \text { SN } & 14.1 & 335 & 4.0 & 2080(22) & 137(\mathrm{~L}) & 71 \mathrm{VA2} & \text { \% }\end{array}$

62SMI5C SN $13.6 \quad 360 \quad 5.50 \quad 2213(23), 203(\mathrm{~L})$ G9VA3

$62 S M 152$ SN $11.6 \quad 400 \quad 2.4 \quad 3079(23) \quad 264(\mathrm{~L})$ G9VA3 $14.7420 \quad 3.4 \quad 69 V A 3 \quad * 7 \mathrm{~L}$

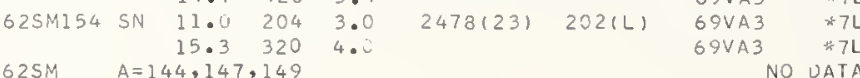

$625 \mathrm{M} \quad \mathrm{A}=144,147,149$

63EU151 SN $14.0 \quad 285 \quad 4.5 \quad 2020(22) \quad 131(\mathrm{~L}) \quad 71 \mathrm{VAL} \quad$ *7L $\begin{array}{llllllll}\text { 63EU153 SN } & 12.3 & 155 & 2.75 & 2310(29) & 166(\mathrm{~L}) & 690 \mathrm{E} 8 & \mathrm{~L} \\ & 15.8 & 222 & 5.83 & & & 698 \mathrm{~EB} & \mathrm{~L}\end{array}$

6460152 SN $12.0 \quad 147 \quad 3.0 \quad 1990(22) \quad 135(L) \quad 71 V A 2 \quad * 7 L$

15. $2593.2 \quad 71 \mathrm{VA2} * 7 \mathrm{~L}$

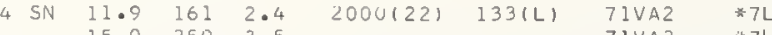

6460156 SN 11.9 $18003.5 \quad 2110(22) \quad 142(\mathrm{~L}) \quad 71 \mathrm{VAC}$

$\begin{array}{llllllll} & 11.9 & 180 & 2.6 & 211(122) & 142(\mathrm{~L}) & 71 \mathrm{VA2} & * 7 \mathrm{~L} \\ & 15.2 & 243 & 3.6 & & & \end{array}$

6460158 SN $11.7 \quad 165 \quad 2.6 \quad 2160(22) \quad 146(\mathrm{~L}) \quad 71 \mathrm{VA2}$ *7L

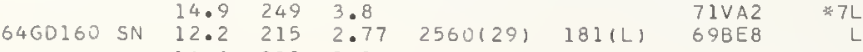
$16.0 \quad 233 \quad 5.28$ G9BE8

$6460 \quad A=155.157$

NO DATA

$\begin{array}{llllllll}65 T B 159 & \text { SN } 12.1 & 205 & 3.25 & 2500(27) & 172(27) & 684 E 5 & \text { L }\end{array}$

$660 Y$

NO DATA
NUCLEUS INT E SIG DEL SIG-O SIG-1 REF REMARKS $Z$ A MEV MO MEV IMEV-MB(NEV) MB(MEV)

$67 H 0165$ SN 12.1 $250 \quad 2.70 \quad 27901251 \quad 1941251 \quad 68 B E 5$ $15.6285 \quad 4.80 \quad$ 68BE5

$\begin{array}{lllllllll}\text { G8ER } & \text { SN } & 12.1 & 225 & 2.90 & 2700(25) & 186(25) & 698 E 6 & \text { L } \\ & & 15.5 & 260 & 5.0 & & & 698 E 6 & \text { L }\end{array}$ 68ER $A=166,167,168,170$ NO OATA

69TM169 SN $13.8 \quad 235 \quad 6.3 \quad 1480(22) \quad 148(\mathrm{~L})$ 58KAl

$70 \times 8$

NO DATA

7ILUI75 SN $\quad 12.4 \quad 230 \quad 2.70 \quad 2650(25) \quad 182(22) \quad 698 E 6$

$72 \mathrm{HA}$

NO DATA

$\begin{array}{lrrrrrrr}\text { 73TA181 SN } & 12.4 & 270 & 2.57 & 2900(25) & 206(25) & 68 B E \\ & & 15.3 & 330 & 4.47 & 60140) & 688 E \\ 73 \text { TA181 P } & & & & 608 A\end{array}$

$\begin{array}{lllllllll}74 W & 186 & \text { SN } & 12.6 & 211 & 2.29 & 3975(29) & 222(\mathrm{~L}) & 698 \mathrm{E} 8 \\ & 14.9 & 334 & 5.18 & & 698 \mathrm{E} 8 & \mathrm{~L}\end{array}$ $74 \mathrm{~W} \quad A=182,183,184^{\circ}$ NO DATA

75RE

NO OATA

7605

NO DATA

77 IR

NO DATA

$789 \mathrm{~T}$

NO DATA

79AU197 SN $13.7 \quad 540 \quad 4.75 \quad 3480(25) \quad 238(25)$ TOVEI

$80 H G$

NO DATA

81TL TOT $14.0 \quad 648 \quad 4.6 \quad 3770(27) \quad 266(27)$ 8ITL203 SN $14.2 \quad 490 \quad 3.7 \quad 2610(20) \quad 185 \quad$ G9AN10 $81 T L 205$ SN $14.1 \quad 490 \quad 3.7 \quad 2780(2 U) \quad 187(L) \quad 69 A N 10$

$1 T 205 \% 20.020103072$

81 TL205 Y 20 OF $A / \dot{P} / N=1 / 100 / 10000$

$82 P 5206$ SN $13.7 \quad 525 \quad 3.75 \quad 2780(28) \quad 208(\mathrm{~L}) \quad 64 \mathrm{HAZ}$ * $5 \mathrm{~L}$ 82 PB 207 SN $13.6 \quad 485 \quad 3.37 \quad 2650(28) \quad 199(\mathrm{~L}) \quad 64 \mathrm{HAC} \quad$ *SL $82 \mathrm{~PB} 208$ SN $13.4 \quad 640 \quad 4.05 \quad 3480(25) \quad 2511251$ 7OVEl L $82 \mathrm{~PB} 208 \mathrm{P} 271331$ 710A1

$8381209 \mathrm{SN} \quad 13.5 \quad 520 \quad 3.83 \quad 2930(28) \quad 213(\mathrm{~L}) \quad 64 \mathrm{HA} 2$ \%5 L

88RA

NO DATA

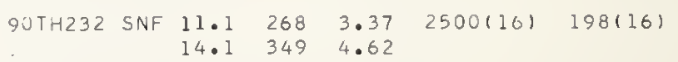

90 TH $A=228,230$

* 8 L NO DATA

$91 P A 231$

NO DATA

$\begin{array}{llllllll}92 U & 233 & \text { XN } & 14.0 & 1670 & 6.0 & 11200(22) & \\ 92 U & 235 & \text { SNF } & 10.9 & 364 & 2.45 & 3560(19) & 303(\mathrm{~L})\end{array}$

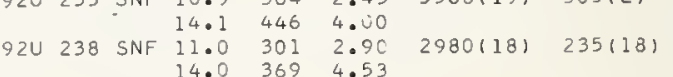

$920 \quad A=234,236$ LUDU3 $64 B 03 \quad$ L L NO DATA

93 NP237 SNF 11.1 $251 \quad 3.16 \quad 2600(16) \quad 204(16)$

$* 8 \mathrm{~L}$
$* 3 \mathrm{~L}$

94 PU239 XN $1396 \quad 1580 \quad 6.3 \quad 11600(22)$

$57 K A] 58 K A 2$ 
*1 E. G. Fuller and Evans Hayward, "The Giant Resonance of the Nuclear Photoeffect" in Nuclear Reactions II, Eds. P. M. Endt and P. B. Smith (North-Holland Publishing Co., Amsterdam, 1962); F. Partovi, Ann. Phys. 27, 79 (1964).

*2 See also - J. S. O'Conne11, "Electromagnetic Interactions of the Few-Nucleon Systems," to be published in the Proceedings of the International Conference on Electromagnetic Interactions of Nuclei at Low and Medium Energies, Moscow, December 1972.

$* 3$ Cross section given is total neutron yield. This includes all reaction cross sections except the ${ }^{6} \mathrm{Li}(\gamma, t)^{3} \mathrm{He}$. Lowest threshold for 2-neutron emissions is $24.2 \mathrm{MeV}$. 0ther data indicate cross section magnitudes may be $1.2 \times$ those quoted. (66Ge3)

*4 Measurement does not extend below $16 \mathrm{MeV}$. The $\sigma_{0}$ and $\sigma_{1}$ values quoted have been corrected to include $\sigma(\gamma, \mathrm{n})$ in energy region froin $1.6-16 \mathrm{MeV}$.

*5 Cross sections may be 1 ow by $20-30 \%$. See $66 \mathrm{Ge} 2,70 \mathrm{Ve} 1$, and other measurements of n-production cross sections.

*6 Cross section for production of .95 isomer. This represents about 0.6 of total ( $\gamma, \mathrm{n}$ ) cross section (see $62 \mathrm{Go} 3,65 \mathrm{Co} 3$ ).

*7 Statistical model used to correct for neutron multiplicity.

*8 R. Bergere, H. Beil, P. Carlos, A. Veyssiere, A. Leprete, Nuclear Structure Studies Using Electron Scattering and Photoreaction, Eds. K. Shoda and H. Vi, p. 273

(Tohoku University, Sendai, Japan, 1972). 


\subsection{Estimating Yield}

Figures 4 and 5, when used in conjunction with the data listed in Table 1 can be used to estimate bremsstrahluig-induced photonuclear reaction yields. The estimate is based on the assumption that the cross sections can be described by Lorentz resonance curves. This is a very good approximation for the heavier nuclei ( $>100)$. It is not a good approximation for light nuclei, particularly those for $A \leq 16$. For these nuclei, $(A<40)$ if yields are required with an accuracy better than a factor of three, detailed calculations should be carried out making use of the best available cross-section data. For the heavier nuclei, yields can usually be estimated to within $\pm 30 \%$ by making use of the Lorentz-line approximation.

It cail be readily shown that for a Lorentz-shaped resonance curve

$$
\begin{gathered}
\sigma_{0}(L)=\int_{0}^{\infty} \frac{\sigma_{i}\left(E \Gamma_{i}\right)}{\left(E^{2}-E_{i}^{2}\right)^{2}+\left(E \Gamma_{i}\right)^{2}} d E=\frac{\pi}{2} \sigma_{i} \Gamma_{i} \\
\sigma_{-1}(L)=\int_{0}^{\infty} \frac{\sigma_{i}\left(E \Gamma_{i}\right)}{\left(E^{2}-E_{i}^{2}\right)^{2}+\left(E \Gamma_{i}\right)^{2}} \frac{d E}{E}=\frac{\pi}{2} \frac{\sigma_{i} \Gamma_{i}}{E_{i}} \frac{1}{\left(1-(2 \alpha)^{-2}\right)^{\frac{1}{2}}}\left[\frac{1}{2}+\frac{1}{\pi} \tan ^{-1}(\alpha) \frac{\left(1-2(2 \alpha)^{-2}\right)}{\left(1-(2 \alpha)^{-2}\right)^{\frac{1}{2}}}\right]
\end{gathered}
$$

where $\alpha=\mathrm{E}_{1} / \Gamma_{1}$. Note that only for $\alpha \gg 1$ does $\sigma_{1}(\mathrm{~L}) \rightarrow \pi \sigma_{1} \Gamma_{1} /\left(2 \mathrm{E}_{1}\right)$. For values of $\alpha$ typical of most giant resonance curves $\sigma_{-1}(\mathrm{~L})$ is approximately 0.8 to 0.9 times the sharp resonance limit. The quantities plotted in Figures 4 and 5 are $\sigma_{0}(x) / \sigma_{0}(L)$ and $\sigma_{1}(x) / \sigma_{1}(L)$ as a function of $x=E / E_{1}$ for various values of $\alpha$.

If $N\left(E, E_{m}\right)=I\left(E, E_{m}\right) / E$ represents the brensstrahlung spectrum (number of photons per $\mathrm{MeV}), \sigma(E)$ the cross section for a reaction, and $\mathrm{n}$ the effective number of nuclei per $\mathrm{cm}^{2}$ in the bremsstrahlung beam, then, $Y\left(E_{m}\right)$, the reaction yield per $\mathrm{MeV}$ in the bremsstrahlung beam, is given by

$$
Y\left(E_{m}\right)=n \int_{0}^{E} \frac{I\left(E, E_{m}\right) \sigma(E)}{E} d E / \int_{0}^{E_{m}} I\left(E, E_{m}\right) d E .
$$

For estimating yields the thin target brensstrahlung spectrim can be approximated by assuming that $I\left(E, E_{\mathbb{W}}\right)$ is independent of $E$ so that

$$
Y\left(E_{m}\right) \simeq \frac{n}{E_{m}} \int_{0}^{E_{m}} \frac{\sigma(E)}{E} d E=\frac{n}{E_{m}} \sigma_{-1}\left(E_{m}\right) .
$$

For any paxticular cross section, values of $\sigma_{-1}\left(E_{\mathrm{u}}\right)$ can be obtained by maling use of Fig. 5 and the data listed in Table 1. With the approximations indicated here, thick target bremstrahlung spectra can be assumed to be an appropriately weighted superposition of thin target spectra. Note that the "cross section per equivalent quantum" $\sigma_{q}\left(E_{\mathbb{u}}\right.$ ) is equal to $\sigma_{-1}$ when $I\left(E, E_{\mathbb{u}}\right)$ is assumed to be constant, i.e.,

$$
\sigma_{q}\left(E_{m}\right)=\frac{E_{m} Y\left(E_{m}\right)}{n}=\frac{E_{m}}{\int^{E_{m}} I\left(E, E_{m}\right) d E} \int_{0}^{E_{n}} \frac{I\left(E, E_{m}\right) \sigma(E)}{E} d E \simeq \sigma_{-1}\left(E_{m}\right) .
$$



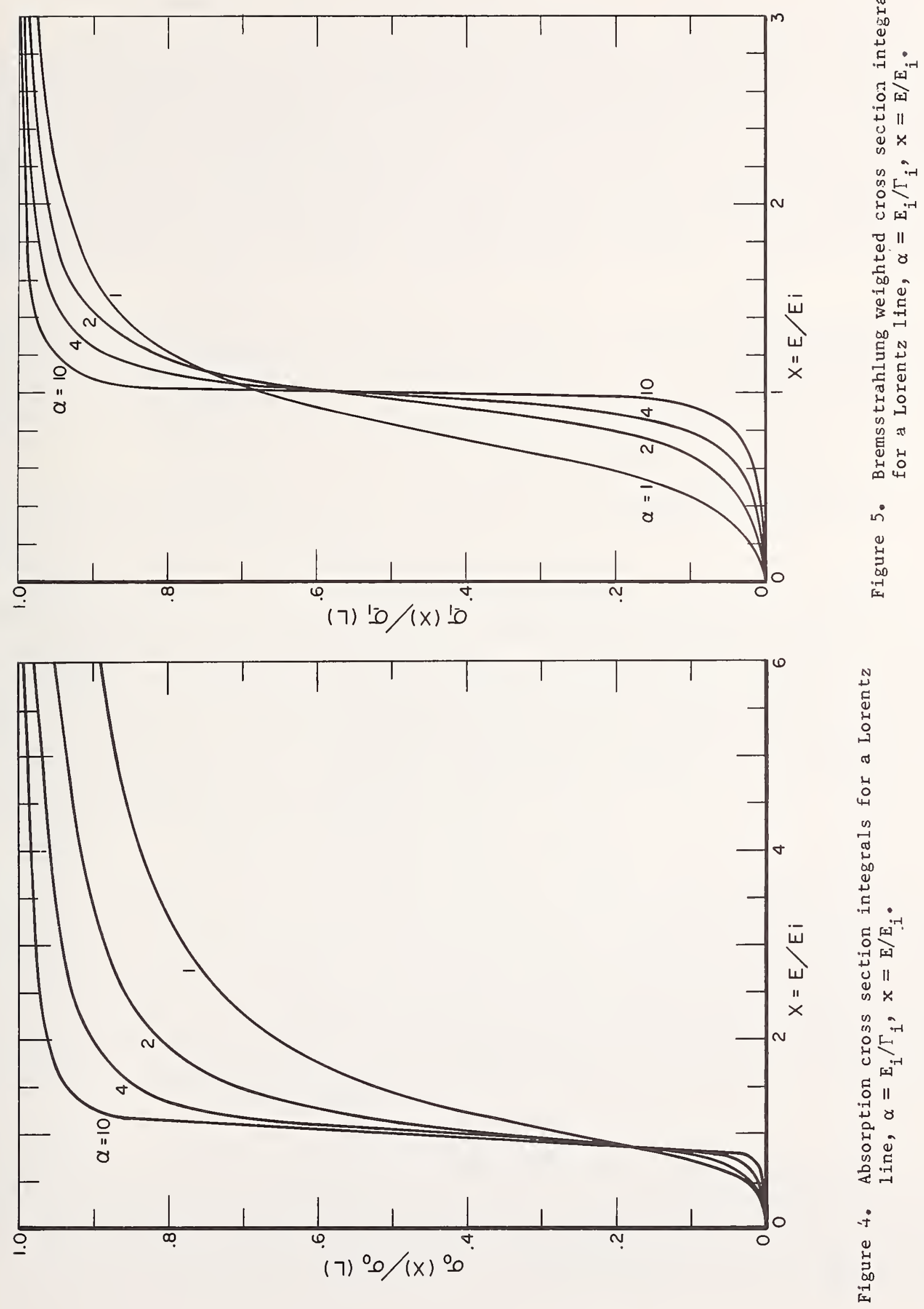


\subsection{Introduction}

This Photonuclear Data Index is the fourth one published by the Photonuclear Data Center. It is a cumulative index and as such supersedes all previous publications of the Center [9]. It is an index to all the experimental data published in the field of photonuclear reactions and entered into the Center's files in the period from January' 1,1955 through January 15, 1973. All of these data are published in regular journals or as rather complete reports as contributions to various international conferences. Nine journals are searched regularly for data. These are listed below along with an indication of the last issue searched for data included in this index. These are the issues that had been received in the National Bureau of Standards Library by January 15, 1973. Nuclear Science Abstracts is used to find papers published in those journals not regularly searched.

Journal
Physical Review, The
Physical Review Letters
Nuclear Physics
Physics Letters
Soviet J. of Nuclear Physics
Il Nuovo Cimento
Il Nuovo Cimento Letters
J. Physical Society Japan
Zeitschrift für Physik
Nuclear Science Abstracts

3.2. Scope of the Data Index

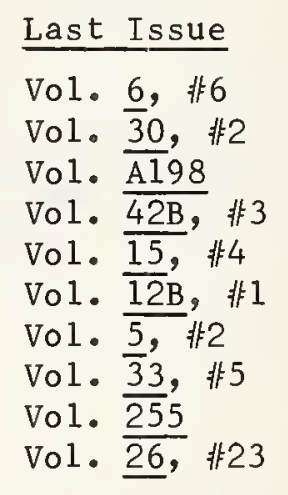

The function of this data index is to furnish a means of readily locating experimental data in the field of photonuclear reactions. This index differs from a bibliography in that it supplies quantitative information about the content of a paper. Each entry in the index corresponds to the measurement of a specific photonuclear reaction for a specific nuclide or group of nuclides. An attempt is made to give as complete a description of each measurement as is possible in a single line. The type of measurement is indicated as well as the range of excitation energies covered, the type of detector used and its energy response, and the type of angular distribution data obtained. The object has been to give a description of each measurement that is complete enough to permit an individual looking for specific types of data in the field to locate the pertinent references without having to go through a large number of irrelevant papers as might be the case if only the title of the paper or the reaction studied were listed for each entry.

For the purposes of this index, the general criterion as to what constitutes a measurement of photonuclear data is that the measurement must give information on the electromagnetic matrix element between the ground state and excited states of some nucleus. The most common type of reactions are: $\left(e, e^{\prime}\right),(\gamma, \gamma),(\gamma, n),(\gamma, p)$, and $\left(p, \gamma_{0}\right)$. Two reactions which fit the matrix element criterion, but which were not included in the compilation because of their rather special nature are heavy particle Coulomb excitation and the thermal neutron capture reaction $\left(n, \gamma_{0}\right)$. While the energy region of particular interest extends from 0 to $150 \mathrm{MeV}$, some papers are indexed which report measurements in the region from $150 \mathrm{MeV}$ to $1 \mathrm{GeV}$. Most of the experiments listed are concerned with the excitation energy range 8 to $30 \mathrm{MeV}$, the region of the photonuclear giant resonance.

\subsection{Description of the Index}

The data index for each element begins with the isotopic abundances for that element and a list of separation energies for each isotope. The abundances were taken from a compilation by Gladys Fuller [10]. This reference should be consulted for remarks concerning the accuracy of these values and possible variations with the source of the sample. The separation energies were taken from the tabulation of Wapstra and Gove [11] which were based on their 1971 Atomic Mass Evaluation. The values given in Ref. [11] have been arbitrarily rounded off to the nearest $0.1 \mathrm{MeV}$ except for those cases where the uncertainties 
quoted are of the order of $1 \mathrm{MeV}$. In these cases, thresholds are given to the nearest MeV.

In the data index which follows, eight columns are used to describe a reaction reported in a specific paper. Where for clarification purposes a brief remark has been included in the index entry for a specific reaction, this remark is listed on the line immediately below the line giving the main index entry. The purpose of this remark is to give additional information that will make the index entry more useful. For example, pertinent energies are more exactly defined, additional information is given on polarization or alignment experiments, residual nuclei are identified, etc. The additional information is selected in a fairly unsystematic way and is limited by the available space. The entries should not be regarded as exhaustive or consistent. The headings of the eight principal columns used for an index entry are: REF (Bibliographic Reference Number), NUCLIDE, REACTION, RES (Result), EXCIT (Excitation Energy), SOURCE, DETECTOR, and NUM (Acquisition Number). These headings are described more completely in the following section.

Under a given element, the index entries are grouped so that those listed jirst are for measurements made on the element, i.e., on samples with naturally occurring isotopic abundances. Following this, measurements pertaining to the various isotopes of an element are listed together. The entries for a given element or isotope are ordered by reaction according to a priority listing of, first, the incoming particle, and second, the outgoing particle. All entries for a given reaction are listed chronologically.

\subsection{Description of Column Heałings}

An alphabetical list of the symbols used under the various columns of the data index is given in Section 6 of this report. The purpose of this section is to describe the meanings of the various column headings.

Column $1 \quad$ REF

Column 2 NUCLIDE

Column 3

REACTION

Column 4

RES

Column 5

EXCIT
Bibliographic Reference Number. This number is made up of the year and the first two letters of the first author's name, plus an additional serial number.

The atomic number ( $Z$ ), chemical symbol, and mass number (A) of the excited nucleus (not necessarily the target nucleus) is given. The mass number is listed only if the isotopic assignment is unambiguous. In general, it was assumed that the mass number was unambiguous if in the target the abundance of a single isotope was $>97 \%$.

The notation used is the usual one. Where necessary, a remark is often used to more fully define some of the more esoteric reactions. The notation $\mathrm{E}, \mathrm{E} / \mathrm{P}$ means the inelastically scattered electron and proton were detected in coincidence in a reaction of the type: $A+e \rightarrow(A-1)+e^{\prime}+p$, while $E$, P means that only the proton was detected. Where the measurement involved the polarization or alignment of either the incoming beam, the target nucleus, or the outgoing particle, a $\$$ is listed to the left of the column.

Result, i.e., the type of information given in the paper.

Excitation Energy Range. The excitation energy range of the nucleus involved in the gamma-ray transition is given in $\mathrm{MeV}$. For reactions initiated by gamma rays, the excitation energy is taken as the ganma-ray energy; for reactions initiated by particles, the binding energy and kinematic corrections are made. The abbreviation THR stands for threshold. Where the 
Column 5

EXCIT

(continued)

Column 6

SOURCE

Column 7

Column 8

NUM scale 1-999 MeV is inappropriate for a measurement, 999 is entered and the actual energy given under REMARKS.

Source Type and Energy Range. The source of incident particles is characterized by the letter $C$ or $D$ indicating that the source was continuous or discrete in energy. The source energy is indicated under MIN-MAX. The usual source of photons is bremsstrahlung which would be marked $C$. The range of endpoint energies is given.

DETECTOR Detector Type, Energy, and Angular Range. The symbols used to indicate the detector used in measurement are defined in the list given in Section 6. The letter $D$ or I under TYPE means that the reaction product was detected differentially or integrally in energy. For example, a scintillator (SCI) is usually used differentially (D) while a $\mathrm{BF}_{3}$ detector (BF3) always integrates over neutron energy (I). The range of detected particle angular distribution is shown under ANG. A single number in the column means the measurement was made at this angle (given in degrees) only. DST means that the measurement was made at two or more angles and $4 \mathrm{PI}$ indicates that the detector used essentially integrated over all outgoing particle directions.

Acquisition Number. This is the number used to identify cross section data that are available in digital form from the Photonuclear Data Center's library. When an acquisition number is followed by a + , digitized data from the paper in question are available for quantities which are normally not indexed, e.g., a $\sigma(S N)$ cross section curve. 
4. Photonuclear Data Index 1955-1972 
HYOROGEN $Z=1$

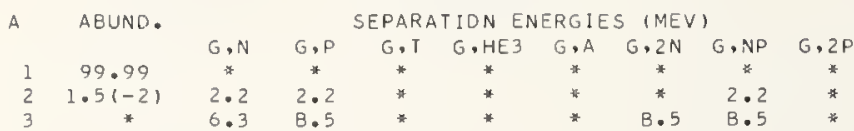

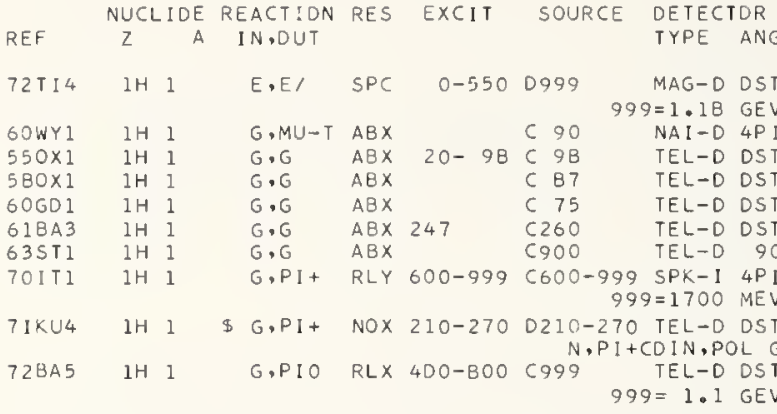

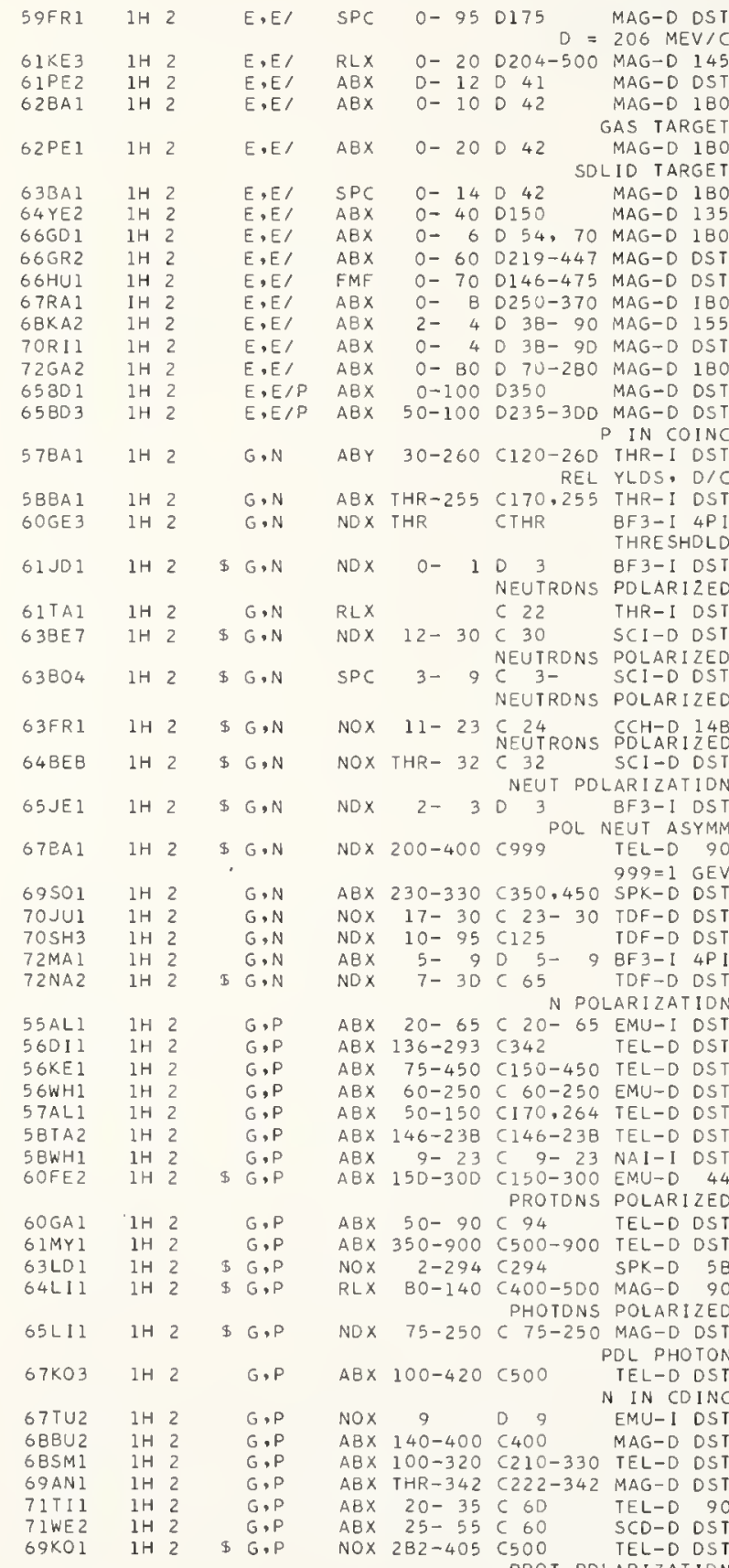
PROT POLARIZATIDN
REF

NUCLIDE REACTION RES EXCIT SOURCE DETECTDR

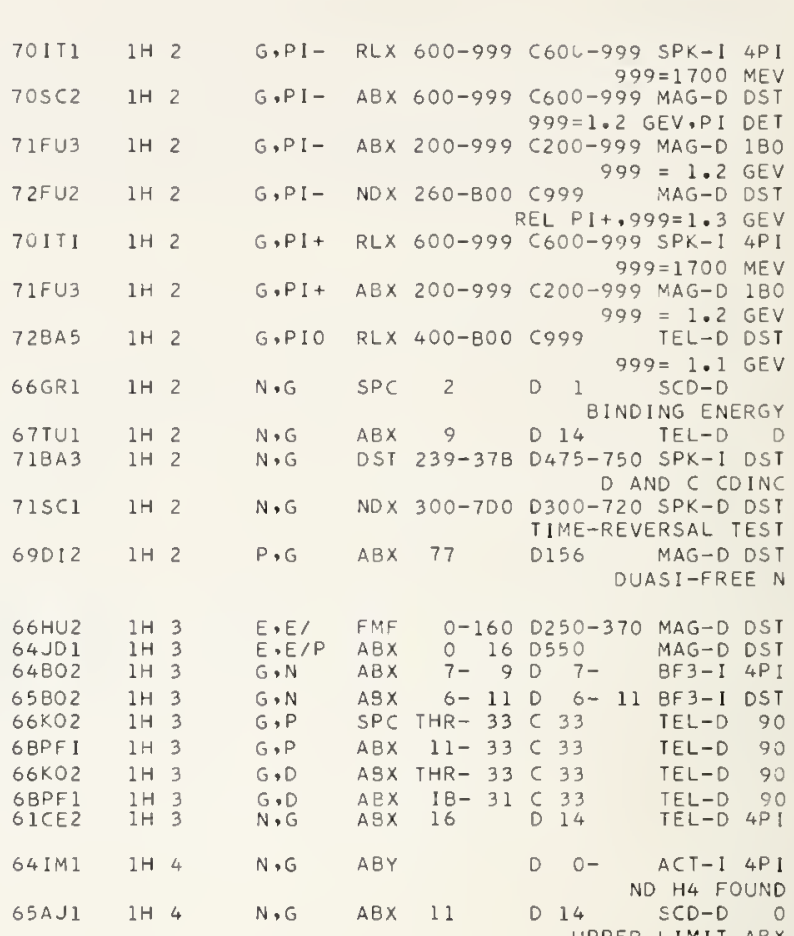

HELIUM $Z=2$

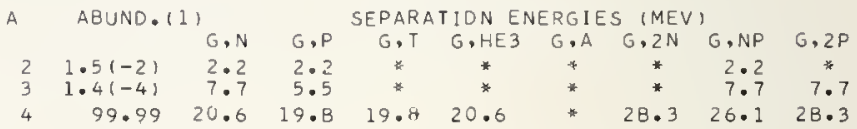

RE

NUCLIDE REACTIDN RES EXCIT SOURCE DETECTOR $Z$ A IN,DUT TYPE ANG NUM

\begin{tabular}{|c|c|c|c|c|c|c|c|c|c|}
\hline $66 \mathrm{HU2}$ & $2 \mathrm{HE}_{3}$ & & $E, E /$ & $A B X$ & $0-150$ & $0250-670$ & $S C I-D$ & DST & \\
\hline $67 F R 1$ & $2 \mathrm{HE} 3$ & & $E, E /$ & $A B X$ & $0-17$ & 020.3 & MAG-D & 60 & \\
\hline $69 \mathrm{CHI}$ & $2 \mathrm{HE} 3$ & & $E, E /$ & $A B X$ & $5-20$ & D 57 & $M A G-D$ & 180 & \\
\hline $69 \mathrm{CH}_{2}$ & $2 \mathrm{HE} 3$ & & $E, E /$ & SPC & $0-14$ & D 56 & MAG-D & 180 & \\
\hline $64 \mathrm{JO} 1$ & $2 \mathrm{HE} 3$ & & $E, E / P$ & $A B X$ & $0-12$ & D550 & MAG-D & DST & \\
\hline $71 K \cup 3$ & $2 \mathrm{HE} 3$ & & $E, D$ & A $9 x$ & $13-45$ & D B6 & $M A G-D$ & DST & \\
\hline $725 \times 1$ & $2 \mathrm{HE} 3$ & & $E, D$ & RLX & $16-27$ & $\begin{array}{l}D \quad 43-87 \\
\text { DETECTE }\end{array}$ & $\begin{aligned} & M A G-D \\
= & D \quad D A N\end{aligned}$ & $\begin{array}{l}\text { DS T } \\
\text { VD P }\end{array}$ & \\
\hline 64604 & $2 \mathrm{HE} 3$ & & $G \cdot N$ & $A B X$ & $5-170$ & $\mathrm{C} 170$ & $\mathrm{CCH}-\mathrm{D}$ & DST & \\
\hline $\begin{array}{l}65 \mathrm{FEl} \\
66 \mathrm{GEI}\end{array}$ & $\begin{array}{l}2 \mathrm{HE} 3 \\
2 \mathrm{HE} 3\end{array}$ & & $\begin{array}{l}G, N \\
G, N\end{array}$ & $\begin{array}{l}A B X \\
A B X\end{array}$ & $\begin{array}{l}\text { THR-170 } \\
\text { THR- } 30\end{array}$ & $\begin{array}{l}\text { CI70 } \\
\text { CTHR- } 30\end{array}$ & $\begin{array}{l}\mathrm{CCH}-\mathrm{D} \\
\mathrm{BF} 3-\mathrm{I}\end{array}$ & $\begin{array}{l}\text { DST } \\
\text { 4PI }\end{array}$ & $\begin{array}{l}70+ \\
63\end{array}$ \\
\hline 66VA3 & $2 \mathrm{HE} 3$ & & $G \cdot N$ & $A B X$ & $T H R-170$ & $C 170$ & $\mathrm{CCH}-\mathrm{D}$ & $4 \mathrm{PI}$ & \\
\hline $70 B E 6$ & $2 \mathrm{HE} 3$ & & $G, N$ & $A B X$ & $8-30$ & $B-30$ & $M O D-1$ & $4 \mathrm{PI}$ & 237 \\
\hline \multirow[t]{2}{*}{$64 B E 5$} & $2 \mathrm{HE} 3$ & & $G, 2 P$ & $A 8 x$ & B 22 & $B-22$ & $S C I-D$ & 90 & \\
\hline & & & & & & \multicolumn{4}{|c|}{ FDRM FACTDR } \\
\hline $65 S T 1$ & $2 \mathrm{HE} 3$ & & $G, 2 P$ & $S P C$ & $9-40$ & C 40 & $S C D-D$ & 90 & \\
\hline $65 \mathrm{VAl}$ & $2 \mathrm{HE} 3$ & & $G, 2 P$ & $A B X$ & $T H R-170$ & $C 170$ & $\mathrm{CCH}-\mathrm{D}$ & DST & \\
\hline $638 E 1$ & $2 \mathrm{HE} 3$ & & $G, D$ & $A B X$ & $B-20$ & C 22 & $5 C 1-0$ & 90 & \\
\hline $63 F=2$ & $2 \mathrm{HE} 3$ & & $G, D$ & $A B X$ & $13-27$ & C 31 & $S C I-D$ & 90 & \\
\hline 3602 & $2 \mathrm{HE} 3$ & & $G \cdot D$ & $A B X$ & $5-90$ & $(170$ & $\mathrm{CCH}-\mathrm{D}$ & 4P I & \\
\hline & & & & & & & ALSO & $G, N$ & \\
\hline $648 E 2$ & $2 \mathrm{HE} 3$ & & $G \cdot D$ & $A B X$ & $T H R-3 D$ & C 34 & $S C D-D$ & 90 & \\
\hline & & & & & & & \multicolumn{3}{|c|}{ SPECTRUM } \\
\hline $64 \mathrm{GO} 1$ & $2 \mathrm{HE} 3$ & & $G, D$ & $A B X$ & $T H R-120$ & C175 & $\mathrm{CCH}-\mathrm{D}$ & DST & \\
\hline $65 \mathrm{STI}$ & $2 \mathrm{HE} 3$ & & $G, D$ & $A B X$ & $9-46$ & $c 40,55$ & $S C D-D$ & 90 & 64 \\
\hline 63WAI & $2 \mathrm{HE} 3$ & & $G \cdot D$ & $A B X$ & 6,7 & D 6,7 & $10 N-D$ & $4 \mathrm{PI}$ & \\
\hline $64 B E 5$ & $2 \mathrm{HE} 3$ & & $G, D$ & $A B X$ & $B-22$ & $B-22$ & SCI $-D$ & 90 & \\
\hline & & & & & & \multicolumn{4}{|c|}{ FDRM FACTOR } \\
\hline $64 G 04$ & $2 \mathrm{HE}_{3}$ & & $G, D$ & $A B X$ & $5-170$ & $\cos 70$ & $\mathrm{CCH}-\mathrm{D}$ & DST & \multirow{8}{*}{$65+$} \\
\hline $65 \mathrm{FE} 1$ & $2 \mathrm{HE} 3$ & & $G \cdot D$ & $A B X$ & THR- 170 & C170 & $C C H-D$ & DST & \\
\hline $65 \mathrm{VAl}$ & $2 \mathrm{HE} 3$ & & $G, D$ & $A B X$ & THR-170 & $\cos 70$ & $\mathrm{CCH}-\mathrm{O}$ & DST & \\
\hline $66 \vee \mathrm{VA} 3$ & $2 \mathrm{HE} 3$ & & $G \cdot D$ & $A B X$ & THR -170 & $\operatorname{ci} 70$ & $\mathrm{CCH}-\mathrm{D}$ & $4 \mathrm{PI}$ & \\
\hline $69 P 13$ & $2 \mathrm{HE} 3$ & & $G \cdot D$ & $A B X$ & $200-500$ & $C 999$ & TEL-D & 90 & \\
\hline & & & & & & & $999=1$ & GEV & \\
\hline 70803 & $2 \mathrm{HE} 3$ & & $G, D$ & NOX & $16-26$ & C 33 & $S P K-1$ & DST & \\
\hline $72 \mathrm{FA} 3$ & $2 \mathrm{HE} 3$ & $\Phi$ & $G \cdot D$ & NOX & $170-290$ & $0170-290$ & $T E L \rightarrow D$ & 90 & \\
\hline & & & & & & POL & 1 PARAM & $M$ G. & \\
\hline $720 F 2$ & $2 \mathrm{HE} 3$ & & $G \cdot D$ & $A B X$ & $40-150$ & $C 250$ & $\begin{array}{l}\text { TEL }-D \\
\text { ND D C }\end{array}$ & DST & \\
\hline
\end{tabular}




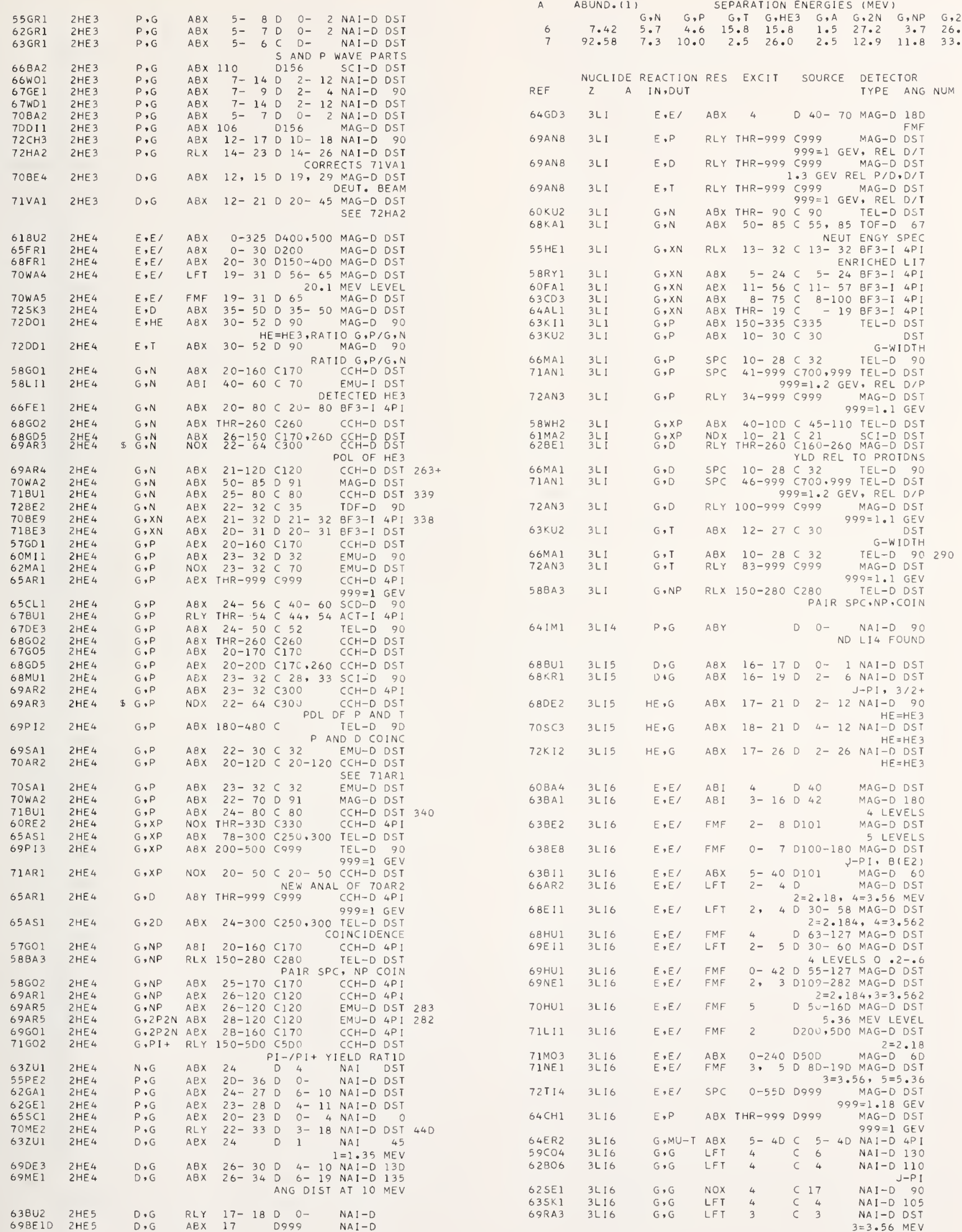




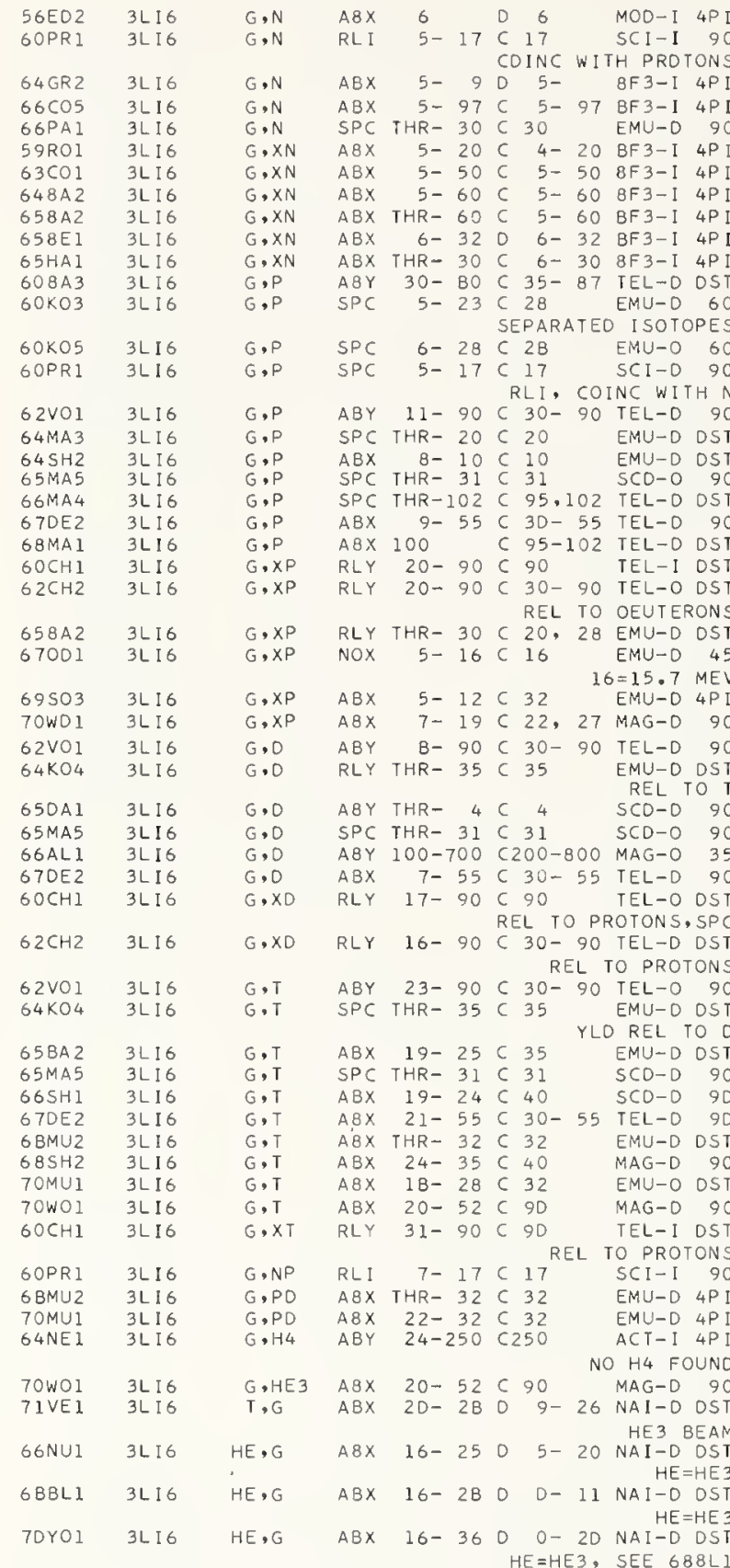

638A1 3LI7 E,E/ ABI $7-14042$ MAG-D 180

S3BE3 3LI7 E,E/ FMF 4- 8 DIDO-1BD MAG-D DST

63BE8 3LI7 E,E/ FMF D- 7 D100-180 MAG-O DST

$64 B 14$ 3L17 E,E/ ABX 1- 60150 J-PI, B BEL

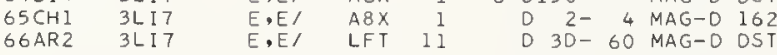
69HU1 3LI7 E,E/ FMF O- 26 D $55-127$ MAG-D DSI

7IVA3 3LI7 E,E/ LFT 1 D 25-90 MAG-O DST

7OWOL 3LI7 E,P A8X 6- 20 C $21-90$ MAG-D 90

SBBE3 3LI7 G, G LFT I C I NAI-D 120

59SWI 3LI7 G,G LFT I D 1 NAI-D DST

60BO3 3LI7 G,G LFT D $\quad$ C 2 NAI-D 100

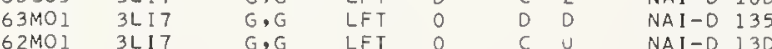

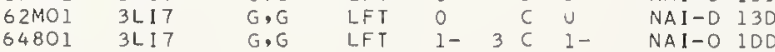

64GR2 3LI7 G,N A8X THR- 11 DTHR- 11 BF3-I 4 P

67BA2 3LI7 G,N ABX THR- 50 C 7-50 8F3-I 4PI

$59 R O 1$ 3LI7 G,XN ABX B- $20 \mathrm{C} 4-208 F 3-0$ 4PI

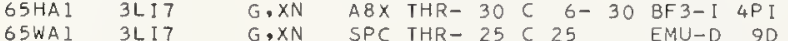

$\begin{array}{llllllll}\text { 65WAI } & \text { 3LI7 } & G, X N & \text { SPC THR- } 25 & C & 25 & \text { EMU-D } & 90 \\ \text { 69GAI } & \text { 3LI7 } & \$ \text { G, XN } & \text { SPC } & 2-B 5 & C & 85 & \text { CCH-D OST }\end{array}$

696A3 3LIT G,XN SPC $8-85685$ POLARIZATION

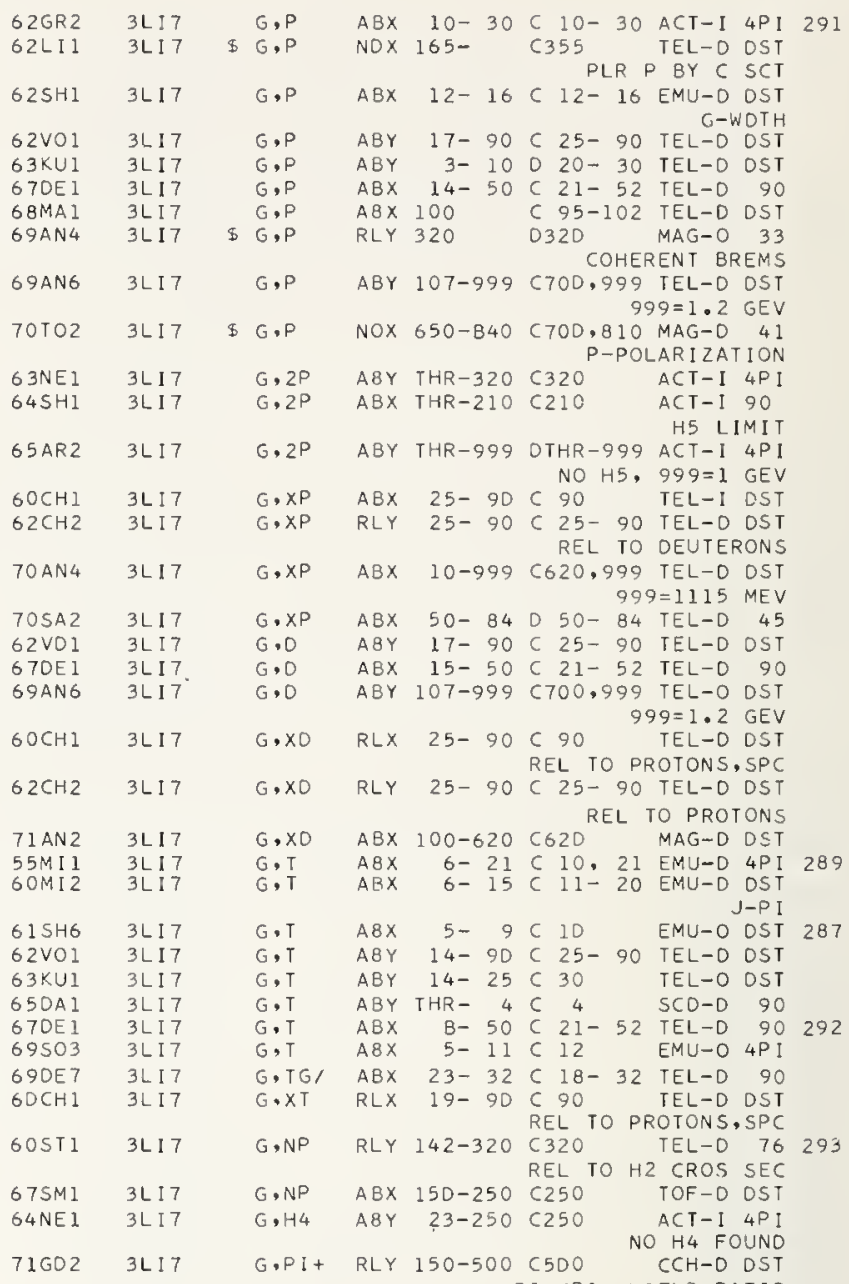

PI-/PI+ YIELO RATIO

BERYLLIUM $\quad Z=4$

ABUND.

A ABPARATION ENERGIES (MEV)
G,N G,P G,T G, HE3 G,A G, $N$ G,NP G, $P$ NUCLIDE REACTION RES EXCIT SOURCE DETECTOR NUCLIDE REACTION RES EXCIT SOURCE DETECTOR
TYPE ANG NUM 69NA2 4 BE7 A,G A8X $1-2$ D D- 1 NAI-D 90 $\begin{array}{lllllllll}\text { S9GEI } & 4 B E B & P, G & \text { ABX } & 21-25 & 0 & 4- & \text { NAI-D } & 90 \\ \text { GDMA2 } & 48 E 8 & \text { P, G } & \text { RLY } & 17-1 B & 0 & C- & \text { NAI-D OST }\end{array}$

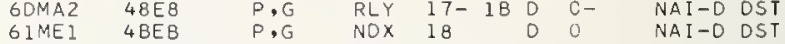

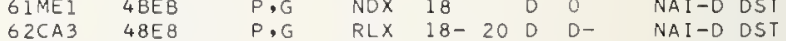
$63 M I 1 \quad 4 B E B$ P, G RLX $19-26 \quad 0$ 2- NAI-D DST 63PE1 $48 E 8$ P, G RLX 18- 270 D $1-11$ NAI-O OST 63REI 4BEB P, G ABX 19- 260 4- NAI-D DST

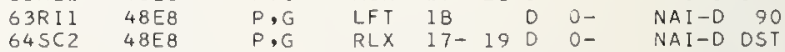

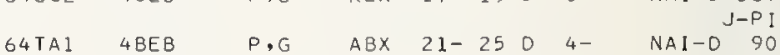
67BL2 48E8 P, G RLX $17-34001-1 B$ NAI-D 90 67NII 4BEB P,G RLY 1B- 19 D 1- 3 NAI-D DST

608A4 48E9 E,E/ ABI 2 D 42 MAG-D 160 G2ED1 $48 E 9$ E,El SPC D- 20042 TEL-D 180 63NGI 4 BE9 E,E/ LFT $1-6$ D $60-250$ MAG-D DST

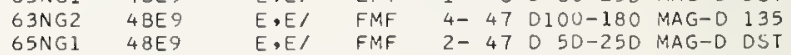
G6AR2 48E9 E,E, LFT $16 \quad 2.47,6.4 \mathrm{MEV}$, CONT S6CL1 $48 E 9 \quad 16=15.97 \mathrm{MEV}$ 67BE1 4BE9 E,E/ FMF 2 O340 MAG-D DST 6BVA1 $48 E 9$ E,E/ ABX $0-26042-6 B$\begin{tabular}{rl}
$2=2.43$ & $M E V$ \\
\hline
\end{tabular} $65 A M 3$ 4BE9 E,E/P RLY B- 6D D510-590 MAG-D 51 
$58 B A 2$ 4BE9 E,N ABX 2- 16 C 2- 16 BF3-I 4PI

$598 A 3 \quad 48 E 9$ E,N ABY THR- 36 D IU- 36 WITH G,N

6BBO2 4BE9 E, XXX RLY THR-119 D119 36 MAG-D 76

$X X X=M A S S$ SPECTRUM

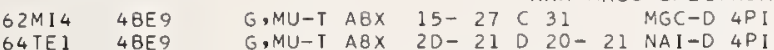

$64 T E 1$ 4BE9 G,MU-T ABX 2D- 21 D 20-2I NAI-D 4PI

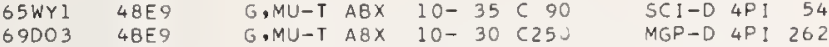

71AHI 4BE9 G, MU-T ABX THR-150 C $10-150$ MGC-D 4PI

64LO3 4 BE9 G,G ABX 10-30 C ID- 30 NAI-D 140

67LOI 4 BE9 G,G/ A8X $12-30$ C 34 NAI-D DST

$55 \mathrm{JOI} \quad 48 E 9$ G,N RLY $12-65 C 65$ SCI-D DST

$56 E D I \quad 48 E 9 \quad G, N \quad A B \times \quad 2-8 D 2-\quad M O D-1 \quad 4 P I$

$56 F A 148 E 9 \quad G, N$ NOX THR- $18 \mathrm{C} 18$ SCI-I DST

58ASI $48 E 9 \quad G, N$ NOX 2- 17 C 17 SCI-I DST

$588 A 2 \quad 48 E 9 \quad G, N$ ABX 2- $16 C 2-168 F 3-14 P I$

$596 I 148 E 9$ G, N ABX 1 COMPARED WITH E, N

$59 \mathrm{THI} \quad 48 E 9 \quad G, N$ ABX 6- $18 \mathrm{C}$ 6- 18 BF 3-1 $4 \mathrm{PI}$

60KU2 $48 E 9$ G, N RLY $12-90 \mathrm{C} 90$ TEL-I DST

6IJAI 4 BE9 G,N ABX THR- 5 CTHR- $8 F 3-14 P I$

$62 B 04$ 4BE9 \$ G.N NOX 3 D 3 8F3-I DST

$6280748 E 9$ G,N NOX $5-905-90$ NEUTRONS POLARIZED
$62501-I D S T$

$62 \mathrm{CUI} 4 \mathrm{BE9}$ G,N ABX $4-32 \mathrm{C} 32$ EMU-D DST

$62 J 02 \quad 48 E 9$ GN ABX $1-1$ D $1-$ MOD-I $4 P 1$

$63804 \quad 4 B E 9$ \$G,N ABX 3- 9 D 5-

$64 C O 14 B E 9 \$ G, N$ NOX $3 \quad$ NEUTRONS POLARIZED

$64 K 0548 E 9 \quad G, N$ RLY $15-32 C 35$ NEUTRONS POLARIZED

$66 C 04 \quad 48 E 9 \quad G, N \quad A B I \quad 6-80 C$ C $6-80$ BF $3-1$ 4PI

66DE3 $48 E 9 \quad G, N \quad$ SPC THR- B5 C 85 CCH-D 135

66THI 4BE9 G,N SPC THR- $17 \mathrm{C} 17$ SCI-D 90

GBKAI $48 E O$ GON ABX $50-85 C 55,05$ TOF D 67

NEUT ENGY SPEC

G4AR2 $46 E 9$ G.2N RLY $21-57 \mathrm{C} 44,57$ ACT-I 4 PI

67GA1 48E9 G,2N ABY THR- 23 C REL TOCI2(G,N)

$55 B A 5$ 4BE9 G,XN ABY $30-200$ C $150-250$ THR-I DST

55DII 48E9 G.XN NOX $2-70 C 70 \quad$ SCI-I DST

59KU1 4BE9 G,XN NOX $12-88 C 8 B$ TEL-I DST

GOBEI $48 E 9 \quad G \times X N$ ABX $2-17$ C 5- 17 TOF-D DST

64AL5 $46 E 9 \quad G, X N$ NOX THR- $34 C 34 \quad$ THR-I DST

$65 \mathrm{CO} 2 \quad 48 \mathrm{E} 9 \mathrm{G}, X \mathrm{~N}$ AB! $6-80 \mathrm{C} 6-80$ 8F3-I $4 \mathrm{PI}$

N POLARIZATION

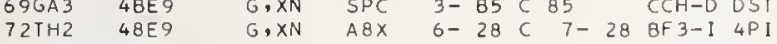

$56 C O 148 E 9$ GP ABY $19-24 C 24$ EMU-D 90

$58 \mathrm{CHI} \quad 48 \mathrm{E} 9 \mathrm{G}, \mathrm{P}$ SPC THR- $44 \mathrm{C} 44$ EMU-D DST 6 ENERGY GROUPS

$62 C L 1 \quad 46 E 9$ G,P ABX 16-54C 16-54 ACT-I 4PI

$62 C U 1$ GBE9 G.P ABX $16-28 C 32$ EMU-D DST

62LII 4BE9 G GP NOX 190 C355 TEL-D DST

$62 \mathrm{VO2} 4 \mathrm{BE9} G . P$ ABX $40-90$ PROTONS POLARIZED

$63 K I 1$ 4BE9 G,P NOX THR $-335 C 335$ TEL-D DST

$64 K O 5 \quad 48 E 9 \quad G, P$ ABX $15-32 C 35$ MAG-D DST

$65 K O 2$ GBE9 G,P ABX $18-31 C 35$ EMU-D DST

660E6 $48 E 9 \quad G, P$ ABX THR- 50 C $2 J-50$ ACT-I $4 P I$

66DEG 48E9 G,P ABX THR- 50 C 20-50 TEL-D 90

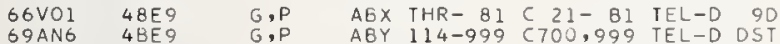

7IANI 4BE9 G,P SPC $51-999$ C700.999 TEL-D DST

$999=1.2$ GEV, REL D/P

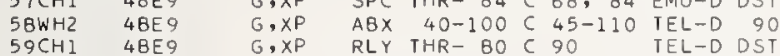

$60 \mathrm{CHI} 48 \mathrm{E} \quad \mathrm{G}, \times P$ RLY $30-90 \mathrm{C} 90^{\text {REL D TO P YLD }}$

REL TO DEUTERONS

$A B \times \quad 4 D-90$ C $40-90$ MAG-D DST

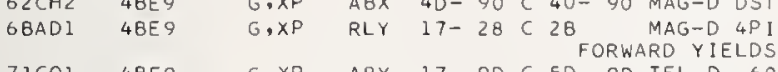

$71 C O 1$ 4BE9 G,XP ABY $17-90$ C 5D-9D TEL-D 60

62BAI 4BE9 G.D ABY 4D- 60 C 45- 62 ACT-I $4 P I$

$64 K 05$ 48E9 G,D RLY $15-32 C 35$ MAG-D DST

66DE6 $48 E 9$ G.D ABX THR- 5D C $20-50$ TEL-D 90

$66 V 01 \quad 4 B E 9 \quad G, D \quad A B X$ THR- 81 C $21-61$ TEL-D $9 D$

69AN6 4BE9 G,D ABY $114-999$ CTDU,999 TEL-D DST $999=1.2$ GEV

$71 A N 1$ 4BE9 G,D SPC $55-999$ C700,999 TEL-D DST

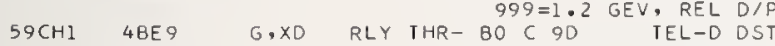

$60 \mathrm{CH} 148 E 9$ G,XD RLY $19-90 \mathrm{C} 90^{\text {REL D TO P YLD }}$

$62 \mathrm{CH} 248 E 9 \quad 6 . \times D$ ABX $40-90 \mathrm{C} \quad$ REL TO PROTONS

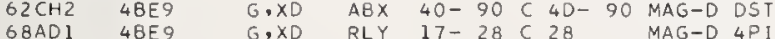

7IAN2 4 BE9 G, XD ABX $107-620 \mathrm{C} 620^{\text {FORWARD YIELDS }}$

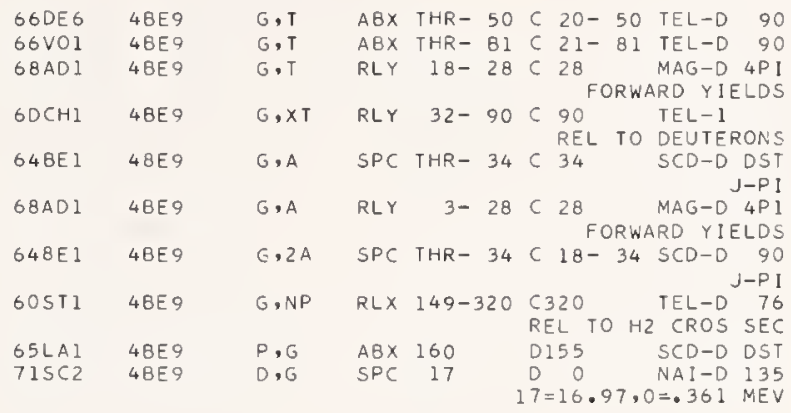

$B O R O N \quad Z=5$

A ABUND. (1) SEPARATION ENERGIES (MEV)

$10 \quad \begin{array}{lllllllll} & \text { G.N } & \text { G.P } & \text { G.T G,HE3 } & \text { G.A } & \text { G.2N } & \text { G.NP } & \text { G. } 2 P\end{array}$

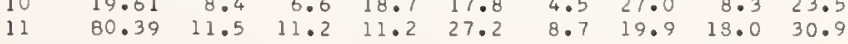

NUCLIDE REACTION RES EXCIT SOURCE DETECTOR

A IN,OUT

TYPE ANG NUM

\begin{tabular}{|c|c|c|c|c|c|c|c|c|}
\hline 2001 & 58 & & $E, P$ & RLY & 30 & & c 30 & $M A G-D$ \\
\hline $\begin{array}{l}59 C O 5 \\
\text { SORE }\end{array}$ & $\begin{array}{l}58 \\
58\end{array}$ & & $\begin{array}{l}G, G \\
G, G\end{array}$ & $\begin{array}{l}A B 1 \\
A B \times\end{array}$ & $\begin{array}{l}5 \\
7\end{array}$ & 7 & $\begin{array}{ll}\text { C } & 5, \\
D & 7\end{array}$ & $\begin{array}{lr}\text { NAI }-D & 130 \\
\text { NAI }-D & 90\end{array}$ \\
\hline 2 SE 1 & 58 & & $G \cdot G$ & RLY & $0-$ & 13 & $C 13$ & NA I-D 90 \\
\hline 801 & 58 & & G, G & LFT & $1-$ & 3 & $1-$ & $\begin{array}{rl}N A I-D & 100 \\
A B I\end{array}$ \\
\hline BKAl & 58 & & $G, N$ & $A B X$ & $50-$ & 85 & $\begin{array}{r}55,85 \\
\text { NEUT }\end{array}$ & $\begin{array}{l}\text { TOF-D } 67 \\
\text { ENGY SPEC }\end{array}$ \\
\hline $3 \mathrm{CO}$ & 58 & & $G, \times N$ & $A B X$ & THR- & 60 & CTHR- 80 & $\mathrm{BF} 3-\mathrm{I} \quad 4 \mathrm{PI}$ \\
\hline $1 C 02$ & 58 & & $G, \times N$ & $A B I$ & $36-$ & 64 & $\begin{array}{r}C 10-64 \\
\text { FAS }\end{array}$ & $\begin{array}{l}B F 3-I \text { 4PI } \\
\text { ST } N \text { YIELD }\end{array}$ \\
\hline $\mathrm{OCH}$ & 58 & & $G . X P$ & RLY & THR- & 90 & $\begin{array}{l}\text { C } 90 \\
\text { REL TO }\end{array}$ & $\begin{array}{l}\text { TEL-I } 90 \\
\text { DEUTERONS }\end{array}$ \\
\hline $3 \times 11$ & 58 & & $G, \times P$ & $A B X$ & THR- & 355 & C 335 & TEL-D DST \\
\hline $\mathrm{OCHI}$ & 58 & & $G \times D$ & RLY & THR - & 90 & $\begin{array}{c}\text { C } 90 \\
\text { REL T }\end{array}$ & $\begin{array}{l}\text { TEL-1 } 90 \\
\text { TO PROTONS }\end{array}$ \\
\hline $\mathrm{OCHl}$ & 56 & & $G, \times T$ & RLY & THR - & 90 & $\begin{array}{l}\text { C } 90 \\
\text { REL TO }\end{array}$ & $\begin{array}{l}\text { TEL-I } 90 \\
\text { DEUTERON }\end{array}$ \\
\hline $7 G A 1$ & 58 & & G,BE 7 & RLY & THR - & 23 & C 23 & $A C T-1 \quad 4 \mathrm{PI}$ \\
\hline 6PA4 & 56 & B & $P, G$ & $A B X$ & $1-$ & 2 & $1-\quad 2$ & $S C D-D \quad 2 P I$ \\
\hline $2 E D 1$ & 58 & 10 & $E, E /$ & $A B X$ & $0-$ & 20 & D 42 & $M A G-D 1 B 0$ \\
\hline $5 F R 2$ & 56 & 10 & $E, E /$ & FMF & 6 & & $0100-220_{6}$ & $\begin{array}{r}\text { MAG-D DST } \\
6=6.02 \text { MEV }\end{array}$ \\
\hline SSP1 & 58 & 10 & $E, E /$ & FMF & 7 & & $\begin{array}{l}\text { D } 55 \\
\text { WIDTH, }\end{array}$ & $\begin{array}{l}\text { MAG-D DST } \\
7.48 \mathrm{MEV}\end{array}$ \\
\hline $6 \mathrm{KO} 1$ & 58 & 10 & $E, E /$ & $A B X$ & $0-$ & 18 & $D 50,60$ & $\begin{array}{c}\text { MAG-D } 180 \\
8 \text { LEVELS }\end{array}$ \\
\hline 6SPI & 58 & 10 & $E, E /$ & $A B X$ & 6 , & 7 & $\begin{array}{r}32-57 \\
6,7=6.01\end{array}$ & $\begin{array}{l}\text { MAG-D DST } \\
1.7 .47 \text { MEV }\end{array}$ \\
\hline $4 \mathrm{LO}_{3}$ & 8 & 10 & $G, G$ & $A B X$ & $10-$ & 30 & C $10-30$ & NAI-D 140 \\
\hline 7L01 & 6 & 10 & $G, G /$ & $A B X$ & $12-$ & 30 & C 34 & NAI I D DST \\
\hline $4 G R 2$ & 58 & 10 & $G, N$ & $A B X$ & $9-$ & 11 & D 9-11 & $\begin{array}{l}\text { BF 3-I } 4 \text { PI } \\
\text { WIDTH }\end{array}$ \\
\hline $5 \mathrm{HA} 1$ & 8 & 10 & . XN & $A B X$ & THR- & 30 & $6-30$ & $B F 3-1 \quad 4 \mathrm{PI}$ \\
\hline $2 \mathrm{VO} 2$ & 8 & 10 & $G, P$ & $A 8 x$ & $23-$ & 90 & C 90 & SCI-D 90 \\
\hline $85 H 6$ & B & 10 & $G, P$ & $A B X$ & $6-$ & 13 & $C 13$ & $\begin{array}{l}\text { EMU-D DST } \\
3=12.5 \text { MEV }\end{array}$ \\
\hline $2 \mathrm{CH} 2$ & 8 & 10 & $G, X P$ & $18 x$ & $23-$ & 90 & c $30-90$ & TEL-D 90 \\
\hline $2 \mathrm{VO} 2$ & $B$ & 10 & , 0 & $8 x$ & $25-$ & 90 & C 90 & SC1-D \\
\hline $2 \mathrm{CH} 2$ & 58 & 10 & .XD & $8 x$ & $22-$ & 90 & C $30-90$ & TEL-D \\
\hline 2ELI & 58 & 10 & $P, G$ & NOX & $7-$ & 8 & D & $\begin{array}{r}N A I-D \\
J-P I I\end{array}$ \\
\hline $3 F \cup 3$ & 58 & 10 & $P, G$ & $A B X$ & $7-$ & 9 & $\begin{array}{ll}\text { D } & 1- \\
& G-W D T H\end{array}$ & $\begin{array}{l}N A !-D \text { DST } \\
H, J-P I, \quad T\end{array}$ \\
\hline $1 L 13$ & 58 & 10 & $T, G$ & LFT & 18, & 19 & $\operatorname{J-PI}_{1} 1-10^{2}$ & $\begin{array}{r}\text { SCD - D DST } \\
4,19.3 \text { MEV }\end{array}$ \\
\hline 5PAI & 56 & 10 & $E, G$ & $A B X$ & $18-$ & 19 & $D-3$ & $\begin{array}{r}N A I-D \text { DST } \\
H E=H E 3\end{array}$ \\
\hline BL I & 58 & 10 & $E \cdot G$ & $A B X$ & $21-$ & 27 & $3-6$ & $\begin{array}{r}\text { NAI }-D \text { DST } \\
H E=H E 3\end{array}$ \\
\hline $6 \mathrm{FO}$ & $5 B$ & 10 & $A, G$ & $S P C$ & $5-$ & 7 & D $1-3$ & $N A !-D \quad 90$ \\
\hline 25 & & 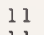 & , É & $A B X$ & 0 & 20 & D 42 & $-D \quad 180$ \\
\hline $48 R 2$ & & 11 & E, El & SPC & $8-$ & 9 & D 54 & $M A G=D \quad 141$ \\
\hline GAR2 & 58 & 11 & $E, E /$ & LFT & 4, & 5 & $\begin{array}{l}D 3 D-60 \\
4=4.46,5\end{array}$ & $\begin{array}{l}M A G-D \text { DST } \\
5=5.04 \mathrm{MEV}\end{array}$ \\
\hline $6 \mathrm{KO}$ & 58 & 1. & $E, E /$ & $A B X$ & $0-$ & 19 & D 50,60 & $\begin{array}{l}\text { MAG-D } 180 \\
7 \text { LEVELS }\end{array}$ \\
\hline $6 R$ & 58 & 11 & E/ & FMF & 2 & 4 & $D_{2,4}=2.13$ & $\begin{array}{r}\text { MAG-D DST } \\
3.4 .46 \text { MEV }\end{array}$ \\
\hline 5 & 58 & 11 & $E, E /$ & $A B X$ & $2-$ & 9 & $\begin{array}{l}D \quad 32-57 \\
2.12,8.56\end{array}$ & $\begin{array}{l}\text { MAG-D DST } \\
6,8.93 \text { MEV }\end{array}$ \\
\hline $75 P 1$ & 58 & 11 & 1 & FMF & 4 & 5 & $\begin{array}{r}35-57 \\
4,5=4.46\end{array}$ & $\begin{array}{r}\text { MAG-D DST } \\
6.5 .04 \mathrm{MEV}\end{array}$ \\
\hline$V L$ & 36 & 1. & $E, E /$ & FMF & $D-3$ & & D592-999 & $M A G-D$ DST \\
\hline
\end{tabular}


NUCLIDE REACTION RES EXCIT SOURCE DETECTOR

$72 N O 35811$ E,PI- RLY $150-999$ C $150-999$ ACT-1 4PI

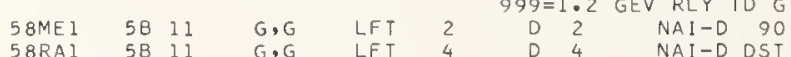

$58 R A 15 B 11$ G,G LFT 4 D 4 NAI-D DST

$\begin{array}{llllllll}60803 & 5811 & G, G & \text { LFT } & 2 & C & 1- & \text { NAI-D } 110 \\ 62806 & 5811 & G, G & \text { LFT } & 2 & C & 1- & \text { NAI-D J1I0 }\end{array}$

63VAl 5811 G,G NDX $0-3 C$ 3 NAL-D 60

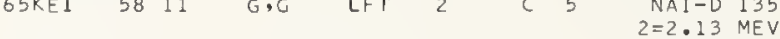

$67 L O 1 \quad 5 B 11 \quad G, G /$ ABX $12-30 C 34 \quad$ NAI-D DST

$69 M U 2$ SB 11 G.NG SPC $11-35$ C 24,35 SCD-D UKN
GAMMA SPECTRUM

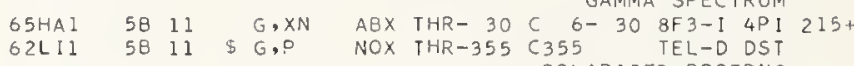

62VO1 5811 G,P RLY THR- 40 POLARIZFD PROTDNS

$69502 \quad 5 B 11$ G,P $A B X \quad 15-32$ C $15-32$ SCD-D 4PI 216

705015811 G,P ABX 11- 19 C 17,19 EMU-O DST $279+$

69MU2 5811 G,PG SPC $11-35$ C 24, 35 SCD-D UKN

58TA1 5811 G.2P ABX THR-320 C100-320 BF $3-1$ 4PI

63NE2 5811 G, 3P A8Y THR-320 C320 DELAYEO NEUTRONS
ACT-I 4PII

66NEI 5B 11 G,3P ABY $45-250 C 250 \quad$ ACT-I $4 P I$

$62 C H 25811$ GOXP RLY $19-40 \mathrm{C} 40$ TEL-D 90

71PAI 5811 G,NG ABX 10-35 C 20-35 SCD-D 90

7IPAI 5811 G.PG A8X 10- 35 C $20-35$ SCD-D 90

$\begin{array}{llllllllll}62 V O 1 & 5 B & 11 & G .0 & R L Y & T H R-40 & C & 40 & \text { TEL-D } & 90 \\ 62 \mathrm{CH} 2 & 58 & 11 & G, X D & R L Y & 24-40 & C & 40 & \text { TEL-D } 90\end{array}$

62VDI $5811 \mathrm{G}, \mathrm{T}$ RLY THR- $40 \mathrm{C} 40^{\text {REL TO TEL }-D} 90$

68NY1 5811 G,PI- ABX 140-700 C140-700 ACT-I 4PI

$72 N O 35 B 11$ G,PI- ABX 150-999 C150-999 ACT-I 4PI

$999=1.2$ GEV

6IKNI 5811 D, G ABX 16-18D 0- 2 THR-I 4PI

$625 U 25811$ D,G RLX $16-20$ D $1-5$ NAI-O 90

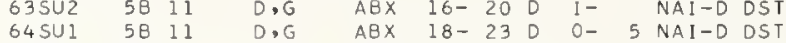

$665 U 15 B 11$ D, G ABX 16- 21 D $1-6$ NAI-D DST

$66 Z I 1 \quad 5 B 11$ D, G ABX 16- 180 1- 2 NAI-D DST

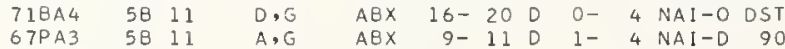

CARBDN $Z=6$

\begin{tabular}{lrrrrrrrrr} 
A & ABUND.11) & \multicolumn{1}{c}{ SEPARATIDN ENERGIES (MEV) } \\
12 & & G.N & G,P & G.T & G,HE3 & G,A & G.2N & G, NP & G.2P \\
13 & 98.89 & 18.7 & 16.0 & 27.4 & 26.3 & 7.4 & 31.8 & 27.4 & 27.2 \\
13 & 1.11 & 4.9 & 17.5 & 23.9 & 24.4 & 10.6 & 23.7 & 20.9 & 31.6
\end{tabular}

\begin{tabular}{cccll}
\multicolumn{1}{c}{ NUCLIOE REACTION RES EXCIT SOURCE } & OETECTOR \\
REF & 2 A IN,DUT & & & TYPE ANG NUM
\end{tabular}

\begin{tabular}{|c|c|c|c|c|c|c|c|c|c|}
\hline 61 JA2 & $6 C$ & 11 & $P, G$ & NOX & 10 & $0-{ }^{j-P_{I}}$ & $\begin{array}{c}N A I-D \\
I, G-W I\end{array}$ & $\begin{array}{r}90 \\
10 T H\end{array}$ & \\
\hline $620 P 2$ & $6 C$ & 11 & $P, G$ & $A B X$ & $11-14$ & $2-$ & NAI I-O & 0 & \\
\hline $70 \mathrm{KUI}$ & $6 C$ & 11 & $P, G$ & $A B X$ & $11-24$ & $2-17$ & NAI - O & DST & 255 \\
\hline $55 F R I$ & SC & 12 & $E, E /$ & $A B X$ & $4-10$ & $\begin{array}{l}\text { O } 80-187 \\
\text { LEVELS } 4 .\end{array}$ & $\begin{array}{l}\text { MAG-0 } \\
.4 .7 .7,\end{array}$ & $\begin{array}{l}\text { DST } \\
9.6\end{array}$ & \\
\hline $56 F R 1$ & $6 C$ & 12 & $E, E /$ & $A B X$ & $0-35$ & 0187 & MAG-D & $\begin{array}{r}\text { DST } \\
J-P I\end{array}$ & \\
\hline $\begin{array}{l}598 \mathrm{Al} \\
60 \mathrm{BA4}\end{array}$ & $\begin{array}{l}6 C \\
6 C\end{array}$ & $\begin{array}{l}12 \\
12\end{array}$ & $\begin{array}{l}E, E / \\
E, E /\end{array}$ & $\begin{array}{l}A 8 \text { I } \\
\text { SPC }\end{array}$ & $\begin{array}{l}0-30 \\
0-30\end{array}$ & $\begin{array}{lll}0 & 43 \\
0 & 42\end{array}$ & $\begin{array}{l}M A G-0 \\
M A G=0\end{array}$ & $\begin{array}{l}160 \\
\text { OST }\end{array}$ & \\
\hline 61804 & $6 C$ & 12 & $E, \subseteq /$ & SPC & $0-164$ & $\begin{array}{l}\text { ABI, MULT } \\
\text { D194 }\end{array}$ & $\begin{array}{l}\text { TIPOLAR } \\
M A G \rightarrow D\end{array}$ & $\begin{aligned} & R \text { RYY } \\
& 135\end{aligned}$ & \\
\hline 61001 & SC & 12 & $E, E$ / & $A B X$ & $13-18$ & $046-154$ & $M A G-O$ & 135 & \\
\hline $62 \mathrm{EOI}$ & $6 C$ & 12 & $E, E /$ & $A B 1$ & 15 & 042 G W & $\begin{array}{l}\text { MAG-O } \\
\text { WIDTH,J }\end{array}$ & $\begin{array}{r}180 \\
J-P 1\end{array}$ & \\
\hline 63803 & $6 C$ & 12 & $E, E /$ & $A B X$ & $4-25$ & 0200 & $M A G-D$ & 135 & \\
\hline 63604 & SC & 12 & $E, E /$ & $A B X$ & $0-30$ & 042 & MAG-O & 180 & \\
\hline 63LEI & $6 C$ & 12 & $E, E /$ & FMF & $0-35$ & 055 & $M A G-0$ & 180 & \\
\hline & & & & & & & -RANGE & $0-1$ & \\
\hline 64BR2 & $6 C$ & 12 & $E, E /$ & $S P C$ & $0-20$ & O 54 & MAG-D & 141 & \\
\hline $64 C R 1$ & SC & 12 & $E, E /$ & $A B X$ & $4-10$ & 0250 & $\begin{array}{l}\text { MAG-D } \\
\text { WID }\end{array}$ & $\begin{array}{l}\text { OST } \\
\text { OTHS }\end{array}$ & \\
\hline $64 G 02$ & $6 C$ & 12 & $E, E /$ & $A B X$ & $10-32$ & D $40-70$ & MAG-O & OST & \\
\hline $64 \mathrm{GO} 3$ & $6 C$ & 12 & $E, E /$ & $A B X$ & 15 & $040-70$ & $M A G-D$ & $\begin{array}{l}\text { FMF } \\
180 \\
F M F\end{array}$ & \\
\hline 64GUI & $6 C$ & 12 & $E, E /$ & FMF & 15 & ${ }^{D} D=0.2-0$ & $\begin{array}{c}\text { NAI -D } \\
0.5 \text {, WI }\end{array}$ & $\begin{array}{l}\text { OST } \\
\text { IOTH }\end{array}$ & \\
\hline 66AR2 & $6 C$ & 12 & $E, E /$ & LFT & 4 & 0 & $\begin{array}{l}M A G-O \\
4=4.03\end{array}$ & 4PI & \\
\hline $66 C R I$ & $6 C$ & 12 & $E, E /$ & FMF & 4,10 & $\begin{array}{c}D 600-800 \\
4,10=4.4\end{array}$ & $\begin{array}{r}\text { MAG-0 } \\
43.9 .6\end{array}$ & $\begin{array}{l}\text { OST } \\
\text { MEV }\end{array}$ & \\
\hline 66PRI & $6 C$ & 12 & $E, E /$ & NDX & $14-21$ & $0100-200$ & MAG-D & 180 & \\
\hline $67 \mathrm{AF} 1$ & $6 C$ & 12 & $E, E \prime$ & $R L x$ & $0-100$ & 0846 & MAG-O & DST & \\
\hline $7 \mathrm{BE} 3$ & $6 C$ & 12 & $E, E /$ & $A B X$ & 4 & D 113-390 & $M A G-0$ & OST & \\
\hline
\end{tabular}

REF NUCLIDE REACTION RES EXCIT SOURCE DETECTOR

GTCRI $6 C 12$ E,E/ FMF $12-100100-200-M A G-D$ DST

67CR2 GC 12 E,EI FMF $19 \quad \begin{array}{r}4.43,7.66 .9 .4 \text { MEV } \\ 0400-800 \text { MAG-O DST }\end{array}$

67PE1 6C 12 E,EI LFT $15 \quad 040-65 \quad 19=19.5$ MEV

688E6 6C 12 E,E/ FMF $18-20 \quad 63-128$ MAG- 12 MEV

68DE3 6C 12 E,E/ FMF 55-202 D580-968 MAG-O DST

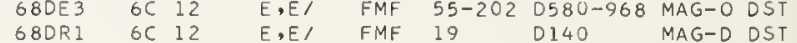

68PR1 6C 12 E,E/ FMF $14-210100-200 \quad 19=19.4 \mathrm{MEV}$

68R11 6C 12 E,E/ FMF 26-35D 60-100 MAG-D DST

$698 E 5$ 6C 12 E,El FMF 20-180 D200-300 MAG-D 60

69DE6 6C 12 E,E/ A8X 300 D580-968 MAG-D DST

69GU2 6C 12 E,EI ABX O- 35 DII5,200 MAG-D DST

69T12 6C 12 E,E/ ABX 0-700 D999 MAG-D 25

69TD2 6C 12 E,E/ FMF 11 999 $=1.126$ GEV

$69 T 04$ 6C 12 E,E/ A8X $18-39$ D 177,250 MAG-D DST

69VAI 6C 12 E,E/ ABX 13-22 D 50-70 MAG-O 180

TOAN1 6C 12 E,E/ A $8 \times 15-30055$ 15.1,19.2 MEV

$70 D E I \quad 6 C 12$ E,E/ ABX 400-999 D690.999 MAG-D DSI

7OLII 6C.12 E,E/ FMF 22-37 999:1115 MEV

E,E/ FMF 22- 37 D 52-102 MAG-D DST

$70 L 12$ 6C 12 E,E/ ABI $17-37$ D 52-102 MAG-O DST

$705 T_{2} 6 C 12$ E,E/ ABX 7 SEE 7OLI

$7=7.650+$

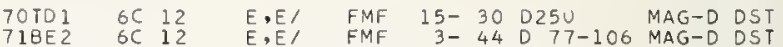

$71 M D 3$ GC 12 E,El ABX $0-240$ D 500 MAG-O 60

$71 N A I$ 6C 12 E, E/ FMF 9- 17 D 250 MAG-D DSI

7INAI 6C 12 E,E/ FMF 9- 17 D250 LELS 14.1,4.43

$71 Y A 2$ GC 12 E,E/ ABX 15-100 BRRATUM 71 NAI

J-PI,FMF, 10 LEVELS

64AMI 6C 12 E,E/P RLX THR-I50 D550 MAG-D 51

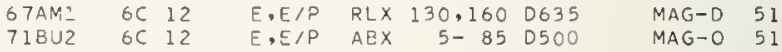

$58 B A 2$ 6C 12 E,N RLY $24-145$ C 24-145 ACT-I $4 P I$

COMPARED WITH G,

598A3 6C 12 E,N ABY THR- $36010-36$ 8F3-I 4PI

$65 H E 1$ 6C 12 E,N RLY THR- 32 D $14-32$ ACT-I 4PI

T1VOI SC 12 E,N ABX THR-266 C150-266 TOF-D 90

72KU6 GC 12 E,N ABX 19-30 D 20-30 ACT-I $4 \mathrm{PI}$

RLY THR- 32 E+/E- YIELD RATIO

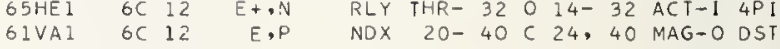

$64 \mathrm{CHI}$ 6C 12 E,P A8X D999 MAG-D DST

69AN8 SC 12 E,P RLY THR-999 C400-999 MAG-O DS

7OAN5 6C 12 E,P RLY $96-999$ C620.099 TEL 9901 GEV, REL O/T

$620016 C \quad 12$ E,XP SPC $25-30<25-30$ MAG-D OST

$70 V Y 1 \quad 6 C 12$ E,XP ABX $31-2000100-200$ MAG-O OST

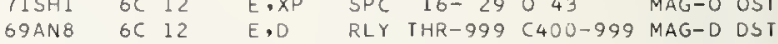

$725 K 7$ 6C 12 E,D ABX $40-6000$ D GEV REL P/O, D/T

69ANB $6 C 12 \quad$ E, T $\quad$ ABX $40-60$ D $40-60$ MAG-D DST

68802 6C 12 E, XXX RLY THR-110 0110 GEV, REL D/T

$X X X=$ MASS SPECTRUM

$\begin{array}{llllllllll}56 T 22 & 6 C & 12 & G, M U-T & \text { LFT } & 23 & C & 22-23 & \text { ACT-I } & 4 P I \\ 59 K O 1 & 6 C & 12 & \text { GMMU-T ABX } & 15-30 & C & 31 & \text { NAI-D } & \text { 4PI }\end{array}$

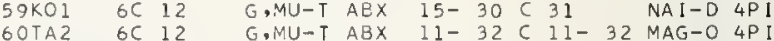

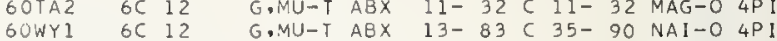

$602 I 1$ GC 12 G,MU-T ABX $10-30$ C $32 \quad$ MGP-O 4 PI

63801 6C 12 G,MU-T A8X 13- 27 C $13-27$ MGP-O $4 P$ I

G,MU-T $A B X 13-27 C 220$ MGP-O $4 P$

65 WYI GC 12 G,MU-T ABX $10-35$ C 90 SCI -0 4PI $52+$

G98E2 GC 12 G,MU-T ABX 10-30C.35 MGC-D 4PI $105+$

$71 \mathrm{AHI}$ GC 12 G,MU-T ABX THR-150 C $10-150$ MGC-O $4 \mathrm{PI}$

$G, G \quad A 8 X \quad 0-19 C 19 \quad N A 1-0120$

5BRAI 6C 12 G,G LFT 4 D 4 NAI-D DST

$59 P A 3-6 C 1215.1$ MEV LEVEL

59PA3 6C 12 G,G ABX $17 \quad 0015,18$ NA1-O 90

$\begin{array}{lllllllll}59 P E 5 & 6 C 12 & G, G & \text { ABX } & 19-61 & C & 19-61 & \text { NAI-O } & 135 \\ \text { 60BU3 } & 6 C 12 & G, G & \text { LFT } & 15 & C & 23 & \text { NAI-D DST }\end{array}$

60HA2 6C 12 15=15.1 MEV

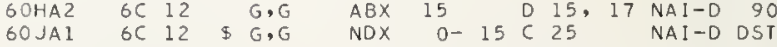

6]BU3 GC 12 G,G ABX 19-36 C 32 NAI-O 140

61W11 SC 12 G,G ABX 40-120 C132 SCI-O DST

S2SEI SC 12 G,G SPC O- 19 C 19 NAI-D 90

$635 C 3$ 6C 12 G,G ABX 15 D 15,18 NAI-O OST

$67 K U 2$ GC 12 G,G LFT 15 O $15 \quad \begin{array}{r}\text { NAI-0 } 135 \\ 15=15.1 \text { MEV }\end{array}$

$\begin{array}{llllllll}70 A H 1 & 6 C & 12 & G, G & A B X & 15-80 C 10 B & \text { NAI-O } & 135 \\ 67 L D 1 & 6 C & 12 & G, G / & A B X & 18-34 & 34 & \text { NAI-O DST }\end{array}$ 
558A1 6C 12 G,N ABX 18-36 C 18-260 ACT-1 4PI $555 P Z$ OC 12 G, N RLY THR- 20 C 17- 20 ACT-1 4P $56 T Z 1 \quad 6 C 12$ G.N RLY $22-24$ C $22-24$ ACT-1 $4 P 1$ $578 A 1$ OC 12 G.N ABY $30-260$ C120-260 THR-I DST G,N ABX 20-31C20-31 ACT-1 4P $588 A 2$ 6C 12 G.N RLY 24-145 O $24-145$ ACT- 4 CP $59 C D 1$ 6C 12 G.N $A B X \quad 20-210020-21 \quad A C T-I \quad 4 P 1$ 595Al 6C 12 G,N NDX THR- 19 C $18-19$ ARTEAKS 59SA1 6C 12 G,N NDX THR- 19 C $18-19$ ACT-I 4 P

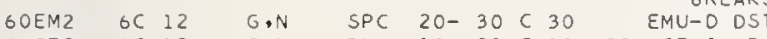
6UGE2 GC 12 G,N RLY $19-20 \mathrm{C} 19,20$ ACT-I 4 P I $A B \times 18-90<18-90 A C T-1$ IP $62081-6 C 12=$ $62 F 12$ GC 12 G.N RLX $21-29$ C 25-32 TOF-D UKN 62FU5 OC 12 G,N A8X 21-31C 31 SCI-D 90

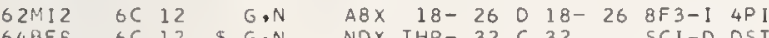
64BEE GC 12 \$ G.N NDX THR- 32 C 32 SCI-D OST 65VE1 GC,12 G.N SPC THR- 33 C 34 TDF-D DST 668A4 GC 12 G.N A8X THR- 52 C $18-52$ 8F3-I 4P $66 C D 2$ 6C 12 G.N A8X THR- 65 CTHR- 70 ACT-I 4P1 11 $G . N \quad A 8 \times 18-70$ C $18-70$ ACT-1 $4 P$ I 66FU1 6C 12 G, N BX $18-37$ D $18-37$ BF $3-1$ 4P1 $66 L D 1$ GC $12 \quad G, N \quad A 8 X \quad 21-27021-27$ ACT-1 4P 66MI2 6C 12 G,N A8X 18-26 D $18-26$ 8F 3-I 4P 67ANI GC 12 G,N RLY THR-999 CTHR-999 ACT-I 4PI 67012 GC 12 G,N ABY $18-999$ C300-999 ACT-1 4PI 67013 SC 12 G.N A8X 300-999 C300-999 ACT-1 4 AP 67GE2 2 GC 12 G.N ABY THR- 27 C $22,27999=1$ GEV

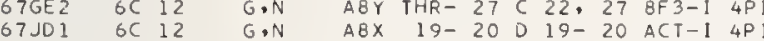
G.N A8I THR-170 C170 CCH-I 4 P 68KAI GC 12 G.N A8X 50-85C 55,85 TDF-D 67

68WU1 6C 12 G.N ABX THR-40 C 20-40 TDF-O $90222+$

G90EI SC 12 G:N ABY THR-999 C 1- 6 ACT-1 4PI

690E4 OC 12 G.V NDX $19-260$ C260 $999=5.5$ GEV

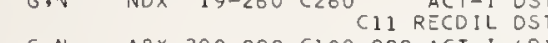
$70 H Y L \quad 6 C 12$ G.N ABX 200-999 C100-999 ACT-1 4P 7OKA2 SC 12 G.N ABX $18-100$ C 20,140 ACT-1 4 4P

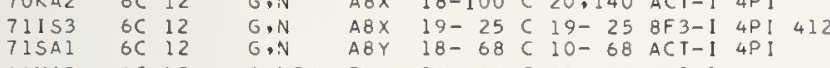
66MA2 GC 12 G.NG/ RLX 21-31 C 21- 31 NAI-D UXN TOME 4 SC 12 G.NG/ ABY $21-42$ C 24- 42 SCO-D 112 $590 \mathrm{CI}$ BC 12 G.2N RLI THR-I00 CTHR-100 ACT-I 4PI

70KA2 GC $12 \quad$ G.2N ABX $30-556$ C $35-130$ REL TD T G T P N $558 A 5$ OC 12 G.XN ABY $30-200$ C150-250 THR-I OSI 55011 GC 12 G.XN NDX THR- $70<70$ SCI-I DST

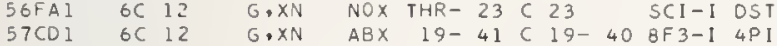

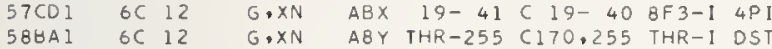
$58 B A 5$ BC 12 G.XN RLY THR- 23 C 18- 23 BF3-I 4PI

$59 K U 1$ GC $12 \quad G \cdot X N$ ABY THR - $88 C 8 B$ TEL-D DS $G, X N$ RLY $30-85 C 30-85$ THR-I $4 P$ $62 M 12$ GC 12 G $X N$ N $A B X 18-26018-26 B F 3-I \quad 4 P I$ $63 \mathrm{CO}_{3} 6 \mathrm{C} 12$ G,XN ABX THR- 120 CTHR- 80 BF $3-\mathrm{I} 4 \mathrm{PI}$

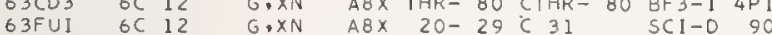

64AL5 OC 12 SPECTRUM

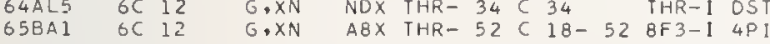
65MII GC 12 G.XN ABX THR- 30 CTHR- 30 8F3-I 4P $668 I 1 \quad 6 C 12 \quad G, X N$ ABX $20-200$ C $20-200 \quad 8 F 3-1 \quad 4 P 1$ 66F 12 6C 12 G.XN SPC THR- 65 C 65 TOF-D 90 GBRA2 GC $12 \quad G \times X N$ ABX $100-150$ C150 $\quad$ BF 3-1 4 P $698 A 1$ GC 12 G.XN ABX $19-25 \mathrm{C} 19-25 \mathrm{BF} 3-1$ - 4 PI 314

$71 C D 2$ GC 12 G.XN $A B I 36-64 C 10-64$ BF $3-1$ LPI $71 M I 1 \quad B C 12 \quad G . X N$ A6X 70- $12064-128$ TDF-O DST 72VA2 GC 12 G.XN ABX 18-32C $18-32$ BF $3-1$ LP $55 \mathrm{JDI}$ GC 12 G,P RLY THR- $65 \mathrm{C} 65$ S 32 SCI-D DST 5SMAI 6C 12 G.P ABX 18 D 18 EMU-O 4P

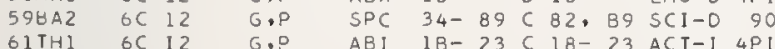
$645 H_{4}$ OC 12 G.P ABX 20-230 20-23 SCI-D 4 PPI 64TA3 GC 12 G.P SPC THR-100 C170 CCH-D 4PI $65 K 12$ 6C 12 G.P ABY $88-720$ D $400-720$ MAG-D 57 65KEI GC 12 \$ G.P ABX $21-22 \quad 0 \quad 21-22$ EMU-O DST 66PA2 GC 12 G.P SPC THR $-55 C 55$ C 5 CIDD DST 67TAI $6 C$ L2 G.0 ABX THR-170 C170 CCH-I DST 68FR2 $6 C 12$ G,P RLX THR- 32 C 30,32 TEL-O DST $G, P \quad A B X 100$ C 95-102 TEL-D DS G8MA2 GC 12 G.P RLX $50-B 0$ C $50-80$ TEL-I 45 GAANS GC 12 G.P ABY $113-999$ C 400999 MAG-D 30 69ANG GC 12 G.P ABY $114-999$ C700.999 TEL-D DET $999=1.2$ GEV

\begin{tabular}{|c|c|c|c|c|c|c|c|c|c|c|}
\hline $69 \mathrm{CA}_{3}$ & $6 C$ & 12 & & $G, P$ & $A B x$ & $70-27$ & $\begin{array}{ll}D & 32 \\
\text { GRD }\end{array}$ & $\begin{array}{l}\text { TEL-D } \\
\text { OUND ST }\end{array}$ & $\begin{array}{l}\text { DST } \\
\text { TATE }\end{array}$ & 316 \\
\hline 69MA3 & $6 C$ & 12 & & $G, P$ & $A B x$ & $36-80$ & C $50-80$ & TEL-D & 45 & \\
\hline 69TAl & $6 C$ & 12 & & $G, P$ & $A B X$ & $16-60$ & 6170 & $\mathrm{CCH}-\mathrm{D}$ & DST & 317 \\
\hline 70102 & $6 C$ & 12 & $\$$ & $G, P$ & NOX & $680-840$ & $\begin{array}{r}X X X=C H R G D \\
C 700-930 \\
P-P D L\end{array}$ & $\begin{array}{l}\text { PARTC } \\
\text { MAG-D } \\
\text { ARIZAT }\end{array}$ & $\begin{array}{r}\text { CLES } \\
41 \\
\text { TIDN }\end{array}$ & \\
\hline $72 \mathrm{ANI}$ & $6 C$ & 12 & & $G, P$ & SPC & $\begin{array}{r}49-999 \\
99\end{array}$ & $\begin{array}{c}C 700,999 \\
99=1.2 \mathrm{GEV}\end{array}$ & $\begin{array}{l}\text { TEL-D } \\
V, R E L\end{array}$ & $\begin{array}{l}\text { OST } \\
\text { O/P }\end{array}$ & \\
\hline $71 G 01$ & $6 C$ & 12 & $\$$ & $G, P$ & NDX & $16-600$ & $\begin{array}{l}\text { C650-800 } \\
\text { PDLARIZE }\end{array}$ & $\begin{array}{l}\text { MAG-D } \\
\text { EO PRDT }\end{array}$ & $\begin{array}{l}\text { UKN } \\
\text { TDNS }\end{array}$ & \\
\hline 66MAZ & $6 C$ & 12 & & G,PGI & $R L X$ & $21-31$ & C $21-31$ & NAI -D & UKN & \\
\hline 7OME 4 & $6 C$ & 12 & & $G, P G /$ & $A B Y$ & $18-42$ & C $24-42$ & $5 C O-O$ & 112 & \\
\hline $67 \mathrm{TAl}$ & $6 C$ & 12 & & $G, 2 P$ & $A 81$ & THR-170 & $C 170$ & $\mathrm{CCH}-\mathrm{I}$ & $4 \mathrm{P!}$ & \\
\hline 58 TA1 & $6 C$ & 12 & & $G, 3 P$ & $A 8 x$ & $100-320$ & $\begin{array}{r}C 100-320 \\
\text { L } 1-9\end{array}$ & $\begin{array}{l}A C T-1 \\
9 N 8 Y\end{array}$ & $\begin{array}{l}4 \mathrm{PI} \\
\mathrm{BF} 3\end{array}$ & \\
\hline $56 C 01$ & $6 C$ & 12 & & $G, X P$ & $A B Y$ & THR- 24 & C 24 & EMU-D & 90 & \\
\hline $57 \mathrm{CHI}$ & $6 C$ & 12 & & $G \times P P$ & SPC & THR - 84 & C 64,94 & EMU-O & OST & \\
\hline $57 L 11$ & $6 C$ & 12 & & $G, \times P$ & SPC & THR - 35 & C 35 & EMU-D & DST & \\
\hline $58 \mathrm{CH} 1$ & $6 C$ & 12 & & $G, X P$ & SPC & THR- 44 & C 30,44 & EMU-D & DST & \\
\hline $598 A 2$ & $6 C$ & 12 & & $G, X P$ & SPC & THR- 89 & C 82,89 & TEL-D & 90 & \\
\hline $59 \mathrm{CHI}$ & $6 C$ & 12 & & $G, \times P$ & RLY & THR - 80 & $\begin{array}{l}C 80 \\
\text { REL } O\end{array}$ & $\begin{array}{c}\text { TEL-D } \\
\text { TO } P\end{array}$ & $\begin{array}{r}90 \\
\text { YLO }\end{array}$ & \\
\hline $59 P \equiv 4$ & $6 C$ & 12 & & $G, \times P$ & $A 8 x$ & $22-62$ & C 22- 90 & $S C 1-0$ & DST & \\
\hline $608 \mathrm{Al}$ & $6 C$ & 12 & & $G, X P$ & $A B Y$ & THR- 90 & C 30,90 & TEL-D & 58 & \\
\hline $60 \mathrm{CHI}$ & $6 C$ & 12 & & $G, \times P$ & RLY & THR - 90 & $\begin{array}{l}C \quad 90 \\
\text { REL TD }\end{array}$ & $\begin{array}{l}\text { TEL-I } \\
\text { OEUTER }\end{array}$ & $\begin{array}{r}\text { DST } \\
\text { RDNS }\end{array}$ & \\
\hline $61 C E 1$ & $6 C$ & 12 & & $G, \times P$ & $A B x$ & THR -342 & $0245-342$ & TEL - O & 60 & \\
\hline GIVA1 & $6 C$ & 12 & & $G, X P$ & NOX & $20-40$ & C 24,40 & $M A G-D$ & DST & \\
\hline $62 \mathrm{CH} 2$ & $6 C$ & 12 & & $G, \times P$ & RLY & THR- 90 & $\begin{array}{l}C \quad 90 \\
\text { REL TD }\end{array}$ & $\begin{array}{l}\text { TEL-D } \\
\text { OEUTER }\end{array}$ & $\begin{array}{l}\text { OST } \\
\text { RDNS }\end{array}$ & \\
\hline $62 \mathrm{HE} 1$ & $6 C$ & 12 & & $G, X P$ & SPC & $19-27$ & C 31 & $5 C D-0$ & UKN & \\
\hline 62411 & GC & 12 & $\$$ & $G \times \times P$ & RLY & $T H R-335$ & $\begin{array}{l}\text { C335 } \\
\text { PRDTDNS }\end{array}$ & $\begin{array}{l}\text { TEL-D } \\
\text { PDLAR I }\end{array}$ & $\begin{array}{l}\text { OST } \\
\text { IZED }\end{array}$ & \\
\hline $62 P A 1$ & $6 C$ & 12 & & $G, \times P$ & $A B Y$ & THR-150 & C 150 & $s \subset I-0$ & 60 & \\
\hline $63 F_{14}$ & $6 C$ & 12 & & $G, \times P$ & SPC & $19-30$ & C 31 & $\begin{array}{l}\text { SCI I D } \\
\text { SPECT }\end{array}$ & & \\
\hline $63 K 11$ & $6 C$ & 12 & & $G, \times P$ & $A B Y$ & THR -335 & C 335 & $T E L-O$ & OST & \\
\hline $63 \mathrm{MUI}$ & $6 C$ & 12 & & $G, X P$ & $A B Y$ & THR- 24 & C 24 & $S C O-D$ & 120 & \\
\hline G3WAZ & $6 C$ & 12 & & $G \times X P$ & $R L Y$ & THR- 65 & C 65 & SC1-0 & DST & \\
\hline 64SE 1 & $6 c$ & 12 & & $G, X P$ & SPC & THR - 24 & C 24 & EMU-D & OST & \\
\hline $68 F R 3$ & SC & 12 & & $G, \times P$ & $R L X$ & $20-38$ & $\begin{array}{ll}C & 38 \\
& 38\end{array}$ & $\begin{array}{l}\text { TEL-D } \\
8=37.7\end{array}$ & $\begin{array}{r}90 \\
\text { MEV }\end{array}$ & \\
\hline $64 K 11$ & $6 C$ & 12 & & $G, D$ & $A B Y$ & $80-800$ & $(400-800$ & TDF $-D$ & 57 & \\
\hline $65 K 12$ & $6 C$ & 12 & & $G, 0$ & $A B Y$ & $70-720$ & $0400-720$ & $M A G=D$ & 57 & \\
\hline 69ANS & $6 C$ & 12 & & 6.0 & $A B Y$ & $219-999$ & $\begin{array}{r}C 400,999 \\
99\end{array}$ & $\begin{array}{l}\text { MAG-D } \\
99=1.4\end{array}$ & & \\
\hline 69ANE & $6 C$ & 12 & & 6.0 & $A B Y$ & $116-999$ & $\begin{array}{r}(700,939 \\
99\end{array}$ & $\begin{array}{l}\text { TEL-D } \\
99=1.2\end{array}$ & $\begin{array}{l}\text { DST } \\
\text { GEV }\end{array}$ & \\
\hline TIA.NI & $6 C$ & 12 & & $G \cdot 0$ & SPC & $\begin{array}{r}55-999 \\
99\end{array}$ & $\begin{array}{c}6700.999 \\
99=1.2 \mathrm{GEV}\end{array}$ & $\begin{array}{l}T E L-D \\
V, R E L\end{array}$ & $\begin{array}{l}\text { DST } \\
\text { D/P }\end{array}$ & \\
\hline 72 AN3 & $6 C$ & 12 & & $G, 0$ & RLY & $105-999$ & $\begin{array}{r}6700,999 \\
99\end{array}$ & $\begin{array}{l}M A G-D \\
99=1.2\end{array}$ & & \\
\hline $59 \mathrm{CHI}$ & $6 C$ & 12 & & $G, \times 0$ & RLY & THR- 80 & $\begin{array}{l}C B O \\
\text { REL D }\end{array}$ & $\begin{array}{l}\text { TEL-D } \\
\text { D TO } P\end{array}$ & $\begin{array}{r}90 \\
\text { YLO }\end{array}$ & \\
\hline $60 \mathrm{CHI}$ & $6 C$ & 12 & & $G, \times D$ & $R L X$ & THR - 90 & C 90 REL T & $\begin{array}{l}\text { TEL-1 } \\
\text { TD PRDT }\end{array}$ & $\begin{array}{l}\text { OST } \\
\text { TDNS }\end{array}$ & \\
\hline $82 \mathrm{CH} 2$ & $6 C$ & 12 & & $6 \times 0$ & $R L Y$ & THR - 90 & C 90 & $\begin{array}{l}\text { TEL }=0 \\
\text { TD PRDT }\end{array}$ & $\begin{array}{l}\text { DST } \\
\text { TDNS }\end{array}$ & \\
\hline 7IAN2 & $6 C$ & 12 & & $G \times \times D$ & $A 8 x$ & $115-999$ & $\begin{array}{r}\mathrm{C} 620,999 \\
999\end{array}$ & $\begin{array}{l}M A G-D \\
9=1.14\end{array}$ & & \\
\hline $67 K R 2$ & $O C$ & 12 & & $G, T$ & $R L x$ & THR- 55 & C $30-55$ & $A C T-1$ & $4 \mathrm{PI}$ & \\
\hline $72 A N 3$ & GC & 12 & & $G, T$ & $S P C$ & $107-999$ & $C 700,399$ & MAG-D & $\begin{array}{l}\text { OST } \\
\text { GEV }\end{array}$ & \\
\hline $67 T A 1$ & $6 C$ & 12 & & 6 , HE 3 & $A B I$ & THR-170 & $C 170$ & $\mathrm{CCH}-\mathrm{I}$ & $4 \mathrm{PI}$ & \\
\hline $\begin{array}{l}55 \mathrm{GLI} \\
70 \mathrm{MUI}\end{array}$ & $\begin{array}{l}6 C \\
6 C\end{array}$ & $\begin{array}{l}12 \\
12\end{array}$ & & $\begin{array}{l}G, A \\
G, A\end{array}$ & $\begin{array}{l}\text { SPC } \\
A B X\end{array}$ & $\begin{array}{l}17 \\
12-32\end{array}$ & $\begin{array}{lll}0 & 14, & 17 \\
C & 32\end{array}$ & $\begin{array}{l}\text { EMU-D } \\
\text { EMU-D }\end{array}$ & $\begin{array}{l}4 \mathrm{PI} \\
4 \mathrm{PI}\end{array}$ & $31 \mathrm{~B}$ \\
\hline $55 C A 2$ & 6C & 12 & & $G, 3 A$ & $\operatorname{AEx}$ & $12-18$ & D 15,18 & EMU-D & $4 \mathrm{PI}$ & \\
\hline $55 C A 2$ & $6 C$ & 12 & & $6,3 \mathrm{~A}$ & $A B X$ & $12-18$ & $015,1 B$ & IDN-D & $4 \mathrm{PI}$ & \\
\hline 55601 & $6 C$ & 12 & & $G, 3 A$ & SPC & THR- 60 & C 60 & EMU-O & OST & \\
\hline $55 \mathrm{HAl}$ & $6 C$ & 12 & & $G, 3 A$ & RLY & $9-33$ & C 27,33 & EMU-D & DST & \\
\hline $57 \mathrm{MUI}$ & 6C & 12 & & $G, 3 A$ & $S P C$ & 15,18 & $\mathrm{O} 15,1 \mathrm{~B}$ & EMU-D & DST & \\
\hline $58 \mathrm{MAl}$ & $6 \mathrm{C}$ & 12 & & $G, 3 A$ & $A B X$ & $12-40$ & C 150.250 & EMU-D & $4 \mathrm{PI}$ & \\
\hline 60GA2 & $6 C$ & 12 & & $G, 3 A$ & $A 8 x$ & 15,28 & D 15,18 & EMU-I & UKN & \\
\hline $615 E 3$ & 6C & 12 & & $G, 3 A$ & $R L Y$ & 18 & 018 & EMU-D & $4 \mathrm{PI}$ & \\
\hline $635+12$ & 6C & 12 & & $6,3 A$ & NO $X$ & $8-70$ & $\begin{array}{l}C 70 \\
Q-V A L U\end{array}$ & $\begin{array}{l}\text { EMU-D } \\
J E \text { OIST }\end{array}$ & $4 P I$ & \\
\hline 64GRI & $6 C$ & 12 & & $G, 3 A$ & $A B Y$ & $7-24$ & C $17-24$ & EMU-1 & $4 \mathrm{PI}$ & \\
\hline 64TDI & $6 c$ & 12 & & $G, 3 A$ & $A 8 X$ & $9-22$ & C 22 & $\begin{array}{l}\text { EMU-D } \\
\text { SPECT }\end{array}$ & $\begin{array}{l}\text { OST } \\
\text { TRUM }\end{array}$ & \\
\hline 64WAL & $6 C$ & 12 & & G, ?A & $A B X$ & 18 & $0 \quad 17$ & $\begin{array}{l}5 C 1-0 \\
8=17.6\end{array}$ & $\begin{array}{l}\text { 4PI } \\
\text { MEV }\end{array}$ & \\
\hline 65RO1 & $6 C$ & 12 & & $G, 2 A$ & $A B x$ & $12-17$ & C $12-17$ & EMU-D & $4 \mathrm{PI}$ & \\
\hline 58 WH2 & $6 C$ & 12 & & $G, N P$ & $A B x$ & THR-110 & $\begin{array}{r}\text { C } 4 v-110 \\
\text { CDINC }\end{array}$ & $\begin{array}{l}\text { TEL-O } \\
C \text { WITH }\end{array}$ & $\begin{array}{l}\text { DST } \\
\text { BF } 3\end{array}$ & \\
\hline 6OST1 & 6C & 12 & & $G, N P$ & $R L x$ & $157-320$ & $\begin{array}{l}\text { C } 320 \\
\text { REL TO H2 }\end{array}$ & $\begin{array}{l}\text { TEL-D } \\
2 \text { CRDS }\end{array}$ & $\begin{array}{r}76 \\
\text { SEC }\end{array}$ & \\
\hline $64 T A 3$ & OC & 12 & & $G, N P$ & SPC & THR-100 & C 170 & $\mathrm{CCH}-\mathrm{O}$ & $4 \mathrm{PI}$ & \\
\hline $675 \mathrm{MI}$ & $6 C$ & 12 & & $G, N P$ & $A B X$ & $150-250$ & $C 250$ & TDF -0 & DST & \\
\hline $67 \mathrm{TAl}$ & 6C & 12 & & $G, N P$ & A8 1 & THR -170 & C 170 & $\mathrm{CCH}-\mathrm{I}$ & $4 \mathrm{PI}$ & \\
\hline $68 \mathrm{TAl}$ & 6C & 12 & & $G, N P$ & $A B X$ & THR-170 & $C 170$ & $\mathrm{CCH}-1$ & OST & \\
\hline $68 \mathrm{TA}]$ & $6 c$ & 12 & & G.NPA & $A B X$ & THR-170 & 6170 & $\mathrm{CCH}-\mathrm{I}$ & DST & \\
\hline $67 T A 1$ & 6C & 12 & & G, NHE 3 & $A B 1$ & THR-170 & $\operatorname{ci70}$ & $\mathrm{CCH}-\mathrm{I}$ & $4 \mathrm{PI}$ & \\
\hline 68TA1 & 6C & 12 & & $G, \mathrm{NHE}_{3}$ & $A B X$ & THR-170 & C170 & $\mathrm{CCH}-\mathrm{I}$ & DST & \\
\hline $67 T A 1$ & $6 C$ & 12 & & $G, N A$ & $A B 1$ & THR-170 & C170 & $\mathrm{CCH}-1$ & $4 \mathrm{PI}$ & \\
\hline $68 \mathrm{TAl}$ & $6 c$ & 12 & & $G, N A$ & $A B X$ & THR-170 & C170 & $\mathrm{CCH}-\mathrm{I}$ & DST & \\
\hline 690W2 & GC & 12 & & G.NA & $A B x$ & $26-31$ & $c 20-71$ & $A C T-I$ & $4 \mathrm{PI}$ & 276 \\
\hline 58MAI & $6 C$ & 12 & & $G, P T$ & $A B X$ & $27-.70$ & C150, 250 & ธMU-D & $4 \mathrm{PI}$ & \\
\hline & 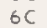 & 1. & & $G, P$ & $A B X$ & & & $\mathrm{CCH}$ & & \\
\hline
\end{tabular}


56LII GC 12 G,PA ABX 30-50 C 25-70 EMU-I UKN 5 BMAI GC 12 G,PA ABX 25- 80 C150,250 EMU-D 4PI $62 \mathrm{MO2}$ 6C 12 G,PA ABI 25-120 C120 EMU-0 4P $67 T A 1$ 6C 12 G.PA ABI THR-170 C170 CCH-I 4P 68TAI 6C 12 G.PA ABX THR-170 C170 CCH-I DST $66 A R 1$ 6C 12 G.BE7 A8X $30-57$ C $30-57$ ACT-I $4 P$ $670 I 2$ GC 12 G.BE7 ABY THR-999 C300-999 ACT-1 4PI

67013 6C 12 G.EE7 ABX 300-999 C300-999 $999=1$ GEV $67 \mathrm{TAl} 6 \mathrm{C} 12 \quad \mathrm{G}, \mathrm{SPL} A \mathrm{ABI}$ THR-170 C170 $999=1 \mathrm{GE}$ $64 M A 4$ OC 12 XXX ABY 150-720 C150-720 ACT-I 4P G9TAI 6C $12 \quad X X X=C 11$ FINAL

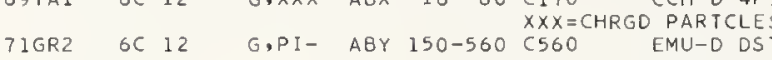
PI- $P I+Y I E L D$ RATIO $G, P I+R L Y 150-500 C 50 D$
PI-/PI+ YIELD RATIO $71 G R 2 \quad 6 C 12$ G.PIt ABY $150-560$ C560 EMU-D DST $59 G E I$ 6C 12 P,G A8X 19- 23 C 4- NAI-0 90 59601 6C 12 P,G RLX 19-26 D 3- 11 NAI-D DST 61601 6C 12 P,G ABX 19- 27 D 3- 11 NAI-D DST $615 E 2$ GC 12 P,G RLY 16 G,P ABI BY OT BAL G-WDTH, MULTIPOLAR

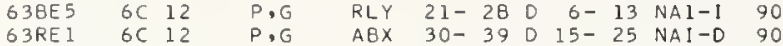

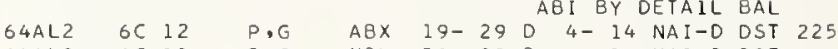
$64 A L 3$ 6C 12 P, NOX $20-30$ D 4 - 14 NAI-D DST $64 \mathrm{HA} 3 \quad 6 C 12$ P, G ABX $17-20 \mathrm{D} 1-4 \mathrm{NAI}-\mathrm{I}$ DST $65 S E 1$ 6C 12 P, A ABX $16-200$ O- 4 NAI-D DST 67FEI GC 12 P, ABX 25-30 D 10- 15 NAI-D 90

69KEI 6C 12 P.G ABX 28- 37 D $13-21$ NAI-O OST $227+$

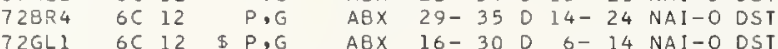

$648 \mathrm{LL} 6 \mathrm{C} 12 \mathrm{HE}, \mathrm{G}$ ABX 28 POLARIZED PROTONS

28L6 6C $12 \quad H E, G \quad H E X=H E 3$

HE=HE3, G-WIDTH, J-P

67PEI 6C 13 E,E/ LFT 15 D 40-65 MAG-D DST

69CAI 6C 13 E.E/ SPC $0-20$ D 140 15=15.11 MEV

69TOI 6C 13 E,EI FMF 3 D $34-65$ MAG-D DS

69WI2 6C 13 E,E/ LFT 3- $15036-65$ MAG-D DS

7OWII 6C 13 E,E/ LFT 3-15 D 36-65 MAG-D DST

$718 E 2$ 6C 13 E,E/ FMF $8-44077-106$ MAG-O DST

7IYAI 6C I3 E,EJ FMF 3- 20 D 40-125 MAG-D DST

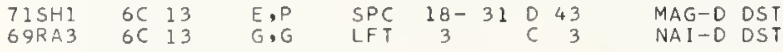

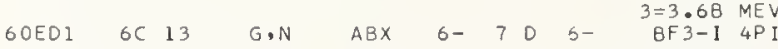

$64 G R 2$ 6C 13 G.N ABX 6- 110 6- 11 BF3-1 4PI

6C 13 G ABX $6-14 \mathrm{C} B-14$ TOF -1 OST

66FU3 6C 13 GN RLY THR- 15 C $5-15$ BF 3-1 4PI

7OFU2 6C 13 G.N ABX 6- 14 C $5-14$ BF 3-I 90

$70 F U 3 \quad 6 C 13 \quad G, N \quad A B X \quad 6-14 C \quad 5-14$ BF 3-1 DST

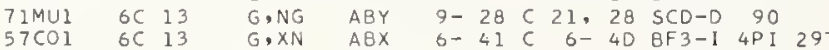

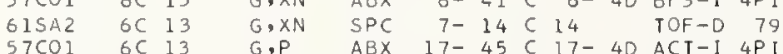

$64 \mathrm{KO2} \quad 6 C 13$ G.P SPC $20-32 C C_{32}$ SCI-D 90

$640 E 2 \quad 6 C 13$ G.XP ABX $18-50$ C $18-50$ ACT-I 4P[ 313

NITROGEN $2=7$

ABUND. 11 SEPARATION ENERGIES (MEV)

G,N G,P G,T G,HE3 G,A G, 2N G, NP G,2P

$\begin{array}{llllllllll}14 & 99.63 & 10.6 & 7.6 & 22.7 & 20.7 & 11.6 & 30.6 & 12.5 & 25.1\end{array}$

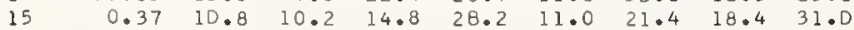
$\begin{array}{lllll}\text { NUCLIDE REACTION RES EXCIT SOURCE } & \text { DETECTOR } \\ Z \text { A IN,OUT } & & & \text { TYPE ANG NUM }\end{array}$

6OHE2 TN 13 P,G ABX 2- 30 D- NAI-O DST

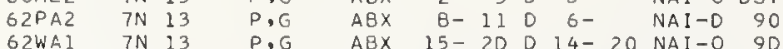
$63 F I 1 \quad 7 N 13$ P.G ABX $11-410$ ID- 49 NAI-D $90266+$ $63 Y 02$ 7N 13 P.G ABX 2- 4 D $1-$ NAI-D DST 680II 7N 13 P,G LFT 15 D 14- 15 NAI-D DST $68 R 127 N 13$ P.G LFT 2 D I NAI-D 4PI 72HAI 7N 13 P,G ABX 10- 17 D $8-17$ NAI-D 90546

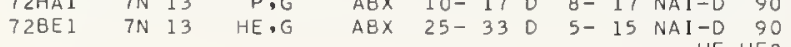
$\begin{array}{llllllll}62 E D I & 7 N 14 & \text { E,E/ } & \text { ABX } & 0-10 & 0 & 42 & \text { TEL-D } 180 \\ 63 B A 1 & 7 N 14 & \text { E,E/ } & \text { ABX } & 0-18 & 0 & 42 & \text { MAG-D } 180\end{array}$

64811 7N 14 E,E/ ABX O- B D120.180 MAG-WDTH $68 C L 17 N 14$ E,E/ LFT $8-12035-58$ MAG-D DST $60 C A 17 \mathrm{~N} 14 \quad G, M U-T$ ABX 7- $21020-17,10.43$ MEV G.MU-T ABI O-170 CI70-21 NAI -0 4P I G.MU-T ABX 10-170 C170 $61 \mathrm{SWI} \quad 7 \mathrm{~N} 14 \quad \mathrm{G}, \mathrm{G}$ LFT 2 D 2 NAI-D DST 64801 7N 14 G,G LFT $1-3 \mathrm{C}$ I- NAI-D 100 $665 W 1 \quad 7 N \quad 14$

$67 \mathrm{LO} \quad 7 \mathrm{~N} 14$ $70 \mathrm{THI} \quad 7 \mathrm{~N} 14$ $59 M U 1 \quad 7 N \quad 14$ GOFAI $\quad 7 \mathrm{~N} 14$ GOGE $17 \mathrm{~N} 14$ SOGE 2 7N 34

$60 \mathrm{KI} 27 \mathrm{~N} 14$ $60 \mathrm{MUI} \quad 7 \mathrm{~N} \quad 14$

$605 A 2 \quad 7 N 14$

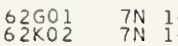

$68 \mathrm{KA}$

$7 \mathrm{~N} 14$

$71 F R 1 \quad 7 N \quad 14$

$71 \mathrm{SAI} \quad 7 \mathrm{~N} 14$

$72 \mathrm{GE} 3 \quad 7 \mathrm{~N} 14$

$590 C_{1} \quad 7 \mathrm{~N} \quad 14$

$\begin{array}{lll}60 \mathrm{BA} 7 & 7 \mathrm{~N} & 14 \\ 63 \mathrm{FU} & 7 \mathrm{~N} & 14\end{array}$

$70 B E B \quad 7 \mathrm{~N} 14$

56WR1 7N 14

57LII 7N 14

$58 \mathrm{CO}_{2} \quad 7 \mathrm{~N} \mathrm{I} 4$

$58 G R 1 \quad 7 \mathrm{~N} 14$

$60 \mathrm{BA} 7 \quad 7 \mathrm{~N} \quad 14$

$60 \mathrm{KOI} \quad 7 \mathrm{~N} 14$

$\begin{array}{lll}60 \mathrm{WAI} & 7 \mathrm{~N} & 14 \\ 62 \mathrm{GOI} & 7 \mathrm{~N} & 14\end{array}$

57 II $7 \mathrm{~N} 14$

6ORE 2 7N 14

$63 F I 4 \quad 7 N \quad 14$

56 WR

$62601 \quad 7 \mathrm{~N} 14$

$\begin{array}{lll}64 \mathrm{TO} 2 & 7 \mathrm{~N} 14 \\ 56 \mathrm{~N} 1 & 7 \mathrm{~N} & 14\end{array}$

56NR1 7N 14

$60 B A 7 \quad 7 N 14$

$60 \mathrm{KOI} \quad 7 \mathrm{~N} 14$

$62601 \quad 7 \mathrm{~N} 14$

$62 \mathrm{KO} \quad 7 \mathrm{~N} 14$

$62 \mathrm{GO} \quad 7 \mathrm{~N} 14$

$70 \mathrm{SHI} 7 \mathrm{~N} 14$

$56 L I 1 \quad 7 \mathrm{~N} 14$

$\begin{array}{lll}56 \mathrm{WRI} & 7 \mathrm{~N} & \mathrm{I} 4 \\ 62 \mathrm{MO2} & 7 \mathrm{~N} & 14\end{array}$

$62 \mathrm{KO2} \quad 7 \mathrm{~N} 14$

$60 \mathrm{HE} 2 \quad 7 \mathrm{~N} 14$

$60 R O 1 \quad 7 N \quad 14$

$60 \mathrm{RO}^{2} \quad 7 \mathrm{~N} 14$

$61 \mathrm{HEI} 7 \mathrm{~N} 14$

$G, G$ LFT

D 7 NAI-D DST

$7=7.11$ MEV
T.

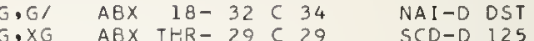
G,N RLY I1- $17 C 11-17$ ACT

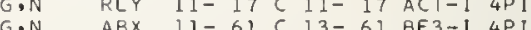
$G, N$ RLX $10-12 C 10-12$ ACT-I $4 P I$ G,N RLX $10-12 C 10-12$ ACT-I $4 P I$ $G, N$ RLY $10-11 C 10-11$ ACT-1 4PI $G, N$ ABX 10- $25 C 10-25$ ACT-I 4PI $G, N$ RLY THR - 20 C $15-20$ ACT-I 4 PI G,N RLY $10-13 C 10-13$ ACT-1 4 PI

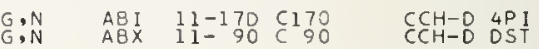
$G, N \quad A B X \quad 50-85 C 55,85$ TOF-D 67 NEUT ENGY SPEC $G, N$ ABY $10-800 \quad 100-800$ ACT -1 4PI $G, N$ ABY $10-6 B C 10-68$ ACT-I 4 PI $G, N$ ABX 1O- 30 C $15-30$ TOF-D 90 $G, 2 N$ RLI THR-100 CTHR-100 ACT-I 4 PI
REL TO G, G. XN RLX THR-200 C2OO CCH-D DST G,XN ABC $10-30$ C 31 - 30 D $10-30$ BF 3-1 4PI $G, P$ ABX 7- 23 C 19- 23 CCH-D 4PI G,P SPC THR- 70 C 30,70 EMU-D

G,P NOX 7- $30 \mathrm{C} 18-30$ EMU-D 90

$G, P$ LFT $B$ D 8 ION-D $4 P$ I $G, P \quad R L X$ THR-200 C200 CCH-D DST G,P ABI THR - 90 C $90 \quad$ CCH-D OST $\begin{array}{lllll}G, P & S P C & 8-16 C 12,16 \text { EMU-D OST } \\ \text { G,P } & \text { ABI } & 7-170 \mathrm{C} 170 & \text { CCH-D } & 4 P\end{array}$ G, XP SPC THR- $70 \mathrm{C} 30,70$ EMU-D DST $G, X P$ C330 CCH-D $4 \mathrm{PI}$

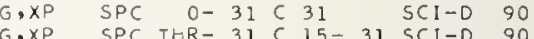
ABX $\begin{array}{llllll}\text { G.A } & A B X & 12-23 & C 19-23 & C C H-D & 4 P I \\ \text { G.A } & A B I & 12-170 & C 170 & C C H-D & 4 P I\end{array}$ G, A ABX THR- 22 C 22 EMU-D DST G.2A ABX 16- 23 C 19- 23 CCH-D 4PI G,NP ABX 12- $23 \mathrm{C} 19-23 \mathrm{CCH}-0$ 4PI G,NP RLI THR-200 C200 CCH-O DST SPC THR- 90 C 90 CCH-D OST G,NP ABI 12-170 C170 CCH-D 4PI G,NP ABX $16-90 \mathrm{C} 90 \quad \mathrm{CCH}-\mathrm{D}$ DST G,NA ABI 20-170 CI7D CCH-D 4PI

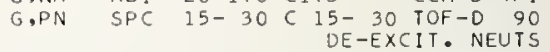
G,PA ABX 25-50 C 25-70 EMU-1 UKN G,PA ABX 17- $23 \mathrm{C} 19-23 \mathrm{CCH}-\mathrm{D} 4 \mathrm{PI}$ G,PA ABI $18-120$ C120 EMU-D 4PI $\begin{array}{llccccc}G, N P 3 A & R L Y & 20-90 & C & 90 & C C H-D & 4 P I \\ P, G & A B X & 8 & 0 & 0- & \text { NAI-D DST }\end{array}$ G-WDTH
G $P, G$ NOX 9 D 2 NAI-D DST P,G ABY 10 D 2- NAI-O DST $P, G$ ABX 8 D $D-\quad N A I-D$ J-PI

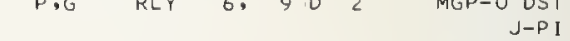
63PRI 7N 14 P,G NOX 9 D 2 NAI-D DST 65DE2 7N 14 P.G LFT 92002 NAI-D DST $7 I R I 1$ 7N 14 P,G ABX 1D- 24 D 3- 18 NAI-D DST $61 \mathrm{KNI} 7 \mathrm{~N} 14$ D.G ABX 10- $12 \mathrm{D}$ D- 2 THR-1 4PI $7 D B L I N 14$ HE, A ABX $21-24 \mathrm{D} 1-3$ NAI-D DST

$688 E 17 N 15$ E.E/ LFT 6 D $50-57$ MAG-D DST $7 D D A I T N 15$ E.E/ ABX 5- 8 D250,4DD MAG-O DST 
$58 R H 1$ TN 15 G,P ABX 11- 25 C 19, 25 EMU-D DST $64 K O 3 \quad 7 N 15$ G,P SPC $12-31$ C $19-31$ SCI-D 90

$72 D E 4 \quad 7 N 15$ G,P A8X $19-39$ C $19-39$ TEL-D 90

$63 F I 3 \quad 7 N 15$ GXXP SPC $13-31 C 25,31$ SCI-D 90

$695117 N 15$ P,C ABX 10 ABI. SEP ISOTOPES

$71 \mathrm{KUI} 7 \mathrm{~N} 15$ P,G ABX 11- SOURCE 250-670 KEV

$72 R A 2$ TN 15 P.G RLY 14 D 4 NAI-D 60

72WE2 7N 15 P,G A8X 13-17D 3- 8 NAI-D DST 547

OXYGEN $Z=8$

A ABUND. (1) G,N G,P SEPARATION ENERGIES (MEV)

$\begin{array}{llrrrlrrrr} & & G, N & G, P & G, T & G . H E 3 & G . A & G, 2 N & G, N P & G, 2 P \\ 16 & 99.76 & 15.7 & 12.1 & 25.0 & 22.8 & 7.2 & 28.9 & 23.0 & 22.3\end{array}$

$\begin{array}{rrrrrrrrrr}16 & 99.76 & 15.7 & 12.1 & 25.0 & 22.8 & 7.2 & 28.9 & 23.0 & 22.3 \\ 17 & 3.71-21 & 4.1 & 13.8 & 18.6 & 18.8 & 6.4 & 19.8 & 16.3 & 25.3\end{array}$

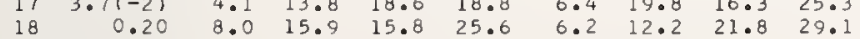

REF NUCLIDE REACTION RES EXCIT SOURCE DETECTOR

$59 \mathrm{CO}_{2} 80 \quad 15 \quad P . G \quad A B X \quad 19-25$ D $14-20$ NAI-D 90

61 COI 8015 P.G A8X 19- 25 D $14-20$ NAI-D 90

$\begin{array}{llllllll}63 B A 2 & 80 & 15 & P, G & \text { NOX } & 8 & D & 1\end{array}$ NAI-D DST

63HE 1 80 15 P.G LFT 8 D D O- NAI-D 55

66EVI 8C 15 P.G SPC 5-9D $1-2$ NAI-D DST

70 KUI 8015 P, G ABX $11-24$ D 2- 19 NAI-D DST $246+$

$72 \mathrm{PHI} 80 \mathrm{15} \quad \mathrm{P}, \mathrm{G}$ ABX $10-13 \mathrm{D} 3-\mathrm{6}$ NAI-D 90

60ISI 8016 E,E/ RLX 20-30 D 60-150 MAG-D DST

61 ISI 8016 E,E/ FMF O-115 D 90-215 MAG=D DST

E, EI FMF 44, 49 DI50 MAG-D UKN

$62 B I 28016$ E,E/ FMF 19 DI40-215 MAG-D DST

62EDI 8016 E,E/ NOX 0- 16 D 41 PI, MULTIPOLE

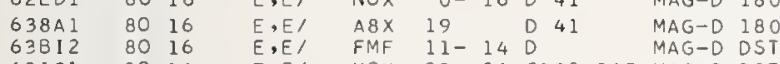

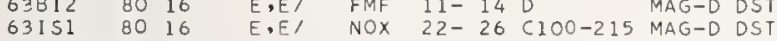

648128016 E,E, ABX O-150 D 90-215 MAG-D DST

$648138016 \quad E, E / A 8 X \quad 6-140100-218$ MAG RULES

FORM FACTORS

640028016 E,EI ABX 17- 32 D 40-70 MAG-D 180
FMF

65VA4 8016 E,E/ ABX 10-30 D 43-69 MAG-D 180

66AR2 8016 E, [/ LFT 7, 12 D 30-60 MAG-D DST

66CRI 8016 E,E, FMF $6 \quad \begin{array}{r}7=6.92,12=11.52 \\ \text { D } 600-800 \text { MAG-D DST }\end{array}$

$66 \mathrm{ST2} \quad 6=6.1 \mathrm{MEV}$

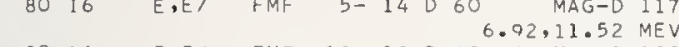

66VA1 8016 E,E/ FMF $10-30043-69$ MAG-D 180

$67 D R 18016$ E,E/ FMF 19- DI28- MAG-D DST

68DRI 8016 E,E/ FMF 19, 20 D J-PI, 19.08 MEV

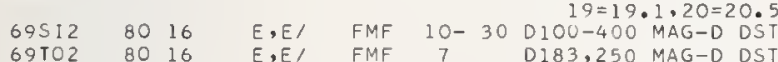

$708 E 18016$ E,E/ FMF $6-8051-105$ MAG-D MST

$70 G 018016 \mathrm{E}, E /$ LFT $19,20.05,6.13,6.91,7.1$

E. 19,20 D 46,54 MAG-D DST

$706048016 \quad E,[/$ FMF $21-26043-58$ MAG-D DST

$70 \mathrm{~K} 11$ 80 $16 \quad \mathrm{E}, \mathrm{E}, \mathrm{FMF} 11-14$ D $40-105$ MAG-D DST

$70 S T 18016$ E,E/ LFT 14-21 D 34-58 MAG-D DST

62814 80 16 E,N ABY 60-150 C $60-150$ ACT-I 4 PI

620018016

$58513 \quad 80 \quad 16$

$59 P E 2 \quad 80 \quad 16$

6OCA1 8016

$\begin{array}{lll}60 W Y 1 & 80 & 16 \\ 60211 & 80 & 16\end{array}$

$628 \cup 18016$

$638 \cup 1 \quad 80 \quad 16$

$65003 \quad 80 \quad 16$

65 WY1 8016

$69 B E 2 \quad 8016$

71AHI $80 \quad 16$

$E, P \quad A 8 \times 16-27 C 30$ MAG-D DST $249+$

G,MU-T RLX $16-25$ C $15-25$ ACT-I 4 P I

G,MU-T A8X 20- 21 D $20-21$ NAI-D $4 P I$

$\begin{array}{lllllll}G, M U-T & A 8 X & 20-21 & D & 20-21 & \text { NAI-D } & 4 P I \\ \text { G,MU-T } & A 8 X & 18-35 & C & 90 & \text { NAII-D } & 4 P I\end{array}$

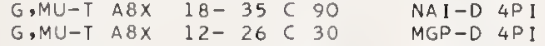

G,MU-T A8X 19- 27 C250 MGP-D 4PI

G,MU-T A8X $12-26 \quad 250 \quad$ MGP-D 4PI

G,MU-T ABX 20- 22 D 20- 22 NAI-D 4 PI

G.MU-T A8X 13- $22 \mathrm{C} \quad$ MGC-D 4PI

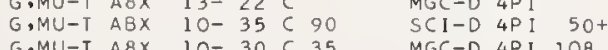

$\begin{array}{llllllll}G, M U-T & A 8 X & 10-30 & C & 35 & \text { MGC-D } & 4 P I & 1 \\ \text { G,MU-T } & A 8 X & \text { THR-150 } & C & 10-150 & \text { MGC-D } & 4 P I & \end{array}$
BO 16 G,G ASX 17 D 15,18 NAI-D 90

59PES 8016 G,G A8X 19-61C $19-61$ NAI-D 135

$\begin{array}{lllllllll}\text { GORE } & 80 & 16 & G, G & A S X & 7 & D & 7 & \text { NAI-D DST }\end{array}$

$\begin{array}{lllllllll}62 S E 1 & 80 & 16 & G, G & \text { NOX } 7 & C & 16 & \text { NAI-D } 90 \\ 64 L A 5 & 80 & 16 & G, G & \text { RLX THR- } 32 \text { C } 24,32 \text { NAI-D DST }\end{array}$

64LO3 80 16 G.G ABX 10-30 C $10-30$ NAI-D 140

68EVI 8016 G,G LFT 6,80 D, 8 SCD-D UKN

TOAHI 8016 G,G ABX 15-80 C108 NAI-D 135

$59 P E 5 \quad 8016 \quad G, G /$ ABX $19-61 C 19-61$ NAI-D 135

65MAI 8016 G,G SPC 19- 16 C 21- 31 NAI-D 140

$8016 \quad G, G / \quad A 8 X \quad 18-32 C 34$ NAI-D DST

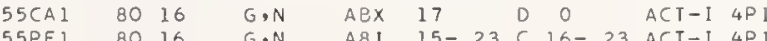

$\begin{array}{llllllll}55 P E 1 & 8016 & 6, N & A 81 & 15-23 C & 16-23 & A C T-1 & 4 P\end{array}$

ABPAKS

$\begin{array}{llllllllll}57 C A 2 & 80 & 16 & G, N & A B X & 16-31 & C & 15-30 & A C T-I & 4 P \\ 57 E R 1 & 80 & 16 & G, N & A B X & 16-32 & C & 16-32 & A C T-I & 4 P\end{array}$

$57 S P 28016$ G,N RLX $16-25$ C 14- 25 ACT-I 4P

$588 E 18016$ G.N RLY 15- 17 C 15- 17 ACT-I 4P

$59 K I 18016 \quad G, N \quad A B I 15-18 C 15-19 A C T-I$ L

NOX THR - $30 C 30 \quad B R K S$

SOMI3 8016 G.N NOX 16 G

$59 P E 3 \quad 8016$ G.N NOX 16-17C $16-18$ ACT-I 4 PI

59SA2 $8016 \quad \mathrm{G}, \mathrm{N}$

GOGEL 8016

SOGE 2 8016

8016

$G, N$

NOX THR- 22 C 15- 22 ACT-I 4 PI $\begin{array}{lllllll}A 8 X & 15-17 & 15-17 & A C T-I & 4 P 1 \\ \text { NOX } & 15-17 C & 15-17 & A C T-I & 4 P 1\end{array}$

$618 R 1 \quad 8016$

6IKE L 8016

$\begin{array}{lll}61 R 02 & 80 & 16 \\ 62 \mathrm{BI} & 80 & 16\end{array}$

$62802 \quad 8016$

$628 R 1$

8016

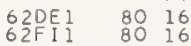

$62 \mathrm{~F} 12 \quad 80 \quad 16$

S2FUS 8016

$62 \mathrm{GOI} \quad 8016$

$62602 \quad 8016$

63 AN $180 \quad 16$

63 CAI $80 \quad 16$

$63 \mathrm{GE} 2$

4BE8

$80 \quad 16$

$\begin{array}{ll}80 & 16 \\ 80 & 16\end{array}$

8016

$64 T A 4$

65GRI $80 \quad 16$

65VEI 8016

$66 \mathrm{CO} 18016$

$66 \mathrm{CO} 1$
$66 \mathrm{FI}$

$66 \mathrm{MI} 2$

67 GE $2-80$

GBKAI $80 \quad 16$

$G, N$
$G, N$
$G, N$

$G, N$

RLY THR- $90 \mathrm{C} 90$ TEL-I DST

NOX $15-16$ C 15- 16 ACT-I 4PI

$A B I \quad 0-34 C 34 \quad A C T-14 P I$

$\begin{array}{lrlllll}A B I & 0-34 & C & 34 & A C T-1 & 4 P I \\ A B X & 17-18 & D & 17-18 & A C T-I & 4 P I\end{array}$

ASY $16-22 \mathrm{C} 22$ ACT-1 4 P

$G, N$

A8 I 60-150 C 60-150 ACT-I 4PI

$G, N$

$A B X 15,30 C 15-30$ BF 3-I $4 \mathrm{PI}$

G,N A8I THR- 32 C 32 ACT-I $4 P$

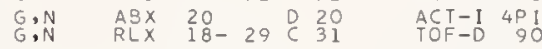

G,N RLX 18-29C26-32 TOF-D UKN

$G, N \quad A 8 \times \quad 21-31 C 31$ SCI-D 90

$G, N$

$G \cdot N$

$A B X 12-75 C 170 \quad C C H-D$ DST

$A B X 15-60 C 15-60 A C T-I 4 P$

$G, N \quad A B X \quad 16-30 D 16-30$ BF $3-I \quad 4 P I$

$G, N \quad A B X \quad 15-23 C 15-23$ ACT-I $4 P$

G.N

NOX $17-32 C 32 \quad$ SCI-D DST

$A B X 15-30 D$ 15- 30 BF $3-1$ 4PI

$\begin{array}{lllllll}\text { A } 8 X & 17-18 & D & 17-18 & \text { ACT-I } & 4 P \text { I } \\ \text { SPC } & 16-26 & D & 34 & \text { TOF-D } & 90\end{array}$

$G, N$ ABX THR- 54 C $10-66$ ACT-I 4 PI

G,N SPC THR- 33 C 34 TOF-D DST

$G, N$

$G, N$

T-I 4 PI $206+$

A8X THR- 65 CTHR- 70 ACT-I 4 PI 113

ASX 16- 27 D $16-278$ F 3-I 4PI

$G, N$ ABY THR - $27 \mathrm{C} \mathrm{22,27} 8 \mathrm{FB}-14 \mathrm{P}$

ABX $50-85$ C 55, 85 TOF-D 67

$\begin{array}{llll}68 W U 1 & 80 & 16 & \mathrm{G}, \mathrm{N} \\ 69 \mathrm{CO} & 80 & 16 & \mathrm{G}, \mathrm{N}\end{array}$

A $8 \times$ THR - 40 C $20-40$ TOF -D 90226

$691 \mathrm{~V} \quad 80 \quad 16$

$69 \mathrm{KHI} \quad 80 \quad 16$

S9NA1 8016

TOFI1 8016

$\begin{array}{lll}70 J U 1 & 80 & 16 \\ 7 \text { IFRI } & 80 & 16\end{array}$

TISAL 8016

57SV1 8016

66FU2 8016

$G, N$

$G, N$

$G, N$
$G, N$
$G, N$

$G, N$

$G, N G$

$660 \mathrm{Wl}$

$80 \quad 16$

G,NG $\begin{array}{lll}660 W 2 & 80 & 16\end{array}$

67MU1 $80 \quad 16$

$G, N G$

$G, N$

G,NG

$69 M U 1$

$6904 \mathrm{LI}$

$7 \mathrm{OHO}$

$500 C 1$

$628 R$

68ME 4

$70 F$ II
, POLARIZATION

RLX 16- 22 C $16-30$ ACT-I 4P I

$A 8 X \quad 16-33<22-33$ TOF-D 98

$A B X \quad 35-65 C 35-65$ TOF $-D \quad 68$

$A B X 16-34$ C I5-120 ACT-I 4PI

$A B X \quad 17-30 C 23-30$ TOF-D DS

A8Y $15-68$ C $10-68$ ACT-I 4 PI

A8X 20- 25 C 16- 30 NAI-D 120

RLY $20-26020-\begin{gathered}I N C L \text { (G, PG } \\ 26 \text { BF } 3-I 4 P]\end{gathered}$

RLY THR - 29 C 20- 29 SCD-D 135 RLX THR - 29 C $20-29$ SCD-D 135 SPC $21-26$ C 26 SCD-D 135 SPC $21-30 C 28,30$ SCD-D 90 SPC $16-30$ C $21-30$ GCD-D 90 ABY THR - 32 C 32 SCD-D 120 RLY $21-27$ C 27,36 NAI-D DSI ABY 22-800 C100-800 SCD-D 135 RLI THR-100 CTHR-100 ACT-I 4PI REL TO G,N ABI THR - $32 C 32$ ACI-I 4P I

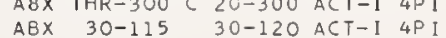
SPC $16-30$ C $21-30$ SPECTRUM 
$63 \mathrm{AUI} \quad 80 \quad 16$

$63 \mathrm{CO}_{3} \quad 80 \quad 16$

$64 \mathrm{FI} \quad 8016$

64 YEI $\quad 8016$

64BA3 8016

64BA5 8016

$65 C A 1 \quad 8016$

$65 \mathrm{HAl} \quad 8016$

$66 \mathrm{FI} 2-80$ I6

$67 \mathrm{FE} 2 \quad 80 \quad 16$

$67 \mathrm{MI2} 8016$

$70155 \quad 80 \quad 16$

$\begin{array}{lll}72 T H 2 & 80 & 16\end{array}$

55 ST1 8016

$56 C 01 \quad 8016$

$\begin{array}{lll}56 \mathrm{LI} & 80 \quad 16 \\ 57101 & 80 \quad 16\end{array}$

$\begin{array}{lll}57 J 01 & 80 & 16 \\ 575 V 1 & 80 & 16\end{array}$

$58 M I 1 \quad 80 \quad 16$

$598 R 2 \quad 8016$

6IFII $8016 \quad G, P$

$615 H^{3} \quad 80 \quad 16 \$ \mathrm{G}, P$

$62601 \quad 8016$

$62 \mathrm{GO} 280 \quad 16$

$\begin{array}{lll}635 C 1 & 80 & 16 \\ 65 M O 1 & 80 & 16\end{array}$

$65512 \quad 80 \quad 16$

$660 E 4 \quad 8016$

67 TH1 8016

$67 \mathrm{TH2} \quad 80 \quad 16$

$67 T U 3 \quad 8016$

$\begin{array}{lll}680 \mathrm{DE} & 80 & 16 \\ 685 \mathrm{Tl} & 80 & 16\end{array}$

68TU1 $80 \quad 16$

698A2 $80 \quad 16$

$69 F R 1 \quad 8016$

$\begin{array}{lll}69 K E 3 & 80 & 16\end{array}$

695T1 8016

66FU2 8016

$660 \mathrm{Wl} \quad 80 \quad 16$

66DW2 8016

67BA5 8016

67 MUI $80 \quad 16$

69MU1 $80 \quad 16$

69ULI $80 \quad 16$

$\begin{array}{llll}70 H D 1 & 80 & 16\end{array}$

$71 A D 1 \quad 8 D \quad 16$

$578 R 1 \quad 80 \quad 16$

$\begin{array}{lll}57 M I 1 & 80 & 16 \\ 61 \mathrm{HEZ} & 80 & 16\end{array}$

$53 F_{14} \quad 8016$

$66 \mathrm{VOl} \quad 8016$

$658 \cup 1 \quad 160$

$\begin{array}{lll}555 C 2 & 80 & 16\end{array}$

$66 \mathrm{GO2} \quad 8016$

$67 K R 2 \quad 8016$

$\begin{array}{lll}57 J 01 & 80 & 16 \\ 62601 & 80 & 16\end{array}$

$64 \mathrm{GRI} \quad 8016$

$64 T 02 \quad B D \quad 16$

$64 T 03 \quad B D 16$

$65 R O 2 \quad 8016$

7 OVDI 8016

55HAI $80 \quad 16$

56DAl 8016

$\begin{array}{lll}58 \mathrm{MAl} & 80 & 16 \\ 64 G R 1 & 80 & 16\end{array}$

$\begin{array}{lll}64 G R 1 & 80 & 16 \\ 64 T D 2 & 80 & 16\end{array}$

$64 T_{03} \quad 8016$

$\begin{array}{lll}65 R 01 & 80 & 16 \\ 56001 & 80 & 16\end{array}$

GOSTI 8016

$62601 \quad 8016$

$65 \mathrm{GAI} \quad 8016$

$\begin{array}{lll}58 \mathrm{MAl} & 80 & 16 \\ 62 \mathrm{MO2} & 80 & 16\end{array}$
G,XN ABX 15- 25 D $15-25$ BF 3-I 4 PI

G,XN ABX $16-75$ C $16-100$ BF $3-1$ 4PI

GOXN SPC THR- 31 C 31 SCI-D 90

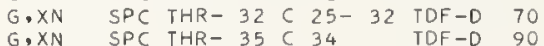

$\begin{array}{llll}G, X N \text { SPC THR- } 35 \mathrm{C} 34 & 34 \\ \text { BRANCHING RATIOS }\end{array}$

$G, X N \quad A B X 15-28 C 15-28$ C 15 (22MEV)

$G, X N \quad A B X \quad 17-28$ D $17-28$ BF $3-1$ IPI $38+$

$G, X N \quad A B X T H R-30 \mathrm{C}$ BRANCH RATIOS

$G, X N$ SPC THR- 65 C 65 TOF-D 90

$G, X N \quad A B X 100-150 C 150 \quad B F 3-1 \quad 4 P I$

G,XN SPC THR- 20 C $23-27$ TDF-D 100

$G, X N \quad A 8 X 16-25$ C $16-25$ BF $3-I$ IPI 34

,XN ABX 16-24C $15-24$ BF 3-I 4PI

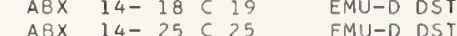

$G, P$ ABX $18-25$ C 25 EMU-D 90

G,P SPC 17- 70 C $30-70$ EMU-D DST

G,P ABX 12- 26 C 20- 26 EMU-D DST

G.P A8X 20- 25 C $16-30$ NAI-D 120

$G$,P ABX 15- 30 C $15-30$ EMU-D 100 $A B I$ I THR - 35 C 35 SCI-D 90 $S P C T H R-21 C 21 \quad E M U-D$ DST

G,P ABI THR-170 C170

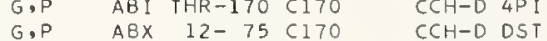

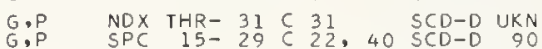

BRANCH RATIOS
G,P ABX 14- 16 C 14- 16 SCD-D 90

G,P ABI THR- 44 C $21-44$ TEL-D 90

G,P ABX THR- 55 C $21-55$ TEL-D 90

G,P RLX $19-26$ C $20-26$ SCD-D DST 202

$G, P \quad R L X \quad 20-25 C 26 \quad$ SCD $-D \quad 135$

G,P SPC THR- 22 D 22 EMU-D DST

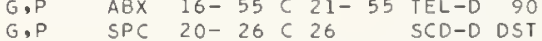

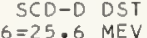

G,P SPC $21,22021,22$ EMU-D DST

G,P ABX $20-30 C^{21} 26,31,31,22=22.16$

G,P ABX $12-33 C 12-33$ SCD-D DST

$\begin{array}{lllllll}\text { G,P } & \text { SPC } & 16-26 & C & 26 & \text { SCD-D } & 90 \\ \text { G.P } & \text { RLX } & 12-27 & C & 23,27 & \text { EMU-D } & \text { DST }\end{array}$

G,P $\quad A B X \quad 21-33 C \quad 33 \quad$ SCD-D DST 194

G,PG RLY 20- 26 D 20- 26 BF3-I 4PI

$$
\text { CDINC }
$$

G.PG RLY THR- 29 C $20-29$ SCD-D 135

G $\$ P G \quad R L X$ THR - 29 C $20-29$ SCD-D 135

G,PG SPC 17- 26 C $26 \quad \begin{aligned} & \text { SCD-D } 135 \\ & \text { S-SPECTRUM }\end{aligned}$

G.PG SPC $17-30 C 28,30$ SCD-D 90

G,PG SPC $12-30 \subset 21-30$ SCD-D 90

ABY THR- 32 C 32 G-SPECTRUM

G,PG PLY 17- $27 \mathrm{C} 27,36 \mathrm{NAI}-\mathrm{D}$ DST

G.PG ABY $18-800 \quad 100-800$ SCD-D 135

$G, X P$ SPC $10-19$ C 28 EMU-D UKN

$G, X P$ NOX THR - 3D C 30 EMU-D DST

$\begin{array}{llllll}G . X P & S P C & 0-35 C & 35 & S C 1-D & 90 \\ G \times P & S P C & 14-30 & 31 & S C 1-D & 90\end{array}$

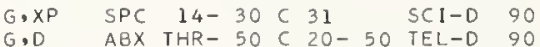

G,T THR 50 C 50 MULTIPOLARITY

$G, T$ ABY THR $-32 C 32$ ACT-I 4 PI

G, T ABX THR - 55 CTHR- 55 ACT-I 4 P I

$G, T$ RLX THR - 55 C $30-55$ ACT-I 4 PI

$G, A \quad A B Y \quad 7-14 C 17,24$ EMU-D $4 P I$

G.A ABX THR- 22 C 22 EMU-D DST

G.A ABX 9- 21 C 22 EMU-D DST

$G, A \quad A B X \quad 12-17 C 12-17$ EMU-D $4 \mathrm{PI}$

G, A RLY $17 \quad$ C 17 EMU-D DST

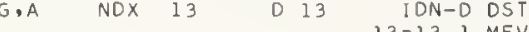

G.4A NOX 2D- 32 C 27, 33 EMU-D DST

G.4A ABI $22-25$ C 70 EMU-D UKN

$G, 4 A$ ABX 20- 40 C150,250 EMU-D 4PI

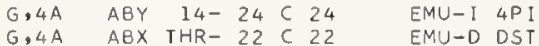

G.4A ABX $9-21$ C 22 EMU-D DST

$\begin{array}{llrlrl}G, 4 A & \text { ABX } & 12-17 & C & 12-17 \text { EMU-D } & 4 P I \\ \text { G,NP } & \text { RLX } & 140-260 & C 340 & & \text { TEL- }\end{array}$

G.NP RLY $153-320 \quad 320$ NP COINCIDENCE

GEL TD H2 CROS SEC

$G, N P \quad A B I$ THR-170 $C 170 \quad C C H-D \quad 4 P I$

G.NP ABX $250 \quad C 300 \quad$ TEL-D DST

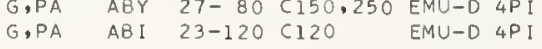

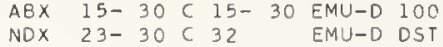

$G, T$ AEY 60-150 C 60-150 ACT-I 4PI

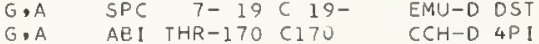

$55 S C 2 \quad 8016 \quad$ G,NA ABY THR- 32 C $32 \quad$ ACT $-14 P$

626018016 GNA ABI THR- 170 C 170 G 16 CCH $4 P$

$62 \mathrm{KOI} 8016$ G,PD RLY THR- $90 \mathrm{C} 90 \quad \mathrm{CCH}-\mathrm{I} 4 \mathrm{PI}$

$62 \mathrm{KO} 18016 \quad \mathrm{G}, \mathrm{ND} \quad \mathrm{RLY}$ THR- $90 \mathrm{C} 90 \quad \mathrm{CCH}-1$ 4PI

65AR3 80 16 G,BE7 RLY $32-57$ C $57 \quad$ ACT-1 $4 P$

66AR1 $8016 \quad$ G,BE7 AEX $30-57$ C $30-57$ ACT-1 $4 P$

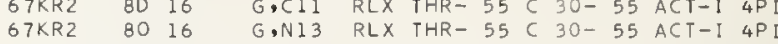

$59 C D 2 \quad 8 D$ 16 P,G AEX 21- 26 D 14- 19 NAI-D 90

59TAI $8016 \quad P, G \quad A B X \quad 16-20$ D $4-\quad$ NAI-D 90

$60 \mathrm{HEI} 8016$ P,G ABX 12 D $0-$ NAI-D UKN

$61 C D 18016 \quad$ P,G ABX 21- 26 D 10- 15 NAI-D 90

$617 A 3$ BD 16 P.G ABX $12-26000-14$ NAI-D 90

64EAI 16 P.G RLY 13- 170 1- NAI-D UKN

64 TA2 8016 P, NOX 13- 25 D 1- 13 NAI-D DST

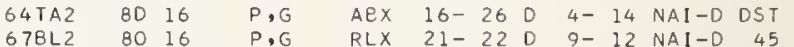

$678 L 1 \quad 80 \quad 16$

$67 E A 1 \quad 8016$

$70 B A 1 \quad 80 \quad 16$

$\begin{array}{lll}625 U 2 & 80 & 16 \\ 635 U 2 & 80 & 16\end{array}$

$\begin{array}{lll}635 U 2 & 80 & 16 \\ 635 U 2 & 80 & 16\end{array}$

645018016

65SU1 8016

$72 W E 1 \quad 8016$

64LA2 $80 \quad 16$

$P, G \quad R L X 20-30$ D $10-18$ NAI-D DST

$P, G \quad A B X \quad 13-2501-14$ NAI-D OST

$P, G \quad A B Y \quad 16-24 D$ 4- 13 NAI-D 90

$D, G \quad R L X \quad 21-250$ 1- 5 NAI-D 90

$D, G$ AEX $21-250$ 1- 11 NAI-D DS

, G ABX 21- 250 1- 4 NAI-D DST

D, G ABX 24- 28 D $1-5$ NAI-D DST

$D, G$ AEX $21-26$ D $1-6$ NAI-D DST

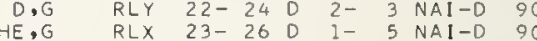

79

$64 M I 1 \quad 8016$

$67501 \quad 80 \quad 16$

$70 \mathrm{JA1} \quad 8016$

$\begin{array}{lll}\text { 70JAl } & 80 & 16 \\ \text { TOVOI } & 80 & 16\end{array}$

$71 K E 1 \quad 80 \quad 16$

$A, G$

$A B X 10-1303-\quad$ NAI-D DS

$A, G \quad R L X 13$ D- J-PI, WIDTHS

$A, G \quad R L X 14-250$ J-PI, WIDTHS

$A, G \quad A B X \quad 8-110$ 1- 5 NAI - D UKN

$A, G \quad A 8 X \quad 9-11001-4$ NAI-D 84

$A, G$ NOX 13 D 8 NAI-D DST

$A, G \quad A B \times \quad 12-140 \quad 7-\quad \begin{aligned} & 13=13.1 \\ & 9 \text { NAI-D DEV }\end{aligned}$

6ILAI 8018 E,E/ FMF 2- 6 DI50 MAG-D DST

65VA2 8018 E,E/ AEX 2- 27069 MAG-D 180

$71 G R I \quad 8018$ E,E/ FMF 2- 6092,106 MAG-D DS

$64 \mathrm{MUI} 8018 \mathrm{G}, \mathrm{N}$ SPC $10-20 \mathrm{C} 20$ LEVELS. BIEL

63FU2 8018 G,XN SPC 11- 31 JIPI, 2189 TRACK

$\begin{array}{lllll}55 R E I & 8 D & 18 & G & \end{array}$

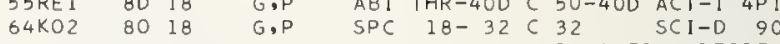

$69 H O 1 \quad 8018$ G.XP ABY THR- 33 C $24-33$ SCD-D 90

FLUORINE $Z=9$

A ABUND.

SEPARATIDN ENERGIES (MEV)

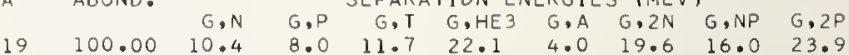

\begin{tabular}{|c|c|c|c|c|c|c|c|}
\hline & NUC & LIDE & REACTION & RES & EXCIT & SOURCE & DETECTDR \\
\hline REF & 2 & A & IN,OUT & & & & TYPE ANG NUM \\
\hline $635 \mathrm{E} \mathrm{I}$ & $9 \mathrm{~F}$ & 17 & $P, G$ & $R L Y$ & 4 & D & $\begin{array}{r}\text { NAI }-0 \text { OST } \\
J-P I,\end{array}$ \\
\hline
\end{tabular}

$71 C D 3$ IF 18 A,G ABX 4- 5 D $0-2 A C T-1 \quad 4 P I$

$638 A 1$ 9F 19 E,EI SPC O- 16 D 42 MAG-D 180

$70 W A 1$ OF 19 E,E/ LFT $1-2$ D 30- 60 MAG-D DST

62001 9F 19 E,P SPC $10-19$ C 18-30 MAG-D DST

66002 IF 19 G.MU-T AEX $8-30$ C260 $\quad$ MGP-D 4P

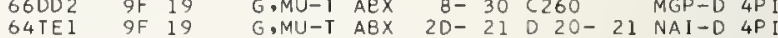

69BE2 IF 19 G,MU-T ABX $10-30 \mathrm{C} 35$ MGC-D 4 PI 107

60603 IF 19 G,G LFT 1 C O- NAI-D 100

6ORE1 $9 F 19$ G.G ABX 7 A 19 D 7 NAI-D 90

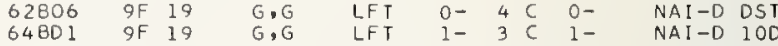

$64 \mathrm{LD} 3$ IF 19 G,G ABX $10-30 \mathrm{C} 10-30 \mathrm{NAI}-\mathrm{D} 140$

$67 \mathrm{LO}$ OF 19 G.G $\mathrm{ABX} 14-32 \mathrm{C} 34-30 \mathrm{NAI}-\mathrm{D} 140$

72SH2 IF 19 G,G SPC $0-14 C_{14} 14$ SCD-D B7

$586 E 1$ IF 19 G,N NOX 10- 11 C $10-11$ ACT-I 4 PI

$60 K 14$ IF 19 G.N ABX 1D- 23 C $10-23$ ACT-I 4PI

60SA2 IF 19 G,N RLY $10-12$ C 10- 12 ACT-I 4PI

60WA2 IF 19 G,N ABX 10-240 C120-240 ACT-I 4PI

IF $19 \quad G, N \quad A B X \quad 20-21$ D 20-21 ACT-I $4 P I$

66DE5 IF 19 G,N NOX THR-260 C260 ACT-I DST

$678 A 3$ IF 19 G,N ABX THR- 25 CTHR- 25 ACT-I $4 P I$

$71 B A 2$ IF 19 G.N ABX $12-15 \mathrm{C} 15$ N 35223 BREAKS

$71 F R I$ IF 19 G.N ABY $10-800$ C $100-800$ ACT-I 4PI

59061 IF 19 G,2N RLI THR-100 CTHR-100 ACT-I 4PI

72VA2 IF 19 G,XN ABX $10-19$ C 10-19 BF3-I 4PI 


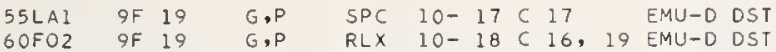
$628 R 3$ OF 19 G,P SPC 18 D 18 EMU-D DST $63 \mathrm{MUI} 9 \mathrm{~F} 19 \mathrm{GP}$ SPC $11-24 \mathrm{C} 24 \quad$ SCD-D 120 $725 \mathrm{HZ}$ IF 19 G,PG SPC $0-14 \mathrm{C} 14 \quad$ SCD-D 87 $55 R E 1$ OF 19 G.2P ABI THR-40D C $8 J-400$ ACT-I 4 PI 64 SE 1 OF 19 G,XP SPC THR- 24 C $14-24$ EMU-D DST $68 A B 1$ OF 19 G,XP SPC THR- 22 C 22 SCD-D 90 $65 \mathrm{HA2}$ OF 19 G,A SPC THR - 31 C 31 EMU-D DST

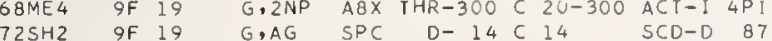

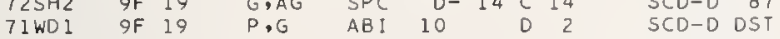
$10=10.129 \mathrm{MEV}$ $72 R 03-9 F \quad 19=A, G=-L F T-5=$ $678 E 7$ SF 20 NGG LFT 6- 70 O- 1 NAI-D 90 NEON $Z=10$

\begin{tabular}{rrrrrllllll} 
A & ABUND. & \multicolumn{1}{c}{ SEPARATIDN ENERGIES (MEV) } \\
& & G.N & G.P & G.T & G,HE3 & G,A & G,2N & G.NP & G, 2P \\
20 & 90.92 & 16.9 & 12.8 & 23.9 & 21.2 & 4.7 & 28.5 & 23.3 & 20.8 \\
21 & 0.26 & 6.8 & 13.0 & 21.6 & 19.9 & 7.3 & 23.6 & 19.6 & 23.6 \\
22 & 8.82 & 10.4 & 15.3 & 21.5 & 26.3 & 9.7 & 17.1 & 23.4 & 26.4
\end{tabular}

\begin{tabular}{|c|c|c|c|c|c|c|c|c|}
\hline \multirow{2}{*}{ REF } & NUCLI I DE & \multirow{2}{*}{$\begin{array}{l}\text { REACT ION } \\
\text { IN,DUT }\end{array}$} & \multirow{2}{*}{ RES } & \multirow[t]{2}{*}{ EXCIT } & \multirow[t]{2}{*}{ SOURCE } & \multicolumn{2}{|c|}{ DETECTDR } & \multirow[b]{2}{*}{ NUM } \\
\hline & $z$ & & & & & TYPE & ANG & \\
\hline $62 \mathrm{GDI}$ & IONE & $G, M U-T$ & $A B I$ & $0-170$ & $C_{1}>0$ & $\mathrm{CCH}-\mathrm{D}$ & 4P I & \\
\hline \multirow[t]{2}{*}{$62 \mathrm{GOI}$} & $1 O N E$ & $G, N$ & $A B I$ & THR -170 & $C 170$ & $\mathrm{CCH}-\mathrm{I}$ & 4PI & \\
\hline & & & & & INCL & LUDES G &, $2 N$ & \\
\hline \multirow{2}{*}{$57 K D 1$} & IONE & $G, P$ & $A B Y$ & THR - 80 & C 80 & $C C H-D$ & DST & \\
\hline & & & & & & & $A B I$ & \\
\hline $62 \mathrm{GO}$ & IONE & $G, P$ & $A B I$ & $T H R-170$ & $C 170$ & $\begin{array}{l}\text { CCH-1 } \\
\text { CLUDES }\end{array}$ & $\begin{array}{l}4 P I \\
G, D\end{array}$ & \\
\hline $57 \mathrm{KD} 1$ & IONE & $G, 2 P$ & $A B Y$ & THR - 80 & C 80 & $\mathrm{CCH}-\mathrm{D}$ & 4PI & \\
\hline $625 U 1$ & IONE & $G, X P$ & $S P C$ & $16-23$ & C 23 & EMU-D & 90 & \\
\hline $63 \mathrm{~F}: 4$ & $1 O N E$ & $G, X P$ & $S P C$ & $15-29$ & C 31 & $S C I-0$ & 90 & \\
\hline $62 \mathrm{GDI}$ & IONE & $G, A$ & $A B I$ & THR -170 & C170 & $\mathrm{CCH}-\mathrm{I}$ & & \\
\hline $57 \mathrm{KDl}$ & IONE & $G, 2 A$ & $A B Y$ & THR - 80 & $C 80$ & $\mathrm{CCH}-\mathrm{D}$ & 4P I & \\
\hline $57 \mathrm{KOI}$ & IONE & G, 5A & $A B Y$ & THR - 80 & C 80 & $\mathrm{CCH}-\mathrm{D}$ & $4 \mathrm{PI}$ & \\
\hline $57 \mathrm{KOl}$ & IONE & $G, N P$ & $A B Y$ & THR - 80 & C 80 & $C C H-D$ & $4 \mathrm{PI}$ & \\
\hline 62601 & IONE & $G, N P$ & $A B I$ & THR -170 & $C 170$ & $\mathrm{CCH}-\mathrm{I}$ & 4P I & \\
\hline $62 \mathrm{GDI}$ & IONE & $G, N A$ & $A B I$ & IHR- 170 & $C 170$ & $\mathrm{CCH}-\mathrm{I}$ & $4 \mathrm{PI}$ & \\
\hline $57 K D 1$ & IONE & $G, P A$ & $A B Y$ & THR - $8 D$ & $C 80$ & $\mathrm{CCH}-\mathrm{D}$ & 4PI & \\
\hline 55REI & IONE & G,N17 & $A 8 I$ & $T H R-400$ & c $90-400$ & $A C T-1$ & 4P I & \\
\hline \multirow[t]{2}{*}{$638 \mathrm{Al}$} & $1 O N E 2 O$ & $E, E \prime$ & SPC & $0-16$ & D 42 & MAG-D & 180 & \\
\hline & & & & & $J-P_{1}$, WI & I DTHS, & $A 8 x$ & \\
\hline $71 B E 1$ & 1ONE 20 & $E, E /$ & $A B X$ & 11 & D $\begin{aligned} 39,56 \\
11.23\end{aligned}$ & $\begin{array}{l}M A G=D \\
.11 .58\end{array}$ & $\begin{array}{l}180 \\
M E V\end{array}$ & \\
\hline \multirow[t]{2}{*}{$71 \mathrm{HOI}$} & IONEZZ & $E, E /$ & FMF & 1,4 & 0183,250 & $M A G-D$ & DST & \\
\hline & & & & & 1.63 & $3,4.25$ & MEV & \\
\hline \multirow[t]{2}{*}{$72 M I 4$} & IONEZZO & $E, E$, & FMF & $6-8$ & $0120-250$ & $M A G-D$ & DST & \\
\hline & & & & & $.72-7.84$ & MEV LEV & VELS & \\
\hline \multirow[t]{2}{*}{62001} & IONEZZO & $E, P$ & $S P C$ & $3-11$ & $C 18-30$ & $M A G-D$ & DST & \\
\hline & & & & & & & $A B I$ & \\
\hline \multirow[t]{2}{*}{$61 \mathrm{CLI}$} & $1 O N E 20$ & $G, G$ & LFT & $1-3$ & $1-$ & NAI I D & 0 & \\
\hline & & & & & $1.63,2.62$ & $2 \cdot 3 \cdot 34$ & MEV & \\
\hline 59HAI & 1ONE 20 & $G, N$ & $A B x$ & 15,18 & D 15,18 & I DN-D & 4P I & \\
\hline $59 \mathrm{HAl}$ & IONE $2 O$ & $G, P$ & $A B X$ & 15.18 & D 15,18 & $I O N-D$ & $4 \mathrm{Pl}$ & \\
\hline 60REZ 2 & IONEZO & $G, X P$ & RLY & $100-240$ & $C 240$ & $C \mathrm{CH}-\mathrm{D}$ & 4PI & \\
\hline $69 \mathrm{HDI}$ & 1 ONEZO & $G, X P$ & $A B Y$ & THR- 33 & $C 24-33$ & $S C 1-D$ & 90 & \\
\hline $59 \mathrm{HAl}$ & IONEZZO & $G, A$ & $A B X$ & 15,18 & D 15,18 & I DN-D & 4P I & \\
\hline $59 \mathrm{HAI}$ & IUNEZ $2 O$ & $G, 2 A$ & $A B X$ & 15,18 & D 15,18 & ION-D & $4 \mathrm{PI}$ & \\
\hline $59 \mathrm{HAI}$ & IONEZ 20 & $G, P A$ & $A 8 X$ & 15,18 & D 15,18 & $I D N-D$ & $4 \mathrm{PI}$ & \\
\hline 60BRI & IONEZ 20 & $P, G$ & RLY & $17-23$ & D $4-11$ & NA! -D & UKN & \\
\hline $64 A L 3$ & $10 N E 2 O$ & $P, G$ & NOX & $17-22$ & D 4- & NAI -D & DST & \\
\hline $64 \mathrm{TAI}$ & $1 O N E Z O$ & $P, G$ & $A B X$ & $17-24$ & D 4- 11 & NAI I-D & 90 & \\
\hline 66PA3 & IONEZO & $P, G$ & NDX & 18 & D & $S C I-D$ & DST & \\
\hline $67 \mathrm{SE} I$ & IONEZZO & $P, G$ & $A B X$ & $16-25$ & $3-13$ & $N A !-D$ & DST & $73+$ \\
\hline \multirow[t]{2}{*}{$72 A L 4$} & $10 N E 2 O$ & $A, G$ & LFT & $7-9$ & $3-5$ & $S C D-D$ & DST & \\
\hline & & & & & & $4 \mathrm{~L}$ & & \\
\hline \multirow[t]{2}{*}{$60 \mathrm{KD} 2$} & 1ONE 22 & $G, P$ & SPC & $T H R-90$ & C 90 & $\mathrm{CCH}-\mathrm{D}$ & DST & \\
\hline & & & & & SEPAR & D ISDT & & \\
\hline $69 \mathrm{HD} 1$ & IONE $2 Z$ & $G, X P$ & $A B Y$ & THR- 33 & C $24-33$ & $5 C I-D$ & 90 & \\
\hline $59 \mathrm{HAI}$ & IONE 22 & $G, A$ & $A B X$ & 15,18 & D 15,18 & $I D N-D$ & $4 \mathrm{PI}$ & \\
\hline \multirow[t]{2}{*}{$60 \mathrm{KD} 2$} & IDNE 22 & $G, N P$ & SPC & $T H R-90$ & C 90 & $C C H-D$ & DS I & \\
\hline & & & & & SEPARATE & D ISDTC & OFt & \\
\hline $68 \mathrm{GR} I$ & $10 N E 22$ & $A, G$ & LFT & 12,13 & D 2, 3 & NAI $-D$ & DST & \\
\hline
\end{tabular}

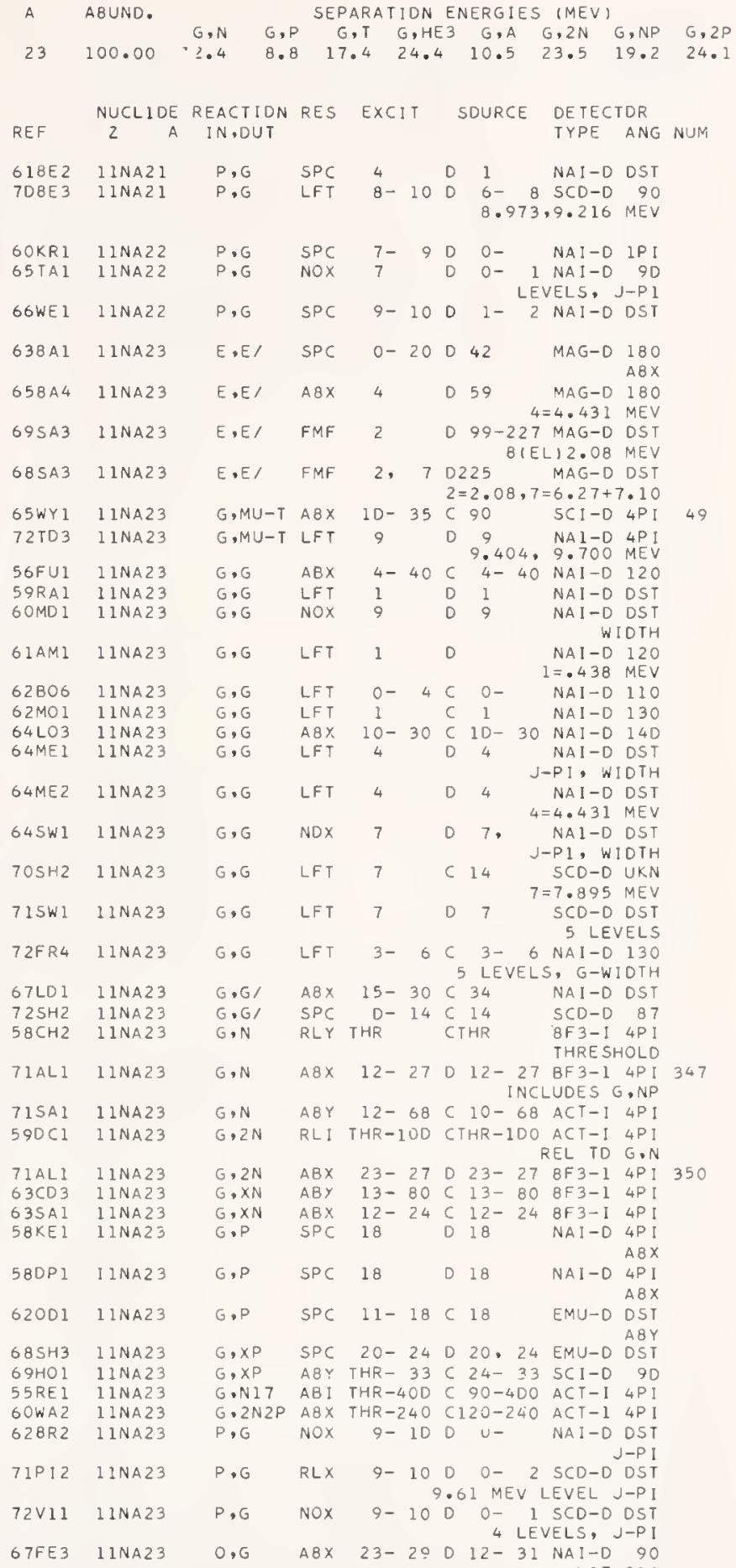

MAGNESIUM $Z=12$

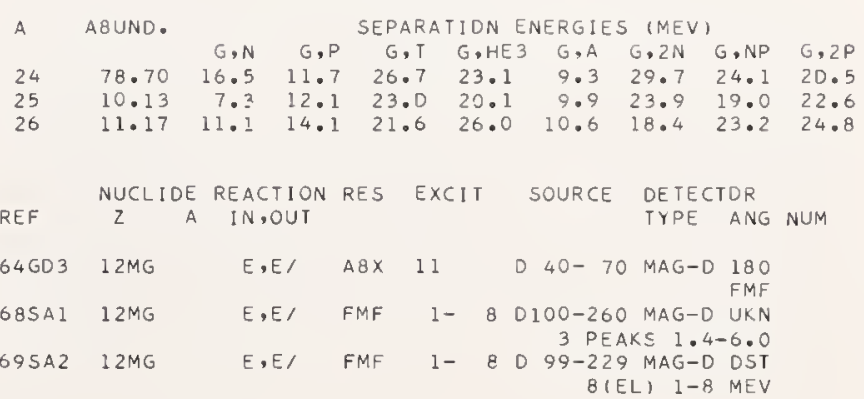




\begin{tabular}{|c|c|c|}
\hline 64002 & $12 M G$ & $G, M U-T$ \\
\hline 65002 & $12 M G$ & $G, M U-T$ \\
\hline $65 W Y 1$ & $12 M G$ & $G, M U-T$ \\
\hline 66002 & $12 M G$ & G,MU-T \\
\hline $56 \mathrm{FUI}$ & $12 M G$ & $G, G$ \\
\hline & & \\
\hline 59 LAI & $12 M G$ & $G, G$ \\
\hline & & \\
\hline $618 \cup 4$ & $12 M G$ & $G, G$ \\
\hline $615 U 1$ & $12 M G$ & $G, G$ \\
\hline $62 \mathrm{SEI}$ & $12 M G$ & $G, G$ \\
\hline $635 \cup 1$ & $12 M G$ & $G \cdot G$ \\
\hline $67 L D 1$ & $12 M G$ & $G, G /$ \\
\hline $58 S P 2$ & $12 M G$ & $G, N$ \\
\hline $64 \mathrm{FI} 2$ & $12 M G$ & $G \cdot N$ \\
\hline 70WE I & $12 M G$ & $G, N$ \\
\hline $56 Y E 1$ & $12 M G$ & $G, \times N$ \\
\hline $63 \mathrm{CD}_{3}$ & $12 M G$ & $G, \times N$ \\
\hline $65 \mathrm{MII}$ & $12 M G$ & $G, \times N$ \\
\hline $66 \mathrm{FI} 2$ & $12 M G$ & $G, \times N$ \\
\hline $71 \mathrm{FU} 2$ & $12 M G$ & $G, \times N$ \\
\hline & & \\
\hline $62 \mathrm{SHII}$ & $12 M G$ & $G, P$ \\
\hline $64 \mathrm{FDI}$ & $12 M G$ & $G, P$ \\
\hline & & \\
\hline $715 A 2$ & $12 M G$ & $G, P$ \\
\hline 64 [ S ] & $12 M G$ & $G, \times P$ \\
\hline $65 \mathrm{MA4}$ & $12 M G$ & $G, X P$ \\
\hline $66 \mathrm{HD} 3$ & $12 M G$ & $G, A$ \\
\hline 55RE I & $12 M G$ & $\mathrm{G}, \mathrm{N} 17$ \\
\hline & & \\
\hline $\begin{array}{l}56 \mathrm{HE} 3 \\
638 \mathrm{Al}\end{array}$ & $12 M G 24$ & $E, E /$ \\
\hline $638 \mathrm{Al}$ & $12 M G 24$ & $E, E /$ \\
\hline & & \\
\hline 66AR2 & $12 M G 24$ & $E, E /$ \\
\hline & & \\
\hline $66 \mathrm{TII}$ & $12 M G 24$ & $E, E /$ \\
\hline & & \\
\hline $67 \mathrm{T!1}$ & $12 M G 24$ & $E, E /$ \\
\hline $68 \mathrm{FAl}$ & $12 M G 24$ & $E, E /$ \\
\hline $69 \mathrm{~T} 11$ & $12 M G 24$ & $E, E /$ \\
\hline $70 \mathrm{FAl}$ & $12 M G 24$ & $E, E /$ \\
\hline & & \\
\hline $70 G 03$ & $12 M G 24$ & $E, E^{\prime}$ \\
\hline $70 \mathrm{KHI}$ & $12 M G 24$ & $\bar{E}, \bar{E} /$ \\
\hline & & \\
\hline $70 S T 2$ & $12 M G 24$ & $E, E /$ \\
\hline $71 \mathrm{HO} 1$ & $12 M G 24$ & $E, E /$ \\
\hline & & \\
\hline $71 M O 3$ & $12 M G 24$ & $E, E /$ \\
\hline $72 \mathrm{NAI}$ & $12 M G 24$ & $E, E /$ \\
\hline & & \\
\hline $588 \cup 1$ & $12 M G 24$ & $G, G$ \\
\hline $608 \cup 2$ & $12 M G 24$ & $G, G$ \\
\hline 60803 & $12 M G 24$ & $G, G$ \\
\hline $60 \mathrm{ME} \mathrm{I}$ & $12 M G 24$ & $G, G$ \\
\hline & & \\
\hline $60 T 01$ & $12 M G 24$ & $G, G$ \\
\hline & & \\
\hline 62806 & $12 M G 24$ & $G, G$ \\
\hline 64801 & $12 M G 24$ & $G, G$ \\
\hline $67 \mathrm{KU} 2$ & $12 M G 24$ & $G: G$ \\
\hline & & \\
\hline $715 W 2$ & $12 M G 24$ & $G, G$ \\
\hline & & \\
\hline $60 \mathrm{KIl}$ & $12 M G 24$ & $G, N$ \\
\hline $66 M 12$ & $12 M G 24$ & $G, N$ \\
\hline $68 \mathrm{COl}$ & $12 M G 24$ & $G, N$ \\
\hline $680 K 2$ & $12 M G 24$ & $G, N$ \\
\hline $718 \mathrm{~A} 2$ & $12 M G 24$ & $G, N$ \\
\hline $71 F \cup 2$ & $12 M G 24$ & $G, N$ \\
\hline & & \\
\hline 55NAI & $12 M G 24$ & $G, X N$ \\
\hline 69 AN2 & $12 M G 24$ & $G, \times N$ \\
\hline & & \\
\hline 72151 & $12 M G 24$ & $G, X N$ \\
\hline 63YAl & $12 \mathrm{MG} 24$ & $G, P$ \\
\hline & & \\
\hline 66 IS1 & $12 M G 24$ & $G, P$ \\
\hline 6OWA2 & $12 M G 24$ & $G, 2 N 3 P$ \\
\hline $63 \mathrm{SH} 3$ & $12 M G 24$ & $G, C 12$ \\
\hline $64 \$ H 6$ & $12 M G 24$ & $G, F$ \\
\hline 61601 & $12 M G 24$ & $P, G$ \\
\hline 62GL1 & $12 M G 24$ & $P, G$ \\
\hline $62 P R I$ & $12 M G 24$ & $P, G$ \\
\hline & & \\
\hline $63 \mathrm{GD} 3$ & $12 M G 24$ & $P, G$ \\
\hline 67 LE I & $12 M G 24$ & $P, G$ \\
\hline $68 B E 3$ & $12 M G 24$ & $P, G$ \\
\hline $68 \mathrm{HII}$ & $12 M G 24$ & $A, G$ \\
\hline & & \\
\hline & & \\
\hline $69 F A 2$ & $12 M G 25$ & $E, E /$ \\
\hline & & \\
\hline & & \\
\hline & & \\
\hline
\end{tabular}


63VA3 13AL27 G,G NOX 2 C 3 NAI-D DST

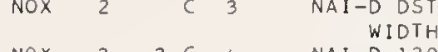

64801 13AL27 G,G LFT 1 - 3 C 1 - NAI-D 100

$64 M E 2$ 13AL27 G.6 LFT 4 D 4 NAI-D DST

65KHI 13AL27 G.G LFT 2,3 D $2, \quad 3$ NAI-D DST

65ME3 13AL27 G.6 LFT $4 \quad 2,3=2.21,2.98 \mathrm{MEV}$

66HO2 13AL27 G,G LFT I C I NAI-D 217

G6VA4 $13 A L 27 \quad G .6 \quad A B I \quad 10 \quad$ D $10 \quad$ NAI-D BO

G.G LFT 3 10 $10.1 \mathrm{MEV}$

72SH2 13AL27 G.6/ SPC $0-14 C^{2.21,2.98,3.0 \mathrm{MEV}}$

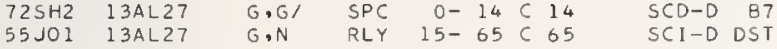

STFE 13 13AL27 G,N RLY $22-31 C 31$ THR-I DST

SBAS1 13AL27 G.N NOX $13-17$ C 17 SCI-I DST

$\begin{array}{llll}5 B C H 2 & 13 A L 27 & G, N & R L Y \\ 5\end{array}$

5BFE1 13AL27 G.N ABX 13-30C 13-30 ACT-I 4PI

$59 K U 1$ 13AL27 G,N NOX $23-8 B C$ BB WUASI-DEUTERON

$60 \mathrm{CH} 213 A L 27$ G.N ABX 13-21 D 12-21 BF3-1 4PI

GOGE3 13 AL27 G.N NOX THR CIHR SF3-I 4 PI

60KU2 13AL27 G.N RLY 23-90C90 THRESHOLD

61BAL $13 A L 27$ GN ABX 13- 19 C $12-19$ BF3-I 4PI

6IPRI 13AL27 G,N RLX $13-85$ C $25-85$ THR-D

$62 B 02$ 13AL27 G,N ABX 13-30 C 14-30 BF3-I 4PI

62MUI 13AL27 G,N ABX 13-24C 13- 24 BF 3-I 4P I

63MI4 13AL27 G.N SPC 15- 30 C 24 , 30 EMU-D 90

$64 \mathrm{TH1} 13 \mathrm{AL} 27 \quad G, \mathrm{~N} \quad \mathrm{ABX} 15-24 \mathrm{C} 15-24 \mathrm{ACT}-\mathrm{I}$ 4PI

65 TH2 13 AL27

$66 B 11 \quad 13 A L 27$

66FU1 $13 A L 27$

$676 E 2 \quad 13 A L 27$

6BKAI I3AL27

66FUI 13AL 27

55BA5 13AL 27

55DII 13AL 27

$5 B B A 5$ I3AL 27

5BFE 1 13AL27

$59 C_{0} 3 \quad$ I3AL27

$63 \mathrm{CO} 3$ 13AL27

67ANE 13AL27

67AN2 13AL27

69AN3 13AL27

$\begin{array}{ll}69 C 02 & 13 A L 27 \\ 69153 & 13 A L 27\end{array}$

69VEI 13AL 27

$71 C 02$ I3AL27

$55 J 01 \quad 13 A L 27$

$598 A 2 \quad 13 A L 27$

$60 \mathrm{CH} 213 A L 27$

$61 M A 113 A L 27$

62SE2 13AL 27

62SHB 13AL27

62SHII 13AL27

$\begin{array}{ll}63001 & 13 A L 27 \\ 66 L 11 & 13 A L 27\end{array}$

$69 \mathrm{CO}$
$70 \mathrm{CO}$
$13 \mathrm{ALL} 27$

$70 C 02213 A L 27$

5 BAUI $13 A L 27$

56DA2 13AL27

$57 B A 2 \quad 13 A L 27$

$5 B 8 A 6 \quad 13 A L 27$

$60 \mathrm{CHI} 13 A L 27$

G1MA2 13AL27

$62 \mathrm{CH} 2$ 13AL27

$63 M 15 \quad 13 A L 27$

64MAZ 13AL27

$6 B A B 3 \quad 13 A L 27$

61MA1 13AL27
$60 \mathrm{CH} 13 \mathrm{AL} 27$

$62 \mathrm{CH} 213 \mathrm{AL} 27$

71AN2 13AL27

$56 \mathrm{HEI} 13$ ALL27

56WAI 13AL 27

$70 C U 1$ 13AL27

60602 13AL27
$G, N$ ABX $13-24 \mathrm{C} 13-25$ ACT-I 4 PI 76

ABX 20-200 C 20-200 BF 3-I 4PI

$G, N \quad A B X \quad 13-37 \quad D \quad 13-37$ BF 3-I $4 P I 115$

$G, N$ RLX THR- 65 C $13-65$ ACT-I $4 \mathrm{PI}$

$A B X \quad 50-85 C 55, B 5$ TOF-D 67

NEUT ENGY SPEC

G, 2N ABX 25- 37 D 25- 37 BF 3-I 4PI 116

$G, X N$ ABY $30-200$ C15U-250 THR-I DST

$6, X N$ NOX $13-70 C 70$ SCI-I DST

$G, X N$ RLY THR- 15 C $12-15$ BF3-I 4 PI

$G, X N \quad A B X \quad 13-30 C 13-30$ BREAKS

SPC 15- $30 C 24$ FAST NEUTRONS

$A B X 12-80 C 24,30$ EMU-D 90

$G, X N$ NOX THR- $34 C 34$ THR-I DST

$G, X N$ SPC THR- 65 C 65 TOF-D 90

$G, X N$ ABX THR- 26 C $13-26 \quad B F 3-1$ 4P I

G, RN RLX 13- 65 C $13-65$ ACT-1 4P !

$G, X N \quad A B X \quad 13-30 \quad C \quad 5-30 \quad B F 3-1 \quad 4 P I \quad 453$

$G, X N$ SPC THR- 33 C 33 TOF-D DST

$G, X N A B I \quad 36-64 C 10-64$ BF 3-I 4PI

G,P RLY THR- 65 C 65 FAST N YIELD

$G, P$ SPC $25-52$ C $18-52$ TEL-D 90

$G, P \quad A B X 12-21<12-21$ ION-I $4 P$ !

G,P RLY $11-35$ C 35 MGP-D UKN

G,P ABX 15, 18 D 15, 1B SCIID

$\begin{array}{lllllll}G, P & A B X & 15,18 & D & 15,1 B & S C I-D \\ G, P & \text { SPC } & 10-24 & C & 24 & & \\ \text { GMU-D DST } & \end{array}$

G,P ABX $10-24$ C $24 \quad$ SCI-1 4 P I

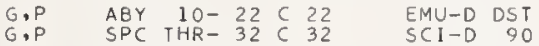

G,P ABY B- B5 C B5

G, P ABX 12- 22 C 15- 22 SCD-1 90

G, 2P ABX 20-63 C 20-63 ACT-1 4PI

$G, X P$ SPC $11-70<70$ EMU-D DST

$G, X P$ SPC THR- $85 C B 5$ C TEL-D ABY

$G, X P$ SPC THR- $85 C B 5,00-T E L-D$ DST

$G, X P$ RLX 24- 90 C 90 TO TEL-1 90

G $X P$ REL TO DEUTERONS

G, XP NOX $8-21$ C 21 SCI-D DST

$G, X P$ RLY $23-35 C 35$ MAG-D DST

$\begin{array}{llll}G, X P \text { ABY 12- } 22 C 22 & C 2 \\ G & \text { SCI-D DST }\end{array}$

$G, \times P$ SPC THR- 20 C 20 SCD-D

G,XP SPC THR- 27 C 22, 27 SCD-D 90

$\begin{array}{llllll}G \cdot D & \text { RLY } & 20-35 & C & 35 & \text { MGP-D DST } \\ G, X D & \text { RLX } & 32-90 & C & 90 & \text { TEL- }\end{array}$

REL TO PROTONS

$G, X D$ RLY $32-35 \subset 35$ MAG-D DST

$G, X D$ ABX 107-999 C999 REL TO PROTONS

G, T RLY THR - 31C $31999=1.14 \mathrm{GEV}$

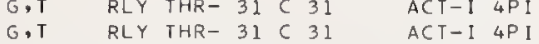

$G$, HE3 ABX $30-260$ C $30-260$ ACT-1 4 PI
$G, N$ ABY THR- 27 C 22,27 BF 3-1 $4 P I$

G.P RLY B- $34 \mathrm{C}$ B5 TEL-D DST

G.T ABY THR- 90 C 90 ACT-I 4 P I

\begin{tabular}{|c|c|c|c|c|c|c|c|}
\hline 801 & $A L 27$ & , $A$ & SPC & THR- 30 & C 31 & EMU-D & \\
\hline TOZ & - & - A & $A B Y$ & $10-$ & & $E M U-I$ & \\
\hline IMA 1 & $13 A L 27$ & , A & RLY & $19-35$ & C 35 & $M G P-D$ & 0 \\
\hline $28 E 3$ & $13 \mathrm{AL}$ & , A & SPC & $16-30$ & $\begin{array}{l}C 30 \\
\text { ASYMME }\end{array}$ & $\begin{array}{l}\text { SCD-D } \\
\text { Y SPECT }\end{array}$ & \\
\hline 5 SHAZ & $13 A L 27$ & , A & $S P C$ & THR- 31 & C 31 & EMU-D & 5 \\
\hline 3 & $13 A L$ & & oc & THR- & & -0 & 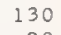 \\
\hline 2 & $13 A L$ & & $8 x$ & 10 & & & 90 \\
\hline & $13 A$ & $N$ & RLX & $149-320$ & $\begin{array}{l}\text { C320 } \\
\text { REL TO H. }\end{array}$ & $\begin{array}{r}\text { TEL-D } \\
12 \text { CROS }\end{array}$ & $\begin{array}{r}76 \\
\text { SEC }\end{array}$ \\
\hline 602 & AL27 & , N2P & $A B X$ & $30-260$ & c $30-260$ & $A C T-1$ & \\
\hline & & & RLX & THR-999 & & $A C T-I$ & $4 \mathrm{PI}$ \\
\hline & $3 A L-\angle C$ & • PD & $A B X$ & 30 & 60 & $A C T-1$ & \\
\hline$B M E 4$ & $13 A L 2$ & NP & $A B X$ & THR & & $A C T-1$ & 4 \\
\hline OWAZ & 13 & $4 \mathrm{P}$ & $A B x$ & THR & & $A C T-1$ & . \\
\hline $\begin{array}{l}55 R E 1 \\
34 M A 4\end{array}$ & $\begin{array}{l}13 \mathrm{Al} \\
13 \mathrm{Al}\end{array}$ & $\begin{array}{l}\text { N17 } \\
F 18\end{array}$ & $\begin{array}{l}A B I \\
A B Y\end{array}$ & $\begin{array}{l}\text { THR } \\
150\end{array}$ & $\begin{array}{l}00 \\
20\end{array}$ & $A C$ & $\begin{array}{l}4 P \\
4 P\end{array}$ \\
\hline 71013 & $13 A L$ & , NA22 & $A B Y$ & THR. & 9 & $A C T-1$ & $4 \mathrm{PI}$ \\
\hline 4MAG & & & & & & $999=1$ & \\
\hline 9NO1 & $\begin{array}{l}13 A L 27 \\
\end{array}$ & , NA24 & $A B Y$ & $31-999$ & C100-999 & $A C T-1$ & $4 P 1$ \\
\hline & & & & & & & \\
\hline 1013 & 13AL27 & ,NA2 4 & $A B Y$ & THR-99 & -999 & $\begin{array}{r}A C T-1 \\
999=1\end{array}$ & $\begin{array}{l}4 P: \\
G E V\end{array}$ \\
\hline ISA & $3 A L 27$ & .NA24 & $A B x$ & 50 & -250 & & $4 \mathrm{PI}$ \\
\hline FU2 & $13 \mathrm{~A}$ & L & RLY & THR & $D$ & $\begin{array}{l}S C D-D \\
999=3\end{array}$ & \\
\hline $1 F U_{4}$ & $13 A L 27$ & - SPL & RLX & THR-999 & D999 & $A C T-1$ & $4 \mathrm{PI}$ \\
\hline $1 \mathrm{x} \cup 2$ & $13 A L 27$ & 1PSTL & $A B Y$ & THR-999 & 999 & $\begin{array}{l}999=5 \\
A C T-1\end{array}$ & $4 \mathrm{PI}$ \\
\hline & & & & & 999 & $=2.1$ & GEV \\
\hline 1 GR2 & $13 \mathrm{AL} 27$ & & $A B Y$ & & $\begin{array}{r}C 560 \\
P I-\end{array}$ & EMU-D & D \\
\hline$m$ & $3 \mathrm{AL}$ & P PIt & r & 20 & 20 & & 4 \\
\hline $6 \mathrm{BNY}$ & $13 \mathrm{Al}$ & & $A B X$ & 140 & C140-700 & $\begin{array}{l}A C T-1 \\
M G-27\end{array}$ & $4 P$ \\
\hline $1 G R 2$ & $3 A L$ & & $A B Y$ & $150-560$ & $C 560$ & EMU-D & D \\
\hline $72 \mathrm{NO}_{3}$ & $13 \mathrm{AL} 27$ & , P I + & $A B X$ & $150-999^{r}$ & C $150-999$ & A & \\
\hline & & & & & & & \\
\hline $1 \mathrm{~N}$ & $13 \mathrm{AL}:$ & & NOX & 9 & $D$ & $-P_{I}, W I$ & \\
\hline $2\lfloor A \mid$ & $13 \mathrm{AL}$ & .6 & NOX & 11 & 3 & NA I-D & \\
\hline $20 \mathrm{~F}$ & $13 \mathrm{AL} 27$ & . 6 & NO $x$ & 9 & D $0-$ & NAI-D & \\
\hline $3 V A$ & $13 A L 27$ & .6 & RLY & $9-10$ & c & NAI I D & \\
\hline iVA & $13 A L 27$ & $P \cdot G$ & NOX & $20-11$ & D 2- & NAI-D & \\
\hline & 3AL 27 & .6 & NOX & 10 & 0 & NAI I D & u \\
\hline & & & & 20 & D & & \\
\hline & & & & $8-11$ & D & & D \\
\hline $7 \mathrm{~V}$ & $13 \mathrm{AL} 2$ & - & NOX & $8-11$ & D $0-$ & $S C D-D$ & 45 \\
\hline $7 B E 7$ & $3 A L 2 B$ & V.G & LFT & 7 & 14 & $A I-D$ & 90 \\
\hline
\end{tabular}

$678 E 7$ I3AL28 N.G LFT 7- B D O- TELD 4PI

SILICON $\quad 2=14$

A ABUND. (1) SEPARATION ENERGIES (MEV)

$\begin{array}{llllllll}0 . N & G, P & G, T & G, H E 3 & G . A & G, 2 N & G, N P & G, 2 P\end{array}$

$\begin{array}{lllllllll}92.21 & 17.2 & 11.6 & 27.5 & 23.2 & 10.0 & 30.5 & 24.6 & 19.9\end{array}$

$\begin{array}{rrrrrrrrrr}29 & 4.70 & 8.5 & 12.3 & 24.6 & 20.6 & 11.1 & 25.7 & 20.1 & 21.9 \\ 30 & 3.09 & 10.6 & 13.5 & 22.2 & 24.8 & 10.6 & 19.1 & 22.9 & 24.0\end{array}$

NUCLIDE REACTION RES EXCIT SOURCE DETECTOR

$6460314 S I$ E,E/ ABX 12 D $40-70$ MAG-D 180

6BSA2 14SI E,EJ FMF $1-130114-260$ MAG-D DST

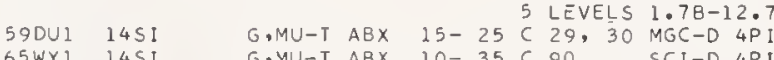

65WY1 14SI G, MU-T ABX $10-35 C 90$ SCI-D 4PI $61+$

$68 B E 4 \quad 145$

GORE 1 14S:

6ISUI 14SI

$61 T 01 \quad 1451$

$62 S E 1$ 14SI

63801 14SI

$635 A 11451$

70 WU1 14S:

$63 \mathrm{CO} \quad 14 \mathrm{SI}$

$66 \mathrm{FI} 214 \mathrm{SI}$

6BGOS 14SI

69AN3 14S:

$\begin{array}{ll}69 \mathrm{CO}^{1} & 145 \mathrm{I} \\ 71 \mathrm{CO} 2 & 14 \mathrm{SI}\end{array}$

G.MU-T ABX $10-30$ C $35 \quad$ MGC-D 4PI 103

G, MU-T ABX THR-150 C $10-150$ MGC-D 4 P I

$\begin{array}{llllllll}G, G & A B X & 7 & D & 7 & \text { NAI-D } & 90 \\ G, G & \text { RLY } & 11 & C & 6-13 & \text { NAI-D } & 120\end{array}$

$G, G$ ABX $4-14$ C 5- 14 NAI-D 120

G.6 NOX 11 C 16 NAI-D 90

$G N \quad A B X \quad 15-25 C 10-30 \quad B F 3-I \quad 4 P I$

$G, N \quad A B X \quad 17-30<17-30$ BF $3-I$ I PI

$G, N \quad A B X 18-21 C 16-22$ TOF-D 90

GROUND STATE

$G, X N$ ABX 13- BO C 13- BO BF3-I 4PI

$G, X N$ SPC THR $-60 C 60$ TOF-D 90

$6 \times X N$ A

$G, X N$ ABX $14-3 B$ C $14-3 B$ BF 3-: 4 PI

$G, X N A B I 36-64 C 10-64$ BF3-I 4 PI

FAST $N$ YIELD 


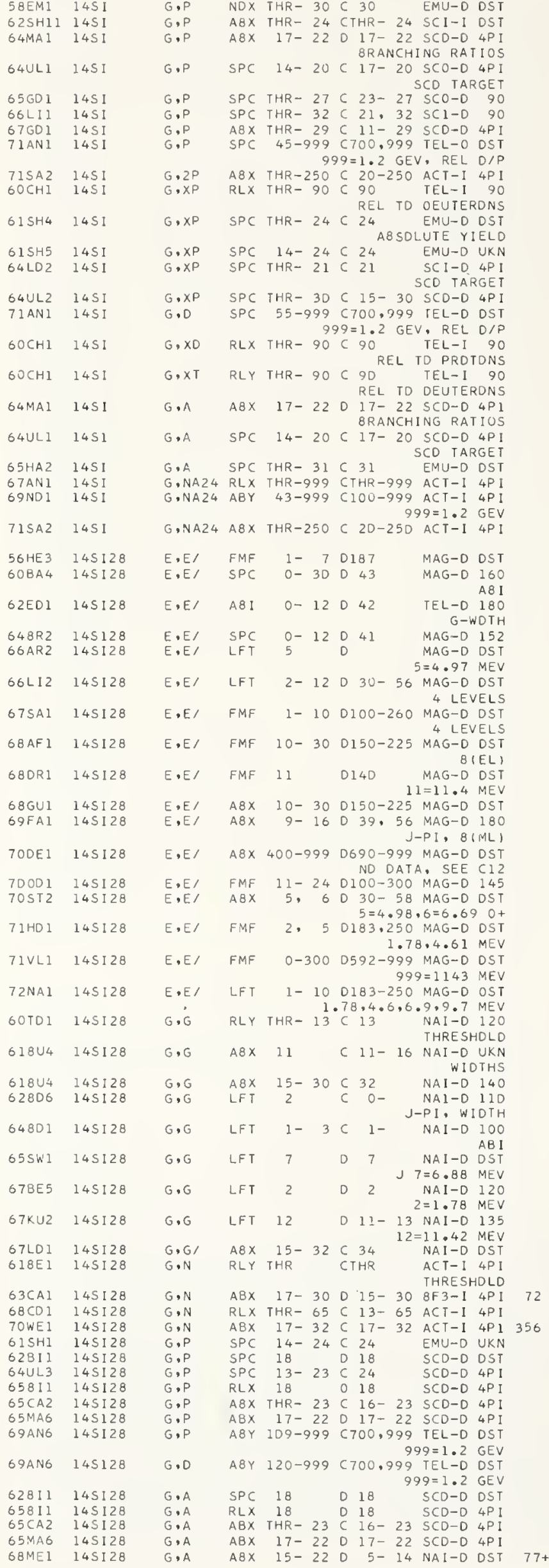

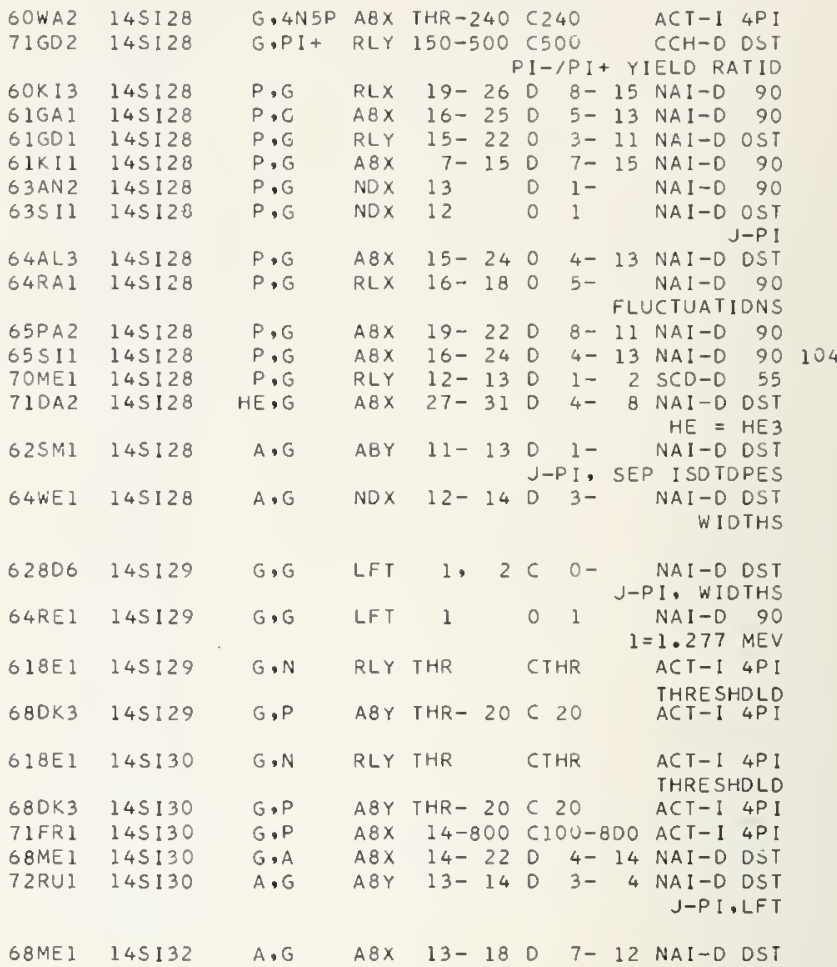

PHDSPHDRUS $z=15$

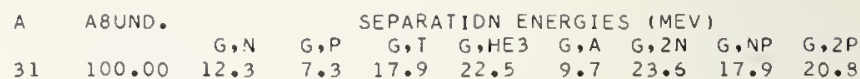

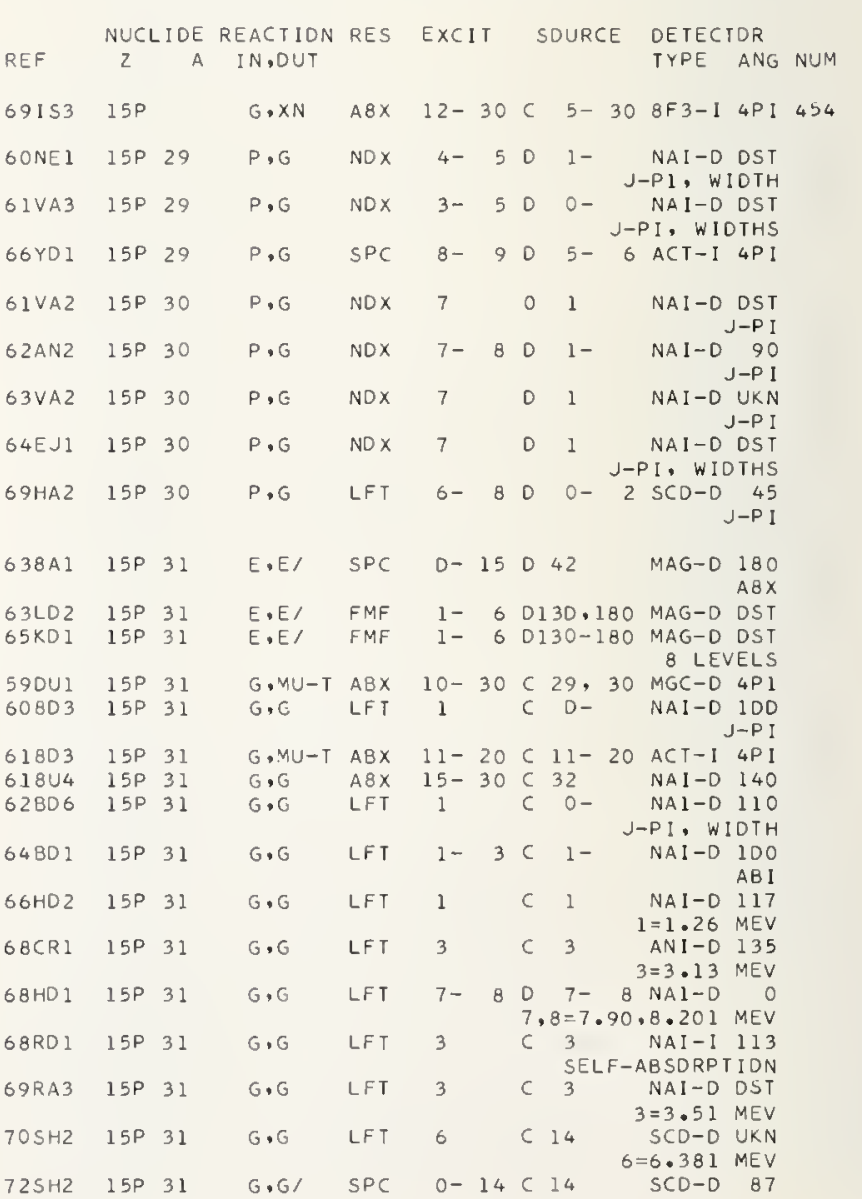




\begin{tabular}{|c|c|c|c|c|c|c|c|}
\hline BA2 & $5 P$ & 31 & $G \cdot N$ & RLY & $12-13$ & C $12-13$ & $T-I \quad 4 P$ \\
\hline $7 B A 3$ & $15 P$ & 31 & $G, N$ & $A B Y$ & $11-14$ & C $11-14$ & $\begin{array}{r}A C T-1 \text { 4PI } \\
\text { BREAKS }\end{array}$ \\
\hline $\mathrm{BCH} 2$ & $15 \mathrm{P}$ & 31 & $G \cdot N$ & RLY & THR & CTHR & $\begin{array}{l}\text { BF } 3-1 \text { 4P I } \\
\text { THRESHOLD }\end{array}$ \\
\hline QGE 3 & $15 P$ & 31 & $G, N$ & NOX & THR & CTHR & $\begin{array}{l}\text { ACT-I } 4 P I \\
\text { THRESHOLD }\end{array}$ \\
\hline ISAI & $15 \mathrm{P}$ & 31 & $G, N$ & RLY & $12-14$ & C $12-14$ & $\begin{array}{l}\text { ACT-1 } 4 P \text { I } \\
\text { THRES SHOLD }\end{array}$ \\
\hline EM & $15 \mathrm{P}$ & 31 & $\mathrm{~N}$ & SPC & $10-30$ & 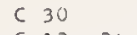 & $U-D \quad D S T$ \\
\hline & $15 P$ & & & & & C $12-24$ & $A C T-1 \quad 4 P I$ \\
\hline 7 & $\begin{array}{l}15 P \\
15 P\end{array}$ & $\begin{array}{l}3 \\
3\end{array}$ & & & THR -27 & $\begin{array}{l}\text { C } 22,27 \\
\text { C100-800 }\end{array}$ & $\begin{array}{ll}\mathrm{BF} 3-1 & 4 \mathrm{PI} \\
\mathrm{ACT}-1 & 4 \mathrm{PI}\end{array}$ \\
\hline $2 M U$ & $\begin{array}{l}15 \mathrm{P} \\
15 \mathrm{P}\end{array}$ & 31 & & & $\begin{array}{l}12-800 \\
12-24\end{array}$ & $\begin{array}{l}C 100- \\
\text { C } 24\end{array}$ & $\begin{array}{ll}A C T-1 & 4 P I \\
B F 3-1 & 4 P I\end{array}$ \\
\hline & $15 \mathrm{P}$ & 3 & & & & c $10-$ & $-14 \mathrm{f}$ \\
\hline & $15 \mathrm{P}$ & 3 & & & & BO & $-140 \mathrm{I}$ \\
\hline & 15 & 3 & & & $\mathrm{THF}$ & C $23-27$ & D 100 \\
\hline & 15 & 31 & & & $10-19$ & C 1 & EMU-D 4PI \\
\hline 1 & $15 P$ & 31 & & & THR & C $1 \mathrm{~B}$, & EMU-D 30 \\
\hline $0 C 1$ & $15 P$ & 31 & & 1 & $T H R-100$ & & $\begin{array}{ll}A C T-1 & 4 P I \\
R E L \text { TO } & G . N\end{array}$ \\
\hline & $15 \mathrm{p}$ & 3 & & & & 63 & $A C T-1 \quad 4 P I$ \\
\hline & $1.7+3$ & & & & & C $20-250$ & $A C T-1 \quad 4$ \\
\hline 15 & $15 P$ & & $\times P$ & $P C$ & 0 & ${ }^{C} 24_{A B S}$ & $\begin{array}{l}\text { EMU-D D } \\
\text { LUTE YIE }\end{array}$ \\
\hline $10 \quad 2$ & & & & & THR- 32 & C $15-$ & $S C 1-D 1$ \\
\hline & 15 & 3 & & & THR & C 22,27 & $S C D-D$ \\
\hline $\begin{array}{l}\text { 8SH3 } \\
9061\end{array}$ & $\begin{array}{l}15 P \\
15 P\end{array}$ & $\begin{array}{l}31 \\
31\end{array}$ & $\begin{array}{l}G \times N \geq P \\
\end{array}$ & $\begin{array}{l}\text { SPC } \\
\text { RLI }\end{array}$ & $\begin{array}{l}\text { THR- } 19 \\
\text { THR-100 }\end{array}$ & $\begin{array}{l}\text { D } 19 \\
\text { CTHR- }\end{array}$ & $\begin{array}{ll}E M U-D & D S \\
A C T-1 & 4 P\end{array}$ \\
\hline & & 3. & & & & 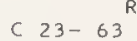 & $A C T-I \quad 4 P$ \\
\hline & & & & & & & $A C T-I \quad 4 P$ \\
\hline & $15 \mathrm{P}$ & & & $A B$ & & C $3 v$ & ACT-I $4 \mathrm{PI}$ \\
\hline NOI & 15 & 31 & NA24 & $A B Y$ & 99 & C100-999 & $\begin{array}{ll}A C T-1 & 4 P \\
9 & =1.2\end{array}$ \\
\hline & 15 & 3. & & & THR & c $20-250$ & $A C T-I \quad 4 P$ \\
\hline & & & & & & D 1 & EMU-D DST \\
\hline$T$ & $15 P$ & 31 & $\$$ & no & 10 & D & D \\
\hline & & & & & & & D D \\
\hline & & & & & & & \\
\hline & & 3 & & & 9 & D & \\
\hline $9 W: 1$ & $15 P$ & 31 & $P, G$ & LF T & $10-11$ & D & $1-$ \\
\hline
\end{tabular}

SULFUR $\quad Z=16$

\begin{tabular}{lrrrrlllll} 
A & ABUND.11) & \multicolumn{10}{c}{ SEPARATION ENERGIES (MEV) } \\
32 & 95.00 & G.N & G.P & G.T & G,HE3 & G,A & G.2N & G,NP & $G, 2 P$ \\
32 & 0.1 & $B .9$ & 24.0 & 19.1 & 6.9 & 28.1 & 21.2 & 16.2 \\
33 & 0.76 & 8.6 & 9.6 & 21.3 & 17.1 & 7.1 & 23.7 & 17.5 & 18.2 \\
34 & 4.22 & 11.4 & 10.9 & 20.4 & 21.9 & 7.9 & 20.1 & 21.0 & 20.4 \\
36 & $1.41-21$ & 9.9 & $*$ & 19.3 & $*$ & 9.0 & 16.9 & 21.2 &
\end{tabular}

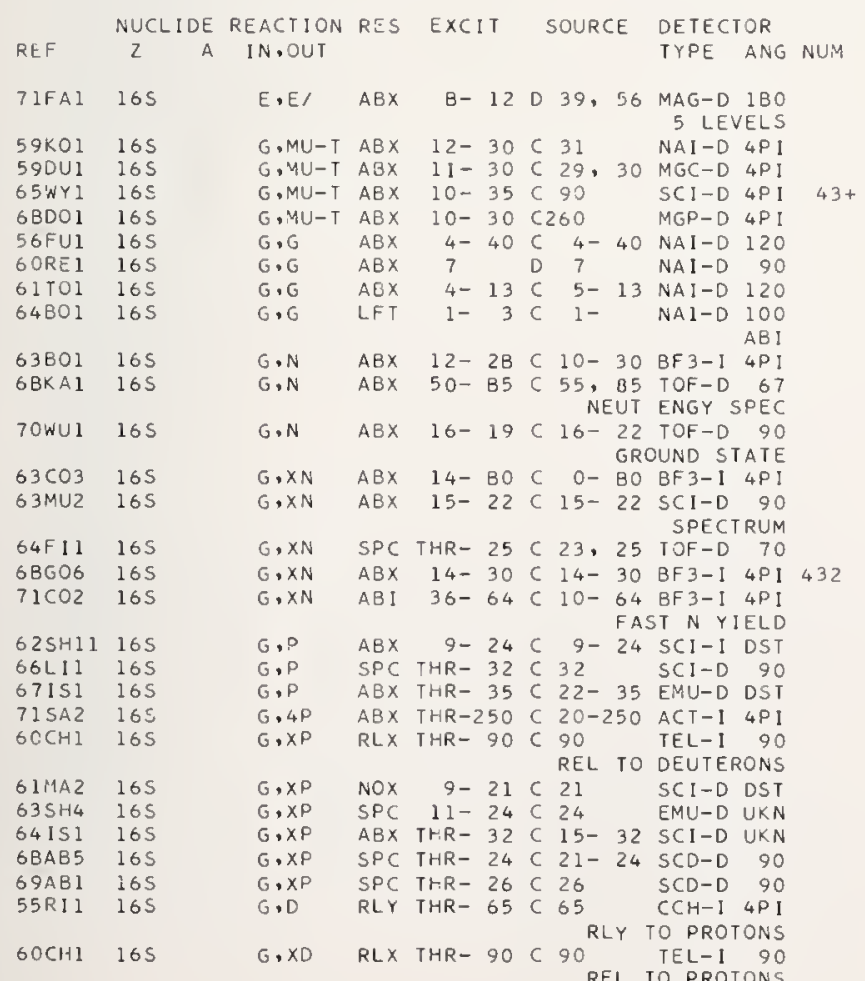

60CH1 16S G.XT RLY THR- $90 \mathrm{C} 90$ TEL-1 90

$66 \mathrm{HO} 316 \mathrm{~S}$

69 NO1 165

$715 A 2 \quad 165$

$56 \mathrm{HE}_{3} \quad 16532$

638416532

64 LOI $165 \quad 32$

$690 E B \quad 165 \quad 32$

69 GU3 16532

TOST2 16532

65AM2 16532

$62806 \quad 16532$

67LO1 16532

$59 F A 1 \quad 165 \quad 32$

GOFEI 16532

$2 \mathrm{~F} 13$ 16S 32

$62 K U 1$ 16S 32

624151653

62 MU2 16532

65THI 16532

$66811 \quad 16532$

$67 W E 2$ 165 32

$6 \mathrm{BCO} \quad 165 \quad 32$

$70152 \quad 165 \quad 32$

$70 \mathrm{THI} \quad 16532$

COFEI $165 \quad 32$

$62 \mathrm{BO} 316532$

GTAN2 16532

TOAN3 16532

$61 \mathrm{FOl} 165$

$70152 \quad 16532$

$70 \mathrm{THI} 16532$

6BSH3 16532

$55 \mathrm{VII} 16 \mathrm{~S} 32$

$5 \mathrm{BGO} 316532$

55DEl 16532

$5 \mathrm{BGO3} 16532$

59FAl 16532

59FAI 16532

COFEL 16532

$62603 \quad 165 \quad 32$

SVA3 16532

$\begin{array}{lll}71 B R 1 & 165 & 32 \\ \text { 6OWA2 } & 165 & 32\end{array}$

$71602 \quad 16532$

62BE4 16532

62NEI 16532

$63 \mathrm{CHI} 16532$

$63 K 1216532$

$3501-16532$

64 SMI 16532

65DEI 16S 32

$66 \mathrm{HOI} 16 \mathrm{~S} 32$

69MAS 16532

69PII 16532

$2 \mathrm{CO} \quad 16532$

$72 E S I \quad 16 S 32$

64 SMI 16S 32

$71 \mathrm{CHI} \quad 165 \quad 32$

$67 B E 7 \quad 165 \quad 33$

$62806 \quad 165 \quad 34$

$65 M C 2 \quad 16534$

$67 \mathrm{WII} \quad 16534$

$66 \mathrm{KO4} \quad 16536$ REL TO DEUTERONS
31

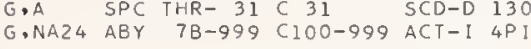
$999=1.2$ GEV
G.NA24 ABX THR-250 C $20-250$ ACT-I 4 PI

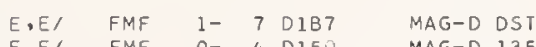

E,E/ FMF O- 4 DI50 MAG-D 135

E,E/ SPC $0-22 D 42$ MULTIPOLARITY

E,E, FMF O- 9 DI20-1BO MAG-D DST

E,E/ ABX O-200 D999 MAG-D DST

OUASI-EL, $999=1.11 \mathrm{G}$
,Ef FMF 13-31 DI20-200 MAG-D DST

E,E, $A B \times \quad 4 \quad 31$ D 58,59 MAG-D 105

$E, E / P$ RLY $0-120 \quad 0500-630 \quad M A G-D \quad 51$

G 100 MEV P COINC

G.G ABX 14- 32 C $34 \quad$ NAI-D DST

$\begin{array}{llllllll}G, G & A B X & 14-32 & C & 34 & \text { NAI-D } & \text { DST } \\ G, N & A B X & 16-30 & C & 30 & A C T-I & 4 P I\end{array}$

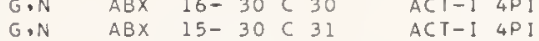

$\begin{array}{llllll}\text { G.N } & A B X & 15-30 & C & 31 & A C T-1 \\ \text { G.N } & R L Y & 16-2 B & C & 32 & \text { TOF-D UKN }\end{array}$

$G, N \quad A B X \quad 15-25$ C $12-27$ ACT-I $4 P$ I

$G, N$ SPC $15-30 C 30$ EMU-D DST

$G, N \quad A B X \quad 15-22$ C $12-24$ BF $3-1$ 4PI

$G, N \quad A B X T H R-22 C 15-22 A C T-14 P$ I

$G, N \quad A B X \quad 20-200$ C $20-200$ BF 3-I $4 P I$

$\begin{array}{llrlll} & A B X & T H R-32 C & 20-32 & A C T-1 & 4 P \\ G & A B X & 20-32 C & 20-32 & A C T-1 & 4 P 1\end{array}$

$G, N$ RLX THR - 65 C $13-65$ ACT-I $4 P$ I

$G, N G$ SPC THR- 32 C 32 SCD-D 135

LEVELS $1.26,2.23$

$G \times X N \quad A B X \quad 12-31 C C_{1} \quad 31$ MOD-I 4 P I

$G, X N$ ABX 16- $90 \mathrm{C} 30-903 F 3-14 P I$

$G, X N$ ABX THR- 26 C $13-26$ BF 3-I 4PI

$G, X N \quad A B X 15-62 C 16-62$ ACT-I 4P! $284+$

G,P SPC $10-30 C 30 \quad$ (G, N)+(G, $2 N)$

G,PG SPC THR- 32 C 32 SCD-D 135

G.PG ABX ThR- 29 C LEVELS $1.26,2.23$

GPG ABX THR- 29 C 29 SCD-D 125

$\begin{array}{lllllll}G, X P & S P C & 17-20 & D & 17,20 & E M U-D & D S T \\ G, 0 & A B X & 19-22 & C & 17-22 & A C T-1 & 4 P I\end{array}$

INCLUDES G,NP

$G, D$ RLY $19-22$ C $16-22$ ACT -1 4 PI

G,NP ABX 1B- 22 C $1 B-22$ ACT-I 4PI

$G, N P$ RLY $21-22 C 16-22$ ACT-I $4 P I$

$G, N P$ ABX 20- 30 REACTION UNCERTAIN

$\begin{array}{lllllll}G, N P & A B X & 20-30 & C & 30 & \text { THR-I } \\ \text { GNP } & A B X & 21-30 & C & 30 & A C T-I & 4 P I \\ G, N P & A B X & 21-31 & C & 31 & A C T-1 & 4 P I\end{array}$

G.NP ABX 20-90C $30-90$ ACT-1 $4 P I$

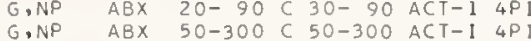

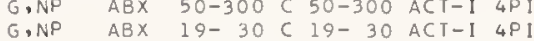

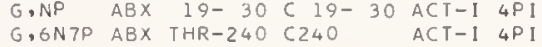

G.PI+ RLY 150-500 C500 CCH-D DST

Pi-1PI+ YIELD RATIO
P ABY $9-10 \mathrm{DO} 0-\quad$ NAI-D DST

, G RLY 9- 10 D O- NAI-I 90

$P, C \quad S P C \quad 9-1000-$ NAI-D J-PI

$P, G \quad A B X \quad 17-22 D$ B- 14 NAI-I DST

P.C NOX $9-10$ D $0-$ NAI-D DST

$P, C$ ABY $9-10 \mathrm{D} 0-\mathrm{J-PI}, \mathrm{G}-\mathrm{WDTH}$

$P, G$ ABX 10- 210 2- J-PI, WIDTHS

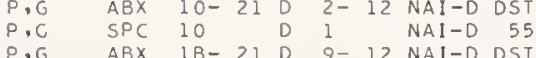

$P, G \quad A B X \quad 1 B-21 D \quad 9-12$ NAI-D DST

P.G LFT $9-110$ O- 2 SCD-D 55

P.G LFT 6- B D 3- 6 STCD-D UKN

LEVEL IS $5.550 \mathrm{MEV}$

$A, G$ ABY 9- $1002-$ NAI-D DST

$A, G$ LFT $10-12 \mathrm{D} \quad 4-\begin{array}{r}J-P I, W I D T H S \\ 6 \text { SCD-D DST }\end{array}$

J-PI

N.G LFT B- 9 D O- I NAI D 90
SOURCE 30,111 KEV

G.G LET O- $4 \mathrm{C} 0-\quad$ NAI-D 110

$A, G$ RLY 11- 12 D $3-4$ NAI-D DST

$A, G \quad L F T$ 11- 12 D $4-5$ NAI-D DST

$P, G$ NOX 10- $1101-2$ NAI-I DST 
A ABUND. SEPARATIDN ENERGIES (MEV)

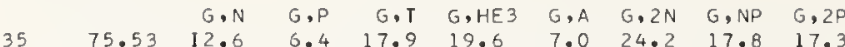

$\begin{array}{llllllllll}37 & 24.47 & 10.3 & 8.4 & 17.9 & 19.6 & 7.0 & 24.2 & 17.8 & 17.3 \\ & & & & 16.8 & 21.9 & 7.8 & 18.9 & 18.3 & \end{array}$

\begin{tabular}{llll}
\multicolumn{1}{c}{ NUCLIDE REACTION RES EXCIT SDURCE DETECTDR } \\
REF \\
Z A IN,OUT & & & TYPE ANG NUM
\end{tabular}

66BE3 $17 C L \quad G, G \quad R L X \quad 5-10 \quad D \quad 5-10$ NAI-D 135

5SERI $17 \mathrm{CL} \quad \mathrm{G}, \mathrm{A}$ ABY THR- $31 \mathrm{C} 32$ EMU-I $4 \mathrm{PI}$

$69 N O 1 \quad 17 \mathrm{CL}$ G.NA24 ABY $104-999$ C $100-999$ ACT-I 4 PI

$999=1.2$ GEV

\begin{tabular}{|c|c|c|c|c|c|c|c|c|}
\hline $69 G R 1$ & $17 C L 34$ & $P, G$ & LFT & 6- & 7 & D & 1 & $\begin{array}{l}\text { SCD-D DST } \\
6 \text { LEVELS }\end{array}$ \\
\hline 62806 & $17 C L 35$ & $G \cdot G$ & LFT & $0-$ & 4 & c & $0-$ & NAI - D $\begin{array}{r}110 \\
J-P I\end{array}$ \\
\hline $66 \mathrm{HO} 2$ & $17 C L 35$ & $G, G$ & LFT & 1 & & c & 1 & $\begin{array}{rr}\text { NAI }-D & 117 \\
1=1.22 & \text { MEV }\end{array}$ \\
\hline $55 B A 3$ & $17 C L 35$ & $G, N$ & RLY & $12-$ & 22 & c & 22 & $\begin{array}{l}\text { ACT-I } 4 P I \\
\text { THRESHOLD }\end{array}$ \\
\hline $55 \mathrm{BD} 2$ & $17 C L 35$ & $G, N$ & $A B X$ & $13-$ & 21 & $c$ & $13-21$ & $A C T-I \quad 4 P I$ \\
\hline $55 D E$ & $17 C L 35$ & $\mathrm{G}, \mathrm{N}$ & $A B X$ & $13-$ & 21 & c & $13-21$ & $\begin{array}{l}\text { ACT-I } 4 P I \\
\text { THRESHOLD }\end{array}$ \\
\hline $57 \mathrm{BA} 3$ & $17 C L 35$ & $G, N$ & RLY & $12-$ & 14 & c & $12-14$ & $\begin{array}{r}\text { ACT-I 4PI } \\
\text { BREAKS }\end{array}$ \\
\hline $59 F E 1$ & $17 \mathrm{CL} 35$ & $G \cdot N$ & $A B X$ & $12-$ & 31 & c & 31 & $A C T-I \quad 4 P I$ \\
\hline 59FEl & $17 \mathrm{CL} 35$ & $G, N$ & $B X$ & $16-$ & 31 & c & 31 & THR-I 4P I \\
\hline 61 SAI & $17 C L 35$ & $G \cdot N$ & $R L Y$ & $12-$ & 14 & c & $12-14$ & $+A C T-1 \quad 4 P I$ \\
\hline $62 K \cup 1$ & 170 & $G, N$ & $A B X$ & $12-$ & 25 & c & 27 & $\begin{array}{l}\text { THRESHOLD } \\
\text { ACT-I } 4 \text { PI }\end{array}$ \\
\hline $67 \mathrm{KO2}$ & $17 C L 35$ & $P, G$ & LFT & B & & D & 2 & $\begin{array}{r}N A I-D \quad D S T \\
J=P I\end{array}$ \\
\hline 67 WAI & $17 C L 35$ & $P, G$ & LFT & B & & D & $2-$ & $S C D-D \quad 4 P$ I \\
\hline 59SE 1 & $17 C L 36$ & $N, G$ & $S P C$ & 9 & & c & 0 & $\begin{array}{rr}\text { NAI I D } & 90 \\
G-W I D T H\end{array}$ \\
\hline $67 B E 7$ & $17 C L 36$ & $N, G$ & LFT & $B-$ & 9 & D & $\begin{array}{c}0-\quad 1 \\
\text { SOURCE }\end{array}$ & $\begin{array}{l}\text { NAI }-D \quad 90 \\
25,50 \mathrm{kEV}\end{array}$ \\
\hline $62 B 06$ & $17 \mathrm{CL} 37$ & $G, G$ & LFT & $0-$ & 4 & $c$ & $0-$ & NAI - $\begin{array}{r}1110 \\
J-P I\end{array}$ \\
\hline $60 G E$ & $17 \mathrm{CL} 37$ & $G \cdot N$ & NOX & THR & & & R & $\begin{array}{l}\text { BF 3- I 4PI } \\
\text { THRESHOLD }\end{array}$ \\
\hline $57 \mathrm{IEI}$ & $17 \mathrm{CL} 37$ & $P, G$ & LFT & $9-$ & 10 & D & $1-$ & $2 \mathrm{NAI}-\mathrm{D} \quad 55$ \\
\hline
\end{tabular}

ARGON $Z=1 B$

A ABUND. SEPARATION ENERGIES (MEV)

$\begin{array}{llrlrlllll}36 & & G, N & G, P & G, T & G, H E 3 & G, A & G, 2 N & G, N P & G .2 P \\ & 0.34 & 15.3 & B .5 & 24.2 & 1 B .6 & 6.6 & 28.0 & 21.2 & 14.9\end{array}$

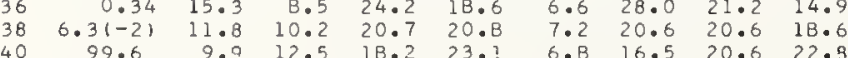

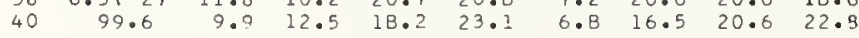

\begin{tabular}{|c|c|c|c|c|c|c|c|c|c|}
\hline & NUCL IDE & REACTIDN & RES & $E \times \subset I$ & & & SOURCE & DETECTDR & \\
\hline REF & 2 & IN.DUT & & & & & & TYPE ANG & $N \cup M$ \\
\hline $72 \mathrm{FAl}$ & $18 A R 36$ & $E, E /$ & $A B X$ & $7-$ & 13 & D & $\begin{array}{l}39,56 \\
, G-W I D\end{array}$ & $\begin{array}{l}\text { MAG-D IBO } \\
T H, B \text { LEVS }\end{array}$ & \\
\hline $59 \mathrm{HO} 1$ & 1 BAR 36 & $G, \times P$ & $A B Y$ & THR - & 33 & c & $24-33$ & SCI-D 90 & \\
\hline 5 ERI & IBAR 36 & $P: G$ & RLY & B- & 10 & D & $2-\quad$ & $\begin{array}{l}\text { NAI -D DST } \\
I, \text { WIDTHS }\end{array}$ & \\
\hline $67 \mathrm{ME} 3$ & IBAR 36 & $P, G$ & $R L X$ & $12-$ & 19 & D & $4-12$ & NAI -D DST & \\
\hline $70 K E 1$ & 18 AR 36 & $P, G$ & RLX & $14-$ & 20 & D & $6-12$ & NAI $-D$ & 244 \\
\hline 64 ER 1 & $18 A R 36$ & $A, G$ & RLY & $8-$ & 10 & D & $2-J-P$ & $\begin{array}{l}\text { NAI-D DST } \\
\text { I, WIDTHS }\end{array}$ & \\
\hline $65 \mathrm{EHI}$ & $18 A R 3 B$ & $G, N$ & $A B Y$ & THIR- & 31 & c & $1 B-31$ & $A C T-I \quad 4 P I$ & \\
\hline 70KEI & $1 B A R 3 B$ & $P, G$ & RLx & $14-$ & 22 & D & $4-12$ & NAI -D 90 & 245 \\
\hline 64ER 1 & $1 B A R 3 B$ & $A, G$ & RLY & $9-$ & 10 & D & $2-J-P$ & $\begin{array}{l}\text { NAI - D DST } \\
\text { I, WIDTHS }\end{array}$ & \\
\hline $64 \mathrm{PHI}$ & IBAR 38 & $A, G$ & RLY & $99-$ & 11 & D & $3-J-P$ & $\begin{array}{l}\text { NAI -D DST } \\
\text { I, WIDTHS }\end{array}$ & \\
\hline $72 \mathrm{CHI}$ & IBAR 3B & $A, G$ & $A B X$ & $10-$ & 12 & D & $\begin{array}{ll}4- & 5 \\
12 & \text { LEV }\end{array}$ & $\begin{array}{l}\text { NAI I D DST } \\
\text { /ELS, J-PI }\end{array}$ & \\
\hline $6 \mathrm{HE} 3$ & $18 A R 40$ & $E, E /$ & FMF & $1-$ & 7 & & 87 & MAG-D DST & \\
\hline $63 \mathrm{BAl}$ & IBAR 40 & $E, E /$ & $S P C$ & $0-$ & 24 & D & 42 & $M A G-D \quad 1 B O$ & \\
\hline 62001 & $18 A R 40$ & $E, P$ & $S P C$ & $13-$ & 30 & $c_{S}$ & $\begin{array}{l}30 \\
\text { EPARATE }\end{array}$ & $\begin{array}{l}\text { MAG-D } 76 \\
\text { D ISOTOPE }\end{array}$ & \\
\hline 59PEI & I BAR4O & $G \cdot N$ & $A B X$ & $10-$ & 23 & c & $14-44$ & $\begin{array}{l}\text { ACT-I } 4 P I \\
\text { SYNTHESIS }\end{array}$ & \\
\hline 60FAI & IBAR 40 & $G \cdot N$ & $A B X$ & $13-$ & 49 & $c$ & $10-50$ & BF 3-I 4PI & \\
\hline $72 \mathrm{LO} 2$ & IBAR 40 & $G, N$ & $A B X$ & $10-$ & I 3 & D & 13 & $T D F-D$ & \\
\hline 59PE & IBAR 40 & $G \cdot 2 N$ & $A B X$ & $17-$ & 23 & c & $14-44$ & $A C T-I \quad 4 P I$ & \\
\hline $55 \mathrm{SP} 3$ & 18 AR40 & $G, P$ & $S P C$ & THR - & 23 & $c$ & 23 & $\begin{array}{l}\text { SYNTHES IS } \\
\text { EMU-D DST }\end{array}$ & \\
\hline $56 \mathrm{KDI}$ & $18 A R 40$ & $G, P$ & NOX & $12-$ & 90 & c & 90 & $\mathrm{CCH}-\mathrm{I} \quad \mathrm{DST}$ & \\
\hline $58 \mathrm{GUI}$ & $18 A R 40$ & $G, P$ & $S P C$ & $13-$ & 15 & $c$ & 15 & $C C H-D \quad D S T$ & \\
\hline $598 R I$ & $1 B A R 40$ & $G \cdot P$ & $A B I$ & $12-$ & 34 & c & 34 & $A C T-I \quad 4 P I$ & \\
\hline 59EM2 & $1 B A R 40$ & $G, P$ & $S P C$ & $10-$ & 30 & c & $23-30$ & EMU-D 90 & \\
\hline 59PE 1 & J $8 \mathrm{AR} 40$ & $G, P$ & $A B X$ & $13-$ & 33 & c & $14-44$ & $\begin{array}{l}\text { ACT-I } 4 \text { P I } \\
\text { SYNTHESIS }\end{array}$ & \\
\hline 60001 & $18 A R 40$ & $G, P$ & $A B I$ & $12-$ & 33 & & 30,34 & $A C T-I \quad 4 P I$ & \\
\hline $\begin{array}{l}\text { 61F I I } \\
65 R E \text { I }\end{array}$ & $18 A R 40$ & $G, P$ & RLY & $0-$ & 35 & c & 35 & SCI-D 90 & \\
\hline & IBAR 40 & & $A B X$ & & & $D$ & & $C C H-D \quad 4 P I$ & \\
\hline
\end{tabular}

\begin{tabular}{|c|c|c|c|c|c|c|c|c|c|}
\hline $57 K D 2$ & I BAR 40 & $G, X P$ & NOX & THR- & 90 & C 90 & & $\mathrm{CCH}-\mathrm{D}$ & DST \\
\hline $5 B \mid A I$ & $1 B A R 40$ & $G, \times P$ & $A B I$ & I $2-$ & 70 & C 70 & & $\mathrm{CCH}-\mathrm{D}$ & DST \\
\hline $9 \mathrm{HOI}$ & I BAR 40 & $G, X P$ & $A B Y$ & THR- & 33 & C $24-$ & 33 & $S C I-D$ & 90 \\
\hline QEM2 & $1 B A R 40$ & $G, A$ & $A B X$ & $6-$ & 12 & C 23- & 30 & $E M U-D$ & 90 \\
\hline KD 1 & IBAR4O & $G, A$ & $S P C$ & $10-$ & 17 & C 70 & & ION-D & 90 \\
\hline RE I & I BAR 40 & $G, A$ & $A B X$ & 9 & & D & & $\mathrm{CCH}-\mathrm{D}$ & $4 P I$ \\
\hline WE 2 & $1 B A R 40$ & $G, A$ & $A B X$ & 7- & 33 & C 33 & & $S C D-D$ & 90 \\
\hline$B R 1$ & IBAR4O & $G, N P$ & $A B I$ & $21-$ & 34 & C 34 & & $A C T-I$ & $4 P I$ \\
\hline PE 1 & $1 B A R 40$ & $G, N P$ & $A B X$ & $19-$ & 40 & C $14-$ & 44 & $\begin{array}{l}\text { ACT }-I \\
\text { SYNTHE }\end{array}$ & $\begin{array}{l}\text { API } \\
\text { ESIS }\end{array}$ \\
\hline & 40 & , NP & $A B I$ & $21-$ & 3 & 30 , & 34 & $A C T-1$ & $4 P I$ \\
\hline EH & IBAR 40 & $G, N P$ & $A B Y$ & IHR- & 31 & C $18-$ & 31 & $A C T-I$ & $4 P I$ \\
\hline
\end{tabular}

PDTASSIUM $Z=19$

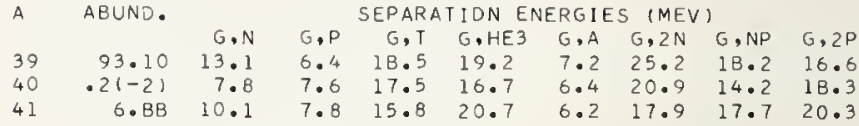

REF NUCLIDE REACTIDN RES EXCIT SDURCE DETECTDR

69SA3 19K E,E/ FMF 3, 7 D 99-227 MAG-D DST

61TO1 19K G,G ABX 6- $12 \mathrm{C} \quad 6-12 \mathrm{NAI}-\mathrm{D} 120$

$59 E M I$ IGK G,XN SPC 7- $31 C 31-12$ NAI-D 120

$63 C D 3$ 19K G,XN ABX 12- $80 C$ O - 80 BF 3-I $4 P I$

$69 N D 1$ I9K G,NA24 ABY 140-999 $100-999$ ACT-I 4PI $999=1.2 \mathrm{GEV}$

$67 G 0219 K 37 \quad P, G \quad$ LFT 2- 4 D O- 6 SCD-D DST

63BAI 19K 39 E,E/ SPC O- 16 D 42 MAG-D 180

TOPEI IOK 39 E,E ABX OAG 180

MAG-D DST
7 LEVELS

$6280619 \mathrm{~K} 39 \mathrm{G}, \mathrm{G}$ LFT O- $4 \mathrm{C} 0-$ NAI-D 110

$67 L O 1 \quad 19 K 39 \quad G, G / \quad A B \times \quad 14-32 C 34 \quad$ NAI-D DST

$55 B D 219 K 39$ G.N ABX 13- 11 C $13-21$ ACT-I $4 P I$

$55 D E 1$ 19K 39 G.N ABX $13-21$ C $13-21$ ACT. I 4PI

60GE3 19K 39 G,N NOX THR CTHR ACT-I 4PI

$6260319 K 39 \quad G, N$ ABX 14- $24 \mathrm{C} 14-24$ THRE SHDLD

$A B X-14-24014-24$ ACT 1 4P

ISDMER RATIO

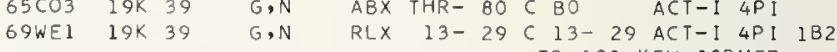

70KA2 19K 39 G,N ABX 15-120 C $15-140$ ACT-I 4PI

$71 W E I$ I9K 39 G,N ABX 13- 30 C $13-30$ ACT-I $4 P$

59KEI 19K 39 G,P ABX 1B D 18 G.N TD I SDMER

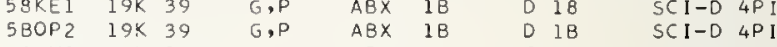

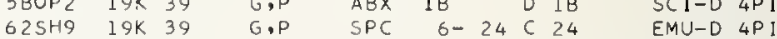

$\begin{array}{lllllllll}62 S H 9 & 19 K & 39 & G, P & S P C & 5-24 C & 24 & \text { EMU-D } & 4 P 1 \\ 69 H O I & 19 K & 39 & G, X P & A B Y & T H R-33 C & 24-33 & \text { SCII-D } & 90\end{array}$

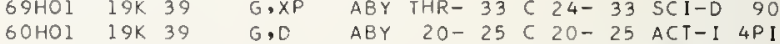

$60 \mathrm{HOI} 19 \mathrm{~K} 39 \mathrm{G}, \mathrm{O}$ ABY $20-25 \mathrm{C} 20-25$ ACT-I $4 P I$
REACTIDN UNCERTAIN

$60 H O 119 K 39$ G,NP ABY $20-25$ C 20- 25 ACT-I 4PI

$65 C_{3} 19 K 39$ G.NP ABX THR- 80 C BO BON UNCERTAIN

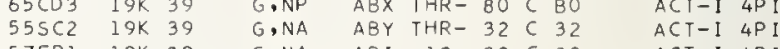

5TERI 19K 39 G.NA ABI $13-32 C 32 \quad$ ACT-I 4PI

$71 B L 1$ 19K41 G.PI+ ABY 250-700 C150-700 ACT-I 4PI

$63 K D 219 K 41$ P,G RLY 9 D O- SEE 6B NY 1 SEPARATED ISDTDPES

CALCIUM $\quad Z=20$

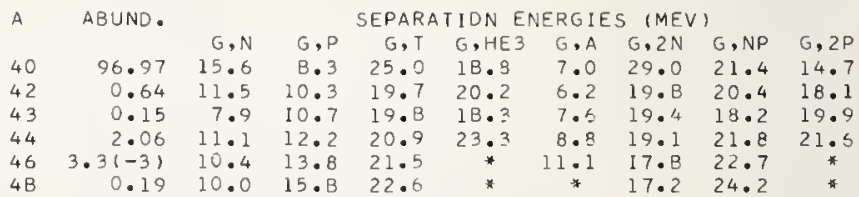

NUCLIDE REACTION RES EXCIT SOURCE DETECTOR

REF $Z$ A IN,DUT TYPE ANG NUM

61PEI 20CA40 E,E/ ABX 11- 26 D120-1BO MAG-D DST

S2BLI 20CA4O E,E/ FMF O- 9 DI20-220 MAG-D DST

62EDI 20CA40 E,E/ NOX O- D $42 \quad \begin{gathered}7 \text { LEVELS } \\ \text { TEL-D } 160\end{gathered}$ ND EXCITATION OBS 
NUCLIOE REACTION RES EXCIT SDURCE OETECTOR REF $Z$ A IN,DUT TYPE ANG NUM

\begin{tabular}{|c|c|c|c|c|c|c|c|c|}
\hline $71 \mathrm{HEI}$ & $22 T 146$ & $E \cdot E /$ & FMF & 1 , & 2 & $\begin{array}{r}0198-300 \\
1=0.8\end{array}$ & $\begin{array}{l}M A G-D \\
85, \quad 2=\end{array}$ & $\begin{aligned} & 0 S T \\
= & 2.0\end{aligned}$ \\
\hline 63KAl & $22 \mathrm{TI} 46$ & $G \cdot G$ & $A 8 x$ & 1 & & $0 \quad 1$ & NAI -D & 108 \\
\hline 58514 & $22 T ! 46$ & $G, N$ & $R L x$ & $13-$ & 22 & CTHR- 22 & $A C T-I$ & $4 \mathrm{PI}$ \\
\hline $625 \mathrm{HS}$ & $22 T ! 46$ & $G, N$ & $A B x$ & $14-$ & 31 & C $14-31$ & $A C T-1$ & $4 \mathrm{PI}$ \\
\hline 69GA2 & $22 T 146$ & $\mathrm{G}, 2 \mathrm{~N}$ & $A B X$ & 30 . & 34 & C $31-39$ & $A C T-I$ & $4 \mathrm{PI}$ \\
\hline $625 \mathrm{SH}$ & $22 \mathrm{TI} 46$ & $G, N P$ & $A E X$ & $22-$ & 31 & C 14- 31 & $A \subset T-I$ & $4 \mathrm{PI}$ \\
\hline 66TA1 & $22 T 146$ & $G \cdot N P$ & RLY & THR- & 48 & c $24-48$ & $A C T-I$ & $4 \mathrm{PI}$ \\
\hline 69GA2 & $22 T 146$ & G.NP & $\mathrm{AEX}$ & 30 , & 34 & C $31-39$ & $A C T-1$ & 4P I \\
\hline 64801 & $22 T ! 47$ & $G, G$ & LFT & $1-$ & 3 & $1-$ & NAI - O & $\begin{array}{l}100 \\
A 8 I\end{array}$ \\
\hline $625 \mathrm{HS}$ & $22 T 147$ & $G, P$ & $A 8 x$ & $14-$ & 31 & C $14-31$, & $A \subset T-1$ & $4 \mathrm{PI}$ \\
\hline 67PA2 & $22 T: 47$ & $G, P$ & RLY & THR- & 30 & C 22,30 & $A C T-1$ & $4 P I$ \\
\hline $680 k 3$ & $22 T I 47$ & $G, P$ & $A B Y$ & THR- & 20 & C 20 & $A C T-1$ & 4PI \\
\hline $690 \mathrm{KI}$ & $22 \mathrm{TI} 47$ & $G, P$ & RLY & THR- & 60 & $\begin{array}{l}30-60 \\
Y L O \text { REL }\end{array}$ & $\begin{array}{l}\text { NA } 1 \text {-O } \\
12 C 10\end{array}$ & $\begin{array}{l}4 \mathrm{PI} \\
\mathrm{O}, \mathrm{NI}\end{array}$ \\
\hline $62 S H 5$ & $22 T 147$ & $G, 2 P$ & $A 8 X$ & $14-$ & 31 & C $14-31$ & $A C T-I$ & $4 \mathrm{PI}$ \\
\hline 67PA2 & $22 \mathrm{~T} 147$ & $G, N P$ & RLY & THR- & 30 & $C 22,30$ & $A C T-1$ & $4 \mathrm{PI}$ \\
\hline $71 \mathrm{HEI}$ & $22 \mathrm{TI} 48$ & $E, E /$ & FMF & $1-$ & 3 & 019 & $\begin{array}{l}M A G-D \\
3 \text { LEV }\end{array}$ & $\begin{array}{l}\text { DST } \\
\text { VELS }\end{array}$ \\
\hline $72 \mathrm{LII}$ & $22 T 148$ & $E, E /$ & FMF & $0-$ & 3 & $\begin{array}{r}060-120 \\
.99\end{array}$ & $\begin{array}{l}M A G-D \\
.2 .44\end{array}$ & $\begin{array}{l}\text { DST } \\
\text { MEV }\end{array}$ \\
\hline $63 \mathrm{AK} 1$ & $22 \mathrm{TI} 48$ & $G, G$ & LFT & 2 & & 0 & NAI -O & 150 \\
\hline $64 \mathrm{BDI}$ & $22 \mathrm{TI} 48$ & $G, G$ & LFT & $1-$ & 3 & $1-$ & NA I - O & 100 \\
\hline $62 S H 5$ & $22 \mathrm{~T}[48$ & G & $A 8 x$ & $14-$ & 31 & $C 16$ & $A C T-1$ & $\begin{array}{l}\mathrm{A} 8 \mathrm{I} \\
4 \mathrm{PI}\end{array}$ \\
\hline $680 K 3$ & 221148 & $G, P$ & $A B Y$ & THR- & 20 & $C 20$ & $A C T-1$ & $4 \mathrm{PI}$ \\
\hline 67PA2 & $22 T 148$ & $G, P$ & RLY & THR- & 30 & C 22,30 & $A C T-I$ & $4 \mathrm{PI}$ \\
\hline $690 \mathrm{KI}$ & $22 T I 48$ & $G, P$ & RLY & THR- & 60 & c $30-60$ & $N A 1-D$ & $4 \mathrm{PI}$ \\
\hline & & & & & & YLO REL & 12616 & $\mathrm{G}, \mathrm{N})$ \\
\hline 6OST1 & $22 T 148$ & $G, N P$ & RLX & $152-3$ & 320 & $\begin{array}{l}\text { C } 322^{\circ} \\
\text { REL TO H2 }\end{array}$ & $\begin{array}{l}\text { TEL-O } \\
\text { CRDS }\end{array}$ & $\begin{array}{r}76 \\
\text { SEC }\end{array}$ \\
\hline $625 \mathrm{H} 5$ & $22 T 148$ & $G \cdot N P$ & A $8 x$ & $14-$ & 31 & C $14-31$ & $A C T-1$ & $4 \mathrm{PI}$ \\
\hline 67PA2 & $22 \mathrm{TI} 48$ & $G, N P$ & RLY & THR- & 30 & C 22,30 & $A C T-I$ & $4 \mathrm{PI}$ \\
\hline $62 \mathrm{SH} 5$ & 22 & $G, P$ & $A B X$ & $14-$ & 31 & C $14-31$ & $A C T-1$ & 4PI \\
\hline 67PAZ & $22 T 149$ & $G, P$ & $R L Y$ & THR- & 30 & C 22,30 & $A C T-I$ & $4 P I$ \\
\hline $680 \times 3$ & $22 T 149$ & $G, P$ & $A 8 Y$ & THR - & 20 & C 20 & $A C T-1$ & $4 \mathrm{PI}$ \\
\hline $690 \mathrm{Kl}$ & $22 T 149$ & $G, P$ & RLY & THR- & 60 & $C 30-60$ & $\begin{array}{l}\text { NAI }=D \\
12 C I G\end{array}$ & $4 \mathrm{PI}$ \\
\hline $625 H 5$ & 221 & $G, N P$ & $A B X$ & $14-$ & 31 & C $14-31$ & $A C T-1$ & $4 \mathrm{PI}$ \\
\hline 67PA2 & 22 TI 49 & G.NP & RLY & THR- & 30 & C 22,30 & $A C T-I$ & 4PI \\
\hline 7 IHEI & 22 TI 50 & $E, E /$ & FMF & $1-$ & 5 & $0198-300$ & $\begin{array}{l}\text { MAG-O } \\
5 \text { LEV }\end{array}$ & $\begin{array}{l}\text { OST } \\
\text { VELS }\end{array}$ \\
\hline 585 & $22 \mathrm{~T}$ & $G, P$ & $R L X$ & $12-$ & 22 & CTHR- 22 & $A C T-I$ & 4PI \\
\hline $625 \mathrm{HS}$ & $22 \mathrm{TI50}$ & $G, P$ & $A 8 x$ & $14-$ & 31 & C 14- 31 & $A C T-I$ & $4 P I$ \\
\hline 67PA2 & $22 \mathrm{~T} 150$ & $G, P$ & RLY & THR- & 30 & C 22,30 & $A C T-I$ & $4 \mathrm{PI}$ \\
\hline $62 S H 5$ & $22 \mathrm{TI} 50$ & $G, N P$ & $A 8 x$ & $14-$ & 31 & C $14-31$ & $A C T-I$ & $4 \mathrm{PI}$ \\
\hline $67 \mathrm{PAZ}$ & 22 TI 50 & $G, N P$ & RLY & THR- & 30 & C 22,30 & $A C T-1$ & $4 \mathrm{PI}$ \\
\hline
\end{tabular}

NUCLIOE REACTION RES EXCIT SDURCE OETECTOR 2 A IN,OUT TYPE ANG NUM
VANADIUM $Z=23$

$67 P A 2 \quad 22 T I 50$ G.NP RLY THR $-30 \mathrm{C} 22,30 \mathrm{ACT}-1$ 4PI

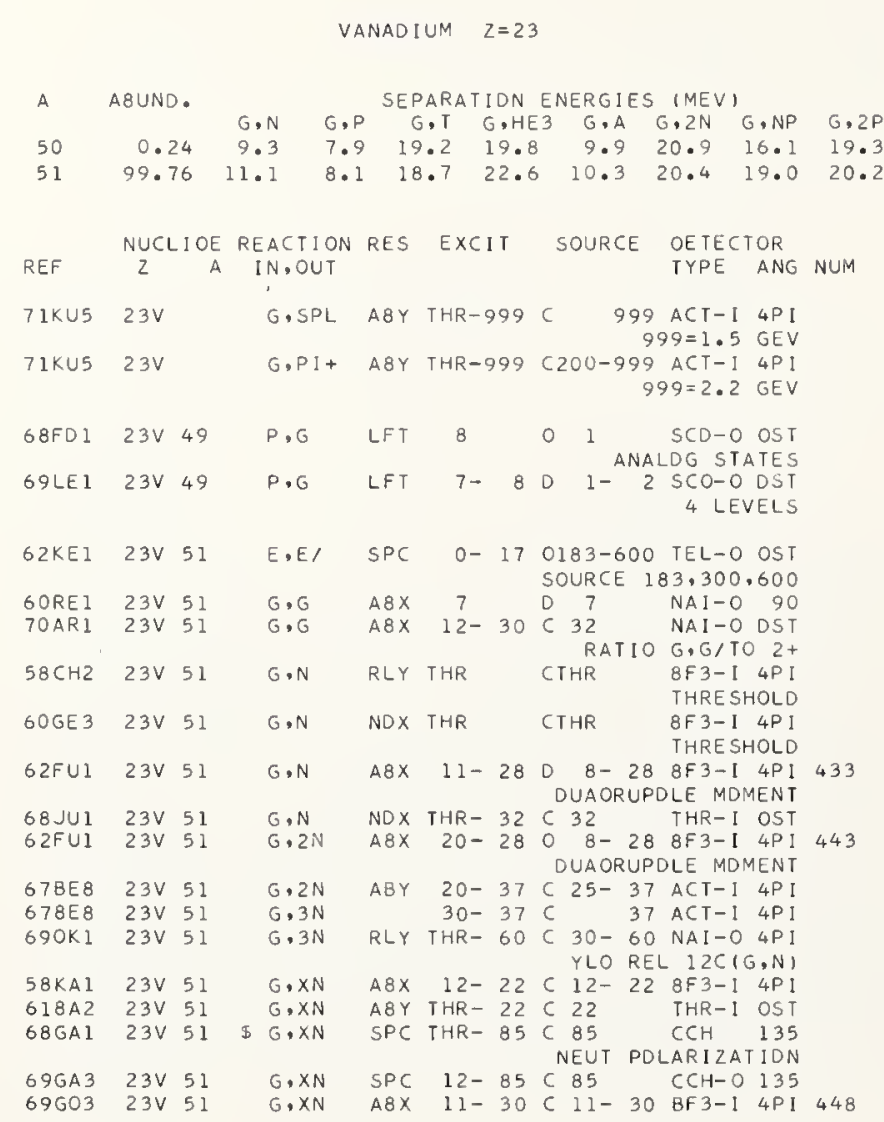

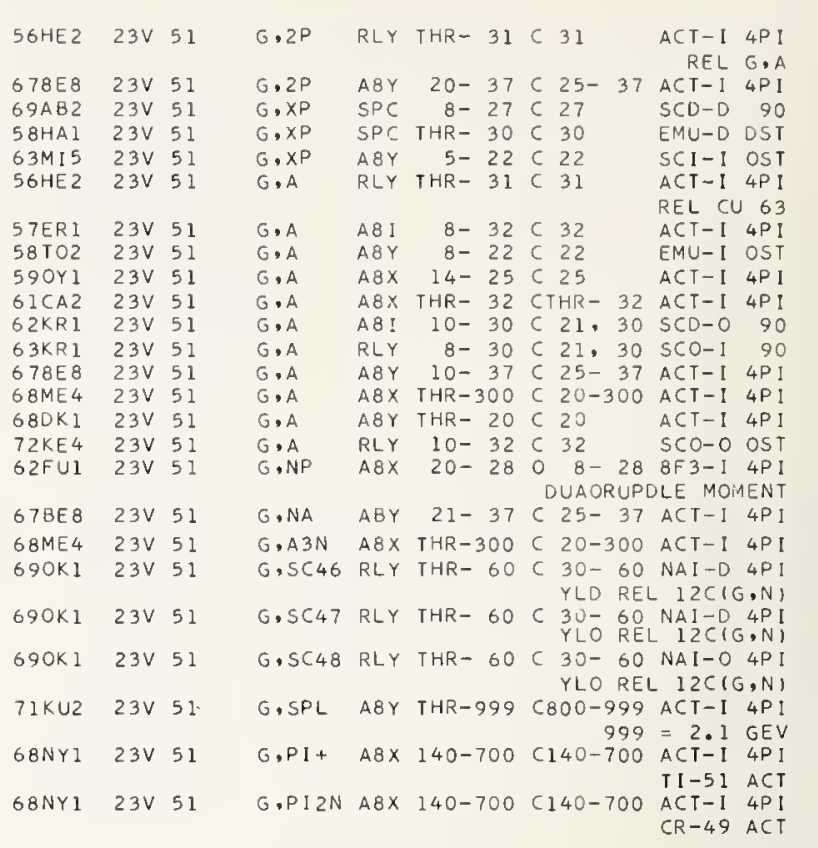

CHROMIUM $Z=24$

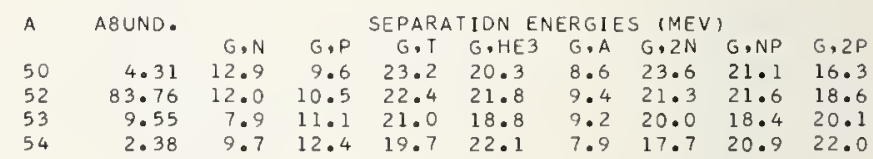

\begin{tabular}{|c|c|c|c|c|c|c|c|}
\hline & NUCL I OE & REACT IDN & RES & EXCIT & SOURCE & DETECTDR & \\
\hline EF & Z A & IN.OUT & & & & TYPE ANG & NUM \\
\hline 6OREI & $24 C R$ & $G, G$ & $A B X$ & 7 & D & NAI $-D$ & \\
\hline OARI & $24 C R$ & $G, G$ & $A 8 X$ & $12-30$ & $C 32$ & $\begin{array}{l}\text { NAI -D OST } \\
G, G / T O \quad 2+\end{array}$ & \\
\hline TFE2 & $24 C R$ & $G, N$ & $A B Y$ & $15-30$ & C $14-30$ & THR-I 90 & \\
\hline $8 \mathrm{CD} 1$ & $24 C R$ & $G, N$ & SPC & $6-30$ & C 20.30 & $\begin{array}{l}\text { EMU-D } 90 \\
\text { THRESHOLD }\end{array}$ & \\
\hline $4 \mathrm{CD} 3$ & $24 C R$ & $G, N$ & $A B I$ & $T H R-80$ & C $10-80$ & $8 \mathrm{~F} 3-14 \mathrm{PI}$ & \\
\hline 8 JUI & $24 C R$ & $G, N$ & NDX & THR- 22 & $c 22$ & THR-I OSI & \\
\hline $18 \mathrm{~A} 2$ & $24 C R$ & $G, \times N$ & $A B Y$ & THR -22 & C 22 & IHR-I DST & \\
\hline G4AL5 & $24 C R$ & $G, X N$ & NDX & THR- 34 & C 34 & THR - I OST & \\
\hline $4 \mathrm{CO} 2$ & $24 C R$ & $G, X N$ & $A B Y$ & THR- 80 & C 80 & $8 F 3-14 P I$ & \\
\hline $68 \mathrm{MCl}$ & $24 C R$ & $G, \times N$ & SPC & THR- 32 & C $22-32$ & 2 THR-I OST & \\
\hline $70 M D 2$ & $24 C R 50$ & $G, G$ & $A B X$ & 9 & $\begin{array}{l}9 \\
9\end{array}$ & $\begin{aligned} & 5 \mathrm{CO}-0 \text { OST } \\
= & 8.88 B \text {, LFT }\end{aligned}$ & \\
\hline $620 E 1$ & $24 C R 50$ & $G, N$ & $A B X$ & 20 & 020 & $A C T-I \quad 4 P I$ & \\
\hline $648 \mathrm{E} 3$ & $24 C R 52$ & $E, E /$ & FMF & $0-\quad 9$ & 0150.180 & $\begin{array}{r}M A G-0 \text { OST } \\
J-P I\end{array}$ & \\
\hline 64801 & $24 C R 52$ & $G, G$ & LFT & $1-3$ & $1-$ & $\begin{array}{rr}N A I-D & 100 \\
& A B I\end{array}$ & \\
\hline 6OGE 3 & $24 C R 52$ & $G, N$ & NOX & THR & CTHR & $\begin{array}{l}\text { 8F3-I 4PI } \\
\text { THRESHDLO }\end{array}$ & \\
\hline $71 B A$ & 24 CR5 2 & $G, N$ & $A 8 X$ & $12-14$ & C 12,14 & + TDF-O 135 & \\
\hline TOWA 3 & $24 C R 52$ & $\mathrm{G}, 2 \mathrm{~N}$ & $R L Y$ & THR-305 & $C 150-305$ & $A C T-I \quad 4 P I$ & \\
\hline 69603 & $24 C R 52$ & $G, X N$ & $A B X$ & $12-30$ & C $12-30$ & $8 \mathrm{~F} 3-\mathrm{I} 4 \mathrm{PI}$ & 449 \\
\hline 70154 & 24 CR52 & $G, X P$ & $A 8 x$ & $10-30$ & C $10-30$ & SCD-O UKN & \\
\hline TOWA3 & 24 CR 52 & $G, P N$ & RLY & THR-305 & $\begin{array}{c}\text { PROB } \\
\text { C150-305 }\end{array}$ & $\begin{array}{l}90 \text { DEGREES } \\
A C T-I \text { 4PI }\end{array}$ & \\
\hline 64801 & $24 C R 53$ & $G, G$ & LFT & $1-3$ & $1-$ & $\begin{array}{rr}\text { NAI I D } & 100 \\
\text { A } 8 \text { I }\end{array}$ & \\
\hline 60GE3 & $24 C R 53$ & $G, N$ & NDX & THR & CTHR & $\begin{array}{l}8 F 3-1 \text { 4P I } \\
\text { THRE SHDLO }\end{array}$ & \\
\hline $18 A 2$ & $24 C R 53$ & $G, N$ & $A B X$ & $9-12$ & 9,12 & IDF-D 135 & \\
\hline $71 \mathrm{JAl}$ & $24 C R 53$ & $G, N$ & $R L X$ & $7-9$ & 7- & TOF-D DST & \\
\hline $680 K 3$ & $24 C R 53$ & $G, P$ & $A B Y$ & THR - 20 & C 20 & $A C T-I \quad 4 P I$ & \\
\hline 71 RAI & $24 C R 53$ & $G, P$ & RLY & $11-14$ & C $11-14$ & $A C T-1 \quad 4 P I$ & \\
\hline
\end{tabular}


A ABUND.

SEPARATION ENERGIES (MEV) NUCLIDE REACTION RES EXCIT SOURCE DETECTOR

\begin{tabular}{|c|c|c|c|c|c|c|c|c|c|}
\hline EF & & , OUT & & & & & TYPE & ANC & NUM \\
\hline TER 1 & $25 M N 51$ & $P, G$ & NOX & $6-$ & $7 \mathrm{~F}$ & $P \quad 1-2=$ & $5<0-0$ & OST & \\
\hline ovU1 & $25 M N 53$ & $P, G$ & RLY & 7- & 90 & $01-21$ & NAI I D & 90 & \\
\hline 69TH1 & $25 M N 55$ & $E, E /$ & $A B X$ & $0-$ & 40 & o $51-61$ & $\begin{array}{c}\text { MAG-D } \\
B ! !\end{array}$ & $\begin{array}{l}\text { DST } \\
\text { (EL) }\end{array}$ & \\
\hline 7HII & $25 M N 55$ & $E, 3 N$ & $A B X$ & $30-6$ & 600 & D $64, B 2 A$ & $A C T-I$ & $4 \mathrm{PI}$ & \\
\hline 90 & MN5 5 & G.MU-T & $A B X$ & $10-3$ & 30 & C $10-260$ & $M A G=0$ & $4 \mathrm{PI}$ & \\
\hline $56 \mathrm{FUI}$ & 25MN55 & $G, G$ & $A B X$ & $4-4$ & 40 & $4-401$ & NAI I-O & 120 & \\
\hline 6ORE I & 25MN55 & $G, G$ & $A B X$ & 7 & & 07 & NAI I D & 90 & \\
\hline $64 B 01$ & $25 M N 55$ & $\mathrm{G}, \mathrm{C}$ & LFT & $1-$ & 3 & c $1-$ & NA I - D & $\begin{array}{l}100 \\
A B I\end{array}$ & \\
\hline 6BAL 1 & 25MN55 & $G, G$ & LFT & $0-$ & 3 & C 4 & $\begin{array}{l}5 C O-0 \\
7 \text { LEV }\end{array}$ & $\begin{array}{r}130 \\
\text { VELS }\end{array}$ & \\
\hline $67\llcorner 01$ & 25 MN5S & $G, G /$ & $A B X$ & $14-3$ & $32 c$ & C 34 & NAI I D & DST & \\
\hline $58 \mathrm{CH} 2$ & 25MNS5 & G.1 & RLY & THR & & CTHR & $\begin{array}{l}\text { BF 3-I } \\
\text { THRESH }\end{array}$ & $\begin{array}{l}4 P I \\
H O L O\end{array}$ & \\
\hline $99 P A$ & $25 M N 55$ & $G, N$ & $A B X$ & $10-2$ & 24 & C $10-24$ & $B F 3-I$ & $4 \mathrm{PI}$ & 439 \\
\hline $606 E 3$ & 25MN55 & $\mathrm{G}, \mathrm{N}$ & NOX & THR & & CTHR & $\begin{array}{l}\text { BF 3-1 } \\
\text { THRESH }\end{array}$ & $\begin{array}{r}4 P I \\
\text { HOLO }\end{array}$ & \\
\hline TAl & $25 t$ & $\mathrm{G}$. & Nox & $16-2$ & 22 & c 22 & THR-1 & 90 & \\
\hline $68 \pi$ & & & $A B$ & THR - 8 & & c $10-80$ & $B F 3-I$ & $4 \mathrm{PI}$ & \\
\hline $6 \mathrm{BJ}$ & $25 \mathrm{MN}$ & $G$, & NOX & THR- 2 & 22 & C 22 & THR-I & DST & \\
\hline 690E I & $25 M N 55$ & $G, N$ & $A B Y$ & THR-99 & 99 & $c^{2} 2-6$ & $\begin{array}{l}A C T-1 \\
9=5.5\end{array}$ & $\begin{array}{l}4 P I \\
\text { GEV }\end{array}$ & \\
\hline 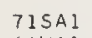 & 2 & $G, N$ & $A B Y$ & 10 & $6 B$ & $C 10-6 B$ & ACT -1 & $4 \mathrm{PI}$ & \\
\hline OWA & $25 M N 55$ & $6,3 \mathrm{~N}$ & $R L Y$ & THR - & 00 & C100-300 & ACT -1 & $\begin{aligned} 4 P I \\
4 N 52\end{aligned}$ & \\
\hline $90 E 1$ & 25 MN55 & $G, 3 \mathrm{~N}$ & $A B Y$ & THR & 99 & $\begin{aligned} & 2- \\
& 69\end{aligned}$ & $\begin{array}{l}A C T-1 \\
99=5.5\end{array}$ & $\begin{array}{l}4 P I \\
\text { GEV }\end{array}$ & \\
\hline $3 K A$ & $25 \mathrm{M}$ & $G, x$ & $A 8 x$ & $11-2$ & 22 & c $11-22$ & $8 F 3-1$ & $4 \mathrm{PI}$ & \\
\hline $60 \mathrm{FL}$ & 25 MNS & $G, \times N$ & $A B X$ & $11-$ & 27 & $\begin{array}{l}12-30 \\
\text { QUADRUPOL }\end{array}$ & $\begin{array}{l}8 F 3-1 \\
\text { LE MOM }\end{array}$ & $\begin{array}{l}4 P I \\
\text { MENT }\end{array}$ & \\
\hline & $25 \mathrm{MN}$ & $G, x$ & $A B Y$ & THR -2 & 228 & C 22 & $T H R-1$ & DST & \\
\hline $64 C$ & $25 \mathrm{MN}$ & $G$, & $A B Y$ & THR- 8 & & & $8 F 3-i$ & $4 \mathrm{PI}$ & \\
\hline $6 B G A 1$ & 25 MN5 5 & 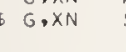 & SPC & THR- 8 & 85 & $\begin{array}{l}C \text { C BS } \\
\text { NEUT POL }\end{array}$ & $\begin{array}{l}C C H-D \\
\text { ARIZAT }\end{array}$ & $\begin{array}{l}135 \\
\text { TION }\end{array}$ & \\
\hline $69 G A$ & $25 \mathrm{MN}$ & G, & STL & $11-8$ & ( & C 85 & $\mathrm{CCH}-\mathrm{O}$ & 135 & \\
\hline 6BSF & $25 \mathrm{MN}$ & & $A 8 x$ & THR -2 & 23 & $\begin{array}{l}\text { C } 13-23 \\
\text { SPECTRA AL }\end{array}$ & $\begin{array}{l}S C I-1 \\
\text { ALSO GI }\end{array}$ & $\begin{array}{l}\text { OST } \\
\text { IVEN }\end{array}$ & \\
\hline $66 \mathrm{~W}$ & $25 \mathrm{MN}$ & $G, 7 N 4 P$ & RLY & $T H R-30$ & 00 & $\begin{array}{l}\text { C100-300 } \\
\text { ISOMER RA }\end{array}$ & $\begin{array}{l}\text { ACT-1 } \\
\text { RATIO }\end{array}$ & $\begin{array}{r}4 \mathrm{PI} \\
5 \mathrm{C} 44\end{array}$ & \\
\hline $71 \mathrm{KU2}$ & 25MNSS & G,SPL & $A B Y$ & THR-99 & 99 & C999 $999=$ & $\begin{array}{l}A C T-1 \\
=1.5\end{array}$ & $\begin{array}{l}\text { API } \\
\text { GEV }\end{array}$ & \\
\hline
\end{tabular}

61CAI $25 M N 56 \quad 0 . G \quad A B X \quad 16-1 B D$ 3- ACT-I $4 P L$
A ABUND. G,N G,P G,T G,HE3 G,A G, $2 N$ G,NP G, $P$ $\begin{array}{llllllllll}54 & 5 . B 2 & 13.4 & 8.9 & 23.0 & 19.7 & B .4 & 24.1 & 20.9 & 15.4\end{array}$ $\begin{array}{lllllllll}91.66 & 11.2 & 10.2 & 20.9 & 20.3 & 7.6 & 20.5 & 20.4 & 18.3\end{array}$ $\begin{array}{lllllllll}1.66 & 11.2 & 10.2 & 20.9 & 20.3 & 7.6 & 20.5 & 20.4 & 18.3 \\ 2.19 & 7.6 & 10.6 & 19.6 & 18.2 & 7.3 & 18.8 & 17.8 & 19.6\end{array}$ $\begin{array}{lllllllll}2.19 & 7.6 & 10.6 & 19.6 & 1 \mathrm{~B} .2 & 7.3 & 1 \mathrm{~B} . \mathrm{B} & 17 . \mathrm{B} & 19.6 \\ 0.33 & 10.0 & 11.8 & 19.4 & 22.0 & 7.6 & 17.7 & 20.6 & 21.5\end{array}$

NUCLIDE REACTION RES EXCIT SOURCE OETECTOR REF 2 A IN,OUT TYPE ANG NUM

$71 F U 1$ 26FE E,SPL A8I THR-999 $0040-999$ SCO-O 4P I

7OFU1 26FE E,F RLY $999 \quad 0999 \quad 999=16$ GEV $999=1.5-16.0$ GEV $9 \mathrm{KO} 26 \mathrm{FE}$ 635UI $26 \mathrm{FE}$

64801 26FE

$64 \mathrm{CO}_{2} 26 \mathrm{FE}$

6BKAI 26FE

55011 26FE

$56 \mathrm{HAl}$ 26FE

$618 \mathrm{A2} 26 \mathrm{FE}$

$64 \mathrm{CO}_{2} 26 \mathrm{FE}$

$67 \mathrm{CO} 226 \mathrm{FE}$

$71 \mathrm{CO} 2$ 26FE

66WAL 26FE

66WAL $26 \mathrm{FE}$

$63 \mathrm{MIS} \quad 26 \mathrm{FE}$

$58 T 102$ 26FE

$63 \mathrm{KR} 1$ 26FE

$72 K E 4$ 26FE G, MUU-T G $G, G$ ABX 7 O 7 NAI-O DSI G.G ABX 4- 14 C 4- 14 NAI-O 120 G,G LFT I- 3 C 1 - NAI-O 100 G.N ABI THR-BOC $10-80$ 8F3-I 4 P I I $G, N \quad A 8 \times 50-B 5 C 55$, B5 TOF-O 67 G XXN ABX 15, 1B D 15, SCI-I OST

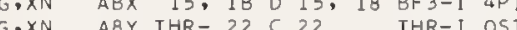

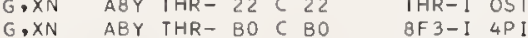
$G, X N$ ABX THR- $24 \mathrm{C} 11-24 \mathrm{BF} 3-\mathrm{I} 4 \mathrm{PI} 436$ $G \cdot X N \quad A B I 36-64 C 10-648 F 3-I$ API ISOMER RATIO MN52 G.XN5P RLY THR-250 C100-250 ACT-I 4PI $G, X P$ ABY $8-22 C 22$ RATIO SC4 G.A ABY 7- $22 C 22$ C 22 EMU-I OST $G, A$ RLY 7- $30 \mathrm{C} 21,305 \mathrm{CO}-\mathrm{T}$ G,A RLY $7-32$ C 32 SCD-D DSI NEUT ENGY SPEC G.XNP RLY THR-250 C100-250 ACT-I 4 P I

\begin{tabular}{|c|c|c|c|c|c|c|}
\hline $69 \mathrm{FU2}$ & $26 \mathrm{FE}$ & $G, S P L$ & RLY & THR-999 & 0999 & SCO-D DST \\
\hline $71 \mathrm{FU}_{4}$ & $26 \mathrm{FE}$ & $G, S P L$ & RLX & $T H R-999$ & D999 & ACT-I 4 P I \\
\hline & & & & & & $999=5$ GEV \\
\hline $71 \mathrm{KU2}$ & $26 \mathrm{FE}$ & G.SPL & $A 8 Y$ & $T H R-999$ & $C 999$ & $A C T-I \quad 4 P I$ \\
\hline $71 \mathrm{KUS}$ & $26 \mathrm{FE}$ & $G, S P L$ & $A B Y$ & & 999 & $\begin{array}{l}=1.5 \text { GEV } \\
A C T-I \text { AP }\end{array}$ \\
\hline & & & & $1 \mathrm{HR}-999$ & & $99=1.5 \mathrm{GEV}$ \\
\hline $67 F \cup 1$ & $26 \mathrm{FE}$ & $G \cdot F$ & $A B Y$ & THR-999 & 0999 & $A C T-1 \quad 4 P I$ \\
\hline $71 \mathrm{KUS}$ & $26 \mathrm{FE}$ & G,PI+ & $A B Y$ & THR-999 & $C 200-999$ & $A C T-1 \quad 4 P I$ \\
\hline $668 \mathrm{E}]$ & $26 \mathrm{FF}$ & $N, G$ & SPC & 15 & D 7 & $\begin{array}{r}99=2.2 \text { GEV } \\
N A I-0 \text { UKN }\end{array}$ \\
\hline & & & & & & \\
\hline $550 E 1$ & $26 \mathrm{FE} 53$ & $G \cdot N$ & $A B X$ & $11-24$ & c $11-24$ & $A C T-I \quad 4 P I$ \\
\hline $550 E_{1}$ & $26 F E 54$ & $G \cdot N$ & $A 8 X$ & $11-24$ & C $11-24$ & $+A C T-I$ \&PI \\
\hline 57 CA2 & $26 F E 54$ & $\mathrm{G} \cdot \mathrm{N}$ & $A B X$ & $12-30$ & C $13-30$ & $A C I-I \quad 4 P I$ \\
\hline $620 E_{1}$ & $26 F E 54$ & $G, N$ & $A 8 x$ & 20 & 020 & $A C T-I \quad 4 P I$ \\
\hline 70WA3 & $26 F E 54$ & $6.2 \mathrm{~N}$ & RLY & THR-305 & C150-305 & $A C T-I$ \&PI \\
\hline 70WA3 & $26 F E 54$ & $G, P N$ & RLY & THR-305 & C150-305 & ACT-I $4 P I$ \\
\hline 58603 & $26 F E 54$ & 6.0 & RLY & $19-22$ & c $16-22$ & $=A C T-I \quad 4 P I$ \\
\hline $5 \mathrm{BGO} 3$ & $26 F E 54$ & $G, N P$ & $R L Y$ & $19-22$ & $\begin{array}{l}\text { REACTION } \\
C \quad 16-22 \\
\text { REACTION }\end{array}$ & $\begin{array}{l}\text { UNCERTAIN } \\
\text { ACT-I } 4 \text { I I } \\
\text { UNCERTAIN }\end{array}$ \\
\hline 7OPEZ & $26 F E 56$ & $E, E \prime$ & FMF & $0-5$ & $\begin{array}{l}060 \\
7 \quad 2+, 1\end{array}$ & $\begin{array}{l}\text { MAG-D DST } \\
3-5 \text { STATES }\end{array}$ \\
\hline $71 \mathrm{HEI}$ & $26 F E 56$ & $E, E /$ & FMF & 1 & $0198-300$ & MAG-D OST \\
\hline 61KE2 & $26 F E 56$ & $G, G$ & Nox & 1 & D 1 & $\begin{array}{l}\text { NAI }=0100 \\
G-W I O T H\end{array}$ \\
\hline 67LOI & $26 \mathrm{FES}$ & $G, G /$ & $A B X$ & $14-32$ & C 34 & NAI -O OST \\
\hline $58 \mathrm{TOI}$ & $26 \mathrm{FES}$ & $\mathrm{G}, \mathrm{N}$ & RLY & $12-22$ & C 22 & $\begin{array}{l}\text { BF3-I 4P I } \\
\text { THRESHOLD }\end{array}$ \\
\hline 60GE 3 & $26 F E 56$ & $G, N$ & NOX & THR & CTH & $\begin{array}{l}8 F 3-1 \text { 4P I } \\
\text { THRE SHOLD }\end{array}$ \\
\hline $66 \mathrm{BE} 4$ & $26 \mathrm{FES}$ & $\mathrm{G} \cdot \mathrm{N}$ & $A 8 X$ & 11 & C 13 & TOF-D 135 \\
\hline 67802 & $26 \mathrm{FE}$ & $G \cdot N$ & $A 8 x$ & 12,13 & C 12,13 & 3 TOF-O 135 \\
\hline $718 A 2$ & $26 \mathrm{~F}$ & $G$, & $A B X$ & $11-13$ & $\left(\begin{array}{lll}11 & 13\end{array}\right.$ & 3 TOF-O 135 \\
\hline $685 \mathrm{H}_{3}$ & $26 \mathrm{~F}$ & G, & $A 8 x$ & THR- 23 & $\begin{array}{l}C 13-23 \\
\text { SPECTRA }\end{array}$ & $\begin{array}{l}\text { SCI-I OST } \\
\text { ALSO GIVEN }\end{array}$ \\
\hline $680 K_{3}$ & 26FE56 & $G, N P$ & $A B Y$ & THR- 20 & C 20 & $A C T-I \quad 4 P I$ \\
\hline $58 \mathrm{TOI}$ & $26 F E 57$ & $G, N$ & $R L Y$ & $12-22$ & C 22 & $\begin{array}{l}8 F 3-1 \quad 4 P 1 \\
\text { THRESHHOLD }\end{array}$ \\
\hline 60GE 3 & $26 F E 57$ & $G, N$ & NOX & THR & CTHR & $\begin{array}{l}\text { BF } 3-I \text { I } 4 \text { P I } \\
\text { THRE SHOLD }\end{array}$ \\
\hline - & $26 \mathrm{FE}$ & $\mathrm{G}$, & $A 8$ & $7-12$ & C $B-12$ & TOF-D 135 \\
\hline $71 \mathrm{JAl}$ & $26 F E 57$ & $\mathrm{G} \cdot \mathrm{N}$ & $R L x$ & $7-8$ & C $7-B$ & 3 TOF-D DST \\
\hline & $26 F E 57$ & $\mathrm{G} \cdot \mathrm{N}$ & $R\llcorner X$ & $\begin{array}{ll}B- & 9 \\
G-\end{array}$ & $\begin{array}{c}C B \\
-W I D T H, 7 .\end{array}$ & $\begin{array}{r}\text { TOF-0 DST } \\
.6-8.4 \mathrm{MEV}\end{array}$ \\
\hline $6 B O K$ & $26 F E 57$ & $G, P$ & $A B Y$ & THR- 20 & $c 20$ & $A C T-1 \quad 4 P 1$ \\
\hline
\end{tabular}

COBALT $Z=27$

\begin{tabular}{|c|c|c|c|c|c|c|c|c|c|}
\hline \multirow{3}{*}{$\begin{array}{l}A \\
59\end{array}$} & \multirow{3}{*}{$\begin{array}{l}\text { ABUNO. } \\
100.00\end{array}$} & \multicolumn{8}{|c|}{ SEPARATION ENERGIES (MEV) } \\
\hline & & \multirow{2}{*}{$\begin{array}{r}G, N \\
10.5\end{array}$} & \multirow{2}{*}{$\begin{array}{l}G . P \\
7.4\end{array}$} & G.T & $\mathrm{G}, \mathrm{HE} 3$ & \multirow{2}{*}{$\begin{array}{r}3, A \\
7.0\end{array}$} & \multirow{2}{*}{\multicolumn{2}{|c|}{$\begin{array}{lll}A & G, 2 N & G, N P \\
0 & 19.0 & 17.4\end{array}$}} & \multirow{2}{*}{$\begin{array}{l}G .2 P \\
19.2\end{array}$} \\
\hline & & & & 5.6 & 20.3 & & & & \\
\hline & NUCLIOE & REACTION & RES & $E \times C I$ & IT & SOURC & DETECT & $T O R$ & \\
\hline REF & $Z \quad A$ & IN,OUT & & & & & TYPE & ANG & NUM \\
\hline 72MA2 & $27 C 055$ & $P, G$ & LFT & 7 & & 02 . & $S C D-D$ & $\begin{array}{l}\text { DST } \\
J-P I\end{array}$ & \\
\hline TOER I & $27 \cos 8$ & $P, G$ & SPC & $8-$ & 9 & $\begin{array}{l}\text { D } 1- \\
\text { OETA }\end{array}$ & $\begin{aligned} 2 S C D-0 \\
A I L S \quad 4 \quad L E V\end{aligned}$ & $\begin{array}{r}45 \\
\text { VELS }\end{array}$ & \\
\hline 6ICRI & 276059 & $E, E \prime$ & NOX & $0-$ & 4 & $\begin{array}{l}\text { O1B3 } \\
\text { G-WDTH }\end{array}$ & $\begin{array}{r}M A G-1 \\
\text { 4. MULTIPG }\end{array}$ & $\begin{array}{r}\text { OST } \\
\text { OLES }\end{array}$ & \\
\hline $65 W Y 1$ & $27 \cos 9$ & $G, M U-T$ & $A B X$ & $10-$ & - 35 & C 90 & $S C I-0$ & $4 \mathrm{PI}$ & 60 \\
\hline 6OREI & $27 \cos 9$ & $G, G$ & $A B X$ & 7 & & 07 & NAI I D & 90 & \\
\hline 64801 & 276059 & $G, G$ & LFT & 1- & 3 & $1-$ & NAI - O & $\begin{array}{l}100 \\
A B[\end{array}$ & \\
\hline 6BALI & $27 \cos 9$ & $G, G$ & LFT & 1 & & c & $\begin{array}{r}5 C 0-0 \\
1=1.187\end{array}$ & $\begin{array}{l}130 \\
\text { MEV }\end{array}$ & \\
\hline $715 w^{3}$ & $27 \cos 9$ & $G, G$ & LFT & $1-$ & 5 & $\begin{array}{l}5 \\
15\end{array}$ & $\begin{array}{l}\text { SCD-D } \\
\text { LEVELS, }\end{array}$ & $\begin{array}{r}\text { OST } \\
J-P I\end{array}$ & \\
\hline $67 \mathrm{LO}_{1}$ & $27 \cos 9$ & $G, G /$ & $A B X$ & $14-$ & - 32 & C 34 & NAI-D & OST & \\
\hline $58 \mathrm{CH} 2$ & $27 \cos 9$ & $G, N$ & RLY & THR & & CTHR & $\begin{array}{l}\text { BF3-I } \\
\text { THRESH }\end{array}$ & $\begin{array}{r}4 P I \\
H O L D\end{array}$ & \\
\hline 60GE 3 & $27 \cos 9$ & $G, N$ & NOX & THR & & CTHR & $\begin{array}{l}\text { BF 3-I } \\
\text { THRES }\end{array}$ & $\begin{array}{r}\text { 4PI } \\
\text { HOLD }\end{array}$ & \\
\hline $62 C A 1$ & $27 \cos 9$ & $G, N$ & NOX & $11-$ & 30 & C 30 & $\begin{array}{r}\text { ACT-I } \\
\text { I SOMER R }\end{array}$ & $\begin{array}{l}\text { 4PI } \\
\text { RATIO }\end{array}$ & \\
\hline $62 \mathrm{FUI}$ & $27 \cos 9$ & $\mathrm{G}, \mathrm{N}$ & $A B X$ & $11-$ & 28 & $9-$ & 28 BF 3-1 & $4 \mathrm{PI}$ & 435 \\
\hline $64 \mathrm{CO}_{3}$ & $27 \cos 9$ & $G, N$ & $A B I$ & THR- & - BO & C $10-$ & $B 08 F 3-1$ & $4 \mathrm{PI}$ & \\
\hline $67 G E 2$ & $27 \cos 9$ & $G, N$ & $A B Y$ & THR- & 27 & C 22 , & $278 F 3-1$ & $4 \mathrm{PI}$ & \\
\hline $\begin{array}{l}67 \mathrm{HUI} \\
67 \mathrm{LII}\end{array}$ & $\begin{array}{l}270059 \\
270059\end{array}$ & $\begin{array}{l}G, N \\
G, N\end{array}$ & A8X & 11 & 54 & $\begin{array}{lll}D & 1 & 1 \\
C & 35\end{array}$ & $5 \begin{array}{l}8 F 3-I \\
54 A C T-I\end{array}$ & 4P I & \\
\hline & & & & & & REL C1 & 11 , ISM R & RATIO & \\
\hline & 25 & $G, 1$ & $A B Y$ & $10-$ & 68 & & 68 & $4 \mathrm{PI}$ & \\
\hline
\end{tabular}


NUCLIDE REACTION RES EXCIT SOURCE DETECTOR REF
$62 F U 127 C D 59$ G, $2 N$ A8X 19- $2 B$ C 9- 28 BF3-I 4 PI 444

67 LII 27 CO59

67 LII 27CD59

67 LII $27 \cos 9$

$56 \mathrm{HA1} 27 \mathrm{COS9}$

$58 K A 127 C 059$

GOFLI 27 CD 59

$618 A 2 \quad 27 C D 59$

$61 \mathrm{EM1} 27 \mathrm{COS9}$

$648 A 4-27 C D 59$

$64 \mathrm{CD} 2 \quad 27 \mathrm{CD} 59$

$658 \mathrm{~A} 3 \quad 27 \mathrm{CD} 59$

$66 \mathrm{FII} 27 \mathrm{CD} 59$

68GAl 27 CD59

$68 \mathrm{RHI} \quad 27 \mathrm{CO} 59$

69GA3 27CO59

$6960327 C D 59$

56FD1 27CD59

$57 R D 1 \quad 27 \operatorname{COS9}$

6OCHI $27 \mathrm{CD} 59$

63 MI5 27 C059

56FD1 $27 C D 59$

6IFO1 27CD59

$61 F 01276059$

$60 \mathrm{CHI} \quad 270059$

$56 \mathrm{HE} 1$ 27CD59

$\begin{array}{ll}\text { 56WAI } & 27 C 059 \\ \text { SOCHI } & 27 C D 59\end{array}$

$\begin{array}{ll}60 \mathrm{CH} 1 & 27 \mathrm{CD} 59 \\ 58102 & 27 \mathrm{CO} 59\end{array}$

SIFDI 270059

$72 K E 4 \quad 27 C D 59$

$\begin{array}{ll}62 F U 1 & 27 C 059 \\ 60 G 02 & 27 C 059\end{array}$

G, $2 N$ RLY THR- 54 C 35,54 ACT-I 4 PI

G,3N RLY THR- $54 \mathrm{C} 35,54$ ACT-I 4 P I

G, $4 \mathrm{~N}$ RLY THR- $54 \mathrm{C} 35,54$ ACT-I $4 \mathrm{PI}$

REL (G, N)

$G, X N \quad A B X \quad 15,18$ D 15,18 BF3-I 4P

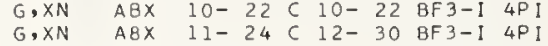

G,XN ABY THR- 22 OUADRUPOLE MOMENT

$\begin{array}{llrllll}G, X N & A B Y & T H R-22 & C & 22 & \text { THR-I } & \text { DST } \\ G, X N & 5 P C & 13-30 & C & 30 & \text { EMU-D } & 9 D\end{array}$

$6, X N \quad A B \times 10-30 \subset 10-30 B F 3-[4 P$ I

$G, X N \quad A B X \quad 10-27 C 10-27$ BF 3-I 4PI

G.XN ABY THR- 80 C 80 S 3 BA SAME DATA

$G, X N$ ABX THR - $28 C 10-30$ BF3-I $4 \mathrm{PI}$

$G, X N$ SPC THR - 60 C 60 TDF - D 90

G,XN SPC THR- BS C 85 CCH-D 135

$G, X N$ NOX THR- $48 C 48$ NEUT POLARIZATIDN

$G, X N$ SPC $11-85$ C 85 C $\quad$ CH-D 135

$G, X N$ ABX $10-30$ C $10-30$ BF $3-I$ I 4 I 450

$G, X N$ ABX $10-22 C 10-22$ BF $3-I$ IPI 247

G,P RLY 7- 30 C 30 EMU-D 90

G,P SPC 15, 18 D 15,18 EMU-D DST

G,XP RLX 22- 90 C 90 TEL-I 90

G, XP ABY $7-22 C 22$ REL TD DEUTERDNS

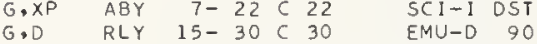

G,D SPC $7-30 \mathrm{C} 30^{\text {YLO REL TD G, G }}$

G.D RLY $15-30$ C 30 EMU-D DST

G. NO DEUTERONS FOUND

RLX $30-90$ C 90 TEL TD PROTONS
REL TD

G.T RLY THR- $31 C 31$ ACT-I 4 PI

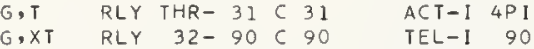

$\begin{array}{lllll}\text { G,A ABY } & 7-22 C 22 & \text { REL DEUTERONS } \\ \text { EMU-I DST }\end{array}$

G,A SPC $9-30$ C 30 EMU-D UKN

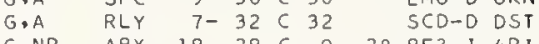

$G, N P \quad A B X \quad 18-28 C 9-28$ BF $3-I$ I PI 441

$G, P D \quad A B X \quad 30-260$ C $30-260$ ACT-I $4 P$ I

$6060227 C 059$ G.HE3 A8X 30-260 C 30-260,ACT-I $4 P$ I

$6060227 C D 59$ G,N2P ABX $30-260$ C $30-260$ ACT-I 4 P I

66WAI $27 \operatorname{CO} 59$ G,5N2P RLY THR-150 CI5D ACT-I 4PI

$67 L I 1276059 \quad$ G,MN56 RLY THR- 54 I 35,54 ACT I 4 P I

71KU2 270059 REL (G,N)

$999=1.5 \mathrm{GEV}$

G6WA2 27 COSO N,G RLY 7 D

NICKEL $\quad Z=28$

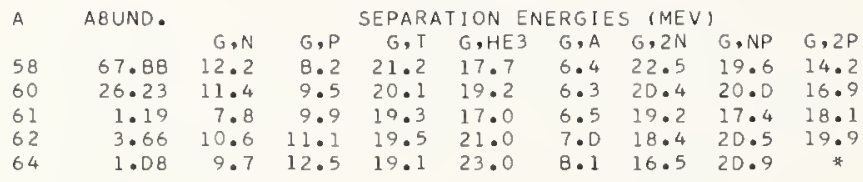

NUCLIDE REACTION RES EXCIT SDURCE DETECTDR

$71 M O 32 B N I \quad E \cdot E \prime$ ABX D-240 DSOD MAG-D 60

$\begin{array}{ll}65 W Y 1 & 8 \mathrm{BN} \\ 56 \mathrm{FUI} & 28 \mathrm{NI}\end{array}$

6DRET $28 \mathrm{NI}$

$61 \mathrm{MII} 2 \mathrm{BN}$

$61 T D 128 N I$

$628 E 2 \quad 28 N I$

$64 \mathrm{GII} 28 \mathrm{BNI}$

$66 B E^{3} 28 \mathrm{NI}$

$69 C E I \quad 28 N I$

$65 \mathrm{GII} 2 \mathrm{BNI}$

$64 \mathrm{CO} 32 \mathrm{BN}$

67 HU2 $28 \mathrm{NI}$

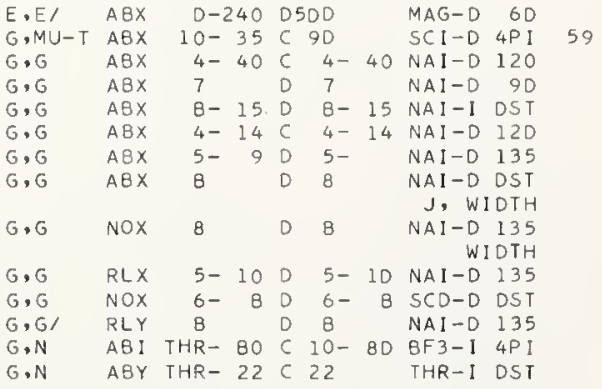

$G, N$ ABY THR- 22 C 22 THR-I DST

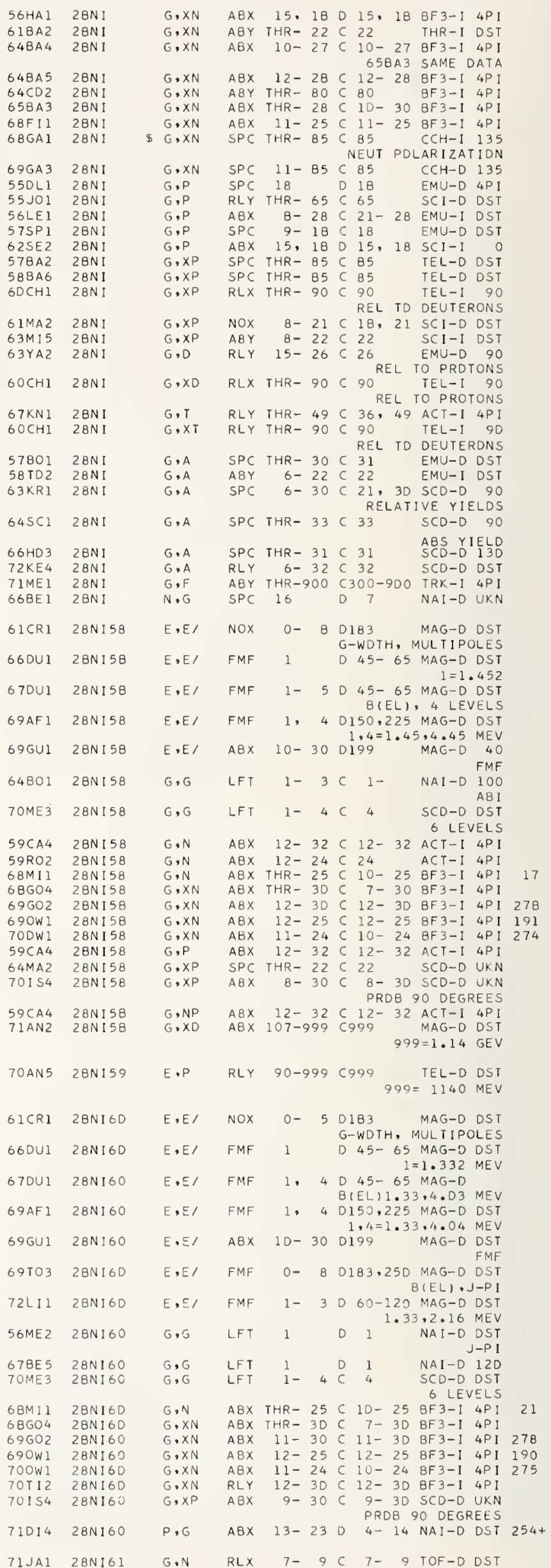


REF

NUCLIDE REACTION

SOURCE DETECTOR

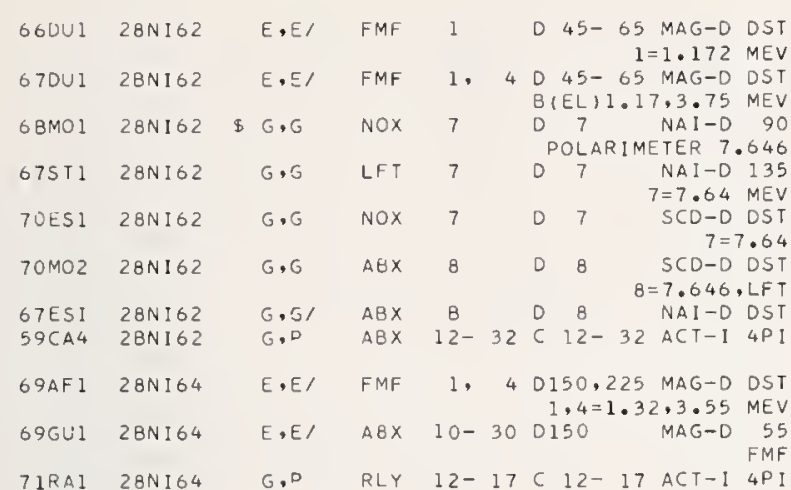

COPPER $Z=29$

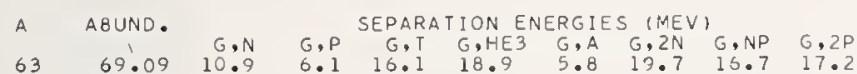

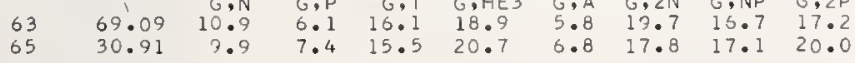

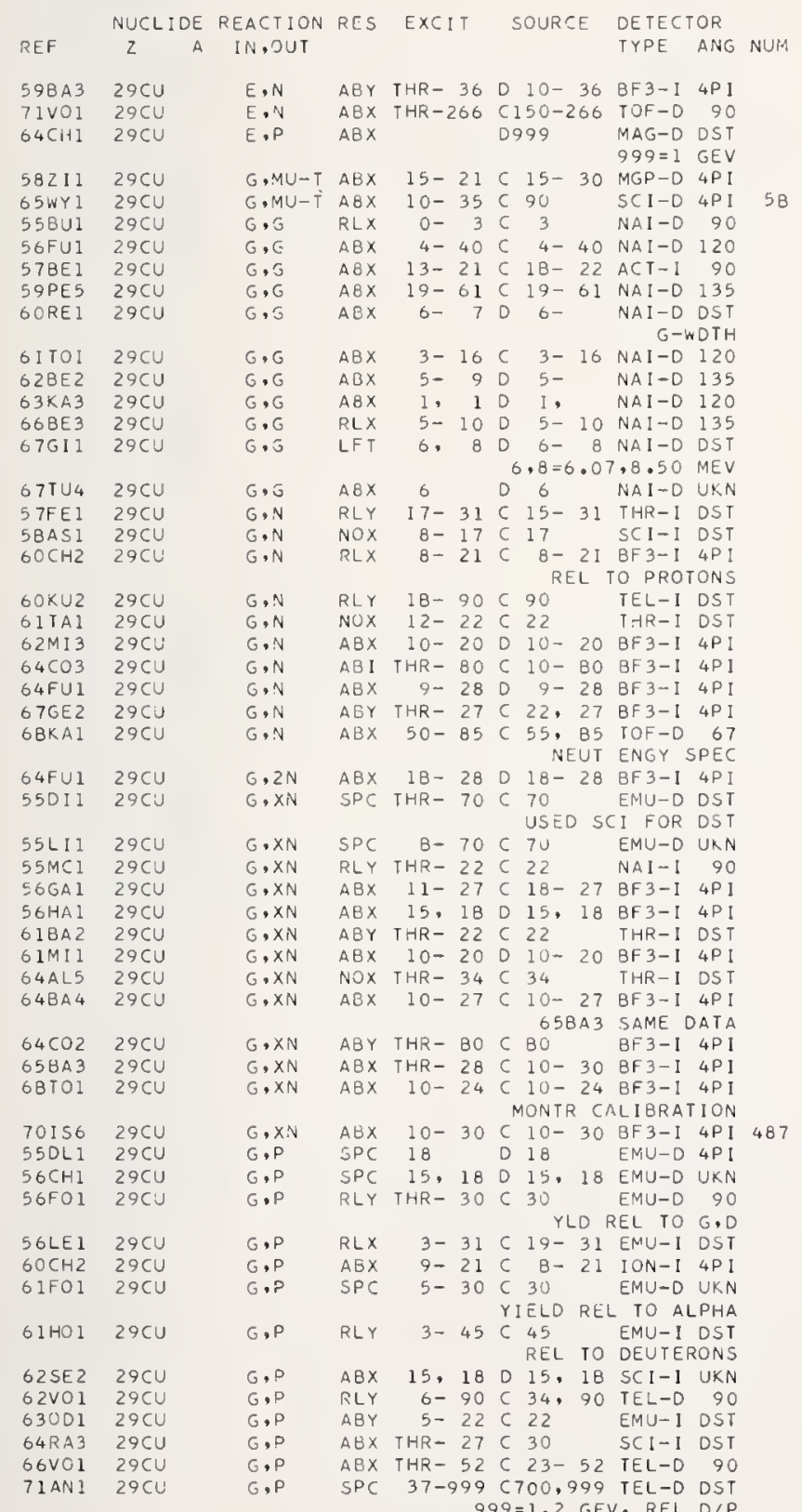

NUCLIDE REACTION RES EXCIT SOURCE DETECTOR RE

2 A IN,OUT

TYPE ANG NUM

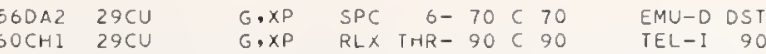

GIMAZ zOCU

$\begin{array}{ll}63 \text { MIS } & 29 C U \\ 56 \text { FOI } & 29 C U\end{array}$

$60 \mathrm{KO} 429 \mathrm{CU}$

$61 \mathrm{HOI} 29 \mathrm{CU}$

$62 \mathrm{VO} 129 \mathrm{CU}$

63YA2 $296 \mathrm{C}$

$645 \mathrm{H3} 29 \mathrm{CU}$

$66 \mathrm{VO} 29 \mathrm{CU}$

$71 \mathrm{ANI} 29 \mathrm{CU}$

$60 \mathrm{CH} 129 \mathrm{CU}$

$56 \mathrm{HE} 129 \mathrm{CU}$

56WA1 29CU

$62 \mathrm{VO} 129 \mathrm{CU}$

$60 \mathrm{CHI} 29 \mathrm{CU}$

$5760129 \mathrm{CU}$

$58 \mathrm{TO2} 29 \mathrm{CU}$

$61 F 0129 C U$

$63 K R 1 \quad 29 C U$

$645 C 1 \quad 29 C 0$

$65 M E 2$ 29CU

T2KE4 $29 \mathrm{CU}$

$605 T 129 C U$

64MA4 29CU

$6901329 \mathrm{CU}$

$71 \mathrm{ME}, 29 \mathrm{CU}$

$71 G R 2 \quad 29 C U$

$716 R 2 \quad 29 C U$

$G, X P$ NOX $6-21 G 21$ TO DEUTERONS

$G, X P$ ABY $7-22 C 22 \quad$ SCI-I DST

$G, D$ RLY THR- 30 C 30 EMU-D 90

G,D RLY $15-35<70$ YLD REL TO G,

$G, D$ RLY $11-45$ C 45 EMU-I DST

G.D RLY $11-90$ C 34, 90 TEL-D 90

G.D RLY $14-26$ C 26 EMU-D DST

G, RLY 15 - 40 YIELD REL TO PROTN

G.D AEX - $A R-52 C 23-52$ TEL-D 9 C

G SPC 45-999 C700,999 TEL-D DST

$099=1.2$ GEV, REL D/P

6.00 RLX THR - $90 \mathrm{C} 90$ TEL TO PROTONS
REL TO

G,T RLY THR- $31 C 31$ ACT-I 4 PI

G, T RLY THR- $31 C 31$ ACT-I 4 P I

G.T RLY $15-90 \mathrm{C} 34,90$ TEL-D 90

GEXT RLY THR- $90 \mathrm{C} 90$ TEL-I 90

G.A SPC THR- 30 C 31 TO DEUTERONS

G,A SPC 7- 22 C 22 EMU-D DST

G,A RLY $7-22$ C 30 EMU-I UKN

G,A SPC $9-30 \mathrm{C} 21,30$ SCD-D 90

RELATIVE YIELO

G.A RLY $5-32$ C 32 SCD-D 90

G.NP RLX $144-320$ C320 TEL-D 76

REL TO HZ CROS SEC G.CU62 ABY 150-720 C150-720 ACT-I 4P G.SPL RLY THR-600 C600 ACT-I 4PI

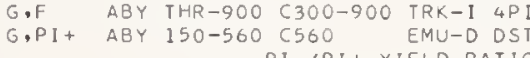

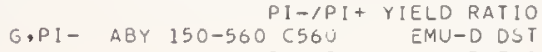
PI- $P I+$ YIELD RATIO

$61 C A 1290460$ D.G ABX 14- 16 D 3- ACT-I 4PI

$57 \mathrm{HI1} 29 \mathrm{CU} 63 \mathrm{E}, \mathrm{N} \quad \mathrm{ABX} 10-\mathrm{B2} D \quad 30-82 \mathrm{ACT}-\mathrm{I} 4 \mathrm{PI}$

65HEI 29CU63 EN RLY THR - 32 MULTIPOLARITIES

72KU6 29 CU63 E.N ABX 11- 30 D 20-30 ACT-I 4PI

65HE] 290463 E+,N RLY THR- 32 D 14- 32 ACT-I 4 PI

$6080329 C U 63$ G.G LFT O- 2 C $0-$ NAI-D 100

6IROI 29CU63 G.G ABX O- 1 D O- NAI-D DST

62806290063 G.G LFT $0-4 \mathrm{C}$ O- LIFETIMES

$63 M C I 29 C U 63$ G.G LFT I D 1 NAI-D 90

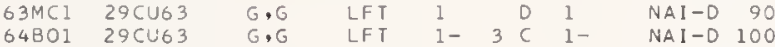

$68 A L I 29 C U 63$ G,G LFT O- $2 C 44$ ABI

6BTA2 29CU63 G,G LFT 1 D $1.414,1.551 \mathrm{MEV}$

69BET 29CUG3 G,G LET 6, 8. 6, B I UKN

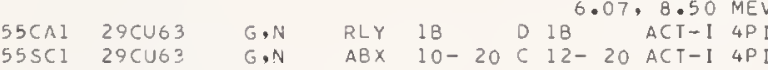

$5 B B E I$ 29CU63 G,N $\quad$ RLY $10-11$ C $10-11$ ACT-I $4 P I$

SBBEI 296063 GN RLY 10-11 C 10- 11 ACT-I 4PI

59NAI 29CU63 G.N ABX $18 \quad 0 \quad 18$ BF3-1 4PI

59PE3 29CU63 G.N RLY $10-11$ C 1J- 11 ACT-I 4PI

$606 E 3-290063$ G,N NOX THR CTHR BF 1 -I 4PI

61002290463 THRESHOLD

$620 E I 29 C U 63$ GNN ABX 21 D 21 ACT-I 4 P

$64 F U 1$ 29CU62 G.N ABX 10-2B D $10-28$ BF3-I 4PI

65 GRI 29CU63 G.N ABX THR- 44 C $10-66$ ACT-I 4 PI

$6 B O W I 29 C U 63 \quad G, N \quad R L X \quad 12-24 C 10-24$ ACT-I $4 P 1$

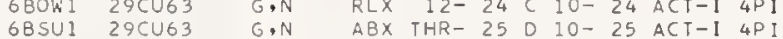

$720 R 2$ 29CU63 G.N ABX $11-26$ C II- 26 ACT-I 4 PI 446

$64 \mathrm{FUI} 29 \mathrm{CU} 63$ G, $2 \mathrm{~N}$ ABX $19-28$ D $10-2 \mathrm{~B}$ BF $3-\mathrm{I} 4 \mathrm{PI} 19$

68 SUI 29CUG3 G.2N ABX THR- 26 D $10-26$ ACT-I 4 PI

GOYA1 29CU63 G,P ABX 15, 18 D 15, 18 ACT-1 4P

G9ANG 29CU63 G.P ABY 103-999 C700,399 TEL-D DST

$62 \mathrm{CH2} 29 \mathrm{CU} 63$ G,XP RLY 6- 90 C $34-90$ TEL-D DST

$64 M A 2$ 29CUG 3 G.XP SPC THR- 17 REL TO DEUTERONS

6BAB2 29CU63 G.XP SPC THR- $27 C 27$ SCD C UKN

63608 29CU63 G.D RPC THR- 37 C 27 SCD-D 90

REL TO PROTONS

69ANG 29CU63 G,D ABY $112-999 \quad 700,999$ TEL-D DST

$62 \mathrm{CHZ} 29 \mathrm{CU} 63 \mathrm{G}, \times D$ RLY $11-90 \mathrm{C} 34-90$ TEL-D DST 
NUCLIDE REACTION RES EXCIT SOURCE DETECTUR 2 A IN,OUT TYPE ANG NUM

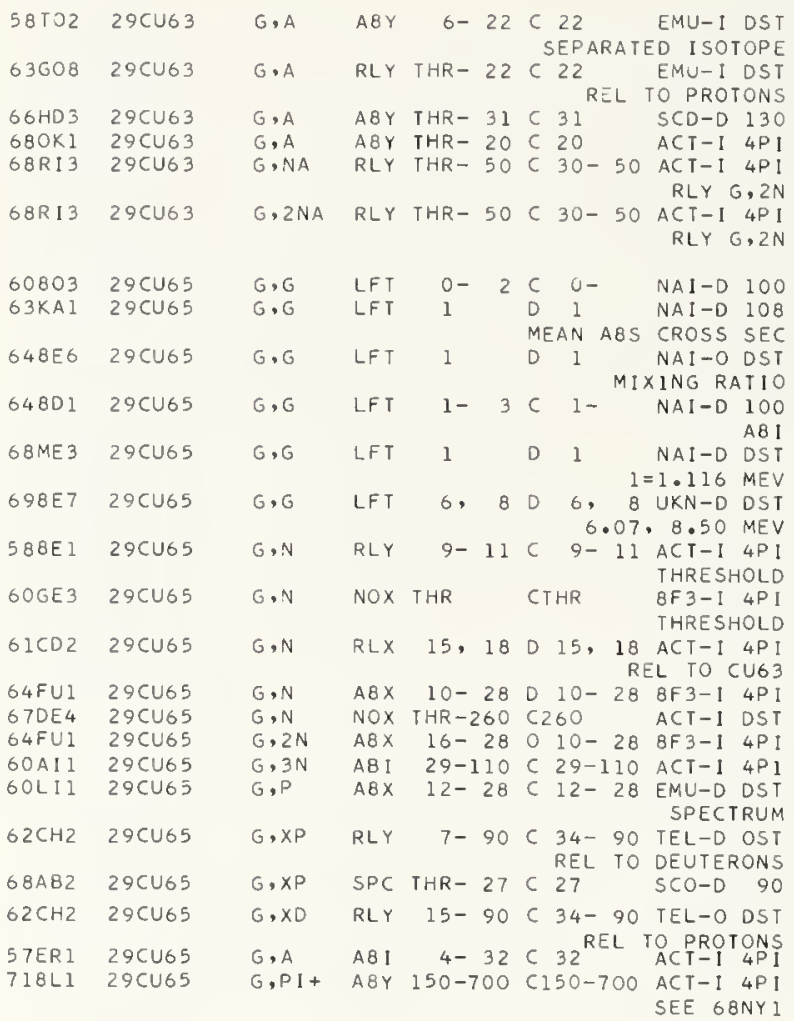

ZINC $Z=30$

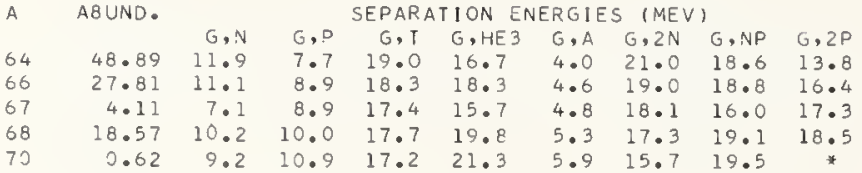

REF NUCLIDE REACTION RES EXCIT SOURCE DETECTOR

\begin{tabular}{|c|c|c|c|c|c|c|c|c|}
\hline 6OREI & $30 Z N$ & $G, G$ & $A B X$ & 7 & & D & 7 & $N A I-D$ \\
\hline 63501 & $302 N$ & $G, G$ & $A 8 x$ & 4- & 14 & $c$ & $4-14$ & NAI-D 120 \\
\hline $668 E 3$ & $302 N$ & $G, G$ & $R L x$ & $5-$ & 10 & 0 & $5-10$ & NAI-D 135 \\
\hline $61 \mathrm{TAl}$ & $302 \mathrm{~N}$ & $G, N$ & NOX & $12-$ & 22 & $c$ & 22 & $T H R-I \quad D S T$ \\
\hline 67 HUZ & $302 N$ & $G, N$ & $A B Y$ & THR - & 22 & $c$ & 22 & THR-I OST \\
\hline 68KAI & $30 Z N$ & $G, N$ & $A B X$ & $50-$ & 85 & $c$ & $\begin{array}{r}55,85 \\
\text { NEUT }\end{array}$ & $\begin{array}{l}\text { TOF-D } 67 \\
\text { ENGY SPEC }\end{array}$ \\
\hline $70 \mathrm{CO} 1$ & $3 O Z N$ & $G, N$ & $R L X$ & $12-$ & 40 & $c$ & $10-40$ & $A C T-I \quad 4 P I$ \\
\hline $70 \mathrm{CO} 1$ & $302 \mathrm{~N}$ & $\mathrm{G}, 2 \mathrm{~N}$ & $R L X$ & $21-$ & 40 & $c$ & $10-40$ & $A C T-1 \quad 4 P I$ \\
\hline $56 \mathrm{GAl}$ & $302 N$ & $G, X N$ & $A B X$ & $7-$ & 27 & $c$ & $9-27$ & $8 F 3-1 \quad 4 \mathrm{PI}$ \\
\hline $56 \mathrm{HAl}$ & $302 \mathrm{~N}$ & $G, \times N$ & $A 8 x$ & 15 & 18 & $D$ & 15,18 & $8 F 3-1 \quad 4 P I$ \\
\hline $64 \mathrm{CO} 2$ & $302 \mathrm{~N}$ & $G, \times N$ & $A 8 Y$ & THR - & 80 & c & 80 & $8 F 3-14 P I$ \\
\hline $67 \mathrm{COI}$ & $302 N$ & $G, \times N$ & $A 8 X$ & $12-$ & 24 & c & 24 & $8 F 3-1 \quad 4 P 1$ \\
\hline $71 \mathrm{CO}^{2}$ & $302 \mathrm{~N}$ & $G, \times N$ & $A 8 I$ & $36-$ & 64 & C & $\begin{aligned} & 10- 64 \\
& \text { FAS }\end{aligned}$ & $\begin{array}{l}8 F 3-1 \text { 4PI } \\
\text { ST N Y IELD }\end{array}$ \\
\hline $550 L 1$ & $302 \mathrm{~N}$ & $G, P$ & $A 8 X$ & 18 & & 0 & 18 & EMU-D 4PI \\
\hline $570 S 1$ & $302 \mathrm{~N}$ & $G, P$ & $A 8 X$ & THR- & 31 & c & $20-31$ & EMU-D DST \\
\hline $63 M I 5$ & $302 N$ & $G, X P$ & A8Y & $10-$ & 22 & $c$ & 22 & $S C I-I \quad O S T$ \\
\hline $66 \mathrm{ACl}$ & $302 N$ & $G, 0$ & $Y L D$ & $16-$ & 22 & & 22 & $\begin{array}{l}\text { MAG-0 } 4 P I \\
\text { PPER LIMIT }\end{array}$ \\
\hline 70 CUI & $302 N$ & $G, T$ & $A B Y$ & THR- & 90 & c & 90 & $A C T-I \quad 4 P I$ \\
\hline $58 \mathrm{TO} 2$ & $302 N$ & $G, A$ & $A 8 Y$ & $2-$ & 22 & c & 22 & EMU-I OST \\
\hline $66 \mathrm{HO} 3$ & $302 N$ & $G, A$ & SPC & THR - & 31 & $c$ & 31 & SCD-D 130 \\
\hline $72 K E 4$ & $302 \mathrm{~N}$ & $G, A$ & RLY & 4- & 32 & $c$ & $16-32$ & SCD-D DST \\
\hline $70 \mathrm{COI}$ & $30 Z N$ & $G, N P$ & $R L x$ & $19-$ & 40 & c & $1 v-40$ & $A C T-1 \quad 4 P I$ \\
\hline 65VA3 & $30 Z N$ & $G, C \cup 64$ & $A 8 I$ & $50-3$ & 00 & $c$ & $50-300$ & $A C T-1 \quad 4 P I$ \\
\hline $70 \mathrm{AF} 1$ & $302 N 64$ & $E, E /$ & FMF & $0-$ & 3 & & $\begin{array}{l}50,225 \\
9,3.0 \mathrm{M}\end{array}$ & $\begin{array}{l}\text { MAG-O DST } \\
\text { MEV, } 8(E L)\end{array}$ \\
\hline $72 \mathrm{LII}$ & $302 N 64$ & $E, E /$ & FMF & $0-$ & 2 & D & $60-120$ & $M A G-D O S T$ \\
\hline $698 E 7$ & $302 N 64$ & $G \cdot G$ & LFT & 7 & & 0 & .99 & $\begin{array}{rr}9,1.80 & \text { MEV } \\
\text { UKN-D DST } \\
7.38 \text { MEV }\end{array}$ \\
\hline $72 M E 3$ & $302 N 64$ & $G, G$ & LF T & $3-$ & 5 & $c$ & 5 & SCD-O DST \\
\hline
\end{tabular}

NUCLIDE REACTIDN RES EXCIT SOURCE DETECTOR

REF $Z$ A IN,OUT TYPE ANG NUM

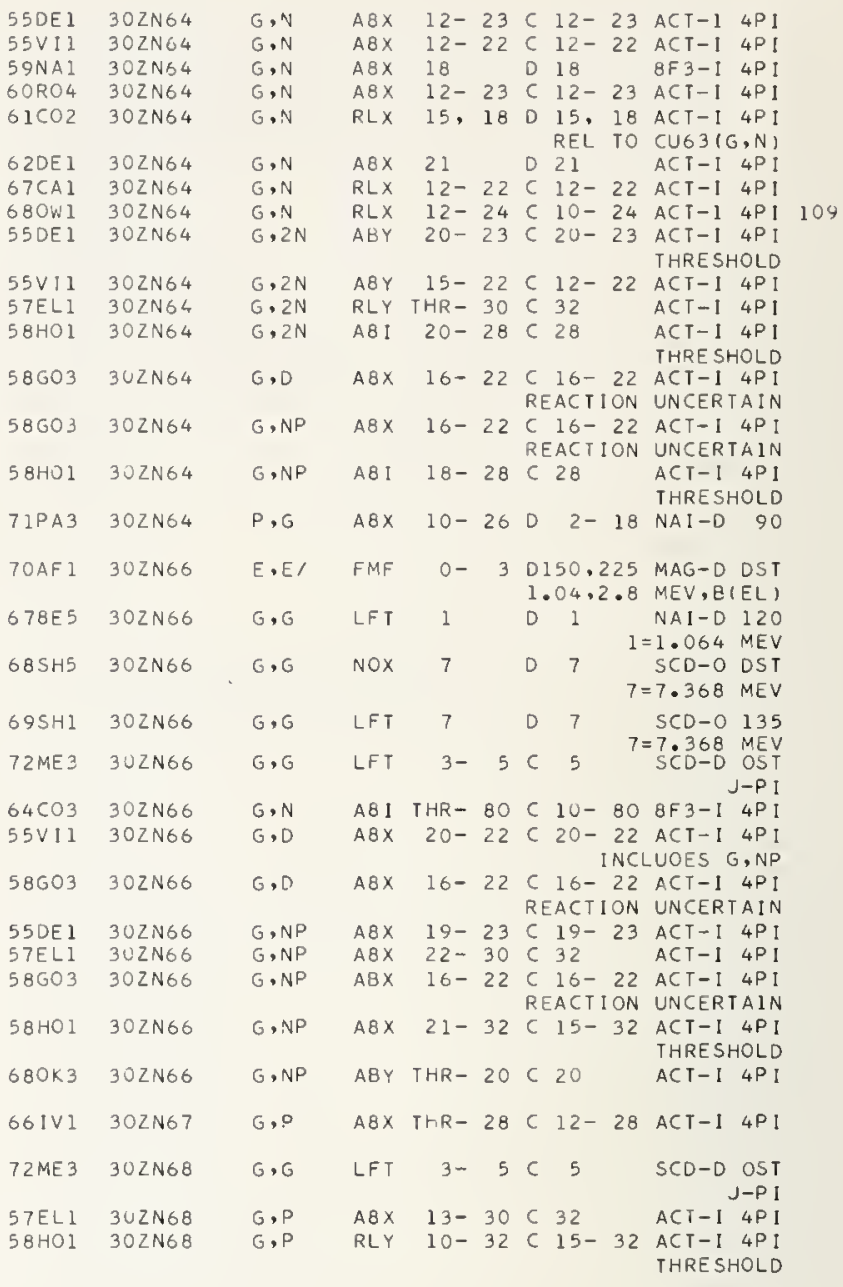

GALLIUM $Z=31$ 
SEPARATION ENERGIES (MEV)

\begin{tabular}{|c|c|c|c|c|c|c|c|c|}
\hline ABUND. & & & DAF & N & & & & \\
\hline & $\mathrm{G}, \mathrm{N}$ & $G, P$ & $G, T$ & G HEZ & $G, A$ & $G, 2 N$ & $G, N P$ & $G, 2 P$ \\
\hline & 11. & 8.5 & 18. & 17.6 & 4.1 & 20.0 & $18 \cdot 8$ & \\
\hline & 10. & 9. & & 19.1 & $5 \cdot 0$ & 18 & 19.0 & .6 \\
\hline & 6.8 & 10.0 & 17 & 16.7 & $.5 \cdot 3$ & 17.5 & 16.5 & $0^{\circ}$ \\
\hline 36.5 & 10.2 & 11.0 & 13 & 21.0 & 6.3 & 17.0 & 20.2 & \\
\hline 7.76 & $9 \cdot 4$ & 12.0 & $8 \cdot 4$ & * & 7.5 & 15.9 & 20.7 & \\
\hline
\end{tabular}

NUCLIDE REACTION RES EXCIT SOURCE DETECTOR

REF $Z$ A IN,OUT

$64 \mathrm{CO}_{3} 32 \mathrm{GE} \quad \mathrm{GN}$ ABI THR- $80 \mathrm{C} 10-80 \mathrm{BF} 3-\mathrm{I}$ 4PI

$64 \mathrm{CO} 232 \mathrm{GE} \quad \mathrm{G}, X N$ ABY THR-

6BKR2 32GE G,A ABX THR- 33 C 33 SCD-D 90

$70 M 0432 G E 70 \quad G, G \quad$ LFT $6 \quad$ D $6 \begin{array}{r}\text { SCD-D DST } \\ 6.018, J-P I, L F T\end{array}$

$55801326 E 70 \quad G, N$ ABX 12- $21612-21$ ACT-1 $4 P I$

$550 E I 32 G E 70$ GN AGX $9-21$ C $9-21$ ACT-1 4 PI

$60 F E I 32 G E 70$ GN ABX 11- 31 C 31 ACT I 4PI

GOFEI $32 G E 70$ GN AB $18-31$ C 31 ACT-I 4 PI

56MEI 32 GE72 G.G LFT 1 D 1 NAI-D DST

58T01 32GE73 G,N RLY 7-22C22 BF3-1 4PI

56MEI $32 G E 74$ G,G LFT 1 D 1 NAI-D DST

70110232 GE 74 G,G ABX 6 D 6 SCD-D DST

$6=6.018$, LFT

ABY THR- $20 \mathrm{C} 20.018,7.632 \mathrm{MEV}$

$32 \mathrm{GE} 76$ G,N ABX 9- $21 \mathrm{C} 9-21 \mathrm{ACT}-14 \mathrm{PI}$

$550 E 1$ 32GEl6 G,N ABX 9- $21 C$ 9- 21 ACT-1 $4 P I$

$680 K 2325 E 76$ ISOMERIC RATIO

ISOMERIC Y $A$ IEI

ARSENIC $Z=33$

\begin{tabular}{|c|c|c|c|c|c|c|c|c|c|}
\hline \multirow{3}{*}{$\begin{array}{l}\text { A } \\
75\end{array}$} & \multirow{3}{*}{$\begin{array}{l}\text { ABUND. } \\
100.00\end{array}$} & \multicolumn{8}{|c|}{ SEPARATION ENERGIES (MEV) } \\
\hline & & $G, N$ & \multicolumn{2}{|c|}{ G, T } & $\mathrm{G}, \mathrm{HE} 3$ & \multirow{2}{*}{$\begin{array}{ll}3, A \\
5.3\end{array}$} & \multirow{2}{*}{$\begin{array}{l}\mathrm{G}, 2 \mathrm{~N} \\
18.2\end{array}$} & \multirow{2}{*}{$\begin{array}{l}G, N P \\
17.1\end{array}$} & \multirow{2}{*}{$\begin{array}{l}G, 2 P \\
17.9\end{array}$} \\
\hline & & 10.2 & & 5.4 & $1 \%$ & & & & \\
\hline & NUCL $1 D E$ & REACTION & RES & $E \times C 1$ & & SOURCE & DETECI & $T O R$ & \\
\hline$E F$ & A & IN,OUT & & & & & TYPE & ANG & NU:M \\
\hline GAM2 & 33 AS75 & $E, E / P$ & SPC & $105-3$ & 365 & D560-880 & $M A G-D$ & $\begin{array}{r}62 \\
5 \\
51\end{array}$ & \\
\hline 62ME2 2 & $33 A S 75$ & $G, G$ & LFT & 1 & & $D^{A} 1$ & NAI - 0 & 0 & \\
\hline $635 \cup 1$ & $33 A \$ 75$ & $G, G$ & $A B X$ & 4- & 14 & $4-14$ & NAI $-D$ & 120 & \\
\hline $64 \mathrm{SHS}$ & 334575 & $G, E$ & LFT & 1 & & D & NA! -D & 122 & \\
\hline 67LAL & 33 AS 75 & $G, G$ & LF T & 1 & & D & NAI I D & DST & \\
\hline & & & & & & $l=.265$ & $5 . .280$ & MEV & \\
\hline $8 A L I$ & $33 A 575$ & $G, G$ & LFT & $0-$ & 1 & $\mathrm{c}$ & SCD -D & OST & \\
\hline & & & & & & $0.86,1.07$ & $7 \cdot 1.35$ & MEV & \\
\hline $69 \mathrm{inO} 3$ & 33 AS 75 & $G, G$ & $A B X$ & B & & ${ }_{J-P I, G-W I} 9$ & $\begin{array}{l}\text { SCD-D } \\
\text { IDTH.7. }\end{array}$ & $\begin{array}{l}\text { DST } \\
.646\end{array}$ & \\
\hline 7OAR 1 & $33 A S 75$ & $G, E$ & $A B X$ & $12-$ & 30 & C 32 GETS & $\begin{array}{l}\text { NAI I D } \\
G, G / T C\end{array}$ & $\begin{array}{l}\text { DST } \\
0 \quad 2+\end{array}$ & \\
\hline 70 MO2 & $33 A 575$ & $G, G$ & $A B X$ & 8 & & D $B$ & $S C D-D$ & DST & \\
\hline & & & & & & & $=7.646$, &, LFT & \\
\hline 6SU1 & 33 AS75 & $G, N$ & RLY & THR -3 & 320 & $C_{140}, 320$ & $A C T-1$ & $4 \mathrm{PI}$ & \\
\hline $57 F E Z$ & 33 AS 75 & $\mathrm{G}, \mathrm{N}$ & $A B Y$ & $14-$ & 30 & C $14-30$ & THR- 1 & $4 \mathrm{PI}$ & \\
\hline $58 \mathrm{CH} 2$ & $33 A 575$ & $G, N$ & $R L Y$ & THR & & CTHR & $\begin{array}{l}\text { BF 3-1 } \\
\text { THRE SH }\end{array}$ & 4PI & \\
\hline $60 \mathrm{GE} 3$ & $33 A S 75$ & $G, N$ & NOX & THR & & CTHR & $\begin{array}{l}\text { BF } 3-1 \\
\text { THRES }\end{array}$ & HPI & \\
\hline 61 TAI & $33 A 575$ & $G, N$ & NOX & $7-$ & 22 & C 22 & THR-I & DST & \\
\hline $64 C 03$ & $33 A S 75$ & $G, N$ & $A B I$ & THR - & 80 & C $10-80$ & $B F 3-1$ & $4 \mathrm{PI}$ & \\
\hline $67 \mathrm{HUI}$ & $33 A 575$ & $G, N$ & $A 8 x$ & 11 & & D 11 & $B F 3-1$ & 4PI & \\
\hline $71 S A 1$ & $33 A 575$ & $G, N$ & $A 8 Y$ & $10-$ & 68 & C $10-68$ & $A C T-I$ & $4 \mathrm{PI}$ & \\
\hline $698 E 1$ & $33 A 575$ & $\mathrm{G}, 2 \mathrm{~N}$ & $A B X$ & THR - & 30 & D $18-30$ & $8 F_{3}-1$ & $4 \mathrm{PI}$ & \\
\hline $565 \cup 1$ & $33 A S 75$ & $G, 3 N$ & RLY & THR -3 & 320 & $C_{140,320}$ & $A C T-I$ & $4 \mathrm{PI}$ & \\
\hline $5 B \times A 1$ & $33 A 575$ & $G, \times N$ & $A B X$ & $10-$ & 22 & C $10-22$ & $B F 3-1$ & $4 \mathrm{PI}$ & \\
\hline $618 A 2$ & 334575 & $G, X N$ & $A B Y$ & THR- & 22 & C 22 & THR - I & DST & \\
\hline $64 \mathrm{CO} 2$ & $33 A 575$ & $G, \times N$ & $A B Y$ & THR - & Bo & 680 & BF $3-1$ & $4 \mathrm{PI}$ & \\
\hline $65 F[1]$ & $33 A 575$ & $G, \times N$ & $A B X$ & $10-$ & 25 & C $10-25$ & $8 \mathrm{~F} 3-1$ & $4 \mathrm{PI}$ & \\
\hline $698 \mathrm{EI}$ & $33 A 575$ & $\mathrm{G}, \times \mathrm{N}$ & $A B X$ & THR- & 30 & $010-30$ & $B F 3-1$ & 4PI & 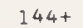 \\
\hline $65 U 1$ & $33 A S 75$ & $G, 2 P$ & RLY & THR - 3 & 320 & $C 140,320$ & $A C T-I$ & $4 \mathrm{PI}$ & \\
\hline
\end{tabular}

$565 U 1$ 33A575 G,N2P RLY THR-320 C1 140,320 ACT-1 4 PI $565 \mathrm{UI} 33 \mathrm{AS7}$ $565 U 1334575$

$565 U 1$ 33AS75

56SU1 $33 A 575$

56SU1 33AS75

56 SUI 33AS75

56SUI $33 A 575$

33AS75

56SUI 33AS75

$565 U 1$ 33AS75 RLY THR-320 C14U,320 ACT-I 4P , 3N4P RLY THR-320 C 140.320 ACT-1 4PI $G, 4 N 5 P$ RLY THR $-320 \quad$ C 140,320 ACT-I 4P: G,5N2P RLY THR-320 C140,320 ACT-I 4PI G,5N5P RLY THR-320 C140,320 ACT-1 4PI G.7N2P RLY THR-320 C140,320 ACT-1 4P 1 G,7N4P RLY THR-320 C140,320 ACT-1 4P G, BNGP RLY THR-320 C140,320 ACT-1 4PI G,CU61 RLY IHR-320 C140,320 ACT-I 4P G,CU65 RLY THR $320 \mathrm{C} 140,320$ ACT $114 \mathrm{PI}$ G. ,SPL A8Y THR-939 C900 ACT-1 $4 \mathrm{PI}$

SELENIUM $Z=34$ SEPARATION ENERGIES (MEV)
G,N G,P G,T G,HE3 G,A G, LN G,NP G, $2 P$

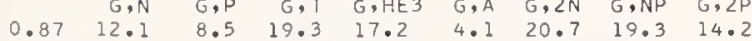

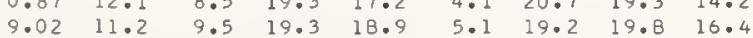
$\begin{array}{lllllllll}7.58 & 7.4 & 9.6 & 18.7 & 16.1 & 5.7 & 18.6 & 16.9 & 17.3\end{array}$

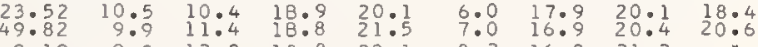
$\begin{array}{llllllll}9.19 & 9.3 & 12.3 & 18.8 & 23.1 & 8.2 & 16.0 & 21.2\end{array}$

NUCLIDE REACTION RES EXCIT SOURCE DETECTOR

REF $Z$ A IN,OUT TYPE ANG NUM

668E3 34SE G.G RLX 5- 10 D 5- 10 NAI-D 135

TOARI $34 \mathrm{SE} \quad G, G \quad A B X \quad 12-30 \mathrm{C} 32$ NAI-D DST

63KAZ 34SE RATIO G,G/TO $2+$

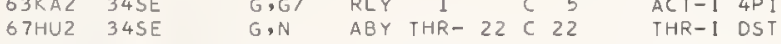

$67 \mathrm{CO} 34 \mathrm{SE} \quad \mathrm{G}, X N$ ABX THR- $24 \mathrm{C} 11-24$ BF3-I $4 \mathrm{PI} 43$

$71 \mathrm{CO} 234 \mathrm{SE} \quad \mathrm{G}, X N$ ABI $36-64 \mathrm{C} 10-64 \mathrm{BF} 3-14 \mathrm{PI} 437$

66WE2 34SE G,A ABX 6- 33 C 33 FAST N Y SEDLD 90

$\begin{array}{llllllll}\text { SODE2 } & 34 \text { SE76 } & \text { G,G } & \text { LFT } & 1 & \text { D } & 1 & \text { NAI-D UKN } \\ \text { G3PR2 } & 34 \text { SE76 } & \text { G.G } & \text { LFT } & 1 & \text { D } & 1 & \text { NAI-O DST }\end{array}$

$69803 \quad 345 E 77$ G, G ABX O- 2 C O- $2 A C T-14 P I$

$63 V E 2345 E 77$ G.G/ ABX $0-1$ D 1 ACT-I $4 P I$

6BOK3 34 SE 77 G,P ABY THR- 20620 ACT-I 4PI

$680 K 2345 E 7 B$ G,N ABY THR- 20 C 20 ACT-I 4PI

$58 H O 1$ 34SEBO G,NP RLY 20-32 C 32 ACT-I 4P:

$56 S I 234$ SE82 G.N ABX 9- 22 C 9- 22 ACT-1 4PI

BROMINE $Z=35$

A ABUND. SEPARATION ENERGIES (MEV)

G.N G.P G,T G.HES G,A G.2N G,NP G,2P

$\begin{array}{llllllllll}79 & 50.54 & 10.7 & 6.3 & 15.8 & 13.7 & 5.5 & 19.0 & 16.8 & 16.7\end{array}$

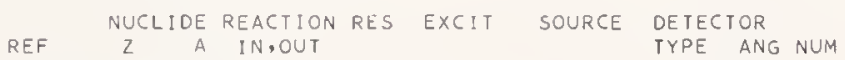

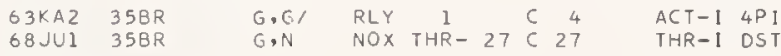

66LAI 35BR79 G,G LFT O- I D O- I NAI-D 123

$1=.834 \mathrm{MEV}$

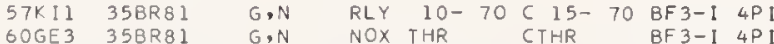

ISOMERIC RATIO

$57 E R I$ 35BR8I G,A ABY THR- 32 C 32 ACT-I 4PI

6BOKI 35BRBI GAA A8I THR- 20 C 20 ACT-I 4PI 


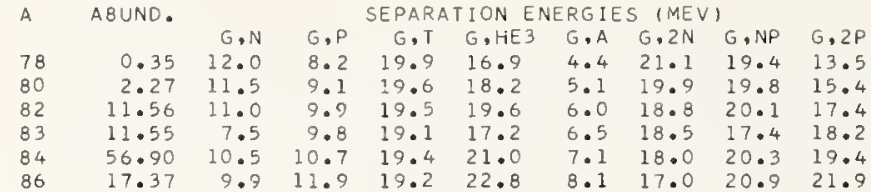

$\begin{array}{lllll} & \text { NUCLIDE REACTION RLS EXCIT SOURCE } & \text { DETECTDR } \\ \text { REF } Z \text { A IN,OUT } & & & \text { TYPE ANG NUM }\end{array}$

69HDI 36KR G.XP A8Y THR- 33 C 24- 33 SCI-D 90

66BE2 36KR82 G,G LFT 1 C I NAI-D 113

69HDI 36KR84 G.XP ABY THR- 33 C 24- 33 SCI-D 90

RU8IDIUM $\quad Z=37$

\begin{tabular}{lrrrrrrrrr} 
A & A8UND. & \multicolumn{8}{c}{ SEPARATION ENERGIES (MEV) } \\
& & G.N & G.P & G, T & G,HE3 & G.A & G.2N & G,NP & G.2P \\
85 & 72.15 & 10.5 & 7.0 & 16.5 & 19.5 & 6.6 & 19.4 & 17.5 & 17.7 \\
87 & 27.85 & 9.9 & 8.6 & 17.1 & 21.8 & 8.0 & 18.6 & 18.5 & 20.5
\end{tabular}

NUCLIDE REACTIDN RES EXCIT SDURCE DETECTOR

REF 2 A IN,OUT RES TYPE ANG NUM

71LEI $37 R 8$ G,N ABX 11- 24 D 11- 24 MOD-I 4 PI 375

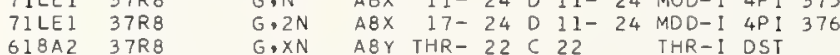

$58 T 0137 R 885$ G.N RLY 10- $11 C 22$ ACT-I 4PI

60GE3 $37 R 885$ G,N NDX THR GTHR 8F3-I 4PI

RLY THRESHDLD

SOMER YIELD

$58 T 0137 R 887$ G.N RLY 9- $11 C 22$ 8F3-I 4PI

57ERI $37 R 887$ GA ABI $8-32$ C 32 ACT-I 4PI

STRONTIUM $Z=38$

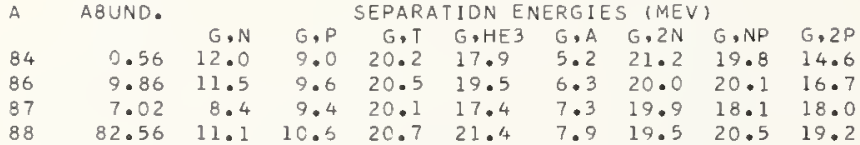

NUCLIDE REACTIDN RES EXCIT SDURCE DETECTOR

63KA2 385R G,G/ RLY I C 6 ACT-I $4 P I$

TILEI 38SR G,N ABX II- 27 D $11-27$ MOD-I 4PI 378

$71 \mathrm{LEI}$ 38SR $\quad$ G.2N $A B X \quad 19-27 \mathrm{D} 11-27 \mathrm{MDD}-\mathrm{I}$ 4PI 379

618A2 $38 \mathrm{SR}$

G.2CAI $385 R 86$

G.XN ABY THR- $22 \quad 622$ THR-I DST

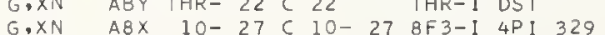

SGYE2 $385 R 86$

$G, N$ NDX $12-30 \subset 30$ ACT-I 4 PI

G3VE2 38SR87

$56 Y E 2 \quad 385 R 87$

$68 D K 3 \quad 385 R 87$

$56 \mathrm{HE} 3 \quad 38 \mathrm{SR} 88$

SBPEI $38 \mathrm{SR} 88$

$695 H 5 \quad 38 S R 88$

GSSH6 38 SRB

S48E 7 38SR8

$56 Y E 2 \quad 385 R 88$

$718 L 1 \quad 385 R 88$

$G, \times N$

A $8 \times \quad 11-23$ C 24

SOMER RATID
8 F $3-1 \quad 4 P$ I

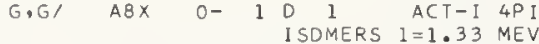

$G, X N$ A8X 9- 23624 8F3-I 4PI

$G, P$ ABY THR- $20 C 20$ ACT-I 4 PI

E,E/ FMF 1- 70187 MAG-D DST

$E, E /$ RLY 1- 7 D 65, 70 MAG-D DST
BiEL), 4 LEVELS

E,P A8X 14- $25016-30$ MAG-D UKN

$\begin{array}{llrrrrr}E, P & \text { SPC } & 14- & 30 & D & 30 & \text { MAG-D UKN } \\ \text { G,G } & \text { LFT } & 2 & & D & 2 & \text { UKN-D UKN }\end{array}$

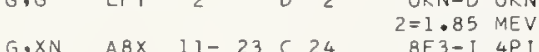

GPI+ ABY $150-700$ C $150-700$ ACT-I 4 PI

G9HAI $38 \mathrm{SR} 88$
$P, G \quad R L X \quad 15-22$ D 4- 12 NAI-D $90207+$
A ABUND. G,N G,P GEPARATION ENERGIES (MEV)

$\begin{array}{llllllllll}89 & 100.00 & 11.5 & 7.1 & 18.1 & 19.9 & 8.0 & 20.3 & 18.2 & 17.7\end{array}$

REF NUCLIDE REACTION RES EXCIT SDURCE DETECTOR

$675 H 139 Y 89$ E,E/ FMF 2- 30225 MAG-D DST

$68 P E 139 Y 89$ E,E/ RLY 1- 3065, 70 MAG-D MEV

5 LEVELS

$71 M O 3$ 39Y 89 E,E/ A8X O-240 D500 MAG-D 60

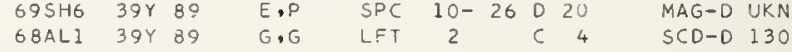

7OARI 39Y 89 G,G A8X 12- $30<32 \quad \begin{aligned} & 2=1.51 \\ & \text { NAI-D DST }\end{aligned}$

$585 I 1$ 39Y 89 G,G/ ABY 2- 21 RATID G,G/TO 2+

63KA2 $39 Y 89$ G.G/ RLY 1 C 5 ACT-I 4PI

$63 V 52$ 39Y 89 G.G/ ABX O- 1 D 1 ACT- $4 P$

RLY THR ISOMERS $1=1.33$ MEV

GOGE3 $39 Y 89$ GN NOX THR CTHR THRESHDLD

62REI 39Y 89 G,N NOX 6- $55 C 55$ THRESHDLD

G3GEI 39Y 39 G,N RLY $11-12$ C $11-12$ SF $3-I$ I 4 PI

$678 E 2$ 39Y 89 . G,N ABX THR- 28 DTHR- 28 THRE SHOLD

$698 E 4$ 39Y 89 G,N ABX 11- 25 D II- 26 MOD-I 4PI 188

$71 \mathrm{LEI} 39 \mathrm{Y} 89$ G,N A8X 11- 27 D $11-27$ MDD-I 4PI 381

$715 A 1$ 39Y $89 \quad G, N \quad$ ABY $11-68 C 10-68$ ACT-I $4 P I$

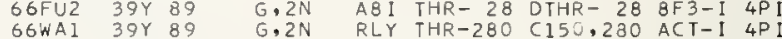

ISOMER RATIO Y 87

$678 E 2$ 39Y 89 G.2N A8X THR- 28 DTHR- 28 BF 3-I 4PI $1+$

$698 E 4$ 39Y $89 \quad \mathrm{G}, 2 \mathrm{~N}$ ABX $21-27 \mathrm{D} 19-28 \mathrm{MDO}-1$ 4PI 189

7OWA3 39Y 89 G,2N RLY THR-305 C150-305 ACT-I 4PI

71LEI 39Y B9 G, 2N ABX 21- 27 D 11- 27 MDD-I 4PI 382

56YE2 39Y 89 G.XN A8X 12- 23 C $24 \quad 8 F 3-I$ 4PI

58XAI 39Y 89 G,XN A8X 12- 22 C 12- 22 8F3-1 4PI

618A2 39Y 89 G,XN A8Y THR- 22 C 22 THR-1 DST

66 FU2 39Y 89 G,XN A8I THR- 28 DTHR- 28 8F3-I 4P

$695 H_{4} 39 Y 89$ G,P A8X 7- 24 C 17- 24 EMU-D DS

67TA2 39Y 89 G, XP SPC THR- 24 C 17,24 EMU-D 4P

TOWA3 $39 Y 89$ G,PN RLY THR-305 C150-305 ACT-I 4PI

68SHI 39Y 89 P, G A8X 13- 15 D 5- 9 NAI-D DST

69RIL 39Y 89 P, G ABX 12 D 5 SCD-D 90

71 UMI 39Y 89 P.G A8X 10- $110 \quad 2-3$ NAI-D 0

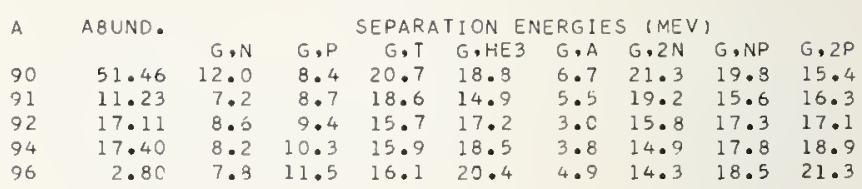

NUCLIDE REACTIDN RES EXCIT SDURCE DETECTDR

GOREI $40 Z R \quad$ G.G ABX 7 A $\quad$ D 7 NAI-D 90

66BE3 40ZR G.G RLX 5- 10 D 5- 10 NAI-D 135

$70 A X 1$ 40ZR G.C ABX 8- 13 D 8- 13 NAI-D 22

S7HU2 40ZR G.N A8Y THR- $22 \quad$ THR-I DST

G4DUI $40 Z R \quad$ G,P $\quad A 8 \times$ THR- 34 C 22- 34 MAG-D DST

650U1 40ZR G,P A8X 18- 26 D 17- 24 EMU-D DST

$695 H 4$ 4OZR G,P ABX $8-24$ C 20, 24 EMU-D DST

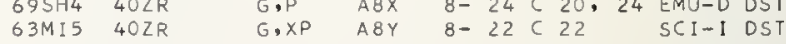

$6980240 Z R 90$ E,E/ FMF 2- 4 D 58 MAG-D DST

$708 E 240 Z R 90$ E,E/ A8X O- 4 D $42-18$ TD 3.84 MEV

$68 S H 4 \quad 402 R 9 O \quad E, P$ SPC $11-20 \quad 020^{2.18,2.74 .3 .84}$

$695 H 5407 R 90$ ANALDG STATES

$69 S H 6$ 40ZR9O E,P SPC 12- 22 D 16- 23 MAG-D DST

$63 A X 1$ 40ZR9O G'G A8X 8- 13 D 8- 13 NAI-D 135

G9RAI 40ZR9O G.G LFT 9 D 9 NAI-D DST

$56 \mathrm{AXI} 40 Z R 90 \quad G, N \quad R L X \quad 12-23 \mathrm{C} 12-23$ ACT-I 4 PI

$59 M U 240 Z R 90$ G.N RLX 12-24C 12-25 ACT $-14 P$

62CAI 40ZR9O G,N NOX 12- $30 \mathrm{C} 30$ ACT-I 4PI

$65 \mathrm{CD} 1402 \mathrm{R} 90$ GN ABX THR- $70 \mathrm{C}$ ISDMERIC RATID

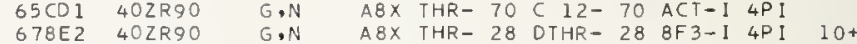

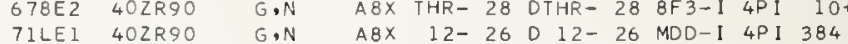

$\begin{array}{llllllll}71 L E I & 402 R 90 & G, N & A 8 X & 12-26 & \text { D } 12-26 & \text { MDD-I } & 4 P I \\ \text { G6FU2 } & 40 Z R 90 & \text { G, } 2 N & \text { ABI THR- } 28 & \text { DTHR- } 28 & 8 \mathrm{~F} 3-\mathrm{I} & 4 \mathrm{PI}\end{array}$

$67 B E 2$ 402R90 G.2N A8X THR- 28 DTHR- 28 BF3-1 4 PI 10

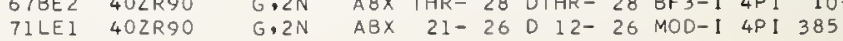




\begin{tabular}{|c|c|c|c|c|c|c|c|c|c|c|}
\hline 6 YYEZ & $40 Z R 90$ & $G, X N$ & $A B X$ & $12-$ & 23 & C 24 & & $B F 3-1$ & $4 \mathrm{PI}$ & \\
\hline 59NAI & $4 \cup Z R 90$ & $G, \times N$ & $A B X$ & $12-$ & 24 & $5-$ & 24 & $\begin{array}{l}\text { BF } 3-1 \\
\text { THRE SH }\end{array}$ & $\begin{array}{l}\text { 4PI } \\
\text { HOLO }\end{array}$ & \\
\hline $66 \mathrm{FU2}$ & $40 Z R 90$ & $G, \times N$ & $A B I$ & THR- & 28 & OTHR - & 28 & $B F 3-1$ & $4 P I$ & \\
\hline 69AN7 & $40 Z R 90$ & $G, \times N$ & $A B X$ & $12-$ & 23 & C $12-$ & 23 & BF 3-I & 4P I & \\
\hline 71152 & 4OZR9O & $G, \times N$ & $A B X$ & $12-$ & 28 & C $12-$ & 28 & $B F 3-1$ & 4P I & 413 \\
\hline $64 B L 2$ & $4 \cup Z R 90$ & $P, G$ & $R L X$ & $13-$ & 17 & 5- & NALC & $\begin{array}{l}\text { NAI I D } \\
\text { DGUE T }\end{array}$ & $\begin{array}{l}\text { DST } \\
=6\end{array}$ & \\
\hline 66081 & $40 Z R 90$ & $P, G$ & $R L x$ & $11-$ & 14 & $2-$ & 6 & NA I - D & 90 & \\
\hline $67 \mathrm{~A} \times 1$ & 40ZR90 & $P, G$ & $A B X$ & $14-$ & 25 & $5-$ & 17 & NAI -D & 90 & \\
\hline 67081 & $40 Z R 90$ & $P, G$ & $A B X$ & $12-$ & 13 & 4- & 5 & NAI $=0$ & 0 & \\
\hline $69 \mathrm{HAl}$ & 4OZR90 & $P, G$ & $R L X$ & $16-$ & 22 & 7- & 14 & NAI -D & UST & \\
\hline 69MA4 & $402 R 90$ & $P, G$ & $A B K$ & $10-$ & 27 & $2-$ & 19 & $N A I-D$ & DST & \\
\hline $71 \cup M 1$ & $40 Z R 90$ & $P, G$ & $A B \times$ & 9- & 10 & $2-$ & 3 & $S C D-D$ & 0 & \\
\hline $678 E 2$ & $40 Z R 91$ & $G, N$ & $A B X$ & THR- & 30 & UTHR- & 30 & BF 3-I & 4P I & \\
\hline $66 \mathrm{FU2}$ & $402 R 91$ & $G, 2 N$ & $A B !$ & THR- & 30 & DTHR- & 30 & BF 3-I & $4 \mathrm{PI}$ & \\
\hline $67 B E 2$ & $40 Z R 91$ & $G, 2 N$ & $A B \times$ & THR- & 30 & DTHR - & 30 & $B F 3-1$ & 4P I & \\
\hline $55 \mathrm{NA} 1$ & $402 R 91$ & $G, X N$ & $A B X$ & 7- & 24 & $5-$ & 24 & $\begin{array}{l}\text { SF } 3-\mathbf{I} \\
\text { THRE SH }\end{array}$ & $\begin{array}{l}4 \mathrm{PI} \\
40 \mathrm{LD}\end{array}$ & \\
\hline S6YE2 & $4 \Delta Z R 91$ & $G, X N$ & $A B x$ & $7-$ & 23 & C 24 & & $B F 3-1$ & $4 P I$ & \\
\hline $56 \mathrm{FU} 2$ & $402 R 91$ & $G, X N$ & $A B I$ & THR - & $3 \mathrm{C}$ & UTtR- & 30 & $B F 3-1$ & $4 \mathrm{PI}$ & \\
\hline $69 \mathrm{AN}^{2}$ & $402 R 91$ & $G, \times N$ & $A B X$ & $12-$ & 23 & $612-$ & 23 & $B F 3-1$ & $4 \mathrm{Pl}_{1}$ & \\
\hline $57 B E 2$ & $402 R 92$ & $G, N$ & $A B X$ & THR- & 28 & DTHR- & 28 & BF 3-I & 4P1 & $10+$ \\
\hline $36 F \cup 2$ & $402 R 92$ & $\mathrm{G}, 2 \mathrm{~N}$ & $A B$ I & THR - & 28 & DTHR & 28 & $B F 3-I$ & 4P 1 & \\
\hline $67 B E 2$ & $402 R 92$ & $G, 2 N$ & $A B X$ & THR- & 28 & DTHR - & 28 & BF 3-I & 4P1 & $10+$ \\
\hline 56YE 2 & $40 Z R 92$ & $G, \times N$ & $A B X$ & $9-$ & 23 & C 24 & & 6F 3-I & $4 P 1$ & \\
\hline $66 \mathrm{FU} 2$ & $402 R 92$ & $G, X N$ & $A B I$ & THR - & 23 & DTHR & 28 & BF 3-I & 4P 1 & \\
\hline 67 UE 2 & $4 \cup Z R 94$ & $G, N$ & $A B X$ & THR- & 30 & DTHR- & 30 & $B F 3-I$ & $4 \mathrm{PI}$ & \\
\hline $6 \mathrm{FU}_{2}$ & $402 R 94$ & $G, 2 N$ & $A 81$ & THR- & 30 & DTHR & 30 & $B F 3-I$ & 4P I & \\
\hline $57 B E 2$ & $4 \cup 2 R 94$ & $\mathrm{G}, 2 \mathrm{~N}$ & $A B X$ & THR- & 30 & DTHR - & 30 & BF 3-I & 4P1 & $12+$ \\
\hline $66 \mathrm{~F} \cup 2$ & $4 \cup Z R 94$ & $G, \times N$ & $A B I$ & THR- & 30 & UTHR & 30 & BF 3-I & 4PI & \\
\hline
\end{tabular}

NIOBIUM $Z=4]$

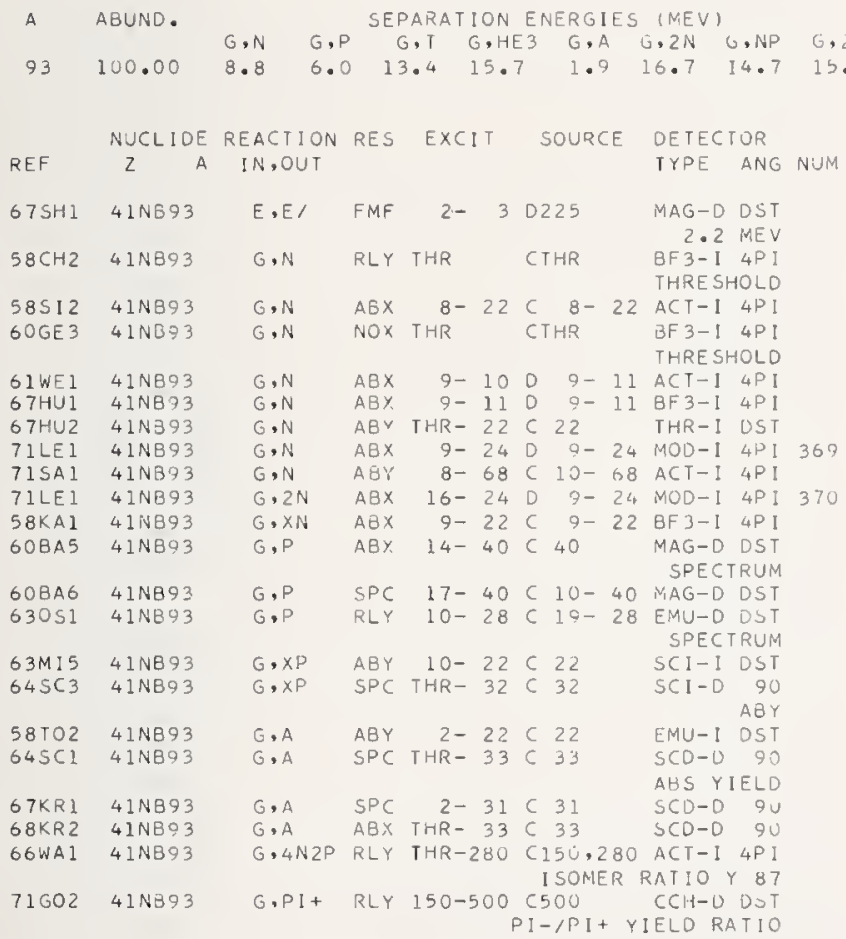

\begin{tabular}{|c|c|c|c|c|c|c|c|c|c|}
\hline & BUNO. & & & $=P A R$ & ON & RG I & IME & & \\
\hline & & $G, N$ & $G, P$ & $G, T$ & $\mathrm{G}, \mathrm{HE} 3$ & $G, A$ & $G, 2 \mathrm{~N}$ & $G, N P$ & $G, 2 P$ \\
\hline 92 & 15.84 & 12. & 7. & 20. & $16.0^{\circ}$ & 5.6 & 22.8 & 19.5 & 12.6 \\
\hline 94 & 9.0 & 9.7 & 8.5 & 16. & 15. & 2. & 17.7 & 17.3 & 14. \\
\hline 95 & 15.72 & & & & 14 & & & & \\
\hline 96 & 16. & 9 & $9 \cdot 3$ & 16 & & & & 17.8 & 16. \\
\hline 97 & 9.46 & 6. & 9.2 & 16 & 15 & 2.8 & & 16.1 & 16.5 \\
\hline 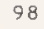 & 23.7 & 8. & 9.8 & 16 & 1 & 3 & & 17.9 & 17. \\
\hline & & 8. & & & & & & 18.0 & \\
\hline
\end{tabular}

\begin{tabular}{|c|c|c|c|c|c|c|c|c|}
\hline & NUCLIDE & REACTION & RES & EXCIT & SOURCE & OETECT & TOR & \\
\hline REF & A & IN,OUT & & & & TYPE & ANG & NUM: \\
\hline GOREI & $42 \mathrm{MO}$ & $G, G$ & $A B X$ & 7 & 0 & NAI I D & 90 & \\
\hline $66 B E 3$ & $42 \mathrm{MO}$ & $G, G$ & $R L X$ & $5-10$ & $5-10$ & $N A !-D$ & 135 & \\
\hline $67 \mathrm{HU2}$ & $42 \mathrm{MO}$ & $G, N$ & $A B Y$ & THR- 22 & C 22 & THR - I & DST & \\
\hline 68 JU1 & $42 M O$ & $G, N$ & NOX & THR- 22 & C 22 & THR-1 & DST & \\
\hline $71 C 02$ & $42 \mathrm{MO}$ & $G, X N$ & $A B I$ & $36-64$ & $\begin{aligned} 610-64 \\
\text { FAS }\end{aligned}$ & $\begin{array}{c}\text { BF 3-1 } \\
\text { ST N YI }\end{array}$ & $\begin{array}{l}4 P I \\
E L D\end{array}$ & \\
\hline 55101 & $42 \mathrm{MO}$ & $G, P$ & RLY & THR- 65 & C 65 & $S C I-D$ & OST & \\
\hline 63MI5 & $42 \mathrm{MO}$ & $G, X P$ & $A B Y$ & $10-22$ & C 22 & $S C I-I$ & DST & \\
\hline 69SH6 & $42 M 092$ & $E, P$ & SPC & $11-18$ & D 20 & MAG-D & UKN & \\
\hline $59 \mathrm{MU2}$ & $42 M 092$ & $G, N$ & $A B X$ & $13-24$ & c $0-25$ & $A C T-I$ & $4 \mathrm{PI}$ & \\
\hline $62 C A 1$ & $42 M 092$ & $G, N$ & NOX & $7-30$ & C 30 ISOME & $\begin{array}{l}\text { ACT-1 } \\
\text { ERIC RA }\end{array}$ & $\begin{array}{l}4 \mathrm{PI} \\
\mathrm{AT} 10\end{array}$ & \\
\hline 62DE I & $42 M O 92$ & $G, N$ & $A B X$ & 21 & D 21 & $A C T-1$ & 4PI & \\
\hline 64GE 1 & $42 M 092$ & $G, N$ & $A B X$ & THR -22 & CTHR- 33 & $A C T-1$ & $4 \mathrm{PI}$ & \\
\hline $65 \mathrm{COl}$ & $42 M 092$ & $G, N$ & $R L X$ & THR - 70 & C $12-70$ & $A C T-1$ & $4 \mathrm{PI}$ & \\
\hline 68GE 1 & $42 M 092$ & $G, N$ & $A B X$ & $12-26$ & CTHR- 26 & $A C T-1$ & $4 \mathrm{PI}$ & \\
\hline $680 K 2$ & $42 M 092$ & $\mathrm{G}, \mathrm{N}$ & $A B Y$ & THR- 20 & C $2 \mathrm{C}_{\text {I SOME }}$ & tACT- ${ }_{t} R I C Y I$ & $\begin{array}{l}4 P 1 \\
E E L D\end{array}$ & \\
\hline T1HA2 & $42 M 092$ & $G, N$ & $R L Y$ & $13-70$ & C $70 \quad 150$ & $\begin{array}{l}\text { ACT - I } \\
\text { OMER RA }\end{array}$ & $\begin{array}{l}4 P I \\
\text { TT } 10\end{array}$ & \\
\hline 64GE 1 & $42 M 092$ & $\mathrm{G}, 2 \mathrm{~N}$ & $A B X$ & THR- 33 & $\begin{array}{r}\text { CTHR }-33 \\
\text { MIXED }\end{array}$ & $\begin{array}{l}A C T-1 \\
W I T H G\end{array}$ & $\begin{array}{l}4 \mathrm{PI} \\
, \mathrm{NP}\end{array}$ & \\
\hline 70WA3 & $42 M 092$ & $\mathrm{G}, 2 \mathrm{~N}$ & $R L Y$ & THR-305 & $C 150-305$ & $A C T-1$ & 4P I & \\
\hline 70151 & $42 M 092$ & $G, X N$ & $A B X$ & THR- 30 & CTHR- 30 & $B F 3-1$ & $4 \mathrm{PI}$ & 429 \\
\hline 57ELI & $42 M 092$ & $G, P$ & RLY & THR- 32 & c 32 & $A C T-I$ & 4PI & \\
\hline $57 E L 1$ & $42 M 092$ & $G, N P$ & RLY & $T H R-32$ & C 32 & $A C T-I$ & 4P! & \\
\hline $58 \mathrm{HO}$ & $42 M 092$ & $G, N P$ & $R L Y$ & $19-32$ & C 32 & $\begin{array}{l}\text { ACT - } 1 \\
\text { THRE SH }\end{array}$ & $\begin{array}{l}4 P ! \\
O L O\end{array}$ & \\
\hline 64GE 1 & $42 M 092$ & $G, N P$ & $A B X$ & THR- 33 & $\begin{array}{r}\text { CTHR- } 33 \\
\text { MIXED }\end{array}$ & $\begin{array}{l}A C T-1 \\
\text { WITHG }\end{array}$ & $\begin{array}{l}4 P I \\
6,2 N\end{array}$ & \\
\hline 7OWA 3 & $42 M 092$ & $G, P N$ & RLY & THR-305 & $C_{1} 50-305$ & $A C T-I$ & 4PI & \\
\hline $71 \mathrm{HA2}$ & $42 M 094$ & $G, 3 N$ & RLY & $31-70$ & I so & $\begin{array}{l}A C T-1 \\
\text { OMER RA }\end{array}$ & $\begin{array}{l}\text { 4PI } \\
\text { ATIO }\end{array}$ & \\
\hline $67 L A 3$ & $42 M 095$ & $G, G$ & LFT & 1 & $1=$ & $\begin{aligned} & \text { NA1 } 1-0 \\
= & 0.766\end{aligned}$ & $\begin{array}{l}\text { DST } \\
\text { MEV }\end{array}$ & \\
\hline $698 E 7$ & $42 M 096$ & $G, G$ & LFT & 6 & $\begin{array}{l}\text { D } 6 \\
6.41,\end{array}$ & $\begin{array}{r}0 \\
.6 .44\end{array}$ & $\begin{array}{l}\text { DST } \\
\text { MEV }\end{array}$ & \\
\hline 69 AN6 & $42 M 096$ & $G \cdot P$ & $A B Y$ & $106-999$ & $\begin{array}{r}(700,999 \\
99\end{array}$ & $\begin{array}{l}\text { TEL-C } \\
99=1.2\end{array}$ & $\begin{array}{l}\text { OST } \\
\text { GEV }\end{array}$ & \\
\hline 69AN6 & $42 M 096$ & $G, D$ & $A B Y$ & $113-999$ & $\begin{array}{r}6700,999 \\
99\end{array}$ & $\begin{array}{l}\text { TEL }-0 \\
99=1.2\end{array}$ & $\begin{array}{l}\text { DST } \\
\text { GEV }\end{array}$ & \\
\hline $62 \mathrm{JAl}$ & $42 M O 96$ & $N \cdot G$ & $S P C$ & 9 & $0-$ & NAI - D & 90 & \\
\hline $64 \mathrm{GE} 1$ & $42 \mathrm{MO} 97$ & $G, P$ & $A B X$ & THR- 26 & CTHR - 33 & $A C T-1$ & 4P! & \\
\hline $68 \mathrm{GE} \mathrm{I}$ & $42 M 097$ & $G, P$ & $A B X$ & $13-26$ & CTHR - 26 & $A C T-1$ & $4 \mathrm{PI}$ & \\
\hline 70151 & $42 M 098$ & $G, X N$ & $A B X$ & THR- 30 & CTHR- 30 & BF 3-I & 4P I & 445 \\
\hline $64 \mathrm{GE} \mathrm{I}$ & $42 M 098$ & G,P & $A B X$ & THR - 26 & CTHR- 33 & $A C T-I$ & $4 \mathrm{Pl}$ & \\
\hline 68GE I & $42 M 098$ & $G, P$ & $A B X$ & $13-26$ & CTHR - 26 & $A C T-I$ & 4PI & \\
\hline 64GE 1 & $42 M 098$ & $G, N P$ & $A B x$ & THR - 32 & CTHR - 33 & $A C T-I$ & 4PI & \\
\hline $71 M O 2$ & $42 M 0100$ & $G, G /$ & LFT & 6 & 6,8 & $\begin{array}{l}S C D-0 \\
6.418\end{array}$ & $\begin{array}{l}\text { DST } \\
\text { MEV }\end{array}$ & \\
\hline $64 \mathrm{GE} 1$ & $42 M O 100$ & $G, N$ & $A B X$ & THR- 20 & $\begin{array}{r}\text { CTHR - } 33 \\
\text { MIXED }\end{array}$ & $\begin{array}{l}A C T-I \\
D \quad W I T H\end{array}$ & $\begin{array}{l}\text { UPI } \\
G, P\end{array}$ & \\
\hline $57 \mathrm{FE}^{2}$ & $42 M 0100$ & $G, P$ & $A B X$ & $14-31$ & C 14- 31 & $A C T-I$ & $4 \mathrm{PI}$ & \\
\hline 64GE I & $42 \mathrm{MO} 100$ & $G \cdot P$ & $A B x$ & THR - 20 & $\begin{array}{r}\text { CTHR- } 33 \\
\text { MIXEO }\end{array}$ & $\begin{array}{r}\text { ACT-I } \\
0 \text { WITH }\end{array}$ & $\begin{array}{l}4 P I \\
G, N\end{array}$ & \\
\hline $86 E I$ & $42 M 010 u$ & $G, N+P$ & $A B X$ & $7-26$ & CTHR- 26 & $A C T-I$ & $4 \mathrm{PI}$ & \\
\hline
\end{tabular}

TECHNET I UM $2=43$

ABUND.

SEPARATION ENERGIES (MEV)
$G, N$ G,P G,T G,HE3 G,A G, $2 N$ G,NP G,2P $\begin{array}{llllllll}12.8 & 4.1 & 1 B .4 & 15.9 & 5.1 & 23.2 & 16.8 & 11.6\end{array}$

REF $Z$ A IN,OUT TYPE ANG NUM

$69 E J 243 T C 93$ P, ABX 9, 10 D 6, 7 SCD-0 DST 


A
96
98
99
100
101
102
104

ABUND.

$G, N G, P$ G,T G,HE3 G,A G, 2N G,NP G,2P $\begin{array}{lllllllll}.51 & 10.7 & 7.4 & 17.4 & 14.2 & 1.7 & 19.6 & 17.3 & 12.2\end{array}$ $\begin{array}{lllllllll}1.87 & 10.3 & 8.3 & 17.2 & 15.4 & 2.2 & 18.3 & 17.7 & 14.0\end{array}$ $\begin{array}{lllllllll}12.72 & 7.5 & 8.4 & 16.7 & 13.8 & 2.3 & 17.7 & 15.8 & 14.7\end{array}$ $\begin{array}{lllllllll}12.62 & 9.7 & 9.2 & 17.0 & 16.6 & 2.9 & 17.1 & 18.1 & 15.7\end{array}$

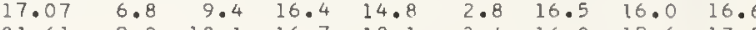
$\begin{array}{lllllllll}31.61 & 9.2 & 10.1 & 16.7 & 18.1 & 3.4 & 16.0 & 18.6 & 17.5 \\ 18.58 & 8.9 & 10.5 & 16.7 & 19.5 & 4.3 & 15.2 & 18.9 & 19.1\end{array}$

NUCLIDE REACTION RES EXCIT SOURCE OETECTDR I A IN,OUT RES EXCIT SOURCE TYPE ANG NUM 68JUI 44RU G.N NOX THR- 27 C $27 \quad$ THR-I OST

\section{RHOOIUM $Z=45$}

ABUND.

SEPARATION ENERGIES (MEV)

G,N G,P G,T G,HE3 G,A G,2N G,NP G,2P

REF NUCLIOE REACTIDN RES EXCIT SOURCE DETECTDR NUTE ANG NUM

\begin{tabular}{|c|c|c|c|c|c|c|c|c|}
\hline 65KRI & $45 \mathrm{RH} 103$ & $E, E /$ & RLY & $7-18$ & D $7-$ & & $\begin{array}{l}A C T-I \text { I } \\
\text { API } \\
M E R \text { YIELO }\end{array}$ & \\
\hline $591 \mathrm{Kl}$ & 45RHID3 & $G, G /$ & $A B X$ & 1 & 1 & & $A C T-1 \quad 4 P I$ & \\
\hline 60802 & $45 R_{H 1 O 3}$ & $G, G /$ & $A B X$ & $6-26$ & $6-$ & 26 & $A C T-I \quad 4 P I$ & \\
\hline 6 $3 V E 2$ & $45 \mathrm{RHIO}_{3}$ & $G, G /$ & $A 8 x$ & $0-1$ & $\begin{array}{l}0 \quad 1 \\
1 \text { SDMER }\end{array}$ & & $\begin{array}{l}A C T-I \quad 4 P I \\
=1.33 \quad M E V\end{array}$ & \\
\hline $65 \mathrm{KRI}$ & $45 \mathrm{RHIO} 3$ & $G, G /$ & RLY & $7-18$ & C $7-$ & $\begin{array}{l}8 \\
5\end{array}$ & $\begin{array}{l}\text { ACT I IP I } \\
M E R \text { YIELO }\end{array}$ & \\
\hline $58 \mathrm{CH} 2$ & 45RHIO3 & $G, N$ & RLY & THR & CTHR & & $\begin{array}{l}\text { BF } 3-\text { I } 4 \text { P I } \\
\text { THRE SHOLD }\end{array}$ & \\
\hline 59PA2 & $45 \mathrm{RH} 103$ & $G, N$ & $A 8 X$ & $9-24$ & C 9- & $\begin{array}{l}24 \\
\text { UP }\end{array}$ & $\begin{array}{l}\text { BF } 3-1 \quad 4 P I \\
\text { LE MOMENT }\end{array}$ & \\
\hline 60GE3 & $45 \mathrm{RHIO} 3$ & $G, N$ & NDX & THR & CTHR & & $\begin{array}{l}\text { BF } 3-I \text { I I } \\
\text { THRE SHDLO }\end{array}$ & \\
\hline 62801 & $45 \mathrm{RH} 103$ & $G, N$ & $A B X$ & $10-23$ & C $10-$ & 23 & $B F 3-I$ - $\mathrm{PI}$ & 428 \\
\hline $67 \mathrm{HUI}$ & 45RH103 & $G, N$ & $A B x$ & 10,11 & D 10 , & 11 & $B F 3-I \quad 4 P I$ & \\
\hline $68 \mathrm{JUI}$ & 45RHIO3 & $G, N$ & NDX & THR -27 & C 27 & & THR-I OST & \\
\hline 69DE 1 & $45 \mathrm{RH} 03$ & $G, N$ & $A B Y$ & THR-999 & C $1-$ & $\begin{array}{l}6 \\
9\end{array}$ & $\begin{array}{ll}A C T-1 & 4 P 1 \\
9=5.5 & \text { GEV }\end{array}$ & \\
\hline 690E 1 & 45RH1O3 & $G, 2 N$ & $A B Y$ & THR-999 & $1-$ & $\begin{array}{l}6 \\
9\end{array}$ & $\begin{array}{ll}A C T-1 & 4 P I \\
9=5.5 & G E V\end{array}$ & \\
\hline $58 \mathrm{KAl}$ & $45 \mathrm{RH}_{103}$ & $G, X N$ & $A B x$ & $9-22$ & $9-$ & 22 & $B F 3-I \quad 4 P I$ & \\
\hline $625 \mathrm{H}_{2}$ & 45RH103 & $G, P$ & AEY & $9-34$ & C 23, & 34 & $\begin{array}{l}\text { EMU-O OST } \\
\text { SPECTRUM }\end{array}$ & \\
\hline $625 \mathrm{H}_{4}$ & 45RHIO3 & $G, P$ & $A B Y$ & $9-34$ & C 23, & 34 & $\begin{array}{l}\text { EMU-O OST } \\
\text { SPECTRUM }\end{array}$ & \\
\hline 65KRI & 45RHID 3 & $\mathrm{G}, 2 \mathrm{P}$ & $A B X$ & $15-40$ & $c 15-$ & 40 & $A C T-I \quad 4 P I$ & \\
\hline $560 A 2$ & $45 \mathrm{RHID}_{3}$ & $G, \times P$ & AEY & $10-70$ & c 70 & & $\begin{array}{l}\text { EMU-D OST } \\
\text { SPECTRUM }\end{array}$ & \\
\hline & $45 R$ & $G \cdot X P$ & $A B X$ & $13-$ & C $14-$ & 2 & SCI-O DST & \\
\hline 10 & 45RHIO3 & $G, A$ & $A B Y$ & THR - 2 & c 22 & & EMU-I OST & \\
\hline
\end{tabular}

\section{PALLADIUM $Z=46$}

\begin{tabular}{rrrrrrrrrr} 
A & ABUNO. & \multicolumn{8}{c}{ SEPARATIDN ENERGIES (MEV) } \\
& & G,N & G.P & G,T & G,HE3 & G,A & G.2N & G,NP & G,2P \\
102 & 0.96 & 10.6 & 7.8 & 17.3 & 15.2 & 2.1 & 18.9 & 17.7 & 13.3 \\
104 & 10.97 & 10.0 & 8.7 & 17.0 & 16.4 & 2.6 & 17.6 & 18.0 & 14.0 \\
105 & 22.23 & 7.1 & 8.8 & 16.6 & 14.2 & 2.9 & 17.1 & 15.8 & 15.7 \\
106 & 27.33 & 9.6 & 9.3 & 16.8 & 17.6 & 3.2 & 16.6 & 18.3 & 16.4 \\
108 & 26.71 & 9.2 & 10.0 & 16.6 & 18.5 & 3.9 & 15.8 & 18.5 & 17.8 \\
110 & 11.81 & 8.8 & 10.5 & 16.4 & 19.6 & 4.4 & 15.0 & 18.7 & 19.2
\end{tabular}

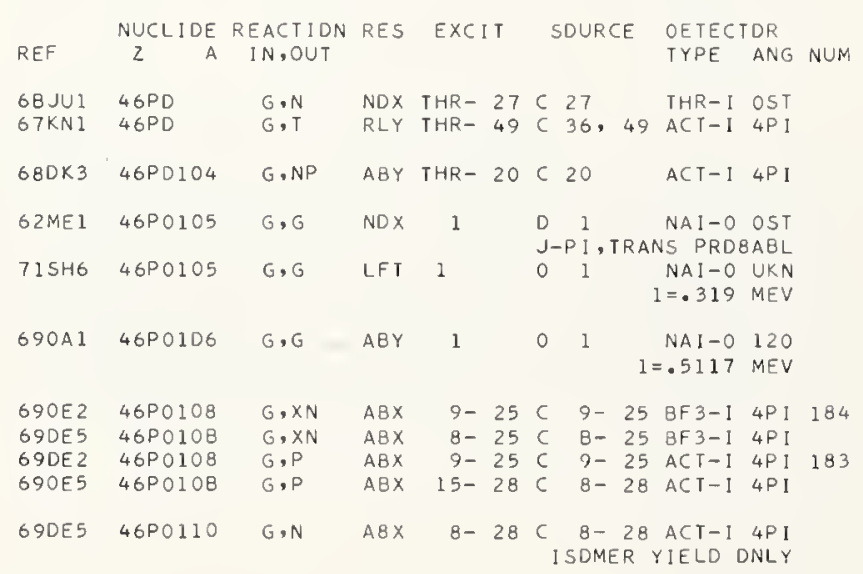

\begin{tabular}{cccccccccc} 
A & ABUNO. & \multicolumn{10}{c}{ SEPARATIDN ENERGIES (MEV) } \\
& & G,N & G.P & G.T & G,HE3 & G,A & G.2N & G.NP & G, 2P \\
107 & 51.35 & 9.6 & 5.8 & 13.9 & 16.4 & 2.8 & 17.5 & 15.4 & 15.1 \\
109 & 48.65 & 9.2 & 6.5 & 13.8 & 17.3 & 3.3 & 16.5 & 15.7 & 16.4
\end{tabular}

NUCLIDE REACTIDN RES EXCIT SDURCE OETECTDR

REF $Z$ A IN,OUT TYPE ANG NUM

65WYI 47AG G,MU-T ABX $10-35$ C $90 \quad$ SCI-0 4PI 57

GOREI $47 A G$ G,G ABX 7 N 077 NAI-O DST

$58 B 01$ 47AG G,G/ ABX 6- 2BC 6- 2B AGT-WOTH

ISDMER YIELD

63KA2 $47 A G$ G,G/ RLY 1 C 5 ACT-I 4 P

$57 F E I \quad 47 A G \quad G, N$ RLY $17-31 C 15-31$ THR-I OSI

$G, N$ NDX $7-22 \subset 22$ THR-I OS

$67 G E 2 \quad 47 A G \quad G, N \quad A B Y T H R-27 C 22,27$ BF $3-I$ I P

67HU1 $47 \mathrm{AG} \quad \mathrm{G}, \mathrm{N}$ A8X 10, $1101 \mathrm{C}, 11 \mathrm{BF} 3-\mathrm{I} 4 \mathrm{PI}$

56HAI 47AG G,XN ABX 15, 18 015,18 BF 3-I 4PI

618A2 47AG G.XN ABY THR- 22 C 22 THR-I OS

$G, X N$ ABX $9-25$ C $9-25$ BF $3-1$ 4P

$G, P \quad A B X 17-31 C 16-32$ SCI-O OSI

$525 E 2-47 A G$

G,P SPECTRU

$G, P \quad A B X \quad 15,18 \quad 0 \quad 15,18$ SCI-1

G, XP ABY 4- 22 C 22 SCI-I OSI

G.A ABY 2- 22 C 22 EMU-I OST

$G, A$ SPC THR - 35 C 35 SCO-D 90

$G, A \quad A B X \quad 3-33 C \quad 33 \quad$ SCD $-D \quad 90$

$G, A$ RLY 2- 32 C 32 SCO-D DST

G, T RLY THR- 31 C 31 ACT-I $4 P$

$G, F \quad A B \times 300-999 \quad 300-999$ EMU-O 4PI

SWAI $47 A C$

64DE $4 \quad 47 A G$

$71 M E 1 \quad 47 \mathrm{AG}$

65HEI $47 \mathrm{AG} 107$

T2KUG 47AG107

65HE 1 47AG107 E+, N

63813 47AG107 G,

69BD $3 \quad 47 A G 107$

$63805 \quad 47 A G 107$

63VE2 47AG107

G6CA1 47AG107

$58 B E 1 \quad 47 A G 107$

$59 M U 2 \quad 47 A G 107$

GOGE3 $47 \mathrm{AGI} 07$

6IWE1 47AG107

$62 B 01 \quad 47 A G 107$

S2CAL 47AG107

62FUG 47AG107

69BE1 47AG107

$69 B E 1 \quad 47 A G 107$

$69152 \quad 47 A G 107$

66WII 47AGIO7

$59803 \quad 47 A G 109$

$70 M O 2 \quad 47 A G 109$

66CAL 47AG109

$588 E 1$ 47AG109

59NAI 47 AGIO9

GOGE3 $37 \mathrm{AG} 109$

$69152 \quad 47 A G 109$

$55 E R 1 \quad 47 A G 1 D 9$

$555 C 2$ 47AG109

STER1 47AG109

$59 R D 3 \quad 47 A G 109$

G8DKI $47 A G 109$
$G, F$

$999=1 \mathrm{GEV}$

OF ABY THR-900 (300-900 TRK-I 4P I

, N RLY THR- 32 D 14-32 ACT-1 4P $A 8 \times 10-3 D 020-30$ ACT-1 $4 P$ $A 8 \times 1$ D 1 ACT I $4 P$ MDSSBAUER

$G, G \quad A B X \quad 0-2 C 0-2$ ACT-1 4 PI G,G/ ABX O- IEPARATED ISOTOPES ISDMERS $1=1.33 \mathrm{MEV}$ $G, G / A B 10-2 C 0-2 A C T-14 P I$ $G, N$ RLY $9-10 \mathrm{C} 9-10$ ACT-1 4 PI THRESHDLD $\begin{array}{llrlrll}G, N & A B X & 9-21 & C & 9-25 & A C T-I & 4 P I \\ G, N & N D X & P H R & C T H R & B F 3-I & 4 P I\end{array}$ THRESHDLD $G, N \quad A B X \quad 9-110$ 9- 11 ACT-I 4PI G.N THRESHOLO SOX 30 C 30 ACT-I 4 PI
ISOMERIC RATIO $G, N$ RLY $9-34 C 34$ ACT-I 4 PI YLO REL TO CU62 , N A8X THR- $30 \mathrm{D} 9-30 \mathrm{BF} 3-\mathrm{I} 4 \mathrm{PI}$ G,2N ABX THR- 30 D $17-30$ BF 3-I 4PI SEP ISDTDPES $G, X N$ ABX 9- 29 C 7- 30 BF 3-1 4PI 455 $G, T$ ABX THR - 56 C $31-56$ ACT-I $4 \mathrm{PI}$ $G, G$ ABX D- $2 C$ D- 2 ACT-I $4 P I$ $G, G \quad A B X \quad B \quad D \quad 8 \quad S C D-D D S T$ $G, G / A B 10-2 C D-\quad \begin{aligned} & 8=7.632, L F T \\ & 2 A C T-14 P I\end{aligned}$ G,N RLY 9- $10 C 9-10$ C LEVELS $G, N \quad A B X \quad 18 \quad 0,18$ THRESHDLO $G, N$ NOX THR CIHR AF NOX THR CIHR BF $3-14 P I$
THRESHDLO $G, X N \quad A B X \quad 9-29 C \quad 7-308 F 3-14 P ! 456$ G, A ABY THR- 31 C 32 ACT-1 4 PI $G, A \quad A B Y T H R-32 C 32 \quad A C T-14 P I$ $G, A \quad A B I T H R-32 C 32 \quad A C T-14 P I$

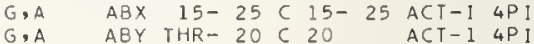
$(E+, N) /(E-, N)$
RLY THR $-32014-32$ ACT-I 4 PI $G, G / A B \times$ 6- 26 C $6-26$ ACT-1 $4 P$ I 


A
106
108
110
111
112
113
114
116

ABUND. SEPARATION ENERGIES (MEV)

$\begin{array}{rrrrrrrrr} & G . N & G, P & G . T & G, H E 3 & G, A & G .2 N & G . N P & G, 2 P \\ 1.22 & 10.9 & 7.3 & 17.3 & 14.6 & 1.6 & 19.3 & 17.2 & 12.3 \\ 0.88 & 10.3 & 8.1 & 17.1 & 15.8 & 2.3 & 18.3 & 17.7 & 13.9 \\ 12.39 & 9.9 & 8.9 & 16.9 & 16.9 & 2.9 & 17.2 & 18.1 & 15.4 \\ 12.75 & 7.0 & 9.1 & 16.6 & 14.7 & 3.3 & 16.9 & 15.9 & 16.2 \\ 24.07 & 9.4 & 9.6 & 16.8 & 17.9 & 3.5 & 16.4 & 18.5 & 16.8 \\ 12.26 & 6.5 & 9.8 & 16.5 & 15.6 & 3.9 & 15.9 & 16.2 & 17.5 \\ 28.86 & 9.0 & 10.3 & 16.7 & 18.9 & 4.1 & 15.6 & 18.8 & 18.3 \\ 7.58 & 8.7 & 11.1 & 16.6 & 16.6 & 4.9 & 14.8 & 19.1 & \end{array}$

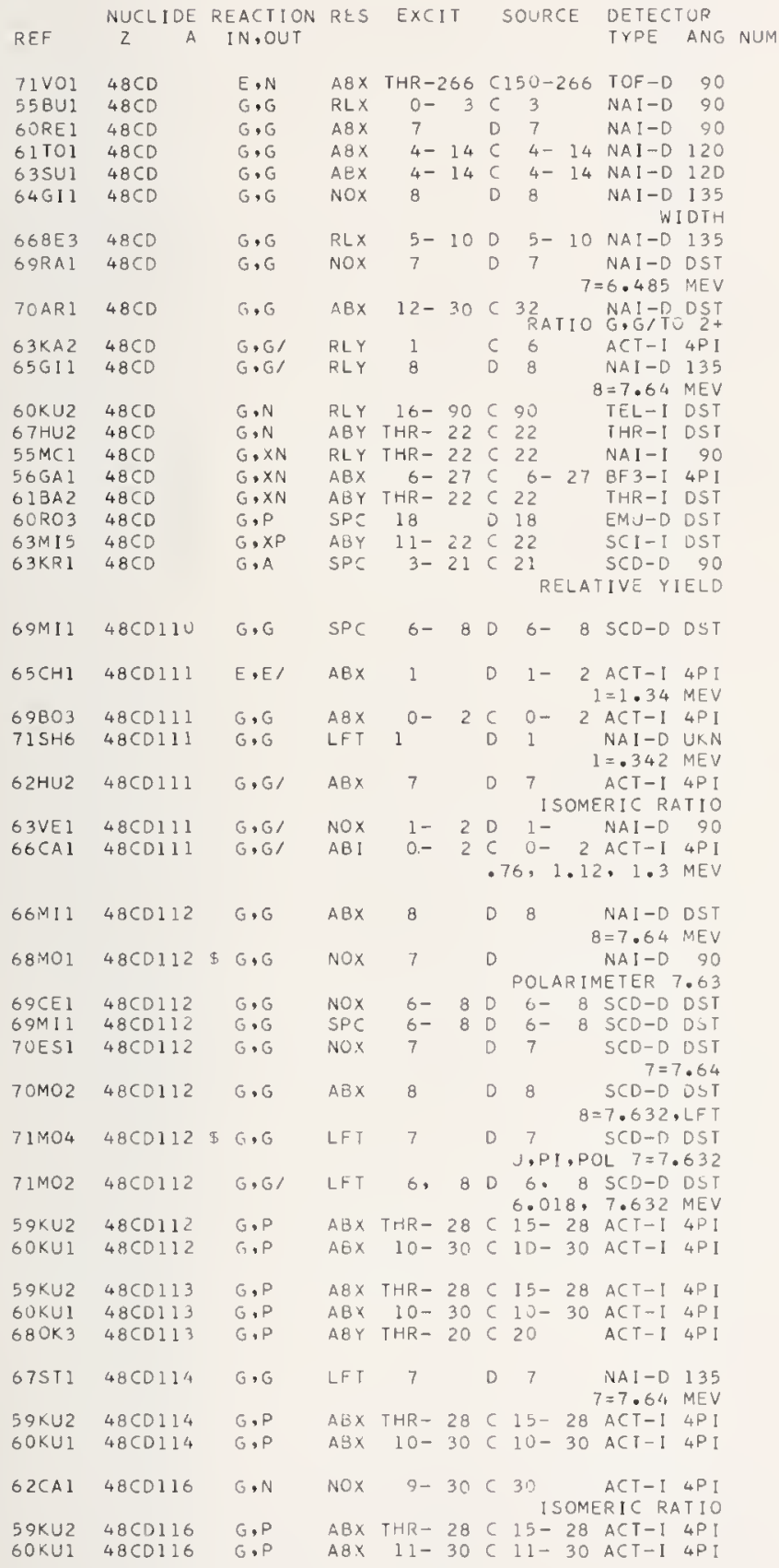

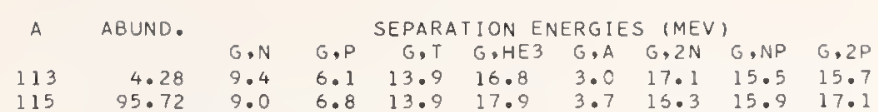

RE

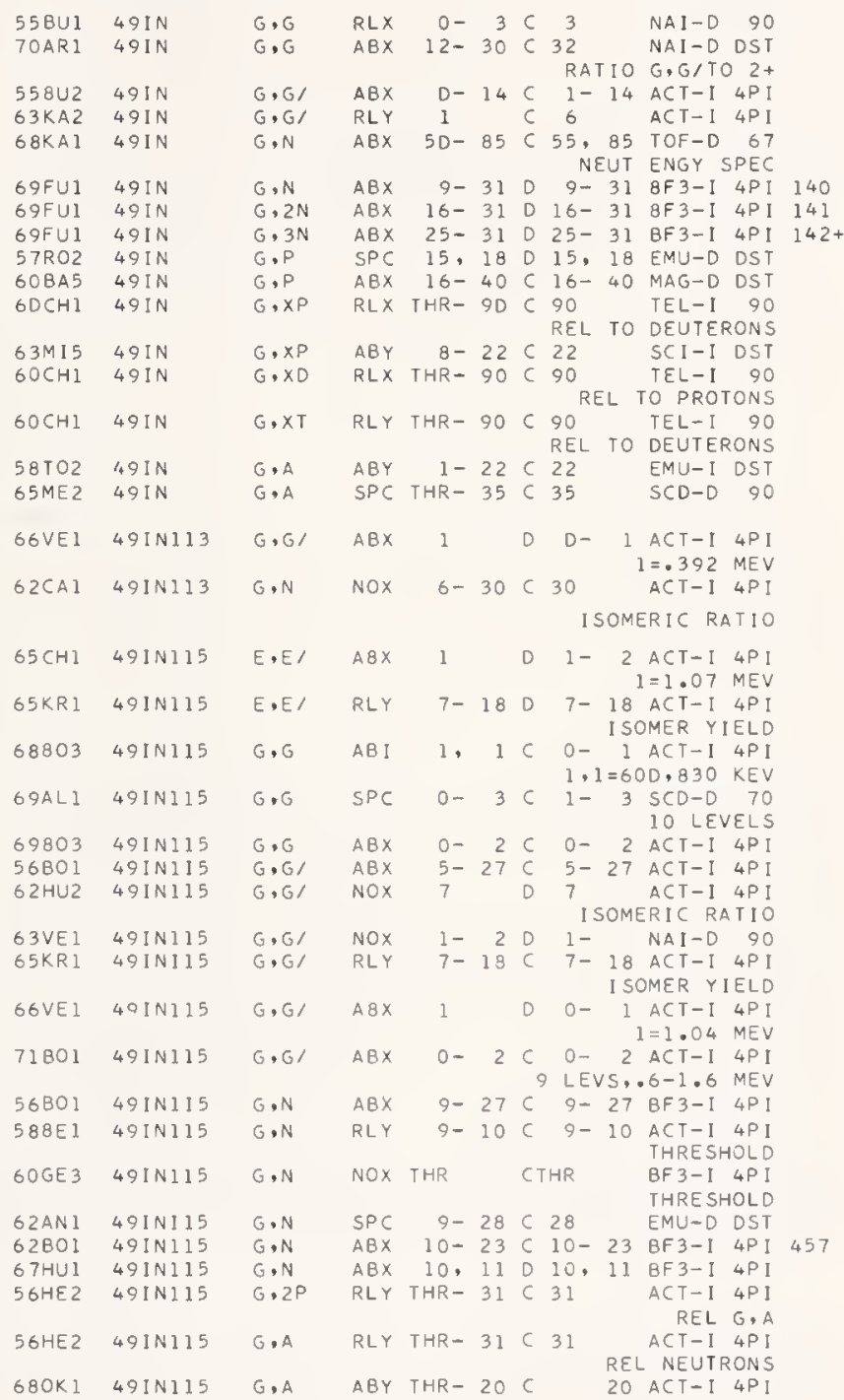

TIN Z $Z=50$

ABUND.

\begin{tabular}{lrrrrrrrrr} 
A & ABUND. & \multicolumn{1}{c}{ G.N } & $G, P$ & $G . T$ & $G, H E 3$ & $G, A$ & $G .2 N$ & $G . N P$ & $G .2 P$ \\
112 & 0.96 & 10.8 & 7.5 & 17.1 & 15.0 & 1.8 & 19.0 & 17.6 & 12.9 \\
114 & 0.66 & 10.3 & 8.5 & 17.1 & 16.2 & 2.6 & 18.1 & 17.9 & 14.6 \\
115 & 0.35 & 7.5 & 8.7 & 17.0 & 14.4 & 3.2 & 17.9 & 16.0 & 15.6 \\
116 & 14.30 & 9.6 & 9.3 & 17.1 & 17.4 & 3.4 & 17.1 & 18.3 & 16.1 \\
117 & 7.61 & 6.9 & 9.4 & 16.8 & 15.3 & .8 & 16.5 & 16.2 & 16.9 \\
118 & 24.03 & 9.3 & 10.0 & 17.1 & 18.5 & 4.1 & 16.3 & 18.8 & 17.5 \\
119 & 8.58 & 6.5 & 9.9 & 16.8 & 16.3 & 4.4 & 15.8 & 16.5 & 18.2 \\
120 & 32.85 & 9.1 & 10.7 & 17.1 & 19.6 & 4.8 & 15.6 & 19.0 & 19.0 \\
122 & 4.72 & 8.8 & 11.4 & 17.2 & 20.7 & 5.7 & 15.0 & 19.8 & $*$ \\
124 & 5.94 & 8.5 & 12.1 & 17.4 & $*$ & $*$ & 14.4 & 20.4 & $*$
\end{tabular}

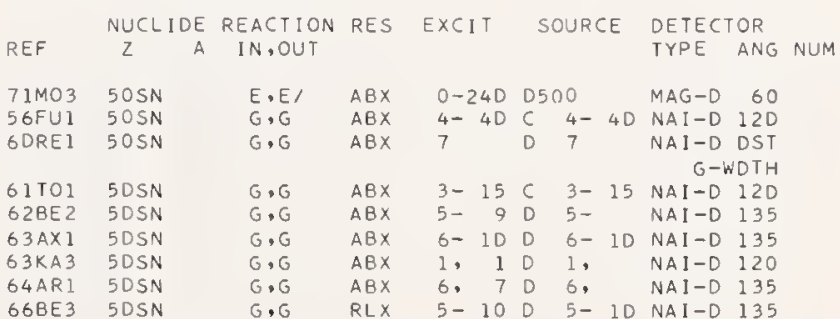




\begin{tabular}{|c|c|c|c|c|c|c|c|c|c|}
\hline 69RAI & $50 S N$ & $G, G$ & NOX & 7 & & D & & $\begin{aligned} & \text { NAI }-D \text { DST } \\
= & 6.988 \mathrm{MEV}\end{aligned}$ & \\
\hline $69 \mathrm{SH}_{2}$ & $505 N$ & $G, G$ & $S P C$ & 6 . & 7 & $\begin{array}{l}6, \\
6=0\end{array}$ & & 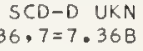 & \\
\hline 70AR 1 & $505 \mathrm{~N}$ & $G, 6$ & $A B X$ & $12-$ & 30 & C 32 & 10 & $\begin{array}{l}\text { NAI I D DST } \\
G, G / T O \quad 2+\end{array}$ & \\
\hline $70 \mathrm{~A} \times 1$ & $505 N$ & $G, G$ & $A B X$ & $6-$ & 10 & $6-$ & 10 & NAI-D 45 & \\
\hline $63 \mathrm{KA2}$ & $505 N$ & $G, G^{\prime}$ & RLY & 1 & & $c$ & & $A C T-I \quad 4 P I$ & \\
\hline $67 \mathrm{HU} 2$ & $50 S N$ & $G, N$ & $A B Y$ & THR- & 22 & C 22 & & THR-I DST & \\
\hline $68 K A 1$ & $505 N$ & $\mathrm{G}, \mathrm{N}$ & $A B X$ & $50-$ & 85 & C 55, & $\begin{array}{l}85 \\
\cup T\end{array}$ & $\begin{array}{l}\text { TOF-D } 67 \\
\text { ENGY SPEC }\end{array}$ & \\
\hline 55011 & $505 \mathrm{~N}$ & $G, X N$ & NOX & THR- & 70 & c 70 & & SCI-I DST & \\
\hline $56 \mathrm{HAl}$ & $505 \mathrm{~N}$ & $G, X N$ & $A B X$ & 15 . & 18 & D 15. & 18 & $B F 3-I \quad 4 P I$ & \\
\hline $5 B F \cup 1$ & $505 \mathrm{~N}$ & $G, X N$ & $A B X$ & $7-$ & 40 & C 7- & 40 & $B F 3-1 \quad 4 P 1$ & \\
\hline $64 A L 5$ & $505 N$ & $G, \times N$ & NOX & THR - & 34 & c 34 & & THR-I OST & \\
\hline 60RO3 & $505 \mathrm{~N}$ & $G, P$ & SPC & 18 & & D 18 & & EMU-D OST & \\
\hline 63MI5 & $505 N$ & $G, X P$ & $A B Y$ & $10-$ & 22 & C 22 & & SCI-I DST & \\
\hline 63YA2 & $50 S N$ & $G, D$ & $R L Y$ & $12-$ & 24 & $\begin{array}{l}C 24 \\
\text { SPC. }\end{array}$ & $Y L L$ & $\begin{array}{c}\text { EMU - D OST } \\
\text { REL TO P }\end{array}$ & \\
\hline 70 CU1 & $50 S N$ & $G, T$ & $A B Y$ & THR - & 90 & C 90 & & $A C T-I \quad 4 P I$ & \\
\hline $71 M E 1$ & SOSN & $G, F$ & $A B Y$ & THR -9 & 900 & D $300-$ & 900 & TRK-I $4 \mathrm{PI}$ & \\
\hline $61 \mathrm{KUI}$ & 5OSN112 & $\mathrm{G}, \mathrm{N}$ & $A B X$ & $10-$ & 21 & C $10^{-}$ & 21 & $A C I-I \quad 4 P I$ & \\
\hline 71501 & $505 N 112$ & $G, X P$ & $A B X$ & $10-$ & 29 & C $8-$ & 29 & SCO-D 4PI & \\
\hline $70 B R 1$ & $505 N 114$ & $G, P$ & $R L Y$ & $12-$ & 22 & C $14-$ & 23 & $A C T-I \quad 4 P 1$ & \\
\hline $67 B A 4$ & $50 S N 116$ & $E, E /$ & FMF & 1 , & 2 & 0150 & & MAG-D DST & \\
\hline $69 C U 1$ & $50 S N 116$ & $E, E /$ & FMF & $1-$ & 12 & D 55 , & 00 & ${ }^{7} \dot{M}^{2} \dot{2}{ }^{2}-2$ MEV & \\
\hline $69 \mathrm{SH} 7$ & SOSN 116 & $E, P$ & $A B Y$ & $14-$ & 19 & $0 \quad 19$ & & MAG-O DST & \\
\hline $725 \cup 4$ & SOSNIIG & $E, P$ & $A B I$ & $13-$ & 19 & D $16-$ & 21 & $M A G-D \quad 4 P I$ & \\
\hline $62 \mathrm{KAl}$ & $505 N 116$ & $G \cdot G$ & LFT & 1 & & D $\quad 1$ & & NAI - D UKN & \\
\hline 62 L. 12 & $505 N 116$ & $G, G$ & LFT & 1 & & D & & NAI I D 132 & \\
\hline $63 \mathrm{BE} 6$ & $505 N 116$ & $G, G$ & LFT & 1 & & D & & NAI -D 100 & \\
\hline $69 \mathrm{FUI}$ & 50 SNII6 & $G, N$ & $A B X$ & $9-$ & 29 & $9-$ & 29 & $B F 3-I \quad 4 P I$ & 117 \\
\hline $69 \mathrm{FUI}$ & $505 N 116$ & $G, 2 N$ & $A B X$ & $17-$ & 29 & D 17- & 29 & $B F 3-I \quad 4 P I$ & \\
\hline $69 \mathrm{FUI}$ & $505 N 116$ & $G, 3 N$ & $A B X$ & $27-$ & 29 & D 27- & 29 & $B F 3-I \quad 4 P I$ & 119 \\
\hline
\end{tabular}

$725 U 4$ 5OSNI17 E,P ABI 9- $20016-21$ MAG-O 90 6OME2 5OSN117 G,G LFT 1 D D 1 I NAI-D 125 $67 \mathrm{GII}$ 5OSNI17 G,G LFT 7 L $6-7$ NAI-D OST

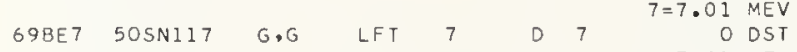

69FUI 50SN117 G,N ABX 6- 31 D $6-31$ BF3-I 4 PI 120

7OWI 2 SOSN117

69FU1 50SN117

G9FU1 50SN117

$680 K 3 \quad 50 S N 117$

69 CUI 5OSN118

$695 H_{7} \quad 505 N 118$

$72 S \mathrm{SL}_{4}$ 5OSNI1B

66HRI SOSNIIB

69CE I 5OSN118

69FU1 5OSNI1B

69FU1 5OSN11B

GIHUI SOSNII8

69FUI 5OSNII9

7OWI2 5OSNII9

69FU1 5OSN119

$\begin{array}{ll}\text { 69FUI } & \text { 5OSN119 } \\ \text { GOSTI } & \text { 5OSN119 }\end{array}$

67BA4 SOSN120

69CUI 50SN12O

$695 H 7 \quad 50 S N 120$

$72 S U 4$ 5OSN12O

G6HRI 5OSN12O

69FUI 5OSN120

69FU1 $50 S N 120$

69FUI 5OSN120

6OKUI $50 S N 120$

72 SU4 50 SN 122

67BA4 50SN124

69 CUI 50 SN124

$695 \mathrm{H} 7 \quad 505 N 124$

$72 S U 4 \quad 50 S N 124$

6 KOFUI $50 \mathrm{SNI} 24$

69FUI 5OSN124

69FUI $50 S N 124$

71501 5OSN124
$G, N \quad A B X \quad 7-9 C 7-9$ TOF-D 130 G. 3N ABX 24- 31 D $24-31$ BF 3-I 4PI $122+$ G,P ABY THR- 20 C 20 ACT-I $4 \mathrm{PI}$

E,E/ FMF 1- 4 D 55, 60 MAG-O DST E,P ABY $15-20020$ MAG-D DST $E, P \quad A B I 14-20 D 16-21 M A G-D$ 4PI G,G NOX 6- $80 \quad 6-\quad 1=1.22$ MEV $G, N \quad A B X \quad 9-300 \quad 9-30$ BF $3-I$ IPI 12 $G, 2 N \quad A B X \quad 16-30 D 16-30$ BF $3-I$ IPI 125 $G, 3 N$ ABX 25- 30 D $25-30$ BF $3-I$ API $126+$ ,P RLY 6- 24 C 24 ACT-I 4 PI

G.N $A B X$ 7- $9 C 6-9$ TOF-D 130 $G, 2 N \quad A B X \quad 15-31 D D_{15}-31$ BF 3-I $4 P I 129$ $G, 3 N \quad A B X \quad 22-31$ D 22- 31 BF 3-I $4 P I 130+$ $G . N P$ RLX $146-320$ C320 TEL-O 76
REL TO H2 CROS SEC

E,E/ FMF 1, 20150 MAG-O OST E,E/ FMF $1-4055,60$ MAG-O OST F,P ABY 15- 20 D 21 MAG-D OST

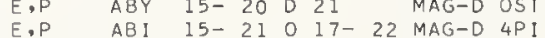
$\begin{array}{lcccccccc}E, P & A B I & 15-21 & 0 & 17-22 & \text { MAG-D } & 4 P I \\ G, G & \text { LFT } & 1 & 0 & 1 & & \text { NAI-D } & 90\end{array}$ $G, N \quad A B X \quad 9-290 \quad 9-29$ BF3-I 4 PI 13 G.2N ABX 15- 29 D $15-29$ BF 3-I 4 PI 133 $\begin{array}{lllllll}\text { G.P } & A B X & 16-27 & C & 16-27 & A C T-I & 4 P I\end{array}$ E P P $E, E /$ FMF 1,20150 MAG-D DST $E, E /$ FMF $1-4$ D 55, 60 MAG-O OST E,P ABY $16-22022$ MAG-D 90 E,P $A B I \quad 16-22$ D $19-24$ MAG-D 90 $G, N \quad A B X \quad B-20 C B-20 A C T-I \quad 4 P$ I $G, N \quad A B X \quad 8-31 \quad D \quad B-31 \quad B F 3-1 \quad 4 P I 136$ $\begin{array}{llllllllll}G, 2 N & A B X & 14-31 & 0 & 14-31 & B F 3-1 & 4 P ! & 137 \\ G, 3 N & A B X & 23-31 & 0 & 23-31 & B F 3-1 & 4 P ! & 138+\end{array}$ $G, X P$ ABX $14-29$ C $12-29$ SCO-D $4 P I$ $G, 2 N \quad A B X \quad 16-31$ D $16-31$ BF 3-I 4PI 121 $G, G$ LFT 1 D 1 D 1 NAI-D 90

$G, N \quad A B X \quad 6-31006-31$ BF3-I $4 P I 128$ G.3N ABX 24- 29 D 24- 29 BF 3-I 4PI $134+$

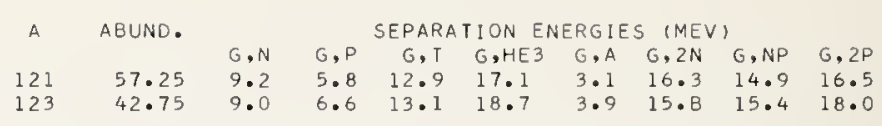

NUCLIDE REACTION RES EXCIT SOURCE OETECTOR

TYPE ANG NUM

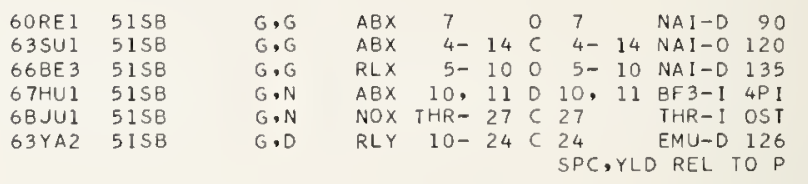

\begin{tabular}{|c|c|c|c|c|c|c|}
\hline 63ME I & 5158121 & $G, G$ & LFT & 1 & D & $\begin{array}{rr}N A I-D & 05 T \\
J-P I\end{array}$ \\
\hline SOGE 3 & $515 B 121$ & $G, N$ & NOX & THR & CTHR & $\begin{array}{l}\text { BF } 3-I \text { I } \quad \text { P } \\
\text { THRE SHOLD }\end{array}$ \\
\hline $61 C 02$ & 5158121 & $G, N$ & $R L X$ & 15,18 & $\begin{array}{l}D \text { 15, } \\
\text { REL }\end{array}$ & $\begin{array}{l}\text { 1B ACT-I } 4 \text { PI } \\
\text { TO CU63 SIGMA }\end{array}$ \\
\hline $620 E 1$ & 5158121 & $G, N$ & $A B X$ & 20 & 020 & $A C T-I \quad 4 P I$ \\
\hline $57 E R 1$ & 5158121 & $G, D$ & $A B X$ & $18-32$ & C $18-$ & $32 \mathrm{ACT}-\mathrm{I}$ 4PI \\
\hline 55ER1 & 5158121 & $G, A$ & $A B Y$ & THR- 31 & C 32 & $A C T-1 \quad 4 P 1$ \\
\hline $56 \mathrm{HE} 2$ & $515 B 121$ & $G, A$ & RLY & THR- 31 & C 31 & $\begin{array}{r}\text { ACT I I } 4 \text { P I } \\
\text { REL NEUTRONS }\end{array}$ \\
\hline $61 W 01$ & 5158121. & $G \cdot A$ & $A B X$ & $15-24$ & C $15-$ & $24 A C T-I \quad 4 P I$ \\
\hline $645 H 5$ & 5158123 & $G \cdot G$ & LFT & 1 & & $\begin{array}{rr}\text { NAI-D } & 122 \\
l=0.161 & \mathrm{MEV}\end{array}$ \\
\hline 6OGE 3 & 5158123 & $G, N$ & NOX & THR & CTHR & $\begin{array}{l}\text { BF 3-I 4P I } \\
\text { THRE SHOLD }\end{array}$ \\
\hline $5 \mathrm{BHOl}$ & $51 S B 123$ & $G, N P$ & RLY & $18-32$ & C 32 & $\begin{array}{l}\text { ACT-I 4P I } \\
\text { THRE SHOLO }\end{array}$ \\
\hline
\end{tabular}

TELLURIUM $Z=52$

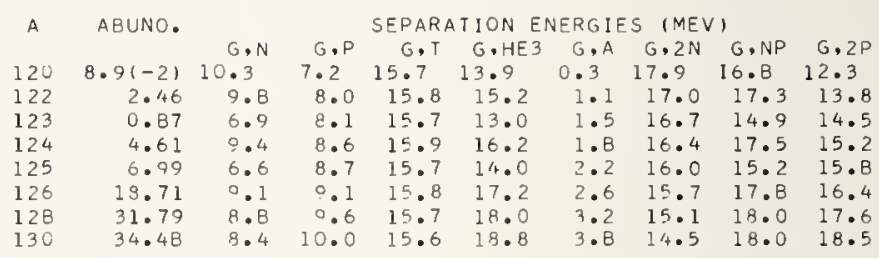

NUCLIOE REACTION RES EXCIT SOURCE OETECTOR

REF $Z$ A IN,OUT TYPE ANG NUM

6OREI 52TE G.G ABX 7 T D 7 NAI-D DST

64PAI 52TE G.G LFT 1 L 0011 NAI-O 90

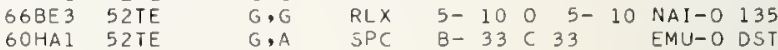

$635 H 5$ 52TE122 G.5 LFT 1 O I NAI-D 105

$\begin{array}{lllllll}\text { 6IAKI } & 52 T E 124 & \text { G.G } & \text { LFT } & 1 & 0 & 1 \\ \text { 65AKI } & 52 T E 124 & \text { G.G } & \text { LFT } & 1 & D & 1\end{array}$

$685 C 152 T E 124$ G.E LFT 1 D I NAI-O 105

66MEI 52TE125 G.S LFT 1 O 1 SCD-O DST

60GE3 52TE125 G,N NOX THR CTHP I B.463 MEV

THRESHOLD

6OGE3 52TE126 G,N NOX THR CTHR BF3-I 4 PI

6OGE3 52TE128 G,N NOX THR CTHR BF3-I 4PI

6OGE3 52TE130 G,N NOX THR CTHR BF3-I 4 PI 
ABUND. SEPARATION ENERGIES (MEV)

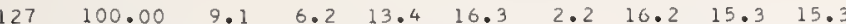
\begin{tabular}{cclll}
\multicolumn{1}{c}{ NUCLIOE REACTION RES EXCIT SOURCE } & DETECTOR \\
REF $Z$ A IN, DUT & & & TYPE ANG NUM
\end{tabular}

56FUI 53I 127 G,G ABX 4- 40 C 4- 40 NAI-D 120

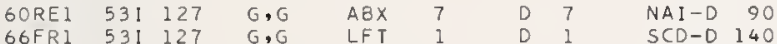

69LAIT $1=.203$ MEV 69LAI 53I 127 G,G LFT I D I NAI-D 130 56ER1 531 127 G N ABX 15, 18 D 15, 18 $1=375$ KEV $57 F E 2$ 531 127 G,N RLY 14-30 C 14- 30 THR-I 90 $58 \mathrm{CH2} 531 \mathrm{I} 27$ GN RLYTHR CTHR BF3-I $4 \mathrm{P}$

SOGE3 531127 G,N NOX THR CTHR THRESHOLD 60KU2 531 127 G,N RLY $16-90 \mathrm{C} 90$ TEL-I DST

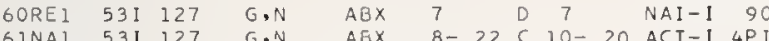
GINAI 531127 GN $\quad$ ABX $12-22 C 22$ C 22 THR-I DST 6ITA1 531 127 GN NOX $12-22 C 22-33$ GF -1 DST $67 D I 1$ 53I 127 GN $\quad$ AEX $300-100$ C100 ACT-I 4PI 67HU1 531127 G.N A8X 20, 11010,11 BF $3=1$ GEV $68 \mathrm{JOI} 531 \mathrm{l} 27$ G,N ABY THR-200 C 50-800 ACT-I 4PI $69 B E 6531127$ GN ABX $9-21$ D $9-31$ MOD-I $4 P I$ 690EI 531127 GN ABY THR-999 C $1-6$ ACT-I $4 P$ I 69VE2 53I $127 \mathrm{G}, \mathrm{N}$ ABX $9-32 \mathrm{D} 9-32 \mathrm{MOD}-\mathrm{I}$ 4P $715 A 153 I 127$ G.N AEY $9-68 C 1 U-68$ ACT-I $4 P I$ 56ERI $53 \mathrm{I} 127 \quad G, 2 \mathrm{~N}$ ABX 15,18 D 15,18 ACT-I $4 \mathrm{PI}$ 6INAI $53 I 127$ G,2N ABX 16- 22 C 10- 20 8F 3-I 4PI $668 R I 53 I 127$ G.2N A8X THR- 33 D $8-33$ BF $3-I$ IPI ABX THR 33 D $8-33$ BF 3-I $4 P$ $67 D I 1 \quad 531127 \quad G, 2 \mathrm{~N} \quad$ ABX $300-999$ C999 ACT-I 4PI $68 \mathrm{JOI} 53 \mathrm{I} 127 \quad 6.2 \mathrm{~N}$ ABY THR-800 C 50-800 ACT-I 4PI $69 B E 6 \quad 53 I 127 \quad 6.2 \mathrm{~N}$ ABX $16-31$ D $9-31$ MOD-I $4 \mathrm{PI}$ 69VE2 $53 \mathrm{I} 127 \quad \mathrm{G}, 2 \mathrm{~N}$ ABX $16-32 \mathrm{D} \quad 16-32 \mathrm{MOD}-\mathrm{I} \quad 4 \mathrm{PI}$ $67011531 \quad 127 \quad$ G.3N ABX $300-9996999$ ACT-I 4 P I $68 J 01531127 \quad G, 3 N$ ABY THR-BOOC $50-800$ ACT -1 4PI 69BE6 53I 127 G, 3N ABX 27-31 D 9- 31 MOD-I 4PI 69DE I $531127 \quad G, 3 N$ ABY THR-999 C 2- 6 ACT-I 4PI G9VE2 531 $127 \quad 999=5.5$ GEV $6 B J 01531.127$ G.4N ABY THR-800 C 50-800 ACT-I 4P $\begin{array}{llllllll}6 B J 01 & 531 & 127 & G .4 N & A B Y & T H R-800 & 50-800 & \text { ACT } \\ 68 J 01 & 531 & 127 & G .6 N & 4 P 1\end{array}$ $7010153 I 127 \quad$ G.6N ABY THR-900 C 75-900 ACT-1 4PI $68 J 0153 I 127 \quad G, 7 N$ AEY THR-800 C 50-800 ACT-I 4 P I

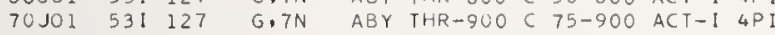
$70 J 0153 I 127 \quad G, 3 N$ ABY THR-900 C 75-900 ACT-I 4PI $70 J 0153 ! 127$ G.9N ABY THR-900 C 75-900 ACT-! 4PI $56 G A I 53 I 127$ G XXN ABX 9- $27 \mathrm{C} 9-27 \mathrm{BF} 3-\mathrm{I} 4 \mathrm{PI}$ 58FUI 53I 127 G.XN ABX 7- $40 \mathrm{C} \quad 7-40$ BF3-I $4 \mathrm{P}$

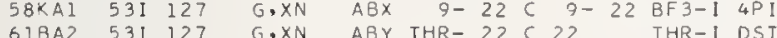

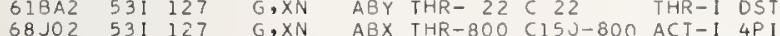

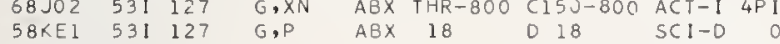
$59801531 \quad 127 \quad G, P \quad A B X \quad 15,18015,1 B \quad$ SCI-D 4PI 6OTAL $53 I 127$ G, P ABX 14- 32 C 14- 32 SCI-D 4PI $615 E 453 I 127 \quad G, P \quad A B X \quad 15,1 B$ D 15, IB SCI-D 4P CSI TARGET, SPC ACT-I 4 P I

XENON $Z=54$

\begin{tabular}{|c|c|c|c|c|c|c|c|c|}
\hline \multirow{2}{*}{ ABUND. } & \multicolumn{8}{|c|}{ SEPARATION ENERGIES (MEV) } \\
\hline & $\mathrm{G}, \mathrm{N}$ & $G, P$ & $G, T$ & G.HE3 & $G, A$ & $\mathrm{G}, 2 \mathrm{~N}$ & G,NP & $G, 2 P$ \\
\hline 3.10 & $10 \cdot 2$ & $6 \cdot B$ & 16.2 & 13.8 & 0.5 & $18 \cdot 5$ & 16.6 & 11.7 \\
\hline 0.09 & $10 \cdot 1$ & $7 \cdot 6$ & 16. & 14.9 & $1 \cdot 3$ & 17.9 & 17.2 & 13.2 \\
\hline 1.92 & 9.6 & 3.2 & 15. & 15.8 & 1.8 & 15.8 & $17 \cdot 3$ & 14.4 \\
\hline 26.44 & 6.9 & 8.2 & 15.7 & 13.6 & 2 . & 16.5 & 15.1 & 15. \\
\hline $4.0 B$ & $9 \cdot 3$ & 8.7 & 15.8 & 16.5 & 2 . & 16.2 & 17 & 15. \\
\hline $21 \cdot 18$ & 5.6 & 8.8 & 15 & 14.4 & ?. & 15.9 & 15 & 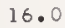 \\
\hline 26.89 & 8.9 & 9.1 & 15.7 & 17.2 & 2.7 & 15.5 & 17.8 & 6 \\
\hline 10.44 & $3 \cdot 5$ & 9.6 & 15.6 & 17.9 & 3.2 & 15.0 & $17 \cdot B$ & $17 \cdot 5$ \\
\hline 8.87 & 8.0 & 9.9 & 15.5 & 18.5 & 3.7 & 14.4 & $17 . \mathrm{B}$ & 18. \\
\hline
\end{tabular}

$\begin{array}{lcll} & \text { NUCLIDE REACTION RES EXCIT SOURCE DETECTOR } \\ \text { REF } 2 \text { A IN,OUT } & \end{array}$

69HOI 54XE GPXP ABY THR- 33 C 24- 33 SCI-D 90

$\begin{array}{ccccccc}70 K E 2 & 54 \times E 130 \quad G, G \quad A B X \quad 1 & D & 1 & \text { SCD-D } 45 \\ \text { LFT }, .536 & \text { MEV LEVEL }\end{array}$

7OBE7 54XE131 G,G LFT O- 1 D O- I NAI-D DST
A ABUND.

$133100.00 \quad G, N \quad G, P$ G,T G,HE3 G,A G,2N G, NP G, 2P NUCLIOE REACTION RES EXCIT SOURCE DETECTUR

\begin{tabular}{|c|c|c|c|c|c|c|c|}
\hline $58 \mathrm{CH} 2$ & $55 C 5133$ & $G, N$ & RLY & THR & CTHR & $\begin{array}{l}\text { BF 3-1 } 4 P \text { I } \\
T H R E S H O L D\end{array}$ & \\
\hline 6OGE 3 & $55 C \$ 133$ & $G, N$ & NOX & THR & CTHR & $\begin{array}{l}\text { BF 3-I 4PI } \\
\text { THRE SHOLD }\end{array}$ & \\
\hline $67 \mathrm{HUI}$ & $55 C S 133$ & $G, N$ & $A B X$ & 10,11 & D 10,11 & $B F 3-14 P I$ & \\
\hline $698 \mathrm{El}$ & $55 \mathrm{CSI}_{133}$ & $G, N$ & $A B X$ & THR - 30 & $D 9-30$ & $B F 3-I \quad 4 P I$ & $250+$ \\
\hline $698 \mathrm{BE}$ & $55 C S 133$ & $G, 2 N$ & $A B X$ & THR - 30 & D $16-30$ & $8 F 3-14 \mathrm{PI}$ & 152 \\
\hline $9 B E 1$ & 5505133 & $G, 3 N$ & $A B X$ & THR - 30 & D $26-30$ & BF $3-I \quad 4 P I$ & 153 \\
\hline $\mathrm{KA3}$ & $55 \operatorname{csi} 33$ & $G, 4 N$ & RLY & $T H R-250$ & C $150-250$ & $\begin{array}{l}A C T-I \quad 4 P I \\
R E L(G, N)\end{array}$ & \\
\hline$\times A 3$ & $55 C \$ 133$ & $G, 6 N$ & RLY & $T H R-250$ & C $150-250$ & $\begin{array}{l}A C T-I \quad 4 P I \\
R E L(G, N)\end{array}$ & \\
\hline $58 \mathrm{KAl}$ & $55 \operatorname{csl} 33$ & $G, \times N$ & $A B x$ & $9-22$ & $9-22$ & $8 F 3-I \quad 4 P I$ & \\
\hline $58 K E 1$ & $55 C 5133$ & $G, P$ & $A B X$ & 18 & D 18 & $\begin{array}{l}\text { SCI-D O } \\
\text { SPECTRUM }\end{array}$ & \\
\hline 3801 & 5505133 & $G, P$ & $A B X$ & 15,18 & D 15,18 & SCI-D $4 \mathrm{PI}$ & \\
\hline & $55 \mathrm{CS}_{133}$ & $G, P$ & $A B X$ & $14-32$ & $\begin{array}{c}14-32 \\
S P C,\end{array}$ & $\begin{array}{l}\text { SCI-D } 4 \text { PI } \\
\text { CSI TARGET }\end{array}$ & \\
\hline $615 E 4$ & 5505133 & $G, P$ & $A 8 x$ & 15,18 & D 15,18 & $\begin{aligned} \text { SCI-D } & 4 P I \\
\text { ARGET, } & \text { SPC }\end{aligned}$ & \\
\hline$K A 3$ & $55 C 5133$ & $G, 2 P$ & RLY & $T H R-250$ & C150-250 & $A C T-I \quad 4 P I$ & \\
\hline $70 \times A 3$ & $55 C S 133$ & $G, 2 P N$ & RLY & THR -250 & C15u-250 & $\begin{array}{r}R E L(G, N) \\
A C T-14 P 1 \\
R E L(G, N)\end{array}$ & \\
\hline $70 K A 3$ & $55 C S 133$ & $G, J N$ & RLY & THR -250 & C150-250 & $A C T-I \quad 4 P I$ & 1 \\
\hline
\end{tabular}

BARIUIM $Z=56$

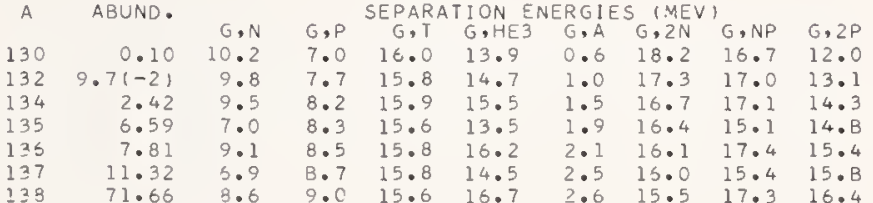

NUCLIDE REACTION RES EXCIT SOURCE DETECTOR

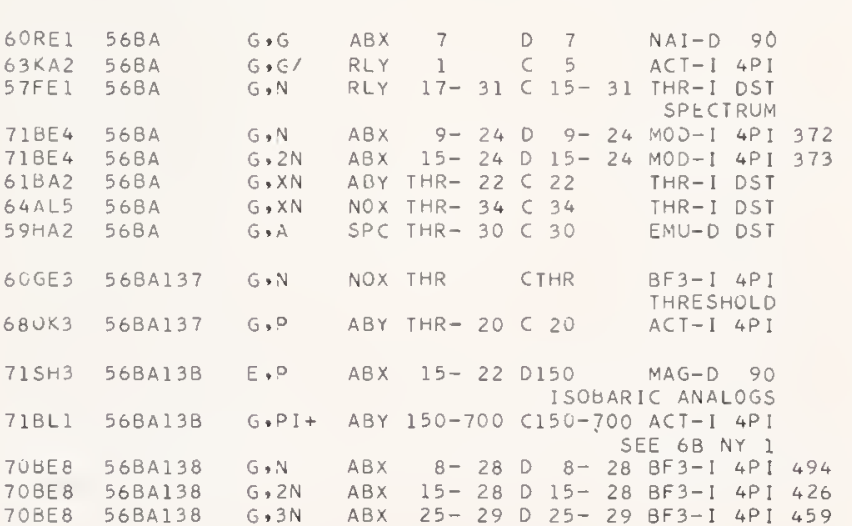

LANTHANUM $z=57$

ABUND.

SEPARATION ENERGIES IMEV

$\begin{array}{llllllllll}13 B & 8.9(-2) & G, N & G, P & G, T & G, H E 3 & G, A & G, 2 N & G, N P & G, 2 P \\ & 7.3 & 6.0 & 13.6 & 13.8 & 2.0 & 16.6 & 12.9 & 14.7\end{array}$

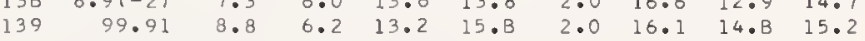

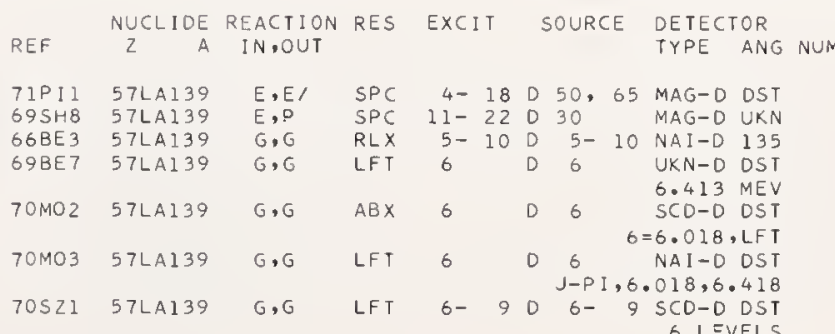


58CH2 57LA139 G, N RLY THR CTHR BF 3-I 4PI THRESHOLO GOGE3 5TLAI39 G,N NOX THR IB C 8- 18 BF 3-I 4P.

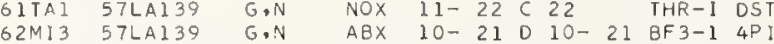
62REI 5TLA139 G,N NOX $11-55$ C 55 THR-1 OST 67HU1 57LA139 G,N ABX 9-11 D 9- 11 BF3-1 4PI GBBES $57 L A 139$ G,N ABX THR- 30 D $7-30$ MOD-I $4 P 1$ GBJUI 57LAL39 G,N NOX THR- 27 C 27 THR-I DST 6BBE5 57LA139 G, $2 \mathrm{~N}$ ABX THR- 30 D 7 - 30 MOD-I $4 \mathrm{PI} 9$

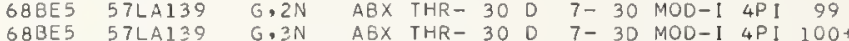
$5 B F U 1$ 57LAI39 G,XN ABY $7-40 \mathrm{C}$ 7- 40 BF3-I $4 \mathrm{PI}$ 58KAI 57LA139 G,XN ABX 9- 22 C 9- 22 BF3-1 4PI 61BA2 $57 L A 139$ G,XN ABY THR- 22 C 22 THR-1 DST GIMI1 57LA139 G.XN ABX 10-22 D 10- 22 BF3-1 4P I 64RI1 57LA139 G,XN ABX 8- 30 C 8 - 30 BF3-I 4PI 72DE3 57LA139 G,XN ABX 9- 24 C 8- 24 BF3-1 4PI 559 T2TH2 57LAL39 G,XN ABX 9- $17 \mathrm{C} 8-16$ BF3-I 4PI

$71 B E 4$ 57LAI39 G.SN ABX 8- 18 D $8-18$ MOD-I $4 P$ I
$\begin{array}{llrrrrr}G . S N & A B X & 8-18 & 0 & 8-18 & \text { MOD-I } & 4 P \text { I } \\ G, F & A B Y & -1 H R-900 & (300-900 & \text { TRK }-1 & 4 P I\end{array}$

\begin{tabular}{rrrrrrrrrr} 
A & ABUND. & \multicolumn{1}{c}{ SEPARATION ENERGIES (MEV) } \\
& & G.N & G.P & G.T & G.HE3 & G.A & G.2N & G.NP & G.2P \\
136 & 0.19 & 10.0 & 6.9 & 15.7 & 13.8 & 0.4 & 17.9 & 16.6 & 12.1 \\
138 & 0.25 & 9.6 & 7.6 & 15.7 & 14.6 & 1.0 & 17.2 & 16.9 & 13.2 \\
140 & 88.48 & 9.2 & 8.1 & 15.8 & 15.2 & 1.6 & 16.7 & 16.9 & 14.3 \\
142 & 11.07 & 7.2 & 8.8 & 12.3 & 14.5 & -1.4 & 12.6 & 15.6 & 15.8
\end{tabular}

PRASEOOYMIUM $Z=59$

ABUNO.

G.N G,P G,T G,HE3 G,A G,2N G,NP G,2P

\begin{tabular}{cccll}
\multicolumn{1}{c}{ NUCLIDE REACIION RES EXCIT SOURCE } & DETECTOR \\
REF & $\mathrm{Z}$ A IN,OUT & & & TYPE ANG NUM
\end{tabular}

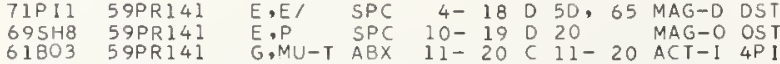
$71 A H 1$ 59PRI4I G,MU-T ABX THR-150 C $10-150$ MGC-O $4 P I$ 62BE2 59PR141 G,C ABX 5- 9 D 5- NAI-D 135 64ARI 59PR141 G,G ABX 6- 90 6- NAI-0 135 66BE3 59PR141 G,G RLX 5- 100 5- 10 NAI-D 135 $68 M 01$ 59PRI41 B G, G NOX 7 D 7 NAI-O 90 69M11 59PRI41 G.6 SPC 6- POLARIMETER 7.632

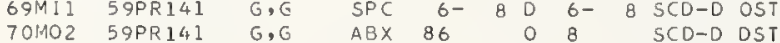

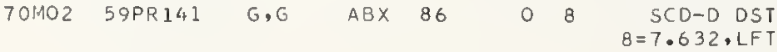
$71 P A 2$ 59PRI4L G.G LFT $6-90$ 6- $9=7.632$. 5 CD OST

\begin{tabular}{|c|c|c|c|c|c|c|c|c|c|}
\hline $58 \mathrm{CH} 2$ & $59 P R 141$ & $G, N$ & RLY & THR & & CTHR & & $\begin{array}{l}\text { BF 3-I 4PI } \\
\text { THRE SHOLD }\end{array}$ & \\
\hline $59 C A 2$ & $59 P R 141$ & $G, N$ & $A B X$ & $10-$ & 32 & C 33 & & $A C T-I \& P I$ & \\
\hline $59 C A 3$ & $59 P R 141$ & $G, N$ & $A B X$ & $10-$ & 32 & C $10-$ & 32 & $A C T-1 \quad 4 P I$ & \\
\hline 59011 & $59 P R 141$ & $G, N$ & RLY & $9-$ & 30 & C 30 & & $A C T-1 \quad 4 P I$ & \\
\hline $59 F E 2$ & $59 P R 141$ & $G, N$ & $A B X$ & $9-$ & 30 & $C 30^{\circ}$ & & $\begin{array}{l}\text { ACT - I } 4 \text { P I } \\
\text { THRESHOLD }\end{array}$ & \\
\hline $60 G E 3$ & $59 P R 141$ & $G, N$ & NOX & THR & & CTHR & & $\begin{array}{l}\text { ACT-I } 4 P \text { I } \\
\text { THRESHOLD }\end{array}$ & \\
\hline $61 \mathrm{BO} 1$ & $59 P R 141$ & $G, N$ & $A B X$ & $9-$ & 29 & C $10-$ & 29 & $A C T-1 \quad 4 P I$ & \\
\hline $61 \mathrm{CO} 2$ & $59 P R 141$ & $G \cdot N$ & $A B X$ & 15 , & 18 & D 15, & 18 & $A C T-I \quad 4 P I$ & \\
\hline $61 \mathrm{TA} 1$ & 59PR 141 & $G, N$ & NOX & $12-$ & 22 & C 22 & & THR-I DST & \\
\hline $620 E 1$ & $59 P R 141$ & $G, N$ & $A B X$ & 20 & & D 21 & & $A C T-1 \quad 4 P I$ & \\
\hline $62 \mathrm{REI}$ & $59 P R 141$ & $G, N$ & NOX & $6-$ & 55 & C 55 & & THR-I DST & \\
\hline 66BR1 & $59 P R 141$ & $G, N$ & $A B X$ & THR- & 33 & $08-$ & 33 & $\mathrm{BF} 3-\mathrm{I} \quad 4 \mathrm{PI}$ & 304 \\
\hline $66 C 03$ & $59 P R 141$ & $G, N$ & $A B X$ & THR - & 65 & CTHR - & 70 & $A C T-I \quad \& P I$ & $114 t$ \\
\hline $67 \mathrm{CA} 1$ & $59 P R 141$ & $G, N$ & $R L X$ & $9-$ & 17 & C 9- & 17 & $A C T-I \quad 4 P I$ & \\
\hline $67 \mathrm{HUI}$ & $59 P R 141$ & $G, N$ & $A B X$ & 10 , & 11 & D 10 , & 11 & $B F 3-1 \quad 4 \mathrm{PI}$ & \\
\hline $68 \mathrm{CAI}$ & $59 \mathrm{PR} 141$ & $6, N$ & $A B X$ & $9-$ & 17 & $9-$ & 17 & $A C T-I \quad 4 P I$ & $69+$ \\
\hline $68 \mathrm{JUI}$ & $59 P R 141$ & $G, N$ & NOX & THR - & 30 & C $30-$ & & THR-I OST & \\
\hline 70 SUI & $59 P R 141$ & $G, N$ & $A B X$ & $9-$ & 24 & D 9- & 24 & $A C T-1$ 4PI & 277 \\
\hline $718 E 4$ & $59 P R 141$ & $G, N$ & $A B X$ & $9-$ & 17 & D 9- & 17 & MOD-I $4 P$ I & 390 \\
\hline $720 R 2$ & $59 P R 141$ & $G, N$ & $A B X$ & $9-$ & 20 & $9-$ & 20 & $A C T-I \quad 4 P I$ & 447 \\
\hline $59 C A 2$ & $59 P R 141$ & $G, 2 N$ & $A B X$ & $18-$ & 32 & C 33 & & $\begin{array}{l}\text { ACT-I } 4 P I \\
\text { THRE SHOLO }\end{array}$ & \\
\hline 59FE2 & $59 P R 141$ & $G, 2 N$ & $A B X$ & $16-$ & 30 & C 30 & & $\begin{array}{l}\text { ACT-I } 4 P I \\
\text { THRE SHOLD }\end{array}$ & \\
\hline 61801 & $59 P R 141$ & $G, 2 N$ & $A B X$ & $16-$ & 29 & C $10-$ & 29 & $A C T-1 \quad 4 \mathrm{PI}$ & \\
\hline $668 R I$ & $59 P R 141$ & $G, 2 \mathrm{~N}$ & $A B X$ & THR- & 30 & $8-$ & 33 & $B F 3-I 4 P I$ & 305 \\
\hline $61 M O 1$ & $59 P R 141$ & $G, 3 N$ & $A B I$ & $27-$ & 33 & C $27-$ & 33 & $\begin{array}{l}\text { ACT-I } 4 P I \\
\text { THRE SHOLD }\end{array}$ & \\
\hline $668 \mathrm{RI}$ & $59 P R 141$ & $G, 3 N$ & $A B X$ & THR - & 30 & $8-$ & 33 & $\mathrm{BF} 3-\mathrm{I} 4 \mathrm{PI}$ & $306+$ \\
\hline $58 \mathrm{KAl}$ & $59 P R 141$ & $G, \times N$ & $A B X$ & $10-$ & 22 & C $10-$ & 22 & $\mathrm{BF} 3-14 \mathrm{PI}$ & \\
\hline $60 \mathrm{THI}$ & 59PR 141 & $G, X N$ & $A B X$ & $9-$ & 18 & $7-$ & 18 & $B F 3-14 P I$ & \\
\hline 64R II & $59 P R 141$ & $G, \times N$ & $A B X$ & $9-$ & 30 & $9-$ & 30 & $B F 3-1 \quad 4 P I$ & \\
\hline $70 M C ?$ & 59PRI41 & $G, \times N$ & $S P C$ & $11-$ & 28 & C 28 & & TOF-D 98 & \\
\hline $720 E^{3}$ & $59 P R 141$ & $G, \times N$ & $A B X$ & $9-$ & 22 & $9-$ & 22 & $B F 3-14 \mathrm{PI}$ & 560 \\
\hline $61 \mathrm{SH} 2$ & $59 P R 141$ & $G, P$ & $A B Y$ & $5-$ & 34 & $C 23$, & 34 & EMU-D DST & \\
\hline $625 H 4$ & $59 P R 141$ & $G, P$ & $S P C$ & $5-$ & 34 & C 23 , & 34 & EMU-O OST & \\
\hline $69 E J 1$ & 59PR 141 & $P \cdot G$ & AEX & 15 & & D $9-$ & $\begin{array}{l}11 \\
15\end{array}$ & $\begin{aligned} & S C 1-0 \text { DST } \\
= & 14.95 \mathrm{MEV}\end{aligned}$ & \\
\hline
\end{tabular}

NEODYMIUM $Z=60$
REF $Z$ A IN,OUT RYPE ANG NUM

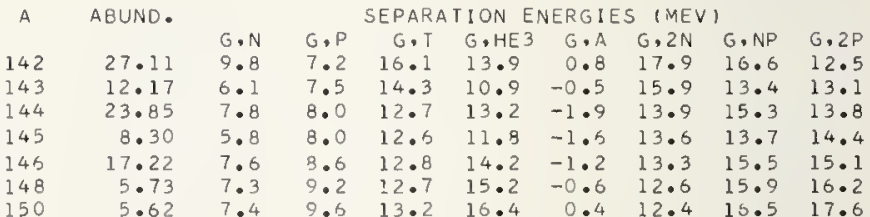

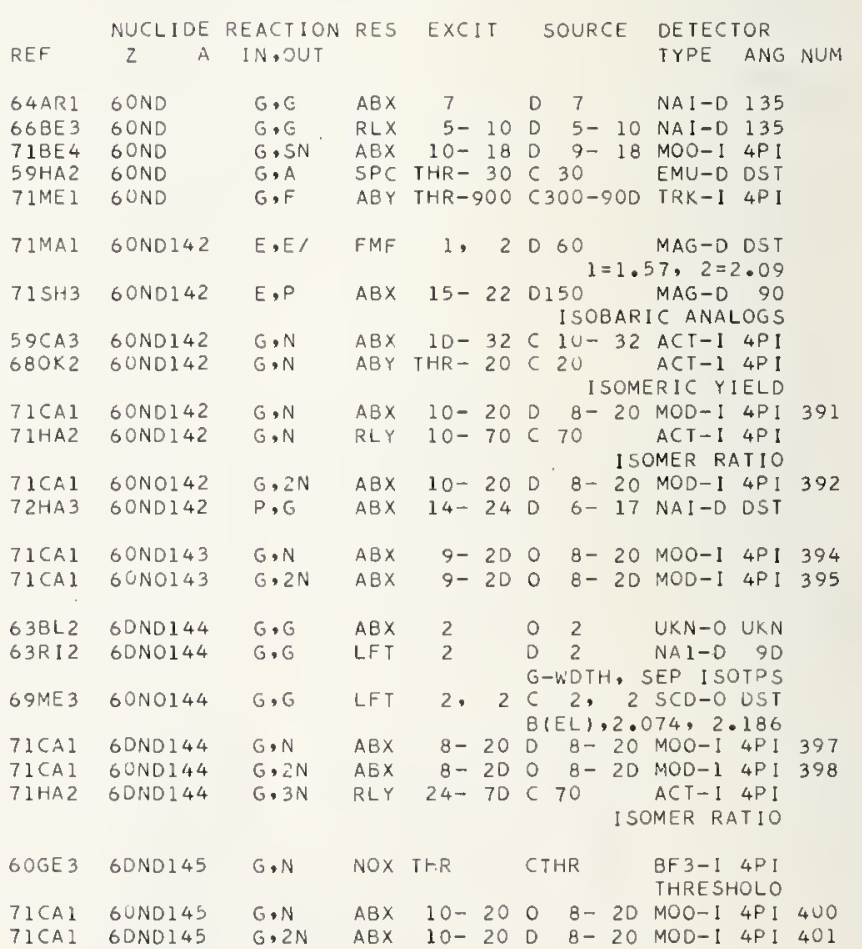




\begin{tabular}{|c|c|c|c|c|c|c|c|c|}
\hline TIMAI & $60 N 0146$ & $E \cdot E /$ & FMF & 0 & D & 41 & $\begin{array}{c}\text { MAG }-D \text { DS T } \\
0=0.45\end{array}$ & \\
\hline $71 C A 1$ & $60 N D 146$ & $G, N$ & $A B X$ & $8-20$ & 0 & $8-20$ & MOD-I 4PI & 403 \\
\hline $71 C A 1$ & 60N0146 & $\mathrm{G}, 2 \mathrm{~N}$ & $98 x$ & $8-20$ & D & $8-20$ & MOO-I $4 \mathrm{PI}$ & 404 \\
\hline $71 V A 2$ & $60 N 0146$ & $G \cdot \times N$ & $A 8 x$ & $7-24$ & c & $7-24$ & $8 F 3-I 4 P I$ & \\
\hline $71 C A 1$ & $60 N O 148$ & $G \cdot N$ & $A B X$ & $8-20$ & 0 & $8-20$ & $M D 0-1 \quad 4 P I$ & 406 \\
\hline $71 C A 1$ & $60 N 0148$ & $G, 2 \mathrm{~N}$ & $A B x$ & $8-20$ & D & $8-20$ & $M D O-I \quad 4 P I$ & 407 \\
\hline 69VA2 & $60 N D 148$ & $G, \times N$ & $A B x$ & $8-23$ & $c$ & $8-23$ & $8 F 3-I \quad 4 P I$ & 208 \\
\hline $69 V A 3$ & $60 N O 148$ & $G, \times N$ & $A B X$ & $8-22$ & $c$ & $8-22$ & $8 F_{3-I} 4 \mathrm{PI}$ & 238 \\
\hline & & & & & & & SEE $69 \mathrm{VA} 2$ & \\
\hline $71 M A 1$ & $60 N D 150$ & $E, E /$ & FMF & 0 & 0 & 60 & $\begin{array}{c}M A G=0 \text { DST } \\
0=0.13\end{array}$ & \\
\hline $59 C A 3$ & $60 N 0150$ & $G, N$ & $R L x$ & $8-32$ & c & $8-32$ & $A C T-I \quad 4 P I$ & \\
\hline $71 C A 1$ & 60NO15O & $G \cdot N$ & $A B x$ & $10-20$ & D & $8-20$ & $M D D-1 \quad 4 P I$ & 409 \\
\hline $71 C A 1$ & $60 N 0150$ & $\mathrm{G}, 2 \mathrm{~N}$ & $A B X$ & $10-20$ & 0 & $8-20$ & $M O D-1 \quad 4 \mathrm{PI}$ & 410 \\
\hline 69VA2 & $60 N D 150$ & $G, \times N$ & $A B X$ & $8-23$ & $c$ & $8-23$ & $8 F 3-I \quad 4 P I$ & $209+$ \\
\hline $69 \vee A 3$ & 60ND15O & $G, \times N$ & $A B X$ & $8-22$ & c & $8-22$ & $8 F 3-14 P I$ & $239+$ \\
\hline
\end{tabular}

SAMARIUM $Z=62$

\begin{tabular}{|c|c|c|c|c|c|c|c|c|c|}
\hline \multirow[t]{2}{*}{ A } & \multirow[t]{2}{*}{ ABUNO. } & \multicolumn{8}{|c|}{ SEPARATION ENERGIES (MEV) } \\
\hline & & $G, N$ & $G, P$ & G.T & G.HE3 & G, A & $\mathrm{G}, 2 \mathrm{~N}$ & $G, N P$ & $G, 2 P$ \\
\hline 144 & 3.09 & 10.6 & 6.3 & 16.4 & 12.7 & $-0 \cdot 1$ & 19.0 & $16 \cdot 2$ & 10 . \\
\hline 147 & 14.97 & 6.4 & 7.1 & $12 \cdot 9$ & 10.5 & $-2 \cdot 3$ & 14.8 & 13.4 & 2 \\
\hline 148 & 11.24 & $8 \cdot 1$ & 7.6 & 13.0 & 12.8 & $-2 \cdot 0$ & 14.5 & $15 \cdot 3$ & 3.0 \\
\hline 149 & 12.93 & 5 . & 7.6 & 12.6 & 11.2 & $-1 \cdot 9$ & 14.0 & 13.5 & 3 \\
\hline 150 & 7.44 & 8.0 & $8 \cdot 3$ & 13.0 & 13.8 & $-1 \cdot 4$ & 13.9 & 15.5 & 4 . \\
\hline $1 \leq 2$ & $26 \cdot 72$ & $8 \cdot 3$ & 8.7 & 13.7 & 15.3 & -0.2 & 13.9 & 16.6 & \\
\hline 154 & 22.71 & 8.0 & 9.0 & 14.0 & 16.5 & 1.2 & 13.8 & 16.5 & 6 . \\
\hline
\end{tabular}

\begin{tabular}{|c|c|c|c|c|c|c|c|c|c|c|}
\hline \multirow[b]{2}{*}{ REF } & NUCLIDE & \multirow{2}{*}{$\begin{array}{l}\text { REACTIDN } \\
\text { IN.DUT }\end{array}$} & \multirow[t]{2}{*}{ RES } & \multirow{2}{*}{\multicolumn{2}{|c|}{ EXCIT }} & \multirow{2}{*}{\multicolumn{2}{|c|}{ SDURCE }} & \multicolumn{3}{|c|}{ DETECT JR } \\
\hline & A & & & & & & & TYPE & ANG & NUM \\
\hline 6OREI & $625 M$ & $G, G$ & $A 8 x$ & 7 & & 0 & 7 & NA I - D & 90 & \\
\hline $62 B E 2$ & $625 \mathrm{M}$ & $G, G$ & $A B X$ & $5-$ & 9 & D & $5-$ & NAI - D & 135 & \\
\hline $64 A R 1$ & $625 M$ & $G, G$ & $A B X$ & 9 & & 0 & 9 & NAI -D & 135 & \\
\hline $698 \mathrm{E} 6$ & $625 \mathrm{M}$ & $\mathrm{G} \cdot \mathrm{N}$ & $A B X$ & $7-$ & 21 & 0 & $7-27$ & MDD-1 & 4P I & \\
\hline 69856 & $625 M$ & $\mathrm{G}, 2 \mathrm{~N}$ & $A 8 x$ & $13-$ & 25 & D & $7-27$ & MDO-1 & $4 \mathrm{PI}$ & \\
\hline $69 B E 6$ & $625 M$ & $\mathrm{G}, 3 \mathrm{~N}$ & $A B X$ & $23-$ & 27 & D & $7-27$ & MDO-1 & 4PI & \\
\hline 58FUl & $625 \mathrm{M}$ & $G, \times N$ & $A 8 x$ & $7-$ & 40 & $c$ & $7-40$ & $8 F 3-1$ & 4P I & \\
\hline 59HAZ & $625 M$ & $G, A$ & $S P C$ & THR- & 30 & c & 30 & EMU-D & OST & \\
\hline 69548 & $62 S M 144$ & $E, P$ & $S P C$ & $11-$ & 20 & 0 & 20 & $M A G-D$ & URN & \\
\hline $715 H_{3}$ & $625 M 144$ & $E, P$ & $A B X$ & $15-$ & 22 & & 150 & MAG-D & 90 & \\
\hline & & & & & & & ISOBAR I & IC ANAL & DGS & \\
\hline $66 \mathrm{eE}^{3}$ & $625 M 144$ & $G, G$ & $R L X$ & $5-$ & 10 & D & $5-10$ & NAI I D & 135 & \\
\hline $72 A R 1$ & $625 M 144$ & G. G & LFT & 9 & & $\begin{array}{l}0 \\
S\end{array}$ & ${ }^{9}{ }^{9} 66 \mathrm{BE} 3$ & $\begin{array}{c}S C D-D \\
3, \quad 9=8 .\end{array}$ & $\begin{array}{r}135 \\
998\end{array}$ & \\
\hline $550 \mathrm{E} 1$ & $625 M 144$ & $G, N$ & $A B X$ & $9-$ & 23 & c & $9-23$ & $A C T-I$ & $4 \mathrm{PI}$ & \\
\hline 56511 & 62 SM: 44 & $\mathrm{G}, \mathrm{N}$ & $A 8 x$ & 9- & 22 & $c$ & 22 & $\begin{array}{l}\text { ACT }-1 \\
\text { THRE SH }\end{array}$ & $\begin{array}{l}4 P I \\
\text { ADLD }\end{array}$ & \\
\hline $59 \mathrm{CA}_{3}$ & $625 M 144$ & $G, N$ & $A B X$ & $10-$ & 32 & c & $10-32$ & $A C T-1$ & $4 \mathrm{PI}$ & \\
\hline 59011 & $625 M 144$ & $G, N$ & RLY & $11-$ & 30 & $c$ & $\begin{array}{l}22,30 \\
\text { REL C }\end{array}$ & $\begin{array}{l}A C T-I \\
C U 63 \quad 16\end{array}$ & $\begin{array}{l}4 P 1 \\
. N 1\end{array}$ & \\
\hline 65MEI & $625 M 148$ & $G, G$ & LFT & 1 & & D & 1 & $\begin{array}{l}\text { NAI }-0 \\
1=1.46\end{array}$ & $\begin{array}{l}\text { DST } \\
\text { MEV }\end{array}$ & \\
\hline 71 VA2 & $625 M 148$ & $G, \times N$ & $A B X$ & $8-$ & 24 & $c$ & $8-24$ & BF 3-I & $4 \mathrm{PI}$ & \\
\hline 6OGE 3 & $625 M 149$ & $G, N$ & NOX & THR & & & THR & $\begin{array}{l}\text { BF } 3-1 \\
\text { THRE SH }\end{array}$ & $\begin{array}{r}4 P I \\
4 D L D\end{array}$ & \\
\hline G9VAZ & $62 S M 150$ & $G, X N$ & $A B x$ & $8-$ & 23 & $c$ & $8-23$ & $B F 3-1$ & 4PI & \\
\hline $728 \mathrm{E} 3$ & $625 M 152$ & $E, E /$ & FMF & $0-$ & 1 & $\begin{array}{r}D \\
12\end{array}$ & $\begin{array}{l}50-105 \\
2 .-367\end{array}$ & $\begin{array}{l}\text { MAG-D } \\
\text { MEV LEV }\end{array}$ & $\begin{array}{l}\text { OST } \\
\text { ELS }\end{array}$ & \\
\hline 65MEI & $625 M 152$ & $G, C$ & LFT & 1 & & D & 1 & $\begin{array}{l}\text { NAI }-D \\
I=0.96\end{array}$ & $\begin{array}{l}105 \\
\text { MEV }\end{array}$ & \\
\hline $678 E 4$ & $625 M 152$ & $G, G$ & LFT & 1 & & D & $1=$ & $\begin{aligned} & N A 1-D \\
= & 0.963\end{aligned}$ & $\begin{array}{l}120 \\
M E V\end{array}$ & \\
\hline 68TA2 & $625 M 152$ & $G, G$ & LFT & 1 & & D & $1=$ & $\begin{aligned} & N A I-D \\
= & 0.963\end{aligned}$ & $\begin{array}{r}90 \\
\text { MEV }\end{array}$ & \\
\hline $69 \mathrm{VAZ}$ & 62SM152 & $G, \times N$ & $A B X$ & $8-$ & 23 & $c$ & $8-23$ & $8 F 3-I$ & 4PI & 211 \\
\hline $69 \mathrm{VA} 3$ & $625 M 152$ & $G, X N$ & $A B X$ & $8-$ & 22 & $c$ & $8-22$ & $\begin{array}{l}8 F 3-1 \\
\text { SEE } 69\end{array}$ & $\begin{array}{l}\text { API } \\
P \vee A 2\end{array}$ & $241+$ \\
\hline $69 \mathrm{MCi}$ & $625 M 154$ & $E, F$ & $A B X$ & $T H R-9$ & 99 & $D$ & $60-999$ & $\begin{array}{l}\text { TRK }-1 \\
999=1\end{array}$ & $\begin{array}{l}\text { OST } \\
\text { GEV }\end{array}$ & \\
\hline $59 C A 3$ & $625 M 154$ & $G, N$ & $A B X$ & $8-$ & 32 & C & $8-32$ & $A C T-1$ & 4PI & \\
\hline $69 \mathrm{VAZ}$ & $625 M 154$ & $G, \times N$ & $A B X$ & $8-$ & 23 & C & $8-23$ & $B F 3-I$ & 4P] & 212 \\
\hline $69 \vee A 3$ & 625,4154 & $G, \times N$ & $A B X$ & $8-$ & 22 & . $c$ & $8-22$ & $\begin{array}{l}B F 3-1 \\
\text { SEE } 69\end{array}$ & $\begin{array}{l}4 P 1 \\
\text { VA2 }\end{array}$ & 212 \\
\hline $62 K D 3$ & $625 M 154$ & $G, P$ & RLX & THR- & 20 & $c$ & 20 & $\begin{array}{l}\text { ACT-I } \\
\text { NEUTR }\end{array}$ & $\begin{array}{r}4 P 1 \\
\text { RDNS }\end{array}$ & \\
\hline 691401 & $625 M 154$ & $G \cdot F$ & $A B X$ & THR -9 & & $c$ & $60-999$ & $\begin{array}{l}\text { TRK-1 } \\
999=1\end{array}$ & $\begin{array}{l}\text { DST } \\
\text { GEV }\end{array}$ & \\
\hline
\end{tabular}

\begin{tabular}{|c|c|c|c|c|c|c|c|c|c|c|c|}
\hline A & ABUNO. & & SE & PARATI & IDN & & JERG I & IES & (MEV) & & \\
\hline & & $\mathrm{G}, \mathrm{N}$ & & $\mathrm{G}, \mathrm{T}$ & G. HE & E3 & $G, A$ & & $\mathrm{G}, 2 \mathrm{~N}$ & $G, N P$ & $\mathrm{G}, 2 \mathrm{P}$ \\
\hline 151 & 47.82 & 8.0 & & 0.3 & 12.7 & & $-2 \cdot 0$ & & 14.4 & 12.9 & 13.2 \\
\hline 53 & 52.18 & 8.6 & & 1.3 & 14.8 & & -0.3 & & 14.9 & 14.2 & 14.6 \\
\hline & NUCLIDE & REACTION & RES & $E \times C 1$ & & & DURC & & DETEC & ETOR & \\
\hline REF & Z A & IN,DUT & & & & & & & TYPE & ANG & NUM \\
\hline $67 \mathrm{HUI}$ & $63 E U$ & $G, N$ & $A B X$ & $9-$ & 11 & D & $9-$ & 11 & $B F 3-I$ & $4 \mathrm{PI}$ & \\
\hline 6OGE 3 & $63 E \cup 151$ & $G \cdot N$ & NDX & THR & & & $H R$ & & $8 F 3-I$ & $4 P I$ & \\
\hline & & & $\Delta 8 X$ & & & 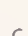 & & & THRES & HOLD & \\
\hline 7OVAI & 63EU151 & $\begin{array}{l}G, X N \\
G, X N\end{array}$ & $A 6 x$ & $8-$ & 24 & $c$ & $8-$ & 24 & $\begin{array}{l}8+3-1 \\
B F=3-1\end{array}$ & $4 P_{1}$ & 488 \\
\hline $71 V 42$ & 63 EUI5I & $G, \times N$ & $A B X$ & $7-$ & 24 & c & $7-$ & 24 & $B F 3-I$ & $4 \mathrm{PI}$ & \\
\hline G6ATI & 63 EU153 & $G, G$ & LFT & 1 & & D & 1 & & $S C D-D$ & UKN & \\
\hline $60 \mathrm{GE}_{3}$ & $63 E U 153$ & $G, V$ & NDX $x$ & THR & & & $H R$ & & $\begin{array}{r}1=97 \\
8=3-1\end{array}$ & KEV & \\
\hline & & & & & & & & & THRE S & HDLD & \\
\hline $698 E 8$ & $63 E U 153$ & $G \cdot N$ & $A 8 X$ & $8-$ & 29 & D & $8-$ & 29 & $\begin{array}{r}B F 3-1 \\
\text { NCLUDE }\end{array}$ & $\begin{array}{r}4 P 1 \\
=5 \quad N P\end{array}$ & $154+$ \\
\hline $698 \mathrm{E} 8$ & $63 E U 153$ & $G, 2 N$ & $A B X$ & $8-$ & 29 & 0 & 8- & 29 & $\begin{array}{r}\text { BF } 3-\text { I } \\
\text { CLUDES }\end{array}$ & $\begin{array}{l}4 \mathrm{PI} \\
2 \mathrm{NP}\end{array}$ & 156 \\
\hline $698 \mathrm{E} 8$ & $63 E U 153$ & $\mathrm{G}, 3 \mathrm{~N}$ & $A B X$ & $8-$ & 29 & D & $8-$ & 29 & $B F 3-I$ & $4 \mathrm{PI}$ & 157 \\
\hline 7OSE? & $63 E \cup 153$ & $G, X N$ & $A B X$ & $8-$ & 24 & c & $8-$ & 24 & $B F 3-1$ & $4 \mathrm{PI}$ & \\
\hline TOVAI & 63 EUI53 & $G, X N$ & $A E X$ & $8-$ & 22 & $c$ & 8- & 22 & $B F 3-1$ & $4 \mathrm{PI}$ & 489 \\
\hline 71 VA2 & 63 EU153 & $G, X N$ & $A B X$ & $8-$ & 24 & $c$ & $8-$ & 24 & $8 F 3-1$ & $4 \mathrm{PI}$ & \\
\hline
\end{tabular}

GADOLINIUM $Z=64$ \begin{tabular}{crrrrrrrrr} 
A & A8UND. & \multicolumn{10}{c}{ SEPARATION ENERGIES (MEV) } \\
152 & 0.20 & G.N & G.P & G.T & G.HE3 & G.A & G.2N & G.NP & G.2P \\
154 & 2.15 & 8.7 & 7.4 & 13.3 & 12.5 & -2.2 & 15.1 & 15.3 & 12.2 \\
154 & 14.73 & 6.4 & 7.5 & 14.0 & 14.1 & -0.9 & 15.1 & 16.2 & 13.5 \\
155 & 20.47 & 8.5 & 8.0 & 14.1 & 12.2 & -0.1 & 15.1 & 14.1 & 14.1 \\
156 & 2.9 & 0.2 & 15.0 & 16.2 & 14.7 \\
157 & 15.68 & 6.4 & 8.0 & 14.1 & 13.3 & 0.7 & 14.9 & 14.4 & 15.2 \\
158 & 24.87 & 7.9 & 8.5 & 13.8 & 15.4 & 0.7 & 14.3 & 16.0 & 15.9 \\
160 & 21.90 & 7.5 & 9.3 & 13.4 & $*$ & 1.0 & 13.4 & 16.0 & $*$
\end{tabular}

\begin{tabular}{|c|c|c|c|c|c|c|c|c|c|}
\hline & NUCL I DE & REACTIDN & RES & EXCIT & \multirow{2}{*}{\multicolumn{2}{|c|}{ SDURCE }} & DETECT & TDR & \multirow{3}{*}{ NUM } \\
\hline EF & $z$ & IN, OUT & & & & & TYPE & ANG & \\
\hline IMEI & $64 \mathrm{GD}$ & $G, F$ & $A B Y$ & THR-900 & \multicolumn{2}{|c|}{$C 300-900$} & TRK-I & $4 \mathrm{PI}$ & \\
\hline OSEI & $64 G 0152$ & $G, \times N$ & $A B X$ & $8-24$ & c & $8-24$ & $B F 3-1$ & 4PI & \\
\hline OVAI & 6460152 & $G, \times N$ & $A B X$ & $8-22$ & $c$ & $8-22$ & $B F 3-I$ & 4P I & 490 \\
\hline$V A$ & $64 \mathrm{GD} 152$ & $G, \times N$ & $A B X$ & $8-24$ & $c$ & $8-24$ & BF 3-1 & 4P I & \\
\hline DSE I & $64 G D 154$ & $G, \times N$ & $A B x$ & $8-24$ & $c$ & $8-24$ & $8 F 3-1$ & $4 P I$ & \\
\hline DVAl & 64 GD 154 & $G, \times N$ & $A B X$ & $8-22$ & $c$ & $8-22$ & $8 F 3-I$ & $4 \mathrm{PI}$ & 491 \\
\hline IVA2 & $64 G 0154$ & $G, \times N$ & $A B X$ & $8-24$ & $c$ & $8-24$ & $8 F 3-1$ & 4P I & \\
\hline UDE 1 & $64 G 0155$ & $G, G$ & LFT & 1 & $\begin{array}{l}\text { D } \\
\text { SE }\end{array}$ & $\begin{array}{l}1 \\
\text { PARA TED }\end{array}$ & $\begin{array}{l}\text { NAI -D } \\
\text { I SOTD }\end{array}$ & $\begin{array}{r}125 \\
\text { DPES }\end{array}$ & \\
\hline 66843 & 6400155 & $G, G$ & LFT & 1 & $D$ & 1 & $N A 1-O$ & UKN & \\
\hline 6STI & $64 G D 155$ & $G, G$ & LFT & 1 & 0 & 1 & $\begin{array}{r}\text { NAI }-0 \\
1=60\end{array}$ & UKN & \\
\hline DSE 1 & $64 G D 156$ & $G, \times N$ & $A B X$ & $8-24$ & $c$ & $8-24$ & $8 F 3-1$ & $4 \mathrm{PI}$ & \\
\hline OVAl & $64 G 0156$ & $G, X N$ & $A B x$ & $8-22$ & $c$ & $8-22$ & BF 3-I & $4 \mathrm{PI}$ & 492 \\
\hline$I \vee A 2$ & $64 \mathrm{GD} 156$ & $G, \times N$ & $A B X$ & $8-24$ & c & $8-24$ & $B F 3-1$ & $4 \mathrm{PI}$ & \\
\hline $60 G E 3$ & $64 \mathrm{GD} 157$ & $G, N$ & NOX & THR & & $H R$ & $\begin{array}{l}\text { 8F } 3-1 \\
\text { THRES }\end{array}$ & $\begin{array}{r}4 P I \\
\text { HDLD }\end{array}$ & \\
\hline OVAl & $64 \mathrm{GO} 158$ & $G, \times N$ & $A B x$ & $8-22$ & $c$ & $8-22$ & $8 F 3-1$ & 4P I & 493 \\
\hline iv & $64 \mathrm{GO} 158$ & $G, \times N$ & $A B X$ & $7-24$ & c & $7-24$ & $8 F 3-1$ & 4PI & \\
\hline $9 c$ & $64 \mathrm{G}$ & $G$, & A8 & $8-32$ & c & $8-32$ & $A C T-1$ & $4 \mathrm{PI}$ & \\
\hline 698 & $64 \mathrm{G}$ & G, & $A B X$ & $8-29$ & D & $\begin{array}{r}8-29 \\
\text { IN }\end{array}$ & $\begin{array}{l}\text { BF } 3-1 \\
\text { VCLUDES }\end{array}$ & $\begin{array}{l}4 P I \\
5 N P\end{array}$ & $158+$ \\
\hline $20 R 5$ & $64 G$ & G,1 & $A B X$ & $6-33$ & $c$ & $6-33$ & $A C T-1$ & $4 \mathrm{PI}$ & \\
\hline & $64 \mathrm{G}$ & $\mathrm{G}, 2 \mathrm{~N}$ & $A B X$ & $8-29$ & 0 & $\begin{array}{r}8-29 \\
I N C\end{array}$ & $\begin{array}{l}\text { BF } 3-1 \\
\text { LUDES }\end{array}$ & $\begin{array}{l}4 \mathrm{PI} \\
2 \mathrm{NP}\end{array}$ & 160 \\
\hline & $64 G 0160$ & $G, 3 N$ & $A B x$ & $8-29$ & D & $8-29$ & $8 F_{3-1}$ & $4 \mathrm{PI}$ & 161 \\
\hline & 6460 & $G_{1}$ & $A 8 X$ & $15-33$ & $c$ & $14-33$ & $\begin{array}{c}A C T-1 \\
\times P=P\end{array}$ & $\begin{array}{r}4 P I \\
P+N P\end{array}$ & \\
\hline
\end{tabular}


SEPARATION ENERGIES (MEV

A ABUND. $\begin{array}{llllllllll}156 & 5.2(-2) & 7.1 & 5.5 & 12.1 & 11.8 & -0.2 & 16.1 & 11.9 & 13.1\end{array}$

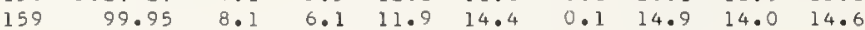
\begin{tabular}{cccll}
\multicolumn{1}{c}{ NUCLIDE REACTION RES EXCIT SDURCE } & DETECTOR \\
REF Z A IN,OUT & & & TYPE ANG NUM
\end{tabular}

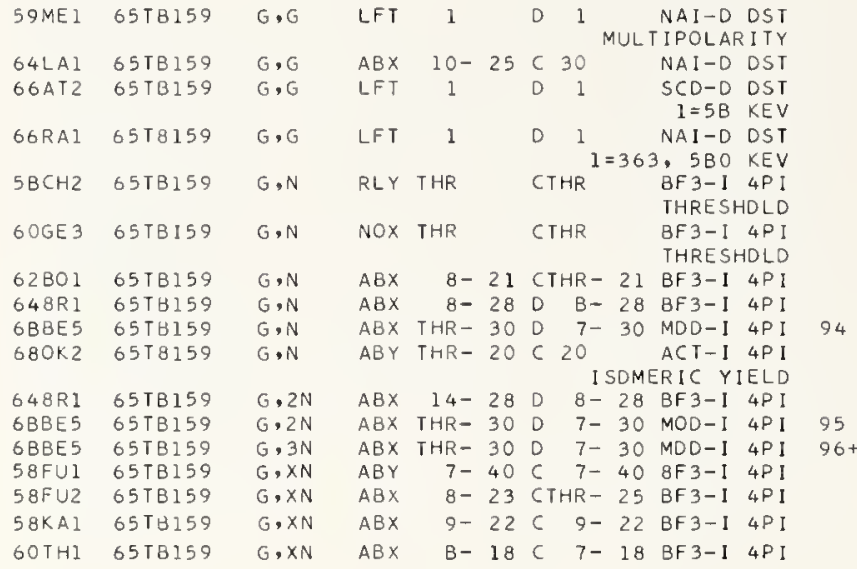

DYSPRDSIUM $Z=66$

\begin{tabular}{|c|c|c|c|c|c|c|c|c|c|}
\hline \multirow[t]{2}{*}{ A } & \multirow[t]{2}{*}{ A8UND. } & \multicolumn{8}{|c|}{ SEPARATIDN ENERGIES (MEV) } \\
\hline & & $G, N$ & $G, P$ & G, T & G, HE 3 & $G, A$ & $\mathrm{G}, 2 \mathrm{~N}$ & $G, N P$ & $\mathrm{G}, 2 \mathrm{P}$ \\
\hline 156 & $5 \cdot 2(-2)$ & 9.4 & 6.6 & 14.1 & $12 \cdot 3$ & -1.8 & 16.3 & 15.6 & 11.4 \\
\hline $15 B$ & $9.0(-2)$ & 9.1 & 6.9 & 14.1 & $13 \cdot 3$ & -0.9 & 16.0 & 15.5 & .4 \\
\hline 160 & 2.29 & 8.6 & 7.4 & $13 . \mathrm{B}$ & 13.8 & -0.5 & 15.4 & 15.6 & 13.5 \\
\hline 161 & $1 \mathrm{~B} \cdot \mathrm{BB}$ & 6.5 & 7.5 & 13.5 & 12.3 & -0.4 & 15.0 & 13.9 & 14.1 \\
\hline 162 & 25.53 & B. 2 & B. O & 13.6 & $14 \cdot 5$ & -0.1 & 14.6 & 15.7 & 14.8 \\
\hline 163 & $24 \cdot 97$ & $6 \cdot 3$ & B. 0 & 13.5 & $13 \cdot 3$ & 0.2 & 14.5 & $14 \cdot 3$ & 5.4 \\
\hline 164 & 28.18 & 7.7 & B. 5 & 13.4 & 15.4 & 0.4 & 13.9 & 15.6 & 16.2 \\
\hline
\end{tabular}

\begin{tabular}{llll}
\multicolumn{1}{c}{ NUCLIDE REACTIDN RES EXCIT SOURCE DETECTOR } \\
REF Z A IN,OUT
\end{tabular}

61BA2 660Y G,XN ABY THR- 22 C 22 THR-I DST

$60 G E 3$ 660Y163 GN NOX THR CTHR BF3-I 4PI

THRESHOLD

HOLMIUM $Z=67$

A ABUND. G,N, G,P GEPARATIDN ENERGIES (MEV)

$\begin{array}{llllllllll}165 & 100.00 & G, N & 6 . P & G, T & G, H E 3 & G, A & G .2 N & G, N P & G .2 P \\ & 8.0 & 6.2 & 11.7 & 14.1 & -0.1 & 14.7 & 13.9 & 14.8\end{array}$

\begin{tabular}{cccl}
\multicolumn{1}{c}{ NUCLIDE REACTIDN RES EXCIT SOURCE } & DETECTDR \\
REF 2 A IN,OUT & & & TYPE ANG NUM
\end{tabular}

$62 \mathrm{FU} 367 \mathrm{HO} 165 \mathrm{G}, \mathrm{G} \quad \mathrm{ABX} \quad 8-28 \mathrm{C} 8-2 \mathrm{~B} N \mathrm{NA}-\mathrm{D} 90$

63LAI 67HO165 G,G ABX 9- 26 C 9- 27 NAI-D DST

64LAI 67HO165 G.G ABX 10- 25 C 27 NAI-D DST

$66 A X 167 H D 165$ G, G ABX 13, 16 D 13,16 NAI-D DST

$63 \mathrm{TI} 1 \mathrm{HHO} 165$ G,G ABX 10- 19 D 10- 19 NAI-D 135

$58 \mathrm{CH} 267 \mathrm{HD} 165 \mathrm{GN}$ RLY THR CTHR BF3-1 4P

60GE3 67HD165 G,N NDX THR CTHR 8F3-I 4PI

6IWEI 67HDI65 GN ABX 8-11 D B- II ACT-I 4P

62 REI 67HO165 G.N NOX $11-55 . \mathrm{C} 55$ THR-I DST

638RI 67HDI65 G,N ABX 8-2BC 8-2B BF3-I 4PI

$66 \mathrm{AX} 167 \mathrm{HO} 165 \mathrm{GN}$ ABX B- 20 D B - 20 8F3-1 4PI

$67 G E 2$ 67HD165 G,N ABY THR- 27 C 22, 27 BF3-1 4P

$67 H U 1$ 67HD165 G.N ABX 9-11 D 9- 11 8F3-I $4 \mathrm{PI}$

$688 E 5$ 67H0165 G,N ABX THR- 30 D $7-30$ MDD-I 4 PI 90

69BE8 67HO165 G,N ABX 8- 29 D B- 29 BF3-I 4PI 163

63BRI 67HD165 G,2N ABX 14-28C 14- 28 BF $3-1$ 4P

$66 A X 167 H 0165$ G.2N ABX $8-20$ QUADRUPOLE MOMENT

67HAI 67HO165 G,2N RLX 14- 29 C 14- 29 ACT-I 4PI

68BE5 67HDI65 G,2N ABX THR- 30 D 7- 30 MDD-1 4PI 91

$69 B E 8$ 67HDI65 G,2N ABX 8- 29 D 8- 29 BF3-I 4PI 164

68BE5 67H0165 G,3N ABX THR- 30 D 7- 30 MDD-I 4 PI $92+$

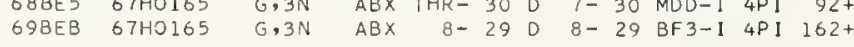

58FU1 67HO165 G,XN ABX 7- $40 \mathrm{C}$ 7- 40 8F3-I 4PI $5 B K A 167110165$ GXXN ABX $9-22 C \quad 9-22$ BF3-I $4 P$ I 6OTHI 67HOI65 G,XN ABX B- 18 C $7-18$ ACT-I 4P $62 \mathrm{FU} 367 \mathrm{HO} 165 \mathrm{G} \times X N$ ABX $8-24 \mathrm{C} B-248 \mathrm{~F} 3-\mathrm{I} 4 \mathrm{P}$ 65 AMI $67 H 0165 \mathrm{D} \mathrm{G}, \mathrm{XN}$ ABY $10-20 \mathrm{C} 10-20 \mathrm{BF} 3-\mathrm{I} 4 \mathrm{P}$ $69 K E 267 \mathrm{HD} 165$ \& $G \times N$ ABX 10- 21 D 10- 1 IENTED TARGE $665 C 167 H O 165$ G.P SPC THR- $70 \mathrm{C} 70$ DRIENTED TARGET

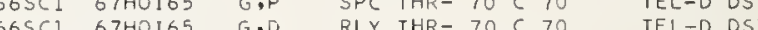
65SCI 67H RLY THR- $70 C 70$ TEL-D DS

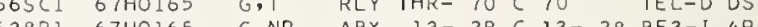
638RI 67H0165 G.NP ABX 13- $2 B$ C $13-28$ BF $3-14 P$ M $71 M E I \quad 67 H O 165$ G,F ABY THR-900 C300-900 TRK-I 4PI

ERB IUM $\quad Z=68$

\begin{tabular}{|c|c|c|c|c|c|c|c|c|}
\hline ABUNO. & & & SEPARA & TIDN EI & NERGIE & CME & & \\
\hline & $G, N$ & $G, P$ & $G, T$ & $G, H E 3$ & $G, A$ & $\mathrm{G}, 2 \mathrm{~N}$ & $G, N P$ &, $2 \mathrm{P}$ \\
\hline 0.14 & 9.2 & 6.4 & I 3.8 & 12.1 & -1.7 & 16.5 & 14.9 & 11.2 \\
\hline 1.56 & 8.9 & 6.9 & 13.7 & $12 \cdot 8$ & $-1 \cdot 3$ & 15.8 & 15.3 & 3 \\
\hline 33.41 & 8.5 & $7 \cdot 3$ & 13.5 & 13. & -0.8 & 15.1 & 15.3 & 6 \\
\hline 22.94 & 6.4 & 7.5 & 13.3 & $12 \cdot 3$ & -2.7 & 14.9 & $13 . B$ & $4 \cdot 3$ \\
\hline 27.07 & 7.8 & 8.0 & 13.0 & 14.3 & -0.5 & 14.2 & 15.3 & 5.0 \\
\hline $14.8 B$ & $7 \cdot 3$ & 8 & 12.7 & * & 0.0 & $13 \cdot 3$ & 15.3 & \\
\hline
\end{tabular}

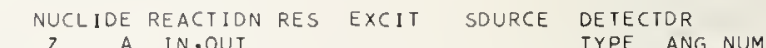

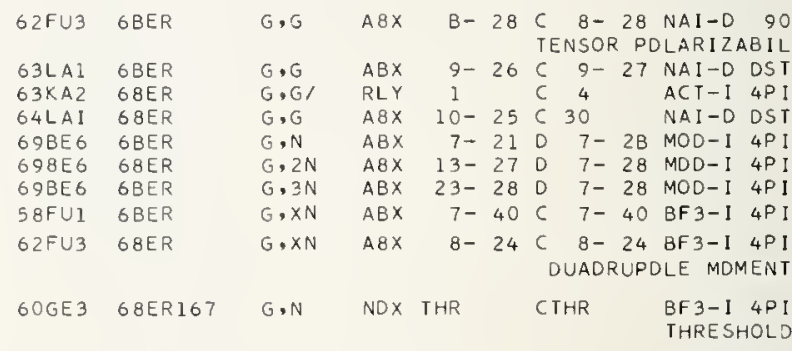

63MI3 68ERI70 G.P NOX THR- $21 C 21 \quad$ ACT-I 4PI

THUL IUM $Z=69$ \begin{tabular}{ccccccccc} 
A & ABUND. & \multicolumn{8}{c}{ SEPARATION ENERGIES (MEV) } \\
169 & 100.00 & G.N & G.P & G.T & G.HE3 G,A & G.2N & G.NP & G.2P \\
169 & 100.1 & 5.6 & 11.3 & 13.1 & -1.2 & 14.9 & 13.3 & 13.5
\end{tabular}

\begin{tabular}{|c|c|c|c|c|c|c|}
\hline & NUCL I DE & REACTIDN & RES & EXCI & SDURCE & DETECTDR \\
\hline REF & $z$ & IN,DUT & & & & TYPE ANG \\
\hline $\mathrm{CH}_{2}$ & 69 TM169 & $G, N$ & RLY & $T H R$ & CTHR & $\begin{array}{l}\text { BF 3-I 4P I } \\
\text { THRE SHDLD }\end{array}$ \\
\hline GE 3 & 69 TMI 69 & $G, N$ & NOX & THR & CTHR & $\begin{array}{l}\text { BF } 3-I \text { I } 4 \text { I } \\
\text { THRE SHDLD }\end{array}$ \\
\hline$K A$ & $69 T M 169$ & $G \times N$ & $A B x$ & $B-22$ & $8-22$ & $8 F 3-1$ \\
\hline
\end{tabular}

YTTERBIUM $Z=70$

\begin{tabular}{|c|c|c|c|c|c|c|c|c|}
\hline \multirow[t]{2}{*}{ ABUND. } & \multicolumn{8}{|c|}{ SEPARATION ENERGIES (MEV) } \\
\hline & $G, N$ & $G, P$ & $G, T$ & $\mathrm{G}, \mathrm{HE}^{\prime}$ & $G, A$ & $\mathrm{G}, 2 \mathrm{~N}$ & $G, N P$ & $G .2 P$ \\
\hline 0.14 & 9.1 & 6.3 & 13.6 & 12.0 & $-1 \cdot 9$ & 16.1 & 15.0 & 11. \\
\hline 3.03 & 8.5 & 6.8 & 13.2 & 12.4 & $-1 \cdot 7$ & 15.3 & 14.8 & 12. \\
\hline 14.31 & 6.6 & 6.8 & 13.0 & 11.3 & $-1 \cdot 6$ & 15.1 & 13.4 & \\
\hline 21.82 & 8.0 & 7.3 & 12.9 & $13 \cdot 3$ & $-1 \cdot 3$ & 14.6 & $14 \cdot 8$ & 3 . \\
\hline 16.13 & 6.4 & 7.5 & 12.7 & 12.4 & -0.9 & 14.4 & 13.7 & 4 . \\
\hline 31.84 & $7 \cdot 5$ & B. 0 & 12.7 & $14 \cdot 2$ & -0.7 & 13.8 & $14 \cdot 9$ & 5 . \\
\hline 12.73 & 6.9 & 8.5 & 12.2 & 15.0 & -0.6 & 12.7 & 15.0 & \\
\hline
\end{tabular}

NUCLIDE REACTION RES EXCIT SDURCE DETECTDR

REF $Z$ A IN.DUT TYPE ANG NUM

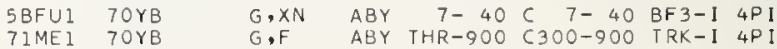

60GE3 7OYBI73 G,N NOX THR CTHR BF3-I 4PI

69MD $70 Y B 174$ E,F ABX THR-999 D 60-999 TRK-I DST

$69 M D 1$ 70YBI74 G.F ABX THR-999 $6 \quad 60-999$ TRK-I DST $999=I$ GEV 
ABUND.

SEPARATION ENERGIES (MEV)

\begin{tabular}{|c|c|c|c|c|c|c|c|c|c|c|}
\hline \multirow{2}{*}{\multicolumn{2}{|c|}{ A ABUNU. }} & \\
\hline & & $\mathrm{G}, \mathrm{N}$ & \multirow{2}{*}{\multicolumn{2}{|c|}{$\begin{array}{r}\text { G.T } \\
10.9\end{array}$}} & \multicolumn{2}{|c|}{ G.HE3 } & $G, A$ & $6,2 \mathrm{~N}$ & $G, N P$ & $G, 2 P$ \\
\hline 175 & 97.41 & 7.7 & & & \multicolumn{2}{|c|}{12.7} & $-1 \cdot 6$ & 14.4 & $13 . D$ & \multirow{2}{*}{$\begin{array}{l}13.5 \\
14.1\end{array}$} \\
\hline & 2.59 & 6.3 & & .8 & 12.1 & & $-1 \cdot 6$ & $14 \cdot 0$ & 11.8 & \\
\hline \multirow[b]{2}{*}{ EF } & NUCLIDE & \multirow{2}{*}{$\begin{array}{l}\text { REACT ION } \\
\text { IN, DUT }\end{array}$} & \multirow[t]{2}{*}{ RES } & \multirow{2}{*}{\multicolumn{2}{|c|}{ EXCIT }} & \multirow{2}{*}{\multicolumn{2}{|c|}{ SDURCE }} & \multicolumn{2}{|c|}{ DETECTOR } & \multirow{5}{*}{ NUM } \\
\hline & & & & & & & & TYPE & ANG & \\
\hline & $71 L U$ & \multirow{2}{*}{$G \cdot N$} & $A B X$ & \multicolumn{2}{|l|}{ 7- } & D & $7-28$ & \multicolumn{2}{|c|}{ MOD-I 4P I } & \\
\hline & $71 \mathrm{LU}$ & & $A B X$ & $14-$ & -28 & D & $7-28$ & \multirow{2}{*}{$\begin{array}{l}\text { MOD-I } \\
\text { MOD-I }\end{array}$} & 4P I & \\
\hline & $71 \mathrm{LU}$ & $\begin{array}{l}G, 2 N \\
G \cdot 3 N\end{array}$ & $A 9 x$ & $24-$ & -28 & D & $7-28$ & & I $4 \mathrm{PI}$ & \\
\hline $20 E 2$ & 71 LU175 & \multirow[t]{2}{*}{$G \cdot G$} & LFT & \multirow{2}{*}{\multicolumn{2}{|c|}{1}} & D & 1 & \multirow{2}{*}{\multicolumn{2}{|c|}{$\begin{array}{l}\text { NAI-D DST } \\
\text { UTIPOLARI T }\end{array}$}} & \\
\hline & & & & & & $J-P$ & PI, MUL & & IULT I POLARI T & \\
\hline \multirow[t]{2}{*}{$8 \mathrm{KII}$} & $71 L \cup 175$ & \multirow[t]{2}{*}{$G \cdot N$} & $A B X$ & \multirow{2}{*}{\multicolumn{2}{|c|}{$8-23$}} & & $8-23$ & \multirow{2}{*}{\multicolumn{2}{|c|}{$\begin{array}{l}\text { BF 3-I 4P I } \\
\text { THRE SHDLD }\end{array}$}} & \\
\hline & & & & & & & & & & \\
\hline \multirow[t]{2}{*}{ DGE 3} & $71 L U 175$ & \multirow[t]{2}{*}{$G, N$} & NOX & \multirow{2}{*}{\multicolumn{2}{|c|}{ THR }} & $\mathrm{CTH}$ & $\mathrm{HR}$ & \multirow{2}{*}{\multicolumn{2}{|c|}{$\begin{array}{l}\text { BF 3- I 4P I } \\
\text { THRE SHDLD }\end{array}$}} & \\
\hline & & & & & & & & & & \\
\hline & $71 L U 175$ & $G, \times N$ & A $8 x$ & $8-$ & -22 & $c$ & $8-22$ & $B F 3-1$ & $14 P 1$ & \\
\hline
\end{tabular}

HAFNIUM $Z=72$

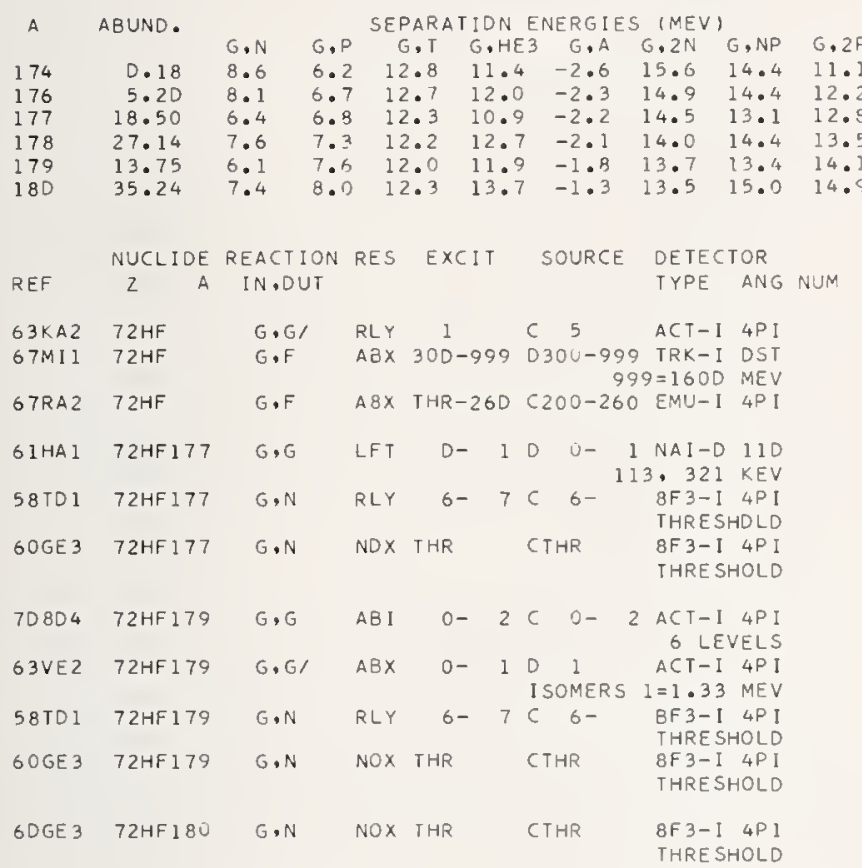

TANTALUM $\quad Z=73$

A ABUND.

G,N G,P G,T G,HEZ G,A G, 2N G,NP G.2P

\begin{tabular}{lllll}
\multicolumn{1}{c}{ NUCLIDE REACTION RES EXCIT SDURCE } & DETECTDR \\
REF & $\mathrm{T}$ A IN,OUT & & \\
TYPE ANG NUM
\end{tabular}

$71 M D 3$ 73TA181 E,E/ A8X D-240 D500 MAG-D 60 $598 A 3$ 73TA181 E,N ABY THR- 36 D $10-36$ BF3-I 4PI $65 \mathrm{HE} 1$ 73TA181 E,N RLY THR- 32 D 14- 32 ACT-I 4 PI 6OGR2 73TAI81 E,N RLY THR-999 C 70-999 ACT-I 4PI

999=1 GEV E-,N/E+, N

60842 73TAl81 EDY 22- $42022-42$ ACT-I $4 P I$

70BU2 73TA181 E,XN SPC 7-140 C14D REL TOF-D 165

69AN8 73TA181 E,P RLY THR-999 C999 THICK TARGET

70AN5 73TAI81 E,P RLY $86-999$ C999 GEV, REL D/T

$999=1140$ MEV
TEL

69AN8 73TA181 E, D RLY THR-999 C999 MAG-D DST

69AN8 73TA181 E,T RLY THR-999 C $999 \quad \begin{array}{r}999=1.3 \mathrm{GEV} \\ \text { MAG }\end{array}$

$990=1$ GEV, REL D/T

$65 H E 1$ 73TA181 E+,N RLY THR- 32 D $14-32$ ACT I $4 P I$

$68 B D I 73 T A 18$ I E,F ABI D250,5DO FMU-I 4PI

$58 F U 3$ 73TAI81 G,G AEX 5- 27 C 5- 27 NAI-D 120

61 BU4 73TAL81 6.6 ABX $10-25$ C 20 NAI-D DST

63LAI 73TAI8I G, G NDX 11- 22 C 27 NAI-D DST

$63 Y O 1$ 73TAI8I G.G ABX $6-880$ 6- NAI-D 120

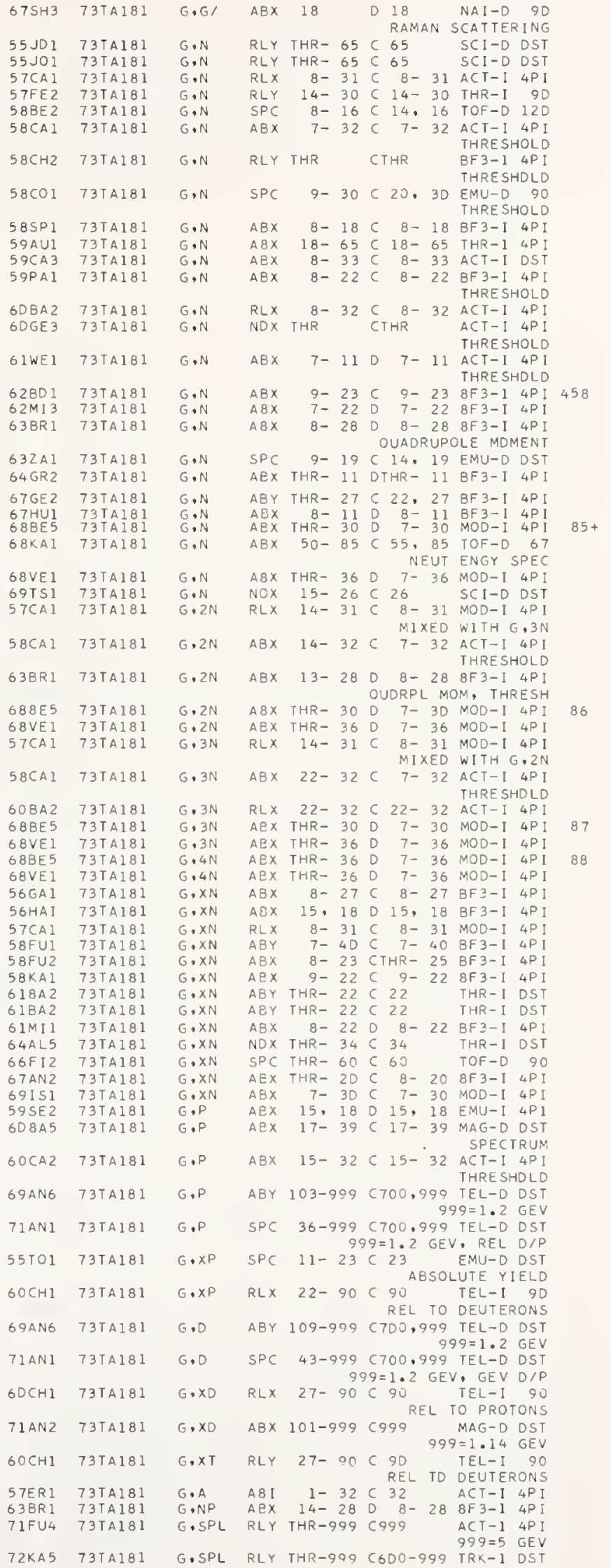

$999=1.7$ 


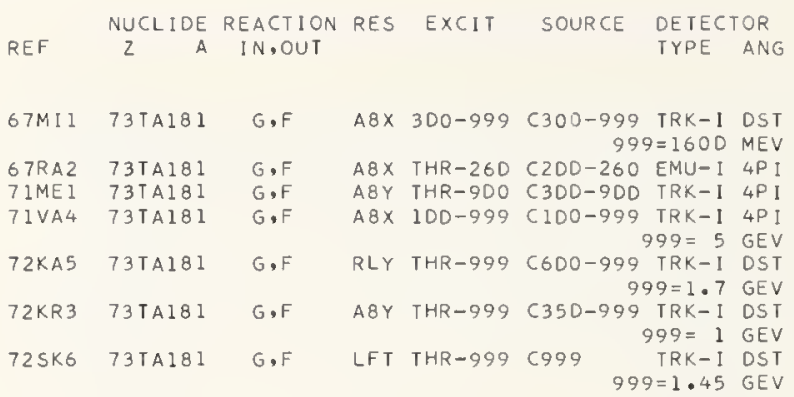

TUNGSTEN (WOLFRAM) $\quad Z=74$ $\begin{array}{ll}\text { A } & \text { ABUND. } \\ 184 & 0.02 \\ 186 & 1.59 \\ 187 & 1.64 \\ 188 & 13.3 \\ 189 & 16.1 \\ 190 & 26.4 \\ 192 & 41.0\end{array}$

REF
SEPARATION ENERGIES (MEV)

$G, N$ G,P SEPARATION ENERGIES TMEV G, NP G,2P

$\begin{array}{llllllll}8.9 & 5.7 & 12.7 & 10.9 & -3.1 & 16.1 & 14.2 & 10.5\end{array}$

$\begin{array}{llllllll}3.3 & 6.5 & 12.1 & 11.6 & -2.8 & 14.9 & 14.3 & 11.9\end{array}$

$\begin{array}{llllllll}6.3 & 6.6 & 12.1 & 10.4 & -2.7 & 14.6 & 12.8 & 12.4\end{array}$

$\begin{array}{llllllll}8.0 & 7.2 & 12.3 & 12.7 & -2.1 & 14.3 & 14.6 & 13.2\end{array}$

$\begin{array}{llllllll}.9 & 7.3 & 12.0 & 11.4 & -2.0 & 13.9 & 13.1 & 13.7\end{array}$

$\begin{array}{llllllll}.8 & 8.0 & 12.4 & 13.7 & -1.4 & 13.7 & 15.1 & 14.6 \\ 7.5 & 8.7 & 12.9 & 15.3 & -0.4 & 13.3 & 15.7 & *\end{array}$

NUCLIOE REACTION RES EXCIT SOURCE DETECTOR

67MII 760S G.F A8X 300-999 $\quad 300-999$ TRK-I DST

67RA2 760S G.F A8X THR-260 C200-260 EMU-I 4PI

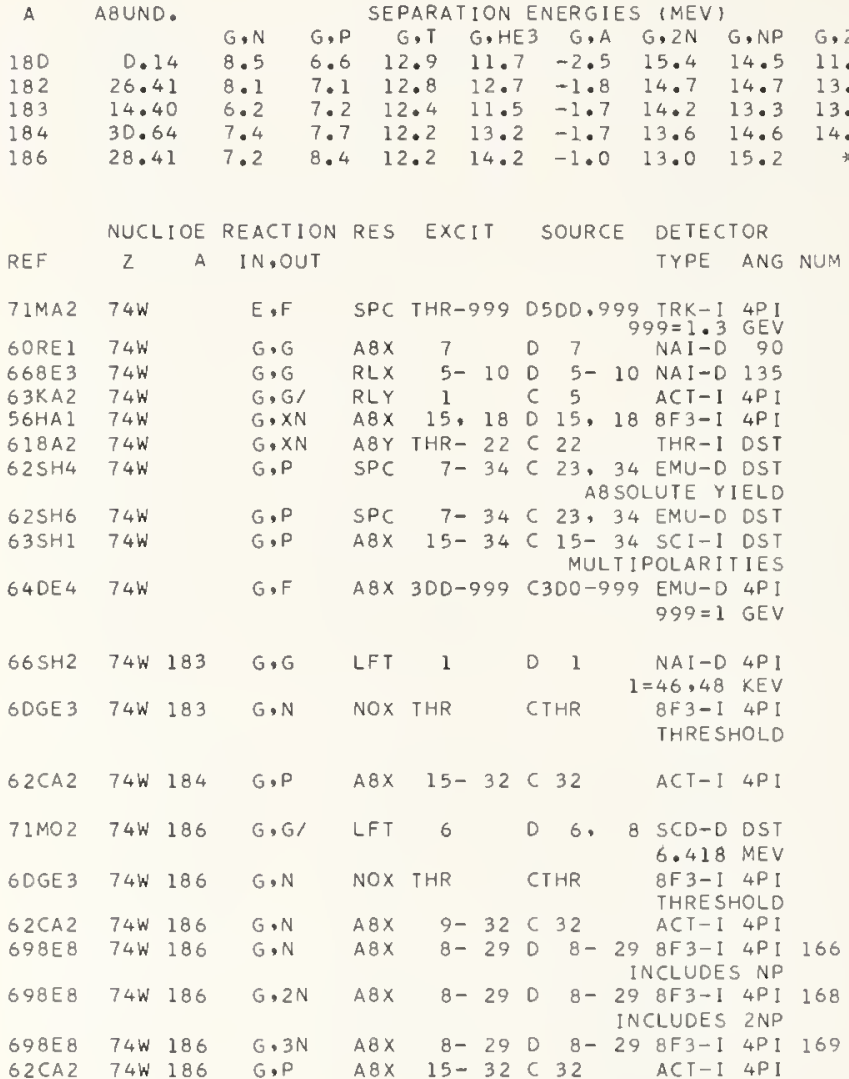

RHENIUM $Z=75$

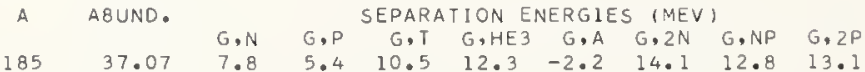

$\begin{array}{llllllllll}185 & 37.07 & 7.8 & 5.4 & 10.5 & 12.3 & -2.2 & 14.1 & 12.8 & 13.1 \\ 187 & 62.93 & 7.4 & 6.0 & 10.5 & 13.5 & -1.7 & 13.6 & 13.2 & 14.4\end{array}$

\begin{tabular}{lclll}
\multicolumn{1}{c}{ NUCLIOE REACTION RES EXCIT SOURCE } & DETECTOR \\
REF & $Z$ A IN,OUT & & & TYPE ANG NUM
\end{tabular}

67MII 75RE G.F A8X 300-999 C300-999 TRK-I OST

67RA2 75RE G.F ABX THR-260 C200-260 EMU-I 4PI

67MEI 75REI85 G.G LFT D- 1 D D- 1 SCO-D OST

64LA3 75REIB7 G,G LFT D- 10 O- I NAI-D DST

64SH5 75RE187 G.G LFT I O I N LEVELS

67LA2 75REI87 G,G LFT D 10 D- 1 SCD-D 115

60GE3 75REI87 G.N NOX THR CTHR 8F3-I 4PI
IRIOIUM $Z=77$

A ABUND. G,N G,P SEPARATION ENERGIES (MEV)

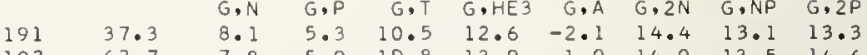

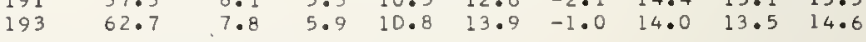

\begin{tabular}{lllll}
\multicolumn{1}{c}{ NUCLIOE REACTION RES EXCIT SOURCE DETECTOR } \\
REF 2 A IN,OUT & & & TYPE ANG NUM
\end{tabular}

63KA2 $77 I R \quad G . G /$ RLY I C 5 ACT-I 4 PI

67SCI 77IRI9I G.G LFT O- 10 O- 1 SCD-D 125

68DAI 77IRI9I S G.G NOX I D I NAI-O DST

MAG MOMENT, $129 \mathrm{KEV}$

64LA4 77IRI9I G.G LFT O- I D O- NAI-D 125

63VE2 77IRI9I G.G/ ABX D- I D I ACT-I 4PI

67ME2 $77 I R 193$ G,G LFT O- 1 D D- I SCD-D IDS

460.557,559 KEV

$0-1$ SCO-D 105
SEE $67 M E 2$

60GE3 77IRI93 G.N NOX THR CTHR SF3-I 4PI

PLATINUM $Z=78$

\begin{tabular}{cccccccccc} 
A & ABUND. & \multicolumn{10}{c}{ SEPARATION ENERGIES (MEV) } \\
190 & $1.31-21$ & G.N & G.P & G.T & G.HE3 & G.A & G.2N & G.NP & G.2P \\
192 & 0.78 & 8.7 & 6.1 & 12.6 & 11.0 & -3.2 & 15.7 & 14.4 & 10.8 \\
194 & 32.9 & 8.4 & 7.5 & 12.8 & 12.2 & -2.4 & 15.1 & 15.0 & 12.2 \\
195 & 33.8 & 6.1 & 7.6 & 12.9 & 13.3 & -1.5 & 14.6 & 15.3 & 13.5 \\
196 & 25.3 & 7.9 & 8.1 & 13.1 & 14.2 & -1.2 & 14.5 & 13.6 & 14.0 \\
198 & 7.21 & 7.6 & 8.8 & 13.0 & 15.0 & -0.1 & 14.0 & 15.5 & 14.8 \\
& 7.21 & & & & & 13.4 & 15.8 & $*$
\end{tabular}

NUCLIDE REACTION RES EXCIT SOURCE DETECTUR

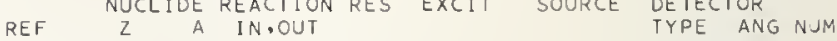

668E3 78PT G,G RLX 5- 10 D 5- 10 NAI-D 135

63KA2 78PT G,G/ RLY 1 G C 5 ACT-I 4PI

64GLI 78PT G,N SPC $16 \quad$ D 16 TOF-D 90

68JU1 78PT G,N NOX THR- $27 \mathrm{C} 27$ THR-1 DST

$62 S H 278 P T$ G.P SPC 7- 34 C 23, 34 EMU-D DST

62SH4 78PT G,P SPC 7- 34 C 23, 34 EMU-O DST

ABSOLUTE YIELD

$67 M I J \quad 78 P T \quad G, F \quad A 8 \times 300-999 \quad 300-999$ TRK-I DST

G7RA2 78PT G,F A8X THR-260 C100-260 EMU-I OST

63VE2 78PTI95 G.G/ A8X D- 1 D 1 ACT-I 4PI

GOGE3 78PT195 G,N NOX THR ISOMERS $1=1.33 \mathrm{MEV}$

GOGE3 78PT196 G,N NOX THR CTHR BF3-I 4PI

$62 \mathrm{CHI} 78 \mathrm{PT} 196 \mathrm{~N} \cdot \mathrm{G}$ NOX 8 O 0 - THRESHOLD REL TRANS PROBAGIL 
$\begin{array}{cccccccccc}\text { A } & \text { ABUND. } & \text { SEPARATION ENERGIES (MEV) } \\ & & \text { G.N } & \text { G.P } & \text { G.T } & \text { G.HEB } & \text { G.A } & \text { G.2N } & \text { G.NP } & \text { G.2P } \\ 197 & 100.00 & 8.1 & 5.8 & 11.4 & 13.6 & -0.9 & 14.8 & 13.7 & 13.9\end{array}$

$\begin{array}{cccccccccc}\text { A } & \text { ABUND. } & \text { SEPARATION ENERGIES (MEV) } \\ & & \text { G.N } & \text { G.P } & \text { G.T } & \text { G.HEB } & \text { G.A } & \text { G.2N } & \text { G.NP } & \text { G.2P } \\ 197 & 100.00 & 8.1 & 5.8 & 11.4 & 13.6 & -0.9 & 14.8 & 13.7 & 13.9\end{array}$

\begin{tabular}{cccll}
\multicolumn{1}{c}{ NUCLIDE REACIION RES EXCIT SOURCE } & DETECTOR \\
REF $Z$ A IN.OUT & & & TYPE ANG NUM
\end{tabular}

6DBA2 79AU197 E,N RLY 14- 38 D 14- 38 ACT-I 4PI

608A2 79AU197 E, 2N RLY 17- 38 D 17- 38 ACT-I 4P.

$58 Z I 179 A U 197$ G,MU-T ABX 9- $20 \mathrm{C}$ 9- 3D MGP-D UKN

56 FUI $79 A U 197$ G.G ABX $4-40$ C 4- 4D NAI-D 120

6OREI 79AU197 G.G ABX 4- 40 C 4- 4D NAI-D 120

620 TI 79AU197

63LAI 79AUI97 G,G ABX $11-18$ D I1- IE NAI-D 135

64LAI 79AU197 G.G ABX $10-25 \mathrm{C} 32$ NAI-D DST

63KA2 79AU197 G,G/ RLY 1 C 4 ACT-I 4P

63VE2 T9AU197 G,G/ ABX O- 1 D I ACT-I $4 P I$

I SOMERS $1=1.33$ MEV

57FEI T9AU197 G.N RLY $15-31$ C $15-31$ THR-I DST

$G, N$ SPC $7-16 C 14,16$ TOF $-D 120$

THRE SHOLD

T.N RLY THR CTHR BF3-I 4PI

59AU1 79AU197 G.N A8X 18-65C 1B-65 THR-I 4PI

YPLD REL TO CU6

G.N SPC 6- $55 C 55$ EMU-D 9

G.N RLX $10-20$ C $10-3 B$ ACT-I $4 P$

NOX THR CTHP BF $3-I 4 P I$

6INAI T9AU197 G.N ABX 10- 22 C $10-22$ ACT-I 4PI

6ITAI 79AU197 G.N NOX 12- 22 C 22 THR-I DST

61WEI 79AUI97 G,N ABX 8- 10 D E- 10 ACT-I 4 P I

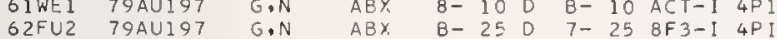

MIXED WITH G, NP

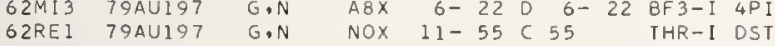

63ZAI 79AU197 G,N SPC 7- 19 C 14, 19 EMU-D DST

$676 E 2$ 79AUI97 G.N ABY THR - 27 C 22, 27 BF3-I 4PI

67HUI 79AUI97 G, N ABX 9- II D 9- 11 BF3-I 4PI

$690 E 1$ T9AUI97 G,N AEY THR-999 C $1-6$ ACT-I $4 P I$

690II 79AU197 G.N ABY THR-900 C400-900 ACT-I 4PI

TDVEI 79AUI97 G.N ABX $8-26$ D $7-36$ BF $3-I$ IPI 334

$710 I 1$ T9AUI97 G,N ABY $8-990$ C300-999 ACT-I 4 PI $999=1$ GEV

7ILI2 79AUI97 G,N ABY THR-90D C 75-900 ACT-I 4PI

71SAl 79AU197 G.N ABY $8-68$ C $10-6 B$ ACT-I 4 PI

60BA2 79AU197 G.2N RLX $15-26$ C $15-38$ ACT-I 4 P

6INAI 79AUI97 G,2N ABX 15- 22 C $10-22$ ACT-I 4 PI

62FU2 79AU197 G,2N ABX 14- 25 D $7-25$ EF3-I 4PI

7OVEI 79AUI97 G, 2N AEX 14- 27 D 7- 36 BF 3-I 4PI 335

$710 I I$ 79AU197 G.2N ABY $15-999$ C30U-999 ACT-I 4PI

$71 L I 2$ 79AU197 G,2N ABY THR-900 C 75-900 ACT-I 4PI

7OVEI 79AUI97 G.3N ABX 24- 27 D $7-36$ BF $3-I$ 4PI $336+$

71112 T9AU197

7ILI 2 T9AUI97

71 LI2 79AUI97

7ILI2 79AU197

$71 L 12$ 79AU197

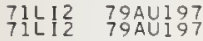

71 LI2 79AUI97

56GAI 79AU197

$56 \mathrm{HAI}$ 79AU197

$58 F \cup 1$ T9AUI97

5 BFU2 79AU197

$58 \mathrm{KAI} 79 \mathrm{AUI} 97$

6IMII TOAUIO

59MA1 T9AU197

$G, 3 N$

G. $4 \mathrm{~N}-\mathrm{ABY}$ THR-9DO C $75-9 D D$ ACT -1

$6.5 \mathrm{~N}$ ABY THR-90C C $75-900$ ACT- I $4 \mathrm{P}$ G,7N ABY THR-900 C 75-900 ACT-I $4 \mathrm{P}$

G,9N ABY THR-900 C 75-9DD ACT-1 4PI

G:IIN ABY THR-90D \& $75-900$ ACT-I $4 P$ P

$G, 15 N$ ABY THR-9DD C $75-900$ ACT-I $4 P I$

$G . X N \quad A B X \quad 7-27$ C $7-27 \quad B F 3-1$ 4PI

$G, X N \quad A B X 15,18$ D $15,1 B$ BF 3-I $4 P I$

$G X N A B Y \quad 7-40$ C $7-40$ BF $3-14 P I$

$G, X N$ AEX B- 23 CTHR- 25 BF3-I $4 P$ I

$G, X N \quad A B X \quad 8-22 C B-22 B F 3-I \quad 4 P I$

$G, X N$ ABX B- 20 D $8-20$ BF3-1 $4 P$

$G, P$ RLY THR- 70 C 70 EMU-D 90

6DBAS $79 A U 197$ G.P SPC $9-40$ C 40 (G.D)/(G,P)

GOMAI 79AUI97 G,P SPC 13-23 ABS INTEG CROS SEC

56DA2 79AUI97 G.XP SPC 11-7D 70 ABSOLUTE YIELD

6DCHI 79AUIO7 G.XP RLX 21- OO 90 ABSOLUTE YIELD

TEL-I 90

$59 M A 179 A U 197$ G.D RLY THR- $70 \mathrm{C} 70$ TO DEUTERONS

6DCHI 79AU197 G,XO RLY 27-90 C 90 (G,D)/(G,P)

6OCH1 79AU197 G.XT RLY $26-90 \mathrm{C} 90^{\text {REL TO PROTONS }}$

$\begin{aligned} & 90-90 \text { TEL-I } 90 \\ & \text { REL TO DEUTERONS }\end{aligned}$

65HA2 79AU197 G,A SPC THR- 31631 EMU-D DST

65ME2 79AU197 G,A SPC THR- 35 C 35 SCD-D 90

62FU2 79AU197 G.NP ABX 14- 25 D 7- 25 BF3-I 4P

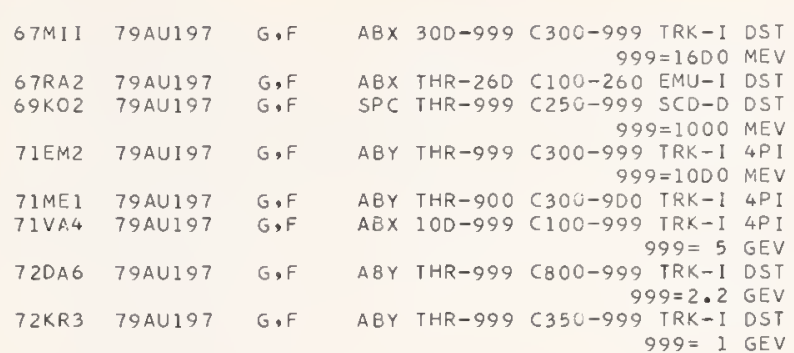

MERCURY $Z=80$

\begin{tabular}{rrrrrrrrrr} 
A & ABUND. & \multicolumn{8}{c}{ SEPARATION ENERGIES (MEV ) } \\
196 & 0.15 & G.N & G.P & G.T & G.HE3 & G.A & G.2N & G.NP & G.2P \\
198 & 10.02 & 8.3 & 6.6 & 13.4 & 12.3 & -2.0 & 15.8 & 15.0 & 11.7 \\
199 & 16.84 & 6.6 & 7.1 & 13.4 & 13.1 & -1.3 & 15.3 & 15.2 & 12.9 \\
200 & 23.13 & 8.0 & 7.7 & 13.3 & 11.8 & -0.8 & 14.9 & 13.8 & 13.7 \\
201 & 13.22 & 6.2 & 7.6 & 13.0 & 12.0 & -0.7 & 14.7 & 15.3 & 14.2 \\
202 & 29.80 & 7.8 & 8.5 & 13.2 & 14.9 & -0.3 & 14.3 & 13.9 & 14.8 \\
204 & 6.85 & 7.5 & 9.2 & 13.5 & 16.1 & 0.5 & 14.0 & 15.4 & 15.3 \\
& & & & & & & & 16.2 &
\end{tabular}

NUCLIDE REACTION RES EXCIT SOURCE DETECTOR

REF $Z$ A IN,OUT TYPE ANG NUM

\begin{tabular}{|c|c|c|c|c|c|c|c|}
\hline ORE I & BOHG & $G . G$ & $A B X$ & 7 & & $D$ & $\begin{array}{r}\text { NAI - D DST } \\
\text { G-WDTH }\end{array}$ \\
\hline $628 E 2$ & $80 \mathrm{OHG}$ & $G, G$ & $A B X$ & $5-$ & 9 & $5-$ & NAI $-D \quad 135$ \\
\hline BFL 1 & OHG & 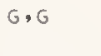 & LFT & 2- & 6 & $2-$ & $\begin{array}{r}\text { NAI -D } 130 \\
\text { G-WIDTH }\end{array}$ \\
\hline $53 Y 01$ & BDHG & $5, G$ & $A B X$ & $6-$ & 8 & $6-$ & NAI - D DST \\
\hline $4 A R I$ & & 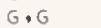 & $x$ & 5 & & D & NAI-D 135 \\
\hline $68 E 3$ & & & L & $5-$ & 10 & $5-10$ & NAI-D 135 \\
\hline 69RAl & $\mathrm{BOHG}$ & .6 & vox & 5 & & $5=$ & 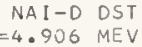 \\
\hline $3 K A 2$ & $8 O H G$ & , G/ & LY & 1 & & $c$ & $A C T-1 \quad 4 P I$ \\
\hline $5 M C 1$ & &,$\times N$ & $-Y$ & TrR- & 22 & C 22 & NA I - I \\
\hline $6 \mathrm{HAl}$ & & $X N$ & $8 x$ & 15 , & 18 & D 15,18 & $B F 3-1$ 4PI \\
\hline $61 B A 2$ & HG & , XN & $B Y$ & THR- & 22 & C 22 & THR-1 DS \\
\hline $71 E M 2$ & BOHG & $G \cdot F$ & $A B Y$ & $T H R-9$ & 999 & $\begin{array}{r}(300-999 \\
999\end{array}$ & $\begin{array}{rr}T R K-1 & 4 P I \\
9=1000 & \mathrm{MEV}\end{array}$ \\
\hline $63 F R 2$ & BDHG I & $G, G$ & $B x$ & 1 & & $1_{\text {LIFET }}$ & $\begin{array}{r}\text { NAI - D OST } \\
\text { TIME, J-PI }\end{array}$ \\
\hline $62 \mathrm{CA}]$ & $80 \mathrm{HG} 198$ & $G, N$ & NOX & THR - & 30 & C 30 ISOME & $\begin{array}{l}\text { ACT-I } \\
\text { ERIC RI } \\
\text { ERTIO }\end{array}$ \\
\hline $57 \mathrm{KN} 1$ & $\mathrm{HC}$ & $G, G$ & & 1 & & D & $S C I-D$ \\
\hline 69803 & BDHGI & G. & $8 x$ & D- & 2 & $0-2$ & $A C T-I \quad 4 P I$ \\
\hline 63VE2 & BDHG199 & $G, G /$ & $B x$ & $0-$ & 1 & $\begin{array}{l}\text { D } 1 \\
\text { I SOMERS I }\end{array}$ & $\begin{array}{rl}A C T-I & 4 P I \\
l=1.33 & \text { MEV }\end{array}$ \\
\hline 60GE 3 & 8DHG & $G \cdot N$ & NOX & THR & & CTHR & $\begin{array}{l}\text { BF 3-I 4PI } \\
\text { THRE SHOLD }\end{array}$ \\
\hline $680 \times 3$ & $8 D H G 199$ & $G \cdot P$ & $A B Y$ & THR - & 20 & C 20 & $A C T-I \quad 4 P I$ \\
\hline $69 C A 2$ & $8 D H G 2 D 1$ & $G, M U-T$ & $A B X$ & 0 & & $\begin{array}{l}D \\
D=32.2\end{array}$ & $\begin{array}{l}\text { SCI-D } 4 P I \\
K E V, J-P I\end{array}$ \\
\hline 6OGE 3 & $8 D H G 2 D$ & $G, N$ & NOX & THR & & CTHR & $\begin{array}{l}\text { BF 3-I } 4 P \text { I } \\
\text { THRE SHOLD }\end{array}$ \\
\hline 62EUI & $80 H G 201$ & $\mathrm{G}$, & $A B$ & $9-$ & 23 & $\begin{array}{l}9-23 \\
\text { RELAT }\end{array}$ & $\begin{array}{l}\text { ACT-I } 4 P I \\
\text { TIVE YIELD }\end{array}$ \\
\hline $62 C A 2$ & $\mathrm{BOHG} 2 \mathrm{C}$ & $G, P$ & $A B X$ & $16-$ & 32 & C $16-32$ & $A C T-I$ 4PI \\
\hline & & & & 1 & & & SST \\
\hline & $\mathrm{BDHC}$ & $G, N$ & NOX & THR & & CTHR & $\begin{array}{l}\text { BF 3-I 4P I } \\
\text { THRE SHOLD }\end{array}$ \\
\hline
\end{tabular}

THALLIUM $Z=8]$

\begin{tabular}{cccccccccc} 
A & AEUND. & \multicolumn{8}{c}{ SEPARATION ENERGIES (MEV) } \\
& & G,N & G,P & G, T & G,HE. & G.A & G,2N & G,NP & G,2P \\
203 & 29.5 & 7.7 & 5.7 & 11.2 & 13.4 & -0.9 & 14.7 & 13.5 & 14.2 \\
205 & 70.5 & 7.5 & 6.4 & 11.4 & 14.9 & 0.1 & 14.2 & 13.9 & 15.6
\end{tabular}

REF NUCLIDE REACTION RES EXCIT SOURCE DETECTOR

$\begin{array}{llllllllll}60 R E 1 & B 1 T L & G, G & A B X & 7 & D & 7 & \text { NAI-D } & 90 \\ 66 B E 3 & 81 T L & G, G & R L X & 5-10 & 0 & 5-10 & \text { NAI-D } & 135\end{array}$

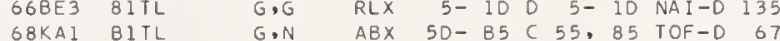

$56 G A 1$ 81TL G.XN ABX $7-27 \mathrm{C} \quad 7-27$ BF $3-14 P I$

$65 \mathrm{MO2}$ BITL G,XN ABX 10-110 C 16-110 ACT-I 4PI

$71 E M 2$ BITL G,F ABY THR-999 C3DO-999 TRK-I 4PI

$67 M I 1$ BITL G,F ABX $300-999 \quad 6300-999$ TRK-I DST 
REF

NUCLIDE REACTION RES EXCIT SOURCE DETECTOR

610E3 81TL2O3 G.G LFT I O I NAI-D DST

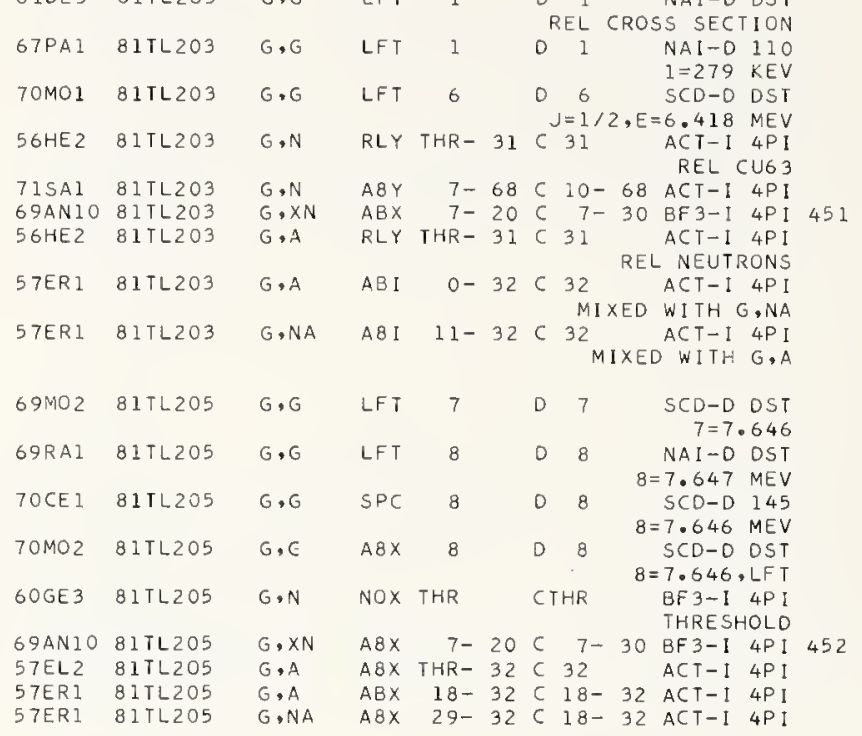

LEAD $\quad Z=82$

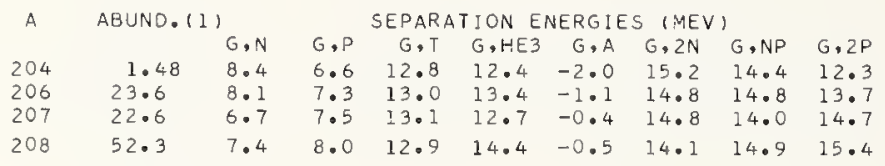

REF NUCLIDE REACTION RES EXCIT SOURCE DETECTOR

59BA3 82P8 E,N A8Y THR- $36010-368 \mathrm{~F} 3-1$ 4PI

$\begin{array}{ll}71 V O I & 82 \mathrm{~PB} \\ 71 \mathrm{MA} & 82 \mathrm{~PB}\end{array}$

$558 \cup 1 \quad 82 \mathrm{~PB}$

$56 \mathrm{FUI} \quad 82 \mathrm{~PB}$

$59006 \quad 82 \mathrm{~PB}$

$\begin{array}{ll}59 P A 3 & 82 \mathrm{~PB} \\ 6 \text { 6RE } & 82 \mathrm{~PB}\end{array}$

6IMI1 $82 \mathrm{~PB}$

$61 T 01$ 82PB

$628 E 2 \quad 82 P B$

$\begin{array}{ll}628 E 2 & 82 \mathrm{~PB} \\ 62 \mathrm{~F} \cup 4 & 82 \mathrm{~PB}\end{array}$

$63802 \quad 82 \mathrm{~PB}$

$63 \mathrm{FLI} 82 \mathrm{~PB}$

$63 Y 01 \quad 82 \mathrm{~PB}$

$\begin{array}{ll}64 \mathrm{ARI} & 82 \mathrm{~PB} \\ 64 \mathrm{GI} & 82 \mathrm{~PB}\end{array}$

$65611 \quad 82 \mathrm{~PB}$

$660 E 1$ 82PB

$55 \mathrm{JO} 182 \mathrm{~PB}$

$55 \mathrm{JO} \quad 82 \mathrm{~PB}$

$5 B A S 1$ B2PB

GOGRI $82 \mathrm{~PB}$

GITAI $82 P B$

$628 R 4$ B2P8

$62 \mathrm{MI} 3$ B2PB

$64 A L 4 \quad 82 \mathrm{~PB}$

64GL1 B2PB

67GE2 $82 \mathrm{~PB}$

S7HU2 B2P8

GBJU1 B2PB

68KA1 $82 \mathrm{~PB}$

69TSI $82 \mathrm{~PB}$

\begin{tabular}{|c|c|c|c|c|c|c|c|}
\hline$E, N$ & $A 8 Y$ & THR - & 36 & 0 & $10-36$ & $8 F 3-1$ & $4 \mathrm{PI}$ \\
\hline $\begin{array}{l}E, N \\
E, F\end{array}$ & $\begin{array}{l}\text { A } 8 X \\
\text { SPC }\end{array}$ & $\begin{array}{l}\text { THR-2 } \\
\text { THR-9 }\end{array}$ & $\begin{array}{l}66 \\
999\end{array}$ & & $\begin{array}{l}50-266 \\
00.999\end{array}$ & $\begin{array}{l}\text { TOF-O } \\
\text { TRK-I }\end{array}$ & $\begin{array}{r}90 \\
4 P \mathrm{P}\end{array}$ \\
\hline & & & & & & $99=1.3$ & GEV \\
\hline$G, \epsilon$ & $R L X$ & $0-$ & 3 & $c$ & 3 & $N A I-D$ & 90 \\
\hline$G, G$ & $A 8 X$ & 4- & 40 & c & $4-40$ & NAI -0 & 120 \\
\hline$G, G$ & A $8 x$ & 6 , & 7 & 0 & 6, 7 & $N A I-D$ & 30 \\
\hline$G, G$ & $A 8 x$ & 17 & & 0 & 15,18 & NAI I D & 90 \\
\hline$G, \epsilon$ & $A 8 X$ & 7 & & 0 & 7 & $\begin{array}{l}N A I-D \\
G \rightarrow W\end{array}$ & $\begin{array}{l}\text { DST } \\
\text { NDTH }\end{array}$ \\
\hline$G, G$ & $A 8 x$ & $10-$ & 22 & D & $10-22$ & NAI I D & DST \\
\hline$G, G$ & $A 8 x$ & $3-$ & 16 & c & $3-16$ & NAI I-D & 120 \\
\hline$G, E$ & A $8 x$ & $5-$ & 9 & D & $5-$ & NAI I D & 135 \\
\hline$G, G$ & $A 8 X$ & 4- & 9 & c & 4- & NAI-D & 120 \\
\hline$G, G$ & $A B X$ & 9 & & 0 & 9 & NAI - D & DST \\
\hline$G, G$ & LFT & $2-$ & 6 & D & $2-$ & $\begin{array}{l}\text { NAI }-D \\
\text { G-WI }\end{array}$ & $\begin{array}{r}30 \\
10 T H\end{array}$ \\
\hline$G, G$ & $A 8 X$ & $5-$ & 8 & D & $5-$ & NA I-D & DST \\
\hline$G, G$ & $A 8 X$ & 7 , & 7 & 0 & 7. & NA I - D & 135 \\
\hline$G \cdot G$ & NOX & 7, & 8 & D & 7 , & $\begin{array}{r}\mathrm{NAI}-\mathrm{D} \\
\text { WI }\end{array}$ & $\begin{array}{r}135 \\
\text { IDTH }\end{array}$ \\
\hline$G, G$ & LFT & 8 & & D & $5-$ & $\begin{array}{r}\text { NAI I D } \\
8=7.28\end{array}$ & $\begin{array}{l}135 \\
M E V\end{array}$ \\
\hline$G, G$ & RLX & $12-$ & 17 & D & $12-17$ & NAI I D & DST \\
\hline$G, N$ & RLY & THR - & 65 & c & 65 & $S C I-D$ & DST \\
\hline$G, N$ & RLY & THR- & 65 & c & 65 & $S C I-D$ & DST \\
\hline$G, N$ & NOX & THR- & 17 & $c$ & 17 & $S C 1-1$ & DST \\
\hline$G, N$ & $A B I$ & $10-$ & 21 & $c$ & $10-21$ & $8 F 3-I$ & 4P I \\
\hline$G, V$ & NOX & $10-$ & 22 & c & 22 & THR-I & DST \\
\hline$G, N$ & $S P C$ & $8-$ & 33 & c & 33 & $S C 1-0$ & 90 \\
\hline$G, N$ & $A B X$ & $6-$ & 22 & D & $6-22$ & $8 F 3-1$ & $4 \mathrm{PI}$ \\
\hline$G, N$ & NOX & THR- & 18 & $c$ & 18 & $5 C I-1$ & OST \\
\hline$G, N$ & $A B X$ & $8-$ & 20 & c & $8-20$ & BF 3-I & $4 \mathrm{PI}$ \\
\hline$G, N$ & SPC & 16 & & 0 & 16 & TOF -D & 90 \\
\hline$G, N$ & $A B Y$ & THR- & 27 & $c$ & $22 \cdot 27$ & $8 F 3-1$ & 4PI \\
\hline$G, N$ & $A B Y$ & THR- & 22 & $c$ & 22 & THR-I & DST \\
\hline$G, N$ & NOX & THR - & 22 & c & 22 & THR-I & DST \\
\hline$G, N$ & $A B X$ & $50-$ & 85 & c & $\begin{array}{r}55,85 \\
\text { NEUT }\end{array}$ & $\begin{array}{l}\text { TOF-D } \\
\text { ENGY S }\end{array}$ & $\begin{array}{r}67 \\
P E C\end{array}$ \\
\hline$G, N$ & NOX & $14-$ & 26 & c & 26 & $S C D-D$ & DST \\
\hline
\end{tabular}

REF

NUCLIDE REACTION RES EXCIT SOURCE DETECTOR

55BA5 82PB

$55011 \quad 82 P B$

$55 \mathrm{MCl} \quad 82 \mathrm{~PB}$

$56 \mathrm{HAl} 82 \mathrm{~PB}$

$57 T 01 \quad 82 \mathrm{~PB}$

$58 F \cup 1 \quad 82 P 8$

$61 B A 2 \quad 82 \mathrm{PE}$

G1BA2 $82 P 8$

6IMII B2PB

64AL5 82P8

MCl $82 \mathrm{~PB}$

$68 T 01 \quad 82 P 8$

$69 G A 3 \quad 82 \mathrm{~PB}$

$70 K 12 \quad 82 \mathrm{~PB}$

$70 M C 1 \quad 82 \mathrm{~PB}$

$62 \mathrm{SH} 2 \quad 82 \mathrm{~PB}$

$62 \mathrm{SH}_{4} \quad 82 \mathrm{PE}$

$71 \mathrm{ANI} \quad 82 \mathrm{~PB}$

71ANI $82 \mathrm{~PB}$

$67 M I 1 \quad 82 \mathrm{~PB}$

GTRA2 $82 P B$

TIEMI $82 P B$

$71 E M 2 \quad 82 \mathrm{~PB}$

$71 M E I \quad 82 \mathrm{~PB}$

$72 \mathrm{KR} 3 \quad 82 \mathrm{~PB}$

$71 G R 2 \quad 82 P 8$

$71 G R 2 \quad 82 P 8$

66BEI 82PB

$66212 \quad 82 P 8206$

$68211 \quad 82 P 8206$

GORE1 $82 P 8206$

$62 F U 4 \quad 82 P B 206$

63AXI 82P8206

66BE3 82PB206

$71 M E 2 \quad 82 P 8206$

6OGE3 82P8206

$64 \mathrm{HA2} \quad 82 \mathrm{P} 8206$

$67 \mathrm{HUI} 82 \mathrm{~PB} 206$

$69801 \quad 82$ PB206

$718 A 2 \quad 82 P 8206$

64HAC $82 P B 206$

62FU4 82PB206

69VEI 82PB206

$66212 \quad 82 P 6207$

$68211 \quad 82 \mathrm{PB207}$

$715 \mathrm{H} 2 \quad 82 \mathrm{~PB} 207$

GOGE3 82PB207

$638 E 4 \quad 82 P B 207$

$64 \mathrm{HA2} \quad 82 \mathrm{~PB} 207$

S6DE2 $82 P B 207$

$69801 \quad$ B2P8207

$69804 \quad 82 P 8207$

$70801 \quad 82 P B 207$

$718 \mathrm{A2} \quad 82 \mathrm{P} 8207$

$64 \mathrm{HA2} \quad 82 \mathrm{PB2} 27$

$62 \mathrm{FU} 4 \quad 82 \mathrm{P} 8207$

$550 E 1 \quad B 2 P B 207$

$62501 \quad 82 P 3207$

605T1 $82 P 8207$
G,XN ABY 30-200 C150-250 THR-I DST

$G, X N$ SPC THR - 70 C 70 EMU-D DST

$G, X N$ RLY THR- 22 C 22 NAI-I 90

,$X N$ ABX 15, 180015,18 BF 3-1 4P!

G.XN A8X 8- 22C $8-22$ BF3-I 4PI

$G, X N$ RLX $7-40$ CPECT RUM WITH EMUL

G,XN A8Y THR- 22 C 22 THR-1 DST

$G, X N$ A8Y THR - 22 C 22 THR-I OST

,$X N \quad A B X \quad 8-20$ D $8-208 F 3-I \quad 4 P I$

GXXN NOX THR $=34 C 34$

GXN NOX THR $=32 C 22-32$ THR 1 DST

OXN $A B X$ IO- $24 C 22-32$ THR-I OST

,XN ABX 10- 24 C $10-24$ BF $3-14 P I$

$G, X N \quad S P C \quad 8-85 C 85$ CCH-D 135

G.XN SPC 7-18C $15-18$ TOF-D OST

$G, X N$ SPC 8- $31 C 31$ THICK TARGETS

G.P SPC $10-34$ C 23, 34 EMU-D DST

$G, P$ SPC 9- 34 C 23, 34 EMU-D DST

GP SPC 37-999 C700,999 TEL-D DST

$999=1.2$ GEV, REL D/P

G.0 SPC 42-999 (701),999 TEL-D DST

G,F AEX 300-999 C 301.2 GEV, REL D/P

$999=1600$ MEV
G.F AEX THR-260 C100-260 EMUI DST

G.F ABY THR-990 C C $300-90$ - 99 TRK-I 4 P I

$999=1000 \mathrm{MEV}$
GF A8Y THR- $999 \quad 300-999 \quad$ TRK -1

A8Y THR $9990300-1000$ MEV
$999=1000$

G,F ABY THR-900 C300-900 TRK-I 4PI

$G, F \quad A B Y$ THR- $999 \quad 350-999$ TRK-I DST

$\begin{array}{rr}999=1 & \text { GEV } \\ & \text { EMU-D DST }\end{array}$

PI-/PI+ YIELD RATIO
G.PI - ABY 150-560 C560 EMU-D OST

N,G SPC $14 \quad$ PI-/PI+ YIELO RATIO

E,E/ RLX 3 O 28-70 MAG-O OST

$\begin{aligned} B(E L) & 3=2.6 \mathrm{MEV}\end{aligned}$

B(EL) 3 LEVELS

$G, G \quad A B X \quad 7 \quad 0 \quad 7 \quad$ NAI-D DST

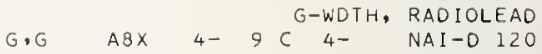

$G, G$ ABX 6- 8 D 6- RADIOLFAD

G.G RLX 5- 10 D 5- 10 NAI-D 135

G.G LFT 2 D 2 SCO-D OST

$2=1.704 \mathrm{MEV}$

$G, N$ NOX THR CTHR BF3-I 4 PI

$G, N$ ASX 6- $27006-268 F 3-\left[\begin{array}{llll}4 & 170\end{array}\right.$

$G, N \quad A B X$ 9- $11 D$ 9- $118 F 3-I$ A I

$G, N$ SPC THR- $10 \mathrm{C} 8-10$ TOF-D 135 G-WIDTH

$G, N$ ABX 8- $10 \mathrm{C} 9,10$ TOF-D 135

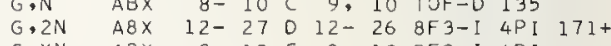

$G, X N \quad A B X \quad 8-19 C 8-19 B F 3-I \quad 4 P I$

ENRICHED P8206

E, E/ RLX 3 O $28-70$ MAG-D DST

$8(E L) \quad 3=2.62 .2 .66$
E,E/ FMF 2- 5 D 28- 73 MAG-D 100

5 LEVELS
$2-5 D 28-73$ MAG-D 100

$\begin{array}{llllllll}E, P & A B X & 12- & 14 & \text { D } 19-21 & \text { MAG-D } & 125 \\ G, N & \text { NOX THR } & \text { CTHR } & \text { BF } 3-1 & 4 P I\end{array}$

G.N SPE 7- 96 THRESHOLD

$\begin{array}{lllrlllll}G, N & S P C & 7- & 9 & C & 9 & \text { TOF }-D & \text { UKN } & \\ \mathrm{GNN} & \mathrm{ABX} & 6-27 & \mathrm{D} & 6-26 & 8 \mathrm{~F} 3-1 & 4 \mathrm{PI} & 173\end{array}$

$\mathrm{G}, \mathrm{N}$ SPC THR- $80 \mathrm{C} B \mathrm{CO} \mathrm{CCH}-\mathrm{D} 135$

$G, N$ SPC THR - 9 C $7-9$ TOF-D 135

G,N GBX THR- GIOTH

25-350 KEV NEUTS

G,N ABX THR- $8 \mathrm{C} 9$ TOF-D 135

$G, N \quad A B X$ ERRATUM FOR $69 \mathrm{BO} 4$

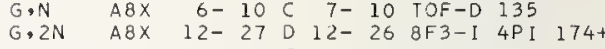

$G, X N \quad A B X \quad 6-19 C$ G 19 BF 3-I 4PI

$G, P \quad A B X 11-21 C 11-21$ ACT-I $4 P I$

MIXED WITH P8208

$G, P A B \times 15-33 C 15-33$ ACT-I 4PI

G,NP RLX 144-320 MIXED WITH P8208

C 320 TEL-D 76
REL TO H2 CROS SEC 


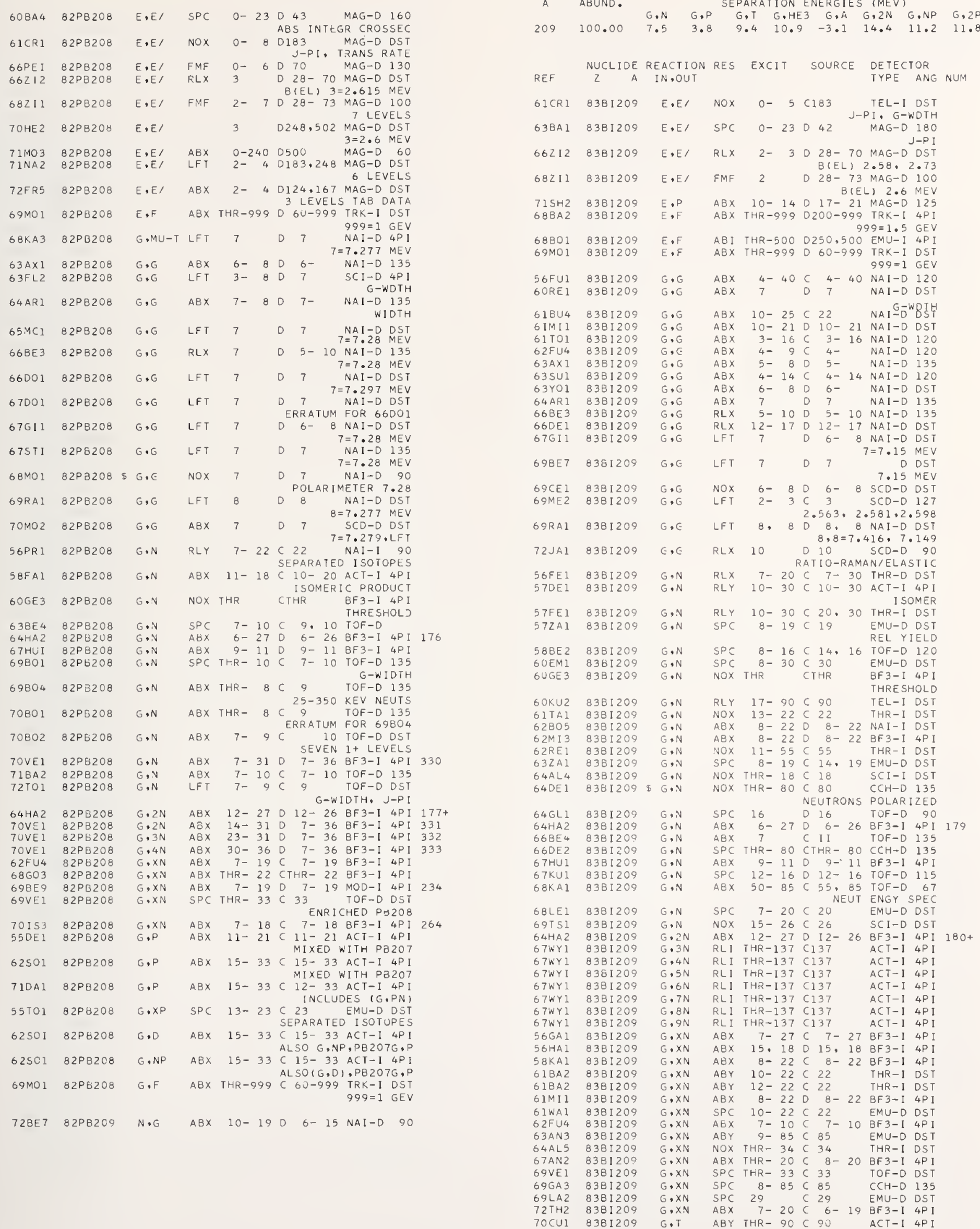




\begin{tabular}{|c|c|c|c|c|c|c|c|}
\hline WYI & $38 I 209$ & , 4NP & RLI & THR-137 & $\mathrm{C}_{137}$ & $A C T-I$ & $4 \mathrm{PI}$ \\
\hline $67 W Y 1$ & 381209 & $5.5 \mathrm{NP}$ & RLI & $T H R-137$ & C137 & $A C T-I$ & $4 P I$ \\
\hline 67WYI & 38 I 209 & - 6NP & RLI & THR- 137 & C137 & $A C T-I$ & 4PI \\
\hline WYI & 8120 & $.7 \mathrm{NP}$ & & $T H R-I 37$ & & $A C T-I$ & \\
\hline 7WY1 & $\mathrm{BI2}$ & AP & RLI & THR-137 & C1 & - I & \\
\hline & & & $A B X$ & $300-999$ & .999 & & \\
\hline OE & & $F$ & $3 x$ & 30 & 99 & $\begin{array}{l}E M U-0 \\
999=1\end{array}$ & $\begin{array}{l}\text { 4P I } \\
\text { GEV }\end{array}$ \\
\hline MI 1 & B 3812 & $G, F$ & $A B X$ & $300-999$ & $\begin{array}{r}999 \\
999\end{array}$ & $\begin{array}{l}\text { TRK - I } \\
9=16000\end{array}$ & $\begin{array}{l}\text { OST } \\
\text { MEV }\end{array}$ \\
\hline$R A$ & & & $3 x$ & TH & $C 100-260$ & EMU-I & \\
\hline WA & & & $3 x$ & $30-40$ & $\begin{array}{r}30-40 \\
\text { FRAGME }\end{array}$ & $\begin{array}{l}\text { ACT-I } \\
\text { ENT YIE }\end{array}$ & $\begin{array}{l}4 P \\
=L O\end{array}$ \\
\hline $\mathrm{KOZ}$ & 8381209 & $G, F$ & SPC & THR-999 & $C 250-999$ & $S C O-D$ & DS \\
\hline MO 1 & 8381209 & $G$, & $A B X$ & THR-999 & 999 & $\begin{array}{r}=1000 \\
\text { TRK-1 } \\
999=1\end{array}$ & $\begin{array}{l}\text { OST } \\
\text { GEV }\end{array}$ \\
\hline S & & & & & & & \\
\hline 7 & & & $A B Y$ & Th & $\begin{array}{r}C 300-999 \\
998\end{array}$ & $\begin{array}{l}\text { TRK - I } \\
S=1000\end{array}$ & $\begin{array}{l}4 P \\
M E V\end{array}$ \\
\hline 14 & 8381209 & G,F & $A B X$ & $100-999$ & C100-999 & TRK-I & 4P I \\
\hline SNR & 8381209 & $P, G$ & $A B X$ & $13-22$ & $09-18$ & NAI-D & 9 \\
\hline & 12 & , & & & & & \\
\hline
\end{tabular}

RADIUM $Z=B B$

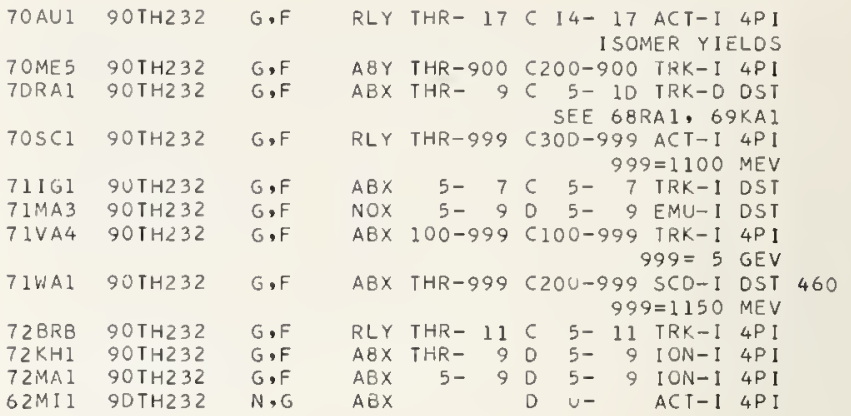

URANIUM $Z=92$

\section{ABUNO. \\ SEPARATION ENERGIES (MEV) \\ $\begin{array}{llllllll}G, N & G, P & G . T & G, H E 3 & G . A & G, 2 N & G, N P & G .2 P \\ 6.4 & 6.9 & 9.7 & * & -4.9 & 11.3 & 13.4 & *\end{array}$ \\ NUCLIOE REACTION RES EXCIT SOURCE OETECTOR

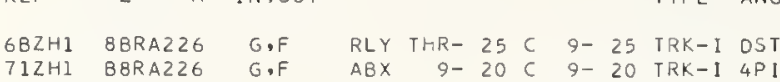 N/F EMISSION WIOTH}

THORIUM $Z=90$

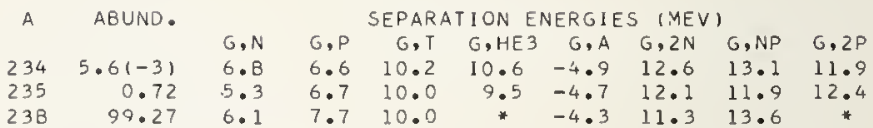

RUCLIDE REACTION RES EXCIT SOURCE OETECTOR
REF AYPE ANG NUM

$71 M A Z \quad 92 \mathrm{U}$

$71 \mathrm{KII} \quad 92 \mathrm{U}$

$57 \mathrm{KAI} \quad 92 \mathrm{~V} 233$

E,F

SPC ThR-999 0500,999 TRK-I 4PI

$G, X N \quad S P C \quad 5-23 C 15-23$ TOF-O 90

$\begin{array}{lll}5 B K A 2 & 92 U & 233 \\ 59 B A 4 & 92 U & 233\end{array}$

$62 \mathrm{HU1} \quad 92 \mathrm{U} 233$

$G, \times N$

A8X 6- 23 C 6- 23 BF3-I $4 \mathrm{PI}$

$70 \mathrm{AUI} \quad 92 \mathrm{U} 233$

$G, F$
$G, F$

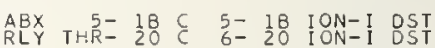

G.F $A B X \quad 6,700,6, \quad$ ION-I 4 P I I SOMER YIELDS

$598 A 4 \quad 920234$

$62 \mathrm{HUI} 92 \mathrm{U} 234$

$70 A U I \quad 92 U 234$

$G, F$
$G, F$
$G, F$ ISOMER YIELDS

$64 B 03 \quad 92 U 235$

$64803 \quad 920235$

64803920235

$728 R B \quad 920235$

$598 A 4 \quad 920235$

$62 \mathrm{HUI} 92 \mathrm{U} 235$

64803920235

$66 \mathrm{KO3} 92 \mathrm{U} 235$

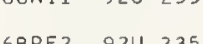

6BPE2 920235

69PEI $92 U 235$

$70 \mathrm{KUZ} \quad 92 \mathrm{U} 235$

TOAUI 924235

71VA4 $92 U 235$

$72 B R 8 \quad 92 U 235$

$72 \mathrm{KHI} \quad 92 \mathrm{U} \quad 235$

598A4 920236

$62 \mathrm{HUI} \quad 92 \mathrm{U} 236$

$70 A U 1 \quad 92 U 236$

59BA3 920238

67RA3 92U 238

68801924238

69GR2 920238

$69 M 0192423 B$

$70 \mathrm{KAL} \quad 92 \mathrm{U} 23 \mathrm{~B}$

56 FUI 924238

63802920238

$71 \mathrm{HAl} 92 \mathrm{U} 23 \mathrm{~B}$

$72 J A 1 \quad 92 U 238$

56011924238

6ITA1 92U 238

64GL1 $92023 B$

690E1 $924 \quad 238$

71012924238

$72 M A 1 \quad 924238$

$725 C 5 \quad 924238$
G.F ABX 6, 70 6, 1 ON-I 4 PI RLY THR- 17 C $14-17$ ACT-1 4 P I I SOMER YIELDS

$G, N \quad A B X \quad B-21008-21 B F 3-1$ API 424

G,2N A8X B- 21 D B- 21 BF 3-I 4 PI 442

$\begin{array}{llrllll}G, T N & A B X & 8-21 & 0 & B-21 & B F 3-I & 4 P I \\ G, X N & R L Y T H R-11 & C & 5-11 & B F 3-I & 4 P I\end{array}$

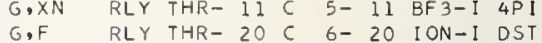

G,F ABX 6, 7 D $6, \quad$ ION-I 4 PI

G,F ABX 6- $19006-19$ ION-I 4 PI 425

G,F RLY THR- 25 C $10-25$ ACT-I 4 PI

G,F RLY THR- 15 C $10-15$ TRK -0 4PI

G,F NOX THR - 25 C 25 DELAYE N YIELOS

G.F SPC THR- 12 C 12 ION-D API

G.F NOX 5- 10 MASS, ENERGY SPC

OELAYEO NEUT YLDS G,F RLY THR- 17 C $14-17$ ACT-I 4PI G,F ABX 100-999 C100-999 TRK-I 4PI G,F RLY THR- II C 5- 11 T99 TRK-1 4 G I I G,F ABX THR- 90 5- 9 ION-I 4 P I G,F RLY THR- 20 C 6- 20 ION-I DST G.F A8X 6, $7006, \quad 10 N-I$ 4PI G,F RLY THR- 17 C $14-17$ ACT-I 4PI ISOMER YIELOS

$E \cdot N$

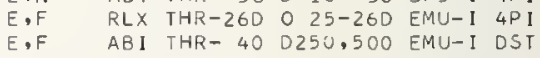

ABY THR- 36 D 10- 36 BF3-1 4PI IF RLY THR-999 C 70-999 ACT-I 4PI E. F ABX THR-999 D 60-999 TRK-I DST $999=1$ GEV

E, F ABX THR-50D C 25-500 TRK-D 4PI G. G ABX $4-40 \mathrm{C}$ 4- 40 NAI-O 120 $G, G$ ABX 9 O 9 NAI-O DST G, G ABX 8- 9 D 8- 9 SCD-D 90 $\begin{array}{rrr}\text { RLX } 10 \text { D } 10 & \text { SCO-D OST } \\ \text { RATIO-RAMAN/ELASTIC }\end{array}$ G,N ABX 8- 16 C 8- 2D ACT-1 4PI G,N NOX THR- 22 C 22 THR-I OST

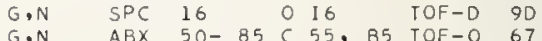
NEUT ENGY SPEC $G, N$ ABY THR-999 C 1- 6 ACT-I 4PI G.N ABY $7-999$ C $30 D-99999=5.5$ GEV $G, N \quad A B Y \quad 7-999 \quad(300-999$
$999=1$ $G, N \quad A B X$ 5- $9 D$ D- $9 B F 3-I \quad 4 P I$ $G, N$ ABY THR-7DO CI5D-7OD ACT-O $4 P I$ 
55BA4 92U 238 G,XN ABY 12 C 19 8F3-1 4PI

$56 G A 1$ $92 U 238$ G $X N$ ABX $5-27 C 5-278 F 3-14 P$

$57 K A 192 U 238$ G,XN ABX 6- 23 C $6-238 F 3-14 P$

61BA2 $92 \mathrm{U} 238$ G,XN ABY THR- 22 C 22 THR-I DST

T2ER8 92U 238 G.XN RLY THR- 11 C $5-11$ 8F3-1 4PI

$\begin{array}{lllllllll}558 A 4 & 92 U & 238 & G, F & A B Y & 12 & C & 19 & \text { [DN-I } 4 P\end{array}$

55KAI $92 \mathrm{U} 238$ G,F ABX THR- 26 C $12-26$ ACT-1 $4 P$

$55 L A 292 U 238$ G,F RLY THR- $19 \mathrm{C} 19$ BF3-1 $4 P 1$

56GII 920238 G,F A8X 8- $20 \mathrm{C} \quad 8-20$ ACT-I 4PI

56KD2 $92 \mathrm{U} 238$ G,F $A B X \quad 8-24 \mathrm{C} \quad 8-24$ IDN-I $4 \mathrm{P}$

$56 K D 392 \mathrm{~L} 238 \quad$ G.F $\quad$ SPC THR- 18 C 18 IDN-D $4 \mathrm{P}$

$578 A 492 U 238$ G,F NDX THR- $27 \mathrm{C} 9-27$ EMU-D DS

$575 C 292 U 238$ G,F RLY THR- 15 C 4- 16 ACT-I 4PI

$588 A 7$ 92U 238 G,F NDX THR- $27 \mathrm{C}$ 6- 27 EMU-I DST

$58 \mathrm{KA2} 92 \mathrm{U} 238$ G.F ABX 5- 18 C 5- 18 IDN-1 DST

$598 A 492 U 238$ G,F RLY THR - $20 \mathrm{C}$ 6- 20 IDN-I DST

$60 B D 192 U 238$ G,F SPC THR- $70 \mathrm{C} 70$ IDN-D 4 P

$60 F D 192 U 238$ G,F NDX 6- 20 C 6- 20 EMU-I DST

GOPR2 924238

GOTA3 920238

6IDEl 920238

$610 E 2 \quad 92 \mathrm{U} 238$

$62808 \quad 920238$

$620 E 3 \quad 920238$

62 HUI $92 \mathrm{U} 238$

$62 K D 4 \quad 924238$

$62 M 03920238$

$63806 \quad 920238$

$30 E 1 \quad 92 U 238$

$63 P E 292 U 238$

65AL1 920238

$65 \mathrm{HDI} 92 \mathrm{U} 238$

$65 H 02920238$

65KI1 920238

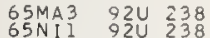

65501924238

$6550292 \mathrm{U} 238$

66801920238

66MA3 $92 \mathrm{~V} 238$

66.MEZ 924238

66NII 924238

66SAl 92U 238

67KA1 $92 \mathrm{U} 238$

67RA3 $92 \mathrm{U} 238$

$68 \mathrm{KUI} 92 \mathrm{U} 238$

68RAI $92 U 238$

69KAl $92 \mathrm{U} 238$

$69 K D 292 U 238$

69MAI $92 \mathrm{U} 238$

$69 M 01 \quad 92 \cup 238$

69WAI 920238

TOAUI 920238

7OKAL 92U 238

$70 K U 2 \quad 92 U 238$

TOMES 920238

TORAI $92 \mathrm{U} 238$

$705 C 1 \quad 920238$

$71001920 \quad 238$

$\begin{array}{lll}711 G 1 & 92 U & 238 \\ 71 P E 1 & 920 & 238\end{array}$

$71 T A 1 \quad 924238$

7IVA4 92U 238

7 IWAI 920238

72AN2 $92 \mathrm{~A} 238$

$72 B R B \quad 92 U 238$

T2UAG 920238

$72 \mathrm{KHI} 92 \mathrm{U} 238$

$72 \mathrm{MAl} 92 \mathrm{U} 238$

$725 C 5920238$
G,F NDX 7 D 7 BF 3-I 4 P I

G,F NDX 6- $20 \mathrm{C}$ 6- 20 EMU-I DST

G,F ABX 300-999 C300-999 EMU-I 4PI

G,F SPC THR- 50 C $17-50$ IDN-D 90

G,F A A X 7 A $\quad D \quad 7$ EMU-I DST

G,F A8X 6, $706, \quad$ IDN-I $4 P$ I

G,F NOX THR- 35 C 35 IDN-D DST

G,F ABY THR- $20 \mathrm{C}$ MASS DISTRIBUTIDN DELAYED $N$ YIELDS

G,F ABX 7 A $\quad 077$ EMU-I DST

G.F RLY THR - 14 C 14 ACT-I 4 P I

G,F RLX 6- 7 D 6- 7 EMU-D DST

A8Y 300-999 C999 EMU-I 4PI

G,F RLY THR- 33 C $33 \quad$ SCD-D 90

NDX 7 D 7 MASS SPC

SDURCE FIO(P,AG)

G,F ABY 6- 7 C 5- 7 ACT-I $4 \mathrm{PI}$

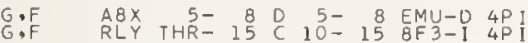

G.F RLX 6, 70 D, 7 TRK-1 DST

G,F NDX THR -9 C 5- 9 EMU-I DST

G, F NDX THR- 9 C 5- 9 TRK-I DST

G,F ABX 5- $9 D$ 5- 9 EMU-I DST

G,F ABY 17 D 17 ACT-1 4 P I

GF RLY THR- IS C SOURCE LIT (P,G)

GLY THR- 15 C $10-15$ TRK
DELAYED N Y Y I I

RLY THR-650 C650 ACT-I 4PI

RLY THR -7 C $5-7$ ACT - I 4 PI

A $8 Y$ THR -7 C $4-7$ ACT-I 4 PI

NDX THR - $8 \mathrm{C} 5-8$ TRK-I DST

$A 8 \times$ THR - 8 C 5- 8 TRK - I DST

SPC THR-999 C250-999 SCD-D DST

$999=1000 \mathrm{MEV}$

$G, F \quad A B \times 5-9 D 5-9$ EMU-I DST

G,F A8X THR-999 C 60-999 TRK-I DST

RLX $0-999 \quad 200-999 \quad 5 C D-D$ DST

RLY THR- 17 C $14-17$ ACT-I 4 PI

OF RLY THR- 17 C $14-$ ISPMER YIELOS
ISDMER

G,F ABY THR-500 C 25-500 TRK-D 4PI

G,F NDX 5- $10 \mathrm{C} 8,108 \mathrm{~F} 3-\mathrm{I}$ 4PI

G,F DELAYED NEUT YLDS

SEE 68RAI, G9KAI

G,F RLY THR-999 C300-999 ACT-I 4 PI

G,F NDX 6- 9 D $6-9$ TRK-I DST

G,F A8X 5- 8 C 5- 8 TRK-1 DST

G,F LFT THR - 53 C 53 131-136 YIELDS

GELAYED FISSIDN

$999=5$ GEV
99 G

G,F ABX THR-999 $\quad 200-999$ SCD-1 DST 46

G,F RLY THR- IIC 5- 11 TRK-I 4PI

G,F ABY THR-999 C800-999 TRK-I DST

$G, F$ ABX 5- $9 D$ D- 9 IDN-I $4 P$ :

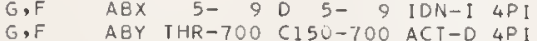

FISSIDN PRDDUCTS
G,F SPC 6 SDURCE FI9(P,AG)

G,F NDX THR- 35 C 35 IDN-D DST

999=1 GEV

RLX THR-260 C 25-260 EMU-I 4PI

G,F RLY THR - 25 C 15,25 MSP-I 4 PI

G.F RLX 5- 80 5- $\begin{array}{r}999=1150 \\ 8 \text { SCD-1 }\end{array}$

G,F ABX THR- $9 D \quad 5-\quad \begin{array}{r}999=2.2 \\ 9 \text { IDN-I }\end{array}$

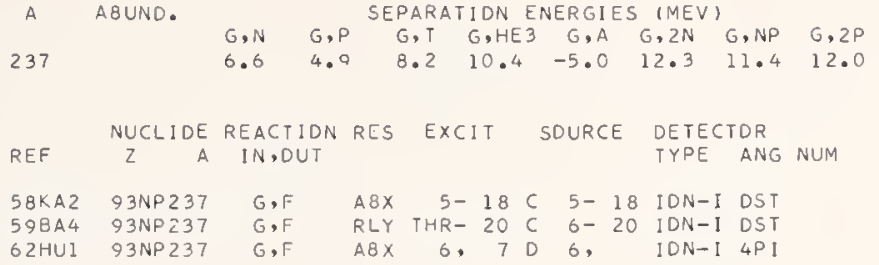

PLUTDNIUY $Z=94$

\begin{tabular}{lllllllll} 
A & ABUND. & \multicolumn{7}{c}{ SEPARATIDN ENERGIES (MEV) } \\
& G,N & G,P & G, T & G,HES & G,A & G, $2 N$ & G,NP & G, 2P \\
238 & 7.0 & 6.0 & 9.8 & 9.7 & -5.5 & 12.9 & 12.6 & 10.9 \\
239 & 5.7 & 6.2 & 9.8 & 8.8 & -5.2 & 12.7 & 11.6 & 11.4 \\
240 & 6.5 & 6.5 & 9.7 & 10.2 & -5.3 & 12.2 & 12.7 & 11.8 \\
242 & 6.3 & 6.9 & 9.5 & 10.8 & -5.0 & 11.5 & 12.9 & 12.6
\end{tabular}

\begin{tabular}{cccll}
\multicolumn{1}{c}{ NUCLIDE REACTIDN RES EXCIT SDURCE } & DETECTDR \\
REF A A IN,DUT & 2 & A INE ANG NUM
\end{tabular}

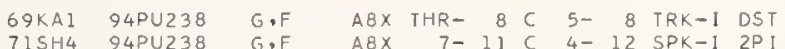

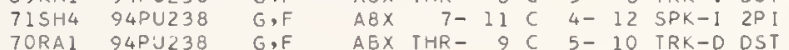

SEE GBRAI, 69KAI

57KAI 94PU239 G,XN ABX 6- 23 C 6- 23 8F3-I 4PI

$728 R 8$ 94PU239 G,XN RLY THR- II C 5- II 8F3-1 4PI

58KA2 94PU239 G,F $\quad$ A8X $5-18 \mathrm{C} 5-18$ IDN-I DST

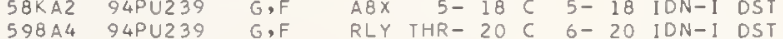

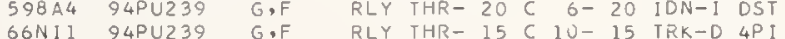

66RA2 94 PU239 G,F NDX 5- 7 C $5-8$ UKN-I DST

68RAI 94PU239 G,F NDX THR- $896 \quad 5, \quad 8$ TRK-I DST

$70 \$ 0294 \mathrm{PU} 239$ G,F ABX 5- 8 C 5- B TRK-D DST

$71 \mathrm{SH} 494 \mathrm{PU} 239$ G.F ABX $7-11 \mathrm{C} 4-12$ SPK-I 4 PI

71TAI 94PU239 G,F LF THR- 53 C 53 SCD-I 90

59EA4 94PU240 G,F RLY THR- $20 \mathrm{C}$ 6- 6 - 20 IDN-I DSI

68RAI 94PU240 G,F NDX THR- 8 C 5.8 TRK-I DST

69KAI 94PIJ240 G,F ABX THR- 8 C 5- 8 TRK-I DST

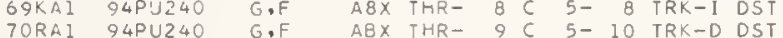

SEE G8RAI, G9KAL

68RAI 94PU242 G.F NDX THR- 865 , 8 TRK-I DST

69KAI 94PU242 G,F A8X THR- 86 5- 8 TRK-I DST

AMERICIUM $Z=95$

A. ABUND.

241

\begin{tabular}{cccccccc}
\multicolumn{1}{c}{ SEPARATIDN ENERGIES (MEV) } \\
G,N & G.P & G.T & $G, H E 3$ & $G, A$ & G, $2 N$ & G, NP & G, $2 P$ \\
6.7 & 4.5 & 8.2 & 9.5 & -5.6 & 12.6 & 11.0 & 11.0
\end{tabular}

NUCLIDE REACTIDN RES EXCIT SDURCE DETECTDR

$\begin{array}{llllrllll}58 K A 2 & 95 A M 241 & G, F & A 8 X & 5-18 & C & 5-18 & \text { IDN-I } & \text { DST } \\ 598 A 4 & 95 A M 241 & G, F & \text { RLY THR }-20 & C & 6-20 & \text { IDN-I } & \text { DST }\end{array}$

$70 G A I$ 95AM24I G,NF THR THR- 14 C 10- 13 SPK-D 4PI PROMPT-DELAYED F

70GAI 95AM243 G.NF THR THR- 14 C $10-13$ SPK-D 4PI 

5. Photonuclear Reaction Data Bibliography 1955-1972 
In the following bibliography Conference Proceedings are indicated as follows;

PICNS-60 Proceedings of the International Conference on Nuclear structure, Kingston, Ontario 1960 (Bromley, D. A. and Vogt, E. W., Eds., University of Toronto Press, 1960)

PICNS-61 Proceedings of the Rutherford Jubilee International Conference Manchester 1961 ( Birks, J. B., Ed., Academic Press Inc., New York, 1961)

PICNS-62 Proceedings of the Conference on Direct Interactions and Nuclear Reaction Mechanisms, University of Padua 1962 (Clementel, E. and Villi, C., Eds., Gordon and Breach, New York-London, 1963 )

PICNS-64 Congres International de Physique Nucleaire, Paris 1964 (Gugenberger, P., Editions Centre Natl. Rech. Sci., Paris, 1964)

PICNS-66 Proceedings of the International Nuclear Physics Conference; Gat $l$ inburg, Tennessee 1966 (Academic Press, New York and London 1967)

PICNS-67 Contributions, International Conference on Nuclear Structure; Tokyo, Japan 1967 (Institute for Nuclear Study, University of Tokyo, Tanashi-shi, Tokyo, Ja pan 1967 )

PICNS-69 Contributions, International Conference on Properties of Nuclear States; Montreal, Canada 1969 (Les Presses de $l$ Universite de Montreal 1969)

55Al1 L. Allen, Jr., Phys. Rev.98,705 ( 1955 )

55 Ba1 W. C. Barber, W. D. George, D. D. Reagan, Phys. Rev. 98, $73(1955)$

55Ba2 R. Basile, C. Schuhl, Compt. Rend, 240, 2399 (1955)

55 Ba3 R. Basile, C. Schuhl, W. Sebaoun, Compt. Rend. 241,387 ( 1955$)$

55Ba4 B. N. Baluev, B. I. Gavrilov, G. N. Zatsepina, L. E. Lazareva; Zhur. Eksp. i Teoret. Fiz. 29, 280 ( 1955 ); Soviet Phys. JETP 2, 106 ( 1956 )

55Ba5 P. S. Baranov, V. U. Gol danskii; Zhur. Eksp. i Teoret. Fiz. 28, 746, (1955); Soviet Phys. JETP 1, 613 (1955)

55Bol O. A. Borello, J. Goldemberg, M. D. S. Santos; An. Acad. Brasil. Cienc: 27,413 (1955)

55Bo2 0. A. Borello, J. Goldemberg, M. D. S. Santos; An. Acad. Brasil. Cilenc. 27,417 ( 1955 )

55Bul J. L. Burkhardt; Phys.Rev. 100, 192 ( 1955 )

55 Bu2 J. L. Burkhardt, E. J. Winhold, T. H. Dupree; Phys. Rev. $100,199-202(1955)$

55Ca1'J. G. Campbell, Australian J.Phys. 8, 449 (1955)

55 Ca2 J. H. Carver, H. J. Hay, E. W. Titterton; Phil. Mag. 46, 841 ( 1955$)$

55De1 M. D. DeSouza Santos, J. Goldemberg, $R$. R. Pieroni, E. Silva, O. A. Borello, S. S. Villaca, J. L. Lopes; Int. Conf. Peaceful Uses of Atomic Energy II (United Nations, N. Y., $1955), 169$

55Dil W. R. Dixon, Can. J. Phys. 33, 785 (1955)

55Dl1 Z. Dlouhy, V. Petrzilka, M. Rozkos; Czech. J.Phys. 5, 193 (1955) 
55Er1 P. Erdos, P. Jordan, P. Stoll; Helv. Phys. Acta 28, 322 (1955)

55Frl J.H. Fregeau and R. Hofstadter; Phys. Rev.99, 1503 ( 1955 )

55 Gl 1 H. Glattli, E. Loepfe, P. Stoll; Helv. Phys. Acta 28,366 ( 1955$)$

55 Go 1 F. K. Goward, J J . Wilkins; Proc. Roy. Soc. (London) A228, 376 ( 1955 )

55 Gr1 G. M. Griffiths, J.B. Warren; Proc. Phys. Soc. A68, 781 ( 1955$)$

55Hal F. I. Havlicek, B. Dobovisek; Phys. Rev. 100,1355 (1955)

55He1 F. Heinrich, R. Rubin; Helva. Phys. Acta 28, 185 ( 1955 )

55Jo1 S. A. E. Johansson; Phys.Rev. 97,434 ( 1955 )

$55 \mathrm{Kal}$ L. Katz, T. M. Kavanagh, A. G. W. Cameron, E. C.

Hailey, J. W. T. Spinks; Phys. Rev.99, 98 ( 1955 )

55 La1 F. B. Lasich, E. G. Muirhead, G. G. Shute; Australian J. Phys. 8, 456 ( 1955 )

55 La2 L. E. Lazareva, B. S. Ratner, I. V. Shtranikh; Zhur. Eksp. i Teoret. Fiz,29,274 (1955); Soviet Phys. JETP 2, 301 ( 1956 )

55Li 1 D. L. Livesey, Can.J. Phys. 33, 391 ( 1955 )

55 Mcl K. G. McNeill; Phil. Mag. 46, 321 ( 1955 )

55Me1 F. R. Metzger, Phys. Rev.98, 200 ( 1955)

55 Mi 1 M. Miwa; J. Phys. Soc. Japan 10, 173 ( 1955)

$55 \mathrm{Na} 1$ R. Nathans, P. F. Yergin, Phys, Rev.98, 1296 ( 1955 )

$558 \times 1$ C. L. Oxley, V. L. Telegdi; Phys. Rev. 100,435 ( 1955 )

55 Pel A. S. Penfold, B. M. Spicer, Phys, Rev. 100, 1377 ( 1955 )

55 Pe2 J. E. Perry, S. J. Bame, Jr.; Phys. Rev.99, 1368 ( 1955 )

55 Re 1 D. Reagan; Phys. Rev. 100, 113 ( 1955 )

55 Ri 1 L. S. Ring, Jr.; Phys. Rev.99, 137 ( 1955 )

55Sa2 D. Sadeh; Compt. Rend.249, 2313 ( 1959 )

55 Sc 1 M. B. Scott, A. H. Hanson, D. W. Kerst; Phys. Rev. 100 , $209(1955)$

55Sc2 J. Schmouker, P. Erdos, P. Jordan, P. Stoll; J. Phys. Radium 16, 169 ( 1955 )

55Sp1 B. M. Spicer, Phys. Rev, 99, 33 ( 1955)

55 Sp2 B. M. Spicer, A. S. Penfold; Phys. Rev. 100, 1375 ( 1955 )

55Sp3 B. M. Spicer; Phys. Rev.100, 791 ( 1955 )

55st1 W. E. Stephens, A. K. Mann, B. J. Patton, E. J. Winhold; Phys. Rev. 98, 839 (1955)

55 To 1 M. E. Toms, W. E. Stephens; Phys.Rev.98, 626 ( 1955 )

55 Val B. N. Valuev, B. I. Gavrilov, G. N. Zatsepina, L. E. Lazareva; Zhur. Eksp. i Teoret. Fiz. 29, 280 ( 1955 ); Soviet Phys. JETP 2, 106 ( 1956 )

55 Vil S. S. Villaca, J. Goldemberg; An. Acad. Brasil. Cienc. 27, 427 ( 1955$)$

56Ax1 P.Axel, J. D. Fox; Phys.Rev.102,400 ( 1956 )

56Bol o. V. Bogdankevich, L. E. Lazareva, F. A. Nikolaev; Zhur. Eksp. i Teoret. Fiz. 31, 405 ( 1956); Soviet Phys. JETP $4,320(1957)$ 
$56 \mathrm{Ch} 1 \mathrm{R}$. Chastel, Compt. Rend 242, 1440 ( 1956 ); Compt. Rend 242, 2337 (1956); J. Phys. Rad. 17, 518 (1956)

56Co 1 L. Cohen, A. K. Mann, B.J.Patton, K. Reibel, W. E. Stephens, E. J.Winhold; Phys. Rev? 104, 108 (1956)

56 Dal 1 . K. Dawson, D. L . Livesey; Can. J P Pys. 34, 241 ( 1956)

56Da2 W. K. Dawson; Can. J. Phys. 34, 1480 (1956)

56Dil D. R. Dixon, K. C. Bandtel; Phys. Rev. 104,1730 (1956)

56 Ed1 R. D. Edge, Nuclear Phys. 2, 485 ( 1956)

56Ed2 R. D. Edge, Australian J. Phys.9, 429 (1956)

56 Er1 J. Ero, L. Keszthelyi, Nuclear Phys.2, 371 ( 1956 )

56Fal B. P. Fabricand, B. A. Allison, J.Halpern; Phys. Rev. $103,1755(1956)$

56 Fel F. Ferrero, A. Hanson, R. Malvano, C. Tribuno; Nuovo Cimento $4,418(1956)$

56Fo1 B. Forkman; Arkiv Fysik 11,265-275 (1956)

56Fr1 J. H. Fregeau; Phys. Rev, 104, 225 (1956)

56Fu1 E. G. Fuller, E. Hayward; Phys. Rev. 101, 692 (1956) See Erratum Phys: Rev. 106,991 (1957)

$56 \mathrm{Ga} 1 \mathrm{~B} . \mathrm{I} \cdot$ Gavrilov, L. E. Lazareva; Zhur. Eksp. i Teoret. Fiz. 30, 855 (1956); Soviet Phys. JETP 3,871 (1957)

56 Gil J. E. Gindler, J. R. Huizenga; Phys. Rev. 104, 425 (1956)

$56 \mathrm{Hal}$. H. Hartley, W. E; Stephens, E. J.Winhold; Phys. Rev. $104,178(1956)$

56 Hel F. Heinrich, H. Waffler; Helv. Phys. Acta 29, 232 (1956) 56 He2 F. Heinrich, H. Waffler, M. Walter; Helv. Phys. Acta 29. $3(1956)$

$56 \mathrm{He} 3 \mathrm{R}$. H. Helm; Phys. Rev. 104, 1466 (1956)

$56 \mathrm{Ke} 1 \mathrm{~J} . \mathrm{C}$. Keck, A. V. Tollestrop, Phys.Rev. 101,360 (1956)

$56 \mathrm{Ko} 1 \mathrm{~A}$. P. Komar, I P. Iavor, Zhur. Eksp. i Teoret.Fiz. 31531 (1956); Soviet Phys. JETP 4, 432 ( 1957 )

56 Ko 2 V. A. Korotkova, P. A Cherenkov, I V . Chuvilo; Dokl. Akad. Nauk SSSR 106,633 (1956); Soviet Phys. Doklady 1, 77 ( 1956$)$

$56 \mathrm{Ko} 3 \mathrm{~V}$. A. Korotkova, P. A. Cherenkov, I. V. Chuvilo; Dokl. Akad. Nauk SSSR 106, 811 (1956); Soviet Phys. Doklady 1, 104 ( 1956$)$

56le1 E. Lejkin, R. Osokina, B. Ratner; Nuovo Cimento 3, Suppl. 1, 105 (1956)

56 Iil 1 D. L. Livesey, Can.J.Phys. 34, 216 (1956)

56 Ii 2 D. L. Livesey, Can. J.Phys. 34, 1022 ( 1956)

$56 \mathrm{Ma} 1 \mathrm{~A} \cdot \mathrm{K} \cdot \mathrm{Mann}, \mathrm{E}$. W. Titterton, Proc. Phys. Soc. 69, 917 (1956)

56Me1 F. R. Metzger, Phys, Rev.101, 286 (1956)

56 Me2 F. R. Metzger, Phys. Rev.103,983 (1956)

56 Me 3 L. Meyer Schutzmeister, V. L. Telegdi; Phys. Rev. 104, 185 ( 1956$)$

$560 d 1$ A. C. Odian, P. C. Stein, A. Wattenberg, B. T. Feld, R. We instein; Phys. Rev, 102, 837 ( 1956 )

$56 \operatorname{Pr} 1$ J. D. Prentice, K. G. McNeill, Phil. Mag. 1, 373 ( 1956)

56Sil E. Silva, J. Goldemberg; Nuovo Cimento 3, 12 ( 1956 ) 
56 Si2 E. Silva, J. Goldemberg; An. Acad. Brasil. Cienc.28, 275 ( 1956$)$

56Su1 T. T. Sugihara, I. Halpern; Phys. Rev. 101, 1768 (1956)

$56 \mathrm{Tz} 1$ C. Tzara; Compt. Rend.242, 2340 ( 1956 )

56 Tz2 C. Tzara; J. Phys. Radium 17, 1001 (1956)

56 Wal H. Waffler, F. Heinrich; Physica 22, 1146 ( 1956 )

56 Wh1 E. A. Whalin, B. D. Schriever, A. O. Hanson; Phys. Rev. $101,377(1956)$

56 Wo1 R. L. Wolke, N. A. Bonner; Phys. Rev. 102, 530 ( 1956 )

56 Wr1 I. F. Wright, D. R. O. Morrison, J, M.Reid, J . R:

Atkinson; Proc. Phys. Soc. A 69, 77 ( 1956 )

56 Ye 1 P. F. Yergin, Phys. Rev. 104, 1340 (1956)

56 Ye2 P. F. Yergin, B. P. Fabricand, Phys. Rev. 104, 1334 ( 1956 )

57 Al 1 Yu. A. Aleksandrov, N. B. Delone, L. I, Slovokhotov, G. A. Sokol, L. N. Shtarkov; Zhur. Eksp. i Teoret. Fiz. 33 , 614 ( 1957 ); Soviet Phys. JETP 6, 472 ( 1958 )

57 Avl N. P. Avdonin, K. A. Petrzhak; Trud. Leningr. Tekhnol.

Inst. Im. Lensoveta 40, 5 (1957)

$57 \mathrm{Ba} 1 \mathrm{P} . \mathrm{S}$. Baranov, V. I. Gol danskii, V. S. Roganov; Zhur.

Eksp. 1 Teoret. Fiz. 33, 1123 ( 1957 ); Soviet Phys.JETP 6 , 865 ( 1958 ); Phys. Rev. 109, 1801 (1958)

57 Ba2 E. B. Bazhanov, Yu, M. Volkov, A. P. Komar, L. A. Kul

chitski , V: P. Chizhov; Dokl: Akad. Nauk SSSR 113,65

( 1957 ); Soviet Phys. Doklady 2, 107 ( 1957 )

57 Ba3 R. Basile; Ann. Phys. (Paris) 2, 267 ( 1957)

57 Ba4 B. P. Bannik, N. M. Kulikova, L. E. Lazareva, V. A.

Yakovlev; Zhur Eksp.i Teoret. Fiz. 33, 53 (1957); Soviet

Phys. JETP 6, 39 ( 1958 )

$57 \mathrm{Be} 1 \mathrm{~J}$. B. Bellicard, J. Miller, C. Tzara; J. Phys. Radium 18, 201 ( 1957 )

57 Bo 1 F. Bobard, G. Boulegue, P. Chanson; Compt. Rend. 244, 1761 (1957)

$57 \mathrm{Br} 1 \mathrm{P} . \mathrm{Brix}, \mathrm{E}$. K. Maschke, Z. Naturforsch, 12, 1013 ( 1957 )

$57 \mathrm{Ca} 1 \mathrm{~J} . \mathrm{H}$. Carver, R. D. Edge, K. H. Lokan; Proc. Phys. Soc. A 70,415 (1957)

57 Ca2 J. H. Carver, K. H. Lokan; Australian J. Phys. 10, 312 ( 1957 )

$57 \mathrm{Ch} 1$ I. V. Chuvilo, V. G. Shevchenko; Zhur. Eksp. i Teoret.

Fiz. 32, $1335^{\circ}$ ( 1957 ); Soviet Phys. JETP 5, i 090 (1957)

57Col B. C. Cook, Phys. Rev. 106, 300 ( 1957 )

57Del S. DeBenedetti, U. Farinelli, F. Ferrero, R. Malvano, G. Pelli, C. Tribuno; Nuovo Cimento 6,682 (1957)

$57 \mathrm{fl}_{1}$ A. El Sioufi, P. Erdos, P. Stoll; Helv. Phys. Acta 30,264 ( 1957 )

57El2 A. El Sioufi, P. Erdos, P. Stoll; Helv. Phys. Acta 30266 ( 1957 )

57Erl P. Erdos, P. Scherrer, P. Stoll; Helva. Phys. Acta 30,639 ( 1957 )

57Fel F. Ferrero, L. Gonella, R. Malvano, C. Tribuno, A. O. Hanson; Nuovo Cimento 5,242 ( 1957 )

$57 \mathrm{Fe} 2 \mathrm{~F}$. Ferrero, R. Malvano, C. Tribuno; Nuovo Cimento 6, 385 (1957) 
57 Fe3 F. Ferrero, A. O. Hanson, R. Malvano, C. Tribuno; Nuovo Cimento 6,585 ( 1957 )

57 Go 1 A. N. Gorbunov, V. M. Spiridonov, Zhur. Eksp. i Teoret. Fiz. 33,21 ( 1957 ); Soviet Phys. JETP 6; 16 ( i 958 )

57 Hal E. Hayward, E. G. Fuller; Phys.Rev. 106,991 (1957)

57 He1 R. M. Henry, D. S. Martin, Jr.; Phys. Rev. 107,772 ( 1957)

57 Hil R. L. Hines, Phys, Rev. 105,1534 ( 1957 )

57 Jo1 S. A. E. Johansson, B. Forkman; Arkiv Fysik 12, 359 ( 1957 )

$57 \mathrm{Kal}$ L. Katz, K. G. McNeill, M. LeBlanc, F. Brown; Can. J.

Phys. 35, 470 ( 1957 )

57 Kil A. M. King, A. F. Voight; Phys . Rev. 105, 1310 ( 1957 )

57 Kn 1 V. Knapp; Proc. Phys. Soc. (London) A70, 142 (1957)

57 Kol A. P. Komar, I. P. I avor; Zhur. Eksp.i Teoret. Fiz. 32,614 ( 1957 ); Soviet Phys. JETP 5,508 ( 1957 )

57 Ko2 A. P. Komar, I. P. Yavor; Zhur. Eksp. i Teoret. Fiz. 31 , 531 ( 1957 ); Soviet Phys. JETP 4, 432 ( 1957 )

57 Li 1 D. L. Livesey; Can.J. Phys.35, 987 ( 1957 )

57 Lo 1 K. H. Lokan, Proc. Phys. Soc. A 70,836 (1957)

$57 \mathrm{Mil}$ C. Milone, R. Ricamo, Nuovo Cimento 5, 1338 ( 1957 )

57 Mi2 C. Milone, R. Ricamo, A. Rubbino; Nuovo Cimento 5, 528 (1957)

57 Mul J. Muto, E. Takekoshi, T. Nakamura, A. Imamura, Y. Tsuneoka; J. Phys. Soc. Japan 12, 109 ( 1957 )

$570 s 1$ R. M. Osokina, B. S. Ratner; Znur. Eksp. i Teoret. Fiz. 32, 20 (1957); Sov. Phys. JETP 5, 1 ( 1957 )

57 Ro 1 M. Rozkos; Czech.J.Phys. 7, 592 ( 1957 )

57 Ro2 M. Rozkos; Czech. J. Phys. 7, 20 ( 1957 )

57 Sc1 F. D. Schupp, C; B. Colvin, D. S. Martin, Jr.; Phys. Rev. 107,1058 (1957)

57 Sc2 R. A. Schmitt, R. B. Duffield; Phys. Rev. 105, 1277 (1957) 57 Spl B. M. Spicer, E. G. Muirhead, G. G. Shute; Australian J.

57Sp2 B. M. Spicer, Australian J. Phys. 10, 326 ( 1957 )

57 Sv1 N. L. Svantesson, Nuclear Phys. 3, 273 (1957)

57 Sw1 C. P. Swann, F. R. Metzger, Phys. Rev. 108,982 ( 1957)

57 To 1 M. E. Toms, W. E. Stephens, Phys.Rev. 108, 77 ( 1957)

$57 \mathrm{Za} 1 \mathrm{G}$. N. Zatsepina, L. E. Lazareva; Zhur. Eksp. i Teoret. Fiz. 32,27 (1957); Soviet Phys.JETP 5, 21 ( 1957 )

$58 A l 1$ L. W. Alvarez, F. S. Crawford, Jr., M. L. Stevenson; Phys: Rev. 112, 1267 ( 1958 )

58As 1 T. Asada, M. Masuda, M; Okumura, J. Okuma; J. Phys. Soc. Japan i3, 1 ( 1958 )

58 Au1 L. B. Aull, W. D. Whitehead; Phys. Rev. 110, 1113 (1958)

58 Ba 1 P. S. Baranov, V. I. Gol danskii, V. S. Roganov; Phys. Rev. 109,1801 ( 1958 )

58 Baz W. C. Barber; Phys. Rev. 111, 1642 (1958)

58 Ba3 M. Q. Barton, J.H. Smith; Phys. Rev. 110,1143 (1958)

58 Ba5 R. Basile, M. Gusakow; Compt. Rend. 246, 588 ( 1958 ) 
58Ba6 E. B. Bazhanov, Yu. M. Volkov, L. A. Kul chitskii; Zhur. Eksp. i Teoret. Fiz 35, 322 ( 1958 ); Soviet Phys. JETP 8 , 224 ( 1959$)$

$58 \mathrm{Ba} 7 \mathrm{~A} \cdot \mathrm{I} \cdot \mathrm{Baz}, \mathrm{N} \cdot \mathrm{M}, \mathrm{Kul}$ ikova, L. E. Lazareva, N. V. Nikitina, V. A. Semenov; Int. Conf. Peaceful Uses of Atomic Energy 15,2037 ( 1958 )

58Be1 W. L. Bendel, J. McElninney, R. A. Tobin; Phys. Rev. 111,1297 ( 1958 )

58 Be2 W. Bertozzi, F. R. Paolini, C. P. Sargent; Phys. Rev. 110 , 790 ( 1958$)$

58Be3 o. Beckman, R. Sandstrom; Nuclear Phys. 5, 595 ( 1958 )

58Bo 1 \%. V. Bogdankevich, B. S. Dolbilkin, L. E. Lazareva, F.

A. Nikolaev; PICNS-58,697 (1958)

58Br1 P. Brix, U, Hegel, K. H. Lindenberger, D. Quitmann; $Z$. Physik 150, 461 ( 1958 )

58 Bu 1 N. A. Burgov, Yu, V. Terekhov; J. Nucl. Energy 7,247 ( 1958$)$

58Ca 1 J. H. Carver, W. Turchinetz; Proc. Phys. Soc. 71,613 ( 1958$)$

$58 C a 2$ S. Cavallaro, V. Emma, C. Milone, A. Rubbino; Nuovo Cimento 9,736 (1958)

$58 \mathrm{Ch} 1$ I. V. Chuvilo, V. G. Shevchenko; Zhur. Eksp. i Teoret. Fiz. 34,593 ( 1958 ); Soviet Phys. JETP7, 410 ( 1958 )

58 Ch2 B. G. Chidley, L. Katz, S. Kowalski; Can. J. Phys. 36, 407 ( 1958$)$

58 Col G. Cortini, C. Milone, A. Rubbino, Nuovo Cimento 9,85 ( 1958$)$

$58 \mathrm{Co} 2 \mathrm{G}$. Cortini, C. Milone, R. Rinzivillo, C. Tribuno; Nuovo Cimento 9, 188 ( 1958 )

$58 \mathrm{Em} 1 \mathrm{~V}$. Emma, C. Milone, A. Rubbino; Nuovo Cimento 9,343 ( 1958 )

58Fal U. Farinelli, F. Ferrero, R. Malvano, S. Menardi, E. Silva; Phys. Rev. 112, 1994 (1958)

58 Fa2 H. Faissner, F. Gonnenwein; Z. Physik 153, 257 ( 1958 )

58 Fel F. Ferrero, R. Malvano, S. Menardi, O. Terracini; Nuclear Phys. 9, 32 ( 1958 )

58Ful E. G. Fuller, B. Petree, M. S. Weiss; Phys. Rev. 112,554 (1958)

58Fu2 E. G. Fuller, M. S. Weiss; Phys.Rev. 112,560 ( 1958 )

58 Fu3 E. G. Fuller, E. Hayward; Phys. Rev. Letters 1, 465 ( 1958 )

58 Go 1 A. N. Gorbunov, V. M. Spiridonov; Zhur. Eksp. i Teoret. Fiz 34, 862 (1958); Soviet Phys.JETP 7, 596 ( 1958 )

58 Go2 A. N. Gorbunov, V. M. Spiridonov; Zhur. Eksp. i Teoret. Fiz 34, 866 ( 1958 ); Soviet Phys. JETP 7, 600 (1958)

58 Go 3 J. Goldemberg, L. Marquez; Nuclear Phys. 7, 202 ( 1958 )

58 Gr1 G. M. Griffiths; Proc. Phys. Soc. (London) 72,337 ( 1958 )

58 Gu1 F. Gudden, J. Eichler; Z. Physik 150, 436 (1958)

$58 \mathrm{Ha} 1 \mathrm{~A}$. Halsteinslid, K. Nybo, R. B. Mollerud; Univ. Bergen Arb. Naturv. R. 15, 1 ( 1958 )

58 Ho 1 A. Hofmann, P. Stoll; Helva. Phys. Acta 31, 591 ( 1958 )

58 Ia 1 I. P. I avor; Zhur. Eksp. i Teoret. Fiz. 34, 1420 ( 1958); Soviet Phys. JETP 7,983 (1958) 
$58 \mathrm{Kal}$ L. Katz, G. B. Chidley; Nuclear Reactions at Low and Medium Energies ( Pub. Academy of Science, USSR) 371 (1958)

$58 \mathrm{Ka} 2$ L. Katz, A. P. Baerg, F. Brown; Peaceful Uses of Atomic Energy, Switzerland ( 1958 )

$58 \mathrm{Ke} 1$ L. Keszthelyi, J. Ero, Nuclear Phys. 8, 650 ( 1958 )

$58 \mathrm{Ki} 1 \mathrm{H}$. J. King, L. Katz; Can.J.Phys. 36, 415 ( 1958 )

$58 L_{i} 1$ D. L. Livesey, I. G. Main, Nuovo Cimento 10, 590 ( 1958)

$58 \mathrm{Mal}$ V. N. Maikov; Zhur. Eksp. i Teoret. Fiz. 34, 1406 (1958); Soviet Phys. JETP 7, 973 ( 1958 )

58 Me 1 F. R. Metzger, C. P. Swann, V. K. Rasmussen; Phys. Rev. 110,906 ( 1958 )

$58 M i 1$ C. Milone, S. Milone-Tamburino, R. Rinzivillo, A. Rubbino, C. Tribuno; Nuovo Cimento 7,729 ( 1958 )

58 epl I. R. Ophel, I. F. Wright; Proc. Phys. Soc. 71,389 (1958)

580 p2 T. R. Ophel, Proc. Phys. Soc. 72, 321 (1958)

$580 \times 1$ C. L. Oxley, Phys. Rev. 110,733 (1958)

58 Ral V. K. Rasmussen, F. R. Metzger, Phys. Rev, 110,154 ( 1958 )

58Rh1 J. L. Rhodes, W. E. Stephens; Phys. Rev. 110, 1415 (1958)

58 Ry 1 T. W. Rybka, L. Katz; Phys. Rev.110, 1123 (1958)

58Sil E. Silva, J. Goldemberg; Phys.Rev.110, 1102 ( 1958)

58 Si2 E. Silva, J. Goldemberg, P. S. Smith; Nuovo Cimento 9, 17 ( 1958$)$

58 Si 3 A. K. M. Siddiq, R. N. H. Haslam; Can.J. Phys. 36, 963 ( 1958$)$

58Si4 E. Silva, J. Goldemberg; An. Acad. Brasil. Cienc. 30, 51 (1958)

58Sp1 B. M. Spicer, H. H. Thies, J. E. Baglin, F. R. Allum; Australian $J$. Phys. 11,298 ( 1958 )

$58 \mathrm{Sp} 2$ B. M. Spicer, F. R. Allum, J. E. Baglin, H. H. Thies; Australian J? Phys: 11,273 ( 1958 )

58 Tal G. W. Tautfest, Phys. Rev. 110,708 ( 1958 )

58 Ta2 C. A. Tatro, T. R. Palfrey, Jr., R. M. Whaley, R. $\theta$. Haxby; Phys. Rev. 112,932 ( 1958 )

58 To1 R. Tobin, J. McElhinney, L. Cohen; Phys. Rev. 110,1388 (1958)

58 To2 M. E. Toms, J. McElhinney; Phys. Rev. 111,561 ( 1958 )

58 Wh 1 A. Whetstone, J. Halpern; Phys. Rev. 109, 2072 ( 1958 )

58 Wh2 C. Whitehead, W. R. McMurray, M. J. Aitken, N. Middlemas, C. H. Collie; Phys. Rev. 110,941 (1958)

58 Hol M. M. Wolff, W. E. Stephens; Phys. Rev. 112, 890 ( 1958)

58 Yal I. P. Yavor, Zhur. Eksp. i Teoret. Fiz. 34, 1420 (1958); Soviet Phys. JETP 7,983 ( 1958)

$58 Z_{i} 1$ B. Ziegler, Z. Physik 152, 566 ( 1958 )

59Au1 L. B. Aull, P. C. Reinhardt, W. D. Whitehead; Nuclear Phys. 13, 292 ( 1959 )

59Ba1 W. C. Barber, F. E. Gudden; Phys. Rev. Letters 3, 219 ( 1959$)$

59Ha2 E. B. Bazhanov, Zhur. Eksp. i Teoret. Fiz. 37, 374 ( 1959); Soviet Phys. JETP 10, 267 ( 1960$)$ 
59 Ba3 W. C. Barber, W. D. George; Phys. Rev. 116,1551 ( 1959 ) 59 Ba4 A. P. Baerg, R. M. Bartholomew, F. Brown, L. Katz, S. B. Kowalski; Can. J Phys. 37, 1418 (1959)

59 Bo 1 M. Bormann, H. Neuert; Z. Naturforsch. 14a, 922 ( 1959 )

59Br 1 P. Brix, A. Kording, K. H. Lindenberger; Z. Physik 154 , $569(1959)$

59Br2 P. Brix, E. K. Maschke; Z. Physik 155, 109 (1959)

59 Bul N. A. Burgov, G. V. Danilyan, B. S. Dolbilkin, L. E. Lazareva, F. A. Nikolaev; Zhur. Eksp. i Teoret, Fiz. 37 1811 ( 1959 ); Soviet Phys: JETP 10, 1278 (1960)

59Ca1 J. H. Carver, G. A. Jones; Nuclear Phys. 11,400 ( 1959)

$59 \mathrm{Ca} 2 \mathrm{~J}, \mathrm{H} \cdot$ Carver, W. Turchinetz; Proc. Phys. Soc.73, 110 ( 1959 )

59 Ca3 J.H. Carver, W. Turchinetz; Proc. Phys. Soc.73,69 ( 1959 )

$59 \mathrm{Ca} 4 \mathrm{~J}$. H. Carver, W. Turchinetz; Proc. Phys. Soc.73,585 ( 1959$)$

$59 \mathrm{Ch} 1$ V. P. Chizhov, L. A. Kul chitskii; Zhur. Eksp. i Teoret. Fiz. 36, 345 (1959); Soviet Phys. JETP 9, 239 ( 1959 )

59 Co1 L. D. Cohen, W. E. Stephens; Phys. Rev. Letters 2, 263 ( 1959 )

59 Co2 S. G. Cohen, P. S. Fischer, E. K. Warburton; Phys. Rev. Letters 3, 433 (1959)

59003 G. Cortini, C. Milone, T. Papa, R. Rinzivillo; Nuovo Cimento 14,54 (1959)

59604 L. Cohen, R. A. Tobin; Nuclear Phys. 14, 243 ( 1959 )

$59 \cos$ L. Cohen, R. A. Tobin, J. McElhinney; Phys. Rev. 114,

59006 S. G. Cohen; Nuovo Cimento 14,931 (1959)

59De1 N. N. Delyagin; J. Exptl. Theoret. Phys. USSR 37,1177 (1959); Sov. Phys. JETP 37, 837 (1960)

59Dil G. Di Caporiacco, M. Mando, F. Ferrero; Nuovo Cimento 13, 522 (1959)

59Dul J. Dular, G. Kernel, M. Kregar, M. V. Mihailovic, G. Pregl, $Y$. Rosina, C. Zupancic; Nuclear Phys. 14, 131 ( 1959 )

59Dy 1 P. Dyal, J P. Hummel; Phys . Rev. 115, 1264 ( 1959 )

$59 \mathrm{Em} 1 \mathrm{~V}$. Emma, C, Milone, R. Rinzivillo; Nuovo Cimento 14 , 1149 ( 1959$)$

59 Em2 V. Emma, C. Milone, R. Rinzivillo, A. Rubbino; Nuovo Cimento 14,62 ( 1959 )

59 Fal U. Farinelli, F. Ferrero, S. Ferroni, R. Malvano, E. Silva; Nuovo Cimento 12,89 ( 1959 )

59 Fel F. Ferrero, S. Ferroni, R. Malvano, S. Menardi, E. Silva; Nuovo Cimento 11,410 (1959)

$59 F e 2$ F. Ferrero, R. Malvano, E. Silva, J, Goldemberg, G. Moscat 1; Nuclear Phys. 10, 423 (1959)

59 Fr1 J. I. Friedman; Phys. Rev. 116, 1257 ( 1959 )

59Ga1 E. L. Garwin; Phys. Rev. 114, 143 (1959)

$59 \mathrm{Gel}$ D. S. Gemmell, A.H. Morton, E. W. Titterton; Nuclear Phys. 10,33 ( 1959$)$ 
59 Gi 1 J. H. Gibbons, R. L. Macklin, J B. Marion, H. W. Schmitt; Phys. Rev. 114, 1319 (1959)

59Go1 H. E. Gove, A. E. Litherland, R. Batchelor; Phys. Rev. Letters 3,177 ( 1959 )

$59 \mathrm{Hal}$ H. J. Hay, J. B. Warren; Can. J. Phys. 37, 1153 ( 1959 )

59 Ha2 F. I. Havlicek; Nuovo Cimento 13,969 ( 1959)

59 Ikl N. Ikeda, K. Yoshihara; Radioisotopes (Tokyo) 8, 24 ( 1959 )

$59 \mathrm{Ki} 1 \mathrm{H}$. King, L. Katz; Can. J. Phys. 37,1357 ( 1959)

$59 \mathrm{Kol} \mathrm{J}$. Kockum, N. Starfelt; Nuclear Instruments 5, 37 (1959)

$59 \mathrm{Ko} 2$ A. P. Komar, T. N. Dragnev; Dokl. Akad. Nauk SSSR:

126, 1234 (1959); Soviet Phys. Doklady 4, 653 (1959)

$59 \mathrm{Ku} 1 \mathrm{~L}$. A. Kul chitskil, V. Presperin; Zhur. Eksp. i Teoret.Fiz. 371524 ( 1959$)$; Sovi et Phys. JETP 10, 1082 (1960)

$59 \mathrm{Ku} 2 \mathrm{Chi}-\mathrm{Ti}$ Kuo, B. S. Ratner; Dokl. Akad. Nauk SSSR 125, 761 (1959); Soviet Phys. Doklady 4, 369 (1959)

59Lal M. Langevin, A. Bussiere de Nercy; J. Phys. Radium 20, 831 ( 1959 ); (See 61Bu4)

59 Lol K. H. Lokan; Proc. Phys. Soc. 73, 697 (1959)

$59 \mathrm{Ma} 1 \mathrm{E}$. D. Makhnovskii; Zhur. Eksp. i Teoret. Fiz. 36, 739 (1959); Soviet Phys. JETP 9, 519 (1959)

59 Me1 F. R. Metzger, W. D. Todd; Nuclear Phys. 13, 177 ( 1959)

59 Mil M. V. Mihailovic, G. Pregl, G. Kernel, M. Kregar; Phys. Rev. 114,1621 (1959)

59 Mi2 C. Milone; Phys. Rev. Letters 3, 43 ( 1959 )

59 Mi3 C. Milone, A. Rubbino. Nuovo Cimento 13, 1035 ( 1959)

59 Mul N. Mutsuro, Y. Bhnuki, K. Sato, K. Kageyama, M. Kimura; J.Phys. Soc. Japan 14, 1457 (1959)

59 Mu2 N. Mutsuro, Y. Ohnuki, K. Sato, M. Kimura; J Phys. Soc. Japan 14, 1649 ( 1959 )

$59 \mathrm{Na} 1 \mathrm{~T}$. Nakamura, K. Takamatsu, K. Fukunaga, M. Yata, S. Yasumi; J. Phys. Soc. Japan 14, 693 ( 1959 )

$598 \mathrm{c} 1 \mathrm{~J}$. Connell, P. Dyal, J.Goldemberg; Phys. Rev.116, 173 ( 1959 )

59 Pal R. W. Parsons, L. Katz; Can. J. Phys. 37, 809 ( 1959)

59 Pa2 R. W. Parsons; Can. J. Phys. 37, 1344 ( 1959 )

59 Pa3 P. Paul, U. Stierlin; Nuclear Phys. 13, 576 (1959)

59 Pe 1 A. S. Penfold, E. L. Garwin; Phys. Rev. 114, 1139 (1959)

59 Pe2 A. S. Penfold, E. L. Garwin; Phys. Rev. 114, 1324 (1959)

59 Pe3 A. S. Penfold, E. L. Garwin; Phys. Rev. 115, 420 (1959)

59 Pe4 S. Penner, J.E. Leiss; Phys. Rev. 114, 1101 ( 1959 )

59 Pe5 A. S. Penfold, E. L. Garwin; Phys. Rev. 116, 120 (1959)

$59 \mathrm{Ra} 1 \mathrm{~V}$. K. Rasmussen, F. R. Metzger, C. P. Swann; Nuclear Phys. 13,95 (1959)

59Ro1 T. A. Romanowski, V. W. Voelker; Phys.Rev. 113, 886 ( 1959 )

59Ro2 J. P. Roalsvig, R.N.H. Haslam, D. J. McKenzie; Can. J. Phys. 37, 607 ( 1959 )

59 Ro3 J. P. Roalsvig, R. N. H. Haslam, L. D. Skarsgard, E. E。 Wuschke; Can. J. Phys. 37,722 (1959)

59Sa1 D. Sadeh; Compt. Rend. 249,531 (1959) 
59Sa2 D. Sadeh; Compt. Rend. 249, 2313 (1959)

59 Se1 R. E. Segel; Phys. Rev. 113, 844 (1959)

59 Se2 W. Sebaoun, H. Gauvin; Compt. Rend. 248, 791 (1959)

59Sm1 C. P. Swann, V. K. Rasmussen, F. R. Metzger; Phys. Rev. $114,862(1959)$

59 Tal N. W. Tanner, G. C. Thomas, W. E. Meyerhof; Nuovo Cimento 14,257 ( 1959 )

$59 \operatorname{Th} 1$ H. H. Thies, B. M. Spicer, J.E.E. Baglin; Australian J. Phys. 12, 21 ( 1959 )

$60 \Lambda i 1$ M. J. Aitken, N. Middlemas; Phys. Rev. 117,1111 (1960)

$60 \mathrm{Al} 1$ Ya. Alksnis, U. Ulmanis; Latv. PSR Zinat. Akad. Vestis 3 ( 152$), 71$ ( 1960$)$

60As1 R. F. Askew, A. P. Batson; Nuclear Phys, 20,408 (1960)

60 Ba 1 E. B. Hazhanov; Zhur. Eksp. i Teoret. Fiz. 38, 267 (1960); Soviet Phys. JETP 11, 193(1960)

60 Ba2 W. C. Barber, T. Wiedling; Nuclear Phys. 18, 575 ( 1960)

$60 \mathrm{Ba} 3 \mathrm{E}$. B. Bazhanov, L. A. Kul chitskii; Zhur. Eksp. i Teoret. Fiz. 38,1685 (1960); Soviet Phys. JETP 11,1215 (1960)

60 Ba4 W. C. Barber, F. Berthold, G. Fricke, F. E. Gudden; Phys. Rev. 120,2081 ( 1960 )

60 Ba5 W. C. Barber, V. J. Vanhuyse. Nuclear Phys. 16, 381 ( 1960$)$

60 Ba6 W. C.Barber, V. J. Vanhuyse. J. Phys. Radium 21,299 ( 1960 )

60 Ba7 D. Balfour, D. C. Menzies; Proc. Phys. Soc. 75, 543 (1960)

60 ba 8 M. A. Bak, K. A. Petrzhak, Tei-Mei Chen; Izv. Akad. Nauk SSSR 24, 818 (1960)

60 Be 1 w. Bertozzi, P. T. Denos, S. Kowalski, F. Paolini, C. P. Sareent; PICNS 746 , (1960)

60 Bo 1 B. A. Bochagov, A. P. Komar, G. E. Solaykin; Zhur. Eksp. i Teoret. Fiz. 38, 1374 (1960); Soviet Phys. JETP 11,990 $(1960)$

60 Bo2 o. V. Bogdankevich, L. E. Lazareva, A. M. Maiseev;

Zhur. Eksp. i Teoret. Fiz. 39, 1224 (1960); Soviet Phys. JETP $12,853(1961)$

60 Ho3 E. C. Booth; Nuclear Phys. 19, 426 (1960)

60Br1 C. Broude, H. E. Gove; PICNS 754 (1960)

60 Bu2 A. Bussiere de Nercy; Compt. Rend. 250, 1252 ( 1960 ); (See $6(\mathrm{Bu} 4)$

$60 \mathrm{bu} 3$ A. Bussiere de Nercy, M. Langevin; J. Phys. Radium 21 , 293 (1960); (See 61 Bu4)

60Cal E. E. Carroll, Jr., W. E. Stephens; Phys. Rev. 118,1256 ( 1960$)$

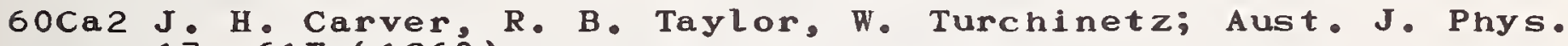
$13,617(1960)$

$60 \mathrm{Ch} 1$ V. P. Chizhov; Zhur. Eksp. i Teoret. Fiz. 38, 809 (1960); Soviet Phys. JETP 11,587 (1960)

$60 \mathrm{Ch} 2$ R. E. Chrien, A. H. Benade; Phys. Rev. 119,748 (1960)

60 De1 B. I. Deutch, F. R. Metzger, F. J. Wilhelm; Nuclear Phys. $16,81(1960)$

60 De2 N. N. Delyagin; Zhur. Eksp. i Teoret. Fiz. 38, 1111 (1960); Soviet Phys. JETP 11, $803(1960)$ 
60 Dol H. G. Dosch, K. H. Lindenberger, P. Brix; Nuclear Phys. $18,615(1960)$

60Ed1 R. D. Edge; Phys. Rev.119, 1643 (1960)

$60 \mathrm{Em} 1$ V. Emma, C. Milone, A. Rubbino, A. Malvano; Nuovo Cimento 17,365 (1960)

60 Em2 V. Emma, C. Milone, A. Rubbino, Phys. Rev, 118, 1297 (1960)

60Fal R. W. Fast, P. A. Flournoy, R. S. Tickle, W. D. Whi tehead; Phys. Rev, 118,535 (1960)

60 Fel F. Ferrero, S. Ferroni, R. Malvano, S. Menardi, E. Silva; Nuclear Phys. 15, 436 ( 1960$)$

$60 \mathrm{Fe} 2 \mathrm{~B}$. T. Feld, B. C. Maglic, J. Parks; Nuovo Cimento 17. Suppi. 2, 241 ( 1960$)$

60Fl 1 P. A. Flournoy, R. S. Tickle, W. D. Whitehead; Phys. Rev. $120,1424\left(1960^{\circ}\right)$

60 Fol B. Forkman, S.A.E. Johansson; Nuclear Phys. 20,136 ( 1960$)$

60 Fo2 B. Forkman, I. Wahlstrom; Arkiv Fysik 18, 339 (1960)

$60 \mathrm{Ga} 1 \mathrm{~J}$. A. Galey; Phys. Rev. 117, 763 (1960)

60 Ga2 M. Garnier, H. Gauvin, W. Sebaoun; J. Phys. Radium 21, $893(1960)$

$60 \mathrm{Ge} 1 \mathrm{~K}$. N. Geller; Phys. Rev. 120, 2147 (1960)

$60 \mathrm{Ge} 2 \mathrm{~K} . \mathrm{N}$. Geller, J. Halpern, E. G. Muirhead; Phys. Rev.119, 716 (1960)

$60 \mathrm{Ge} 3 \mathrm{~K} \cdot \mathrm{N}$. Keller, J. Halpern, E. G. Muirhead; Phys . Rev. 118 , $1302(1960)$

60Gol V. I. Gol danskil, O. A. Karpukhin, A.V.Kutsenke, V.V. Pavlovskaya; Zhur? Eksp. i Teoret. Fiz. 38, 1695 ( 1960 ); Soviet Phys. JETP 11, 1223 (1960)

60 Go2 A. N. Gorbunov, F. P. Denisov, V. A. Kolutkhin; Zhur. Eksp. i Teoret. Fiz. $38,1084^{\circ}$ ( 1960 ); Soviet Phys. JETP 11 , 783 ( 1960$)$

60 Gr1 V. M. Grizhko, D. I. Sikora, V. A. Shkoda-Ul yanov, A. D. Abramenkov, B. I. Shramenko, A. N. Fisun; Zhur.

Eksp. i Teoret. Fiz. 38, 1370 (1960); Soviet Phys. JETP 11 , 987 ( 1960$)$

60 Hal F. I. Havli cek; Nuovo Cimento 18,65 (1960)

6 CHa2 S. S. Hanna and R. E. Segel; Proc. Royal Soc. (London) A259, 267 ( 1960 )

60 He1 D. F. Hebbard; Nuclear Phys. 15, 289 (1960)

$60 \mathrm{He} 2 \mathrm{D}$. F. Hebbard, J. L. Vogl; Nuclear Phys. 21, 652 ( 1960)

$60 \mathrm{Hol}$ P. Horvat, J.Pahor, M. Vakselj; Nuclear Phys. 16, 90 (1960)

60 Is 1 D. Isabelle, G. Bishop; Compt. Rend. 251,697 (1960)

60 Jal D. Jamnik, D. Axel; Phys. Rev.117, 194 (1960)

$60 \mathrm{Ki} 1 \mathrm{~J}$. D. King, W. J. McDonald; Nuclear Phys . 19, 94 ( 1960 )

$60 \mathrm{Ki} 2 \mathrm{~J}$. D. King, R. N. H. Haslam, R. W. Parsons C Can. J . Phys. 38,231 ( 1960 )

$60 \mathrm{Ki} 3 \mathrm{M}$. Kimura, K. Shoda, N. Mutsuro, T. Tohei, K. Sato, K. Kuroda, K. Kuriyama, T. Ariba; J. Phys. Soc. Japan 15, 1128 ( 1960$)$

$60 \mathrm{Ki} 4 \mathrm{~J}$. D. King, R. N. H. Haslam, W. J . McDonald; Can. J . Phys. 38, 1069 (1960) 
60 Ko 1 A. P. Komar, Ya. Krzhemenek, I. P. Yavor; Dokl. Akad. Nauk SSSR 131,283 (1960); Soviet Phys. Doklady 5, 295 ( 1961$)$

$60 \mathrm{Ko} 2 \mathrm{~A} . \mathrm{P}$. Komar, Ya. Krzhemenek, I. P. Yavor; Dokl. Akad. Nauk SSSR 135, 291 (1960); Soviet Phys. Doklady 5, 1234 ( 1961$)$

$60 k \circ 3$ A. P. Komar, E. D. Makhnovskii; Dokl. Akad. Nauk SSSR 135,52 ( 1960 ); Soviet Phys. Doklady 5, 1229 ( 1961 )

$60 \mathrm{Ko} 4$ A. P. Komar, E. D. Makhnovskii, V. P. Puddubnov; Dokl. Akad. Nauk SSSR 133,797 (1960); Soviet Phys. Doklady 5, $824(1961)$

60 Ko5 A. P. Komar; PICNS-60, 494 ( 1960 )

$60 \mathrm{Kr} 1$ R. W. Krone, J. J. Singh; Phys.Rev. 117,1562 ( 1960 )

$60 \mathrm{Kul}$ Kuo Ch i-di, B. S. Ratner; Zhur. Eksp. i Teoret. Fiz. 39 , 1578 ( 1960 ); Soviet Phys JETP 12, 1098 ( 1961 )

$60 \mathrm{ku} 2 \mathrm{~L}$. A. Kul chitskii, V. Presperin; Zhur. Eksp. i Teoret. Fiz. 39, 1001 (1960); Soviet Phys. JETP 12,696 (1961)

$60 \mathrm{Li} 1 \mathrm{~N}$. V. Linkova, R. M. Osokina, B. S. Ratner, Sh. Amirov, v. V. Akindinov; Zhur. Eksp. I Teoret. Fiz? 38, 780 ( 1960$)$; Soviet Phys. JETP 11,566 (1960)

$60 \mathrm{Li} 2 \mathrm{~K}$. H. Lindenberger, J. A. Scheer; Z. Physik 158, 111 (1960)

$60 \mathrm{Mal}$ E. D. Makhnovski ; Zhur. Eksp. i Teoret. Fiz. 38, 96 ( 1960 ); Soviet Phys. JETP 11,70 ( 1960)

$60 \mathrm{Ma} 2$ B. Maingbridge; Nuclear Phys. 21, 1 ( 1960 )

60 Mel F. R. Metzger, C. P. Swann, V. K. Rasmussen; Nuclear Phys. 16, $568(1960)$

$60 \mathrm{Me} 2 \mathrm{~F}$. R. Metzger; J. Franklin Institute 270, 138 (1960)

60 Mil C. Milone; Phys. Rev.120, 1302 (1960)

$60 \mathrm{Mi} 2 \mathrm{M}$. Miwa, M. Yamanouchi; J. Phys. Soc. Japan 15, 947 (1960)

60Mol W. L. Mouton, P. B. Smith; Nuclear Phys. 16, 206 ( 1960 ) 60 Mul N. Mutsuro, K. Sato, M. Mishina; J. Phys. Soc. Japan 15, $358(1960)$

$6 \mathrm{CNe} 1 \mathrm{~J} .0$. Newton; Nuclear Phys. 21,529 ( 1960 )

60 Pr1 D. G. Proctor, W. H. Voelker; Phys. Rev. 118, 217 (1960) 60 Pr2 L. I. Prokhorova, G. N. Smirenkin; Atom. Energiya 8,457 ( 1960$)$

60 Re 1 K. Reibel, A. K. Mann; Phys. Rev. 118, 701 (1960)

$60 \mathrm{ke} 2 \mathrm{~J}, \mathrm{M}$. Reid, B. Lalovic; Proc. Phys. Soc. (London) 76, 65 (1960)

60Ro1 H. J. Rose; Nuclear Phys. 19, 113 (1960)

60 Ro2 H. J. Rose, F. Riess, W. Trost; Nuclear Phys. 21,367 (1960)

60 Ro3 M. Rozkos, M. Smrcka, O. Jakubcek; Czech. J.Phys. 10, 129 ( 1960$)$

60 Fo 4 J. P. Roalsvig, R. N. H. Haslam, J. L. Bergsteinsson; Can. J. Phys. 38, $320(1960)$

60sal D. Sadeh; Phys. Rev. Letters 4,75 (1960)

60 Sa2 D. Sadeh; Compt. Rend. 250, 1632 ( 1960 )

60st1 P. C. Stein, A. C. Odian, A. Wattenberg, R. Weinstein; Phys. Rev. 119, 348 (1960)

60 Ta1 R. B. Taylor; Nuclear Phys. 19, 453 (1960) 
60 Ta2 G. Tamas, J. Miller, C. G. Schuhl, C. Tzara; J.Phys. Radium 21, 532 ( 1960$)$

60 Ta3 E. Takekoshi, J. Phys. Soc. Japan 15, 2129 (1960)

60 Ih 1 H. H. Thies, B. M. Spicer; Australian J. Phys. 13, 505 ( 1960$)$

60 To 1 R. A. Tobin; Phys. Rev.120, 175 (1960)

60 Val V. J Vanhuyse, G. J. Vanpraet; J. Phys. Radium 21,290 ( 1960$)$

60 Hal I. Wanlstrom, B. Forkman; Arkiv Fysik 18, 83 ( 1960 )

60 Wa2 T. G. Walker, W. T. Morton; Proc. Phys. Soc. 75,4 (1960)

60 Wy 1 J.M. Wyckoff, H. W. Koch; Phys.Rev. 117, 1261 (1960)

60 Yal S. Yasumi, M. Yata, K. Takamatsu, A. Masaike, Y.

Masuda; J. Phys. Soc. Japan 15, 1913 (1960)

$60 Z 11$ B. Ziegler; Nuclear Phys. 17, 238 (1960)

61 Ak 1 A. F. Akkerman, D. K. Kaipov, Yu. K . Shubnyi; Zhur.

Eksp.i Teoret. Fiz.40, 1031 (1961); Soviet Phys.JETP 13 , $725(1961)$

61 An 1 B. Ambrozy, A. Faudrowicz, A. Jasinski, J Kownacki, H. Lancman, J. Ludziejewski; PICNS-61 281 ( 1961 )

$61 \mathrm{Hal} \mathrm{J}$. E. E. Baglin, M. N. Thompson, B. M. Spicer; Nuclear Phys. 22, 207 (1961)

$61 \mathrm{Ba} 2$ R. G. Baker, K. G. McNeill; Can. J.Phys.39, 1158 ( 1961 )

$61 \mathrm{Ba} 3$ P. S. Baranov, L. I. Slovokhotov, G. A. Sokol, L: N. Shtarkov; Zhur. Eksp. il Teoret.Fiz.41, 1713 (1961); Soviet Phys. JETP 14, 1219 ( 1962 )

$61 \mathrm{Be} 1$ A. K. Berzin, R. P. Meshcheryakov; Zhur. Eksp. 1 Teoret. Fiz. 41, 1013 ( 1961 ); Soviet Phys. JETP 14,721 ( 1962 )

$61 \mathrm{Be} 2 \mathrm{R}$. E. Benenson, L.J.Lidofsky; Phys. Rev. 123,939 (1961)

61 Bil G. R. Bishop, G. A. Proca; J. Phys. Radium 22, 541 ( 1961)

$61 \mathrm{Bol}$ A. Borello, S. Costa, F. Ferrero; Nuclear Phys. 27, 25 (1961)

61 Bo2 B. A. Bochagov, A. P. Komar, G. E. Solyakin, V. I. Fadeev; Atom. Energiya 11, 540 (1961)

61 Bo3 o. A. Borello; Rev. Un. Mat. Argent. As. Fis. Argent.19, 251 ( 1961 )

61 Bo4 P. Bounin, G. R. Bishop; J. Phys. Radium 22, 555 ( 1961 )

$61 \mathrm{Br} 1 \mathrm{P}$. Brix, H. Fuchs, K. H. Lindenberger, C. Salander; Z. Physik 165, 485 (1961)

61 Bul J. W. Butler; Phys. Rev.123,873 (1961)

61 Bu2 G. R. Burleson; Phys. Rev. 121, 624 (1961)

61 Bu3 A. B. DeNercy; J Phys. Radium 22, 535 ( 1961 ) (see 61 Bu4)

61 Bu4 A. Bussiere de Nercy; Ann. Phys. (Paris) 3rd Series, Vol. 6, $1379(1961)$

$61 \mathrm{Cal}$ J. H. Carver, G. A. Jones; Nuclear Phys. 24, 607 ( 1961 )

$61 \mathrm{Ca} 2 \mathrm{~J}$. H. Carver; Proc. Phys. Soc.77, 417 (1961)

$61 \mathrm{Ce} 1 \mathrm{R}$. J. Cence, B. J. Moyer; Phys.Rev.122, 1634 ( 1961 ) (1961)

$61 \mathrm{Ce} 2 \mathrm{M}$. Cerineo, K. I Lakovac, I. Slaus, P. Tomas; Phys. Rev. 124,1947 (1961)

$61 \mathrm{Cl} 1 \mathrm{M}$. A. Clark, H. E. Gove, A. E. Litherland; PICNS-61 807 (1961) 
61 Co1 S. G. Cohen, P S. Fisher, E. K. Warburton; Phys. Rev. $121,858(1961)$

$61 \mathrm{Co} 2 \mathrm{G}$. E. Coote, W.E.Turchinetz, I.F.Wright; Nuclear Phys. 23, $468(1961)$

$61 \mathrm{Cr} 1 \mathrm{H}$. Crannell, R. Helm, H. Kendall, J. Oeser, M. Yearian; Phys. Rev. 123,923(1961)

$61 \mathrm{De} 1 \mathrm{H} . \mathrm{G}$. DeCarvalho, A. G. Dasilva, J. Goldemberg; Nuovo Cimento 19,1131 ( 1961 )

61 De2 H. G. DeCarvalho, A. Celano, G. Cortini, R. Rinzivil[o, G. Ghigo; Nuovo Cimento 19, 187 ( 1961 )

$61 \mathrm{De} 3$ B. I. Deutch, F. R. Metzger; Phys. Rev. 122,848 ( 1961 )

61 Du 1 B. Dudelzak, R. E. Taylor; J. Phys. Radium 22, 544 ( 1961 )

61 Ec 1 A. C. Eckert, E. F. Shrader; Phys. Rev. 124, 1541 ( 1961 )

$61 \mathrm{Em} 1 \mathrm{~V}$. Emma, C. Milone, A. Rubbino, S. Janelli, F. Mezzanores; Nuovo Cimento 22, 135 ( 1961 )

61Fil E. Finch, U. Hegel; Z, Physik 162, 154 (1961)

61 Fol B. Forkman; Nuclear Phys. 23, 269 (1961)

$61 \mathrm{Ga1}$ C. C. Gardner, P. C. Gugelot; PICNS-61, 263 ( 1961 )

$61 \mathrm{Gol}$ H. E. Gove, A. E. Litherland, R. Batchelor; Nuclear Phys. $26,480(1961)$

$61 \mathrm{Ha} 1$ w. D. Hamilton, B. S. Sood; Nuclear Phys. 27, 66 ( 1961 )

61 He1 R. E. Hester, W. A. S. Lamb; Phys. Rev. 121, 584 ( 1961 )

61 He2 U. Hegel, E. Finckh; Z. Physik 162, 142 (1961)

61 Ho1 C. P. Ho, E. L. Iloff; Nuclear Phys. 27, 234 (1961)

61 Hul J. P. Hummel, Phys. Rev.123,950 (1961)

61 Is 1 D. S. Isabelle, G. R. Bishop; J. Phys. Radium 22,548 ( 1961 )

$61 \mathrm{Ja} 1 \mathrm{M} \cdot \mathrm{J}$. Jakobson; Phys. Rev.123, 229 ( 1961 )

$61 \mathrm{Ja} 2$ A. N. James; Nuclear Phys. 24, 675 (1961)

61 Jo1 1 . John, F. V. Martin; Phys. Rev. 124, 830 (1961)

$61 \mathrm{ke} 1 \mathrm{~L}$. Keszthelyi, I. Berkes, I. Demeter, I. Fodor; Nuclear Phys. 23, $513(1961)$

$61 \mathrm{ke} 2$ W. H. Kelly, G. B. Beard; Nuclear Phys. 27, 188 ( 1961 )

$61 \mathrm{Ke} 3 \mathrm{H}$. W. Kendall, J. I. Friedman, E. F. Erickson, P. A. M. Gramm; Phys. Rev.124, 1596 (1961)

$61 \mathrm{Ki} 1 \mathrm{M}$. Kimura, K. Shoda, N. Mutsuro, T. Tohei, K. Sato, K.Kuroda, K.Kuriyama, T.Akiba; Nuclear Phys. 23,338 (1961)

$61 \mathrm{Kn} 1$ H. H. Knitter, H. Waftler; PICNS-61, 823 ( 1961 )

$61 \mathrm{Ko} 1 \mathrm{~A}$. P. Komar, B. A. Bochagov, G. E. Solyakin; Dokl. Akad. Nauk SSSR 141, 1339 (1961); Soviet Phys. Doklady 6, $1088(1962)$

$61 \mathrm{Ku} 1$ Kuo Ch i-di, B. S. Ratner, B. V. Sergeev; Zhur. Eksp. i Teoret Fiz. 40, 85 (1961); Soviet Phys. JETP 13, 60 ( 1961$)$

61 La1 F. Lacoste, G. R. Bishop; Nuclear Phys. 26, 511 ( 1961 )

$61 \mathrm{Ma} 1 \mathrm{E}$. D. Makhnovskil; Zhur. Eksp. i Teoret. Fiz. 41,1091 ( 1961 ); Soviet Phys. JETP 14,779 (1962)

$61 \mathrm{Na2}$ M. Masuda; J. Phys. Soc. Japan 16, 1801 ( 1961 )

61 Me1 V. Meyer, H. Muller, H. H. Staub, R. Zurmunle; Nuclear Phys. 27, $284(1961)$ 
61 Mil J.Miller, C. Schuhl, C. Tzara; J.Phys. Radium 22, 529 (1961)

61 Mol G. Moscati; Nuclear Phys. 26, 321 (1961)

$61 \mathrm{My} 1 \mathrm{H}$. Myers, R. Gomes, D. Guinier, A. V. Tollestrup; Phys. Rev. 121, 630 (1961)

$61 \mathrm{Na} 1 \mathrm{I}$. C. Nascimento, G. Moscati, J. Goldemberg, Nuclear Phys. 22, 484 ( 1961 )

61 No1 R. Nordhagen; Nuclear Phys. 27, 112 (1961)

61 Pel J. P. Perez y Jorba, H. Nguyen Ngoc; J. Phys. Radium 22,

61 Pe2 G. A. Peterson, W. C. Barber; PICNS-61, 831 (1961) 61 Pol R. A. Pope, D. V. Freck, W. W. Evans; Nuclear Phys.

$61 \mathrm{Pr} 1 \mathrm{~V}$. Presperin, L. A Kul chitskii; Zhur. Eksp. i Teoret. Fiz. 41, 60 (1961); Soviet Phys. JETP 14, 46 ( 1962 )

61 Ra 1 V. K. Rasmussen, F. R. Metzger, C. P. Swann; Phys. Rev. 123, 1386 ( 1961$)$

61 Fol I. Rothem, F, R. Metzger, C. P. Swann; Nuclear Phys. 22, 505 ( 1961$)$

61 Ro2 J. P. Roalsvig, I. C. Gupta, R. N. H. Haslam; Can.J. Phys. 39, 643(1961)

61 Sal D. Sadeh; Phys. Rev. 123, 855 (1961)

61 Sa2 C. P. Sargent, W. Hertozzi, P. T. Demos, W. Turchinetz; Proceedings of the Symposium on Neutron Time-of-Flight Methods, p. 353 (1961)

61 Se1 R. E. Segel, J W. Daughtry, J.W. Olness; Phys. Rev. $123,194(1961)$

61 Se2 R. E. Segel, M. J. Bina; Phys. Rev. 124, 814 ( 1961 )

$61 \mathrm{Se} 3$ W. Sebaoun, H. Gauvin; J. Phys. Radium 22,771 (1961)

61 Se4 W. Sebaoun, J. Phys. Radium 22, 735 ( 1961 )

$61 \mathrm{Sh} 1 \mathrm{~K}$. Shoda, K. Kobayashi, S. Si na, K. Abe, M. Kimura; J. Phys. Soc. Japan 16, 1031 ( 1961 )

$61 \mathrm{Sh} 2 \mathrm{~V}$. G. Shevchenko, B. A. Yur ev; Zhur. Eksp. i Teoret. Fiz. 41, 1421 ( 1961 ); Soviet Phys. JETP 14, 1015 ( 1962 )

61 Sh3 K. Shoda; J. Phys. Soc. Japan 16, 1841 (1961)

$61 \mathrm{Sh} 4 \mathrm{~K}$. Shoda, S. Si ina, K. Kobayashi, K. Abe, M. Kimura; J . Phys. Soc. Japan 16, 1807 ( 1961 )

61 Sh5 K. Shoda, K. Kobayshi, S. Siina, K. Abe, M. Kimura; J. Phys. Soc. Japan 16, 1031 ( 1961 )

61 Sh6 V. G. Shevchenko, B. A. Yur ev; Izv. Akad Nauk SSSR 25, $1269(1961)$

61 Su 1 M. Sugawara; J.Phys.Soc. Japan 16, 1857 (1961)

61 Sw1 C. P. Swann, V K. Rasmussen, F. R. Metzger; Phys. Rev. $121,242(1961)$

61 Ta1 F. Tagliabue, J. Goldemberg, Nuclear Phys. 23, 144 (1961)

61 Ta2 N. W. Tanner, G. C. Thomas, E. D. Earle; PICNS-61 297 (1961)

61 Ta3 N, W. Tanner, G. C. Thomas, E. D. Earle; PICNS-61 295 (1961)

61 Th1 I. M. Thorson, L. Katz; Proc. Phys. Soc. 77, 166 ( 1961 )

61 Tol T. Tohei, M. Sugawara, S. Mori, M. Kimura; J. Phys. Soc. Japan 16, 1657 (1961) 
61 Val V. J. Vanhuyse, W. C. Barber; Nuclear Phys 26, 233 ( 1961$)$

61 Va2 A. K. Val ter, Yu. P. Antur ev, E. G. Kopanets, A. N, L vov, P. Tsytko; Zhur. Eksp. i Teoret. Fiz. 41, 1499 ( 1961 ); Soviet Phys. JETP 14, 1035 ( 1962)

$61 \mathrm{Va} 3 \mathrm{~K}$. J. Van Oostrum, N: Hazewindus, A. H. Wapstra, J.W. Olness, J. L. Parker; Nuclear Phys. 25, 409 (1961)

61 Wal A. Wataghin, R. B. Costa; A. M. Freire, J. Goldemberg; Nuovo Cimento 19,864 (1961)

61 We 1 R. E. Welsh, D. J. Donahue; Phys. Rev. 121, 880 ( 1961 )

61 Wil 1 W. S. C. Williams, H.S. Caplan, D. T. Stewart; Proc. Phys. Soc. (London) 78, 1125 ( 1961 )

61 tiol J.H. Wolfe, J. P. Hummel; Phys. Rev. 123, 898 ( 1961)

62An1 E. S. Anashkina; Zhur. Eksp. i Teoret. Fiz. 43, 1197 (1962); Soviet Phys. JETP 16, 847 ( 1963)

62 An2 Yu. P. Antuf ev, A. K. Val ter, A. N. L vov, E. G. Kopanets, S. P. Tsytko; Zhur. Eksp.i Teoret. Fiz. 42,386 ( 1962 ); Soviet Phys. JETP 15, 268 ( 1962 )

$62 \mathrm{Ba} 1$ V. V. Balashov, V. N. Fetisov; Izv. Akad. Nauk SSSR Ser. Fiz. 26,1188 ( 1962$)$

62Be1 A. S. Belousov, S. V. Rusakov, E. I. Tamm; Zhur. Eksp. i Teoret. Fiz. 43, $813(1962)$; Soviet Phys. JETP 16, 576 ( 1963$)$

62 Be2 G. Ben-David (Davis), B. Huebschmann; Phys. Letters 3, 87 ( 1962$)$

62 Be3 C. Becchi, L. Meneghetti, S. Vitale; Phys. Letters 3,89 ( 1962 )

$62 \mathrm{Be} 4$ I. Berkes, I. Dezsi, I Fodor, L. Keszthely; Nuclear Phys. 39,631 (1962)

62Bil P. G. Bizzeti, A. M. Bizzeti-Sona, M. Bocciolini, G. DiCapoliacco, M. Mando; Nuovo Cimento 26, 1412 (1962)

62Bi2 G. R. Bishop, D. B. Isabelle; Phys. Letters 1, 323 (1962)

$62 \mathrm{Bi} 3 \mathrm{G}$. R. Bishop, D. B. Isabelle; Phys. Letters 3, 74 ( 1962 )

62 Bi4 G. R. Bishop, B. Grossetete, J. C. Risset; J . Phys. Radium 23, 31 (1962)

62Bl1 D. Blum, P. Barreau, J.Bellicard; PICNS-62761 (1962)

62Bol o. V. Bogdankevich, B. I. Goryachev, V. A. Zapevalov;

Zhur. Eksp. i Teoret. Fiz. 42, 1502 ( 1962 ); Soviet Phys. JETP 15, 1044 ( 1962 )

62 Bo 2 L. N. Bolen, W. D. Whitehead; Phys. Rev. Letters 9, 458 (1962)

62 Bo3 G. Bonazzola, $\theta$. A. Borello, S. Costa, S. Ferroni; Nuclear Phys. 34, 637 ( 1962 )

62504 R. Bosch, J. Lang, R. Muller, W. Wolfli; Phys. Letters 1 , 114 (1962)

62 Bo5 $\theta$. Borello, F. Ferrero, R. Malvano, A. Molinari; Nuclear Phys. $31,53(1962)$

62Bo6 E. C. Booth, K. A. Wright; Nuclear Phys. 35, 472 ( 1962 )

62 Bo7 R. Bosch, J. Lang, R. Muller, W. Wolfli; Helv. Phys. Acta 35, $288(1962)$

62 Bo8 B. A. Bochagor, A. P. Komar, G. E. Solyakin; Zhur. Eksp. i Teoret. Fiz. 43, 1611 (1962); Soviet Phys. JETP 16, 135 ( 1963 )

62Br1 H. Breuer, W. Pohlit; Nuclear Phys. 30, 417 ( 1962 ) 
$62 \mathrm{Br} 2 \mathrm{D}$. Wraben, L. L. Green, J. C. Willmott; Nuclear Phys. 32, $584(1962)$

$62 \operatorname{Br} 3$ E. Braun; Z. Physik 166, 62 (1962)

$62 \mathrm{Br} 4 \mathrm{H}$. Breuer; Z. Naturforsh. $17 \mathrm{~A}, 584$ ( 1962 )

$62 \mathrm{Bu} 1 \mathrm{~N}$. A. Burgov, G. V. Danilyan, B. S. Dolbilkin, L. E.

Lazareva, F. A. Nikolaev; Zhur Eksp. i Teoret. Fiz. 43, 70 (1962); Soviet Phys. JETP 16,50 (1963)

62 Cal J. H. Carver, G. E. Coote, T. R. Sherwood; Nuclear Phys. 37. $449(1962)$

62 Ca2 J.H. Carver, D. C. Peaslee, R. B. Taylor; Phys. Rev. 127, 2198 ( 1962$)$ 62 Ca3 S. Cavallaro, R. Potenza, A. Rubbino; Nuclear Phys. 36,

62Ch1 R. E. Chrien, H. H. Bolotin, H. Palevsky; Phys. Rev. 127 , $1680(1962)$

62 Ch2 V. P. Chizhov, A. P. Komar, L. A. Kul Chitskil, A. V. Kulikov, E. D: Makhnovsky, Yu. M. Volkov; Nuclear Phys. 34, 562 ( 1962 )

$62 \mathrm{Cl}_{1} \mathrm{~F}$. M. Clikeman, A. J. Bureau, M. G. Stewart; Phys. Rev. $126,1822(1962)$

62Cu1 B. Cujec; Nuclear Phys. 37, 396 ( 1962 )

62Del W. E. Del Bianco, W. E.Stephens; Phys. Rev. 126, 709 ( 1962$)$

62De2 B. I. Deutch; Nuclear Phys. 30, 191 ( 1962 )

$62 \mathrm{De} 3$ H. G. DeCarvalho, A.Manfredini, M.Muchnik, M.Severi, H. Bosch, J. Lang, R. Muller, W. Wolfli; Nuovo Cimento 25, $534(1962)$

62 Do 1 W. R. Dodge, W. C. Barber; Phys.Rev. 127, 1746 ( 1962 )

62Drl T. N. Dragnev, B. P Konstantinov; Zhur. Eksp. i Teoret. Fiz. 42, 344 ( 1962 ); Soviet Phys.JETP is, 236 ( 1962$)$

62 Ed1 R. D. Edge, G. H. Peterson; Phys. Rev. 128, 2750 ( 1962)

$62 E I_{1}$. J. Ellison, B. Dickinson; Nuclear Phys. 35, 606 ( 1962 )

$62 \mathrm{Em} 1 \mathrm{~V}$. Emma, C. Milone; S. Jannelli, F. Mezzanares; Nuovo Cimento 26, 890 ( 1962 )

62Eul P. Euthymiou, P. Axel; Phys . Rev. 128, 274 ( 1962 )

62Fil F. W. K. Firk; Phys. Rev. Letters 8, 321 ( 1962 )

$62 \mathrm{Fi2}$ F. W. K. Firk, K. H. Lokan, E. M. Bowey; PICNS-62 804 ( 1962$)$

$62 \mathrm{Fi} 3 \mathrm{~F}$. W. K. Firk, E. R. Rae; PICNS-62 807 ( 1962 ) $62 F u 1$ S.C.Fultz, R.L.Bramblett, J.T.Caldwell; N. E. Hansen C.

62 Fu2 S. C. Fultz, R. L. Bramblett, J. T. Calawell, N. A. Kerr; Phys. Rev. 127, 1273 (1962)

62Fu3 E. G. Fuller, E. Hayward; Nuclear Phys. 30, 613 ( 1962 )

62 Fu4 E. G. Fuller, E. Hayward; Nuclear Phys. 33, 431 ( 1962 )

$62 \mathrm{Fu} 5 \mathrm{H}$. Fuchs, D. Haag, K. H. Lindenberger, U. Meyer-

Berkhout; Z: Naturforsch $17 a, 439$ (1962) 62 Fu6 ${ }_{590}^{\text {H. Fuchs, }}$ ( 1962 . Kosiek, U. Meyer-Berkhout; Z Physik 166 ,

62Gal C. C. Gardner, J. D. Anderson; Phys. Rev. 125, 626 ( 1962 ) 62Ge1 D. S. Gemmell, G. A. Jones; Nuclear Phys. 33, 102 ( 1962 ) 
62 Gl1 P. W. M. Glaudemans, P. M. Endt; Nuclear Phys. 30, 30 ( 1962$)$

62 Go1 A. N. Gorbunov, V. A. Dubrovina, V. A. Osipova, V. S. Silaeva, P. A. Cerenkov; Zhur. Eksp. i Teoret. Fiz. 42,747 (1962); Soviet Phys. JETP 15, 520 (1962)

62 Go2 A. N. Gorbunov, V. A. Osipova; Zhur. Eksp. i Teoret. Fiz. 43, 40 (1962); Soviet Phys. JETP 16, 27 (1963)

62 Go3 D. Goldman, A. F. T. Piza, E. Silva; Nuovo Cimento 25, $41(1962)$

62 Go4 A. N. Gorbunov, D. K. Kaipov, K. G. Kuvatov; Trudy Inst. Yadernoi Fiz,, Akad. Nauk Kazakh. SSR 5, 135 (1962)

62 Gr1 G. M. Griffiths, E. A. Larson, L. P. Robertson; Can. J. . Phys. 40, 402 ( 1962 )

62 Gr2 A. G. Gregory, T. R. Sherwood, E. W. Titterton; Nuclear Phys. 32, $543(1962)$

62 He1 K. Hermann, J. A. Scheer; Z. Physik 170, 162 ( 1962 )

62 Hul J.R.Huizenga, K.M.CLarke, J.E.Girdler; R.Vandenbosch; Nuclear Phys. 34, 439 ( 1962 )

62 Hu2 J. R. Huizenga, R. Vandenbosch; Nuclear Phys. 34,457 (1962)

62 Ja 1 H. E. Jackson; Phys. Rev. 127, 1687 (1962)

62 Jo1 S.A.E.Johansson, B.Forkman; Nuclear Phys. 36, 141 (1962)

62 Jo2 W. John, J. M. Prosser; Phys. Rev. 127,231 ( 1962 )

62 Ka 1 D. K. Kaipov, Yu. K. Shubnyi, R. B. Begzhanov, A. A. Islamov, Zhur. Eksp. i Teoret. Fiz. 43, 808 (1962); Soviet Phys. JETP 16,572 ( 1963 )

$62 \mathrm{Ke} 1$ H. W. Kendall, I. Talmi; Phys. Rev. 128, 792 ( 1962 )

62 Kol A. P. Komar, A. V. Kulikov, V. P. Chizhov, I. P. Yavor, Yu. M. Volkov; Zhur. Eksp. i Teoret. Fiz. 43, 1657 ( 1962 ); Soviet Phys. JETP 16, 1168 (1963)

62 Ko2 A. P. Komar, Ya. Krzhemenek, I. P. Yavor; Nuclear Phys. 34, 551 ( 1962$)$

$62 \mathrm{Ko} 3 \mathrm{~K}$. Kotajina; Nuclear Phys. 39, 89 ( 1962 )

62 Ko4 A. P. Komar, B. A. Bochagov, V. I. Fadeev; Dokl Akad. Nauk SSSR 146, 1051 (1962); Soviet Phys. Doklady 7,913 ( 1963$)$

$62 \mathrm{Kr} 1$ N. Kregar, B. Povh; Phys. Letters 2, 103 (1962)

62 Kul K. Kuri yama; J. Phys. Soc. Japan 17, 1681 (1962)

$62 \mathrm{La} 1 \mathrm{~B} . \mathrm{T}$. Lawergren, T. R. Ophel; Phys. Letters 2, 265 ( 1962 )

62 Li 1 F. F. Liu, F. J. Loeffler, T.R. Palfrey, Y. S. Kim; Phys. Rev. 128,2784 ( 1962$)$

$62 \mathrm{Li} 2 \mathrm{~N}$. Lingappa, E. Kondaiah, C. Badrinathan, M. D. Deshpande, M. Balakrishnan; Nuclear Phys. 38, 146 ( 1962 )

$62 \mathrm{Ma} 1$ I. G. Main; Nuovo Cimento 26, 884 ( 1962 )

62Me1 F. R. Metzger; Phys. Rev.128, 2332 (1962)

62 Me2 F. R. Metzger; Phys. Rev. 127, 220 ( 1962 )

$62 \mathrm{Mil} \mathrm{J}$. A. Miskel, K. V. Marsh, M. Lindner, R. J. Nagle; Phys. Rev. 128,2717 ( 1962$)$

62 Mi 2 J. Miller, C. Schuhl, G. Tamas, C. Tzara; Phys. Letters 2, 76 ( 1962$)$

62 Mi3 J. Miller, C. Schuhl, C. Tzara; Nuclear Phys. 32, 236 ( 1962 ) 
$62 M i 4$ U. Miklavzic, N, Bezic, D.Jamnik, G. Kernel, Z, Milavc, J. Snajder; Nuclear Phys. 31,570 ( 1962)

62 Mis C. Milone, S. Milone Tamburino, V. Emma, S. Femino, S. Jannelli; PICNS-62 817 ( 1962 )

62Mol W. L. Mouton, J.P. F. Sellschop, R. J. Keddy; Phys. Rev. 128, 2745 (1962)

62 Mo2 W. T. Morton, T. G. Walker; Phil. Mag. 7, 741 ( 1962)

62 Mo3 G. Moscati, J. Goldemberg; Phys. Rev. 126, 1098 ( 1962 )

$62 \mathrm{Mu} 1 \mathrm{~N}$. Mutsuro, K. Kageyama, M. Mishina, T. Nakagawa,. E. Tanaka, M. Kimura; J. Phys. Soc. Japan 17, 1672 (1962)

62 Mu2 N. Mutsuro, K. Kageyama, M. Mishina, E. Tanaka, M. Kimura: J. Phys. Soc. Japan 17, 1673 ( 1962)

62 Ne 1 E. B. Nelson, R. R. Carlson, L. D. Schlenker; Nuclear Phys. 31, $65^{\circ}(1962)$

62 Ne2 D. R. Neher, F. W. Prosser, Jr., R. W. Krone; Nuclear Phys. 31, 231 ( 1962$)$

62 Oc1 J. S. O Connell, P. A. Tipler, P. Axel; Phys.Rev. 126, $228(1962)$

62 9d1 M. Odera; J.Phys. Soc. Japan 17, 1675 (1962)

62 epl T. R. Ophel, B. T. Lawergren; Nuclear Phys. 30,215 (1962)

$620 \mathrm{p} 2 \mathrm{~T}$. R. Ophel, R. N. Glover, E. W. Titterton; Nuclear Phys. 33, $198(1962)$

62 Pal V. Parikh; Nuclear Phys. 38, 529 ( 1962 )

62 Pa2 A. W. Parker, G. G. Shute; Australian J.Phys. 15, 443 ( 1962 )

62 Pel G. A. Peterson, W. C. Barber; Phys. Rev. 128, 812 ( 1962 )

62 Pr 1 F. W. Prosser, Jr., W. P. Unruh, B. W. Wildenthal; R. W. Krone; Phys. Rev. 125, 594 (1962)

62 Rad L. K. Rangan, G. I. Farris, L. W. Seagondollar; Phys. Rev. 127, 2180 ( 1962$)$

$62 \operatorname{Re} 1 \mathrm{G}$. C. Reinhardt, W. D. Whitehead; Nuclear Phys. 30, 201 (1962)

62Se1 F. D. Seward; Phys. Rev.125, 335 (1962)

62Se2 W. Sebaoun; J. Phys. Radium 23, 989 ( 1962 )

$62 \operatorname{Sh} 1$ A. K. Shardanov, V. G. Shevchenko, Zhur. Eksp. i Teoret. Fiz. 42, 1438 ( 1962 ); Soviet Phys: JETP 15, 996 (1962)

$62 \operatorname{sh} 2$ V. G. Shevchenko, B. A. Yur ev; Zhur. Eksp. i Teoret. Fiz. 42, 707 ( 1962 ); Soviet Phys.JETP 15,492 (1962)

$62 \operatorname{sh} 3$ V. G. Shevchenko, B. A. Yur ev; Zhur. Eksp. 1 Teoret. Fiz. 43, 860 ( 1962 ); Soviet Phys. JETP 16, 609 (1963)

$625 h 4$ V. G. Shevchenko, B. A. Yur ev; Nuclear Phys. 37,495 ( 1962$)$

62 Sh5 T. R. Sherwood, W. E. Turchinetz; Nuclear Phys. 29, 292 ( 1962$)$

62 Sh6 V. G. Shevchenko, B. A. Yur ev; Vestn. Mosk. Univ., Ser. III, No. 3, 90 ( 1962$)$

$62 \operatorname{sh} 8$ K. Shoda, T. Ishizuka, K. Shimizu, M. Akashi; J. Phys. Soc. Japan i7, 1536 ( 1962 )

$62 \operatorname{sh} 9$ K. Shoda, H. Nilzeki, N. Fujiwara, A. Okiguchi, A. Watanabe, M. Midera; J. Phys. Soc: Japan 17 , 1083 ( 1962 ) 
62 Sh10 K. Shoda, T. Ishizuka, K. Shimizu, M. Akashi; J. Phys. Soc. Japan 17,407 (1962)

$62 \operatorname{Sh} 11 \mathrm{~K}$. Shoda, K. Abe, T. Ishizuka, N. Kawamura, M. Kimura; J: Phys. Soc. Japan 17,735 ( 1962 )

62 Sh 12 K. Shoda, T. Ishizuka, N. Kawamura, K. Abe, M. Kirura; J: Phys. Soc. Japan 17,401 ( 1962 )

62Sm1 P. J. M. Smulders, P. M. Endt; Physica 28, 1093 ( 1962 )

62 So 1 Yu. I. Sorokin, V, G. Shevchenko, B, A. Yur ev; Zhur. Eksp. i Teoret? Fiz.43, 1600 (1962); Soviet Phys. JETP 16, 1127 ( 1963 )

62Su1 B. N. Sung; J. Phys. Soc. Japan 17, 1811 (1962) 842 (1962)

62 Su2 M. Suffert, G. Costa, D. Magnac-Valette; PICNS-62

62 Tu1 P. M. Tutakin; Zhur. Eksp. 1 Teoret. Fiz. 43, 1140 (1962); Soviet Phys. JETP 16,805 ( 1963 )

62 Vol Yu. M. Volkov, L. A. Kul chitskil; Zhur. Eksp. i Teoret. Fiz. 42, 108 ( 1962 ); Soviet Phys. JETP is, 77 ( 1962 )

62 Vo2 Yu. M. Volkov, A. V. Kulikov, V. P. Chizov; Zhur. Eksp. I Teoret. Fiz. 42, 61(1962); Sóviet Phys. JETP 15, 42 ( 1962 )

62 Wa1 E. K. Warburton, H. 0 . Funsten; Phys. Rev. 128, 1810 ( 1962$)$

63 Ak 1 A. F. Akkerman, V. L. Kochetkov, V. N. Chekanov, V. V. Suvorov, A. K. Shtolts; Zhur. Eksp. i Teoret. Fiz. 45, 1778 (1963); Soviet Phys. JETP 18, 1218 ( 1964)

63 An 1 D. W. Anderson, A. J . Bureau, B. C. Cook, J. E. Griffin, J. R. McConneli, K. H. Nybo; Phys. Rev. Letters 10,250 (1963)

63 An2 Y. P. Antoufiev, L. M. El-Nadi, D. A. E. Darwish, O. E. Badawy, P. V. Sorokin; Nuclear Phys.46, 1 ( 1963 )

63 An 3 E. S. Anashkina; Zhur. Eksp. i Teoret. Fiz. 45, 404 (1963); Soviet Phys. JETP 18,279 (1964)

63 Aul R. H. Augustson, N. N. Kaushal, W. R. Moyer, E. J. Winhold, P. F. Yergin; Helv. Phys. Acta 36, 816 ( 1963 )

63 Ax1 P. Axel, K. Min, N. Stein, D. C. Sutton; Phys. Rev. Letters $10,299^{\circ}(1963)$

$63 \mathrm{ba} 1$ W. C. Barber, J. Goldemberg, G. A. Peterson, Y. Torizuka; Nucl ear Phys. 41, 461 ( 1963 )

$63 \mathrm{Ba} 2$ G. N. Hailey, D. R. Hebbard; Nuclear Phys. 46, 529 ( 1963 ) $63 \mathrm{Ba3}$ M. V. Ballariny; Notes de Fisica 10(No. 12), 205 ( 1963 ) 63 Be 1 B. L. Berman, L. J Koester, Jr., J. H. Smith; Phys. Rev. Letters 10,527 (1963)

63He2 M. Bernheim, G. R. Bishop; Phys. Letters 5, 270 ( 1963 )

$63 \mathrm{Be} 3 \mathrm{M}$. Bernheim, G. R. Bishop; Phys. Letters 5, 294 ( 1963 )

63 Be4 W. Bertozzi, C. P. Sargent, W. Turchinetz; Phys. Letters $6,108(1963)$

63Be5 J. A. Becker, J. D. Fox; Nuclear Phys. 42, 669 ( 1963 )

63Be6 G. B. Beard, W. H. Kelly; Nuclear Phys. 43, 523 ( 1963 )

63Be7 W. Bertozzi, P. T. Demos, S. Kowalski, C. P. Sargent, W. Turchinetz, R. Fuilwood, J. Russell; Phys. Rev. Letters $10,106(1963)$

63 Be 8 M. Bernheim, G. R. Bishop; J d de Physique 24,970 ( 1963 ) 63Bil G. R. Bishop, M. Bernheim; Phys. Letters 5, 140 ( 1963 ) 
63Bi2 G. R. Bishop, C. Betourne, D. B. Isabelle; J. de Physique 24, $973(1963)$

$63 \mathrm{Bi} 3 \mathrm{G}$. E. Bizina, A. G. Beda, N. A. Burgov, A. V. Davydov; Zhur. Eksp. i Teoret. Fys.45, 1408 (1963); Soviet Phys. JETP $18,973(1964)$

638l1 D. Blum, P. Barreau, J. Bellicard; Phys. Letters 4,109 ( 1963$)$

63 Bl2 J. P. Blanc, M. Lambert, C. F. Perdrisat; Helva Phys. Acta $36,820(1963)$

63 Bo 1 L. N. Bolen, W. D. Whitehead; Phys . Rev. 132, 2251 ( 1963$)$

63 Bo2 R. Bosch, J. Lang, R. Muller, W. Wolfli; Helva. Phys. Acta 36,625 (1963)

63Bo3 P. Bounin, G. R. Bishop; J. de Physique 24,974 ( 1963 )

63 Bo4 R. Bosch, J Lang, R. Muller, W. Wolfli; Helva. Phys. Acta 36,657 (1963)

63 Bo5 5 . V. Bogdankevich, B. S. Dolbilkin, L. E. Lazareva, F. A. Nikolaev; Zhur. Eksp. i Teoret. Fiz. 45, 882 (1963); Soviet Phys. JETP 18,606 (1964)

63806 B. A. Bochagov, A. P. Komar, V. I. Fadeev; Atomnaya Energiya 15, 191 ( 1963); Soviet Atomic Energy 15, 891 $(1963)$

$63 \mathrm{Br} 1$ R. L. Bramblett, J . T. Caldwell, G. F. Auchampaugh, S. C. Fultz; Phys. Rev. 129, 2723 (1963)

63 But N. A. Burgov, G. V. Danilyan, D. S. Dolbilkin, L. E. Lazareva, F. A. Nikolaev, IZv. Akad. Nauk. SSSR 27, 866 (1963)

63 Bu2 W. Buss, H. Waeffler, B. Ziegler; Phys. Letters 4,198 ( 1963$)$

638u3 N. A. Burgov, G. V. Danilyan, B. S. Dolbilkin, L. E. Lazareva, F. A. Nikolaev; Zhur. Eksp. i Teoret. Fiz. 45 , 1693 ( 1963 ); Soviet Phys. JETP 18, 1159 ( 1964 )

63Cal J. T. Caldwell, R. R. Harvey, R. L. Bramblett, S. C. Fultz. Phys. Letters 6, 213 (1963)

63 Ch1 P. R. Chagnon, P. A. Treado; Nuclear Phys. 40, 195 (1963)

63Co 1 S. Costa, S. Ferroni, V. Wataghin, R. Malvano, Phys. Let ters 4, 308 ( 1963$)$

63 Co3 S. Costa, F. Ferrero, S. Ferroni, B, Minetti, C. Molino, R. Malvano; Phys. Letters 6, 226 ( 1963 )

$63 \mathrm{Cv} 1 \mathrm{~F}$. Cvelbar, A. Hudoklin, M. V. Mihailovic, M. Najzer, V. Ramsak; Phys. Letters 3, 364 ( 1963 )

$63 \mathrm{Dc} 3 \mathrm{H}$. Schmid, W. Scholz; Z. Physik 175, 430 (1963)

$63 \mathrm{De} 1 \mathrm{H}$. G. DeCarvalho, A. Manfredini, M. Muchnik, M. Severi, R. Bosch, W. Wolfli; Nuovo Cimento 29,463 (1963)

63 Fil P. S. Fisher, D. F. Measday, F. A. Nikolaev, A. Kalmykov, A. B. Clegg; Nuclear Phys. 45, 113 (1963)

$63 \mathrm{Fi} 2 \mathrm{E}$. Finckh, R. Kosiek, K. H. Lindenberger, U. MeyerBerkhout, N. Nucker, K. Schlupmann; Phys: Letters 7 , 271 ( 1963$)$

63Fi3 E. Finckh, R. Kosiek, K. Schlupmann; Naturwiss.50, 326 ( 1963$)$

$63 \mathrm{Fi} 4 \mathrm{E}$. Finckh, R. Kosiek, K. H. Lindenberger, K. Maier, U. Meyer-Berkhout, M. Schechter, J.Zimmerer; Z Physik $174,337(1963)$ 
$63 \mathrm{Fl} 1 \mathrm{H}$. H. Fleischmann; Ann. Physik12, 133 (1963)

63 Fl2 H.H. Fleischmann, F. W. Stanek; Z. Physik 175, 172 ( 1963$)$

63Fr1 D. E. Frederick. Phys - Rev.130, 1131 (1963)

$63 F r 2$ E. Friedland, H. R. Lemmer; Z, Physik 174, 507 ( 1963)

63Fu 1 H. Fuchs, D. Haag; Z Physik 171, 403 ( 1963 )

63Fu2 H. Fuchs; Z. Physik 171, 416 (1963)

63 Fu3 B. Furubayashi, E. Teranishi, M. Kageyama; J. Phys. Soc. Japan 18, 1235 (1963)

63Gal N. H. Gale, N. Tubbs, N. Nath, A. T. G. Ferguson; Phys. Letters 5,345 (1963)

$63 \mathrm{Ge} 1 \mathrm{~K}$. N. Geller; Nuclear Phys. 40, 177 ( 1963 )

$63 \mathrm{Ge} 2 \mathrm{~K}$. N. Geller; Phys. Rev. Letters 11, 371 (1963)

63 Go2 A. N. Gorbunov, A. T. Varfolomeev; Phys. Letters 5, 149 ( 1963$)$

63Go3 H. E. Gove; Nuclear Phys.49, 279 ( 1963 )

63 Go4 J. Goldemberg, Y. Torizuka, W. C. Barber, J. D. Walecka; Nuclear Phys. 43,242 (1963)

63 Go5 S. Gorodetzky, W. Benenson, P. Chevallier, D. Disdier, F. Scheibling; Phys. Letters 6, 269 ( 1963 )

63Go8 J. Goldemberg, E. W. Hamburger, A. Szily; An. Acad. Brasil. Cienc. 35, i69 (1963)

63Grl G. M. Griffiths, M. Lal, C. D. Scarfe; Can. J. Phys.41, $724(1963)$

63He1 D. F. Hebbard, G. M. Bailey; Nuclear Phys. 49,666 ( 1963 )

63Hol P. Horvat, P. Kump, B. Povh; Nuclear Phys. 45, 341 (1963)

63 Is 1 D. B. Isabelle, G. R. Bishop; Nuclear Phys. 45, 209 (1963)

63 Is2 B. S. Ishkhanov, E, N Kornienko, Yu. I Sorokin, V. G. Shevchenko, B. A. Yur ev; Zhur. Eksp. i Teoret. Fiz. 45 , 38 ( 1963 ); Soviet Phys. JETP 18, 29 ( 1964 )

$63 \mathrm{Kal}$ D. K. Kaipov, R. B. Begzhanov, A. V. Kuz minov, Yu. K. Shubnyi; Znur. Eksp. i Teoret. Fiz. 44, 1811 (1963); Soviet Phys. JETP 17,1217 ( 1963 )

63Ka2 T. Kaminishi, C. Kojima; Jap. J. Appl. Phys. 2, 399 (1963)

$63 \mathrm{Ka} 3 \mathrm{D}$. K. Kaipov, Yu, K. Shubnyi, Yu, G. Kosyak, R, B. Begzhanov; Zhur. Eksp. i Teoret. Fiz. 45, 443 (1963); Soviet Phys. JETP 18, 305 ( 1964 )

$63 \mathrm{Ke} 1 \mathrm{H}$. W. Kendall, J. Oeser; Phys. Rev. 130, 245 ( 1963 )

$63 \mathrm{Ki} 1 \mathrm{Y} . \mathrm{S}, \mathrm{Kim}, \mathrm{F}, \mathrm{F}, \mathrm{Liu}, \mathrm{F}, \mathrm{J}$. Loeffler, T. R. Palfrey; Phys. Rev. 129,1362 ( 1963 )

$63 \mathrm{Ki} 2 \mathrm{M}$. Kimura, K. Shoda, N. Mutsuro, M. Sugawara, K. Abe, K. Kageyama, M. Mishina, A. Ono, T. Ishizuka, S. Mori, N. Kawamura, T. Nakagawa, E. Tanaka; J. Phys. Soc. Jap an $18,477(1963)$

63kol A. P. Komar, B. A. Bochagov, V. I. Fadeev; Dokl. Akad. Nauk SSSR 152, 858 (1963); Soviet Phys. Doklady 8, 978 ( 1964 )

$63 \mathrm{Ko} 2$ I. Kohno; J. Phys. Soc. Japan 18, 1709 (1963)

$63 \mathrm{Kr} 1 \mathrm{M}$. Kregar, B. Povh; Nuclear Phys. 43, 170 ( 1963)

$63 \mathrm{ku} 1 \mathrm{~L}$. A. Kul chitskii, Yu. M. Volkov; Zhur. Eksp. i Teoret. Fiz. 44, 1153 ( 1963 ); Soviet Phys. JETP 17,780 (1963) 
63 Ku2 L. A. Kul chitskii, Yu, M. Volkov, V. P, Denisov, V. I. Orgurtsov; Izv. Akad. Nauk. SSSR 27, 1412 (1963)

63 La 1 M. Langevin, J. M. Loiseaux; J. de Physique 24, 1027 ( 1963 )

$63 \mathrm{Lel} \mathrm{F.} \mathrm{H.} \mathrm{Lewis,} \mathrm{Jr.,} \mathrm{J.} \mathrm{D.} \mathrm{Walecka,} \mathrm{J} \mathrm{.} \mathrm{Goldemberg,} \mathrm{W.} \mathrm{C.}$ Barber, Phys. Rev. Letters 10,493 ( 1963 )

63 Lo $1 \mathrm{~F} . \mathrm{J}$. Loeffler, T. R, Palfrey, Jr., T. Q. White, Jr., Phys. Rev. 131, 1844 (1963)

63 Lo2 R. Lombard, P. Kossanyi, G . R. Bishop; J de Physique $24,968$ ( 1963$)$

$63 \mathrm{Mcl}$ J. A. McIntyre, G. K. Tandon; Phys. Letters 4, 117 (1963)

$63 \mathrm{Mc} 2 \mathrm{G} . \mathrm{J} . \mathrm{McCa} L \mathrm{um} ; \mathrm{Can}$. J.Phys.41,923 (1963)

$63 \mathrm{Mc} 3 \mathrm{~W} . \mathrm{J} . \mathrm{McD}$. Mald, E. Buchholz, R. N. H. Haslam; Can. J. Phys. 41, 180 ( 1963 )

63 Me 1 F. R. Metzger, H. Langhoff; Phys. Rev. 132,1753 (1963)

63 Mil I. V. Mitchell, R. B. Taylor; Nuclear Phys. 44,664 (1963)

63 Mi2 K. Min, L. N. Bolen, W. D. Whitehead; Phys. Rev. 132 , $749(1963)$

$63 \mathrm{Mi} 3$ K. Miyano, T. Kuroyanagi, Nuclear Phys. 49, 315 (1963)

63 Mi4 C. Milone; Nuclear Phys. 47,607 ( 1963 )

63 Mis d. M. M. Mitchell, K. G. McNeill; Can. J.Phys.41, 871 ( 1963 )

63 Mo 1 W. L. Mouton, J. P. F. Sellschop, G. Wiechers; Phys. Rev. 129,361 ( 1963 )

63 Mul R. M. Murray, W. L. Bendel; Phys. Rev. 132, 1134 (1963)

$63 \mathrm{Mu} 2 \mathrm{~N}$. Mutsuro, K. Kageyama, M. Mishina, E. Tanaka, T. Aizawa, M. Kimura; J. Phys. Soc. Japan 18, $599^{\text {( } 1963 \text { ) }}$

63 Nel B. M. K. Nefkens; Phys. Rev. Letters 10,55 (1963)

$63 \mathrm{Ne} 2 \mathrm{~B} . \mathrm{M}$. K. Nefkens; Phys. Rev. Letters 10, 243 ( 1963 )

$63 \mathrm{Ng} 1 \mathrm{H}$. Nguyen Ngoc, M. Hors, J. Perez y Jorba; Nuclear Phys. 42, 62 (1963)

$63 \mathrm{Ng} 2 \mathrm{H}$. Nguygen Ngoc, J. Perez y Jorba; J de Physique 24 , $965(1963)$

63 od 1 M. Odera, N. Yamamuro, J. Phys. Soc. Japan 18, 325 ( 1963$)$

$630 s 1$ R. M. Osokina; Zhur Eksp. i Teoret. Fiz. 44, 444 ( 1963); Soviet Phys. JETP 17, 303 ( 1963 )

63 Pel R. R. Perry, B. Mainsbridge, J. Richards; Nuclear Phys. 45, $586(1963)$

63 Pe2 K. A. Petrzhak, R. V. Sedletskii; Atomnaya Energiya 15, 308(1963); Soviet Atomic Energy 15, 1025 (1963)

63 Pr1 F. W. Prosser, Jr., R. W. Krone, J J S ingh; Phys. Rev. $129,1716(1963)$

63 Pr2 P. R. Pruett; Phys. Rev.129, 2583 (1963)

$63 \operatorname{Re} 1 \mathrm{~N}$. W. Reay, N. M. Hintz, L. L. Lee, Jr., Nuclear Phys. $44,338(1963)$

63 Ril v. Riech; Phys. Letters 6, 267 (1963)

63Ri 2 P. Rice-Evans; Proc. Phys. Soc. 82, 914 ( 1963 )

63 Sa 1 K. Sato; J. Phys. Soc. Japan 18, 1353 (1963) 
63Sc1 J.A. Scheer, K. Schlupmann, F. Triantafyllidis; Phys. Letters 7,269 (1963)

$63 \mathrm{Sc} 3 \mathrm{H}$. Schmid' and W. Scholz; Z. Physik 175,430 ( 1963 )

63 Se 1 R. E. Segel, P. P. Singh, R. G. Allas, S. S. Hanna; Phys. Rev. Letters 10,345 (1963)

63 Sh1 V. G. Shevchenko, B. A. Yur ev, B. P. Levkin; Zhur. Eksp. i Teoret. Fiz. 44, 808 (1963); Soviet Phys. JETP 17 , 547 ( 1963 )

63Sh2 N. K. Sherman; Phys. Letters 4, 113 (1963)

$63 \mathrm{Sh} 3 \mathrm{~N}$. K. Sherman; Phys. Letters 4, 138 ( 1963 )

63 Sh4 K. Shoda, B. N. Sung, N. Kawamura, M. Oyamada, K. Abe, T. Ishizuka, M. Kimura; J. Phys. Soc. Japan 18, 152 $(1963)$

63Sh5 Yu. K. Shubnyi; Zhur. Eksp. i Teoret. Fiz. 45, 460 (1963); Soviet Phys. JETP 18, 316 (1964)

63Si1 L. Simons, K. E. Nysten, M. Koskelin, $\theta$. Siltanen, E. Spring, G. Wendt; Phys. Letters 3, 306 ( 1963 )

63 Si2 L. Simons, K. E. Nysten, E. Spring, L. Kald; H, Junger, P. Holmberg, I. Forsblom; Phys. Letters 7,344 (1963)

63 Sk1 S. J. Skorka, R. Hubner, T, H. Retz-Schmidt, H, Wahl Nuclear Phys. 47, 417 (1963)

63Spl E. Spring; Soc. Sci. Fennica, Commentationes PhysMath. 28, 1 (1963)

63st 1 R. F. Stiening, E. Loh, M. Deutsch; Phys. Rev. Letters 10 , 536 (1963)

63 Sul M. Sugawara, S. Mori, A. Ono, A. Hotta, M. Kimura; J. Phys. Soc. Japan 18, 17 ( 1963$)$

635u2 M. Suffert, G. Costa, D. Magnac-Valette; J de Phys. 24, 1029 ( 1963 )

63 ill P. A. Tipler, P. Axel, N. Stein, D. C. Sutton; Phys. Rev. $129,2096(1963)$

63 Val V.J.Vanhuyse, G.T.Vanpraet; Nuclear Phys. 43, 344 ( 1963 )

$63 \mathrm{Va} 2 \mathrm{~A}, \mathrm{~K}$. Val ter, E. G. Kopanets, A. N, L vov, S. P. Tsytko; Izv. Akad. Nauk. SSSR 27, 232 ( 1963 )

63 Va 3 V. J. Vanhuyse, G. J . Vanpraet; Nuclear Phys. 45, 602 ( 1963$)$

63 Va 4 V. J . Vanhuyse, G. J.Vanpraet, K. J. Van Camp; Phys. Letters $5,89(1963)$

63 Va5 A. K. Val ter, E. G. Kopanets, A. N. L vov, A. Stegner, S. P. Isytko; Izv. Akad. Nauk SSSR 27, 1419 (1963)

63Ve1 A. Veres; Int. J. Appl. Rad. I sotopes 14,123 (1963)

63Ve2 A. Veres; Acta Phys. Acad. Sci. Hung. 16,261-273 (1963)

63 Kal J. B. Warren, K. L. Erdman, L. P. Robertson, D. A. Axen, J.R. MacDonald; Phys. Rev. 132, 1691 (1963)

63 Wa2 H. D. Warren, A. P. Batson; Nuclear Phys. 48, 361 (1963)

63 Yal N. Yamamuro; J. Phys. Soc. Japan 18, 11 (1963)

63 Ya2 M. Yamanouchi; J. Phys. Soc. Japan 18,638 (1963)

63 Yo1 C. S. Young, D. J. Donahue; Phys. Rev. 132,1724 ( 1963 )

63 Yo2 F. C. Young, J. C. A rmstrong, J. B. Marion; Nuclear Phys. 44, 486 ( 1963 ) (Errata: Nuclear Phys. 49,695 (1963))

$63 \mathrm{Za} 1 \mathrm{G}$. N. Zatsepina, V. V. Igonin, L. E. Lazareva, A. I. Lepestkin; Zhur. Eksp. i Teoret. Fiz. 44,1787 (1963); Soviet Phys. JETP 17,1200 (1963) 
63 Zu1 R. W. Zurmuhle, W. E. Stephens, H. H. Staub; Phys. Rev. 132,751 (1963)

64Al1 F. R. Allum, G. M. Crawley, B. M. Spicer; Nuclear Phys. $51,177(1964)$

64 Al2 R. G. Allas, S. S. Hanna, L. Meyer-Schutzmeister, R. E. Segei; Nuclear Phys. 58, 122 (1964)

64 Al3 R. G. Allas, S. S. Hanna, L. Meyer-Schutzmeister, R. E. Segel, P. P. Singh, Z. Vager; Phys. Rev. Letters 13, 628 ( 1964 )

64 Al 4 F. F. Allum, T. W. Quirk, B. M. Spicer; Aust. J. Phys. 17 , 420 ( 1964$)$

64Al5 F. R.Allum, T. W. Quirk, B. M. Spicer; Nucl. Phys. 53, 545 ( 1964$)$

$64 \mathrm{Am} 1 \mathrm{I}$. Amaldi, Jr., G. Campos Venuti, G. Cortellessa, C. Fronterotia, A. Reale, P. Salvadori, P. Hillman; Phys. Rev. Letters 13,341 ( 1964$)$

64 Ar 1 B. Arad (Huebschmann), G. Ben-David(Davis), I. Pelah, Y. Schlesinger; Phys. Rev. 133, B684 (1964)

64 Ar2 H. Artus, G. Fricke, D. E. von Stein; Z. Physik 178, 109$112(1964)$

64Bal J. E. E. Baglin, B. M. Spicer; Nuclear Phys. 54, 549 (1964)

$64 \mathrm{Ba}$ E. B. Bazhanov, A. P. Komar, A. V. Kulikov; Zhur. Eksp. i Teoret. Fiz, 46, 1497 (1964); Soviet Phys. JETP 19,1014 ( 1964 )

64 Ba 3 G. Baciu; Rev. Roum. Phys.9, 783 ( 1964 )

$64 \mathrm{Ea} 4 \mathrm{G}$. Baciu, A. Bonazzola, B. Minetti, C. Molino, L. Pasqualini, G. Piragino; Rev. Roum. Phys.9,9977 ( 1964 )

$64 \mathrm{Ba} 5 \mathrm{G}$. Baciu, C. Molino, B. Minetti, L. Pasqualini, G. Piragino; Stud. Cercetari Fiz. 16, 903 (1964)

$64 \mathrm{Hel} \mathrm{C.} \mathrm{Becchi,} \mathrm{L.} \mathrm{Meneghetti,} \mathrm{M.} \mathrm{Sanzone,} \mathrm{S.} \mathrm{Vitale;} \mathrm{Nuclear}$ Phys. 59, 375 ( 1964 )

64Be2 C. Becchi, G. E. Manuzio, L. Meneghetti, S. Vitale; Phys. Letters 8,322 ( 1964 )

$64 \mathrm{Be} 3 \mathrm{~J}$. Bellicard, P. Barreau, D. Blum; Nuclear Phys.60, 319 ( 1964 )

64 Be 4 R. B. Begzhanov, A. A. Islamov; Zhur. Eksp. i Teoret. Fiz. 46, 1486 (1964); Soviet Phys. JETP 19, 1005 ( 1964 )

64 He5 B. L. Berman, L, J. Koester, Jr., J H. Smith; Phys. Rev. $133, \operatorname{B1} 17(1964)$

64 Be6 G. B. Beard; Phys. Rev. 135, B577-580 (1964)

64Be7 R. B. Begzhanov, A. A. Islamov; Zhur. Eksp. i Teoret. Fiz. 47, 768 (1964); Soviet Phys. JETP 20, 513 (1965)

64 Be8 w. Bertozzi, P. Demos, F. Hanser, S. Kowalski, C. Sargent, W. Turchinetz, R. Fullwood, J. Russell; PICNS$641026^{\circ}$ ( 1964 )

64Bil G. R. Bishop, M. Bernheim, P. Kossanyi Demay; Nuclear Phys. 54, 353 ( 1964 )

64Bi2 G. R. Bishop, D. B. Isabelle, C. Betourne; Nuclear Phys. 54, 97 ( 1964$)$

64Bi3 G. R. Bishop, C. Betourne, D. B. Isabelle; Nuclear Phys. 53, 366 (1964)

64Bi4 G. R. Bishop, M. Bernheim; Phys. Letters 8, 48 ( 1964 )

$64 \mathrm{Bl} 1 \mathrm{~J} . \mathrm{L} . \mathrm{Black}, \mathrm{G}$. A. Jones, P. B. Treacy; Nuclear Phys. 54, 689 (1964) 
648l2 J. L. Black, N. W. Tanner; Phys. Letters 11, 135 ( 1964 ) 64 Bo1 E. C. Booth, B. Chasan, K. A. Wright; Nuclear Phys. 57, $403(1964)$

64802 R. Bosch, J. Lang, R. Muller, W. Wolfli; Phys. Letters 8, $120(1964)$

64 Bo3 C. D. Bownan, G. F. Auchampaugh, S. C. Fultz; Phys. Rev. 133, B676 (1964)

64Br1 R. L. Bramblett, J.T. Caldwell, R. R. Harvey, S. C. Fultz; Phys. Rev. 133, B869 (1964)

$64 \mathrm{Br} 2 \mathrm{P}$. Brix, H. G. Clerc, R. Engfer, G. Fricke, F. Gudden, H. Liesem, E. Spamer; PICNS-64 372 ( 1964 )

$64 \mathrm{Chl} \mathrm{K}$. W. Chen, J. R, Dunning, Jr., J. R. Rees, W. Shlaer, J. K. Walker, R. Wilson; Phys. Rev. 135, 4B Bio30 (1964)

64Co1 E. G. Corman, R. W. Jewell, W. John, J. E. Sherwood, D. White; Phys. Letters 10, 116 (1964)

64Co2 S. Costa, F. Ferroni, S. Ferroni, C. Molino, R. Malvano; Phys. Letters 11,324 ( 1964 )

64 Co3 S. Costa, F. Ferrero, S. Ferroni, R. Malvano; PICNS-64 1034 ( 1964$)$

64Cr1 H. L. Crannell, T. A. Griffy; Phys. Rev. 136, B1580 ( 1964 ) 64 De1 A. DeMarco, R. Garfagnini, G. Piragino; Phys. Letters 10, $213(1964)$

64 De2 V. P. Denisov, A. V. Kulikov, L. A. Kul chitskii; Zhur.

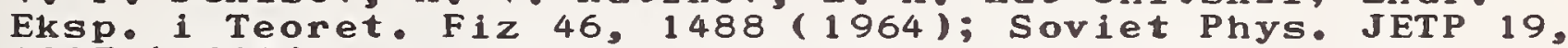
1007 ( 1964 )

64De3 W. Del Bianco, W. E. Stephens, J. Wiza; Phys. Rev. 136 , B418 ( 1964$)$

$64 \mathrm{De} 4$ H. G. De Carvalho, G. Cortini, E. Del Gi udice, G. Potenza, R. Rinzivillo, G. Ghigo; Nuovo Cimento 32, 293 ( 1964 )

64 Do 1 M. M. Dorosh, A. M. Parlag, V. A. Shkoda Ulyanov, L. A. Shabal i na; Zhur. Eksp. i Teoret. Fiz. 46, 1540 (1964); Soviet Phys. JETP 19, 1042 ( 1964 )

64 Do2 B. S. Dolbilkin, V. A. Zapevalov, V. I. Korin, L. E. Lazareva, F. A. Nikolaev; PICNS-64 1060 ( 1964 )

64 Du1 I. I. Dushkov, B. S. Ishkhanov, I. M. Kaitonov, B. A. Yur ev, V. G. Shevchenko; Phys. Letters 10,310 (1964)

64 Eal E. D. Earle, N. W. Tanner, G. C. Thomas; PICNS-64 385 (1964)

$64 E_{j} 1 \mathrm{H}$. Ejiri, Y. Nakjima, K. Horie, S. Matsumoto, Y. Nogami; Nuclear Phys. 51,470 ( 1964 )

64 Er 1 F. C. Erne, C. Van der Leun; Nuclear Phys. 52, 515 ( 1964) 64 Er2 R. A. Eramzhyan; Izv. Akad. Nauk. SSSR, Ser. Fiz., 28, $1181-1184$ ( 1964$)$

64Fi1 F. W. K. Firk; Nuclear Phys. 52, 437 ( 1964)

64 Fi2 F. W. K. Firk, E. M. Bowey; PICNS-641013 ( 1964 )

64 Fo'1 B. Forkman, W. Stiefler; Nuclear Phys. 56, 604 ( 1964)

64 Ful S. C. Fultz, R. L. Bramblett, J. T. Caldwell, R. R. Harvey; Phys. Rev. 133, B1149 (1964)

64Ge1 R. W. Gellie, K. H. Lokan; Nuclear Phys. 60, 343 ( 1964 )

64Gi1 M. Giannini, P, Oliva, D. Prosperi, S. Sciuti; Nuovo Cimento $34,1116(1964)$ 
$64 \mathrm{Gl} 1 \mathrm{Yu}$. Ya.Glazunov, M. V. Savin, I. N. Safina, E. F. Fomushkin, Yu. A. Khokhlov; Zhur. Eksp. i Teoret. Fiz. 46, 1906 (1964); Soviet Phys. JETP 19, 1284 ( 1964 )

64Go1 A. N. Gorbunov, A. T. Varfolomeev; Phys. Letters 11, 137 (1964)

64Go2 J. Goldemberg, W. C. Barber; Phys. Rev.134, B963 (1964) 64 Go3 J. Goldemberg, W. C. Barber, F. H. Lewis, Jr., J.W. Wal ecka; Phys. Rev. 134, B1022 (1964)

64 Go4 A. N. Gorbunov, A. T. Varfolomeev; PICNS -641071 ( 1964 )

$64 \mathrm{Grl}$ G. H. Greenberg, J. P. Roalsvig, R. N. H. Haslam; Can. J. Phys. 42, 731 ( 1964 )

64Gr2 L. Green, D. J Donahue; Phys. Rev. 135, B701-705 ( 1964 ) 64Gu1 F. Gudden; Phys. Letters 10, 313 ( 1964 )

$64 \mathrm{Hal} \mathrm{J}$. C. Hafele, F. W. Bingham, J. S. Allen; Phys. Rev. 135 , B365 ( 1964 )

$64 \mathrm{Ha} 2 \mathrm{R}$. R. Harvey, J. T. Caldwell, R. I. Hramblett, S. C. Fultz; Phys. Rev. 136, ( 1964 )

$64 \mathrm{Ha} 3$ S. S. Hanna, R. E. Segel, R. G. Allas; PICNS-64 1022 ( 1964 )

64 Ho 1 M. Hors, H. Nguyen Ngoc, J. Perez y Jorba; Phys. Letters 9, $40(1964)$

64 Im 1 W. I. Imhof, F, J. Vaughn, L. F. Chase, Jr., H. A. Grench, M. Walt; Nuclear Phys. 59, 81 (1964)

64 Is 1 B. S. I shkanov, I. M. Kapitonov, V, G. Shevchenko, B. A. Yur ev; Phys. Letters 9, 162 ( 1964 )

64 Is2 B. S. Ishkhanov, I . M. Kapitonov, E. N Kornienko, V. G. Shevchenko, B. A. Yur ev; Zhur. Eksp. i Teoret. Fiz. 46, 1486 ( 1964 ); Soviet Phys: JETP 19, 1003 (1964)

64 Is3 B. S. Ishkhanov, I. M. Kapitonov, B. A. Yur ev, V. G. Shevchenko; PICNS-64 io42 (1964)

64 Jo1 A. Johansson; Phys. Rev.136, B1030 (1964)

$64 \mathrm{Ki} 1 \mathrm{M}$. Kihara, K. Baba, K. Miyake, T. Nakamura, T. Yamaki, Y. Yoshimura; J. Phys. Soc. Japan 19, 2236 ( 1964 )

64 Ko 1 R. Kosiek, K. Maier, K. Schlupmann; Phys. Letters 9,260 ( 1964$)$

$64 \mathrm{Ko} 2 \mathrm{R}$. Kosiek, K. Schlupmann, H. W. Siebert, $R$. Wendling;

Z. Physik 179, 9 ( 1964 )

$64 \mathrm{Ko} 3 \mathrm{R}$. Kosiek; Z. Physik 179, 544 ( 1964 )

$64 \mathrm{Ko} 4 \mathrm{~A}$. P. Komar, E. D. Makhnovskii ; Dokl. Akad. Nauk SSSR 156, 774 ( 1964 ); Soviet Phys. Dokl.9, 463 ( 1964 )

64 Ko5 A. P. Komar, Yu. D. Makhnovsky; Nucl. Phys. 65, 662 ( 1964 )

$64 \mathrm{La} 1 \mathrm{M}$. Langevin, J. M. Loiseaux, J. M. Maison; Nuclear Phys. 54, $114(1964)$

64 Ia2 J. D. Larson, R. H. Spear; Nuclear Phys. 56, 497 ( 1964 )

$64 \mathrm{La} 3$ H. Langhoff; Phys. Rev . 135, B 1 (1964)

64 Ia 4 H. Langhoff; Phys. Rev. 136, ( 1964 )

64 La5 M. Langevin, J . M. Loiseaux, J. M. Maison; PICNS-64 1045 (1964)

$64 \mathrm{Ii} 1$ F. F. Liu; Phys. Letters 11, 306 ( 1964 ) 
64 Lol R. Lombard, P. Kossanyi Demay, G. R. Bishop; Nuclear Phys. 59, 398 ( 1964$)$

64 Lo2 K. H. Lokan, G. R. Hogg, P. H. Cannington, R. J . Stewart; Phys. Letters 11, 73 (1964)

64 Lo3 J.M. Lolseaux, M. Langevin, J. M. Maison; PICNS-64 $1048(1964)$

$64 \mathrm{Mal}$ S. Matsumoto, H. Yamashita, T. Kamae, Y. Nogami;

Phys. Letters 12, 49 ( 1964)

$64 \mathrm{Ma} 2 \mathrm{M}$. Masuda, M. Kondo, S. Takeda, M. Okumura, J.

Ookuma; J. Phys. Soc. Japan 19, 2339 (1964)

$64 \mathrm{Ma} 3 \mathrm{E}$. D. Makhnovskii; J.Expt. Teoret.Phys. (USSR) 461136 (1964); Soviet Phys. JETP 19,769 (1964)

64 Ma 4 Akira Masaike; J. Phys. Soc. Japan 19, 427 (1964)

$64 \mathrm{Me} 1 \mathrm{~F}$. R. Metzger; Phys. Rev. 136, B374 (1964)

$64 \mathrm{Me} 2 \mathrm{~F} . \mathrm{R}$. Metzger; PICNS-64 392 ( 1964)

64Mil I. V. Mitchell, T. R. Ophel; Nuclear Phys. 58, 529 ( 1964 ) 64 Mul S. F. Mughabghab, W. E. Stephens; Phys. Rev. 133, B660

64Ne1 B. M. K. Nefkens, G. Moscati; Phys. Rev. 133, B17 (1964)

$64 \mathrm{Pal}$ J. C. Palathingal; Phys. Rev. 136, B1553 (1964)

64 Ph1 W. R. Phillips; Nuclear Phys.60,544 ( 1964 )

64 Ra 1 F. Rauch, E. Rossle; Phys, Letters 12, 217 (1964)

64 Ra2 B. S. Ratner; Zhur. Eksp. i Teoret. Fiz. 46, 1480 ( 1964 ); Soviet Phys. JETP 19, 1000 ( 1964 )

64 Ra3 B. S. Ratner; Zhur. Eksp. i Teoret. Fiz. 46, 1157 (1964); Soviet Phys. JETP 19,783 (1964)

$64 \operatorname{Re} 1 \mathrm{~T}, \mathrm{R}$. Retz-Schmidt, S. J . Skorka, J . Morgenstern, PICNS-64 396 (1964)

64R11 L. B. Rice, L. N. Bolen, W. D. Whitehead; Phys. Rev. $134, B 557$ ( 1964 )

64Sc1 J. A. Scheer, K. Schlupmann, F. Triantafyllidis; Nuclear Phys. 56, $113(1964)$

64 Sc2 D. J. Schlueter, R. W. Krone, F. W. Prosser, Jr.; Nuclear Phys. 58, 254 ( 1964$)$

$64 \mathrm{Sc} 3 \mathrm{~K}$. Schlupmann, R. Wendling; Naturwissenschaften 16,380 (1964)

64Sel S. Seki, M. Yamanouchi, M. Miwa; J. Phys. Soc. Japan 19,1999 ( 1964 )

64 Sh 1 N. K. Sherman, P. Barreau; Phys. Letters 9, 151 ( 1964 )

$64 \mathrm{Sh} 2 \mathrm{~A}$. Kh. Shardanov, V. G. Shevchenko, B. A. Yur ev; Izv. Akad. Nauk SSSR 28,60 (1964)

64 Sh3 J. Shannon, W. E. Stephens, J. S. O Connel L; Phys. Rev. 134, B $113(1964)$

64Sh4 Y. M. Shin, W. E. Stephens; Phys. Rev. 136, 660 (1964)

64 Sh5 Yu. K. Shubnyi, D. K. Kaipov, R. B. Begzhanov; Zhur.

Eksp. i Teoret. Fiz, 47, 16 (1964); Soviet Phys. JETP 20, $11(1965)$

64 Sh6 N. K. Sherman; PICNS-641051 ( 1964 )

64 Sil L. Simons, E. Spring, L. Kald, H. Jungner, P. Holmberg, I. Forsblom; Acta Polytech. Scand., Phys. Nucl. Ser., No. 29, $1-19(1964)$

$64 \operatorname{Sin} 1$ P. J. M. Smulders; Physica 30, 1197 ( 1964 ) 
64 Sul M, Suffert, G. Costa, D. Magnac-Valette; PICNS-64 1054 (1964)

64Sw1 C. P. Swann; Phys. Rev. 136, B1355 ( 1964) 64 Tal N. W. Tanner, G. C. Thomas, E. D. Earle; Nuclear Phys.

64 Ta2 N. W. Tanner, G. C. Thomas, E. D. Earle; Nuclear Phys. $52,45(1964)$

$64 \mathrm{Ta} 3 \mathrm{G}$. G Taran, A N. Gorbunov; Zhur. Eksp. i Teoret. Fiz. 46, 1492 (1964); Soviet Phys. JETP 19, 1010 (1964)

64 Ta 4 N. Tanner, E. D. Earle; Phys. Rev. Letters 13,410 (1964)

64 Tel G. Tessler, W. E. Stephens; Phys. Rev. 135, B129 ( 1964)

64 Th1 M. N. Thompson, J. M. Taylor, B. M. Spicer; PICNS-64 1008 ( 1964$)$

64To1 M. E. Toms; Nuclear Phys.50, 561 (1964)

64To2 M. E. Toms; Nuclear Phys. 54, 625 ( 1964 )

64 To3 M. E. Toms; PICNS-641004 (1964)

64 Tul P. M. Tutakin; Zhur. Eksp. i Teoret. Fiz, 46, 10 ( 1964); Soviet Phys. JETP 19,7 ( 1964$)$

64 UL 1 H. ULlrich; Phys. Letters 12, 114 (1964)

64 Ul2 H. ULZrich; Z. Physik 177, 514 (1964)

$64 \mathrm{UL3}$ H. Ullrich; PICNS-641038 (1964)

64 Val A. K. Val ter, E. G. Kopanets, A. N. L vov, S. P Tsytko; Izv. Akad. Nauk. SSSR, Ser. Fiz, 28, 271 ( 1964 )

$64 \mathrm{Va} 2 \mathrm{~A}$. R. Val ter, E. G. Kopanets, A. N. L vov, S. P. Tsytko; Izv. Akad. Nauk. SSSR, Ser. Fiz, 28, 268 (1964)

64 hal G. Walter, A. Coche; Compt. Rend. Acad. Sci. Paris 259, $2817(1964)$

64 the $1 \mathrm{~J}$. A. Weinman, L. Meyer-Schutzmeister, L. L. Lee, Jr.; Phys. Rev.133, B59C (1964)

64 Ye 1 P. F. Yergen, R. H. Augustson, N. N. Kaushal, H. A. Medicus, W. R. Moyer, E. J. Winhold; Phys. Rev. Letters $12,733$ ( 1964$)$

64 Ye2 M. R. Yearian, E. B. Hughes; Phys. Letters 10, 234 ( 1964 )

65 Ajl 1 . Ajdacic, M. Cerineo, B. Lalovic, G. Paic, I. Slaus, P. Tomas; Phys. Rev. Letters 14,444 (1965)

65 Ak 1 A. F. Akkerman, V L . Kochetkov, V. N. Chekanov; J . Nucl: Phys. (USSR) 1; 738(1965); Sov. J. Nucl. Phys. 1, 527 (1965)

65 Al 1 E. Albertsson, B. Forkman; Nucl. Phys. 70,209 ( 1965 )

65 Am 1 E. Ambler, E. G.Fuller, H. Marshak; Phys. Rev. 138, Bi 17 ( 1965 )

65 Am2 U. Amaldi, Jr., G. Campos Venuti, G. Cortellessa, G. Fronterotta, $A$. Reale, P. Salvadori; Rend. Acc. Naz. Lincei 39, $470(1965)$

65Am3 U. Amaldi, Jr., G. Campos Venuti, G. Cortellessa, G. Fronterotta, A. Reale, P. Salvadori; Rend. Acc. Naz. Lincei $38,499(1965)$

55Ar 1 P. E. Argan, G. Mantovani, A. Piazzoli; Nuovo Cimento 37 376 ( 1965$)$

65Ar2 P. E. Argan, L. Meneghetti, S. Vitale; Nuovo Cimento 38, $1489(1965)$

65 Ar3 H. Artus; Z. Naturforschg. 20 A, 320 (1965) 
65As1 J.G. Asbury, F.J.Loeffler; Phys.Rev.137, B1214 (1965)

65Bal E. B. Bazhanov, A. P. Komar, A. V. Kulikov, V. I.

Ogurtzov; Phys. Letters 19, 239 ( 1965 )

65 Ba2 E. B. Bazhanov, A. P. Komar, A. V. Kulikov, E. D. Makhnovsky; Nucl. Phys. 68, 191 (1965)

$65 \mathrm{Ea3}$ G. Baciu, G. C. Bonazzola, B. Minetti, C. Molino, L. Pasqualini, G. Piragino; Nucl. Phys.67, 178 (1965)

65 Ba4 W. C. Barber, G. J. Vanpraet; Nucl. Phys.72, 63 ( 1965 )

65Be1 B. L. Berman, R. L. Bramblett, J, T. Caldwell, R. R. Harvey, S. C. Fultz; Phys. Rev. Letters 15, 727 ( 1965 )

65 Be 2 W. Bertozzi, P. T. Demos, S. Kowalski, F. R. Paolini, C. P. Sargent, W. Turchinetz; Nucl. Instr. and Methods 33 , $199(1965)$

65Bi 1 P. G. Bizzeti, A. M. Bizzeti Sona, M. Bocciolini, G. Di Caporiacco, T. Fazzini, M. Mando; Nucl. Phys.63, 161 $(1965)$

65Bo 1 P. Bounin, M. Croissiaux; Nucl. Phys. 70,401 ( 1965 )

65 Bo2 R. Bosch, J. Lang, R. Muller, W. Wolfli; Helv. Phys. Acta $38,753$ ( 1965$)$

65Bo3 Paul Bounin; Ann. Phys. 10,475 (1965)

65 Bu 1 H. v. Buttlar, A. Goldmann; Z. Physik 187, 355 (1965)

65 Cal J. T. Caldwell, R. L. Bramblett, B. L. Berman, R. R. Harvey, S. C. Fultz; Phys. Rev. Letters 15, 976 (1965)

$65 \mathrm{Ca} 2$ P. H. Cannington, R. J. J. Stewart, G. R . Hogg, K. H. Lokan, D. G. Sargood; Nuci. Phys.72, 23 ( 1965 )

$65 \mathrm{Ca} 3 \mathrm{~F}$. Carbonara, H. G. DeCarvalho, R. Rinzivillo, E. Sassi, G. P. Murtas; Nucl. Phys.73, 385 (1965)

$65 \mathrm{Ch} 1$ B. T. Chertok, E. C. Booth; Nucl. Phys. 66, 230 ( 1965 )

$65 C l 1$ H. G. Clerc, R. J. Stewart, R. C. Morrison; Phys. Letters $18,316(1965)$

65Co1 S. Costa, F. Ferrero, S. Ferroni, L. Pasqualini, E. Silva; Nucl. Phys. 72, 158 ( 1965$)$

$65 \mathrm{Co} 2 \mathrm{~S}$. Costa, B. Minetti, C. Molino, L. Pasqualini, G. Piragino; Atti Cl. di Sci. Fis.99, 1157 (1965)

65 Co3 S. Costa, F. Ferrero; Mat. Nat.99, 85 ( 1965)

$65 \mathrm{Cr} 1 \mathrm{M}$. Croissiaux, R. Hofstadter, A. E. Walker, M. R. Yearian, D. G. Ravenhall, B. C. Clark, R. Herman; Phys. Rev. 137, B865 (1965)

65Dal P. J. Dallimore, K. S. Lam, H. H. Thies; Aust. J. Phys. $18,389(1965)$

65 De 1 G. Dearnaley, D. S. Gemmell, B. W. Hooton, G. A. Jones; Nucl. Phys.64, $177(1965)$

65De2 R. W. Detenbeck, J C.Armstrong, A. S. Figuera, J. B. Marion; Nucl. Phys: 72,552 ( 1965 )

65 Do1 B. S. Dolbilkin, V. I. Korin, L. E. Lazareva, F. A. Nikolaev; Phys. Letters 17,49 ( 1965 )

65 Do2 B. S. Dolbilkin, V. I. Korin, L. E. Lazareva, F. A. Nikolaev, V. A. Zapevalov; Nucl. Phys.72, 137 (1965)

$65 \mathrm{Do} 3 \mathrm{~B}$. S. Dolbilkin, V.I. Korin, L. E. Lazareva, F. A. Nikolaev; ZnETF Pisma 1, (1965); JETP Letters 1,148 $(1965)$ 
65 Dul I. I. Dushkov, B. S. Ishkhanov, I. M. Kapitonov, V. G. Shevchenko, B. A. Yur ev; Izv. Akad. Nauk Fiz. 29, 213 ( 1965 ); Bull. Acad. Sci. USSR-Phys.29, 213 ( 1965 )

55 Ehl D. Ehhalt, R. Kosiek, R. Pfeiffer; Z. Physik 187,210 (1965)

65 Fel V. N. Fetisov, A. N, Gorbunov, A. T. Varfolomeev; Nucl. Phys. $71,305^{\circ}(1965)$

65 Fil D. S. Fielder, J. Le Tourneux, K. Min, W. D. Whitehead; Phys. Rev. Letters 15, 33 (1965)

65 Frl R. Frosch, R. E. Rand, M. R. Yearian, H. L. Crannell, L. R. Suelzle; Phys. Letters 19, 155 (1965)

65Fr2 G. Fricke, G. R. Bishop, D. B. Isabelle; Nucl. Phys. 67 , $187(1965)$

65Gal J. Garvey, B. H. Patrick, J. G. Rutherglen, I. L. Smith; Nucl. Phys.70, 241 ( 1965 )

65Gil M. Giannini, P. Oliva, D. Prosperi, S. Sciuti; Nucl. Phys. $65,344(1965)$

65Go1 B. I. Goryachev, B. S. Ishkhanov, I . M. Kapitonov, V. G. Shevchenko, B. A. Yur ev; J. Nucl. Phys. (USSR) 1, 1005 (1965) ; Sov.J. Nucl. Phys. 1,716 (1965)

65 Gr1 I. A. Grishaev, D. I . Sikora, V. A. Shkoda-Ul yanov, B. I. Shramenko; Atomnaya Energiya 18, 28 (1965); Soviet Atomic Energy 18,29 ( 1965 )

65Hal E. Hayward, T. Stovall; Nucl. Phys. 69, $241 \cdot(1965$ )

$65 \mathrm{Ha} 2 \mathrm{~F}$. I. Havlicek, M. Modesto; Energia Nucleare 12,77 (1965)

65 He1 D. F. Herring, I. C. Nascimento, R. B. Walton, R. E. Sund; Phys. Rev. 139, B562 (1965)

65 Ho 1 G. R. Hogg; Nucl. Phys.72, 167 (1965)

65 Ho2 M. Holmberg, H. Conde; Arkiv Fysik 29, 301 ( 1965 )

65 Is 1 B. S. Ishkhanov, I. M. Kapitonov, E. N. Kornienko, V. G. Shevchenko, B. A. Yur ev; Izv. Akad. Nauk Fiz. 29, 221 ( 1965 ); Bull. Acad. Sci.USSR-Phys.29, 221 ( 1965 )

$65 \mathrm{Je} 1 \mathrm{R}$. W. Jewe Ll, W. John, J. E. Sherwood, D. H. White; Phys. Rev. 139, B71 ( 1965 )

$65 \mathrm{Ke} 1 \mathrm{E} . \mathrm{F}$. Kennedy, W. C. Miller; Nucl. Phys.63,634 (1965)

$65 \mathrm{Kh} 1$ N. A. Khan, V. K. Rasmussen; Phys. Rev. 138, B1385 ( 1965 )

$65 \mathrm{kil}$ T. Kivikas, B. Forkman; Nucl. Phys. 64, 420 ( 1965 )

$65 \mathrm{Ki} 2$ Motohiro Kihara; J. Phys. Soc. Japan 20, 1313 ( 1965 )

65 Ko 1 P. Kossanyi-Demay, R. M. Lombard, G. R. Bishop; Nucl. Phys. 62, $615(1965)$

$65 \mathrm{Ko} 2$ A. P. Komar, E. D. Makhnovskii; Dokl. Akad. Nauk SSSR 160,1300 (1965); Sov. Phys. Dokl. io, 150 (1965)

$65 \mathrm{Kr} 1 \mathrm{P}$. Kruger, T. M C Crawford, J Goldemberg, W. C. Barber; Nucl. Phys. 62, 584 ( 1965 )

65Ia1 H. LangevinfJoliot, C. Stephan, G. Johnson, J.Vernotte; Phys. Letters 14,208 ( 1965 )

65 Li 1 F. F. Liu; Phys. Rev.138, B1443 (1965)

$65 \mathrm{Ma} 1 \mathrm{~J}$. M. Maison, M. Langevin, J.M. Loiseaux; Phys. Letters $19.308(1965)$ 
$65 \mathrm{Ma3}$ A. Manfredini, M. Muchnik, L. Fiore, C. Ramorino, H. G. De Carvalho, J. Lang, R. Mulier; Nucl. Phys. 74,377 ( 1965$)$

$65 \mathrm{Ma} 4 \mathrm{G}$ Manuzio, G. Ricco, M. Sanzone; Nuovo Cimento 39 , $1057(1965)$

65 Ma5 G. Manuzio, R. Malvano, G. Ricco, M. Sanzone; Nuovo Cimento $40 \mathrm{~B}, 300$ ( 1965$)$

65 Ma6 S. Matsumoto, H. Yamashita, T. Kamae, Y. Nogami; J. Phys. Soc. Japan 20, 1321 ( 1965 )

65 Mc 1 J. A. McIntyre, J. D. Randall; Phys. Letters 17,137 ( 1965 )

$65 \mathrm{Mc} 2$ V. R. McMurray, I. J. Van Heerden, G. Wi echers; Nucl. Phys. 72, $113(1965)$

65Me1 F. R. Metzger; Phys. Rev. 137, B1415 ( 1965 )

65 Me2 L. Meneghetti, S. Vitale, Gruppo CNEN; Nucl. Phys. 61, $316(1965)$

65Ne3 F. R. Metzger; Phys. Rev.139, B1464 ( 1965 )

65 Mil K. Min, W. D. Whitehead; Phys. Rev. 137, B301 ( 1965 )

65Mol R. C. Morrison, J.R. Stewart, J. S. O Connell; Phys. Rev. Letters 15, 367 ( 1965 )

65 Mo2 J. Moffatt, D. Reitmann; Nucl. Phys.65, 130 ( 1965 )

65Ng1 H. Nguyen Ngoc; Ann. Phys. 10, 315 (1965)

65 Nil 1 . P. Nikotin, K. A. Petrzhak; Atom. Energ. 19, 185 ( 1965 ) Sov. At. Energy 19, 1080 ( 1965 )

65Fal P. Paul, S. L. Blatt, D. Kohler; Phys. Rev. 137 B493 ( 1965)

65 Pa2 A. F. Parker, R. R. Whitehead, G. G. Shute; Aust. J. Phys. 18, 15 ( 1965 )

$65 \operatorname{Re} 1$ M. A. Reimann, J.R. MacDonald, J. B. Warren; Nucl. Phys. 66,465 ( 1965 )

65 Ro 1 J. P. Roalsvig; Can. J. Phys.43, 330 ( 1965 )

65 Ro2 J. P. Roalsvig; Can.J.Phys.43, 1015 ( 1965 )

65Sa1 C. P. Sargent, W. Bertozzi, P. T. Demos, J. L. Matthews, W. Turchinetz; Phys. Rev. 137, B89 ( 1965 )

65 Sc 1 R. Schrack, D. Kohler, N, G. Puttasmamy, W. E. Meyerhof; Phys. Letters 18,327 (1965)

65Se1 R. E. Segel, S. S. Hanna, R. G. Allas; Phys. Rev. 139 B8 18 ( 1965$)$

65Sil P. P. Singh, R. E. Segel, L. Meyer-Schutzmeister, S. S. Hanna, R. G. Allas; Nucl. Phys. 65, 577 (1965)

65So1 A. S. Soldatov, Z. A. Aleksandrova, L. D. Gordeeva, G. N. Smirenkin; J. Nucl. Phys. (USSR) 1, 471 ( 1965 ); Sov. J. Nuc l. Phys. 1, 335 ( 1965 )

65502 A. S. Soldatov, G. N. Smirenkin, S. P. Kapitza, Y. M. Tsipeniuk; Phys. Letters 14, 217 ( 1965)

65Sp1 E. Spamer, F. Gudden; Phys. Letters 14, 210 ( 1965 )

65St1 J. R. Stewart, R. C. Morrison, J. S. O Connell; Phys. Rev. $138, B 372(1965)$

65 St2 R. J. J. Stewart, P. H. Cannington, B. M. Spicer; Aust. J. Phys. 18, 66i ( 1965 )

65 Sw1 C. P. Swann; Phys. Rev. 139, B271 ( 1965 )

65 Ta1 N. W. Tanner; Nucl. Phys.61, 297 ( 1965 )

65 Ih 1 M. N. Thompson, J.M. Taylor, D. V. Webb; Phys. Let ters 14, 223 ( 1965 ) 
65 Th2 M. N. Thompson, J. M. Taylor, B. M. Spicer, J. E. E. Baglin; Nucl. Phys. 64, 486 (1965)

65Val A. T. Varfoloweev, A. N. Gorbunov; Zhur. Eksp. i Teoret. Fiz. 47, 30 (1964); Soviet Phys.JETP 20, 20 ( 1965 )

65 Va2 G. J. Vanpraet; Phys. Letters 17, 120 (1965)

65 Va3 J.R. Van Hise, R. A. Meyer, J. P. Hummel; Phys. Rev. $139, \mathrm{B5} 54(1965)$

65 Va4 G.J.Vanpraet; Nucl. Phys.74, 219 ( 1965 )

65 Va5 A. K. Val ter, E. G. Kopanets, S. P. Tsytko; Izv. Akad. Nauk SSSR Ser. Fiz.29, 803 (1965) ; Bull. Acad. Sci. USSR ( Phys. Sci.) 29,806 (1965)

65 Ve1 V. V. Verbinski, J.C. Courtney; Nucl. Phys. 73 398 (1965)

65 hal A. Wataghin, M. Scotto, G. Paoli; Nuovo Cimento 40B, 441 ( 1965$)$

65 Wy 1 J. M. Wyckoff, B. Ziegler, H. W. Koch, R. Uhlig; Phys. Rev. 137, B576 (1965)

66 AC 1 J. C. A cquadro, R. $\theta$. Cesar, J . Goldemberg, E. W.

Hamburger; An. Acad. Brasil Cienc. 38, $257^{\circ}$ ( 1966 )

56 All E. Allton, S. Ferroni, V. G. Gracco, B. Merkel, C. Schaerf; Nuovo. Cimento $46 \mathrm{~A}, 407$ ( 1966 )

66Am1 U. Amaldi Jr., G. Campos Venuti, G. Cortellessa, E. De Sanctis, S. Frullani, R. Lombard, P. Salvadori; Phys. Letters 22,593 (1966)

66 Am2 U. Amaldi, Jr., G. Campos Venuti, G. Cortellessa, E. De Sanctis, S. Frullani, R. Lombard, P. Salvadori; LinceiRend. Sc. Fis. Mat. E Nat.41, 494 (1966)

66An1 D. W. Anderson, B. C. Cook, J. A. Kadlecek; Nucl. Phys. 83, $584(1966)$

66Ar1 H. Artus; Z. Physik 189, 355 ( 1966 )

66 Ar2 H. Artus, P. Brix, H. G. Clerc, F. Eigenbrod, A. Goldmann, F. Gudden, E. Spamer, P. Strehl, M. Stroetzel, o. Titze, K: J. Wetzel; PICNS-66, 314 (1967)

66 At 1 U. Atzmony, S. Ofer; Phys. Rev. 145, B915 (1966)

66 At 2 U. Atzmony, E. R. Bauminger, S. Ofer; Nucl. Phys. 89, $433(1966)$

66Axl P. Axel, J. Miller, C. Schuhl, G. Tamas, C. Tzara; J. Phys. et Rad.27, 262 (1966)

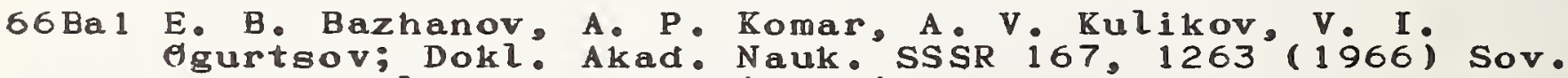
Phys. Dokl ady i1, 332 ( 1966 )

$66 \mathrm{Ba} 2 \mathrm{D}$. Bachelier, M. Bernas, I. Brissaud, C. Detraz, J.P. Didelez, H. Langevin-Joliot, J. Lee, P. Radvanyi; Phys. Letters 21, 697 (1966)

66Ha3 A. E. Balabanov, N. N. Delyagin, H. el Sayes; J. Nucl. Phys. (USSR) 3, 209 (i 966 ); Sov. J. Nucl. Phys. 3, 150 ( 1966 )

66Ba4 E. B. Bazhanov, A. P. Komar, A. V. Kulikov, V. I. Ogurtsov; J. Nucl. Phys.o(USSR) 3, 711 (1966); Sov. J. Nuc l. Phys. 3, 522 ( 1966 )

66Ba5 G. Baciu, D. Catana, C. Deberth, I. Raileanu; Rev. Roum. Phys. 11, 301 ( 1966$)$

66 Be1 I. Bergqvist, B. Lundberg, L. Nilsson, N. Starfelt; Phys. Letters 19,670 ( 1966$)$

66 He2 G. B. Beard; Phys. Rev. 145,862 ( 1966 ) 
$66 \mathrm{Be} 3 \mathrm{G}$. Ben-David, B. Arad, J. Balderman, Y. Schlesinger; Phys. Rev. 146, 852 ( 1966 )

66Be4 B. I. Berman, G. S. Sidhu, C. D. Bowman; Phys. Rev. Letters 17,761 ( 1966$)$

66 Bi 1 G. Bishop, S. Costa, S. Ferroni, R. Malvano, G. Ricco; Nuovo Cimento 42B, 158 ( 1966 )

66Bo 1 I. E. Bocharova, V. G. Zolotukhin, S. P. Kapitza, G. N. Smirenkin, A. S. Soldatov, Yu. M. Tsipenyuk; Zhur. Eksp. i Teoret. Fiz. 49, 476 (1965); Sov.Phys.JETP 22, 335 ( 1966 )

66Br1 R. L. Bramblett, J T. Caldwell, B. L. Berman, R. R. Harvey, S. C. Fultz; Phys. Rev.148, 1198 (1966)

$66 \mathrm{Ca} 1$ Y. Cauchois, Y. Heno, M. Boivin; C. R. Acad. Sci: Paris 262, B503 ( 1966 )

$66 \mathrm{Cl}_{1}$ H. G. Clerc, K. J. Wetzel, E. Spamer; Phys. Letters 20, 667 ( 1966$)$

66Co1 B. C. Cook, J. E. E. Baglin, J. N. Bradford, J. E. Griffin; Phys. Rev. 143, $712(1966)$

66 Co2 B. C. Cook, J. E. E. Baglin, J. N. Bradford, J. E. Griffin; Phys. Rev, 143,724 ( 1966 )

$66 \mathrm{Co} 3 \mathrm{~B}, \mathrm{C}$. Cook, D. R. Hutchinson, R. C. Waring, J N .

Bradford, R. G. Johnson, J. E. Griffin; Phys. Rev. 143, 730 ( 1966 )

66004 S. Costa, L. Pasqualini, G. Piragino, L. Roasio; Nuovo Cimento $42 \mathrm{~B}, 306$ (1966)

$66 \mathrm{Co5}$ S. Costa, F. Ferrero, C. Manfredotti, L. Pasqualini, L. Roasio; Nuovo Cimento $42 \mathrm{~B}, 382$ ( 1966 )

66Cr1 H. Crannell; Phys. Rev. 148, 1107 ( 1966)

66De1 N. De Botton, J. Miller, C. Schuhl, G. Tamas, C. Tzara; Nucl. Phys.75, 396 ( 1966$)$

66 De2 A. De Marco, R. Garfagnini, G. Piragino; Nuovo Cimento $44 \mathrm{~B}, 172(1966)$

$66 \mathrm{De} 3 \mathrm{~A}$. De Marco, R, Garfagnini, G. Piragino; Nuovo Cimento $42 \mathrm{~B}, 355(1966)$

66 De 4 V. P. Denisov, L. A. Kul chitskii; J. Nucl. Phys. (USSR) 2,70 (1965); Sov. J. Nucl. Phys.2, 48 (1966)

66De5 V. P. Denisov, A. Duisebaev, K, V. Kosareva, P. A. Cerenkov; J. Nuci. Phys. (USSR) 1; 82 (1965) Sov. J. Nucl. Phys. 2, 58 ( 1966$)$

66De6 V. P. Denisov, L. A. Kul chitskii; J. Nucl. Phys. (USSR) 3. 268 (1966); Sov. J. Nucl. Phys. 192 ( 1966)

66 Do 1 E. J. Dowdy, J. A. McIntyre; Phys. Rev. 145, 982 ( 1966 )

66 Do2 B. S. Dolbilkin, V. A. Zapevalov, V. I. Korin, L. E. Lazareva, F. A. Nikolaev; Izv. Akad. Nauk. Fiz. 30, 349 (1966); Buli. Acad. Sci. USSR-Phys.30, 354 ( 1966 )

$66 \mathrm{Du} 1 \mathrm{M}$. A. Duguay, C. K. Bockelman, T. H. Curtis, R. A. Eisenstein; Phys. Rev. Letters 17, 28 (1966)

66Du2 J. Dubois, S. Maripuu, đ. Almen; Ark. Fysik 31, 65 (1966)

66Ev1 A. E. Evans, B. Brown, J. B. Marion; Phys. Rev. 149, 863 (1966)

$66 \mathrm{Fe} 1 \mathrm{~F}$. Ferrero, C. Manfredotti, L. Pasqualini, G. Piragino, P. G. Rama; Nuovo Cimento 45B; 273 (1966)

66Fil F. W. K. Firk; Nucl. Instr. and Methods 43, 312 (1966)

$66 \mathrm{Fi} 2 \mathrm{~F}, \mathrm{~N}, \mathrm{~K}, \mathrm{Firk}$; PICNS-66,352 (1967) 
66Fol G. M. Fossa, G; Ricco. C. Manfredotti; Nuovo Cimento $43 \mathrm{~B}, 347(1966)$

66Fo2 P. D. Forsyth, H. T. Tu and W. F. Hornyak; Nucl. Phys. $82,33(1966)$

66 Frl L. Frevert, W. Schott, H. Langhoff, A. Flammersfeld; Z. Physik 194, 248 ( 1966 )

66Ful S. C.Fultz, J.T. Caldwell, B. L. Berman, R. L. Bramblett, R. R. Harvey; Phys. Rev. 143, 790 (1966)

66Fu2 S. C. Fultz, R. L. Bramblett, B. L. Berman, J. T. Caldwell, M. A. Kelly; PICNS-66, 397 ( 1967 )

66 Fu3 R. Fukuda, S. Okabe, Y. Sato; Ann. Rep. Radiat. Center Osaka Prefect. 7, 38 (1966)

$66 \mathrm{Ge} 1 \mathrm{H}$. M. Gerstenberg, J.S. O Connell; Phys. Rev. 144, 834 (1966)

66Go1 J. Goldemberg, C. Schaerf; Phys. Letters 20, 193 (1966)

66 Go2 A. Goldmann, P. Kneisel, H. v. Buttlar; Z. Physik 192, 282 (1966)

66Go3 B. I. Goryachev, B. S. Ishkhanov, V. G. Shevchenko, B. A. Yur ev; ZhETF Pisma 4, 491 (1966); JETP Letters 4 , $330(1966)$

66Gr1 R. C. Greenwood, W. W. Black; Phys. Letters 21,702 ( 1966$)$

66Gr2 B. Grossetete, S. Jullian, P. Lehmann; Phys. Rev. 141, $1435(1966)$

66Hol P. Holmberg; Comm. Phys. Math. Ed. Soc. Sci. Fennica $31,1(1966)$

66 Ho2 J. H. Hough, W. L. Mouton; Nucl. Phys. 76, 248 ( 1966 )

$66 \mathrm{Ho}$ H. Hoffmann, B. Prowe, H. Ullrich; Nucl. Phys. 85, 631 (1966)

66Hrl B. Hrastnik, V. Knapp, M.Vlatkovic; Nucl. Phys. 89, 412 ( 1966$)$

66 Hul E. B. Hughes, T. A Griffy, R. Hofstadter, M. R, Yearian; Phys. Rev. 146, 973 ( 1966 )

66Hu2 E. B. Hughes, M. R. Yearian, R. Hofstadter; Phys. Rev. $151,841(1966)$

66 Is 1 B. S. Ishkhanov, I. M. Kapitonov, V. G. Shevchenko, B. A. Yur ev; Izv. Akad. Nauk Fiz. 30, 378 (1966); Bull. Acad. Sci . USSR-Phys. 30,383 ( 1966 )

66 Is2 B. S. Ishkhanov, I. M. Kapitonov, Zh. M. Seliverstova, V. G. Shevchenko, B. A. Yur ev; Izv. Akad. Nauk. SSR Fiz. 30, $1385(1966)$; Bull. Acad. Sci. USSR 30, 1446 (1966)

66 Iv 1 V. G. Ivanchenko, B. S. Ratner; ZhETF Pisina 3, 452 (1966); JETP Letters 3,296 (1966)

$66 \mathrm{Kel}$ E. M. Kellogg, W. E. Stephens; Phys. Rev. 149,798 (1966)

66 Ko 1 P. Kossanyi-Demay, G. J. Vanpraet; Nucl. Phys. 81, 529 (1966)

66 Ko2 R. Kosiek, D. Muller, R. Pfeiffer, O. Merwitz; Phys. Letters 21, 199 ( 1966$)$

$66 \mathrm{Ko} 3 \mathrm{M}$. Ya. Kondrat ko, K. A. Petrzhak; Atomnaya Energija 20, 514 ( 1966 ); Atomic Energy 20,594 (1966)

$66 \mathrm{Ro} 4$ A. A. Koval, E. G. Kopanets, Yu. S. Korda, L. N. Sukhotin, S. P. Tsytko; Izv. Akad. Nauk Fiz. 30,416 ( 1966 ); Bull. Acad. Sci. USSR Phys. 30, 423 (1966)

66 Lal H. Langhoff, L. Frevert, W. Schott, A. Flammersfeld; Nucl. Phys.79, 145 (1966) 
66Le1 H. P. Leenhouts, P. M. Endt; Physica 32, 322 ( 1966 )

66Iil H. Lichtblau, B. M. Spicer; Aust. J. Phys. 19, 297 (1966)

66 Ii2 H. Liesem; Z. Physik 196, 174 ( 1966 )

66 Lo1 W. A. Lochstet, W. E. Stephens; Phys. Rev. 1411002 ( 1966 )

66 Mal G. E. Manuzio, G. Ricco, M. Sanzone; Nuovo Cimento $42 \mathrm{~B}, 348(1966)$

$66 \mathrm{Ma} 2 \mathrm{~J} \cdot \mathrm{M}$. Maison, M. Langevin, J. M. Loiseaux; J . Physique Colloq. 1,36 ( 1966$)$

$66 \mathrm{Ma3}$ A. Manfredini, M. Muchnik, L, Fiore, C. Ramorino, H. G. De Carvalho, R. Bosch, W. Wolfli; Nuovo Cimento $44 \mathrm{~B}$, 218 (1966)

$66 \mathrm{Ma} 4 \mathrm{~J}$. L. Matthews, W. Bertozzi, S. Kowalski, C. P. Sargent, W. Turchinetz; PICNS-66, 386 ( 1967 )

66Me1 F. R. Metzger, R. S. Raghavan; Phys. Rev 1 145, 968 ( 1966 )

66 Me2 J. L. Meason, P. K. Kuroda; Phys. Rev. 142, 691 (1966)

66 Me 3 F. R. Netzger, G. K. Tandon; Phys. Rev. 148, 1133 ( 1966 )

66Mil K. Min; Phys. Rev. 152, 1062 (1966) $66 \mathrm{Mi} 2 \mathrm{~J}, \mathrm{Mill}$ er, C. Schuhl, G. Tamas, C. Tzara; J. de Physique

$66 \mathrm{Ne} 1 \mathrm{~B} . \mathrm{M}$. K. Nefkens, D. C. Sutton, M. N. Thompson; Nucl. Phys. 88, $523(1966)$

66 Ni 19 . P. Nikotin and K. A. Petrzhak; Atom. Energiya 20, 268 (1966)

$66 \mathrm{Nul} \mathrm{F}$. Nusslin, H. Werner, J. Zimmerer; Z Naturforschg. $21 \mathrm{~A}, 1195$ ( 1966$)$

$560 b 1$ E. Obst, F. Rauch, E. Rossle; Phys. Letters 21,50 (1966) 66 ew 1 R. O. Owens, J. E. E. Baglin; Phys. Rev. Letters 17,1268 (1966)

660 w2 R. O. Owens, J. E. E. Baglin; PICNS-66, 337 ( 1967 )

$66 \mathrm{~Pa} 1 \mathrm{G}$. Paoli, M. Scotto, A. Wataghin; Nuovo Cimento $43 \mathrm{~B}$, $189(1966)$

66 Pa2 V. Parikh; Nucl. Phys. 77, 157 (1966)

$66 \mathrm{~Pa} 3$ A. . Parker, R. R. Whitehead, G. G. Shute; Aust. J. Phys: $19,147^{\prime}(1966)$

66 Pa 4 P. D. Parker; Phys. Rev. 150, 851 ( 1966 )

$66 \mathrm{Pe} 1$ G. A. Peterson, J. F. Ziegler; Phys. Letters 21, 543 (1966)

66 Prl G. Proca, D. Isabelle, J Goldemberg; J. Physique Colloq. 1. 105 (1966)

66 Pu1 N. G. Puttaswamy, D. Kohler; Phys. Letters 20, 288 (1966)

66 Ka 1 R. S. Raghavan; Phys. Rev. 143, B947 (1966)

66 Ra2 N. S. Rabotnov, G. N. Smirenkin, A. S. Soldatov, L. N. Usachov, S. P. Kapitza, Yu. M. Tsipenyuk; Nucl. Phys. $77,92$ ( 1966$)$

66Ril R. Riskalla, D. Vinciguerra; J. Physique Colloq.1, 106 (1966)

66Sa 1 K. Sakamtoto, P. J. Kuroda; J. Inorg. Nucl. Chem. 28, 679 ( 1966$)$

66Sc1 P. J. Scanlon; Nucl. Phys. 88, 424 (1966)

66 Sh 1 N. K. Sherman, J R. Stewart, R. C. Morrison; Phys. Rev. Letters 17,31 ( 1966 ) 
66Sh2 N. Shikazono, H. Takekoshi, T. Shoji; J. Phys. Soc. Japan $21,829(1966)$

66 Spl E. Spamer; Z. Physik, 191, 24(1966)

66 St1 R. R. Stevens, Jr., Y. K. Lee, J. C. Walker; Phys. Letters $21,401$ ( 1966$)$

66st2 M. Stroetzel, F. Gudden; Phys. Letters 22, 485 (1966)

66Sul M. Suffert; Nucl. Phys.75, 226 (1966)

66 Sw1 C. P. Swann; Phys. Rev. 148, 1119 (1966)

$66 \mathrm{Ta} 1 \mathrm{~J}, \mathrm{R}$. Tatarczuk, H. A. Medicus; Phys. Rev. 143, B818 ( 1966 )

66Th1 M. N. Thompson, J. M. Taylor; Nucl. Phys.76, 377 (1966)

66 Ii 1 O. Titze, E. Spamer; Z. Naturforschg. $21 \mathrm{~A}, 1504$ ( 1966 )

66 Val G. J. Vanpraet, W. C. Barber; Nucl. Phys.79, 550 (1966)

66 Va2 H. A. Van Rinsvelt, P. M. Endt; Physica 32, 513 ( 1966)

$66 \mathrm{Va} 3 \mathrm{~A}$. T. Varfolomeev, A. N. Gorbunov, G. G. Taran; J. Nucl. Phys. (USSR) 3,647 (1966); Sov. J.Nucl. Phys. 3, 473 (1966)

66 Va4 C. Van der Leun, N. C. Burhoven Jaspers; Nucl. Phys. 88,235 (1966)

66 Vel A. Veres, I. Favlicsek; Int. J. App. Rad. and Isotopes 17, $69(1966)$

66 Vo 1 Yu. M. Volkov, A. P. Komar, V. P. Chizhov; J Nucl. Phys. (USSR) 3, $277(1966)^{\circ}$; Sov. J. Nucl. Phys. 3, 198 ( 1966$)$

66 Vul P. H. Vuister; Nucl. Phys. 83, 593 (1966)

66 Hal W. B. Walters, J. P. Hummel; Phys. Rev. 150, 867 ( 1966)

66 Ma2 o. A. Wasson, M. R. Bhat, R. E. Chrien, M. A. Lone, M. Beer; Phys. Rev. Letters 17, 1220 ( 1966 )

66 Wel E. Wernbom-Selin, S. E. Arnell; Ark. Fysik 31,113 ( 1966$)$

66 He2 R. Wendling, R. Kosiek; Z. Physik 192, 502 (1966)

66 Wil Bjorn Wiik; Z. Physik 189,423 (1966)

66 Wo1 W. Wolfli, R. Bosch, J. Lang, R. Muller, P. Marmier; Phys. Letters 22, 75 (1966)

66 Yo 1 D. H. Youngblood, G. C. Morrison, R. E. Segel; Phys. Letters 22, 625 (1966)

$66 Z 11$ B. Ziegler, W. Buss, H. Waffler; Nucl. Phys. 83, 145 (1966)

66 Zi2 J.F. Ziegler, G. A. Peterson; PICNS-66, 319 (1967)

67 Af 1 N. G. Afanas ev, V. A. Gol dshtein, S. V. Dementii, V. G. Vlasenko, I. M. Arkatov, Yu. I Titov; J. Nucl Phys. (USSR) 5, 1134 (1967); Sov. J.Nucl. Phys. 5, 808(1967)

67 Am 1 U. Amaldi Jr., G. Campos Venuti, G. Cortellessa, E. De Sanctis, S. Frullani, R. Lombard, P. Salvadori; Phys. Letters 25B, 24 (1967)

67 An 1 Yu. P. Antuf ev, I. I. Miroshnichenko, V. I. Noga, P. V. Sorokin; Yad. Fiz.6, 431 (1967); Sov, J: Nucl.Phys.6, 312 (1968)

67 An2 G. P. Antropov, I E. Mitrofanov, V. S. Russkikh; Izv. Akad. Nauk SSR Fiz, 31, 336 (1967); Bull. Acad. Sci. USSR 31,320 (1967)

$67 \wedge \times 1$ P. Axel, D. M. Drake, S. Whetstone, S. S. Hanna; Phys. Rev. Letters 19, 1343 (1967) 
$67 \mathrm{Bal}$ G. Barbiellini, C. Bernardini, F. Felicetti, G. P. Murtas; Phys. Rev. 154,988 (1967)

$67 \mathrm{Ba}$ E. B. Bazhanov, A. P. Komar, A. V. Kulikov; Dokl. Akad. Nauk. SSSR 171, 549 (1966); Sov. Phys. Doklady 11, 953 ( 1967 )

$67 \mathrm{Ba} 3$ G. Baciu, D. Catana, C. Deberth, I. Raileanu; Rev. Roum. Phys, 12, 385 ( 1967 )

$67 \mathrm{Ba} 4$ P. Barreau, J. B. Bellicard; Phys, Rev. Letters 19, 1444 ( 1967 )

67 Ha5 J. E. E. Baglin, M. N. Thompson; PICNS-67, 388 ( 1967 )

67Bel M. Bernheim, T. Stovall, D. Vinciguerra; Nucl. Phys. A97, $488(1967)$

67 Be2 B. L. Berman, J. T. Caldwell, R. R. Harvey, M. A. Kelly, R. L. Bramblett, S. C. Fultz; Phys. Rev. 162, 1098 (1967)

67 Be 3 M. Bernheim, T. Stovall, D. Vinciguerra; Phys. Letters $25 B, 461(1967)$

67 Be4 R. B. Begzhanov, A. A. Islamov, S. V. Starodubtsev; J. Nucl. Phys. (USSR) 5, 250 ( 1967 ); Sov. J. Nucl. Phys.5, $176(1967)$

67 Be5 R. B. Begzhanov, A. A. Islamov; J. Nucl. Phys. (USSR) 5 , 483 ( 1967 );Sov. J. Nucl. Phys.5, 339 (1967)

67 He6 B. L. Berman, R. L. Van Hemert, C. D. Bowman; Phys. Rev. 163, 958 (1967)

67He7 I. Bergquist, J. A. Biggerstaff, J. H. Gibbons, W. M. Good; Phys. Rev. 158, 1049 (1967)

67 Be 8 H. Bernatowicz, H. A. Medicus; PICNS-67, 384 ( 1967 )

$67 B l 1$ J. L. Black, W. J. O Connell, S. S. Hanna, G. L. Latshaw; Phys. Letters 25B, 405 (1967)

67 Bl2 J. L. Black, F. Riess, P. Paul, W. J. O Connell, G. A. Fisher, S. S. Hanna; PICNS-67, 375 (1967)

67Bol C. H. Bornman, M. A. Meyer, D. Reitmann; Nucl. Phys. A $99,337(1967)$

67 Bo2 C. D. Bowman, G. S. Sidhu, B. L. Berman; Phys. Rev. $163,951(1967)$

67 Bul H. v. Buttlar, F. Freund, G. Gammel; Z. Physik 200, 1 ( 1967 )

$67 \mathrm{Ca} 1 \mathrm{P}, \mathrm{H}$, Cannington, D. G. Owen, R. J . J . Stewart, E. G. Muirhead, B. M. Spicer; PICNS-67, 379 ( 1967 )

$67 \mathrm{Ca} 2 \mathrm{~J} . \mathrm{T}$. Caldwell, Ph.D. thesis, University of California, 1967 ; UCRL -50287 .

67Ch1 C. Chasman, K. W. Jones, R. A. Ristinen, J. T. Sample; Phys. Rev. Letters 18, 219 (1967)

67col S. Costa, F. Ferrero, C. Manfredotti, L. Pasqualini, G. Piragino, H. Arenhovel; Nuovo Cimento $48 \mathrm{~B}, 460$ (1967)

67 Co2 S. Costa, F. Ferrero, C. Manfredotti, L. Pasqualini, G. Piragino, H. Arenhovel; Nuovo Cimento $51 \mathrm{~B}, 199$ (1967)

67Cr1 H. Crannell, T. A. Griffy, L. R. Suelzle, M. R. Yearian; Nuc l. Phys. A90, 152 (1967)

67 Cr2 H. Crannell, H. A. Dahl, F. H. Lewis, Jr.; Phys. Rev. 155, $1062(1967)$

67Del V. P. Denisov, L. A. Kul chitskii; J Nucl. Phys. (USSR) 5, 490 (1967); Sov. J. Nucl. Phys. 5, 344 ( 1967 ) 
67 De2 V. P. Denisov, A. P. Komar, L. A. Kul chitskii, E. D. Makhnovskii; J. Nucl. Phys. (USSR) 5, 498 (1967) Sov. J. Nuc 2. Phys. 5, 349 (1967)

67 De3 V. P. Denisov, L. A. Kul chitskii; J. Nucl. Phys. (USSR) 6, 437 (1967); Sov. J. Nucl. Phys. 6, 318 (1968)

67 De4 F. P. Denisov, A. Duisehaev, P. A. Cerenkov; ZhETF Pisma 5, 249(1967); JETP Let ters 5, 200 (1967)

67Dil V. Di Napoli, F. Dobici, F. Salvetti, H. G. De Carvalho; Nuovo Cimento $48 \mathrm{~B}, 1$ ( 1967 )

67Di2 V. Di Napoli, F. Dobici, F. Salvetti, O. Forina, H. G. De Carvalho; PICNS-67, 389 ( 1967 )

67Di3 V. Di Napoli, F. Dobici, O. Forina, F. Salvetti, H. G. de Carvalno; Notas Fis. Cent. Brasil Pis. Fis. 13,46 ( 1967 )

67Do 1 E. J. Dowdy, J. A. McIntyre; Phys. Rev. 157, 1166 ( 1967)

67Dr1 T. E. Drake, R. M. Hutcheon, V. W. Stobie, G. A. Beer, H. S. Caplan; Phys. Rev.163,947 (1967)

67 Dr2 T. N. Dragnev, A. P. Komar; J. Nucl. Phys. (USSR) 5 , 1170 (1967); Sov.J. Nucl. Phys. 5, 836 (1967)

67Dul M. A. Duguay, C. K. Bockelman, T. H. Curtis, R. A. Eisenstein; Phys. Rev.163, 1259 ( 1967 )

67 Eal E. D. Earle, N. W. Tanner; Nucl. Phys. A95, 241 (1967)

67 Er1 B. Erlandsson; Arkiv Fysik 34, 285 (1967)

67 Es 1 G. P. Estes, K. Min; Phys. Rev. 154, 1104 (1967)

67 Ev1 A. E. Evans; Phys. Rev. 155, 1047 ( 1967 )

67 Fe1 L. Feldman, B. B. Baliga, M. Nessin; Phys. Rev. 157, 921 (1967)

67 Fe2 S. Ferroni, V. Gracco, D. B. Isabelle, R. Malvano, M. Sanzone; Nucl. Phys. A98,409 (1967)

67 Fe3 W. Feldman, D. W. Heikkinen, W. E. Meyerhof; Phys. Letters 25B, 593 ( 1967 )

$67 \mathrm{Frl}$ R. F. Frosch, H. L. Crannell, J. S. McCarthy, R. E. Rand, R. S. Safrata, L. R. Suelzle, M. R Yearian; Phys. Letters $24 \mathrm{~B}, 54(1967)$

67Ful C. B. Fulmer, I. R. Williams, T. H. Handley, G. F. Dell, L. N. Blumberg; Phys. Rev. Letters 19,522 (1967)

67Gal \%. L.Garzon, G. G. Rocco; J.Inorg. Nucl. Chem. 29, 1 (1967)

67 Ge1 K. N. Geller, E. G. Muirhead, L. D. Cohen; Nucl. Phys. A96, 397 (1967)

$67 \mathrm{Ge} 2 \mathrm{H}$. M. Gerstenberg, E. G. Fuller; NBS Technical Note 416 , Study of a Halpern-Type Neutron Detector; U.S. Government Printing Office, Washington, D.C., June 1967 .

67 Gil M. Giannini, P. Oliva, D. Prosperi, G. Toumbev; Nucl. Phys. A 101,145 ( 1967 )

67Go1 B. I. Goryachev, B. S. Ishkhanov, I . M . Kapitonov, Zh. M. Sel iverstova, $V$. G. Shevchenko, B. A. Yur ev; J. Nucl. Phys. (USSR) 4,505 (1966); Soviet J. Nucl. Phys. 4, 359 (1967)

67 Go2 D. R. Goosman, R. W. Kavanagh; Phys. Rev.161,1156 ( 1967 )

67 Go3 B. I. Goryachev, B. S. Ishkhanov, B. G. Shevchenko, B. A. Yur ev; J. Nucl. Phys. (USSR) 5, 1138 (1967); Sov.J. Nucl. Phys. 5, 811 ( 1967 ) 
67 Go4 B. N. Goryachev, B. S. Ishkhanov, I. M. Kapitonov, I. M. Piskarev, V. G. Shevchenko, $\theta$. P. Shevchenko; JETP Pisma 5, 225 (i967); JETP Letters 5, 180 ( 1967 )

67 Go5 A. N. Gorbunov; PICNS-67, 376 ( 1967)

67 Hal J. R. Harrington, B. M. Spicer; PICNS-67, 380 (1967)

67 Hu1 R. R. Hurst, D. J. Donahue; Nucl. Phys. A91, 365 ( 1967)

67 Hu2 S. M. Hussain, K. G. Mc Neill; Can. J. Phys. 45, 2851 ( 1967 )

67 Ie 1 Yu. P. Ievlev, A. A. Koval, E. G. Kopanets, Yu, S. Koraa L. N. Sukhotin, S. P. Tsytko; Uk. Fiz. Zhur. 12,743 ( 1967 )

67 Isl B. S. Ishkhanov, I. M. Kapitonov, V. G. Shevchenko, B. A. Yur ev; J. Nucl: Phys. (USSR) 4, 765 ( 1966 ); Sov. J. Nucl. Phys. 4, 544 (1967)

$67 \mathrm{Jol}$ S. A. E. Johansson, L. Nilsson; Arkiv Fysik 35, 403 (1967)

$67 \mathrm{Ka} 1 \mathrm{~S}$. P. Kapitsa, V. I Novgorodtseva, V. A. Pchelin, G. N. Smirenkin, Yu. M. Tsipenyuk, V. M. Shubko; ZhETF Pisma 6, 495 (1967); JETP Letters 6, 27 (1967) $67 \mathrm{Kn} 1$ P. Kneisel, A. Goldmann, H. v. Buttlar; Z. Physik 199 ,

67 Kol A. P. Komar, V. P. Denisov, L. A. Kul chitskii; Dokl. Akad. Nauk. SSSR 169, 1307 (1966); Sov. Phys. Doklady $11,728(1967)$

$67 \mathrm{Ko} 2$ E. G. Kopanets, Yu. S. Korda, A. A. Koval, L. N. Sukhoton, S. P. Tsytko; J. Nucl. Phys. (USSR) 6,233 ( 1967 ); Sov. J. Nucl. Phys.6, 170 (1968)

$67 \mathrm{Ko} 3 \mathrm{R}$. Kose, W. Paul, K. Stockhorst, K. H. Kissler; Z, Physik $202,364(1967)$

$67 \mathrm{Kr} 1 \mathrm{G}$. Kraft, R. Kosiek; Natur. Heft 13, 337 (1967)

$67 \mathrm{Kr} 2 \mathrm{~K}$. Kramer, H. v. Buttlar, A. Goldmann, B. Huber; Z. Phys ik 207, 1 ( 1967 )

67 Ku 1 F. T. Kuchnir, P. Axel, L. Criegee, D. M. Drake, A. 0 . Hanson, D. C. Sutton; Phys. Rev.161, 1236 ( 1967 )

$67 \mathrm{Ku} 2 \mathrm{H}$. W. Kuehne, P. Axel, D. C. Sutton; Phys. Rev. 163, $1278(1967)$

$67 \mathrm{La} 1 \mathrm{H}$. Langhoff, M. Schumacher; Phys. Rev. 155, 1246 (1967)

$67 \mathrm{La} 2 \mathrm{H}$. Langhoff; Phys. Rev.159, 1033 (1967)

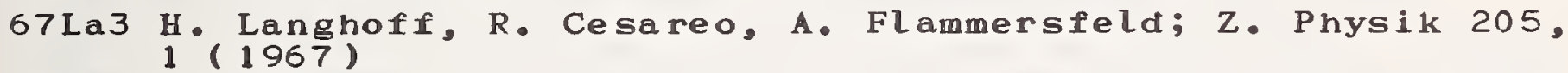

67 Le 1 J. R. Leslie, I. G. Main; Nuovo Cimento 50B, 389 ( 1967 )

67 Li 1 H. Lichtblau, A. Goldmann; Z. Physik 205, 47 ( 1967 )

67 Lol J. M. Loiseaux, J. M. Maison, M. Langevin; J. de Physique 28, 1 i ( 1967 )

67 Lo2 R. M. Lombard, G. R. Bishop; Nucl. Phys, A101, 601 (1967)

67 Me1 F. R. Metzger; Phys. Rev. 157, 1060 (1967)

67 Me2 F. R. Metzger; Phys. Rev.161, 1249 (1967) (See F. R. Metzeer; Phys. Rev. C2, 2024 ( 1970 )

67 Me3 L. Meyer-Schutzmeister, D. S. Geminell, R. C. Bearse, N. G. Puttaswamy; PICNS-67, 385 (1967)

$67 \mathrm{Mi} 1 \mathrm{~A}$. V. Mitrofanova, Yu, N. Ranyuk, P. V. Sorokin; J. Nucl. Phys. (USSR) 6,703 (1967); Sov.J. Nucl. Phys.6, 512 (1968) 
$67 \mathrm{Mi} 2 \mathrm{M}$. Mishina, E. Tanaka, K. Kageyama, N. Mutsuro, A. Asami, Y. Kawarasaki, Y. Nakajima; J.Phys. Soc. Japan 23, $919(1967)$

67 Mul K. M. Murray, J. C. Ritter; PICNS-67, 383 ( 1967 )

$67 \mathrm{Ni} 1$ L. Nilsson, I. Bereqvist; Arkiv Fysik 35, 411 ( 1967 )

67 ebl E. Obst, F. Rauch, H. G. Hahsweiler; Nucl. Phys. A103,

67 Ed 1 Y. Oda, H. Hirabayasi, K. Fujioka; PICNS-67, 374 ( 1967)

67 Pal J. C. Palathingal, M. L. Wiedenbeck; Nucl. Phys. A101, $193(1967)$

67 Paz H. L. Pai, K. G. Mc Neill; Can. J. Phys. 45, 3235 ( 1967 )

67 Fa3 P. Paul, N, G. Puttaswamy, D. Kohler; Phys. Rev. 164. $1332(1967)$

$67 \mathrm{Pe} 1$ G. A. Peterson; Phys. Letters 25B, 549 (1967)

67 Ral R. E. Rand, R. F. Frosch, C. E. Littig, M. R. Yearian; Phys. Rev. Letters 18,469 (1967)

67 ka2 Yu. N. Ranyuk, P. V. Sorokin; J . Nucl. Phys. (USSR) 5, 37 ( 1967 ); Sov. J .. Nucl. Phys. 5, 26 ( 1967 )

67 Ra3 Yu. N. Ranyuk, P. V. Sorokin; J . Nucl. Phys. (USSR) 5 , 531 ( 1967 ); Sov. J. Nucl. Phys. 5, 377 (1967)

$675 a 1$ G. A. Savitskii, N. G. Afanas ev, I. S. Gul karov, V. D. Kovalev, A. S. Omelaenko, V. M, Khvastunov, N. G. Shevchenko; J. Nucl Phys. (USSR) 6, 685 ( 1967 ) Soviet J. Nucl. Phys. 6, 499 (1968)

67 Sc1 M. Schumacher, H. Langhoff; Phys. Rev. 162, 1153 ( 1967 )

67 Se1 R. E. Segel, Z, Vager, L. Meyer-Schutzmeister, P.P. Singh, R. G. Allas; Nucl. Phys. A93, 31 (1967)

$67 \operatorname{Sh} 1$ N. G. Shevchenko, N. G. Afanas ev, G. A. Savitskil, V. M. Khvastunov, $V$ : D. Kovalev, A S Omeiaenko, I. S. Gul karov; J Nucl. Phys; (USSR) 5, 948 (1967) Sov. J. Nucl. Phys. 5, 676 ( 1967 )

67 Sh2 D. M. Sheppard, C. Van der Leun; Nucl. Phys. A100,333 ( 1967 )

$67 \operatorname{Sh} 3$ s. Shimizu, Y. Isozumi, Y. Nakayama; Phys. Letters $25 B$, $124(1967)$

$67 \operatorname{Sm} 1$ I. I. Smith, J. Garvey, J G. Rutherglen, G. R. Brookes; Nucl: Phys. B1, 483 ( 1967 )

67 Sp1 E. Spamer, H. Artus; Z. Physik 198, 445 (1967)

67 st 1 S. V. Starobdubtsev, R. B. Begzhanov, A. A. Islamov; Dokl: Akad. Nauk SSSR i 74,332 (1967); Sovil Phys? Dokl. $12,472(1967)$

67 Su1 M. Suffert, W. Feldman; Phys. Letters 24B, 579 ( 1967 )

67 Ta1 G. G. Taran, A. N. Gorbunov; Yad, Fiz. 6, 1124 (1967) Sov. J. Nucl. Phys. 6, 816 (1968)

67 Ta2 H. Taneichi, K. Shoda; J. Phys. Soc. Japan 22, 664 (1967)

67 Th 1 M. N. Thompson, J. E. E. Baglin; Phys. Letters 25B, 256 (1967)

67Th2 M. N. Thompson, J. E. E. Baglin; PICNS-67, 386 (1967)

67 Til A. Titze, E. Spamer, A. Goldmann; Phys. Letters 24 B, 169 ( 1967 )

67Tu1 J. Tudoric-Ghemo; Nucl. Phys. A92, 233 (1967)

67 Tu2 P. M. Tutakin; J Nucl. Phys. (USSR) 5, 701 ( 1967 ) Sov. J. Nuc l. Phys. 5, 497 (1967) 
67 Tu3 P. M. Tutakin; J. Nucl. Phys. (USSR) 5, 1325 ( 1967 ); Sov. J. Nucl. Phys. 5, 946 (1967)

67 Tu4 G. Khr. Tumber; Compt. Rend. Acad. Bulg. Sci.,20, 541 ( 1967 )

67 Val C. Van der Leun, D. M. Sheppard, P. M. Endt; Nucl. Phys. A 100, 316 (1967)

67 thal D. D. Watson, J C. Manthuruthil, F. D. Lee; Phys. Rev. $164,1399(1967)$

67 Tel D. V. Webb; B. M. Spicer, H. Arenhovel; Phys. Rev. 164, $1397(1967)$

67 We2 D. V. Webb, B. M. Spicer, H. Arenhovel, W. Greiner; PICNS-67, $377(1967)$

67 Hil 1 G. Wiechers, W. R. McMurray, I. J.Van Heerden; Nucl. Phys. A92, 175 (1967)

67 Hol W. Wolfli, R. Bosch, J. Lang, R. Muller, P. Marmier; Helv. Phys. Act 40,946 (1967)

67 Hyl J. M. Wyckoff; Phys. Rev.159, 953 (1967)

$68 \mathrm{Ab} 1 \mathrm{~K}$. Abe, E. Tanaka, N, Kawamura, M, Kanazawa, N. Mutsuro; J. Phys. Soc. Japan 25, 1507 ( 1968 )

$68 \mathrm{Ab} 2 \mathrm{~K}$. Abe, N. Kawamura, H. Nihei, H. Tsubota, N. Mutsuro; J. Phys. Soc. Japan 25, 1723 ( 1968 )

$68 \mathrm{Ab3}$ K. Abe, N. Kawamura, N. Mutsuro; J. Phys. Soc. Japan 25, $1724(1968)$

$68 \mathrm{Ab} 4 \mathrm{~K}$. Abe, N. Kawamura, N. Mutsuro; J. Phys. Soc. Japan 25, 1725 (1968)

68 Ab5 K. Abe, N. Kawamura, M. Kanazawa, N . Mutsuro; J . Phys. Soc. Japan 25, 1728 ( 1968$)$

68Adl Jan-8lof Adler and Bengt Forkman; Arkiv Fysik 38, 389 ( 1968 )

68Af1 N. G. Afanas ev, I S. Gul karov, G. A. Savitskii, V. M. Khvastunov, N. G. Shevchenko; Yad. Fiz, 7, 13 (1968); Sov.J. Nucl. Phys. 7, 8 (1968)

68Al1 W. J. Alston III, H. H. Wilson, E. C. Booth; Nucl. Phys. A $116,281(1968)$

68Bal J. Bartko, T. T. Thwaites; Phys. Letters 27B, 212 (1968)

68 Ba2 A. V. Babchenko, V. V. Petrenko, Yu. N. Ranyuk, P. V. Sorokin; Ukr. Fiz. Zhur. 13, 863 (1968); Ukr. Phys. J. 13, 615 (1968)

68Be 1 G. A. Beer, P. Brix, H. G. Clerc, B. Laube; Phys. Letters $268,506(1968)$

68 Be2 W. I. Bendel, L. W. Fagg, R. A. Tobin, H. F. Kaiser;

Phys. Kev. 173, 1103 (1968)

$68 \mathrm{Be} 3$ R. C. Bearse, L.Meyer-Schutzmeister, R. E. Segel; Nucl. Phys. A 116,682 ( 1968 )

$68 \mathrm{Be} 4 \mathrm{~N}$, Bezic, D. Jamnik, G, Kernel, J. Krajnik, J. Snajder; Nucl. Phys. A117, 124 ( 1968 )

68 Ae5 R. Bergere, H. Beil, A. Veyssiere; Nucl. Phys. A121, 463 (1968)

68 Be6 G. A. Beer, T. E, Drake, R. M. Hutcheon, V. W. Stobie, H. S. Caplan; Nuovo Cimento $53 \mathrm{~B}, 7$ (1968)

$68 \mathrm{Bl} 1 \mathrm{~S}$. L. Blatt, A. M. Young, S. C. Ling, K, J. Moon, C. D. Porterfield; Phys. Rev. 176, 1147 (1968) 
68Bo1 H. R. Bowman, R. C. Gatti, R. C. Jared, G. Kilian, L. G . Moretto, S. G. Thompson, M. R. Croissiaux, J. H. Heisenberg, $R$. Hofstadter, $L, M, M i d d l e m a n, M, R$. Yearian Phys. Rev.168, 1396 (1968)

68 Bo2 V. F. Borzhkovskil, A. S. Cherkasov, N. G. Afanas ev, I. A. Grishaev, I. I. Zalyubovski i Yad. Fiz. 7, 261 ( 1968 ); Sov. J. Nucl. Phys.7, 181 (1968)

68 Bo3 M. Boivin, Yvonne Heno; Compt. Rend. Acad. Sci. Paris $266,437(1968)$

$68 \mathrm{Bu} 1$ W. Buss, W. Del Bianco, H. Waffler, B. Ziegler; Nucl. Phys. A112, 47 (1968)

$68 \mathrm{Bu} 2 \mathrm{~J}$. Buon, V. Gracco, J. Lefrancois, P. Lehmann, B. Merkel, Ph. Roy; Phys. Letters 26B, 595 (1968)

$68 \mathrm{Ca} 1 \mathrm{P}$. H. Cannington, R. J, J Stewart, B. M. Spicer, M. G. Huber; Nucl. Phys. A109, 385 (1968)

$68 \mathrm{Cl} 1 \mathrm{H} . \mathrm{G} . \mathrm{Clerc}$. E. Kuphal; Z. Physik 211,452 (1968)

68 Co 1 B. C. Cook, D. W. Anderson, T. J. Englert; Phys. Letters $26 B, 341$ ( 1968$)$

$68 \mathrm{Cr} 1$ W. L. Creten, R. J J Jacobs, H. M. Ferdinande; Nucl. Phys. A 107,347 ( 1968 )

68Da1 A. V. Davydov, G. R. Kartashov, Yu, V. Khrudev; Yad. Fiz.7,735 (1968); Sov.J.Nucl. Phys.7, 447 (1968)

68De1 V. P. Denisov, A. P. Komar, L. A. Kulchitsky; Nucl. Phys. A113, 289 (1968)

68De2 W. Del Bianco, F. Lemire, R. J. A. Levesque, J. M. Poutissou; Can. J. Phys.46, 1585 ( 1968 )

$68 \mathrm{De} 3 \mathrm{~S}$. V. Dementi , N. G. Afanas ev, I. M. Arkatov, V. G. Vlasenko, V. A. Gol dshtein, E. L. Kuplennikov; Yad. Fiz. 8, 429 ( 1968 ); Sov. J. Nucl. Phys. 8, 248 ( 1969 )

68Dil F. S. Dietrich, M. Suffert, A. V. Nero, S. S. Hanna; Phys. Rev. 168,1169 (1968)

$68 \mathrm{Do} 1 \mathrm{~B}$. S. Dolbilkin, A. I. Isakov, V. I Kor in, L. E. Lazareva F. A. Nikolaev; Yad. Fiz. 8, i080 (i968) Sov.J.Nucl. Phys. 8, 626 (1969)

68 Drl T. E. Drake, E. L. Tomusiak, H. S. Caplan; Nucl. Phys. A $118,138(1968)$

68 Ei 1 F. Eigenbrod; Z. Naturforsch. 23, 1671 (1968)

$68 \mathrm{Ev} 1 \mathrm{D}$. Evers, G. Flugge, J Morgenstern, T W Retz-Schmid, H. Schmidt, J. W. Schmidt, S. J. Skorka; Phys. Letters $27 B, 423(1968)$

$68 \mathrm{Fa} 1$ L. W. Fagg, W. L. Bendel, R. A. Tobin, H. F. Kaiser; Phys. Rev. $171,1250(1968)$

68Fil D. S. Fielder, K. Min, W. D. Whitehead; Phys. Rev. 168, $1312(1968)$

68Fol I. Fodor, I. Demeter, L. Keszthelyi, I. Szentpetery, Z. Szoketalvi-Nagy, J. Szucs, L.Varga, J . Zimanyi; Nucl. Phys. A116, $167^{\circ}(1968)$

68Frl R. F. Frosch, R. E. Rand, H. Crannell, J. S. McCarthy, L. R. Zuelzle, M. R. Yearian; Nucl. Phys. A110,657(1968)

68 Fr2 D. E. Frederick, A. D. Sherick; Phys. Rev. 176, 1177 ( 1968 )

68Fr3 D. E. Frederick; Nucl. Phys. A119, 347 (1968)

$68 \mathrm{Ga} 1 \mathrm{R}$. Garfagnini, G. Piragino; Nucl. Phys. A122, 49 ( 1968 )

$68 \mathrm{Ge} 1$ R. W. Gellie; Aust. J.Phys.21,765 (1968) 
68Go1 B. I. Coryachev, B. S. Ishkhanov, I. M. Kapitonov, I. M. Piskarev, $\theta$. P. Shevchenko, V. G. Shevchenko; Yad. Fiz. 7, 944 (1968); Sov.J.Nucl. Phys. 7, 567 (1968)

68 Go2 A. N. Gorbunov; ZnETF Pis. Red. 8, 148 (1968); JETP Letters $8,88(1968)$

68 Go3 B. I. Goryachev, V. S. Ishkhanov, I. M. Kapitonov, V. G. Shevchenko; ZhETF Pisma 7,210 (1968); JETP Letters 7 , $161(1968)$

68 Go4 B. I. Goryachev, B. S. Ishkhanov, I. M. Kapitonov, I. M. Piskarev, V. G. Shevchenko, $\theta$. P. Shevchenko; ZhETF Pisma Red. 8, $76(1968)$; JETP Letters 8,46 (1968)

68 Go5 A. N. Gorbunov; Phys. Letters 27B, 436 (1968)

68606 B. I. Goryachev, B. S. Ishkhanov, V. G. Shevchenko, B. A. Yur ev; Yad. Fiz. 7, $1168(1968)$; Sov.J. Nucl: Phys. 7, 698 ( 1968$)$

68 Gr1 J. Graft, G. Chouraqui, M. Port, J. M. Thirion, S. Jang, T. Muller; J Physique 29, 141 (1968)

68Gu1 I. S. Gulkarov, N. G. Afanasyev, G. A. Savitsky, V. M. Rhvastunov, N. G. Shevchenko; Phys. Letters $27 \mathrm{~B}, 417$ ( 1968 )

$68 H i 1$ G J Highland, T. T. Thwaites; Nucl. Phys. A109, 163 ( 1968 )

68 Ho $1 \mathrm{~J}$. H. Hough, Z, B. du Toit, W. L. Mouton; Nucl. Phys. A 109,393 ( 1968$)$

68 Hul R. M. Hutcheon, T. E. Drake, V. W. Stobie, G. A. Beer,

H. S. Caplan; Nucl. Phys. Alo7, 266 (1968)

$68 \mathrm{Jo} 1 \mathrm{G}$. G. Jonsson, B. Forkman; Nucl. Phys. A107, 52 ( 1968)

$68 \mathrm{Jo} 2 \mathrm{G}$ G. Jonsson, B. Forkman, K. Lindgren; Phys. Letters $26 \mathrm{~B}, 508(1968)$

6EJul J.W. Jury, J. S. Hewitt, K. G. McNeill; Can. J. Phys. 46, 1823 ( 1968 )

$68 \mathrm{Kal} \mathrm{N}$. N. Kaushal, E. J. Winhold, P. F. Yergin, H. A. Medicus, R. H. Augustson; Phys. Rev. 175, 1330 (1968)

$68 \mathrm{Ka} 2 \mathrm{~L}$ Katz, G. Ricco, T, E. Drake, H. S. Caplan; Phys. Letters $28 \mathrm{~B}, 114(1968)$

$68 \mathrm{Ka} 3 \mathrm{C} . \mathrm{J}$. Kapadia, V. E. Michalk, J. A. McIntyre; Nucl. Instr. and Meth. 59, $197(1968)$

$68 \mathrm{Kr} 1$ L. Kraus, M. Suffert, D. Magnac-Valette; Nucl. Phys. A109, 593 ( 1968$)$

$68 \mathrm{Kr} 2 \mathrm{G}$. Kraft, R. Kosiek, R. Mundhenke, J. Winter; Nucl. Phys. A 118,25 (1968)

$68 \mathrm{Ku} 1$ B. V. Kurchatov, V. I. Novgorodtseva, V. A. Pchelin, G. N. Smirenkin, Yu. M. Tsipenyuk, V. M, Shubko; Yad. Fiz. 7, 521 (1968); Sov. J. Nucl. Phys. 7, 326 (1968)

$68 \mathrm{La} 1$ H. Langhoff, L. Frevert; Nucl. Phys. A111, 225 ( 1968 )

68 Le1 A. I. Lepestkin; Yad. Fiz. 8, $433(1968)$; Sov. J. Nucl. Phys. 8, 251 ( 1969 )

68 Li1 S. C. Ling, A. M. Young, S. L. Blatt; Nucl. Phys. A108, 221 ( 1968 )

$68 \mathrm{Mal} \mathrm{J}$. L. Matthews, W. Bertozzi, S. Kowalski, C. P. Sargent, W. Turchinetz; Nucl. Phys. A112, 654 ( 1968 )

$68 \mathrm{Ma} 2 \mathrm{G}$. Manuzio, G. Ricco, M. Sanzone, L. Ferrero; Phys. Rev. Letters 21,1266 (1968) 
$68 \mathrm{MCl}$ K. G. McNeill, J. S. Hewitt, J.W.Jury; Can.J.Phys.46, $1974(1968)$

68 Ne1 L. Meyer-Schutzmeister, Z. Vager. R. E. Segel, P. P. Singh; Nucl. Phys. A 108 , i 80 (1968)

$68 \mathrm{Me} 2 \mathrm{~F}$. R. Metzger; Phys. Rev.165, 1245 (1968)

$68 \mathrm{Me} 3$ F. R. Metzger; Phys. Rev.171, 1257 (1968)

68 Me4 K. A. Meyer, W. H. Walters, J. P. Hummel; Nucl. Phys. A122, 606 (i968)

68 Mil K. Min, T. A. White; Phys. Rev. Letters 21, 1200 (1968)

68 Mol R. Moreh, M. Friedman; Phys. Letters 26B, 579 (1968)

68 Mo2 G. C. Morrison, D. H. Youngblood, R. C. Bearse, R. E. Segel; Phys. Rev. 174, 1366 ( 1968 )

68 Mul R. Mundhenke, R. Kosiek, G. Kraft; Z. Physik 216,232 ( 1968 )

68 Mu2 A. Murakami; Nuovo Cimento 55B, 604 ( 1968 )

68Nyl G. Nydahl and B. Forkman; Nucl. Phys. B7, 97 (1968)

68ek1 Y. Oka, T. Kato, K. Nomura, T. Saito, H. T. Tsai; Bull. Chem. Soc. Japan 4i, 2660 (1968)

$680 k 2$ Y. Oka, T. Kato, A. Yamadera; Bull. Chea. Soc. Japan $41,1606(1968)$

$68 \mathrm{ek} 3$ Y. Oka, T. Kato, K. Nomura, T. Saito, H. Tsai; Bull. Chem. Soc. Japan 41,380 (1968)

68 ew 1 D. G. Owen, E. G. Muirhead, B. M. Spicer; Nucl. Phys. A $122,177(1968)$

68Pe1 G. A. Peterson, J.Alster; Phys. Rev.166, 1136 (1968)

68 Pe2 K. A. Petrzhak, G. A. Tutin; Yad. Fiz. 7, 970 (1968) Sov.

J. Nuc 2. Phys. 7, 584 (1968)

68Pf1 R. Pfeiffer; Z. Physik 208, 129 (1968)

68frl G. A. Proca, D. B. Isabelle; Nucl. Phys. A109, 177 (1968)

$68 \mathrm{Ra} 1 \mathrm{~N}$. S. Rabotnov, G. N, Smirenkin, A. S. Soldatov, L. N. Ysachev, S. P. Kapitza, Yu. M. Tsipeniuk; Phys. Letters $26 \mathrm{~B}, 218(1968)$

$68 \mathrm{Ra} 2 \mathrm{~J}$. A. Rawlins, C. Glavina, S. H. Ku, Y. M. Shin; Nucl. Phys. A122, 128 (1968)

68 Rhl C. E. Rhoades, Jr., H. A. Medicus; Phys. Rev.167, 1049, ( 1968 )

68Ril G. Ricco, H. S. Caplan, R. M. Hutcheon, R. Malvano; Nucl. Phys. A1 14,685 ( 1968 )

68 Ki2 F. Riess, P. Paul, J. B. Thomas, S. S. Hanna; Phys. Rev. $176,1140(1968)$

68 Ri3 J. C. Ritter, R. E. Larson, J. I. Hoover; Nucl. Phys. A110, 463 ( 1968$)$

68Ro $1 \mathrm{~S}$. Wobinson, C. P. Swann, V.K. Rasmussen; Phys. Rev. $174,1320^{\circ}(1968)$

$68 \mathrm{Sa} 1 \mathrm{G}$. A. Savitskil, N. G. Afanas ev, I. S. Gul ka rov, V. M. Khvastunov, A. A. Khomich, N. G. Shevchenko; Yad. Fiz. 1181 ( 1968 ); Sov. J. Nucl. Phys.7,705 (1968)

$68 \mathrm{Sa} 2 \mathrm{G}$. A. Savitskil, N, G. Afanas ev, I. S. Gul karov, V. D. Kovalev, A: S. ome Laenko, V, M. Khvastunov, N. G. $8,376(1969)$ 
$68 \mathrm{Sa} 3 \mathrm{G}$. A. Savitskii, N, G. Afanas ev, I. S. Gul karov, V. D. Kovalev, I. V. Andreeva, V. M. Khvastunov, N. G .

Shevchenko; Ukr. Fiz. Zhur. 13, 1648 ( 1968 ); Ukr. J. Phys. $13,1174(1969)$

68Sc1 M. Schumacher; Phys. Rev.171, 1279 (1968)

68 Sh 1 S. M. Shafroth, G. J.F. Legge; Nucl. Phys. A107, 181 ( 1968 )

68 Sh2 N. K. Sherman, J. E. E. Baglin, R. O. Owens; Phys. Rev. $169,771(1968)$

$68 \mathrm{Sh} 3 \mathrm{~K}$. Shoda, K. Abe, T. Ishizuka, N. Kawamura, M. Oyamada, Baik Nung Sung; J. Phys. Soc. Japan 25, 664 ( 1968 )

68 Sh4 K. Shoda, M. Sugawara, T. Saito, H. Miyase; Phys. Let ters; 28B, 30 ( 1968 )

68 Sh5 N. Shikazono, Y. Kawarasaki; Nucl. Phys. A118, 114 ( 1968 )

$68 \operatorname{sh} 6$ A. Kh. Shardanov, B. A. Yur ev; Yad. Fiz. 8, 424 ( 1968) Sov. J. Nucl. Phys. 8, 245 (1969)

68Sm 1 A. M. Smith, S. J. Hall, B. Mann, D. T. Stewart; J. Phys. A. (Proc. Phys. Soc.) 1, 553 (1968)

68St 1 R. J.J. Stewart; Aust. J. Phys. 21, 107 (1968)

68Sul R. E. Sund, M. P. Baker, .L. A. Kull, R. B. Walton; Phys. Rev. 176, i366 (1968)

$68 \mathrm{Tal}$ G. G. Taran; Yad. Fiz. 7, 478 (1968); Sov. J. Nucl. Phys. 7, 301 ( 1968$)$

$68 \mathrm{Ta} 2 \mathrm{G} . \mathrm{K}$. Tandon, J.A. McIntyre; Nucl. Instr. and Meth. 59, $181(1968)$

68To1 T. Tomimasu; J. Phys. Soc.Japan 25, 655 (1968)

68 Tul P. M. Tutakin; Yad. Fiz. 8, 661 (1968); Sov. J. Nucl. Phys. 8, 383 ( 1969 )

$68 \mathrm{Val}$ G. J. Vanpraet, W. C. Barber; Z. Physik 211,213 (1968)

$68 \mathrm{Vel}$ A. Veyssiere, H. Beil, R. Bergere; Compt . Rend. Acad. Sci. Paris 267,234 (1968)

68 tal R. V. Warnock, R. C. Jensen; J. Inorg. Nucl. Chem. 30 , 2011 (1968)

68Wo1 A. C. Wolff, M. A. Meyer, P. M. Endt; Nucl. Phys. A107, 332 (1968)

68 tul C. P. Wu, F. W. K. Firk, T. W. Phillips; Phys. Rev. Rev. Letters $20,1182(1968)$

$68 \mathrm{Zhl}$ E. A. Zhagrov, Yu. A. Nemilov, Yu. A. Selitskii; Yad. Fiz. 7. 264 (1968) ; Sov.J.Nucl. Phys.7, 183 (1968)

$68 Z 11$ J.F. Ziegler, G. A. Feterson; Phys.Rev. 165, 1337 (1968)

68 Zi2 P. D. Zimmerman, M. R. Yearian, T. W. Donnelly; Phys. Rev. Letters 21,1392 ( 1968 )

$69 \mathrm{Abl}$ K. Abe, N. Kawamura, M. Kanazawa, N. Mutsuro; J . Phys. Soc. Japan 26, 203 (1969)

$69 \mathrm{Ab} 2 \mathrm{~K}$. Abe, N. Kawamura, H. Nihei, T. Osawa, N. Mutsuro; J. Phys. Soc. Japan 26,577 ( 1969 )

69Afl V. D. Afanas ev, N. G. Afanas ev, I. S. Gul karov G. A. Savitskii, V. M. Khvastunov, N, G. Shevchenko, A. A. Khomich; Yad. Fiz. 10, 33 (1969); Sov, J. Nucl. Phys. 10 , 18 (1970)

69Al 1 William J. Alston, III; Phys. Rev. 188, 1837 (1969) 
69An 1 R. I. Anderson, R. Prepost, B. H. Wiik; Phys. Rev. Let ters 22,651 (1969)

69 An2 D. W. Anderson, B. C. Cook, T. J. Englert; Nucl. Phys. A 127,474 ( 1969$)$

69 An 3 D. W. Anderson, A. J Bureau, B. C. Cook, T. J. Englert; Phys. Rev. 183, 978 (1969)

69An4 Yu. P. Antuf ev, Yu. V. Zhebrovskii, L. Ya. Kolesnikov, V. S. Kuz menko, I. I. Miroshnichenko, A. L. Rubashkin, P. V. Sorokin; Yad. Fiz.9,680 (1969); Sov. J. Nucl. Phys. 9. 394 ( 1969$)$

69An5 Yu. P. Antuf ev, V. L. Agranovich, V. B. Ganenko, V. S. Kuz menko, I. I: Moroshnichenko, $P$. V. Sorokin, V. M. Sanin; Yad. Fiz.9,921 (1969); Sov.J. Nucl. Phys.9, 538 ( 1969$)$

69An6 Yu. P. Antuf ev, V. L. Agranovich, V. B. Ganenko, V. S. Kuzmenko, I. I . Miroshnichenko, and P. V Sorokin; Ukr. Fiz. Zh. 14, $248(1969)$

69 An7 G. P. Antropov, I. E. Mitrofanov, A. I Prokof ev and V. S. Russkikh; Izv. Akad. Nauk Fiz, 33, 700 ( 1969), Bull. Acad. Sci. U.S.S.R.- Phys. 33, 645 (1969)

69 An 8 Yu. P. Antufyev, V. L. Agranovich, V. B. Ganeko, V. S. Kuzmenko, I. I . Mi roshnichenko, P. V. Sorokin; Ukr. Fiz. $-4,496(1969)$

69 An 10 G. P. Antropov, I. E. Mitrofanov, A. I. Prokof ev, V. S. Russkikh; Izv. Akad. Nauk. Fiz.34, 116 ( 1969 ); Bull. Acad. Sci. USSR-Phys. 34, 108 ( 1970$)$

69Ar1 Yu. M. Arkatov, A. V. Bazaevam, P. I. Vatset, V. I. Voloshchuk, A. P. Klyucharev, A. F. Khodyachikh; ZhETF Pis. Red.9, 462 ( 1969 ) ; JETP Letters 9, 278 (1969)

69Ar2 Yu. M. Arkatov, P.I. Vatset, V.I. Voloshchuk, V. V. Kirichenko, I. M. Prokhorets, A. F. Khodyachikh; ZhETF Pis. Red.9, 574 ( 1969 ) ; JETP Letters 9, 350 ( 1969 )

69 Ar3 Yu, M. Arkatov, P. I . Vatset, V. I. Voloshchuk, V.L. Marchenko, A. F. Khodyachikh, V.I.Chmil; ZhETF Pis. Red.9,626 (1969); JETP Letters 9, 384 (1969)

69 Ar4 Yu. M. Arkatov, P. I . Vatset, V.I. Voloshchuk, A. P. Klyucharev, V. L. Marchenko, A. F. Khodyachikh; Yad. Fiz.9, 473 ( 1969 ); Sov.J. Nucl. Phys.9, 271 (1969)

69 Ar5 Yu. M. Arkatov, A. V. Bazareva, P. I. Vatset, V.I. Voloshchuk, A. P. Klyucharev, A. F. Khodyachikh; Yad. Fiz. 10, 1123 ( 1969 ); Sov.J? Nucl. Phys. 10,639(1970)

$69 \mathrm{Ba} 1 \mathrm{G}$. Baciu, D. Catana, C. Deberth, C. Iliescu, B. Cirstoiu; Z. Physik 227, 336 ( 1969 )

$69 \mathrm{Ba} 2 \mathrm{~J} . \mathrm{E} \cdot \mathrm{E} \cdot$ Baglin, M. N. Thompson; Nucl. Phys.A138, 73 ( 1969$)$

69Be1 B. L. Berman, R. L. Bramblett, J. T. Caldwell, H. S. Davis, M. A. Kelly, S. C. Fultz; Phys. Rev. 177,1745 ( 1969 )

69Be2 N. Bezic, D. Brajnik, D. Jamnik, G. Kernel; Nucl. Phys. A $128,426(1969)$

69 Be3 B. L. Berman, R. L. Van Hemert, C. D. Bowman; Phys. Rev. Letters 23,386 (1969)

$69 \mathrm{Be} 4$ R. Bergere, P. Carlos, A. Veyssiere, H. Beil; PICNS-69 $352(1969)$

69Be5 J. Berthot, P. Y. Bertin, J. P. Girardeau-Montaut, D. B. Isabelle; PICNS-69, 138 (1969) 
69 Be6 R. Bergere, H. Beil, P. Carlos, A. Veyssiere; Nucl. Phys. A $133,417(1969)$

69Be7 R. B. Begzhanov, S. M. Akhrarov; ZhETF Pis. Red. 10, 39 (1969); JETP Letters 10,26 (1969)

69 Be 8 B. L. Berman, M. A. Kelly, R. L. Bramblett, J. T. Caldwell, H. S. Davis, S. C. Fultz; Phys. Rev•185, 1576 (1969)

69Be9 H. Beil, R. Bergere, P. Carlos and A. Veyssiere; C.R. Acad. Sci. Paris 269, 216 (1969)

69Be10 V. M. Bezotosnyi, V. A. Zhmailo, L. M. Surov, and M. S. Shvetsov; Yad. Fiz. 10, 225 (1969), Sov. J. Nucl. Phys. 10, $127(1970)$

69Bol C. D. Bowman, B; L. Berman, H. E. Jackson; Phys. Rev. $178,1827(1969)$

69Bo2 C. K. Bockelman, T. H. Curtiss, R. A. Eisenstein, D. W. Madsen; PICNS-69, 132 ( 1969 )

69Bo3 M. Boivin, Y. Cauchois, Y. Heno; Nucl. Phys. A137, 520 (1969)

69Bo4 C. D. Bowman, R. J Baglan, B. L. Berman; Phys. Rev. Letters 23, 796 ( 1969 )

$69 \mathrm{Ca} 1$ H. S. Caplan, R. K. Gupta, E. L. Tomusiak, C. S. Yang; PICNS-69, 134 ( 1969$)$

69 Ca2 D. E. Carlson, A. A. Temperley; Phys. Letters 30 B, 322 (1969)

69 Ca 3 P. H. Cannington and J. M. Dixon; Aust.J. Phys. 22, 679 (1969)

69Ce1 R. Cesareo, M. Giannini, P. Oliva, D. Prosperi, M. C. Ramorino; Nucl. Phys. A132, 512 ( 1969 )

69Ch1 B. T. Chertok, E. C. Jones, W. L. Bendel, L. W. Fagg; Phys. Rev. Letters 23, 34 (1969)

$69 \mathrm{Ch} 2 \mathrm{~B} . \mathrm{T}$. Chertok, E. C. Jones, W. L. Bendel, L. W. Fagg, H. F. Kaiser, S. K. Numrich; PICNS-69,60 ( 1969 )

69Co1 S. Costa, L. Ferrero, L. Pasqualini, F. Ferrero, C. Manfredotti; Lettere al Nuovo Cimento 1, 346 (1969)

69002 S. Costa, L. Ferrero, L. Pasqualini, C. Manfredotti; Lettere al Nuovo Cimento 2, 318 ( 1969 )

69003 G. W. Cole Jr., F. W. K. Firk, T. W. Phillips; Phys. Letters $30 \mathrm{~B}, 91$ ( 1969 )

69004 S. Costa, L. Ferrero, R. Garfagnini, G. Piragino, and A. Zanini; Lettere Al Nuovo Cimento 2,336 ( 1969 )

69 Cul T. H. Curtis, R. A. Eisenstein, D. W. Madsen, C. K. Bockelman; Phys. Rev. 184, 1162 (1969)

69Dal A. V. Davydov, G. R. Kartashov, I. N. Vishnevsky; Phys. Letters 30B, 616 ( 1969 )

69De1 H. G. De Carvalho, V. Di Napoli, D. Margadonna, F. Salvetti, K. Tesch; Nucl. Phys.A126,505 (1969)

69De2 T. K. Deague, E. G. Muirhead, B. M. Spicer; PICNS-69, 350 ( 1969 )

69De3 W. Del Bianco, J. M. Poutissou; Phys. Letters 29B, 299 ( 1969 )

69De4 F. P. Denisov, A. Duisebaev, P. A. Cerenkov; Yad. Fiz. 9 , 3 ( 1969 ); Sov. J. Nucl. Phys.9, 1 (1969)

69 De5 T. K. Deague, E. G. Muirhead, B. M. Spicer; Nucl. Phys. A $139,501(1969)$ 
69 De6 S. V. Dementil, N. G. Afanas ev, I. M. Arkatov, V. G. viasenko, V. A. Gol dshtein, E. L. Kuplennikov; Yad. Fiz. 9, 241 (1969); Sov. J. Nucl. Phys.9, 142 (1969)

$69 \operatorname{De} 7$ V. P. Denisov, A. P. Komar, L. A. Kul chitskii, I. Ya. Chubukov; Yad, Fiz. 10, 1116 (1969); Sov. J. Nucl. Phys. $10,635(1970)$

$69 \mathrm{De} 8 \mathrm{~S}$. V. Dementii, M. G. Afanas ev, - M. Arkatov, V. G. viasenko, $v, \theta$. Gol dshtein, E, L. Kuplennikov, $v . I$. Startsev; Ukr. Fiz. Zhur. 14, 1911 (1969)

69Di 1 V. Di Napoli, D. Margadonna, F. Salvetti, H. G. De Carvalno, J. B. Martins; Lettere al Nuovo Cimento 1,121 ( 1969 )

69Di2 J. P. Didelez, H. Langevin-Joliot, G. Duhamel, L. Marcus, P. Narboni; Nucl. Phys. A139, 69(1969)

$69 \mathrm{Di} 3 \mathrm{~V}$. DiNapoli, D. Margadonna, F. Salvetti; Ricerca Sci. 39 , $133(1969)$

69 Do1 B. S. Dolbilkin, A. I. Isakov, V. I. Korin, L. E. Lazareva; ZhETF Pis. Red. 10, 365 (1969); JETP Letters 10,231 ( 1969 )

69002 B. S. Dolbilkin, A. I. Isakov, V. I Korin, L. E. Lazareva, N. V. Lin kova, B. A. Tulupov; Yad. Fiz.9, 675 ( 1969 ); Sov. J. Nucl. Phys.9, 391 (1969)

69003 B. S. Dolbilkin, A. I. Isakov, V. I. Korin, L. E. Lazareva, N. V. Lin kova, and F. A. Nikolaev; Yad. Fiz. 9, 914 ( 1969 ), ; Sov. J. Nucl. Phys.9,534 (1969)

$69 \mathrm{Ei}_{1} \mathrm{R}$. A. Eisenstein, D. W. Madsen, H. Theissen, $-\mathrm{L}$. S. Cardman, C. A. Bockelman; Phys. Rev. 188, 1815 (1969)

69 Ei2 F. Eigenbrod; Z. Physik 228, 337 (1969)

$69 \mathrm{Ej} 1$ H. Ejiri, P. Richara, S, Ferguson, R. Heffner, D. Perry; Nucl. Phys. A128, 388 ( 1969 )

$69 \mathrm{Ej} 2 \mathrm{H}$. Ejiri, S. M. Ferguson, I. Halpern, R. Heffner; Phys. Letters $29 \mathrm{~B}, 111$ (1969)

69 Fa 1 L. W. Fagg, W. L. Bendel, E. C. Jones, Jr., S. Numrich; Phys. Rev. 187,1378 ( 1969 )

$69 \mathrm{Fa} 2 \mathrm{~L}$. W. Fagg, W. L. Bendel, E. C. Jones, Jr, H. F. Kaiser, T. F. Godlove; Phys. Rev? 187, 1384 (1969)

69 Fr 1 D. E. Frederick, R. J. J . Stewart, R. C. Morrison; Phys. Rev. 186,992 (1969)

69Fu1 S. C. Fultz, B. L. Berman, J. T. Caldwell, R. L. Bramblett, M. A. Kelly; Phys. Rev. 186, 1255 ( 1969 )

69 Fu2 C. B. Fulmer, I. R. Williams, K. S. Toth, G. F. Dell; Phys: Rev. 188,1752 ( 1969 )

$69 \mathrm{Gal}$ R. Gartaenini, G. Piragino, A. Zanini; Nuovo Cimento $63 \mathrm{~B}$, 670 ( 1969 )

69 Ga2 D. B. Gayther, J. W. McMillan, H. A. Medicus; PICNS69365 (1969)

$69 \mathrm{Ga} 3 \mathrm{R}$. Garfagnini, G. Pi ragino, A. Zanini; Atti Accad. Naz. Lincei, Rend. Cl. Sci. Fis: Mat. Natur. 47, 33 (1969)

69Go1 A. N. Gorbunov; Yad.Fiz.10, 469 (1969) Sov. J. Nucl. -Phys. 10,268 ( 1969 )

$69 \mathrm{Go} 2 \mathrm{~B}$. I. Goryachev, B. S. Ishkhanov, I. M. Kapitonov, I. M. Piskarev, V. G. Shevchenko, $\theta$. P. Shevchenko; Yad: Fiz. 10, 252 (1969); Sov.J.Nucl. Phys.11, 141 (1970) 
$69 \mathrm{Go} 3 \mathrm{~B}$. I. Goryachev, B. S. Ishkhanov, Piskarev, V. G. Shevchenko, $\theta$. Akad. Nauk. Fiz. 33, 1736 (1969); Bull. Acad. Sci. USSRPhys. 33, 1588 ( 1969 )

69Gr1 H. D. Graber and Gale I. Harris; Phys. Rev. 188, 1685 ( 1969$)$

69Gr2 I. A. Grishaev, V. P. Efimov, V. I. Kasilov, V. I. Noga, Yu. N. Ranyuk, P. V. Sorokin, A. N. Fisun; Ukr. Fiz. Zhur. 14, 1818 (1969)

69 Gul I. S. Gul karov, N. G. Afanas ev, V. M. Khvastunov, N. G. Shevchenko, V. D. Afanas ev, G. A. Savitskil, A. A. Khomich; Yad. Fiz.9, 478 (1969); Sov. J. Nucl. Phys.9, $274(1969)$

69 Gu2 I. S. Gulkarov, N. G. Afanas ev, A. A. Khomich, V. D. Afanas ev, V. M. Khvastunov, G. A. Savitskii, N. G. Shevchenko; Yad. Fiz.9, 1138 (1969), Sov. J. Nucl. Phys. 9, 666 (1969)

69 Gu3 I. S. Gul karov, N. G. Afanas ev, A. A. Khomich, V. M. Khvastunov, V. D. Afanas ev, G. A. Savitskii, N. G . Shevchenko; Yad. Fiz. 10,694 (1969); Sov. J. Nucl. Phys. $10,400(1970)$

$69 \mathrm{Hal}$ M. Hasinoff, G. A. Fisher, H. M. Kuan, S. S. Hanna; Phys. Letters 30B, 337 (1969)

$69 \mathrm{Ha} 2 \mathrm{G} . \mathrm{I}$. Harris, A. K. Hyder,Jr., J. Walinga; Phys. Rev. 187 , $1413(1969)$

69 Hol W. Hofmann, R. Kosiek, G. Kraft, R. Mundhenke; Z。 Physik 225, 303 ( 1969 )

69 Hul R. M. Futcheon, H. S. Caplan; Nucl. Phys. A127, 417 ( 1969$)$

69 Is 1 B. S. Ishkhanov, I. M. Kapitonov, E. V. Lazutin, I. M. Piskarev, 9. P. Shevchenko; ZhETF Pis. Red. 10,80 (1969); JETP Letters 10,51 (1969)

69 Is2 B. S. Ishkhanov, I. M. Kapitonov, E. V. Lazutin, I. M. Piskarev, $\theta$. P. Shevchenko; Izv. Akad. Nauk. Fiz. 33 , 2074 ( 1969 ); Bull. Acad. Sci. USSR-Phys.33, 1889 ( 1969 )

69 Is 3 B. S. Ishkhanov, I. M. Kapitonov, E. V. Lazutin, I. M. Piskarev, V. G. Shevchenko; Izv. Akad. Nauk. Fiz. 33, 1742 ( 1969 ); Bull. Acad. Sci.USSR-Phys. 33, 1594 ( 1969 )

69 Iv1 V. G. I vanchenko and B. S. Ratner; Izv. Akad. Nauk. Fiz. 34, i 75 (1969); Bull. Acad. Sci. USSR-Phys. 34, 159 ( 1970 )

$69 \mathrm{Ka} 1$ S. P. Kapitza, N. S. Rabotnov, G. N. Smirenkin, A. S. Soldatov, L. N. Usachev, Yu. M. Ysipenyuk; ZnETF Pis. Red.9, 128( 1969 ); JETP Letters9, 73 (1969)

$69 \mathrm{Ke} 1 \mathrm{G}$. Kernel, W. M. Mason; Nucl. Phys. A123, 205 ( 1969)

$69 \mathrm{Ke} 2 \mathrm{M} . \mathrm{A} \cdot$ Kelly, B. L. Berman, R. L. Bramblett, S. C. Fultz; Phys. Rev. i79, 1194 ( 1969 )

$69 \mathrm{Ke} 3 \mathrm{~N} . \mathrm{K}$. Keller, J.K. P. Lee, D. B. McConnell; Can. J. Phys. 47, 611 ( 1969 )

$69 \mathrm{Kh1}$ T. A. Khan, J. S. Hewitt, K. G. McNeill; Can. J. Phys. 47, $1037^{\circ}(1969)$

$69 \mathrm{Kn} 1 \mathrm{U}$. Kneissl, H. Schneider, R. Volpel, K. Wolcken; Nucl. Phys. A 135, 395 ( 1969 )

69 Kol R. Kose, B. Martin, R. Runkel, H. Wahlen, K. H. Kissler; Z. Physik 220, $305^{\circ}(1969)$ 
69 Ko2 A. P. Komar, B. A. Bochagov, A. A. Kotov, Yu. N. Ranyuk, G. G. Semenchuk, G. E. Solyakin, and $P$. V. Sorokin; Yad. Fiz. 10,51 (1969), Sov.J. Nucl. Phys. 10, 30 ( 1970 )

69la1 H. Langhoff, D. Gfoller; Nucl. Phys. A127, 379 ( 1969)

$69 \mathrm{La} 2 \mathrm{~L}$. E. Lazareva and A. I. Lepestkin; Yad. Fiz. 10, 266 ( 1969 ); Sov. J. Nucl. Phys.11, 149 (1970)

$69 \mathrm{La} 3 \mathrm{R}$. E. Larson and C. M. Gordon; J.Geophys. Res. 74,6023 (1969)

69 Le1 J. C. Legg, D. G. Megli, D. R. Abraham, L. D. Ellsworth, S. Hechtl; Phys. Rev.186.1138(1969)

$69 \mathrm{Ma} 1 \mathrm{~A}$. Manfredini, L. Fiore, C. Ramorino, H. G. De Carvalho, W. Wolfli; Nucl. Phys.A123, 664 (1969)

$69 \mathrm{Ma2}$ A. Manfredini, L. Fiore, C. Ramorino, H. G. De Carvalho, W. Wolfli; Nucl. Phys. A127, 687 (1969)

$69 \mathrm{Ma} 3 \mathrm{G}$. Manuzio, G. Ricco, M. Sanzone, L. Ferrero; Nucl. Phys. A 133,225 ( $\left.1969^{\circ}\right)$

69 Ma4 W. M. Mason, G. Kernel, J. L. Black, N. W. Tannner; Nucl. Phys. A135, 193 ( 1969 )

69Ma5 W. M. Mason, N.W. Tanner, G. Kernel; Nucl. Phys. A 138,253 ( 1969 )

$69 \mathrm{Me} 1$ W. E. Meyerhof, W. Feldman, S. Gilbert, W. OConnell; Nucl. Phys. A131, 489 (1969)

69Me2 F. R. Metzger; Phys. Rev. 187, 1680 (1969)

$69 \mathrm{Me} 3 \mathrm{~F}$. R. Metzger; Phys. Rev.187, 1700 ( 1969 )

69 Mil V. E. Michalk, J.A. McIntyre; Nucl. Phys. A137, 115 ( 1969 )

69 Mo 1 L. G. Moretto, R. C. Gatti, S. G. Thompson, J. T. Routti, J. F. Heisenberg, L. M. Middleman, $M$. R. Yearian, R. Hofstadter; Phys: Rev. 179, 1176 (1969)

69Mo2 R. Moreh, A. Wolf; Phys. Rev. 182, 1236 (1969)

69 Mo3 R. Moreh and $\theta$. Shahal; Phys. Rev. 188, 1765 ( 1969 )

69 Mu 1 K. M. Murray, J. C. Ritter; Phys. Rev. 182,1097 ( 1969)

69 Mu2 Kenneth M. Murray; Phys. Rev. Letters 23, 1461 ( 1969)

$69 \mathrm{Na} 1 \mathrm{P} . \mathrm{C}$. Nagle, E. J. Winhold, P. F. Yergin, P. Stoler, S. J. Mecca; Nucl: Phys. Al27, 669 ( 1969 )

$69 \mathrm{Na} 2 \mathrm{~K}$. Nagatani, M. R. Dwarakanath, D. Ashery; Nucl. Phys. Ai28, 325 ( 1969 )

69 Nel Reiner Neuhausen; Z. Physik 220, 456 ( 1969)

69 No 1 V. I. Noga, Yu. N. Ranyuk, and P. V. Sorokin; Yad. Fiz. 9, 1152 (1969), Sov. J. Nucl. Phys.9,673 (1969)

69 ok 1 Y. Oka, T. Kato, N. Sato; Bull. Chem. Soc. Japan 42,387 ( 1969 )

690w 1 D. G. Owen, E. G. Muirhead, B. M. Spicer; PICNS-69, $349(1969)$

$690 w 2$ D. G. Owen, B. M. Spicer; Phys. Letters 30 B, 242 ( 1969)

69 Pe1 K. A. Petrzhak and G. A. Tutin; Yad. Fiz.9, 949 ( 1969 ), Sov. J. Nucl. Phys.9, 556 (1969)

69Pil C. J. Piluso, G. C. Salzman, D. K. McDaniels; Phys. Rev. 181,1555 ( 1969 ) 
69 Pi2 P. Picozza, C. Schaerf, R. Scrimaglio, G. Goggi, A. Piazzoli, D. Scannicchio; Lettere al Nuovo Cimento II, 445 ( 1969 )

69 Pi 3 P. Picozza, C. Schaerf, R. Scrimaglio, G Goggi, A. Piazzoli, D. Scannicchio; PICNS-69, 70 ( 1969 )

69Ral S. Ramchandran, J. A. McIntyre; Phys. Rev.179, 1153 ( 1969$)$

69 Ra3 V. K. Rasmussen, C. P. Swann; Phys. Rev. 183, 918 (1969)

69 Fil P. Richard, F. Gabbard, A. C. Porter, F. F. Hopkins; Phys. Letters 29B, 646 ( 1969)

69Sa 1 J. Sanada, M. Yamanouchi, N. Sakai, S. Seki; J. Phys. Soc. Japan 26, 850 (1969)

$695 a 2$ G. A. Savitskii, N. G. Afanas ev, I. V. Avdreeva, I. S. Gul karov, L. M. Krugovaya, V. M. Khvastunov, A. A.

Khomich, N. G. Shevchenko; Izv. Akad. Nauk. Fiz.33, 53

( 1969 ), Bull. Acad. Sci. USSR-Phys. 33, 50 ( 1969 )

69 Sa 3 G. A. Savitskii. N. G. Afanas ev, I. S. Gul karov, V. D. Kovalev, V. M. Khvastunov, N, G. Shevchenko, I, V'. Sci. USSR-Phys. 33, 56 (1969)

69 Shl N. Shikazono, Y. Kawarasaki; J. Phys. Soc. Japan 27,273 ( 1969 )

69 Sh2 N. Shikazono, Y. Kawarasaki; J. Phys. Soc. Japan 26, $1319(1969)$

69 Sh3 T. Shintomi, M. Masuda; J. Phys. Soc. Japan 26, 607 ( 1969$)$

69 Sh4 K. Shoda, H. Taneichi; J. Phys. Soc. Japan 26, 217 (1969)

69 Sh 5 K. Shoda, M. Sugawara, T. Saito, H. Miyase; Phys. Rev. Letters 23,800 (1969)

$69 \operatorname{sh} 6$ K. Shoda, M. Sugawara, T. Sa1to, H. Milyase; PICNS-69 (Asilomar), 125 ( 1969 )

69 Sh7 K. Shoda, M. Sugawara, T. Saito, H. Miyase; PICNS-69 (Asilomar), 131 ( 1969 )

69 Sh8 K. Shoda, M. Sugawara, T. Saito, H. Mi yase; PICNS-69 (Asilomar), 137 (1969)

69Si 1 H. F,. Siefken, P. M. Cockburn, R. W. Krone; Nucl. Phys. A128, 162 ( 1969 )

$695 i 2$ I. Sick, E. B. Hughes, T. W. Donnelly, J. D. Walecka and G. E. Walker; Phys. Rev. Letters 23, 1117 (1969)

69 So 1 D. I. Sober, D. G. Cassel, A. J. Sadoff, K. W. Che n, P. A. Crean; Phys. Rev. Letters 22,430 (1969)

69 So 2 Yu. I. Sorokin, V. G. Shevchenko, B. A. Yur ev; Yad. Fiz. 9, 254 ( 1969 ); Sov. J. Nucl. Phys.9, 149 ( 1969 )

$69503 \mathrm{Yu}$. I. Sorokin, A. Kh. Shardanov, V. G. Shevchenko and B. A. Yur ev; Izv Akad. Nauk. Fiz, 33, 721 (1969), Bull. Acad. Sci. USSR-Phys. 33, 663 (1969)

69st1 R. J. J. Stewart, R. C. Morrison, D. E. Frederick; Phys. Rev. Letters 23, 323(1969)

69 Tal G. G. Taran; Yad. Fiz. 10, 211 (1969), Sov. J. Nucl. Phys. $10,119(1970)$

69 Th1 H. Theissen, R. J. Peterson, W. J.Alston, III, J. R. Stewart; Phys. Rev. 186, 1119 (1969)

69 Til Otto Titze; Z. Physik 220,66 ( 1969 ) 
$69 T i 2$ Yu. I Titov, E. V. Stepula, R, V. Akhmerov, S. A. Byvalin, N. F. Severin, E. M, Smelovand L.'D: $9,772(1969)$

69 To 1 M. Tomaselli, L. Grunbaum, G. A. Beer, H. G. Clerc, G. Witwer; Phys. Letters 29B, 579 ( 1969 )

69 To2 Y. Torizuka, M. Oyamada, K. Nakahara, K. Sugiyama, Y. Kojima, T. Terasama, K. Itoh, A. Yamaguchi, M. Kimura; Phys. Rev. Letters 22, 544 (1969)

69 To3 Y. Torizuka, Y. Kojima, M. Oyamada, K. Nakahara, K. Suk i yama, T. Te rasawa, K. I toh, A. Yamaguchi, M. Kimura; Phys. Rev. 185, 1499 ( 1969 )

69 To4 Y. Torizuka, A. Yamaguchi, K. Nakahara, N. Oyamada, I. Terasawa, K. Itoh, $Y$. Kojima and M. Kimura; Phys. Rev. $188^{2}, 1745^{\circ}(1969)$

69 Tsi H. Tsubota, N. Fujiwara, H. Ishimaru, E. Tanaka, T. Aizawa, M. Kanazawa, N. Mutsuro; J. Phys. Soc. Japan 26, 1 ( 1969$)$

69021 H. Ullrich, H. Krauth; Nucl. Phys. A123, 641 ( 1969 )

69Va1 G. J. Vanpraet, P. Kossanyi; Z. Physik 222, 455 ( 1969 )

$69 \mathrm{Va} 2$ \&. V. Vasilijev, G. N. Zalesny, S. F. Semenko, V. A. Semenov; Phys. Letters $30 \mathrm{~B}, 97^{\circ}$ ( 1969 )

69 Va 3 o. V. Vasil ev, G. N. Zalesnyi, S. F. Semenko, and V. A. Semenov; Yad. Fiz. 10, 460 (1969), Sov. J. Nucl. Phys. io, $263(1970)$

69Ve1 V.V. Verbinski, W. R. Burrus; Phys. Rev . 177, 1671 ( 1969 )

69 Ve2 A. Veysiere, H. Beil, R, Bergere, P. Carlos; C. R. Acad. Sc. Paris 268,981 (1969)

69 Wa 1 Y. Wakuta, M. Sonada, A. Katase, H. Tawa ra, M. Hyakutake; J. Phys. Soc. Japan 26, 851 ( 1969 )

69 the 1 D. V. Webb, E. G. Muirhead, B. M. Spicer; PICNS-69, $351(1969)$

69 Wi 1 G. Hiechers, W. R. McMurray, I. J. Van Heerden; Nucl. Phys. A 124,165 ( 1969 )

69 Wi2 G. Wittwer, H. G. Clerc, G. A. Beer; Phys. Letters $30 B$, $634(1969)$

69 wu 1 C. P. Wu, J. E. E. Baglin, F. W. K. Firk, T. W. Phillips; Phys. Letters 29B, 359 ( 1969 )

70Af $1 \mathrm{~V}$. D. Afanas ev, N. G. Afanas ev, A. Yu. Buki, G. A. Savitskii, V. M. Khvastunov, N . G. Shevchenko; Yad. Fiz. 12, 885 (1970); Sov. J. Nucl. Phys. 12, 480 ( 1971 )

7 CAhl J. Ahrens, H. Borchert, K. H. Czock, D. Mehlig and B. Ziegler; Phys. Letters $31 \mathrm{~B}, 570$ ( 1970 )

70 An 1 P. Antony-Spies, P. P. Delsanto, E. Spamer, A. Goldmann and $\theta$. Titze; Phys. Letters 31 B, 632 (1970)

70 An2 D. W. Anderson and S. C. Swift; Nucl. Phys. A150, 625 (1970)

$70 \mathrm{An} 3 \mathrm{D}$. W. Anderson, A. J Bureau, B. C. Cook, T. J. Englert, R. E. Schramm; Nucl. Phys. A156,74 ( 1970 )

70 An $4 \mathrm{Yu}$. P. Antufe ev, V. B. Ganenko, V. S. Kuz menko, V. L. Agranovich, I. I Miroshnichenko, V.M. Sanin, and $P$. V. Sorokin; Yad. Fiz. 11, 948 (1970) Sov. J.Nucl. Phys. 11 , 527 ( 1970$)$ 
70 An5 Yu. P. Antuf ev, V. L. Agranovich, V. G. Ganenko, V. S. Kuz menko, I, I: Miroshnichenko, $P$. V . Sorokin; Yad. Fiz. 12, 1143 (1970); Sov.J. Nucl. Phys. 12, 627 (1971)

70 Arl H. Arenhovel, J.M. Maison; Nucl. Phys. A147, 305 ( 1970 )

70 Ar2 Yu. M. Arkatov, P. I. Vatset, V. I. Volshchuk, V. V. Kirichenko, I. M. Prokhorets, A. F. Khodyachikh; Yad. Fiz. 12, 227 ( 1970 ); Sov.J.Nucl. Phys.12, 123 (1971)

70 Aul D. C. Aumann, J.E. Gindler; J. Ionorg. Nucl. Chem. 32, $731(1970)$

70 Ax1 P.Axel, K. K. Min, D. C. Sutton; Phys. Rev. C2, 689 ( 1970 )

70 Bal A. R. Barnett and N. W. Tanner; Nucl. Phys. A152, 257 ( 1970$)$

$70 \mathrm{Ba} 2$ G. M. Hailey, G. M. Griffiths, M. A. Olivo; R. L: Helmer; Can. J.Phys:48, $3059(1970)$

$70 \mathrm{Be} 1 \mathrm{~J}$. C. Hergstrom, H. Bertozzi, S. Kowalski, X. K. Maruyama, J. W. Lightbody, Jir, S. P. Fivozinsky, S. Penner; Phys. Rev. Letters 24, 152 (1970)

$70 \mathrm{Be} 2 \mathrm{~J}$. Bellicard, P. Leconte, T. H. Curtis, R. A. Eisenstien, D. Madsen and C. Bockelman; Nucl. Phys. A143, 213 (1970)

$70 \mathrm{Be} 3$ R. C. Bearse, J. C. Legg, G. C. Morrison, and R. E. Segel; Phys. Rev. C1, 608 ( 1970 )

70 Be4 B. D. Belt, C. R. Bingham, M. L. Halbert, A. Van Der Woude; Phys. Rev: Letters 24, 1120 (1970)

$70 \mathrm{Be} 5$ B. L. Berman, R. J Baglan, and C. D. Bowman; Phys. Rev. Letters 24, $319(1970)$

70 Be6 B. L. Berman, S. C. Fultz, and P. F. Yergin; Phys. Rev. Letters 24,1494 (i970)

$70 \mathrm{Ee} 7$ M. Berman and G. B. Beard; Phys. Rev. C2, 1506 ( 1970)

70 Be8 B. L. Berman, S. C.Fultz, J. T. Caldwell, M. A. Kelly, S. S. Dietrich; Phys. Rev. C2, 2318 ( 1970 )

70 Be 9 B. L. Berman, S. C. Fultz, and M. A. Kelly; Phys. Rev. Letters 25, $938(1970)$

$70 \mathrm{Bl} 1$ J. L. Black, W.J. Caelli, W. F. Davidson, R. B. Watson; Nucl. Phys. A153, 233 (1970)

70Bo1 C. D. Bowman, R. J.Baglan, B. L. Berman; Phys. Rev. Letters $24,193(1970)$

70 Bo2 C. D. Bowman, R. J.Baglan, B. L. Berman, T. W. Philips; Phys. Rev. Letters 25, 1302 ( 1970 )

$70 \mathrm{Bo} 3 \mathrm{H}$. Bock and H. Walenta; Z. Physik 238, 56 (1970)

70 Bo 4 M. Boivin, V. Zecevic; C. R. Acad. Sc. Paris 270, 1627 $(1970)$

$70 \mathrm{Br} 1 \mathrm{~V}$. K. Brovtsyn and R. M. Osokina; Izv. Akad. Nauk SSSR Ser. Fiz. 34,927 ( 1970 )

$70 \mathrm{Bu} 1 \mathrm{~L}$. Busso, S. Costa, L. Ferrero, R. Garfagnini, L. Pasqualini, G. Piragino, S. Rochi Della Rocca and $A$. Zanini; Lettere AL Nuovo Cimento III, 423 ( 1970 )

$70 \mathrm{Bu} 2 \mathrm{C}$. E. Burgart, E. A. Straker, T. A. Love, R. M. Freestone, Jr.; Nuci. Sci. and Engr.42, 421 (i970)

$70 \mathrm{Ce} 1 \mathrm{R}$. Cesareo, M. Giannini, P. R. Oliva, D. Prosperi, M. C. Ramorino; Nucl. Phys. Ai41; 561 ( 1970 )

70 Co1 B. C. Cook, R. C. Morrison, F. H. Schamber; Phys. Rev. Letters 25,685 (1970) 
$70002 \mathrm{~S}$. Costa, L. Ferrero, G. Garfagnini, L. Pasqualini, G. Piragino, A. Zanini; Nuovo Cimento $72 \mathrm{~A}, 517$ ( 1970 )

$70 \mathrm{Cu} 1 \mathrm{~L}$. A. Currie and R. H. Rodriguez-Pasques; Nucl. Phys. A 157, 49(1970)

$70 \mathrm{Dal}$ E. B. Dally, M. G. Croissiaux, B. Schweitz; Phys. Rev. C2, $2057(1970)$

70Del S. V. Dementii, N. G. Afanas ev, I. M. Arkatov, V. G. viasenko, V. A. Gol dshtein, E. L. Kuplennikov; Yad. Fiz. 11,19 ( 1970 ); Sov. J. Nucl. Phys. 11, 10 (1970)

$70 D i 1$ J. P. Didelez, H. Langevin-Joliot, Z, Maric and v. Radojevic; Nucl. Phys. A 143,602 (1970)

7CDo1 T. W. Donnelly Jr, J. D. Walecka, G. E. Walker, I. Sick; Phys. Letters $32 \mathrm{~B}, 545$ ( 1970 )

70 Erl B. Erlandsson and A. Marcinkowski; Nucl. Phys. A146, 43 ( 1970$)$

70 Es 1 G. P. Estes and K. Min; Phys.Rev. C1, 201 (1970)

70Fal L. W. Fagg, W. L. Bendel, S. K. Numrich and B. T. Chertok; Phys. Rev. C1, 1137 ( 1970 )

70Fil P. Filss, W. Collin, S. Gulabakke, K. Kayser, H, Reich, J. ब. Trier, W. Witschel; Z. Physik239, 461 ( 1970 )

70 Ful C. B. Fulmer, K. S. Toth, I. R. Williams, T. H. Handley, G. F. Dell, E. L. Callis, T. M. Jenkins, J.M. Wyckoff; Phys. Rev. C2, 1371 ( 1970 )

70 Fu2 K. Fukuda; Nucl. Phys. A 156, 10 (1970)

70Fu3 K. Fukuda, S. Okabe, Y. Sato; Ann. Rept Rad. Center of Osaka Prefecture 11,75 ( 1970 )

$70 \mathrm{Ga} 1 \mathrm{Yu}$. P. Gangrski1, B. N. Markov, Yu, M. Tsipenyuk; Yad. Fiz. 11, 54 (1970); Sov.J.Nucl. Phys. 11,30 (1970)

70 Gol A. Goldmann and M. Stroetzel; Phys. Letters $31 \mathrm{~B}, 287$ ( 1970$)$

7 CGo2 D. R. Goosman, E. G. Adelberger and K. A. Snover; Phys. Rev. C1, 123 ( 1970$)$

$70 \mathrm{Go}$ A. Goldmann; Z. Physik 234, 144 (1970)

70 Go4 A. Goldmann and M. Stroetzel; Z. Physik 239, 235 ( 1970)

70 Hel F. Heimlich and W. Mausberg; Z. Physik 231,397 (1970)

70 He2 J.H. Heisenberg and I. Sick; Phys. Letters 32B, 249 ( 1970$)$

70 il R. S. Hicks and B. M. Spicer; Nucl. Phys. A159, 265 ( 1970$)$

70 Ho 1 Y. S. Horowitz, D. B. McConnell, J. Ssengabi, N. Keller; Nucl. Phys. Al51, 161 ( 1970 )

$70 \mathrm{Hul}$ R. M. Hutcheon, R. Neuhausen, F. Eigenbrod; $Z$. Naturforsch 25A, 973 ( 1970$)$

70 Hyl Gunnar Hylten; Nucl. Phys. A158, 225 (1970)

70 Is 1 B. S. Ishkhanov, I. N. Kapitonov, E. v. Lazutin, I M. Piskarev, $\beta$. P. Shevchenko; Yad. Fiz. 11,702 (1970); Sov. J. Nucl.Phys. 11, 394 ( 1970 )

$70 I s 2$ B. S. Ishkhanov, I. M. Kapitonov, E. V. Lazutin, I. M. Piskarev, V. S. Sopov, V. G. Shevchenko; Yad. Fiz. 12 , 224 ( 1970); Sov.J. Nucl. Phys.12, 121 ( 1971 )

$70 I s 3 \mathrm{~B}$. S. Ishkhanov, I. M. Kapitonov, E. V. Lazutin, I. M. Piskarev, $\theta$. P. Shevchenko; Yad. Fiz. 12, 682 (1970); Sov. J. Nucl. Phys. 12, 370 (1971) 
$70 I s 4$ B. S. Ishkhanov, I M. Kapitonov, I. M. Piskarev, V. G. Shevchenko, $\theta$. P. Shevchenko; Yad. Fiz. 11, 485 (1970); Sov. J. Nucl. Phys. 11, 272 (1970)

70 Is5 B. S. Ishkhanov, I. M. Kapitonov, E. V. Lazutin,; I. M. Piskarev, V.S. Sopov and V. G. Shevchenko; Yad. Fiz. 12,892 ( 1970 ) Sov. J. Nucl. Phys. 12,484 ( 1971 )

70 Is6 B. S. Ishkhanov, I. M. Kapitonov, E. V. Lazutin, I. M. Piskarev, V. G. Shevchenko; Ser. 3 Fiz: Astron. 11:606 ( 1970$)$

$70 I t 1$ A. Ito, R. Loe, E. C. Loh, A. Ramanauskas, D. Ritchie, W. Schmidt; Phys. Rev. Letters 24,687 (1970)

$70 \mathrm{It} 2 \mathrm{~K}$. Itoh, M. Oyamada, Y. Torizuka; Phys, Rev, C2, 2181 ( 1970$)$

$70 \mathrm{Ja} 1 \mathrm{R}$. J Jaszczak, J H. Gibbons, R. L. Macklin; Phys. Rev. C2, $63(1970)$

$70 \mathrm{Ja} 2 \mathrm{~K}$. J.Jaszczak and R. L. Macklin; Phys. Rev. C2, 2452 ( 1970$)$

70 Jo1 G. G. Jonsson and K. Lindgren; Nucl. Phys. A141, 355 ( 1970 )

$70 J 02$ G. G. Jonsson and B. Persson; Nucl. Phys. A153, 32 ( 1970)

$70 J u 1$ J.W. Jury, J. S. Hewitt, K. G. McNeill; Can. J. Phys. 48 , 1635 ( 1970 )

70Kal V. I. Kasilov, Yu, N. Ranyuk, P. V. Sorokin; Yad. Fiz. 11, 1324 ( 1970 ); Sov. J.Nucl. Phys. 11,736 ( 1970 )

$70 \mathrm{Ka} 2 \mathrm{~K}$. Kayser, W. Collin, P. Filss, S. Guldbakke, G. Nolte, H, Reich, J.' $\theta$. Trier, W. Witschei; Z. Physik 239, 447 ( 1970 ) $70 \mathrm{Ka} 3 \mathrm{~T}_{576(1970)} \operatorname{Kato}_{\text {. }}$. T. Tsai, Y. Oka; Bull. Chem. Soc. Japan 43,

$70 \mathrm{Ke} 1 \mathrm{~K}$. G. Kernbach; Lettere Al Nuovo Cimento 3,461 (1970)

70 Ke2 P. F. Kenealy, G. B. Beard, K. Parsons; Phys. Rev. C2, 2009 ( 1970$)$

7CKhl V. M. Khvastunov, N. G. Afanas ev, V. D. Afanas ev, E. V. Bondarenko, I: S. Gul karov, G, A. Savitskii, $N$. G. Shevchenko; Yad. Fiz. 12,9 (1970); Sov. J. Nucl. Phys. 12, 5 ( 1971$)$

70 Kil J. C. Kim, R. P. Singhal, H. S. Caplan; Can. J. Phys. 48, $83(1970)$

$70 \mathrm{Ki} 2$ I. Kimura, S. A. Hayashi, K. Kobayashi, S. Yamamoto, T. Shibata; Ann. Repts Res. Reactor Inst. Kyoto University 3 , 75 ( 1970$)$

7CKol A. Kosiara and H. B. Willard; Phys. Letters 32B, 99 (1970)

$70 \mathrm{Ku} 1 \mathrm{H} . \mathrm{M}$. Kuan, M. Hasinoff, W. J. $\theta$ Connell, S. S. Hanna; Nucl. Phys. A151, 129 (1970)

$70 \mathrm{Ku} 2 \mathrm{~L} . \mathrm{A}, \mathrm{Kull}, \mathrm{R}$. L. Bramblett, T, Gozani, D. E. Rundquist; Nucl. Sci. and Eng. 39, $163^{\circ}$ ( 1970 )

$70 \mathrm{Li} 1 \mathrm{~J}$. W. Lightbody, Jr., S. Penner; Phys. Rev. Letters 24,274 ( 1970$)$

$70 \mathrm{Li} 2 \mathrm{~J}$. W. Lightbody Jr.; Phys. Letters 33B, 129 ( 1970 )

70 Mcl K. G. McNeill, J.W. Jury, J. S. Hewitt; Can. J. Phys. 48, $950(1970)$

$70 \mathrm{Mel}$ M. A. Meyer, N. S. Wolmarans, D. Reitmann; Nucl. Phys. A144, 261 ( 1970 )

70 Me2 W. E. Meyerhof, M. Suffert and W. Feldman; Nucl. Phys. A $148,211(1970)$ 
70 Ne3 F. R. Metzger; Nucl. Phys. A158, 88 ( 1970 )

$70 \mathrm{Me} 4 \mathrm{H}$. A. Medicus, E. M. Bowey, D. B. Gaythe r, B. H.

Patrick, E. J. Winhold; Nucl. Phys. A156, 257 (1970)

70 Me5 T. Methasiri; Nucl. Phys. A158, 433 ( 1970 )

70 Ne6 F. R. Metzger; Phys. Rev. C2, 2024 (1970)

70 Mo1 R. Moreh, A. Nof, A. Wolf; Phys. Rev. C2, 249 ( 1970)

70 Mo2 R. Moreh, S. Shlomo, A. Wolf; Phys. Rev. C2, 1144 ( 1970 )

$70 \mathrm{Mo3}$ R. Moreh and A. Nof: Phys. Rev. C2, 1938 (1970)

70 Mo4 R. Moreh and $\theta$. Shahal; Phys.Rev. C2, 2217 (1970)

70 Mu 1 A. Murakami; J Phys. Soc. JAP AN 28, 1 ( 1970 )

70 ew 1 D. G. Owen, E. G. Muirhead, B. M. Spicer; Nucl. Phys. A 140,523 ( 1970$)$

$700 w 2 \mathrm{D}, \mathrm{G}$. Gwen, Ph.D. Thesis, University of Melbourne, 1970 .

$70 P e 1$ R. J. Peterson, H: Theissen, and W. J. Alston; Nucl. Phys. A 143, 337 ( 1970$)$

$70 P e 2$ R. J. Peterson, H. Theissen, W. J. Alston; Nucl. Phys. A153, $610(1970)$

70Pi 1 R. Pitthan; Z. Naturforsch. 25A, 1358 (1970)

70 Ka 1 N. S. Rabotnov, G. N. Smirenkin, A. S. Soldatov, L. N. Usachev, S. P. Kapitza, Yu. M. Tsi penyuk; Yad. Fiz. 11 , 508 (1970); Sov. J. Nucl. Phys. 11, 285 (1970)

70 Ri 1 G. Ricco, T. E. Drake, L. Katz, and H. S. Caplan; Phys. Rev. C1, 391 (1970)

$70 S a 1$ J. Sanada, M. Yamanouchi, N. Sakai and S. Seki; J. Phys. Soc. JAPAN 28, 537 (1970)

70 Sa 2 M. Sanzone, G. Ricco, S. Costa, L. Ferrero; Nucl. Phys. A 153,401 ( 1970$)$

70 Sc1 B. Schroder, G. Nydahl, and B. Forkman; Nucl. Phys. A 143,449 (1970)

70 Sc2 P. E. Scheffler, P. L. Walden; Phys. Rev. Letters 24,952 (1970)

$70 S c 3$ H. Schroder and W. Mausberg; Z. Physik 235.234 (1970)

70 Sel S. F. Semenko, $\theta$. V. Vasilijev and V. A. Semenov; Phys. Letters $31 \mathrm{~B}, 429(1970)$

7osh 1 N. K. Sherman, R. W. Gellie, K. H. Lokan and R. G. Johnson; Phys. Rev. Letters 25, 114 (1970)

70 Sh2 N. Shikazo no and Y. Kawarasaki; Phys. Letters 32B, 473 (1970)

$70 \sin 3$ Y. M. Shin, J.A. Rawlins, W. Buss, A. Q. Evwaraye; Nucl. Phys. A154, 482 (1.970)

70 Sol Yu. I. Sorokin, A. Kh. Shardanov, V. G. Shevchenko, B. A. Yur ev; Yad. Fiz. 11, 8 (1970); Sov: J. Nucl. Phys. 11, 4 ( 1970$)$

70 So2 A. S. Soldatov, Yu, M. Tsipenyuk, G. N. Smirenkin; Yad. Fiz. 11,992 (1970) Sov.J. Nucl. Phys. 11, 552 (1970)

70St 1 M. Stroetzel and A. Goldmann; Z. Physik 233, 245 ( 1970)

70 t 2 P. Strehl; Z. Physik 234, 416 (1970)

70 Sul R. E. Sund, V. V. Verbinski, H. Weber, L. A. Kull; Phys. Rev. C2, $1129(1970)$

$70 S z 1$ H. Szichman, Y. Schlesinger, G. Ben-David, D. Pavel; Nucl. Phys. A148, 369 (1970) 
70 Th 1 M. N. Thompson, R. J. J . Stewart and J E. M. Thompson; Phys. Letters $31 \mathrm{~B}, 211$ (1970)

$70 \mathrm{Til}$ d. Titze, A. Goldmann and E. Spamer; Phys. Letters $31 \mathrm{~B}$, 565 ( 1970$)$

$70 \mathrm{Ii}$ V. G. Tikhonov, V. G. Shevchenko, V. Ya. Galkin et al. Ser. III. Fiz. Astron.11,208 (1970)

7CTo1 Y. Torizuka, A. Yamaguchi, T. Terasawa, Y. Kojima, K. Nakahara, M. Ayamada, K. Itoh, A. Nakada, S. Mitunobu, M. Kimura; Phys. Rev. Letters 25, 874 ( 1970 )

70 To2 S. G. Tonapetyan, $\theta$, G. Konovalov, A. I. Derebchinskii, A. A. Zybalov, V. M. Khvorostyan, N. V. Goncharov, V. A. Gol dshtein; ZhETF Pis. Red. 11,165 (1970); JETP Letters 11,101 ( 1970$)$

$70 \mathrm{Val} \theta$. V. Vasil ev and V.A. Semenov; ZhETFPis. Red. 11,520 (1970); JETP Letters 11,356 ( 1970 )

70 Vel A. Veyssiere, H. Beil, R. Bergere, P. Carlos, A. Lepretre; Nuc 2. Phys.A159, 561 ( 1970 )

70 Vo $1 \mathrm{U}$. Von Wimmersperg, G. Kernel, B. W. Allardyce, W. M. Mason, N. W. Tanner; Phys. Letters 33B, 291 (1970)

70 Vyl A. V. Vysotskaya and N. G. Afanas ev; Yad. Fiz. 11,942 ( 1970); Sov. J. Nucl. Phys. 11, 523 (1970)

70 Wa 1 T. Walcher and P. Strehl; Z. Physik 232, 342 ( 1970)

70 Wa2 G. D. Wait, S. K. Kundu, Y. M. Shin, W. F. Stubbins; Phys. Letters 33B, 163 (1970)

70 ha3 w. B. Walters, J. R. Van Hise, W. L. Switzer, J.P. Hummel; Nucl. Phys. A157, 73 ( 1970 )

70 Ta 4 Th. Walcher; Z. Physik 237, 368 (1970)

70 tas Ih. Walcher; Phys. Letters $31 \mathrm{~B}, 442$ (1970)

70 the 1 D. V. Webb, E. G. Muirhead, B. M. Spicer; Nucl. Phys. A $159,81(1970)$

70 Wil G. Wittwer, H. G. Clerc and G. A. Beer; Z. Physik 234, $120(1970)$

70W12 E. J. Winhold, E. M. Bowey, D. B. Gayther, B. H. Patrick; Phys. Letters 32B, 607 (1970)

70 Wol C. F. Wong, R. M. Hutcheon, Y. M. Shin, H. S. Caplan; Can. J. Phys. 48, 1917 (1970)

70Wul C. P. Wu, F. W. K. Firk, T. W. Phillips; Nucl. Phys. A $147,19(1970)$

70 tu2 C. P. Wu, F. W. K. Firk, B. L. Berman; Phys. Letters $32 \mathrm{~B}, 675^{\circ}(1970)$

70 Yo1 A. M. Young, S. L. Blatt, R. G. Seyler; Phys. Rev. Letters 25, $1764(1970)$

71 Ad 1 J. O. Adler, G. Andersson, B. Forkman, G. G. jonsson, $K$. Lindgren; Nucl. Phys. A171,560 (1971)

71 Ahl J. Ahrens, H. Borchert, H. B. Eppler, H. Gimm, H. Gundrum, P. Riehn, G. Sita Ram, A. Zieger, B. Ziegler; Elba-71, Tagungsbericht Elektronen Beschleuniger Arbeits Gruppen ( Sept. 1971); Justus Liebig Universitat Giessen.

$71 A L 1$ R. A. Alvarez, B. L B Berman, D. R. Lasher, T. W. Philitips, S. C. Fultz; Phys. Rev. C4, 1673 ( 1971 )

71 An 1 Yu. P. Antuf ev, V. L. Agranovich, V. B. Ganenko, V. S. Kuz menko, I. I. Miroshnichenko, P. V. Sorokin; Yad. Fiz. 13.473 (1971); Sov. J. Nucl. Phys. 13, 265 (1971) 
71 An2 Yu. P. Antuf ev, V. L. Agranovich, V. B. Ganenko, V. S. Kuz menko, I. I, Miroshnichenko, P. V. Sorokin; Yad. Fiz. 14, 898 ( 1971 ); Sov. J. Nucl. Phys. 14,502 (1972)

71 Ar 1 Yu. M. Arkatov, P. I. Vatset, V. I. Voloshchuk, V. V. Kir ichenko, I. M. Prokhorets, A. G. khodyachikh, Yad. Fiz. 13, 256 (1971); Sov.J.Nucl.Phys.13, 142 (1971)

$71 \mathrm{Ba} 1 \mathrm{G}$. Baciu, D. Catana, C. Deberth, C. Iliescu, B. Carstoiu; Nucl. Phys. A167, 177 ( 1971 )

$71 \mathrm{Ba} 2 \mathrm{R}$. J. Baglan, C. D. Bowman and B. L. Berman; Phys. Rev. C3, 672 (1971)

$71 \mathrm{Ha3}$ D. F. Bartlett, C. E. Friedberg, P. e. Goldhagen K. Goulianos; Phys. Rev. Letters 27,881 ( 1971 )

$71 \mathrm{Ba} 4 \mathrm{~K}$. Battleson, D. K. McDaniels; Phys.Rev. C4, 1601 (1971)

71 Be 1 W. L. Bendel, L. W. Fagg, S. K. Numrich, -E. C. Jones Jr., and H. F. Kaiser; Phys. Rev. C3, 1821 ( 1971 )

$71 \mathrm{Be} 2 \mathrm{~J}$. C. Bergstrom, H. Crannell, F. J. Kline, J. T. g Brien, J. W. Lightbody,Jr., S. P. Fivozinsky; Phys. Rev, C4, 1514 (1971)

71 Be3 B. L. Berman, S. C. Fultz, M. A. Kelly; Phys. Rev. C4, $723(1971)$

71 Be 4 H. Beil, R. Bergere, P. Carlos, A. Lepretre, A. veyssiere, A. Parlag; Nucl. Phys. A172, 426 ( 1971 )

71 Fl I I. Blomquist, G. Nydahl and B. Forkman; Nucl. Phys. A $162,193(1971)$

71 Bol M. Boivin, Y. Cauchois, Y. Heno; Nucl. Phys. A176, 626 (1971)

$71 \mathrm{Br} 1$ E. Bramanis; Nucl. Phys. A175, 17 (1971)

$71 \mathrm{Bu} 1 \mathrm{~L}$. Busso, R. Garfagnini, G. Piragino, S. Ronchi della Rocca, A. Zanini; Lettere al Nuovo Cimento 1, 941 ( 1971 )

$71 \mathrm{Bu} 2$ A. Bussiere, J. Mougey, Phan Xuan Ho, M. Priou, I. Sick; Lettere al Nuovo Cimento 2, 1149 ( 1971 )

71 Cal P. Carlos, H. Beil, R. Bergere, A. Lepretre, A. Veyssiere; Nucl. Phys. A172, 437 ( 1971 )

$71 \mathrm{Ch} 1 \mathrm{~A}$. Chevallier, E. Bozek, A. Pape, J.C. Sens, R. Armbruster. M. Langevin, J. Vernotte; Nucl. Phys. A176, 401 ( 1971$)$

71 Col S. Costa, L. Ferrero, L. Pasqualini, M. Sanzone; Lettere al Nuovo Cimento 1,448 ( 1971 )

$71 \mathrm{Co} 2 \mathrm{~s}$. Costa, L. Ferrero, L. Pasqualini, E. Mancini; Lettere al Nuovo Cimento 2, $665^{\circ}$ ( 1971 )

71 Co3 R. G. Couch, H. Spinka, T. A. Tombrello, T. A. Weaver; Nuc 2. Phys. A175, 300 ( 1971 )

$71 \mathrm{Da} 1 \mathrm{H}$. Dahmen, F. Dreyer, J. Staude and H. H. Thies; Nucl. Phys.A164, 140 ( 1971 )

$71 \mathrm{Da} 2$ W. F. Davidson, J. L, Black. M. R. Najam, and B. M. Spicer; Nucl. Phys. A 168,399 ( 1971 )

$71 \mathrm{Di} 1 \mathrm{~V}$. DiNapoli, A. M. Lacerenza, D. Margadonna, F. Salvetti, H. G. De Carvalho, J. Benuzzi Martins; Lettere al Nuovo Cimento 1,65 ( 1971 )

71 Di2 V. DiNapoli, F. Salvetti, H. G. DeCarvalho, J. Benuzzi Martins; Lettere al Nuovo Cimento 1, 538 ( 1971 )

$71 \mathrm{Di} 3 \mathrm{~V}$. DiNapoli, A. M. Lacerenza, Carvalho, J: Benuzzi Martins; $1,835$ ( 1971$)$

F. Salvetti, H. G. de

Lettere al Nuovo Cimento 
$71 \mathrm{Di} 4 \mathrm{E} . \mathrm{M}$. Diener, J. F. Amann, P. Paul, S. L. Blatt; Phys Rev. C3, $2303(1971)$

71 Do1 E. J. Dowdy, T. L. Krysinski; Nucl. Phys. A175, 501 ( 1971$)$

$71 \mathrm{Em} 1 \mathrm{~V}$. Emma, S. Lo Nigro, C. Milone; Lettere al Nuovo Cimento 2, $117(1971)$

$71 \mathrm{Em} 2 \mathrm{~V}$. Emma, S. Lo Nigro, C. Milone; Lettere al Nuovo Cimento $2,271(1971)$

$71 \mathrm{Fa} 1 \mathrm{~L}$. W. Fagg, W. L. Bendel, L. Cohen, E. C. Jones, Jr., H. F. Kalser, H. Uberall; Phys. Rev. C4, 2089 ( 1971 )

71 Frl B. Friberg, G. Andersson, B. Forkman; Nucl. Phys. A171, 551 ( 1971 )

$71 \mathrm{Ful} \mathrm{C.} \mathrm{B.} \mathrm{Fulmer,} \mathrm{K.} \mathrm{S.} \mathrm{Toth,} \mathrm{I.} \mathrm{R.} \mathrm{Williams,} \mathrm{G.} \mathrm{F.} \mathrm{Dell,} \mathrm{a} \mathrm{nd}$ T. M. Jenkins; Phys. Rev? C3, 1955 (1971)

71 Fuz S. C. Fultz, R. A. Alvarez, B. L, Berman, M. A. Kelly, D. R. Lasher, T. W. Phillips, J. McElninney; Phys, Rev. C4, $149(1971)$

$71 \mathrm{Fu} 3 \mathrm{~T}$. Fuji $\mathrm{i}$, Hkuno, S. Orito, H, Sasaki, T, Nozaki, F. Takasaki, K. Takikawa, K. Amako, I. Endo, K. Yoshida, M. Higuchi, M. Sato, Y. Sumi; Phys. Rev. Letters 26, $1672(1971)$

71 Fu4 C. B. Fulmer, K. S. Toth, I. R. Williams, G. F. Dell; Phys. Rev. C4, 2123 (1971)

71 Go 1 N. V. Goncharov, A. I Derebchinski i, A. A. Zybalov, 0. G. Konoval ov, A. E T enishev, S. G. Tonapetyan, V. M. Khvorostyan; Yad. Fiz. 14,673 (1971); Sov. J? Nucl. Phys. $14,377$ ( 1972$)$

$71 \mathrm{Go} 2 \mathrm{~N}$. V. Goncharov, A. I. Derebchinski i, O. P. Konovalov, S. G. Tonapetyan, V. M. Knvorostyan; Yad. Fiz. 14, 31 (1971) Sov. J. Nucl. Phys.; 14, 18 ( 1972 )

$71 \mathrm{Gr} 1 \mathrm{~J}$. L. Groh, R. P. Singhal, H. S. Caplan, B. S. Dolbilkin; Can. J. Phys. 49, 2743 (1971)

$71 \mathrm{Gr} 2$ I. A. Grishaev, A. N. Krinitsyn, N. I. Lapin, V. I. Nikiforov, G. D. Pugachev, B. I. Shramenko; Yad: Fiz. 14 , 35 (1971); Sov. J. Nucl. Phys. 14, 20 (1972)

$71 \mathrm{Hal}$ M. Hass, R. Moreh, D. Salzmann; Phys. Letters 36B, 68 (1971)

$71 \mathrm{Ha} 2 \mathrm{P}$. E. Haustein, A. F. Voigt; J.Inorg. Nucl. Chem. 33, 289 ( 1971 )

71 He $1 \mathrm{~J}$. Heisenberg, J. S. McCarthy and I. Sick; Nucl. Phys. A164, $353(1971)$

71 Hol Y. Horikawa, Y. Torizuka, A. Nakada, S. Mitsunobu, Y. Kojima, M. Kimura; Phys. Letters 36B, 9 (1971)

71 Ig 1 A. V. Ignatyuk, N. S. Rabotnov, G. N. Smirenkin, A. S. Soldatov, Yu. M. Tsipenyuk; Zh. Eksp. Teor. Fiz, 61, 1284 ( 1971 ); Sov. Phys. JETP 34, 684 (1972)

71 Is 1 B. S. I shkhanov, I. M. P1skarev, I. M. Kapitonov, E. V. Lazutin, V. S. Sopov, V. G. Shevchenko; Yad. Fiz. 13 , 1141 ( 1971 ); Sov. J. Nucl. Phys.13,655 (1971)

$71 \mathrm{Is} 2$ B. S. Ishkhanov, I. M. Kapitonov, I. M. Piskarev, $\theta$. P. Shevchenko; Yad. Fiz. 14, 27 (1971); Sov. J. Nucl. Phys. $14,16(1972)$

71 Is 3 B. S. Ishkhanov, I. M. Kapitonov, I. M. Piskarev, V. G. Shevchenko; Yad. Fiz. 14, 253 (1971); Sov.J. Nucl. Phys. 14, $142(1972)$

$71 \mathrm{Ja} 1$ H. E. Jackson, E. N. Strait; Phys, Rev, C4, 1314 (1971) 
$71 \mathrm{Ja}$ H. E. Jackson, E. N. Strait; Statistical Properties of Nuclei, ed. J. B. Garg, Plenum Publishing Corporation, New York ( 1971$)$ p. 601 .

$71 \mathrm{Ke} 1 \mathrm{G}$. Kernel, W. M. Mason, U. Von Wimmersperg; Nucl. Phys. A 167,352 ( 1971 )

$71 \mathrm{Ki} 1$ I. Kimura, S. A. Hayashi, K. Kobayashi, S. Yamamoto, T. Shibata; J. Nucl. Sci. and Tech. 8, 173 (1971)

$71 \mathrm{Ku} 1 \mathrm{H}$. M. Kuan, C. J. Umbarger and D. G. Shirk; Nucl. Phys. A160, 211 ( 1971$)$

$71 \mathrm{Ku} 2 \mathrm{G}$. J. Kumbartzki, Ung Kin and Ch. K. Kwan; Nucl. Phys. A $160,237(1971)$

$71 \mathrm{Ku} 3 \mathrm{~S}, \mathrm{~K}$. Kundu, Y. M. Shin, G. D. Wait; Nucl. Phys, A171, 384 (1971)

$71 \mathrm{Ku} 4 \mathrm{~V}$. M. Kuznetsov, O. I. Stukov, E. V. Repenko, V. D. Epaneshnikov, $V$. N . Zabaev, A. P. Potylitsyn; Yad. Fiz. 13, 1052 (1971); Sov.J. Nucl. Phys.13,603 (1971)

$71 \mathrm{Ku} 5$ G. Kumbartzki, U. Kim; Nucl. Phys. A176, 23 (1971)

71 Le 1 A. Lepretre, H. Beil, R. Bergere, P. Carlos, A. Veyssiere; Nuc l. Phys. A175, 609 ( 1971 )

$71 \mathrm{Li} 1 \mathrm{G}$. C. Li, I. Sick, R. R. Whitney and M. R. Yearian; Nucl. Phys. A 162, 583 ( 1971 )

$71 \mathrm{Li} 2 \mathrm{~K}$. Lindgren and G. G. Jonsson; Nucl. Phys. A166, 643 ( 1971 )

$71 \mathrm{Li} 3$ S. C. Ling, S. L. Blatt; Nucl. Phys.A174, 375 (1971)

$71 \mathrm{Ma} 1 \mathrm{D}$. W. Madsen, L. S. Cardman, J. R. Legg, C. K. Bockelman; Nucl. Phys.A168,97 (1971)

$71 \mathrm{Ma}$ J. Maly; Phys. Letters 35B, 148 (1971)

$71 \mathrm{Ma} 3 \mathrm{~A}$. Manfredini, L. Fiore, C. Ramorino, W. Wolfli; Nuovo Cimento $4 \mathrm{~A}, 421$ ( 1971 )

71 Mel T. Methasiri and S. A. E. Johansson; Nucl. Phys. A167, $97(1971)$

71 Me2 F. R. Metzger; Ann. Phys.66, 697 ( 1971 )

$71 \mathrm{Mi} 1 \mathrm{H}$. G. Miller, W. Buss, and J.A. Rawlins; Nucl. Phys. A 163,637 (1971)

$71 \mathrm{Mo2}$. Moreh, A. Nof, O. Shahal, A. Wolf; Phys. Letters 36B, $71(1971)$

$71 \mathrm{Mo} 3 \mathrm{E}$. J. Moniz, I. Sick, R, R. Whitney, J R. Ficenec, R, D. Kephart, W. P. Trower; Phys. Rev. Letters 26,445 ( 1971 )"

71 Mo4 R. Moreh, A. Nof; Phys. Rev. C4, 2265 (1971)

71 Mul K. M. Murray and M. E. Toms; Lettere al Nuovo Cimento $1,571(1971)$

$71 \mathrm{Nal} \mathrm{A.} \mathrm{Nakada,} \mathrm{Y.} \mathrm{Torizuka,} \mathrm{Y.} \mathrm{Horikawa;} \mathrm{Phys.} \mathrm{Rev.} \mathrm{Letters}$ 27,745 (1971); See Erratum- Phys, Rev. Letters 27, 1102 ( 1971 )

$71 \mathrm{Na} 2 \mathrm{M}$. Nagao, Y. Torizuka; Phys. Letters 37B, 383 ( 1971)

71 Nel 1 . Neuhausen and R. M. Hutcheon; Nucl. Phys. A164, 497 ( 1971 )

$71 \mathrm{~Pa} 1$ B. H. Patrick, H. A. Medicus, G. K. Menta, E. M. Bowey, and D. B. Gayther; Phys. Letters $34 \mathrm{~B}, 488$ ( 1971 )

$71 \mathrm{~Pa} 2 \mathrm{D}$. Pavel, G. Ben-David, Y. Schlesinger, H. Szichman; Nucl. Phys. A160, 409 ( 1971 )

71 Pa3 P. Paul, J F. Amann, K. A. Snover; Phys. Rev Letters $27,1013(1971)$ 
$71 \mathrm{Pe} 1$ R. A. Petrzhak, V. F. Teplykh, M. G. Pan yan, V. A. Demin; Yad. Fiz. 14,950 (1971); Sov.J. Nucl. Phys. 14, 532 ( 1972 )

$71 \mathrm{Pi} 1$ R. Pitthan and Th. Walcher; Phys. Letters 36B, 563 (1971)

$71 \mathrm{Fi} 2 \mathrm{M}$. Piiparinen, A. Anttila, M. Viitasalo; Z. Physik 247, 400 (1971)

71 Ral B. S. Ratner; ZhETF Pis. Red.13,628 ( 1971 ); JETP Letters 13,447(1971)

71 Kil F. Riess, W. J. O Connell, P. Paul; Nucl. Phys. A175, 462 (1971)

71 Sal Tatsuya Saito; Nippon Kagaku Zasshi 92, 164 (1971)

$71 \mathrm{Sa}$ Tatsuya Saito; Bull. Chem. Soc. Japan 44, 1800 ( 1971 )

71 Sc1 B. L. Schrock, R. P. Haddock, J. A. Helland, M. J. Longo S. S. Wilson, K. K. Young, D, Cheng, V. Perez-Mendez;

Phys. Rev. Letters 26, 1659 ( 1971 )

71 Sc2 M. Schaeffer, M; Suffert, D. Magnac-Valette; Nucl. Phys. A $175,217(1971)$

71 Sh1 Y. M. Shin, C. F. Wong, H. S. Caplan; Nucl. Phys. A166, $162(1971)$

$71 \mathrm{Sh} 2 \mathrm{~K}$. Shoda, A. Suzuki, M. Sugawara, T. Saito, H. Miyase and S. Oikawa; Phys. Rev. C3, 1999 (1971)

$71 \mathrm{Sh} 3 \mathrm{~K}$. Shoda, T. Saito, M. Sugawara, H. Miyase, S. Oikawa, A. Suzuki; Phys. Rev. C4, 1842 (1971)

71 Sh4 A. Shapiro, W. F. Stubbins; Nucl. Sci. and Engr. 45,47 (1971)

71 Sh5 T. Shintomi, C. Yamaguchi, M. Masuda; J. Phys. Soc. Japan 31, 1297 ( 1971 )

71 Sh6 Yu. K. Shubnyi, Yu. A. Lysikov; Izv. Akad. Nauk SSSR Fiz. 35, 1651 (1971)

71 Sil J J Simpson, W. R. Dixon, R. S. Storey; Phys. Rev. C4, $443(1971)$

71 Sn 1 K. A Snover, J. M. McDonald, D. B. Fossan; Phys. Rev. C4, $398(1971)$

71 sn2 K. A. Snover, J. F. Amann, W. Hering, P. Paul; Phys. Letters $37 \mathrm{~B}, 29(1971)$

71 So 1 Yu. I. Sorokin, V. A. Khrushchev, B. A. Yur ev; Yad. Fiz. 14, 1118 (1971); Sov.J. Nucl. Phys.14,622 (1971)

71 Sw 1 C. P. Swann; Nucl. Phys. A167, 201 (1971)

71 Sw2 C. P. Swann; Phys. Rev.C4, 1489 ( 1971 )

71 Sw3 C. P. Swann; Nucl. Phys. A 172,569 ( 1971 )

71 Tal B. Tamain, B. Pfeiffer, H. Wollnik, E. konecny; Nucl. Phys. A $173,465(1971)$

$71 \mathrm{Ti} 1 \mathrm{~K}$. Tietze, H. Riech and J. O. Trier; Z. Physik 242, 328 ( 1971 )

$71 \mathrm{Um} 1 \mathrm{C}$. J. Umbarger, D. A. Close, W. L. Sievers, and R. C. Bearse; Phys. Rev. C3, 199 ( 1971 )

$71 \mathrm{Va} 1 \mathrm{~A}$, van der Woude, $M$. L Halbert, $C$, R. Bingham, and B. D. Belt; Phys. Rev. Letters 26,909 ( 1971 )

$71 \mathrm{Va}$ o. V. Vasil ev, V. A. Semenov, S. f. Semenko; Yad. Fiz. 13, 463 (1971); Sov.J. Nucl. Phys. 13, 259 ( 1971 )

$71 \mathrm{Va} 3 \mathrm{G} . \mathrm{J} . \mathrm{C}$. Van Niftrik, L. Lapikas, H. DeVries, G. Box; Nucl. Phys. A174, 173 ( 1971 ) 
71 Va4 G. A. Vartapetyan, N. A. Demekhina, V. I. Kasilov, Yu. N. Ranyuk, P. V. Sorokin, A. G. Khudaverdyan; Yad. Fiz. 14, 65 (1971); Sov.J. Nucl. Phys.14, 37 (1972)

71 Vel E. Ventura, C. C. Chang, W. E. Meyerhof; Nucl. Phys. A $173,1(1971)$

$71 \mathrm{Vl} 1 \mathrm{~V}$. G. Vlasenko, N. G. Afanas ev, V. a. Gol dshtein, S. V. Dementi i, E. L: Kupl ennikov; Yad. Fiz. 13, 259 (1971); Sov. J. Nucl. Phys. 13, 144 ( 1971 )

71 Vol H. J. Von Eyss, H. Schier, B. Schoch; Elba-71, Taungsbericht Elektronen Beschleuniger Arbeits Gruppen; ( Sept. 1971) Justus Liebig-Universitat Giessen.

71 Wal Yoshihisa Wakuta; J. Phys. Soc. Japan 31, 12 ( 1971)

71 Wel D. V. Webb, E. G. Muirhead, B. M. Spicer; Nucl. Phys. Ai $71 ; 324$ ( 1971 )

71 We 2 B. Weissman and H. L. Schultz; Nucl. Phys. A174, 129 (1971)

71 Hol M. R. Wormald, I. F. Wright; Reprinted from Particles and Nuclei: 2, 168 ( 1971 )

71 Yal C. S. Yang, E. L. Tomusiak, R. K. Gupta, H. S. Caplan; Nuc 2. Phys: Ai62, 71 (1971)

71 Ya2 A. Yamaguchi, T. Terasawa, K. Nakahara, and $\mathrm{Y}$. Torizuka; Phys. Rev. C3, $1750^{\circ}$ (1971)

$71 \mathrm{Zhl}$ E. A. Zhagrov, Yu, A. Nemilov, N. V. Nikitina, Yu. A. Selitskii; Yad. Fiz. 13,934(1971); Sov.J. Nucl. Phys.13, 537 ( 1971 )

$72 A l 4$ T. K. Alexander, B. Y. Underwood, N. Anyas-Weiss, N. A. Jelley, J. Szucs, S. P. Dolan, M. R. Wormald, K. W. All en; Nucl. Phys. A197, i (1972)

72 An2 R. A. Anderl, J. E. Hall, R. C. Morrison, R. G. Struss, M. V. Yester, D. J. Zaffarano; Nucl. Instr. and Methods 102 , 101 ( 1972$)$

72 An $3 \mathrm{Yu}$. P. Antuf ev, V. L. Agranovich, V. B. Ganenko, V. S. Kuz menko, I. I . Miroshnichenko, P.V.Sorokin, $S_{\text {. }}$. Shalatskil; Yad. Fiz. 15, 643 (1972); Sov. J. Nuci. Phys. 15, 357 ( 1972 )

72 Ar1 B. Arad, G. Ben-David, Y. Schlesinger, M. Hass; Phys. Rev. C6, 670 (1972)

$72 \mathrm{Ba} 5 \mathrm{C}$. Bacci, R. Baldini-Celio, B. Esposito, C. Mencuccini, A. Reale, G. Sciacca, M. Spinetti, A. Zallo; Lettere al Nuovo Cimento 4, 5 ( 1972 )

72 Be 1 R. A. I. Bell, I. G. Graham, J.V. Thompson; Nucl. Phys. A $179,408(1972)$

72 Be2 B. L. Berman, F W. K. Firk, C. P. Wu; Nucl. Phys. Al79, 791 ( 1972 )

72 Be3 W. Bertozzi, T. Cooper, N. Ensslin, J.Heisenberg,. Kowalski, M. Mills, W. Turchinetz, C. Williamson, $S$. P. Fivozinsky, J. W. Lightbody, Jr., S. Penner; Phys. Rev. Letters 28, 1711 (1972)

72Be7 I. Bergquist, D. M. Drake, D. K. McDaniels; Nucl. Phys. A191, 641 ( 1972 )

72 Bl 6 S. L. Blatt, K. J. Moon, D. Kohler; Phys. Rev. C6, 1536 (1972)

$72 \operatorname{Br} 8$ R. L. Bramblett, T. Gozani, R. O. Ginaven, D. E. Rundquist; Nucl: Technology 13, 33 (1972)

72 Br4 C. Brassard, H. D. Shay, J. P. Coffin, W. scholz, D. A. Bromley; Phys. Rev. C6, 53 (1972) 
72 Chl A. Chevallier, E. Bozek, J, Chevallier, A. Pape, R. Armbruster; Nucl. Phys. A191, 201 ( 1972 )

72 Ch3 C. C. Chang, E. M. Diener, E. Ventura; Phys. Rev. Letters 29, 307 ( 1972 )

72 Co 2 W. F. Coetzee, M. A. Meyer, D. Reitmann; Nucl. Phys. A 185,644 ( 1972$)$

72 Da6 P. David, J Debrus, U. Kim, G. Kumbartzki, H. Mommsen, W. Soyez, K. H. Speidel, G. Stein; Nucl. Phys. A 197,163 ( 1972$)$

72 De 3 T. K. Deague, R. J. J. Stewart; Nucl. Phys. A191, 305 ( 1972$)$

72De4 V. P. Denisov, L. A. Kul chitskii, I. Ya. Chubukov; Yad. Fiz. 14, 889 ( 1971 ); Sov. J. Nucl? Phys. 14, 497:( 1972 )

72Do1 W. R. Dodge, J.J. Murphy,II; Phys. Rev. Letters 28, 839 ( 1972$)$

72 Dr2 F. Dreyer, H. Dahmen, J. Staude, H. H. Thies; Nucl. Phys. A 181,477 ( 1972 )

72 Dr5 F. Dreyer, H. Dahmen, J . Staude, H. H. Thies; Nucl. Phys. A 192, 433 ( 1972 )

72 Es 1 M. A. Eswaran, M. Ismail, N. L Ragoowansi; Phys. Rev. C5, $1270(1972)$

$72 \mathrm{Fa} 1 \mathrm{~L}$. W. Fagg, W. L. Bendel, E. C. Jones, Jr., L. Cohen, H. F. Kaiser: Phys. Rev. C5, 120 (1972) See Erratum - Phys. Rev. C5, 1143 ( 1972 )

72 Fa 3 F. L. Fabbri, P. Picozza, C. Schaerf; Lettere al Nuovo Cimento 3,63 ( 1972 )

72 Fr4 D. L. Friesel, T. J. Lewis, W. C. Miller; Phys. Rev. C6, $846(1972)$

72Fr5 J Friedrich; Nucl. Phys. A191, 118 ( 1972 )

$72 \mathrm{Fu} 2 \mathrm{~T}$. Fujii, S. Homma, K. Huke, S. Kato, H. Okuno, F. Takasaki, T. Kondo, S. Yamada, I. Endo, H. Fujii ; Phys. Rev. Letters 28, 1672 ( 1972 )

72 Ga2 D. Ganichot, B. Grossetete, D. B. Isabelle; Nucl. Phys. A178,545 (1972)

72 Ge3 R. W. Gellie, K. H. Lokan, N. K. Sherman, R. G. Johnson, J. I. Lodge; Can. J. Phys. 50, 1689 (1972)

72 Gil H. F. Glavish, S. S. Hanna, R. Avida, R. N, Boyd, C. C. Chang, E. Diener; Phys. Rev. Letters 28, 766 ( 1972 )

72 Ha 1 M. Hasinoff, D. Johnson, D. F. Measday; Phys. Letters $39 \mathrm{~B}, 506(1972)$

$72 \mathrm{Ha} 2 \mathrm{M}$. L. Halbert, E. K. Warburton; Proceedings of International Conference on Few Particle Problems in the Nuclear Interaction; UCLA (Aug. 28-Sept. 1, 1972)

$72 \mathrm{Ha} 3 \mathrm{M}$. Hasinof $\mathrm{f}, \mathrm{G}$. A. Fisher, P. Kurjan, S. S. Hanna; Nucl. Phys. A195, 78 ( 1972 )

72 Is 1 B. S. Ishkhanov, I M. Kapitonov, E. V. Lazutin, I. M. Piskarev, V. G. Shevchenko; Nucl? Phys. A186, 438 ( 1972 )

72Ja 1 H. E. Jackson, K. J. Wetzel; Phys. Rev. Letters 28,513 ( 1972$)$

72 Ka5 V. I. Kasilov, A. V. Mitrofanova, Yu. N. Ranyuk, P. V. Sorokin; Yad. Fiz. 15, 406 (1972); Sov. J. Nucl. Phys. 15, $228(1972)$

$72 \mathrm{Ke} 4$ N. A. Keller and D. B. McConnell; Can. J. Phys. 50, 1554 ( 1972$)$

72 Kh 1 A. M. Khan, J. H. Knowles; Nucl. Phys. A179, 333 ( 1972 ) 
$72 \mathrm{Ki} 2 \mathrm{H}, \mathrm{T}$. King, W. E. Meyerhof, R. G. Hirko; Nucl. Phys. $A 178,337$ ( 1972$)$

$72 \mathrm{Kr} 3$ I. Kroon, B. Forkman; Nucl. Phys. A179, 141 ( 1972 )

$72 \mathrm{Ku} 6$ G. Kuhl, U. Kneissl; Nucl. Phys. A195, 559 ( 1972)

$72 \mathrm{Li} 1 \mathrm{~J}$. W. Lightbody Jr.; Phys. Letters 38B, 475 ( 1972 )

72 Lo2 K. H. Lokan, N. K. Sherman, R. W. Gellie, J. W. Jury, J . I. Lodge, R. G. Johnson; Phys. Rev. Letters 28, 1526 ( 1972$)$

$72 \mathrm{Ma} 10 . \mathrm{Y}$. Mafra, $\mathrm{S}$. Kuniyoshi, J. Goldemberg; Nucl. Phys. A186, 110 ( 1972$)$

$72 \mathrm{Ma} 2 \mathrm{D} \cdot \mathrm{J}$. Martin, J. R. Leslie, W. McLatchie, C. F. Monahan, L. E. Carlson; Nuci. Phys. A187, 337 (1972)

$72 \mathrm{Me} 3$ F. R. Metzger; Nucl. Phys. A189,409 (1972)

72 Mi4 S. Mitsunobu and Y. Torizuka; Phys. Rev. Letters 28, 920 ( 1972 )

72Nal A. Nakada and Y. Torizuka; J. Phys. Soc. Japan 32, 1 ( 1972$)$

72Na2 R. Nath, F. W. K. Firk, H. L. Schultz; Nucl. Phys. A194, 49 ( 1972$)$

72 No3 V. I. Noga, Yu. N. Ranyuk, P. V. Sorokin, V. A. Tkachenko; Yad. Fiz. 14,904 (1971); Sov. J. Nucl. Phys. $14,506$ ( 1972$)$

72 edl 1 . Oda, M. Kondo, H. Hirabayashi; J. Phys. Soc. Japan $32,869(1972)$

$720 \mathrm{f} 2$ N. M. OFallon, L. J. Koester,Jr., J.H. Smith; Phys. Rev. C5, $1926(1972)$

72Phl G. W. Phillips, P. Richard, D. O. Elliott, F. F. Hopkins, A. C. Porter; Phys. Rev. C5, 297 ( 1972)

72 Ka2 J. J. Ramirez, R. A. Blue, H. R. Weller; Phys. Rev. C5 $17,(1972)$

72 Ro3 D. W. $\theta$. Rogers, R. P. Beukens, W. T. Di amond; Can. J. Phys. 50, 2428(1972)

72Rul J. P. Russell, W. E. Taylor, F. E. Dunnam, H. A. Van Rinsvelt; Nucl. Phys. A187, 449 (1972)

72 Sc5 B. Schroder, B. Nordgren, A. A lm; Nucl. Phys. A193, 555 ( 1972$)$

72 sh2 N. Shikazono and Y. Kawarasaki; Nucl. Phys. A188, 461 (1972)

72Sk 1 D. M. Skopik, Y. M. Shin; Can. J. Phys. 50, 392 ( 1972 )

72 Sk3 D. M. Skopik and W. R. Dodge; Phys. Rev. C6, 43 ( 1972 )

72 sk6 N. A. Skakun, N. P. Dikii; Yad. Fiz. 15, 615 ( 1972); Sov. J. Nucl. Phys. 15, 341 ( 1972 )

$725 k 7$ D. M. Skopik, Y. M, Shin, J, W. Horniachek, S. K. Kundu; Nucl. Phys. A197, 185 ( 1972)

72 Su4 M. Sugawara, K. Shoda, T. Saito, H. Miyase, A. Suzuki, S. Eikawa, R. Bergere; Phys. Rev. C5, $1705^{\circ}$ ( 1972 )

72 Th2 B. W. Thomas, D. M. Crawford, H. H. Thies; Nucl. Phys. A196, 89 (1972)

72114 Yu. I. Iitov, E. V. Stepula; Yad. Fiz. 15, 649 (1972); Sov. J. Nucl. Phys. is, 361 (1972)

72 Tol R. E. Toohey, H. E. Jackson; Phys. Rev, C6, 1440 ( 1972)

72 To3 Z. B. du Toit, P. R. de Kock, J. H. Hough, W. L. Mouton; Z. Physik 255, 97 ( 1972 ) 
$72 \mathrm{Va} 2 \mathrm{R}$. E. Van de Vyver, H. Ferdinande, G. Knuyt, R.

Carchon, J. Devos; Nucl. Phys. A198, 144 ( 1972 )

$72 V_{i} 1$ M. Viitasalo, M. Pilparinen, A. Anttila; Z. Physik 250,387 (1972)

72 tel 1 H. R. Weller, R. A. Blue, S. L. Blatt; Phys. Rev. C5, 648 (1972)

72 the 2 H. R. Weller, R. A. Blue, E. M. Bernstein, J. J. Ramirez; Nucl. Phys. A 185,284 ( 1972 ) 
6. Definitions of Abbreviations and Symbols

6.1. Symbols Used to Define Incoming Beam and Reaction Products

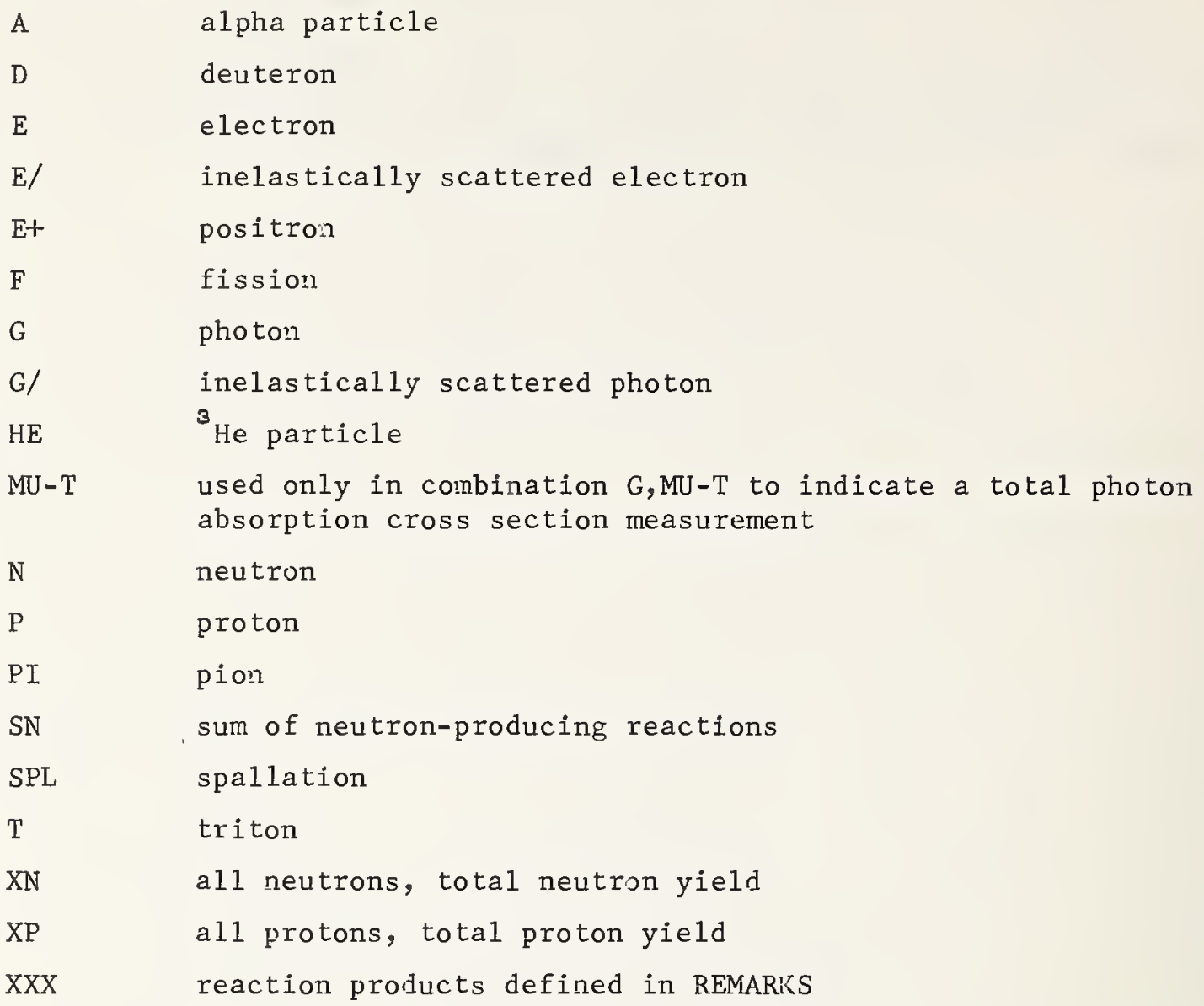

6.2. Symbols and Abbreviations (Table 1 and Data Index - Columns 4-7 and REMARKS

A

$\mathrm{ABI}$

$\mathrm{ABX}$

$\mathrm{ABY}$

$\mathrm{ACT}$

ANG

BBL

$B(E L)$

BF 3

BREAKS

C

$\mathrm{CCH}$

CF

COINC

$\mathrm{D}$ nuclear mass number

absolute integrated cross-section data $\int \sigma \mathrm{dE} \gamma$

absolute cross-section data

absolute yield data

measurement of radioactivity of the target

angle. Symbols listed under ANG indicate type of angular distribution data available for a reaction.

bubble chamber

reduced radiative transition probability

$\mathrm{BF}_{3}$ neutron counter with moderator, e.g., Ha1pern detector, long counter

levels located by "breaks" in the yield curve

continuous. Used to describe a photon source or a detector response function. Contrast with $\mathrm{D}=$ discrete.

cloud chamber

compared with

coincidence

deuteron 
6.2. Symbols and Abbreviations (Continued)

D

DEL

DST

DT BAL

E

EMU

EXCIT

F

FMF

G-WIDTH

INT

ION

J-PI

LFT

MAG

MGC

MGP

MOD

MSP

N

$\mathrm{N}+\mathrm{P}$

NAI

NOX

NUM

P

PI

POL

Q-SQUARE

REF

REL

RES

RLI

RLX discrete. Used to describe a photon source or a detector response function. Contrast with $\mathrm{C}=$ continuous.

delta, full width of cross-section curve at half maximum. May also be width of Lorentz line fit to cross-section curve.

an angular distribution was ineasure:

detail balance

energy. Usually energy at which cross-section curve peaks or resonance energy of Lorentz line fit.

emulsions (photographic plates)

excitation. Usually the excitation energy of the nucleus in which a gamma-ray transition is made.

fission

form factor

$\Gamma_{\gamma}$, gamma-ray transition width

interaction or type of reaction

ionization chamber

spin and parity assignments of levels are inate

excited state lifetime

magnetic spectrometer

magnetic Compton spectrometer

magnetic pair spectrometer

moderated neutron detector not employing a $\mathrm{BF}_{3}$ çounter; e.g., rhodiun foil, Szilard-Chalmers reaction, ${ }_{\mathrm{He}}{ }^{6} \mathrm{Li}$ reactions, Gd loaded liquid scintillator, ets.

mass spectrometer

neutron

neutron plus proton. Usually means the sum of the $(\gamma, n)$ and $(\gamma, p)$ reactions.

$\mathrm{NaI}(\mathrm{T} 1)$ spectrometer

no cross-section data

number. The acquisition number for data available in the digitized cross-section library of the Photonuclear Data Center.

proton

pionl

polarization

momentum transfer squared $\left(q^{2}\right)$

reference. The bibliographic reference number assigned to a paper by the Photonuclear Data Center.

relative

result. Symbols listed under RES indicate type of measurement made for a given reaction.

relative integrated cross-section data

relative cross-section data 
6.2. Symbols and Abbreviations (Continued)

\begin{tabular}{|c|c|}
\hline RLY & relative yield data \\
\hline$S C D$ & semiconductor (solid state) detector \\
\hline $\mathrm{SCI}$ & $\begin{array}{l}\text { scintillator detector other than } \mathrm{NaI}, \mathrm{e} \cdot \mathrm{g} \cdot, \mathrm{CsI}, \mathrm{KI} \text {, } \\
\text { organic (liquid or solid), stilbene, } \mathrm{He}\end{array}$ \\
\hline SEP ISOTP & separated isotope used \\
\hline SIG & $\begin{array}{l}\text { cross section. The maximum value of the cross section as } \\
\text { a function of photon energy. }\end{array}$ \\
\hline SIG-0 & $\sigma_{0}$ \\
\hline SIG-1 & $\sigma_{-1}$ \\
\hline SN & $\begin{array}{l}\text { used alone or as } \sigma(S N) / \sigma(\gamma, S N) \\
\sigma(S N)=\sigma(\gamma, n)+\sigma(\gamma, n p)+\sigma(\gamma, 2 n)+\sigma(\gamma, 3 n)+\ldots\end{array}$ \\
\hline SNF & $\begin{array}{l}\text { used alone or as } \sigma(S N F) / \sigma(\gamma, S N F) \\
\sigma(S N F)=\sigma(S N)+\sigma(\gamma, f)\end{array}$ \\
\hline SPC & phoion or particle energy spectrum \\
\hline SPK & sparik chamber \\
\hline $\mathrm{T}$ & triton \\
\hline TEL & counter telescope \\
\hline THR & threshold detector, e.g., ${ }^{29} \mathrm{Si}(\mathrm{n}, \mathrm{p})^{29} \mathrm{Al}$ \\
\hline TOF & time-of-flight detector \\
\hline TOT & total nuclear absorption cross section for photons \\
\hline TRK & $\begin{array}{l}\text { tracks of particles or fragments observed in solid } \\
\text { materials (glass, mylar, etc.) }\end{array}$ \\
\hline $\mathrm{XN}$ & $\begin{array}{l}\text { all neutrons. Used alone or as } \sigma(\mathrm{XN}) / \sigma(\gamma, \mathrm{XN}) \\
\sigma(\mathrm{XN})=\sigma(\gamma, \mathrm{n})+\sigma(\gamma, \mathrm{np})+2 \sigma(\gamma, \mathrm{n}) \cdots+{ }^{\prime} \ldots(\gamma, \mathrm{f})+\ldots .\end{array}$ \\
\hline $\mathrm{XP}$ & $\begin{array}{l}\text { all photons. Used alone or as } \sigma(\mathrm{XP}) / \sigma(\gamma, \mathrm{XP}) \\
\sigma(\mathrm{XP})=\sigma(\gamma, \mathrm{p})+\sigma(\gamma, \mathrm{np})+2 \sigma(\gamma, \mathrm{p})+\cdots\end{array}$ \\
\hline Z & atomic number (number of protons) \\
\hline $4 \mathrm{PI}$ & $\begin{array}{l}\text { a } 4 \pi \text { geometry was used or a method like radioactivity } \\
\text { or a total absorption measurement }\end{array}$ \\
\hline 999 & energy defined in REMARKS \\
\hline$\$$ & $\begin{array}{l}\text { indicates the measurement involved either beams or targets that } \\
\text { were polarized/aligned, or that the polarization of the reaction } \\
\text { products was determined }\end{array}$ \\
\hline
\end{tabular}




\section{References}

[1] J. Chadwick and M. Goldhaber, Nature 134, 237 (1934).

[2] D. W. Kerst, Phys. Rev. 58, 841 (1940).

[3] G. C. Baldwin and G. S. Klaiber, Phys. Rev. 71, 3 (1947); Phys. Rev. 73 , 1136 (1948).

[4] C. Schuhl and C. Tzara, Nucl. Instr.\& Methods 10, 217 (19ól); C. P. Jupiter, N. E. Hansoin, R. E. Shafer, S. C. Fultz, Phys. $\overline{\operatorname{Rev}} \cdot 121,866$ (1951)。

[5] W. C. Barber, Ann. Rev. Mod. Sci. 12, 1 (1952).

[6] J. S. Levinger and H. A. Bethe, Phys. Rev. 78, 115 (1950).

[7] B. M. Spicer, The Giant Dipole Resonance, in Advances in Nuclear Physics, Vol. 2 (Plenum Press, 1969); Evans Hayward, Photonuclear Reactions, NBS Monograp' 118, August 1970; F. W. K. Firk, Low-Energy Photonuclear Reactions, in Ann. Rev. Nucl. Sci., Vol. 20, 1970 .

[8] J. S. Levinger, Nuclear Photodisintegration, Oxford University Press (1960).

[9] N. V. Baggett, T. M. Collins, E. G. Fuller, J。C. Holland, J. H. Hubbell, and J. S. O'Connell, Photonuclear Data Index, (1955-1965), NBS Misc. Publ. 277, April 1966; T. M. Collins, E。G.Fuller, J. D. Murphy, and J. S. O'Connell, Photonuclear Data Index (January 1965 through April 1967), NBS Misc. Publ. 277Supplement 1, October 1967; E. G. Fuller, H. M. Gerstenberg, and T. M. Collins, Photonuclear Data Index (1965-1970), NBS Spec. Publ. 322, May 1970.

[10] Gladys Fuller, 1959 Nuclear Data Tables (NUCLEAR DATA PROJECT) - National Academy of Sciences, National Research Council, Washington, D. C.

[11] A. H. Wapstra and N. B. Gove, Nuclear Data Tables, 9, 303 (1971). 

FORM NBS-114A (1-71)

\begin{tabular}{|c|c|c|}
\hline $\begin{array}{l}\text { U.S. DEPT. OF COMM. } \\
\text { BIBLIOGRAPHIC DATA } \\
\text { SHEET }\end{array}$ & $\begin{array}{l}\text { 1. PUBLICATION OR REPORT NO. } \\
\text { NBS SP }-380\end{array}$ & 3. Recipient's Accession No. \\
\hline 4. TITLE AND SUBTITLE & 4. TITLE AND SUBTITLE & $\begin{array}{l}\text { 5. Publication Date } \\
\text { March } 1973\end{array}$ \\
\hline \multicolumn{2}{|c|}{ Photonuclear Reaction Data, 1973.} & 6. Performing Organization Code \\
\hline $\begin{array}{llll}\text { 7. AUTHOR(S) } & \text { E. G. } & \text { F } \\
& \text { T. } & \text { C. } & \text { D }\end{array}$ & ler, H. M. Gerstenberg, H. Vander Molen, & 8. Performing Organization \\
\hline \multicolumn{2}{|c|}{ 9. PERF ORMING ORGANIZATION NAME AND ADDRESS } & $\begin{array}{l}\text { 10. Project/Task/Work Unit No. } \\
2410232\end{array}$ \\
\hline \multicolumn{2}{|c|}{$\begin{array}{l}\text { NATIONAL BUREAU OF STANDARDS } \\
\text { DEPARTMENT OF COMMERCE } \\
\text { WASHINGTON, D.C. } 20234\end{array}$} & 11. Contract/Grant No. \\
\hline \multirow{2}{*}{\multicolumn{2}{|c|}{ 12. Sponsoring Organization Name and Address }} & $\begin{array}{l}\text { 13. Type of Report \& Period } \\
\text { Covered } \\
\text { Final }\end{array}$ \\
\hline & & 14. Sponsoring Agency Code \\
\hline
\end{tabular}

15. SUP PLEMENTARY NOTES

This publication supersedes NBS Miscellaneous Publication 277 and NBS Special Publication 322 which also embraced Supplement 1 to Misc. Publ. 277.

16. ABSTRACT (A 200-word or less factual summaty of most significant information. If document includes a significant bibliography or literature survey, mention it here.)

A brief summary is given of the available data on the gross features of the photonuclear giant resonance. Data are presented in tabular form for all nuclei where measurements have been made. In addition, a comprehensive, annotated data index and bibliography are given which cover experimental data for the field of photonuclear reactions published in scientific and technical journals in the period from 1955 through 1972. Organized by element and isotope, each entry in the index is for a specific reaction reported in a given reference. Information is given on the type of measurement, excitation energies studied, source type and energies, detector type and angular ranges covered for each reaction entry.

17. KEY WORDS (Alphabetical order, separated by semicolons) Bibliography; data index; data summary; elements; isotopes; nuclear physics; photonuclear giant resonance; photonuclear reaction.

18. AVAILABILITY STATEMENT

X] UNL IMITED.

FOR OFFICI AL DISTRIBUTION. DO NOT RELEASE TONTIS.

\begin{tabular}{|l|c|}
$\begin{array}{l}\text { 19. SECURITY CLASS } \\
\text { (THIS REPORT) }\end{array}$ & 21. NO. OF PAGES \\
UNCL ASSIFIED & 131 \\
\hline $\begin{array}{l}\text { 20. SECUR ITY CLASS } \\
\text { (THIS PAGE) }\end{array}$ & $\begin{array}{l}\text { 22. Price } \\
\$ 2.10 \text { Domestic Postpaid } \\
\text { UNCL ASSIFIED }\end{array}$ \\
\hline
\end{tabular}



PERIODICALS

JOURNAL OF RESEARCH reports National Burcau of Standards research and development in physics, mathematics, and chemistry. Comprehensivc scientific papers give complete details of the work, including laboratory data, experimental procedures, and theoretical and mathematical analyses. Illustrated with photographs, drawings, and charts. Includes listings of other NBS papers as issued.

Published in two sections, available separately:

\section{- Physics and Chemistry (Section A)}

Papers of interest primarily to scientists working in these fields. This section covers a broad range of physical and chemical research, with major emphasis on standards of physical measurement, fundamental constants, and properties of matter. Issued six times a year. Annual subscription: Domestic, $\$ 17.00$; Foreign, \$21.25.

\section{- Mathematical Sciences (Section B)}

Studies and compilations designed mainly for the mathematician and theoretical physicist. Topics in mathematical statistics, theory of experiment design, numerical analysis, theoretical physics and chemistry, logical design and programming of computers and computer systems. Short numerical tables. Issued quarterly. Annual subscription: Domestic, $\$ 9.00$; Foreign, $\$ 11.25$.

\section{TECHNICAL NEWS BULLETIN}

The best single source of information concerning the Bureau's measurement, research, developmental, cooperative, and publication activities, this monthly publication is designed for the industry-oriented individual whose daily work involves intimate contact with science and technology-for engineers, chemists, physicists, research managers, product-development managers, and company executives. Includes listing of all NBS papers as issued. Annual subscription: Domestic, $\$ 6.50$; Foreign, $\$ 8.25$.

\section{NONPERIODICALS}

Applied Mathematics Series. Mathematical tables, manuals, and studies.

Building Science Series. Research results, test methods, and performance criteria of building materials, components, systems, and structures.

Handbooks. Recommended codes of engineering and industrial practice (including safety codes) developed in cooperation with interested industries, professional organizations, and regulatory bodies.

Special Publications. Proceedings of NBS conferences, bibliographies, annual reports, wall charts, pamphlets, etc.

Monographs. Major contributions to the technical literature on various subjects related to the Bureau's scientific and technical activities.

National Standard Reference Data Series. NSRDS provides quantitative data on the physical and chemical properties of materials, compiled from the world's literature and critically evaluated.

Product Standards. Provide requirements for sizes, types, quality, and methods for testing various industrial products. These standards are developed cooperatively with interested Government and industry groups and provide the basis for common understanding of product characteristics for both buyers and sellers. Their use is voluntary.

Technical Notes. This series consists of communications and reports (covering both other-agency and NBS-sponsored work) of limited or transitory interest.

Federal Information Processing Standards Publications. This series is the official publication within the Federal Government for information on standards adopted and promulgated under the Public Law 89-306, and Bureau of the Budget Circular A-86 entitled, Standardization of Data Elements and Codes in Data Systems.

Consumer Information Series. Practical information, based on NBS research and experience, covering areas of interest to the consumer. Easily understandable language and illustrations provide useful background knowledge for shopping in today's technological marketplace.

\section{BIBLIOGRAPHIC SUBBSCRIPTION SERVICES}

The following current-awareness and literature-survey bibliographies are issued periodically by the Bureau:

Cryogenic Data Center Current Awareness Service (Publications and Reports of Interest in Cryogenics). A literature survey issued weekly. Annual subscription: Domestic, $\$ 20.00$; foreign, $\$ 25.00$.

Liquefied Natural Gas. A literature survey issued quarterly. Annual subscription: $\$ 20.00$.

Smperconducting Devices and Materials. A literature survey issued quarterly. Annual subscription: $\$ 20.00$. Send subscription orders and remittances for the preceding bibliographic services to the U.S. Department of Commerce, National Technical Information Service, Springfield, Va. 22151.

Electromagnetic Metrology Current Awareness Service (Abstracts of Selected Articles on Measurement Techniques and Standards of Electromagnetic Quantities from D-C to Millimeter-Wave Frequencies). Issued monthly. Annual subscription: $\$ 100.00$ (Special rates for multi-subscriptions). Send subscription order and remittance to the Electromagnetic Metrology Information Center, Electromagnetics Division, National Bureau of Standards, Boulder, Colo. 80302.

Order NBS publications (except Bibliographic Subscription Services) from: Superintendent of Documents, Government Printing Office, Washington, D.C. 20402. 
U.S. DEPARTMENT OF COMMERCE

National Bureau of Standards

Washington, D.C. 20234

OFFICIAL BUSINESS

Penalty for Private Use, $\$ 300$
PDSTAGE AND FEES PAID U.S. DEPARTMENT DF COMMERCE 215

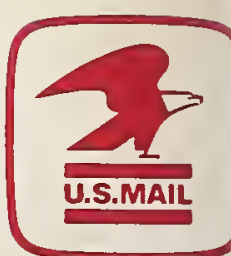

